\title{
Quarterly Report of RCRA Groundwater Monitoring Data for Period July 1 through September 30, 1995
}

\author{
Earth and Environmental \\ Technical Services \\ Westinghouge Haniord Company
}

\author{
Dipto Publinghed \\ January 1996
}

\section{DISCLAIMER}

This repart rast prepured as an accosul of work spomeared by an ageacy af the Unitod Statcs Grovetiment. Neither the United Slates Government oor any agency thaseof, hor acy of their

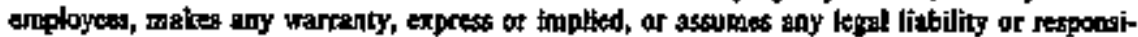
billyy for the aceuracy, compltteptess, or usefulnoss of ary information, apparalus, product, of process diselosed, or tepresents that it bye would not infrings privotely owned fiphts. Refor-

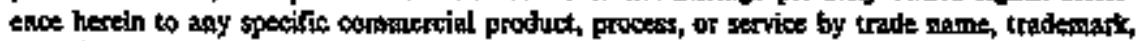

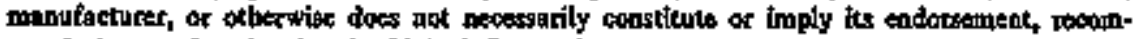
mendation, of faworing by the United Siates Gowemmenl or any agency thereof. The witws and opinions of aullors expressed herein to not accessarily stale of rellect thos of the Uaited Stak Gowernment or any agency thereof.

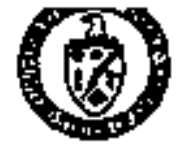

\section{United otates}

Department of Energy

P.O. Box 550

fichiand, Wastingtan 99352 



\section{LIST OF TERNS}

\begin{tabular}{|c|c|}
\hline $\begin{array}{l}\text { A-10 Crib } \\
\text { A-29 Ditch } \\
\text { A-36B Crib } \\
\text { APT } \\
\text { Basins } \\
\text { CBNL } \\
\text { CFR } \\
\text { CIP } \\
\text { CRQL } \\
\text { DOE } \\
\text { DWS } \\
\text { ECO]ogy } \\
\text { EPA } \\
\text { ICP } \\
\text { IHC } \\
\text { ITAS } \\
\text { LERF } \\
\text { LLBG } \\
\text { LLWHA } \\
\text { LWDF } \\
\text { NOC } \\
\text { HOL } \\
\text { HCR } \\
\text { NRDHL } \\
\text { NTU } \\
\text { PHHL } \\
\text { PUREX } \\
\text { QA } \\
\text { QC } \\
\text { RADE } \\
\text { RCRA } \\
\text { RCW } \\
\text { REDOX } \\
\text { RL } \\
\text { RPD } \\
\text { S-10 Facility } \\
\text { SST } \\
\text { TOC } \\
\text { TOX } \\
\text { Tri-Party } \\
\text { Agreement } \\
\text { U-12 Crtb } \\
\text { WAC } \\
\text { WHC } \\
\text { WHA } \\
\text { WP } \\
\text { WS }\end{array}$ & 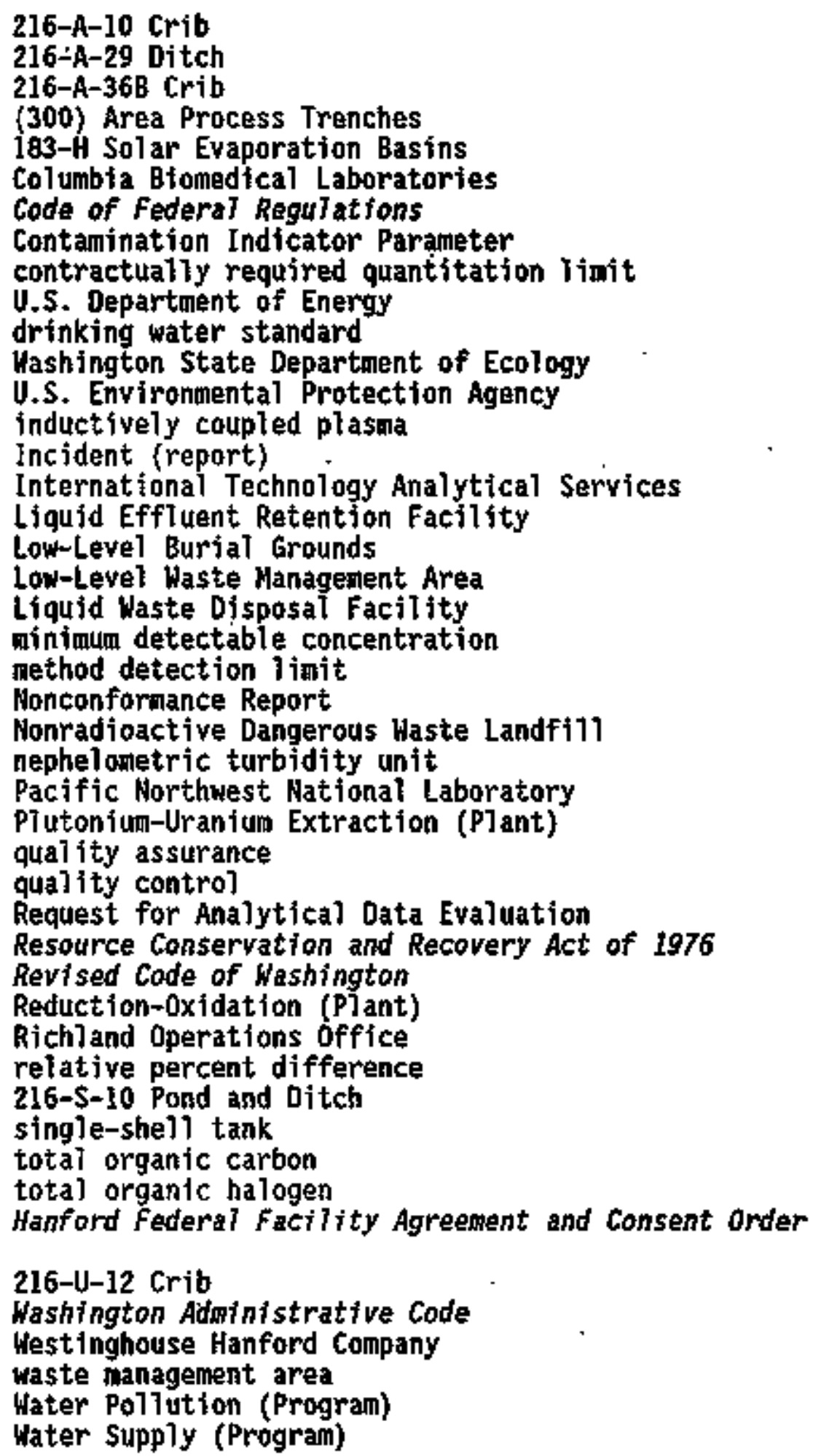 \\
\hline
\end{tabular}


DOE/RL-95-69-3

This page intentionally left biank. 
DOE/RL-95-69-3

\section{CONTENTS}

1.0 InTRDDUCTION ...................... 1-1

$2.0100-0$ PONDS . . . . . . . . . . . . . . . 2-1

$3.0183-H$ SOLAR EVAPORATION BASINS $\ldots \ldots \ldots \ldots$ 3-1 . . . . .

$4.0100-N$ RCRA SITES . . . . . . . . . . . . . . . 4-1

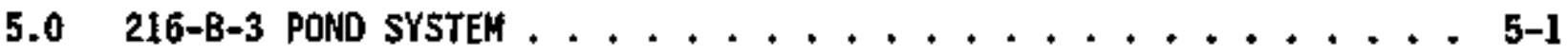

$6.0216-A-29$ DITCH $\ldots \ldots \ldots \ldots$ 6-1

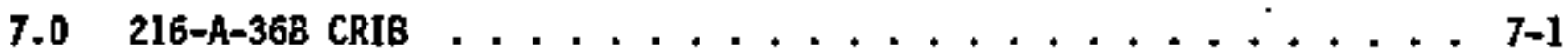

8.0 216-A-10 CRIB . . . . . . . . . . . . . . . 8-1

9.0 216-B-63 TRENCH ...................... . . . . .

$10.0216-5-10$ POND AND DITCH . . . . . . . . ..... 10-1

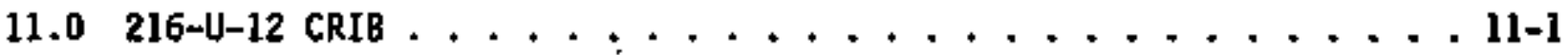

12.0 LIQUID EFFLUENT RETENTION FACILITY . . . . . . . . . . 12-1

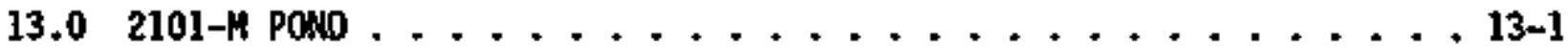

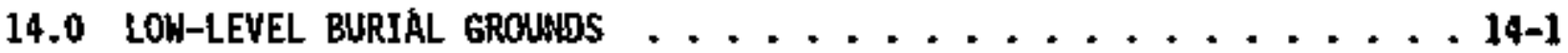

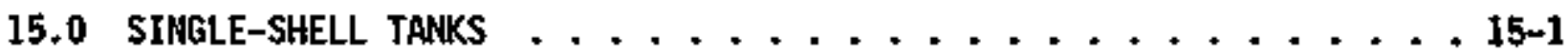

16.0300 AREA PROCESS TRENCHES . . . . . . . . . . . 16-1

17.0 NONRADIOACTIVE DANGERONS WASTE LANDFILL .......... 17-1

18.0 REFERENCES $\ldots \ldots \ldots \ldots \ldots \ldots \ldots \ldots \ldots$ 
COE/RL-95-69-3

This page intentionally left blank.

vi 
DOE/RL-95-69-3

\section{COKTERTS}

1.0 INTRODUCTION . . . . . . . . . . . . . 1-1

1.1 LABORATORY AND SAMPLIMG STATUS ............. 1-1

1.2 QUALITY CONTROL PROGRAM ................. 1-3

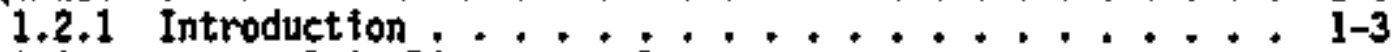

1.2.2 Externa1 Quality Control Program .......... 1-5

1.2 .3 Internal Quality control Program . . . . . . . 1-8

1.3 QUALITY CONTROL DEFINITIONS .................

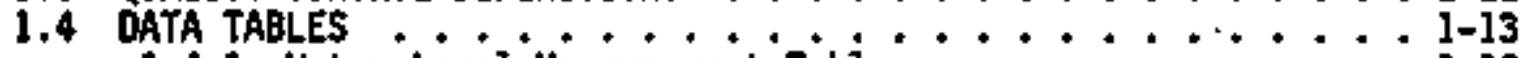

1.4.1 Water Level Measurement Table ......... I-13

1.4.2 Constituent List and Summary of Results Tabie : : . 1-13

1.4.3 Constituents with at Least One Detected Value Table . 1-14

1.4.4 Contamination Indicator Parameters Table . . . . . 1-15

1.4.5 Codes and Abbreviations ............ 1-15

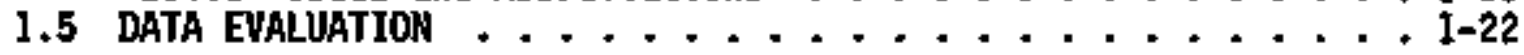


DOE/RL-95-69-3

\section{LIST OF FIEURES}

1-1 Locations of the RCRA Groundwater Honitoring

Projects and Landmarks on the Hanford Site . . . . . . . . . . 1-2

\section{LIST OF TABLES}

1-1 Completeness . . . . . . . . . . . . . . . . . . . . .

1-2 Field Duplicates and Field Blanks Evaluation Results, Third Quarter 1995 ................... 1-6

1-3 Breakdown of Fjeld Dupljcates . . . . . . . . . . . . . . . 1-7

1-4 Breakdown of Fjeld Btanks . . . . . . . . . . . . . . . 1-8

1-5 Radiochemistry Quarterly Report Sumary . . . . . . . . . . . . 1-9

1-6 Analysis Hethod Code Definitions................. . . . . . . . . . .

1-7 Drinking Water Standards............................... 
DOE/RL-95-69-3

QUARTERLY REPORT OF RCRA GROUMDLATER MONTTORING DATA FOR PERIOD JULY 1, 1995 THROLAh SEPTEMBER 30, 1995

\subsection{IHTREDUCTIOH}

Nineteen Resource Conservation and Recovery Act of 1976 (RCRA) groundwater monitoring projects are conducted at the Hanford Site. These projects fnclude treatment, storage, and disposal facilities for both solid and liguid waste. The location of each facility is shown in Figure 1-1. The groundwater monitoring programs described in this report comply with the interim- and final-status federal (Title 40 Code of Federal Regulation [CFR] Parts 264 and 265) and state (Washington Administrative Code [WAC] 173-303-400 and 173-303-645) regulations. The RCRA projects are monitored under one of the following prograns: background monitoring, indicator parameter eva]uation, or groundwater quality assessment or detection.

Westinghouse Hanford Company (Hic) manages the RCRA groundwater monitoring projects on the Hanford site. Perforwing project management, preparing groundwater monitoring plans, well network design and instal fation, specifying groundwater data needs, perforsing quality control (QC) oversight, data management, and preparing project sappling schedules are all parts of this responsibility. Pacific Northwest National Laboratory (PWNL) administers the contract for analytical services to WHC for the RCRA groundwater monitoring program.

This quarterly report contains data recejved between Juty 1 and September 30, 1995, which are the cutoff dates for this reporting period. This report may contain not only data from the July through September quarter, but also data from earlier sampling events that were not prevfously reported.

\subsection{LABORATORY AD SANPLING STATUS}

DataChem Laboratories of Salt Lake Cjty, Utah, performs most of the hazardous chemicals analyses for the Hanford Site. Quanterra, St. Louls, Lockheed Environmental, and Quanterra, Richiand also perform hazardous chemical analyses for the Hanford Site. Analyses for coliform bacteria are performed by Columbia Btomedical Laboratory (CBHL) and Quanterra, Richland. Analyses for dloxtn are performed by TWS Analytical Services, Inc. Quanterra, Richland performs the radiochemical analyses. 


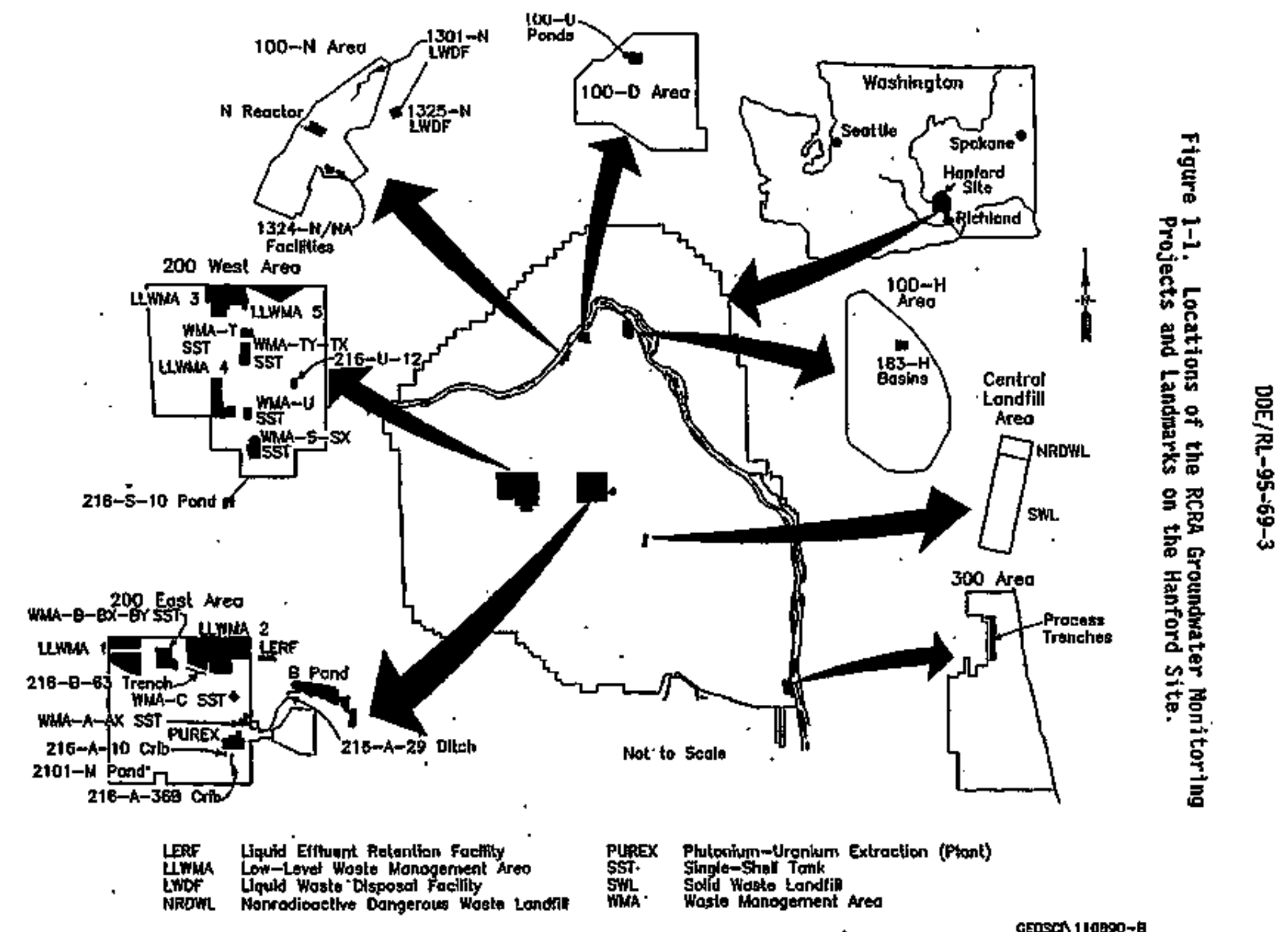




\section{2 qUALITY CONTROL PROGRAY}

H. Heapt

Westinghouse Hanford Company

\subsubsection{Introduction}

The QC Program is based on guidance from the U.S. Environienta] Protection Agency (EPA), the RCRA Groundwater Nonftoring Technical Enforcement Guidance Docuant (EPA 1986a), and Chapter 1.0, "Qual ity Control, " from 7est Nethods for Evaluating Solid Waste (EPA 1986b).

1.2.1.1 Date Quality leasures. The QC Prograin uses these five measures of data quality: precision, accuracy, representativeness, completeness, and comparability, along with applicable program-specific quality parameters to evaluate the quality of the data and the analytical laboratories analyzing the sawples. Target values for precision and accuracy are specified in the Qualty Assurance Project Plan for RCRA Groundwater Monitoring Activities (WHC 1992a).

1. Precision is evaluated using data results from laboratory duplicates, matrix spike duplicates (see Section 1.2.3), field duplicates, and blind samples.

2. Accuracy is evaluated using data results from laboratory matrix spikes; laboratory control samples; EPA Water Pollution (WP), Nater Supply (WS), and Interlaboratory Performance Evaluation Programs (see Section 1.2.3); and bilind samples.

3. Representativeness expresses the degree to which RCRA facility groundwater monitoring data represent the real composition of the groundwater in the aquifer. Goals for data representativeness for groundwater monitoring programs are addressed qualitatively by the specification of wl construction, sampling locations, sampling intervals, and sampling and analysis techniques in the groundwater monitoring plan for each RCRA facility.

4. Completeness is defined as the percentage of measurements that are fudged to be valid. Completeness is deternined by the number of data unflagged during validation, divided by the total number of data evaluated, and multiplied by 100 . The calculated percentages used in reporting completeness are conservative figures and are based on the data flags ' $P$,' ' $F$,' 'Y,' 'R,' ' $Q$,' and 'H.' Table 1-1 illustrates project completeness for the data presented in this report.

5. Comparability is the confidence with which one data set can be compared to another. It is evaluated using repifcates to ensure that samples analyzed by different laboratories or by the same laboratory over different time periods are comparable. For this reporting perjod, samples were analyzed in accordance with Test Hethods for Evaluating Solid Waste (EPA 1986b) and other applicable approved methods. To attain maximum comparab11ity among fleld measurements, samplers follow approved sampling 
procedures. Rigorous adherence to these procedures can ensure the desired consistency among sampling events.

Table 1-1. Completeness.

\begin{tabular}{|c|c|c|c|}
\hline Project & $\begin{array}{l}\text { Total nuwber } \\
\text { of evaluated } \\
\text { data }\end{array}$ & $\begin{array}{l}\text { Number of } \\
\text { unf Tagged } \\
\text { results }\end{array}$ & $\begin{array}{c}\text { Percent } \\
\text { complete } \\
\text { (unflagged) }\end{array}$ \\
\hline $100-\mathrm{D}$ & 200 & 195 & 98 \\
\hline $100-N$ & 950 & 932 & 98 \\
\hline $183-4$ & 243 & 222 & 91 \\
\hline $2101-H$ & - & $\cdots$ & $\mathrm{MA}$ \\
\hline $216-A-10$ & $=$ & $=$ & M \\
\hline $216-A-29$ & 1,596 & 1,443 & 90 \\
\hline $216-A-36 B$ & - & - & $\mathrm{MA}$ \\
\hline 216-B-3 & 987 & 944 & 96 \\
\hline $216-8-63$ & - & $=$ & NA \\
\hline $216-\$-10$ & $\cdots$ & - & Na \\
\hline $216-\mathrm{U}-12$ & 299 & 284 & 95 \\
\hline 300 APT & - & - & $N / A$ \\
\hline LERF & 172 & 172 & 100 \\
\hline LEBG WAA-1 & 22 & 19 & 86 \\
\hline LLBGG MWA-2 & 209 & 209 & 100 \\
\hline LLEG LMA-3 & 1,396 & 1,350 & 86 \\
\hline LLEG WH-4 & 95 & 95 & 100 \\
\hline LLEBG WHA-5 & 929 & 876 & 94 \\
\hline NRONL & 663 & 577 & 87 \\
\hline SST A/AX & 120 & 119 & 99 \\
\hline SST $\mathrm{B} / \mathrm{BX} / \mathrm{BY}$ & 168 & 164 & 98 \\
\hline \$ST C & 120 & 119 & 99 \\
\hline SST S/SX & 161 & 160 & 99 \\
\hline SST T & 201 & 185 & 92 \\
\hline SST TX/TY & 185 & 179 & 97 \\
\hline SST U & 157 & 157 & 100 \\
\hline
\end{tabular}

APT * Area Process Trench.

LERF = Liquid Effluent Retention Facility.

LLBG = Low-Level Burial Grounds.

NROWL = Nonradioactive Dangerous Waste Landfill.

SST = single-shell tank.

WHA = waste management area. 
1.2.1.2 Progra-specific Validation Flags. Program-specific validation flags are assigned during the process of evaluating RCRA groundwater data. These flags include:

1. H--Data are flagged with an ' $\mathrm{H}$ ' flag wen method-required hold times have been exceeded. Hold times stated in the jaboratory contract are those hold times required by the method in use by the laboratory. The H-flagged data may be used qualjtatively, but no regulatory decisions should be made based on a single-flagged data point.

2. Q-Data are flagged with a ' $Q$ ' flag when results of field blanks indicate contanination and/or resuits of field duplicate samples have greater than $20 \%$ relative percent difference (RPD). This flag means that the field QC data associated with the sample data were outside the 1 imfts estab] ished in the Environmental Engineering and Geotechnology function Procedures (WHC-CM-7-8).. The Q-flagged data can be used qualitatively, but no regulatory decisions shoutd be made based on a single-fiagged data point.

3. F--Data are flagged with an ' $F$ ' when the data point is subritted for review through the RADE system. The RADE system is a process used to track and evaluate data that appear to be inconsistent with the current pattern or with historical trends.

4. $Y$ or $R$-Data are flagged with a ' $Y$ ' or ' $R$ ' when Nonconfornance Reports (NCR) and Incident Reports (INC) are received from the laboratory and/or RADEs have been resolved. NCRs and INCs document occurrences that may affect data quality.

5. 6-Data points subwitted to the RADE system that have been deterwined to be acceptable are assigned a ' 6 ' flag.

6. P--Data points related to isolated occurrences determined by the specific project scientist are flagged with a ' $p$, ' indicating a potential problem with the data. Explanations for use of the flag wit] be discussed in the specific project chapter.

\subsubsection{Externa1 Quality Control Program}

The external QC progran uses three kinds of QC samples to evaluate the quality of data generated in the field and in the laboratory. These are field dupl icates, field blanks, and b7ind samples. (See Section 1.3 for definitions of the QC samples.)

The analytical results of QC samples are judged to be acceptable if the following evaluation criteria are met.

- Fieid duplicates-Results of field duplicate pairs must have precision as measured by RPD within $\pm 20 \%$. Hazardous chenical results below the Practical Quantitation Limit (PQL) and radiological results below the Liwit of quantitation (LOQ) are not evaluated. 
- Blanks--Two kinds of blanks are used to check for contamination resulting from field activities and/or bottle preparation. These are full trip blanks and field trip blanks.

Except for comson laboratory contaminants, results above the threshold of two $t$ lmes the method detection 1 imit (MDL) are identified as suspected contamination. For common laboratory contaninants, such as acetone, methylene chloride, 2-butanone, toluene, and phthalate esters, sample results less than five times the MDL are qualiffed as nondetects.

Table 1-2 summarizes the analytical results of QC samples avafiable for the July through September 1995 quarter.

Samples associated with duplicate or biank anaiytes that exceeded the QC 1 inits are discussed in this section and are flagged with $a$ ' $Q$ ' in the data tables.

Table 1-2. Field Duplicates and Field Blanks Evaluation Results, Third Quarter 1995.

\begin{tabular}{|l|c|c|c|}
\hline $\begin{array}{c}\text { Qual ity control } \\
\text { sample }\end{array}$ & $\begin{array}{c}\text { Number of } \\
\text { analyses }\end{array}$ & $\begin{array}{c}\text { Number of results } \\
\text { outside of criteria }\end{array}$ & $\begin{array}{c}\text { Acceptable } \\
(\%)\end{array}$ \\
\hline Duplicates & 900 & 9 & 99.0 \\
\hline Blanks & 785 & 26 & 96.7 \\
\hline
\end{tabular}

Preciston based on field duplicate analysis was determined to be $99.0 \%$ acceptable. See Table 1-3 for a complete breakdom of field dupi ficates that did not meet QC acceptance criteria.

of the 785 blanks analyzed during the July through Septenber' 1995 quarter, 26 exceeded the QC Yimit for $96.7 \%$ acceptability. Table 1-4 shows a breakdown of the constituents detected in the blanks.

Field QC samples collected for the July through September 1995 quarter were duplicates, field trip blanks, and full trip bianks. Duplicates were collected at approximately every 20th well. Field trip blanks were collected only on days when samples were collected for VOA. Fuil trip blanks were collected at approximately every 20 th well. 
Table 1-3. Breakdown of Field Ouplicates. - $(2$ sheets)

\begin{tabular}{|c|c|c|c|c|}
\hline $\begin{array}{l}\text { Method } \\
\text { analysts } \\
\text { code }\end{array}$ & Method description & $\begin{array}{l}\text { Number of } \\
\text { flagged } \\
\text { results }\end{array}$ & $\begin{array}{l}\text { Total } \\
\text { number of } \\
\text { results }\end{array}$ & $\begin{array}{l}\text { Percent of } \\
\text { total out } \\
\text { of } 1 \text { imits }\end{array}$ \\
\hline $17 / 1199$ & Pesticides/PCBs & 0 & 56 & 0 \\
\hline $19 / 1206$ & Seni-YOA & $\mathbf{0}$ & 259 & 0 \\
\hline 25 & VOA (GC) & 0 & 34 & 0 \\
\hline $30 / 1197$ & Phenols & 0 & 32 & 0 \\
\hline $34 / 1139$ & $\begin{array}{l}\text { Induct jvely coupled } \\
\text { plasna thetais }\end{array}$ & 1 & 201 & 0.5 \\
\hline $40 / 1170$ & Lead & 0 & 2 & 0 \\
\hline $41 / 1174$ & Mercury & 0 & 2 & 0 \\
\hline $43 / 1148$ & Arsenic & 0 & 2 & 0 \\
\hline 48 & Selenitum & 0 & 2 & 0 \\
\hline $49 / 1204$ & Herbicides & $\underline{0}$ & 8 & 0 \\
\hline $65 / 1039$ & Total Dissolved Solids & 2 & 7 & 28.6 \\
\hline 67 & Total Organic Halides & 0 & 6 & 0 \\
\hline 113 & Total Carbon & 0 & 2 & 0 \\
\hline $122 / 1230$ & Total Organic Carbon & 0 & 11 & 0 \\
\hline $124 / 1083$ & Anions & 0 & 70 & 0 \\
\hline $135 / 1247$ & Gross alpha & 1 & 8 & 12.5 \\
\hline $136 / 1252$ & Gross Beta & 0 & 8 & 0 \\
\hline $140 / 1249$ & Gamma Scap & 0 & 18 & 0 \\
\hline $14 !$ & Strontiun-90 & 0 & 2 & 0 \\
\hline $142 / 1255$ & Tritiug & 0 & 8 & 0 \\
\hline 143 & Technetium-99 & $I$ & $\underline{2}$ & 50 \\
\hline 145 & Uraniun & 2 & 4 & 50. \\
\hline $182 / 1300$ & VOA GC/HS & 0 & 112 & 0 \\
\hline 356 & Chemtca] oxygen demand & 0 & 1 & 0 \\
\hline $357 / 1086$ & Alkalinity & 0 & 6 & 0 \\
\hline 1040 & Iotal suspended solids & 0 & 2 & 0 \\
\hline 1097 & Total cyanide & 0 & $\underline{2}$ & 으 \\
\hline 1103 & Ammonia & 0 & 2 & 0 \\
\hline 1114 & Nitrite nitrate & 0 & 2 & 0 \\
\hline 1188 & Tin & 0 & 2 & 0 \\
\hline 1194 & Honha.logenated VOA & 0 & 12 & 0 \\
\hline 1216 & Total halogens . & 0 & 1 & 0 \\
\hline 1220 & Sulfide & 0 & 2 & 0 \\
\hline 1235 & OiT Grease & 1. & 2 & 50 \\
\hline 1270 & Plutonium & 0 & 4 & 0 \\
\hline
\end{tabular}


Table 1-3. Breakdown of Field Duplicates. (2 sheets)

\begin{tabular}{|c|l|c|c|c|}
\hline $\begin{array}{c}\text { Method } \\
\text { analysis } \\
\text { code }\end{array}$ & Methad description & $\begin{array}{c}\text { Mumber of } \\
\text { flagged } \\
\text { results }\end{array}$ & $\begin{array}{c}\text { Total } \\
\text { number of } \\
\text { results }\end{array}$ & $\begin{array}{c}\text { Percent of } \\
\text { total out } \\
\text { of 7imits }\end{array}$ \\
\hline 1272 & Radium-226 & 0 & 2 & 0 \\
\hline 1273 & Radium-228 & 0 & 2 & 0 \\
\hline 1295 & Gasol tne & 1 & 2 & 50 \\
\hline
\end{tabular}

Table 1-4. Breakdown of Field Blanks.

\begin{tabular}{|c|l|c|c|c|}
\hline $\begin{array}{c}\text { Method } \\
\text { analysis } \\
\text { code }\end{array}$ & \multicolumn{1}{|c|}{ Method description } & $\begin{array}{c}\text { Number of } \\
\text { flagged } \\
\text { results }\end{array}$ & $\begin{array}{c}\text { Total } \\
\text { number of } \\
\text { results }\end{array}$ & $\begin{array}{c}\text { Percent } \\
\text { of totai } \\
\text { out of } \\
\text { 1imits }\end{array}$ \\
\hline 19 & Semi-Y0A & 0 & 15 & 0 \\
\hline 25 & $\begin{array}{l}\text { Volat1le Organics (GC } \\
8010 / 8020)\end{array}$ & 2 & 17 & 11.8 \\
\hline 34 & $\begin{array}{l}\text { Induct Ively coupled plasma } \\
\text { meta]s }\end{array}$ & 14 & 196 & 7.1 \\
\hline 40 & Lead & 0 & 2 & 0 \\
\hline 41 & Mercury & 1 & 2 & 50 \\
\hline 65 & Tota] Dissolved Solids & 3 & 3 & 100 \\
\hline 67 & Tota1 Organic Halogen & 0 & 21 & 0 \\
\hline 122 & Total Organic Carbon & 0 & 22 & 0 \\
\hline 124 & Anions & 0 & 63 & 0 \\
\hline 135 & Gross Alpha & 0 & 5 & 0 \\
\hline 136 & Gross Beta & 0 & 5 & 0 \\
\hline 138 & Iodine-129 & 0 & 1 & 0 \\
\hline 142 & Tritium & 0 & 5 & 0 \\
\hline 143 & Technetium-99 & 0 & 3 & 0 \\
\hline 145 & Urantum & 0 & 2 & 0 \\
\hline 182 & Volatile organics (GC/MS 8260) & 6 & 419 & 1.4 \\
\hline 357 & Alka1inity & 0 & 5 & 0 \\
\hline
\end{tabular}

\subsubsection{Interna1 Quality Control Program}

The internal QC program uses five types of QC data to estabi ish and monitor performance in the laboratory. These data are laboratory blanks, matrix spikes, matrix duplicates, matrix spłke duplicates, and EPA studjes (WP, US, and radiochemical intercomparisons). 
Every quarter each contracted laboratory supplies fts own QC report, which includes data quality information on matrix spikes, matrix duplicates, matrix spike duplicates, and b]anks in the form of precision and accuracy. Each contracted laboratory also supp]ies to wHC a report of its results of EPA's WP, WS, and radiochemical intercomparison studies on a quarteriy basis. The resujts of EPA studies independently verify the continuation of laboratory performance and are expressed as the percentage of EPA-accepted restults. In addition, once a year each contracted laboratory supplies an $\mathrm{MDL} / \mathrm{minimum}$ detectabie concentration (NDC) report. WHC reviews each of these reports. The MDLs and F.BCs are required to be below the contractually required quantitation limit (CRQL). The CRQL is not associated with a quantitation limit as the name suggests; it is intended to be the lowest analyte concentration in a given matrix that a laboratory can be expected to detect consistently. The CRQL is agreed on under the contractual statement of work. A Taboratory NCR is issued when the $\mathrm{KDL}$ is greater than the CRQL.

The hazardous chemistry laboratory provided precision and accuracy figures in the July through September 1995 quality assurance quarteriy report. Last quarter, problems with blank contanination for ICP metals were noted; however, whC does not believe that additional follow-up is necessary. The percentage of samples affected by 'B' qualifiers was only slightly higher than usual during the April through June quarter. The percentage of ' $B$ ' qual ifiers for the July through September quarter is similar to what is typically seen. The July through september 1995 quarteriy quality control report noted blank contamination for zinc in 5 of 16 sample groups. WHC will request an explanation and follow up if necessary.

Radfochemistry precision and accuracy figures are provided in the radiochenistry laboratory's guarterly report, with percent acceptability calculated from duplicates and spikes, respectively. These results, along with radiochemistry blank data, are summarized in Table 1-5.

Precision and accuracy results from the radiochemistry laboratory, summarized in Table 1-5, indicate the perforeance of all customers submitting water matrix samples. WHC samples represent oniy a part of the performance sumary. These figures represent sampies analyzed during the period of July through September 1995.

Table 1-5. Radiochemtstry Quarterly Report Surmary.

\begin{tabular}{|c|c|c|c|}
\hline $\begin{array}{c}\text { Third } \\
\text { quarter 1995 }\end{array}$ & $\begin{array}{c}\text { Ouplicates } \\
\text { (precision, } \\
\text { \& acceptable) }\end{array}$ & $\begin{array}{c}\text { Spikes } \\
\text { (accuracy, } \\
\text { \%cceptable) }\end{array}$ & $\begin{array}{c}\text { Blanks } \\
\text { (\% acceptable) }\end{array}$ \\
\hline $\begin{array}{c}\text { July through } \\
\text { September }\end{array}$ & 99.0 & 97.4 & 99.1 \\
\hline
\end{tabular}

EPA intercomparison data were not reported for the July through September quarter.

EPA Water Pollution (WP) samples were analyzed by the hazardous chemistry laboratory during August 1995. The EPA sent blind samples for the 
analysis of 143 anatytes for performance evaluation 4P034. The blind samples were analyzed for metals, minerals, nutrients, TOC, COD, PCBs, pesticides, volatile halocarbons, volatile aronatjcs, total cyanide, non-filterable residue, oil and grease, total phenolics, and total residual chlorine. of the 143 results, 137 were acceptable. Results for non-filterable residue, oil and grease, and total residual chlorine were unacceptable. Hone of the analytes out of jimits are of particular concern to the RCRA program.

Environoental Sciences sent performance evaluation (PE) samples to five laboratories during June 1995. PE samples were also sent out in September 1995.

AII PE samples were sent out in dupticate and contained known amounts of particular analytes. The laboratories that participated in the analysis of these samples were the primary hazardous chenistry laboratory (Lab 1) and five comparison 1aboratories (Labs 2, 3, 4, 5, and 6).

Lab 1 's results were within the 11mits of the 95\% confldence interval estab1 1shed in accordance with WHC-CH-7-8, Section 2.4 for carbon disulfide, benzene, toTuene, zinc, manganese, and Toc (September 1995 only). Lab 1 had unacceptable results twice for TOX. Lab 1 also had unacceptable results for iron and chromlun. WHC will send additfonal performance evaluation samples as appropriate. WHC will also consider selecting a new laboratory for TOX analysis. 


\section{3 qUALITY CONTROL OEFIMITIOLS}

Accuracy--The closeness of agreement between an observed value and a true value. Accuracy is assessed by means of reference samples and percent recoveries.

B1ind sampie-A sample that contains a concentration of analyte that is known to the supplier but unknown to the analyzing laboratory. The analyzing laboratory is informed that the sample is a QC sample and not a field sample. The bilind, double blind, and matrix-matched double blind samples are used to assess accuracy and monitor the performance of the analytical laboratory(ies) with prepared or purchased waterials from EPA QC samples/concentrates or primary materials.

Contractually required quantitation Iinit-A value intended to be the lowest analyte concentration in a given matrix that the laboratory can be expected to achieve consistently; agreed upon under the contract statement of work.

Double blind sample-A sample that contains a concentration of analyte that is known to the supplier but is unknown to the analyzing laboratory. The analyzing laboratory is not informed that the sample is a QC sample. All attempts are made to make this sample appear like a field sample. For example, the double blind sample should be subwitted to the laboratory within the same time period and with a sample identification number similar to that of the field samples. The double blind sample does not include matrix matching.

External quality control sample--Any oc sample prepared without the knowledge of the analytical laboratory.

Field duplicate sample--A sample used to deternine repeatability of an analytical measurement on identical samples collected as close as possible to the same time at the same location. These samples are stored in separate containers and are analyzed independently by the same laboratory.

Field trip blank-A sample that contains only Type II reagent water. At the time of sample collection, the field blank is filled at the sampling site by pouring Type II reagent water from a cleaned container into volatile organic analysis (VOA) vials. After collection, the field trip blank is treated in the sawe manner as the other samples collected during the sampling event. Field trip blanks are collected only on days when samples are collected for VOA.

Full trip blank-A sample that contains only Type II reagent water and preservative, as required. A full trip blank is used to check for contamination in sample bottles and sample preparation. The full trip b]ank is analyzed for all constituents of interest on all types of sample bott\}es used during that sampling period. The frequency of collection for a full trip blank is 1 per 20 samples, or 1 per sampling batch. A full trip blank is fit.led in the analytical laboratory under the sample preparation procedures. The full trip blank is not opened in the field. 
Internal quality control sanple--Any QC sample prepared by the analytical laboratory and used to establish and monitor the quality of the analytical iaboratory.

Linit of detection--The lowest concentration level that is statistically different from a blank. This level is calculated by the average blank signal plus three standard deviations of the bTank analyses.

Linit of quantitation--The level above which quantitative results may be obtained with a specific degree of confidence. This level is calculated as the blank mean plus 10 standard deviatiens of the blank.

Matrix-atched double blind sample--A matrix-matched double blind sample contains a concentration of analyte that is known to the supplier but unknown to the analyzing laboratory. The saaple atrix has been altered to closely match that of the field samples.

Method detection limit--The miniman concentration of a substance that can be weasured and reported with $99 \%$ conftdence that the analyte concentration is greater than zero and is determined from analysis of a sample in a given matrix type containing the analyte.

Hinimer dotectable concentration--Required level of analytical detection for radiochenical samples.

Practical quantitaion Limit-The lowest concentration that can be reliably achieved within specified 1 imits of precision and accuracy during routine laboratory operating conditions. The PQL is generally 5 to 10 times the $\mathrm{NOL}$.

Precision--The agreement among a set of individuat measurements of the same property, usually under prescribed similar conditions. Precision is calculated by using the RPO of the duplicate/replicate analyses. These samples should contain concentrations of analyte above the MDL and may involve the use of matrix spikes.

Reliable detection Ievel-A detection 1 imit set at two times the concentration of the $\mathrm{mLL}$, so the risk of both false positives and false negatives falls below $1 \%$.

Type II reagent water--Distilled or deionized water that is free of contaminants that may interfere with the analytical test in question. 
DOE/RL-95-69-3

\subsection{DATA TABLES}

J. A. Serkowski

Westinghouse Hanford Company

Four different formats are used to display water level measurements and groundwater sample analytical results. This section describes the meaning of each table colurin heading, defines the abbreviatjons used, explains the data qualifiers and flags, and 1 ists analysis method codes and relevant regulatory standards.

\subsubsection{Water Level Measurenent Table}

The water level measurenent table has the following four columns:

- We]]--Weit in which measurement was made.

- Date--Date of measurement.

- Depth to water--Depth, in feet, fron well casing reference point to top of water in well.

- Water leve]--Elevation, in feet above mean sea level, of water leve] computed by subtracting depth to water from casing reference elevation.

Wells are grouped according to the zone that they monitor, beginning with the top of the unconfined aquifer and continuing downard to the conffned aquifer.

Only measurements made during the reporting quarter are included in the tables. Heasurenents made during sampling are reported with an asterisk (*). Heasurenents are also routinely perforwed at other times to permit collection of data for an entire network within a 1- or 2-day tine period.

Data that are judged to be suspect by the project scientist are flagged with a ' + ' in the tabile. Before data are designated as suspect, checks are conducted to deteraine if a transcription error occurred between the field sheet and the electronic database.

\subsubsection{Constituent List and Sunpary of Results Table}

The Constituent List and Sumnary of Results tab]e ("Sumary" table) is the first of three tables that present the results of groundwater sample analyses. The "Surnary" table displays statistics based on the complete data set for the reporting period. The complete data set consists of all analysis results requested by the project associated with the sampiles collected before the end of the current reporting quarter that arrived at WHC before the cutoff date for the current quarterly report. (Novenber 17, 1995) and have not been reported in a prevlous quarterly report. Therefore, data collected during previous quarters may appear in this document if the laboratory did not report. the data intil the current reporting perfod. 
The fields in the "Sumary" table are as follows:

- Constituent Hame--Naqe of the analyzed constituent being surnarized; the Short name is an abbrevtation used in the Constituents with at Least One Detected Value table (Section 1.4.3); the (Method), when present, is a code (Section 1.4.5) used to distinguish between different analytical methods for the same constituent.

- Units--Units in which the MDL and drinking water standards (DWS) are reported in this table.

- Lab MDL--The MDL computed by the laboratory for a constituent. This fijeld is biank for radionuclides where the MDL is defined as the value of the total error reported by the laboratory for each result.

- DWS Limit/Agency-- The DWS used for comparison to the reported results; see Section 1.4.5 for Agency definitions and a complete listing of standards used.

- Number of Samoles--Total is the number of sample results from all wells in the project contained in the complete data set for that constituent; $>M D L$ is the number of results that exceed the MDL; $\geq$ DWS is the number of results that exceed the listed DWS.

Summarized constituents are grouped as Contamfnation Indicator Parameters (CIP), Drinking Water Paraneters, Groundwater Quality Parameters, and Site-Specific and Other Constituents. Within groups, rows are ordered a]phabeticaliy by the full constituent name.

If a constituent has an MOL that exceeds the ONS, exceedance of the DWS cannot be determined, so an asterisk (*) is placed in the 2BMS field. Some radionuclides do not have an explictt WOL; the asterisk is used if the DWS exceeds the total error assoctated with at least one of the results.

\subsubsection{Constituents with at Lesst One Betected Value Table}

The Constituents with at Least One Detected Value table ("Detected" table) is a subset of the complete data set. Only constituents that were detected above the MDL in at least one sample collected for the project during the current reporting period are reported in the "Oetected" table.

The first three colum headings are as follows:

- Nell Name--Wel1 from which sample was collected.

- Collection Date-Date on which the sample was collected.

- Sample_Number--Unique number assigned to a well sample. 
The renaining columns contain analytical results and quatifiers for the constituent. The structure of the coluen header is as follows.

const 1tuent short name

method code/reporting units

$M D L / D W S$ (suffix).

The "Sunmary" tab]e (Section 1.4.2) shows the relationship between the full constituent name and the short name. The analyttcal method code is defined in Tab]e 1-6. Abbreviations used for reporting units are 7isted in Section 1.4.5. On the third line, a period appears in place of the MDL or DWS if a value does not extst. The one-letter suffix that may be added to the DWS identifies the regulation associated with the standard and is described in Section 1.4.5.

Analysis results are displayed with two digits to the right of the decimal point. This is a consequence of the generating software and does not reflect the precision of the results. The laboratories nornally report the result with three significant digits. Radionuclide values may be negative because the laboratory subtracts a background reading from the measured result. The letter(s) that may follow the result are data qualifters and flags. A complete explanation of what each flag means is provided in Section 1.4.5.

\subsubsection{Contamination Indicator Parameters Table}

The "CIP" table has a format similar to the Detected table (see Section 1.4.3) but includes onTy data for pH, specific conductance, TOC, and ToX. All results for these parameters, whether detected or not, are ifsted in the "CIP" table. The analys is method code is not part of the column header in this report.

Because more than one laboratory, each with jts own MDL may perform some of the TOC and TOX analyses, values in those columns of the "CIP" table may reflect different HDLs. In such cases, an asterisk appears in the MDL portion of the column header.

\subsubsection{Codes and Abbreviations}

Abbreviations--The abbreviations used in the data tables are as follows:

COL coliformi colonies per 100 militiliters

OHS drinking water standard

ft. feet

MDL method detection 7 imit

mas7 mean sea level

NTU nepheionetric turbidity unit

$\mathrm{pC} / \mathrm{L}$ picocuries per $\mathrm{liter}$

ppb parts per billion

ppm parts per mition

$\mu$ Thio $/ \mathrm{cm}$ micromhos per centimeter. 
Table 1-6. Analysis Method Code Definitions.

(2 sheets)

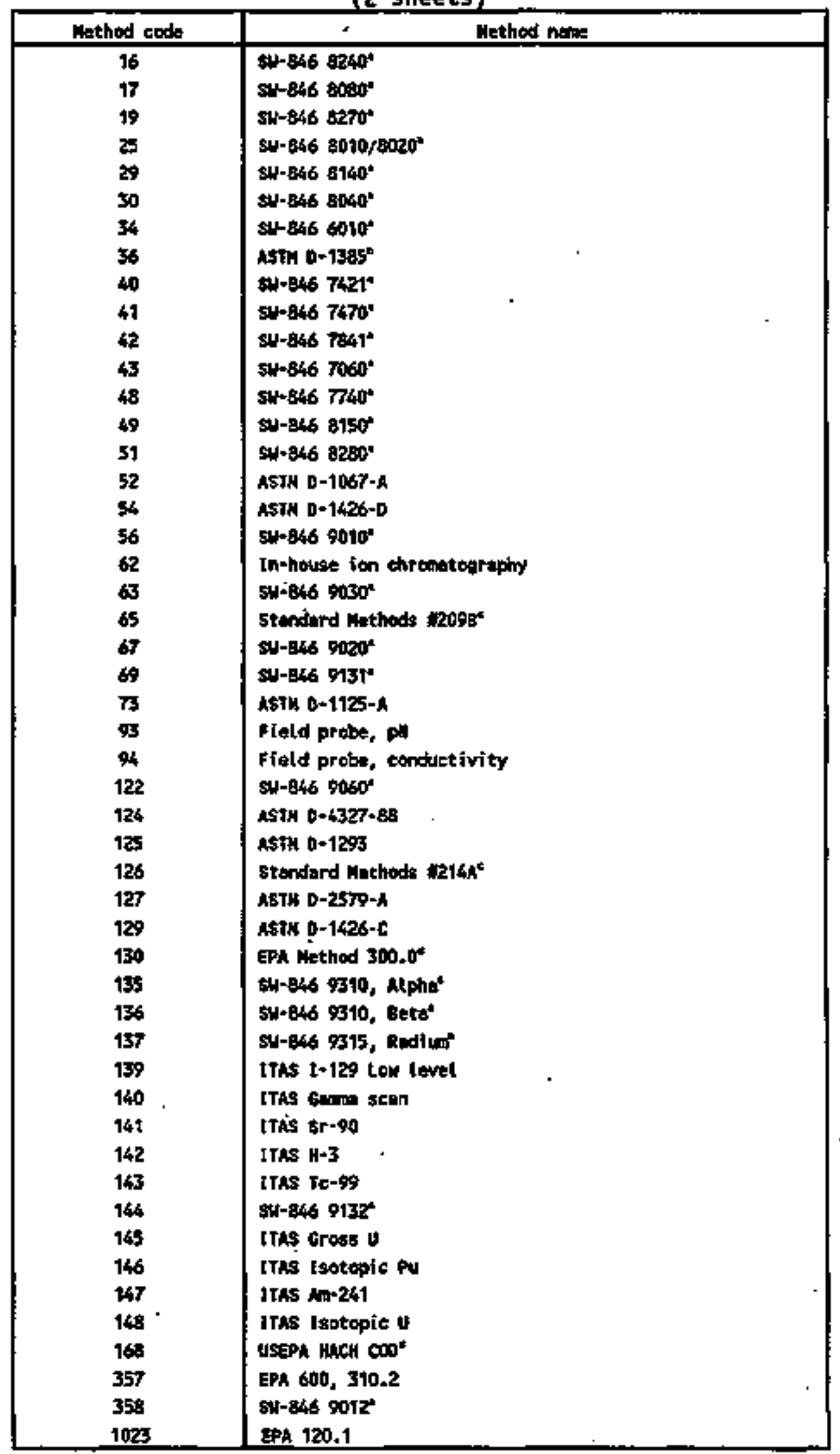


Table 1-6. . Analysis Method Code Definitions.

(2 sheets)

\begin{tabular}{|c|c|}
\hline Inthod eods & nethad mos \\
\hline $\begin{array}{l}1039 \\
1044 \\
1083 \\
1066 \\
1139 \\
1148 \\
1197 \\
1199 \\
1206 \\
1216 \\
1225 \\
1230 \\
1247 \\
1269 \\
1252 \\
1255 \\
1270 \\
1276 \\
1281 \\
1203 \\
1288 \\
1300\end{array}$ & 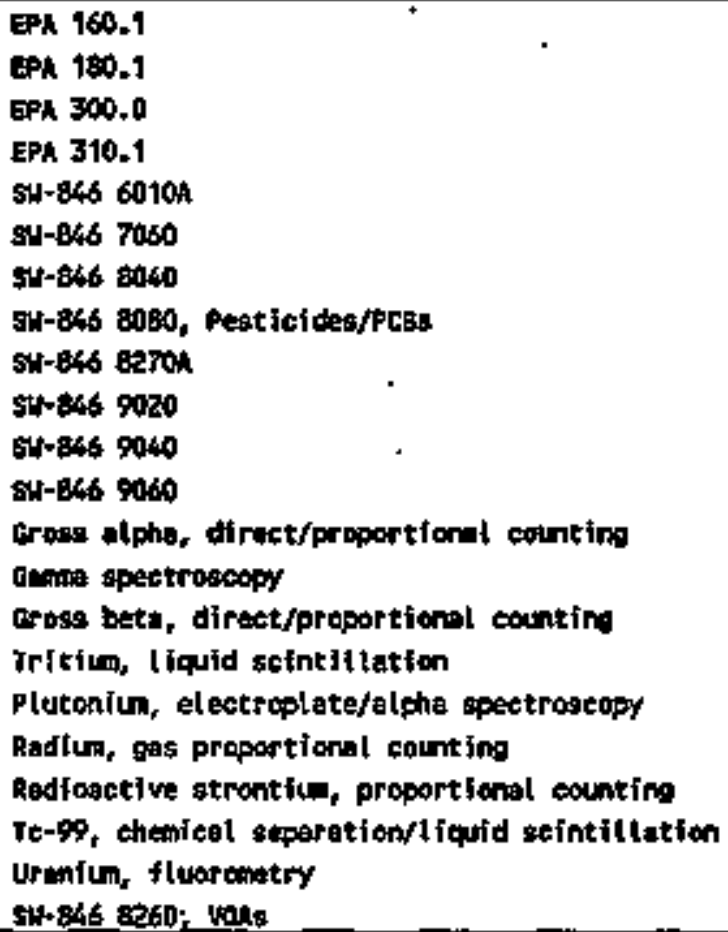 \\
\hline
\end{tabular}

"CepA 1986b).

"Chstr 1991.

"EPA 1970).

(CAPHA 1989). 
Agencies and suffixes--Agency codes and DNS suffixes identify the regulatory origin of the standard of the biss. Agency codes are used in the "Sumary" tables, and OuS suffixes are used in the "Detected" and "CIP" tables. The codes are defined as follows:

\begin{tabular}{|c|c|c|}
\hline Agency & Suffix & Regulatory basis \\
\hline EPA & Mone & $\begin{array}{l}\text { Maximum contaminant leve]s in } 40 \text { CFR } 141 \text {, } \\
\text { "Hational Prieary Orinking Water Regulations." }\end{array}$ \\
\hline EPAA & $\mathbf{a}$ & 40 CFR 141 action leve]. \\
\hline EPAS & $\mathbf{s}$ & $\begin{array}{l}\text { Secondary maximii contaminant levels in } \\
40 \text { CFR 143, "Nat Ional Secondary Drinking Water } \\
\text { Regulations." }\end{array}$ \\
\hline
\end{tabular}

Data qualifiers and flags--Data qualifiers and flags used in the "Detected" and "CIP" tables are assigned by the laboratory and WHC personnel, respectively. Qualifiers reflect conditions occurring in the laboratory relating to the analytical procedure. Flags serve a wider function of alerting the data user to the 11 mitations of the reported value. Qualifiers and flags can be appended to each other to form a string of letters when severa] factors apply to a result. The qualifiers and flags used are as follows:

Laboratory qualifiers:

B - Blank associated with analyte is contaminated

D - Analyzed sample is diluted

E - Concentration is out of instrument calibration range

J - Concentration is estimated

$L$ - Concentration is below the contractually required quantitation limit but above the MDL

$U$ - Undetected; concentration is below the indicated value.

Data flags:

F - Suspect data currently under review

H - Laboratory holding tíme exceeded

G - Revieved data that are considered valjd

P - Potential problem; see text associated with tabie

Q - Result associated with suspect QC data

$R$ - Reviewed data that have been rejected

$Y$ - Reviewed data that continue to be suspect

+ - Suspect water level data

* - MOL is greater than DWS, so exceedance of DNS is undetermined.

Hore complete descrtptions of some of these qualifiers and flags are presented in Section 1.2, Qual ity control Program.

Analysis method codes--Analysis method codes are used as an abbreviation for the laboratory method used to perform an analysis. A complete listing of the analysis method codes used in the Sumary and Detected tables is shown in Table 1-6. 
DHSS-DWS are used in all of the chenistry tables to provide a standard with which to compare sample results. The DWS are based on standards found in 40 CFR 141 and 40 CFR 143. Where there is overlap in constituents addressed by these regulations, 40 CFR 141 takes precedence over 40 CFR 143. A complete listing of the OHSs used in the Summary, Detected, and CIP tables is shown in Table 1-7. 
Table 1-7. Drinking Water Standards. (Sheet 1 of 2)

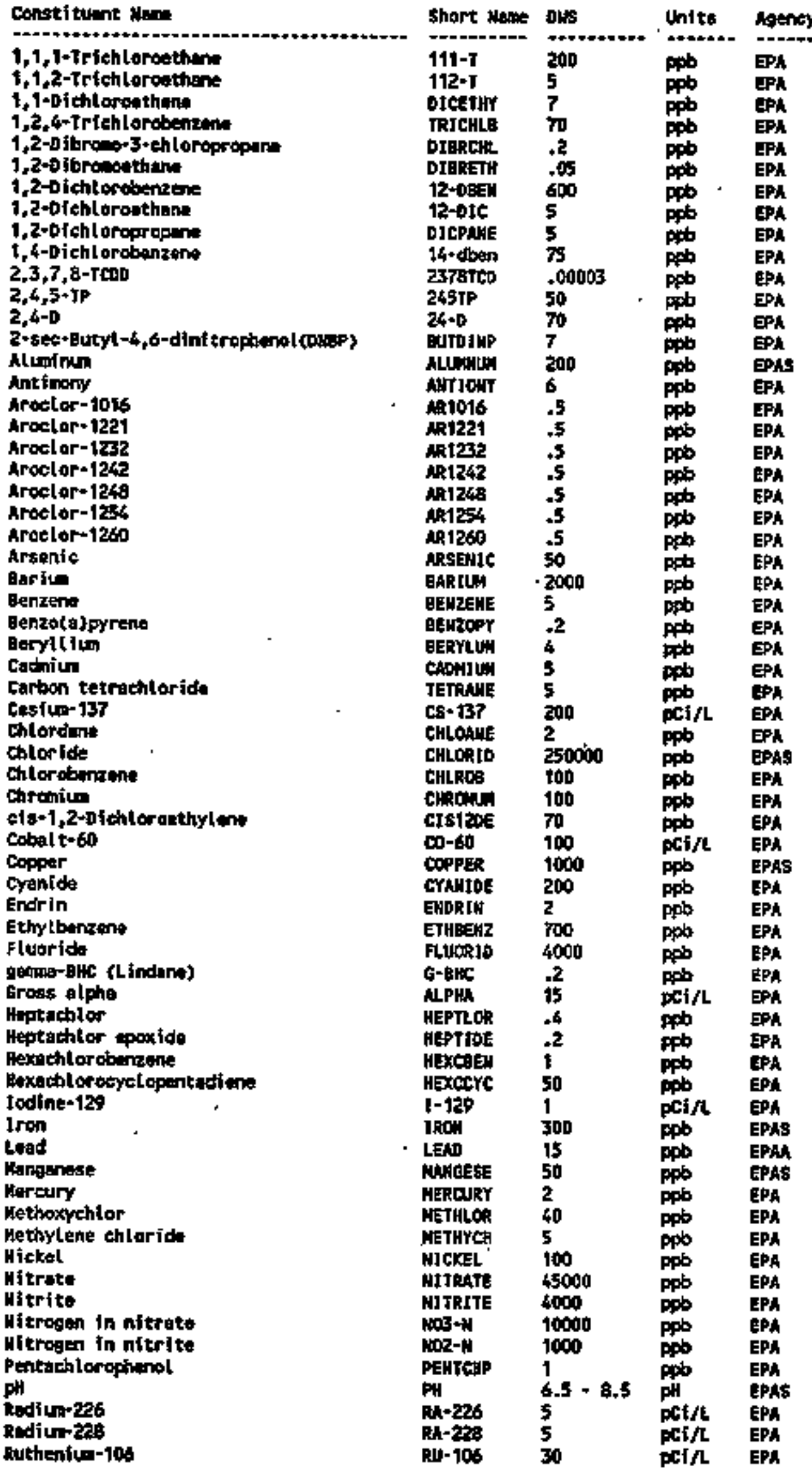


Table 1-7. Drinking Water Standards. (Sheet 2 of 2)

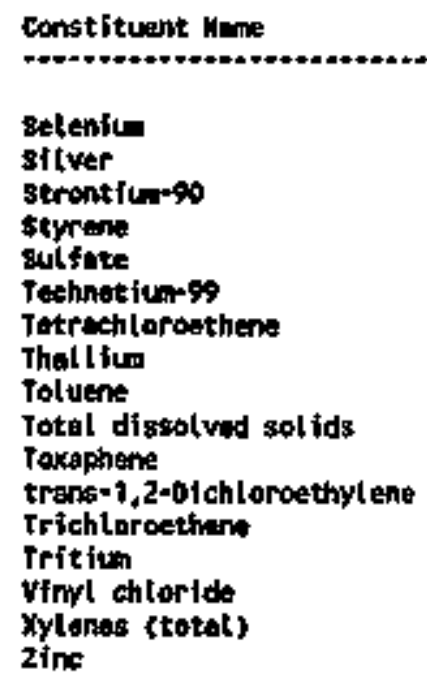

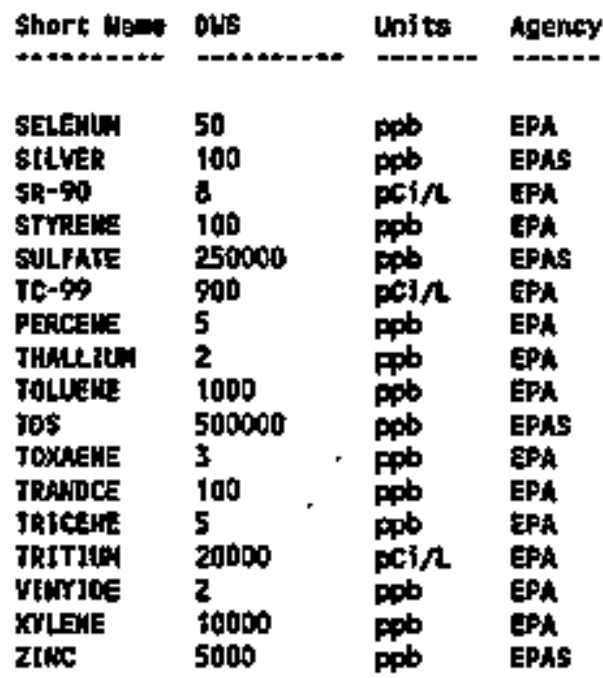




\subsection{DATA EVALUATIOH}

\section{R. V. Gray}

Westinghouse Hanford Company

Data evaluation is a process through which suspect data are identified and/or investigated. At present, the data evaluation process consists of the evaluating data called out in RADEs, which are subitited by data users and/or identified when performing the required statjstical evaluation of CIP data. The statistical evaluations of CIP data are presented within the chapters for individual sites.

Thirty-six RADEs were subnitted during the July through Septenber quarter of 1995. Of the submjtted RADEs, 21 are for ICP metals, 7 are for radionuclides, 6 are for anjons, 4 are for field parameters (pH and conductivity), 2 are for TOX, 2 are for turbidjty, 1 is for organics, 1 is for mercury and $i$ is for $1 \mathrm{ab} \mathrm{pH}$.

An $F$ flag is used to indicate that a RADE has been subnitted. Suspect analytical data shajl be evaluated for the following, as appljcable:

historical trends, site geohydrology, contaminant distribution (e.g., plumes), and internal consistency to determine if the data are acceptable. After evaluation, the F flag will be changed to ' $G$,' indicating acceptable data; ' $Y$, ' indicating suspect data; or 'R,' indicating untsable data. A summary of the results will be presented in the annual report. 
DOE/RL-95-69-3

COLTERTS

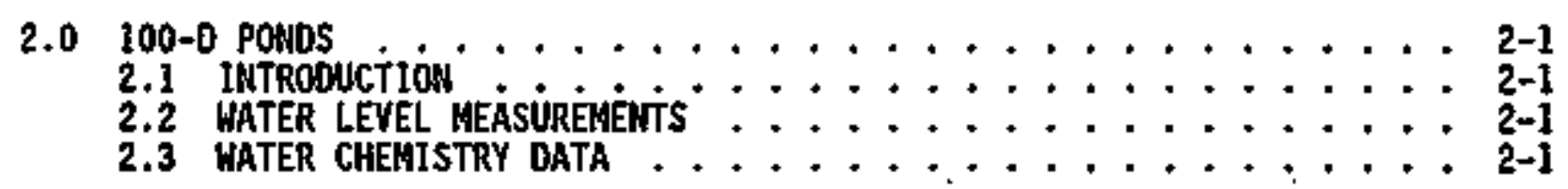




\section{LIST OF FIGtRES}

2-1 Monitoring Well Location Map for the 100-0 Ponds . . . . . . 2-2

\section{LIST OF TABLES}

2-1 Monitoring Well Purpose and Sampling Schedule

In the 100-D Ponds Network .............. 2-3

2-2 RCRA Water Leve1 Measurenent Report 100-0 Ponds,

Third Quarter $1995 \ldots \ldots \ldots$. . . . . . . . . . . .

2-3 Constituent List and Sumnary of Results for the 100-D Pond

Data for Reporting Period July 1 through September 30, 1995 ... . 2-7

2-4 Constituents with at Least One Detected Value for the 100-D Pond

Data for Reporting Period July 1 through September 30, 1995 . . . 2-9

2-5 Contanination Indicator Parameters for the 100-D Pond

Bata for Reporting Period July 1 through September 30, $1995 \ldots$. . 2-11 


\title{
2.0 100-D POHDS
}

\author{
A. J. Hartman \\ Nestinghouse Hanford company
}

\subsection{IHTRODUCTION}

The 100-D Ponds system is an interim-status RCRA disposal unit located in the 100-0 Area of the Hanford Site (Figure 2-1). Four wells make up the 100-D Ponds sampling network (Table 2-1). The 100-D Ponds are monitored under an indicator evaluation program (40 CFR 265). .

The 100-D Ponds unit was constructed in 1977 for disposal of nonradioactive effluents derived from 100-D Area operating facilities. The 100-D Ponds are located in the former 18B-D ash dfsposal basin and constst of a settiing pond and a percolation pond, separated by a dike.

Effluent to the 100-D Ponds originated from two sources: a filter plant and engineering testing laboratories. Some past discharges may have included corrosive waste or mercury (Hartman 1991). All discharges to the 100-D Ponds ceased in Kay 1994.

\subsection{MATER LEVEL MEASIREMEMTS}

Water levels are measured monthiy in wetls in and near the 100-D Area to track changes caused by river-stage fluctuations and the cessation of effivent discharge. Water level data are listed in Table 2-2.

\subsection{MATER CHEIISTRY DATh}

Al1 four wells were sappled as scheduled last quarter. Data are reported in Tables 2-3 through 2-5. Data flags are explained in Sections 1.2 and 1.4. We11s DB-4 and D8-6 exceeded the upper DWS for PH. Requests for analytical data evaluation were made for well 05-13 (tritium) and well D8-6 (field pH) because the resuits did not follow historical trends.

Downgradient we17s did not exceed critical mean values for conductivity, TOC, or TOX. Downgradient well DB-6 exceeded the critical range for field $\mathrm{pH}$, but the value was suspect. Laboratory $\mathrm{pH}$ was $0.5 \mathrm{pH}$ unit lower, which is within the critical range and more in trend with historical data. The well was resampled for field pH using two meters, but one meter gave an average value above the critical range and the other gave an average value within the critical range. The well will be sampled again for pH until conclusive results are obtained. 


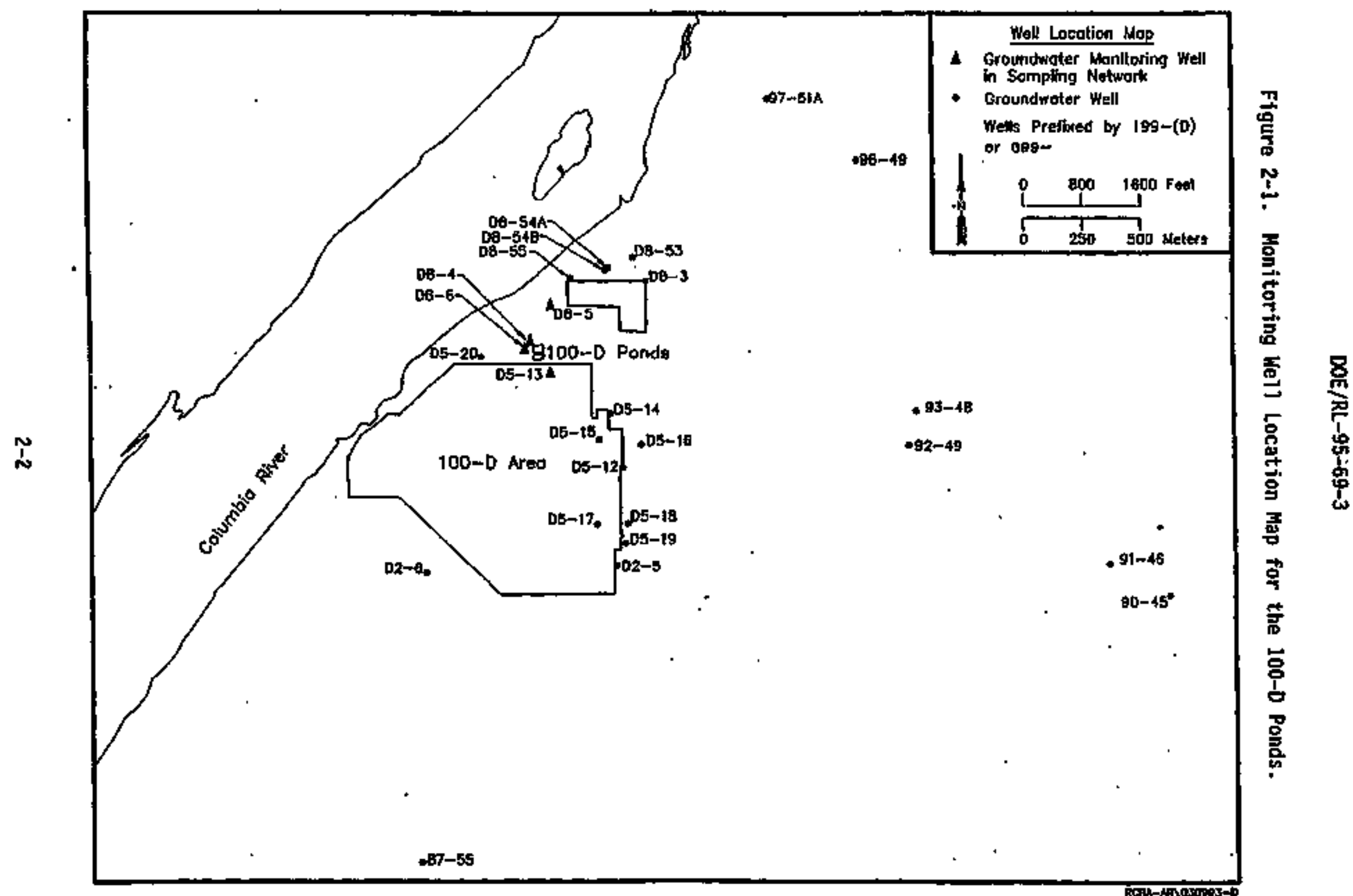


Table 2-1. Monitoring Nell Purpose and Sampling Schedule in the 100-D Ponds Network.

\begin{tabular}{|c|c|c|c|}
\hline $\begin{array}{c}\text { Nell no. } \\
(199-)\end{array}$ & $\begin{array}{c}\text { Relative } \\
\text { position }\end{array}$ & $\begin{array}{c}\text { Sample } \\
\text { frequency }\end{array}$ & $\begin{array}{c}\text { Sample date, } \\
\text { 3rd Qtr 1995 }\end{array}$ \\
\hline $05-13$ & Upgradient & Quarterly & $8 / 16 / 95$ \\
\hline $08-4$ & Downgradient & Seniannually & $8 / 16 / 95$ \\
\hline $08-5$ & Downgradient & Semi annually & $8 / 16 / 95$ \\
\hline $08-6$ & Downgradient & Semi annually & $8 / 16 / 95$ \\
\hline
\end{tabular}

Note: The hydrogeologic unit monttored is the uppermost portion of the unconfined aquifer, which comprtses unconsolidated sand and gravels of the Ringold Formation. 
Table 2-2. RCRA Water Level Heasurement Report 100-D Ponds, Third Quarter 1995. (sheet 1 of 3 )

\begin{tabular}{|c|c|c|c|c|c|}
\hline Wel1 & & Date & $\begin{array}{l}\text { Depth to } \\
\text { water (ft) }\end{array}$ & $\begin{array}{l}\text { Water } \\
\text { elevation } \\
\text { (ft) }\end{array}$ & $\begin{array}{c}\text { Ievel } \\
\text { above } \mathrm{ms} 1 \\
\text { (m) }\end{array}$ \\
\hline \multicolumn{6}{|c|}{ Wells Monitoring the Top of the Unconfined Aquifer } \\
\hline $199-02-5$ & & $\begin{array}{l}7 / 19 / 95 \\
8 / 15 / 95 \\
9 / 28 / 95\end{array}$ & $\begin{array}{l}75.21 \\
75.10 \\
75.11\end{array}$ & $\begin{array}{l}385.09 \\
385.20 \\
385.19\end{array}$ & $\begin{array}{l}117.38 \\
117.41 \\
117.41\end{array}$ \\
\hline 199-D2-6 & & $\begin{array}{l}7 / 19 / 95 \\
9 / 15 / 95 \\
9 / 28 / 95\end{array}$ & $\begin{array}{l}93.90 \\
84.07 \\
85.06\end{array}$ & $\begin{array}{l}385.38 \\
385.21 \\
384.22\end{array}$ & $\begin{array}{l}117.46 \\
117.41 \\
117.11\end{array}$ \\
\hline $199-05-12$ & & $\begin{array}{l}7 / 19 / 95 \\
8 / 15 / 95 \\
9 / 28 / 95\end{array}$ & $\begin{array}{l}85.83 \\
85.31 \\
85.54\end{array}$ & $\begin{array}{l}383.78 \\
384.30 \\
384.07\end{array}$ & $\begin{array}{r}116.98 \\
117.13 \\
117.06\end{array}$ \\
\hline 199-05-13 & - & $\begin{array}{l}7 / 19 / 95 \\
8 / 15 / 95 \\
8 / 16 / 95\end{array}$ & $\begin{array}{l}87.40 \\
87.69 \\
87.75 *\end{array}$ & $\begin{array}{l}384.09 \\
383.80 \\
383.74\end{array}$ & $\begin{array}{l}117.07 \\
116.98 \\
116.96\end{array}$ \\
\hline 199-D5-17 & 、 & $\begin{array}{l}7 / 19 / 95 \\
8 / 15 / 95 \\
9 / 27 / 95\end{array}$ & $\begin{array}{l}84.70 \\
84.51 \\
84.67\end{array}$ & $\begin{array}{l}384.79 \\
384.98 \\
384.82\end{array}$ & $\begin{array}{l}117.28 \\
117.34 \\
117.29\end{array}$ \\
\hline $199-05-20$ & & $\begin{array}{l}7 / 19 / 95 \\
8 / 15 / 95 \\
9 / 28 / 95\end{array}$ & $\begin{array}{l}83.67 \\
84.58 \\
85.95\end{array}$ & $\begin{array}{l}384.43 \\
383.52 \\
382.15\end{array}$ & $\begin{array}{l}117.17 \\
116.90 \\
116.48\end{array}$ \\
\hline $199-08-3$ & . & $\begin{array}{l}7 / 19 / 95 \\
8 / 15 / 95 \\
9 / 28 / 95\end{array}$ & $\begin{array}{l}65.83 \\
67.02 \\
68.69\end{array}$ & $\begin{array}{l}383.13 \\
381.94 \\
380.27\end{array}$ & $\begin{array}{l}116.78 \\
116.42 \\
115.91\end{array}$ \\
\hline $199-08-4$ & & $\begin{array}{l}7 / 19 / 95 \\
8 / 15 / 95 \\
8 / 16 / 95 \\
9 / 28 / 95\end{array}$ & $\begin{array}{l}84.61 \\
85.24 \\
85.28^{*} \\
86.37\end{array}$ & $\begin{array}{l}384.12 \\
383.49 \\
383.45 \\
382.36\end{array}$ & $\begin{array}{l}117.08 \\
116.89 \\
116.88 \\
116.54\end{array}$ \\
\hline $199-08-53$ & & $\begin{array}{l}7 / 19 / 95 \\
8 / 15 / 95 \\
8 / 16 / 95 \\
9 / 28 / 95 \\
7 / 19 / 95 \\
8 / 15 / 95\end{array}$ & $\begin{array}{l}68.73 \\
69.71 \\
69.78^{*} \\
71.30 \\
52.97 \\
54.16\end{array}$ & $\begin{array}{l}383.76 \\
382.78 \\
382.71 \\
381.19 \\
383.06 \\
381.87\end{array}$ & $\begin{array}{l}116.97 \\
116.67 \\
116.65 \\
116.19 \\
116.76 \\
116.39\end{array}$ \\
\hline
\end{tabular}


Table 2-2. RCRA Water Level Measurement Report 100-D Ponds, Third Quarter 1995. (sheet 2 of 3 )

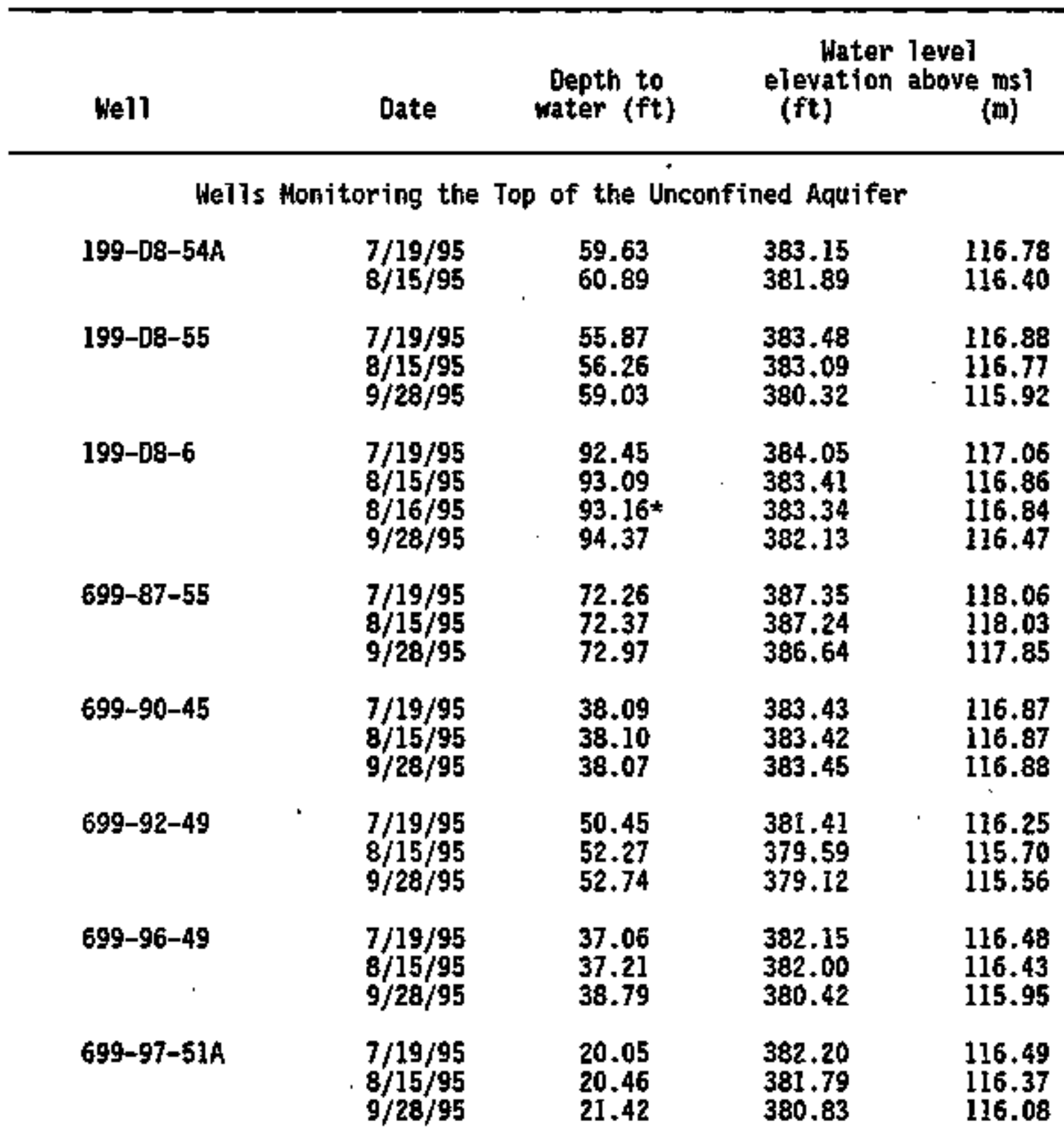


Table 2-2. RCRA Water Level Measurement Report

100-D Ponds, Third Quarter 1995.

(sheet 3 of 3 )

\begin{tabular}{ccccc}
\hline Wel1 & Date & $\begin{array}{c}\text { Mater level } \\
\text { Depth to } \\
\text { water (ft) }\end{array}$ & $\begin{array}{c}\text { Mlevation above ms1 } \\
\text { (ft) }\end{array}$ & (m) \\
\hline \multicolumn{5}{c}{ Wells Monitoring the Confined Aquifer } \\
$199-08-548$ & $7 / 19 / 95$ & 59.14 & 383.37 & 116.85 \\
& $8 / 15 / 95$ & 60.12 & 382.39 & 116.55 \\
& $9 / 28 / 95$ & 61.41 & 381.10 & 116.16 \\
\hline
\end{tabular}

NOTES: 1. Water level elevations are calculated by subtracting the measured depth-to-water from the surveyed elevation for the well.

2. Depth-to-water values are transcribed frow fleld records.

3. Heasurements aarked with an ' $*$ ' were taken at the time of sanpling.

4. Measurements narked with a ' + ' are outside of the expected range, and are suspected of error. 
Table 2-3. Constituent List and Summary of Results for the 100-D Pond Data for Reporting Period

July 1 through September 30, 1995.

(sheet 1 of 2)

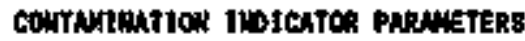

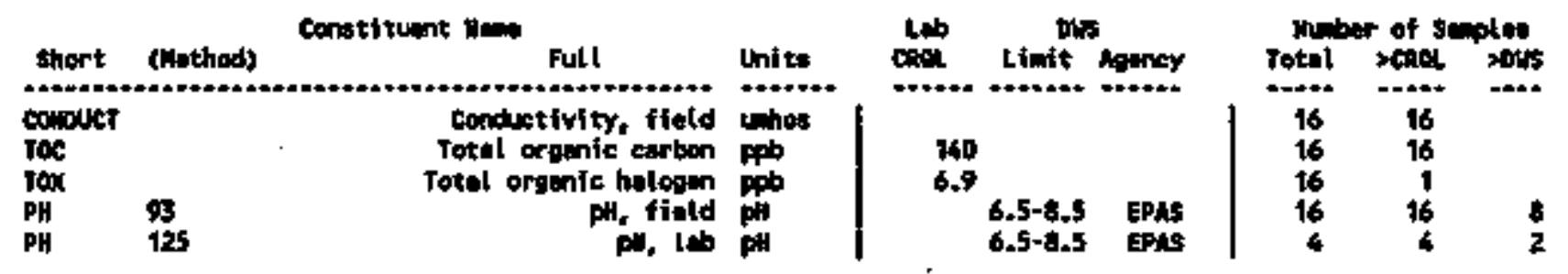

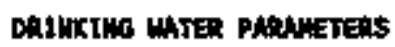

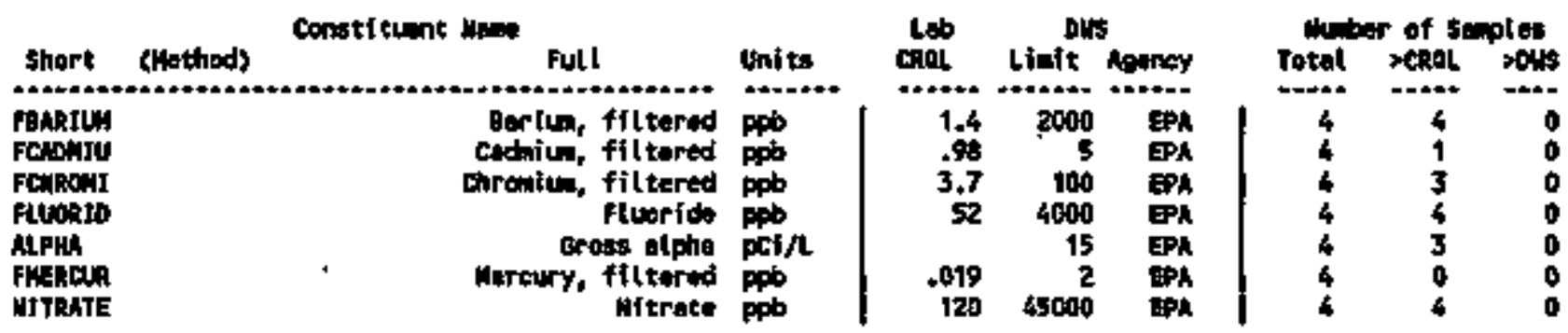

GROC:DWTER OHALITY PARUETERS

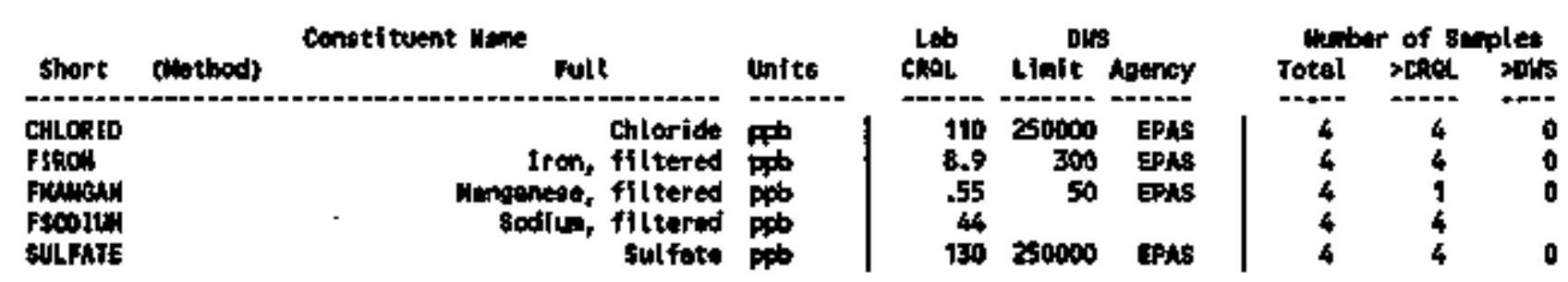

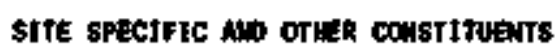

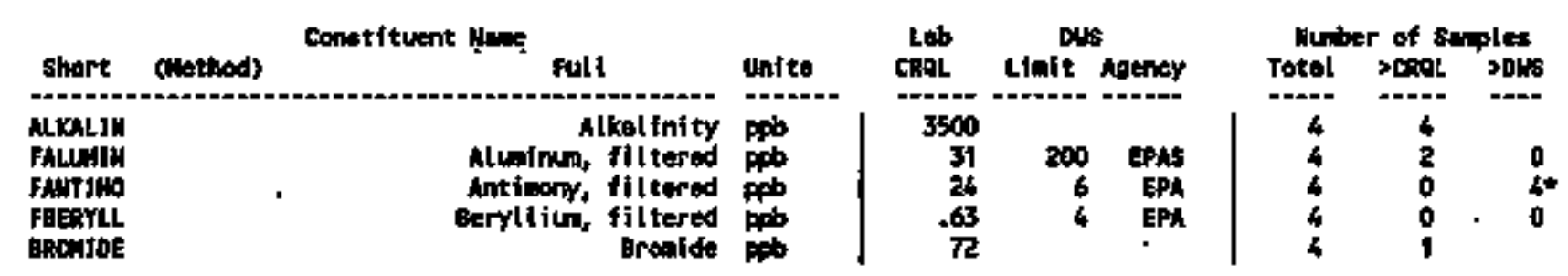


Table 2-3. Constituent List and Sumary of Results for the 100-D Pond Data for Reporting Period

July 1 through September 30, 1995.

(sheet 2 of 2)

\begin{tabular}{|c|c|c|}
\hline short (Wethob) & theme fut & Unite \\
\hline 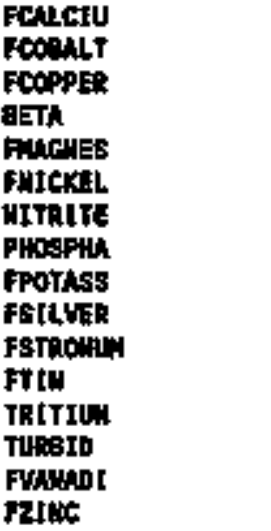 & 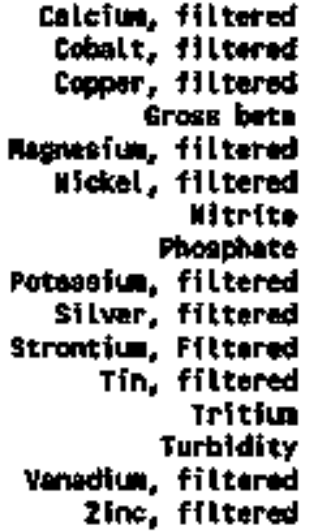 & $\begin{array}{l}p p b \\
p o b \\
p p b \\
p G i / c \\
p p b \\
p p b \\
p p b \\
p p b \\
p p b \\
p p b \\
p p b \\
p p b \\
p c i f l \\
p m u \\
p p b \\
p p b\end{array}$ \\
\hline
\end{tabular}

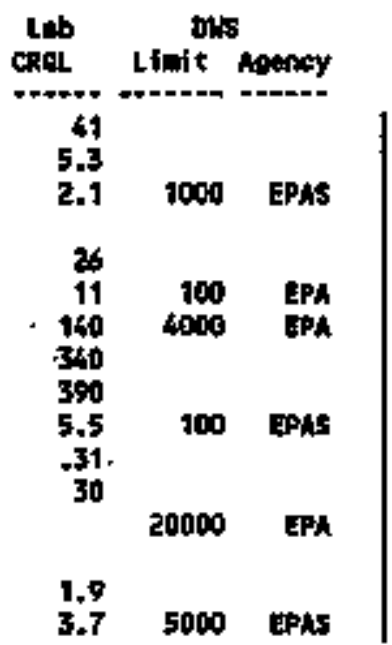

\begin{tabular}{ccc}
\multicolumn{4}{c}{ Muber of semples } \\
Total & $\rightarrow$ [ace & >ous \\
4 & 4 & \\
4 & 0 & \\
4 & 0 & 0 \\
4 & 3 & \\
4 & 4 & \\
4 & 0 & 0 \\
4 & 0 & 0 \\
4 & 0 & \\
4 & 6 & \\
4 & 0 & 0 \\
4 & 4 & \\
4 & 0 & \\
4 & 2 & 0 \\
4 & 4 & \\
4 & 4 & \\
4 & 0 & 0
\end{tabular}

For explantion of this table, stes section T.6 of raport. 
Table 2-4. Constituents with at Least One Detected Value for the 100-D Pond Data for Reporting Period July 1 through Septeriber 30, 1995.

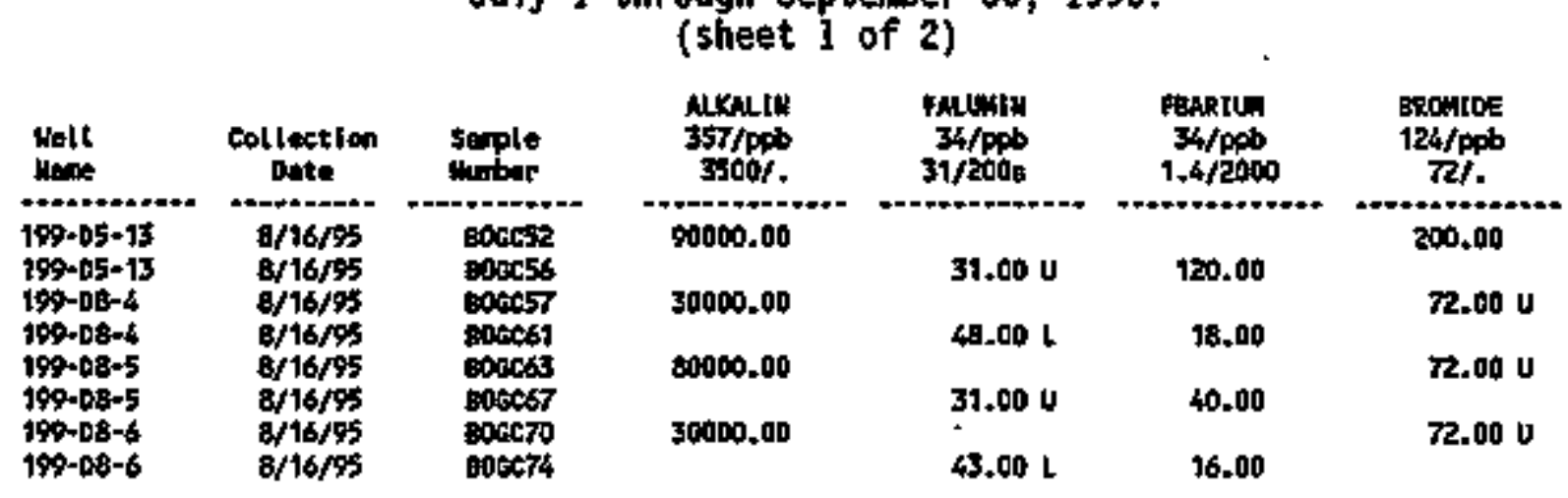

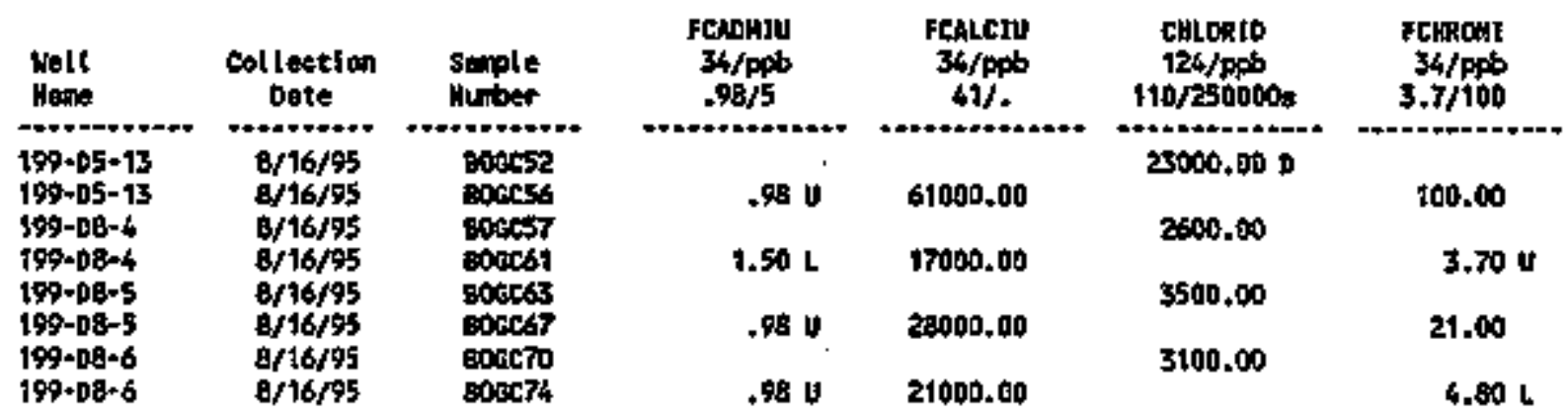

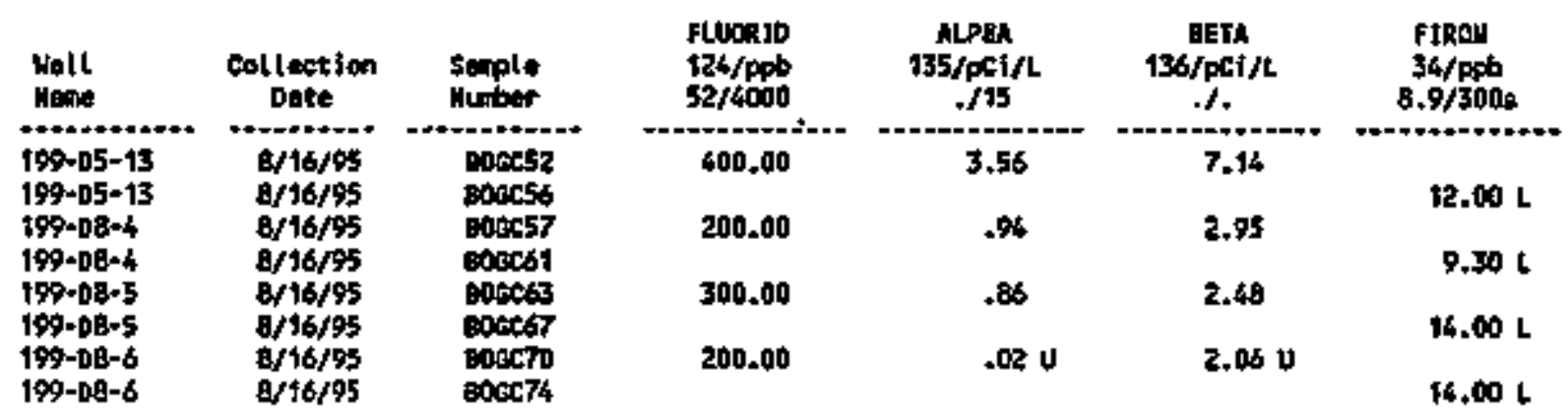


Table 2-4. Constituents with at Least One Detected Value for the 100-0 Pond Data for Reporting Period July 1 through September 30, 1995. (sheet 2 of 2 )

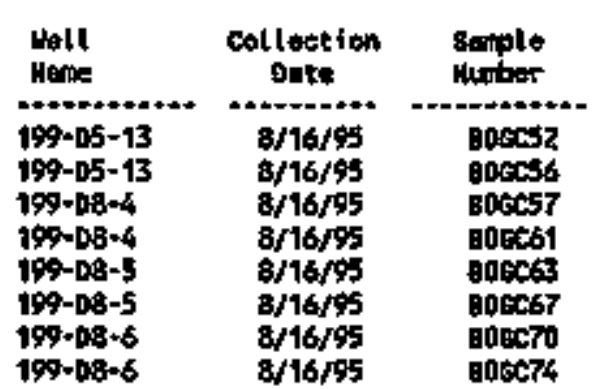

Fwhics
$34 / \mathrm{ppb}$
26.6
17000,00
750.00
7060,00
930.00

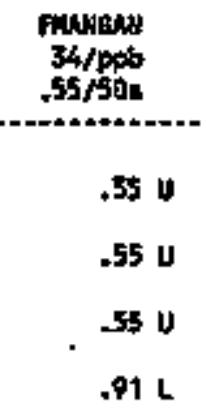

N1TRATE
$124 / 650$
$120 / 45000$
38000.000
2200.00
2500.00
4600.00

\section{FPOTASS}

$34 / \mathrm{ppb}$ $590 \%$.

4400.00

3100.00

3300.00

2200.00

\section{(2)}

well
$199-05-13$
$199-05-13$
$199-08+4$
$199-08-4$
$199-08-5$
$199-08-5$
$199-18-6$
$199-08-6$

\begin{tabular}{|c|c|}
\hline $\begin{array}{l}\text { Cof tettion } \\
\text { Dete }\end{array}$ & $\begin{array}{l}\text { strple } \\
\text { Mubar }\end{array}$ \\
\hline $\begin{array}{l}8 / 16 / 95 \\
8 / 16 / 95 \\
8 / 16 / 95 \\
8 / 16 / 95 \\
8 / 16 / 95 \\
8 / 16 / 95 \\
8 / 16 / 95\end{array}$ & 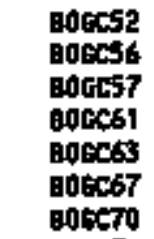 \\
\hline
\end{tabular}

$8 / 16 / 95 \quad$ 800674

$\begin{array}{cc}\text { rsootum } & \text { Fstroutin } \\ 34 / \mathrm{pHb} & 34 / \mathrm{ppb} \\ 44 / \mathrm{B} & .31 / .\end{array}$

sufate TRITIUH
124/ppb
142/pCi/L $130 / 250000$ s .$/ 20000$

- 73000,00 0

$1100.00 \mathrm{~F}$

$7600,400 \quad \$ 10,00$

3700.00 a $\quad 140.00$

5300.00 a

190.00

$\mathbf{4 0 0 . 0 0} \quad 290.00$

17000.000

$173.00 \mathrm{U}$

390.00

196.00 is

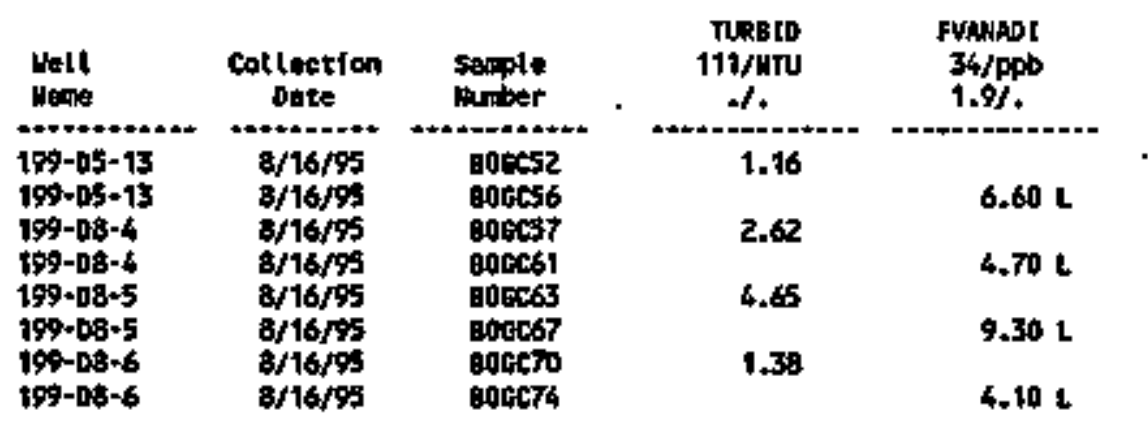

For explanstion of this trible, ste section 1.4 of report. 
Table 2-5. Contamination Indicator Parameters for the 100-D Pond Data for Reporting Period July 1 through September 30, 1995.

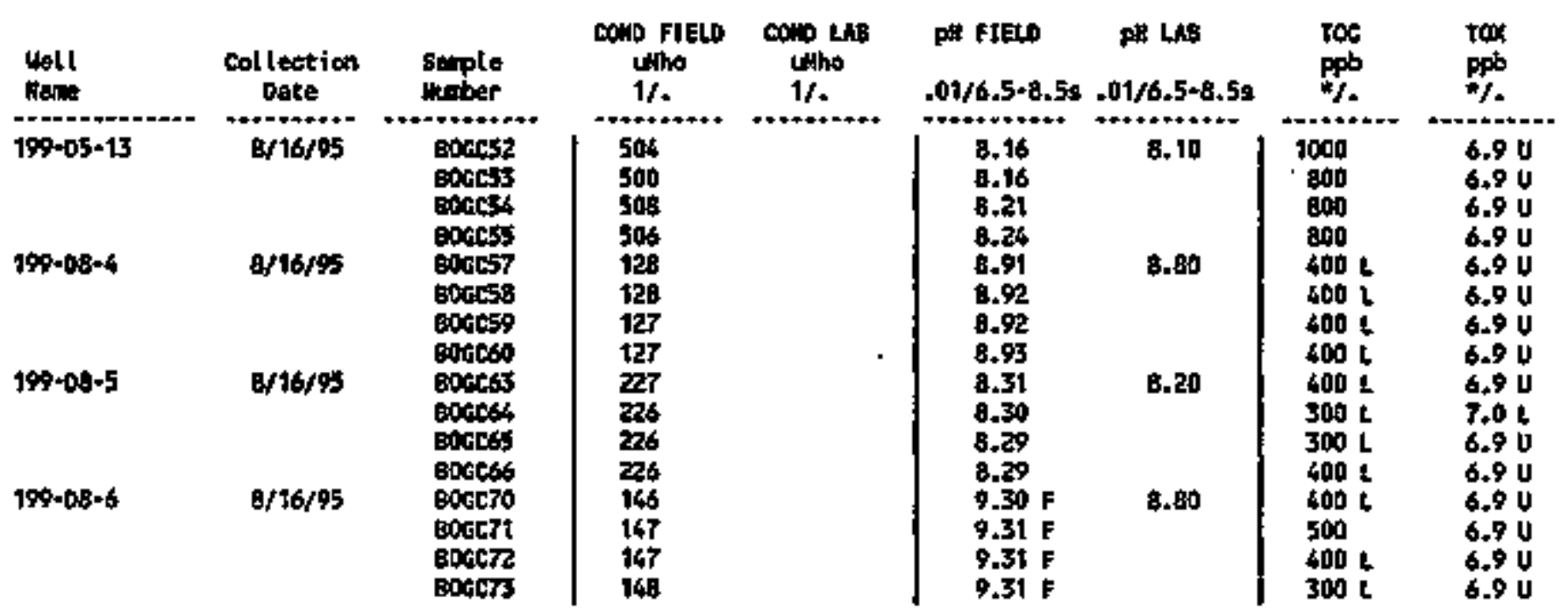

Detipetion linits for Toc and tox vary depending on the perforing laboratory. Far explanation of thie table, ste section 1.4 of report. 
COE/RL-95-69-3

This page intentionally left blank. 
DOE/RL-95-69-3

conturs

$3.0183-H$ SOLAR EYAPORATIOH BASIMS ..............

3.1 INTRODUCTIOH $\ldots \ldots \ldots \ldots \ldots \ldots \ldots \ldots$

3.2 WATER LEYEL MEASUREMEMTS $\ldots \ldots \ldots \ldots \ldots \ldots$

3.3 MATER CHEMISTRY DATA $\ldots \ldots \ldots \ldots \ldots$ 


\section{LIST OF FIGURES}

3-1 Mell Location Map for the 183-H Solar Evaporation Basins Metwork . 3-2

\section{LIST OF TABLES}

3-1 Monitoring Hell Position and Sampling Schedule for the 183-H Solar Evaporation Basins Network . . . . . . . 3-3

3-2 RCRA Water Leve] Measurement Report 183-H Solar Evaporation Basins, Third Quarter $1995 \ldots \ldots \ldots \ldots$ 3-4

3-3 Constituent List and Sumary of Results for 183-H Solar Evaporation Basins Data for Reporting Period July 1 through September 30, $1995 \ldots . . . . . . . .36$

3-4 Constituents with at Least one Detected VaTue for the 183-H solar Evaporation Bastins Data for Reporting Period JuTy 1 through September $30,1995 \ldots \ldots$ 3-7

3-5 Contaminatton Indicator Parameters for the $183-\mathrm{H}$ Solar Evaporation Basins Data for Reporting Period JuTy 1 through September 30, $1995 \ldots \ldots$ 3-9

3-6 Drinking Water Standards Exceeded for the $183-H$ Basins . . . . 3-10

3-7 Requests for Analyt fcal Data Evaluation for the 183-H Solar
Evaporation Basins, 3rd Quarter, 1995. . . . . . . . . . . . 
DOE $/ R L-95-69-3$

\subsection{3-H SOLAR EYAPORATIOA BASINS}

i. J. Hartman

Hestinghouse Hanford Company

\subsection{IHTROOUCTIOH}

The 183-H Solar Evaporation Basins (Basins) are a RCRA-regulated facility located near the Colunbia River in the 100-H Area of the Hanford Site. Originally the Basins were part of the water treatment factlity that prepared coolant for the 105-H Reactor. Subsequent to that mission, between 1973 and 1985, the Basins were used for storage and volume reduction (by solar evaporation) of liquid waste from nuclear fuel processing activities conducted in the 300 Area. The predoninant waste consisted of nitric acid solutions that had been neutralized with sodiun hydroxide before discharge into the Basins. The soluttons contained various metals and radioactive constituents (e.g., chromium, uranium, and technetium). Hazardous waste has been removed from the Basins and most of the walls have been decontaminated and demol ished. Additional decontamination and demolition is in progress.

Groundwater quality has been affected by leakage from the Basins. Until now, groundwater monitoring has been conducted as an assessmentit-level progran under 40 CFR 265 interin-status standards, as mandated by a consent agreement and comp] iance order (Ecology and EPA 1986). A final-status compliance monitoring program was initiated in Septenber 1995 (Hartman and Chou 1995) under WAC $173-303-645$.

The purpose of each RCRA well in the 183-H network, its sampling frequency, and the dates sampled during the quarter are summarized in Table 3-1. Well locations are shown in Figure 3-1.

\subsection{WATER LEVEL, MEASUREMEMTS}

Water levels were recorded before the 183-H network wells were sampled. Rout ine measurements also are made in a larger network of wells on a single day ance each quarter. Water levels are presented in Table 3-2.

\subsection{MATER CHEHISTRY DATA}

Under the final-status comp 1 fance monitoring progrant at 183-H, each senifannua] sampling ovent comprises four jndependent samples from each well, collected at monthiy intervals. The first of the four sample sets for the fall sampling event was collected in Septenber. Results are presented in Tables 3-3 through 3-5. Table 3-6 lists concentration limits that were exceeded. Table 3-7 1ists RADEs issued on suspect data. 
Figure 3-1. Well Location Map for the 183-H Solar Evaporation Basins Hetwork.

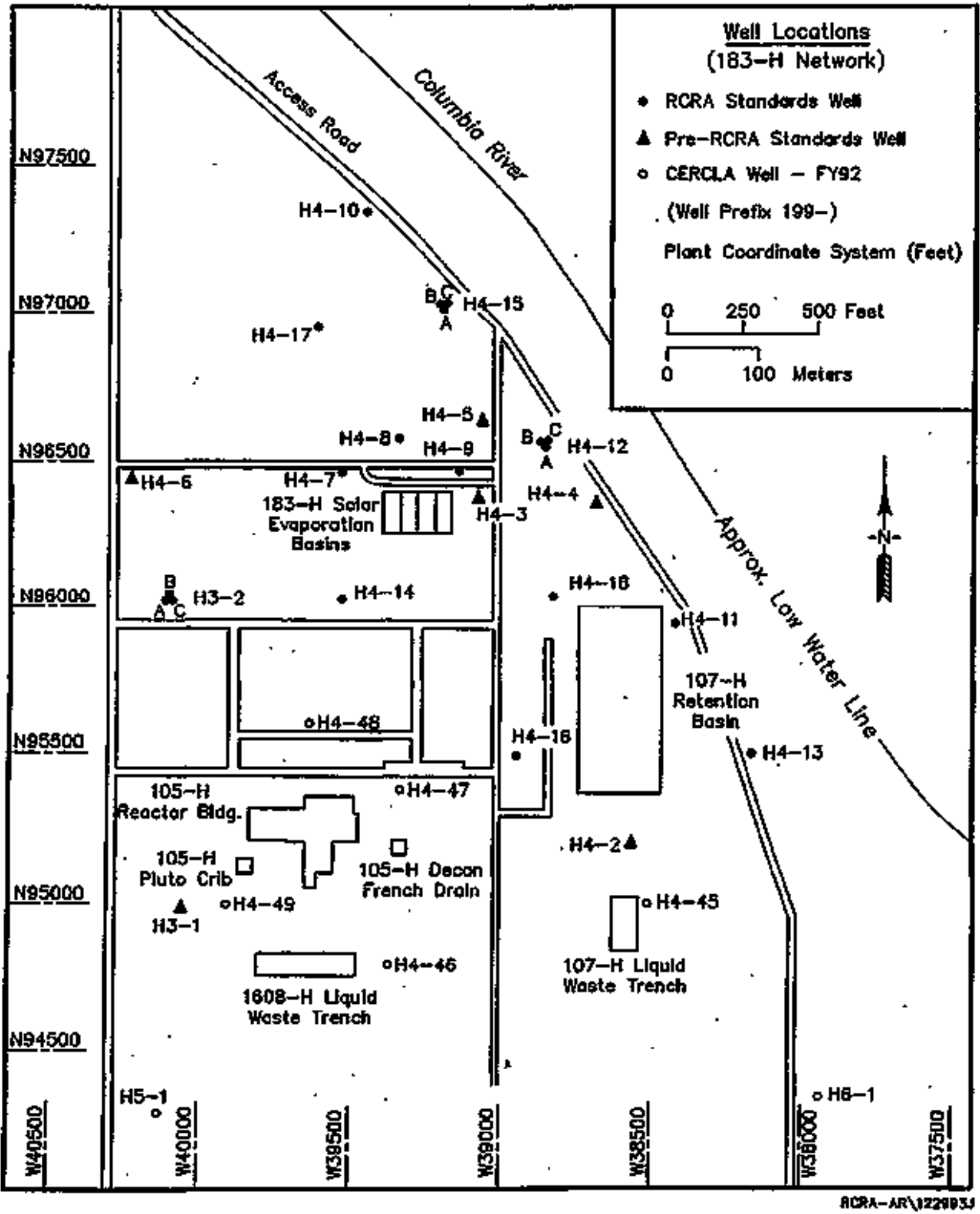


Table 3-1. Honitoring Well Position and Sampling Schedule for the 183-H Solar Evaporation Basins Metwork.

\begin{tabular}{|c|c|c|c|}
\hline $\begin{array}{l}\text { Nel] no. } \\
\text { (199-) }\end{array}$ & $\begin{array}{l}\text { Relative } \\
\text { position }\end{array}$ & $\begin{array}{c}\text { Sample } \\
\text { frequency }\end{array}$ & $\begin{array}{c}\text { Sample date, 3rd } \\
\text { Qtr } 1995\end{array}$ \\
\hline H3-2A & Upgradient & Semi annual1y & $9 / 19 / 95$ \\
\hline H4-12A & Downgradient & Semi annually & $9 / 20 / 95$ \\
\hline $\mathrm{H} 4-12 \mathrm{C}$ & Downgradjent & Semi annual1y & $9 / 20 / 95$ \\
\hline H4-18 & Downgradient & Semi annua $11 \mathrm{y}$ & $9 / 20 / 95$ \\
\hline $\mathrm{H} 4-3$ & Downgradjent & Semi annua 11y & $9 / 19 / 95$ \\
\hline H4-4 & Downgradient & Semiannually & $9 / 20 / 95$ \\
\hline $\mathrm{H} 4-6$ & Upgradient & Semiannual1y & $9 / 19 / 95$ \\
\hline $\mathrm{H} 4-\mathrm{g}$ & Downgradjent & Semtannual1y & $9 / 20 / 95$ \\
\hline
\end{tabular}

Note: Hydrogeologic units include the sandy gravels of the Hanford formation (seven we]1s) and silty sands of the Ringold Formation (we11 H4-12C).

'The semiannual sampling event comprises four independent sample sets from each well collected at monthiy intervals. The fall samples are collected in September, October, November, and December. The spring samples are collected in Harch, April, Hay, and June. 
Table 3-2. RCRA Water Level Heasurement Report 183-H Solar Evaporation Basins, Third Quarter 1995.

(sheet 1 of 2)

Wel1 Date $\begin{gathered}\text { Depth to } \\ \text { water (ft) }\end{gathered} \begin{gathered}\text { Water Tevel } \\ \text { elevation above ms) } \\ \text { (ft) }\end{gathered}$

Wel1s Wonitoring the Top of the Unconfined Aquifer (Hanford Foration)

\begin{tabular}{|c|c|c|c|c|}
\hline I99-H3-1 & $9 / 14 / 95$ & 45.75 & 375.73 & 114.52 \\
\hline $199-H 3-2 A$ & $\begin{array}{l}9 / 14 / 95 \\
9 / 19 / 95\end{array}$ & $\begin{array}{l}42.42 \\
42.54 *\end{array}$ & $\begin{array}{l}375.90 \\
375.78\end{array}$ & $\begin{array}{l}114.57 \\
114.54\end{array}$ \\
\hline $199-13-2 B$ & $9 / 14 / 95$ & 43.02 & 375.89 & 114.57 \\
\hline $199-H 4-10$ & $9 / 14 / 95$ & 31.57 & 372.87 & 113.65 \\
\hline 199-H4-11 & $9 / 14 / 95$ & 44.83 & 372.01 & 113.39 \\
\hline $199-H 4-12 \mathrm{~A}$ & $\begin{array}{l}9 / 14 / 95 \\
9 / 20 / 95\end{array}$ & $\begin{array}{l}40.82 \\
40.92^{\star}\end{array}$ & $\begin{array}{l}372.68 \\
372.58\end{array}$ & $\begin{array}{l}113.59 \\
113.56\end{array}$ \\
\hline 199-H4-12B & $9 / 14 / 95$ & 40.84 & 372.68 & 113.59 \\
\hline $199-H 4-13$ & $9 / 14 / 95$ & 46.02 & 372.18 & 113.44 \\
\hline 199-H4-14 & $9 / 14 / 95$ & 45.51 & 375.57 & 114.47 \\
\hline 199-H4-15A & $9 / 14 / 95$ & 34.37 & 373.34 & 113.79 \\
\hline $199-H 4-15 B$ & $9 / 14 / 95$ & 34.09 & 372.83 & 113.64 \\
\hline $199-14-16$ & $9 / 14 / 95$ & 49.68 & 374.55 & 114.16 \\
\hline $199-14-17$ & $9 / 14 / 95$ & 45.46 & 374.13 & 134.03 \\
\hline $199-H 4-18$ & $\begin{array}{l}9 / 14 / 95 \\
9 / 20 / 95\end{array}$ & $\begin{array}{l}48.11 \\
48.18^{k}\end{array}$ & $\begin{array}{l}374.21 \\
374.14\end{array}$ & $\begin{array}{l}114.06 \\
114.04\end{array}$ \\
\hline $199-H 4-3$ & $\begin{array}{l}9 / 14 / 95 \\
9 / 19 / 95\end{array}$ & $\begin{array}{l}46.85 \\
46.90^{*}\end{array}$ & $\begin{array}{l}373.44 \\
373.39\end{array}$ & $\begin{array}{l}113.82 \\
113.81\end{array}$ \\
\hline $199-H 4-4$ & $\begin{array}{l}9 / 14 / 95 \\
9 / 20 / 95\end{array}$ & $\begin{array}{l}41.29 \\
41.39 *\end{array}$ & $\begin{array}{l}372.95 \\
372.85\end{array}$ & $\begin{array}{l}113.6 \mathrm{~B} \\
113.64\end{array}$ \\
\hline $199-14-5$ & $9 / 14 / 95$ & 43.42 & 372.79 & 113.63 \\
\hline
\end{tabular}


Table 3-2. RCRA Water Leve] Measurement Report IB3-H Solar Evaporation Basins, Third Quarter 1995.

(sheet 2 of 2)

Well Date $\quad \begin{gathered}\text { Depth to } \\ \text { water (ft) }\end{gathered}{\underset{(f t)}{\text { eTation above msl }}}_{(\mathrm{ft})}^{\text {Water Tevel }}$

Wells Manitoring the Top of the Unconfined Aquifer (Hanford Formation)

199-H4-6

9/14/95

44.19

$44.29 *$

375.39

375.29

114.42

$9 / 19 / 95$

46.15

374.44

114.39

199-H4-7

9/14/95

46.10

373.90

114.13

199-H4-8

9/14/95

44.74

44.86*

373.84

373.72

113.96

199-H4-9

$9 / 14 / 95$
$9 / 20 / 95$

Wells Monitoring Upper Levels in the Ringold Formation

199-H3-2C

$9 / 14 / 95$

43.01

375.73

114.52

$199-\mathrm{H} 4-12 \mathrm{C}$

$9 / 14 / 95$

41.15

372.37

372.38

113.50

$9 / 20 / 95$

41.14*

372.69

113.50

199-H4-15CS

$9 / 14 / 95$

34.75

113.60

Well Monitoring Mid-Level in the Ringold Formation

199-H4-15CR

$9 / 14 / 95$

32.91

374.46

114.14

NOTES: 1. Water level elevations are calculated by subtracting the measured depth-to-water from the surveyed elevation for the well.

2. Depth-to-water values are transcribed from field records.

3. Measurements marked with an ' $k$ ' were taken at the time of sampling.

4. Heasurements marked with a ' + ' are outside of the expected range, and are suspected of error. 
Table 3-3. Constituent List and Sunary of Results for 183-H Solar Evaporation Basins Data for Reporting

Period July 1 through September 30, 1995.

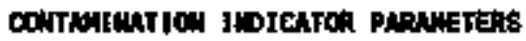

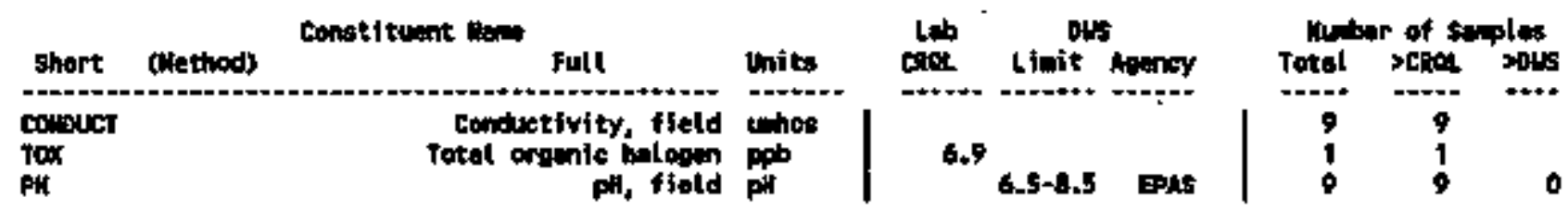

DRIMKIWO WATER PHDUETERB

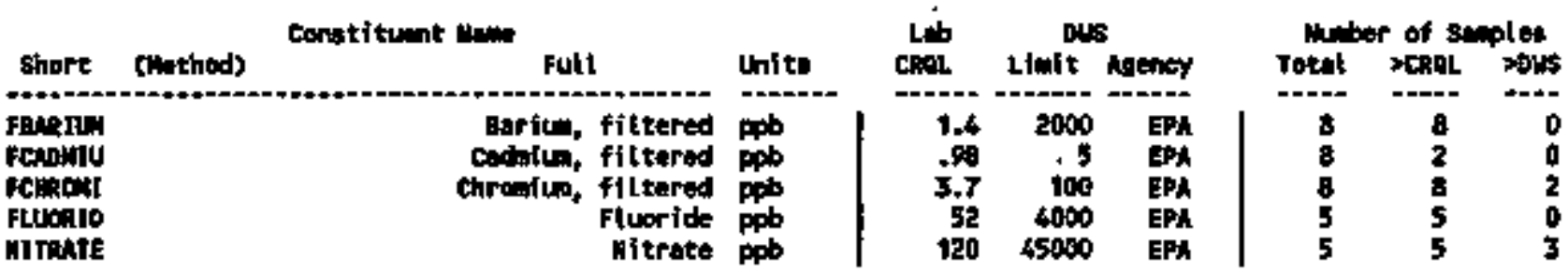

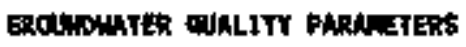

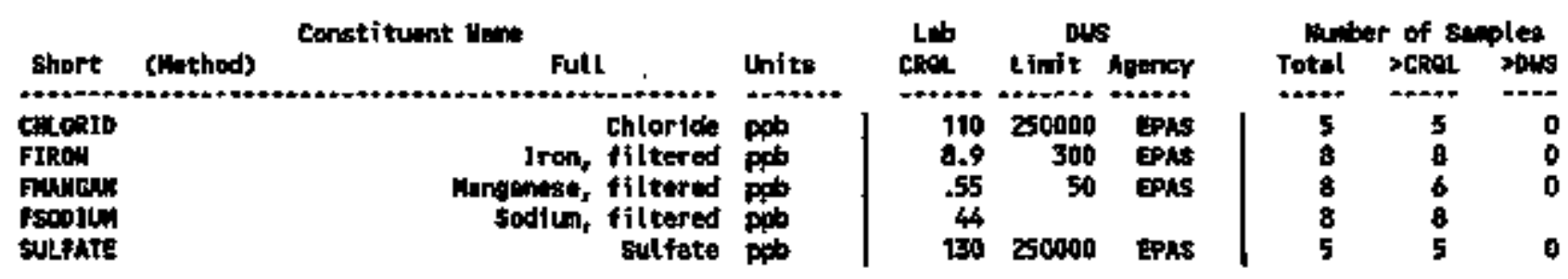

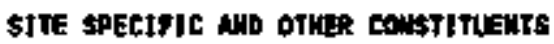

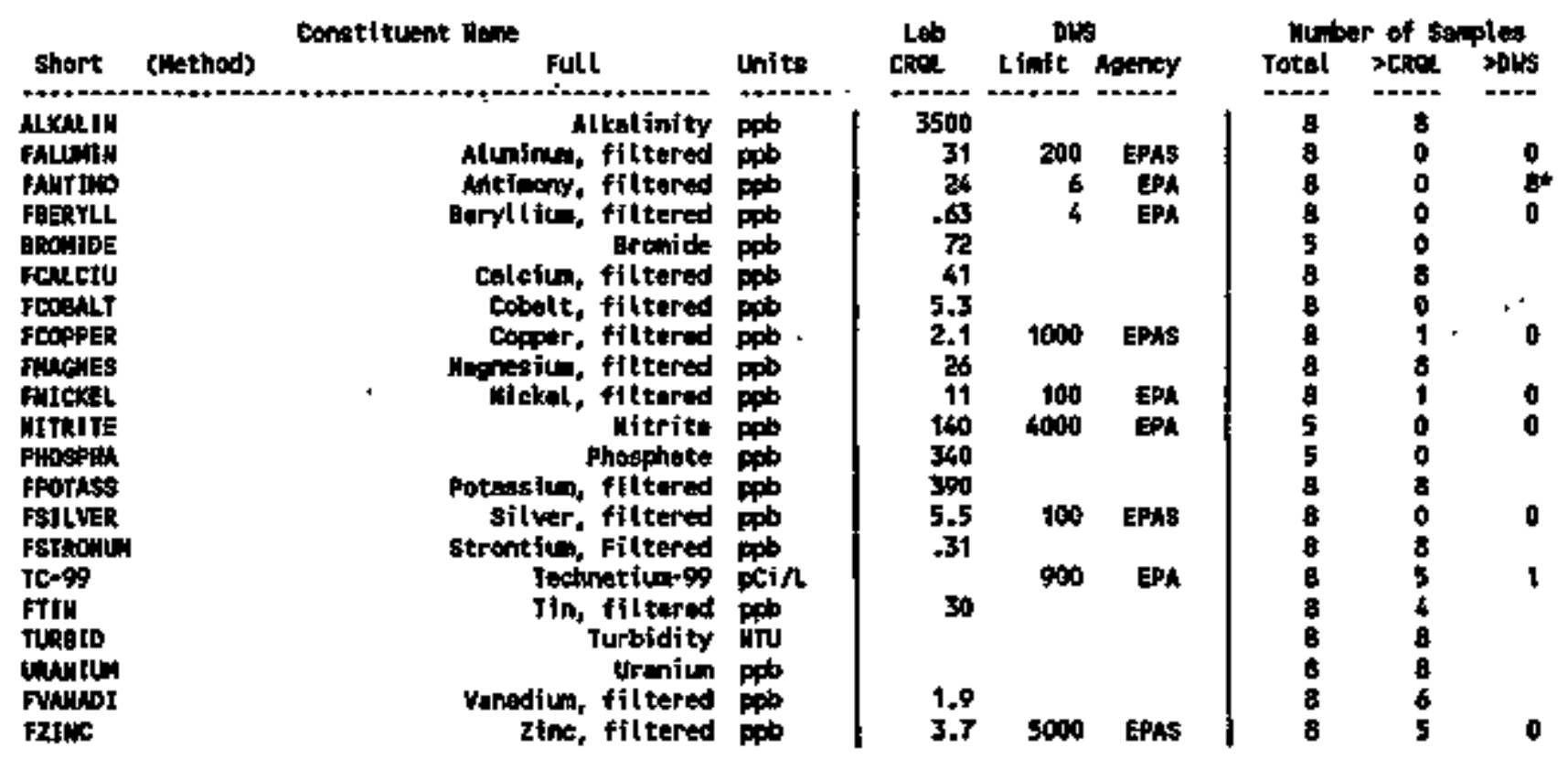

For explanatien of this teble, see Section 1.4 of repoft. 
Table 3-4. Constituents with at Least One Detected Value for the 1B3-H Solar Evaporation Basins Data for-Reporting Period July 1 through Septenber 30, 1995.

(sheet 1 of 3)

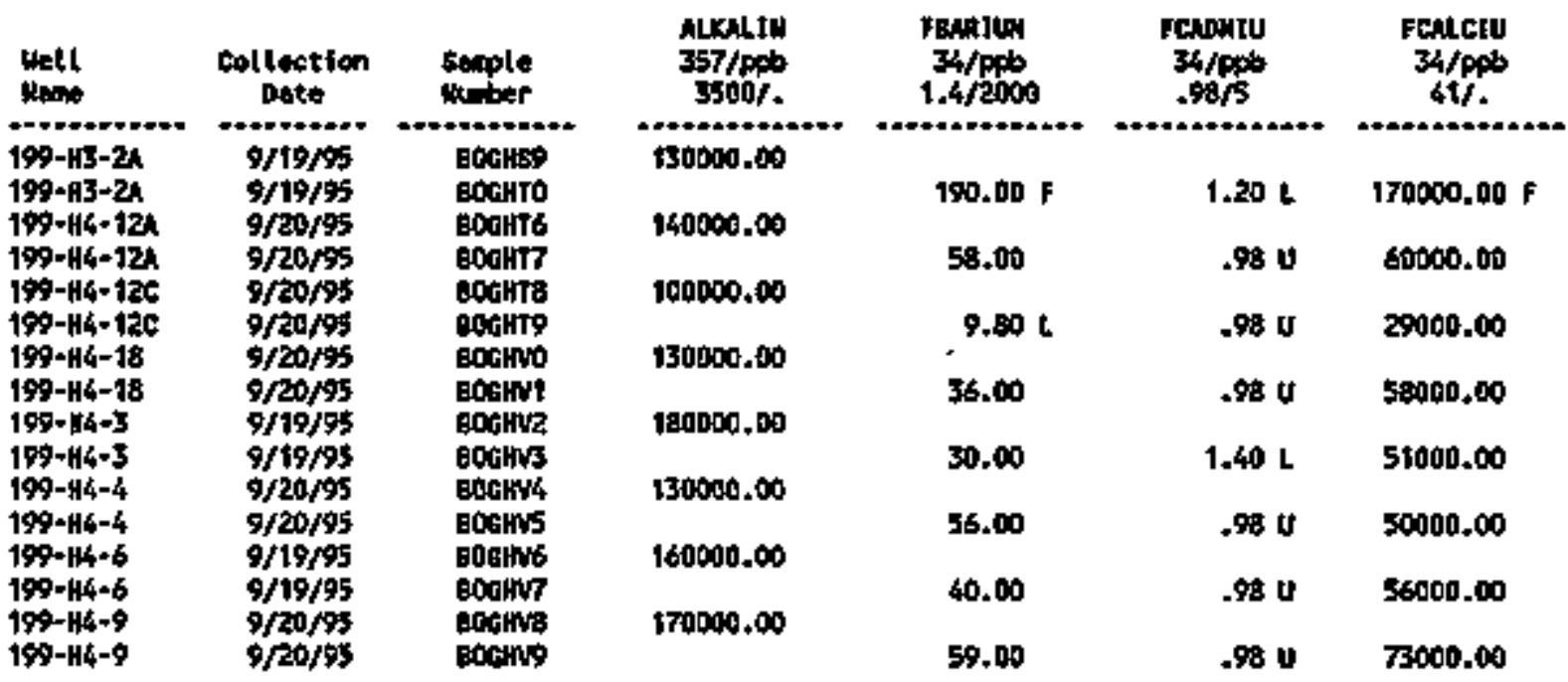

\begin{tabular}{|c|c|c|}
\hline Welt & $\begin{array}{l}\text { Collection } \\
\text { Dote }\end{array}$ & $\begin{array}{l}\text { semple } \\
\text { thitor }\end{array}$ \\
\hline 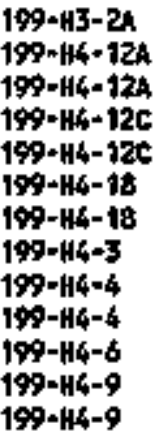 & $\begin{array}{l}9 / 19 / 95 \\
9 / 20 / 95 \\
9 / 20 / 95 \\
9 / 20 / 95 \\
9 / 20 / 95 \\
9 / 20 / 95 \\
9 / 20 / 45 \\
9 / 19 / 95 \\
9 / 20 / 95 \\
9 / 20 / 55 \\
9 / 19 / 95 \\
9 / 20 / 95 \\
9 / 20 / 95\end{array}$ & 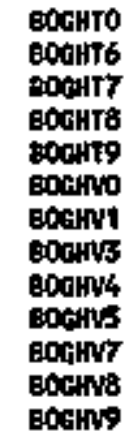 \\
\hline
\end{tabular}

\begin{tabular}{|c|c|c|c|}
\hline $\begin{array}{c}\text { chlokto } \\
\text { t24/ppb } \\
110 / 2 \text { soonos }\end{array}$ & $\begin{array}{r}\text { Fch:R4) } \\
34 / \mathrm{ppb} \\
3.7 / 100\end{array}$ & $\begin{array}{l}\text { FCOPPER } \\
34 / \mathrm{pob} \\
2.1 / 100 \mathrm{gs}\end{array}$ & $\begin{array}{l}\text { Fturonjo } \\
124 / \mathrm{pt}^{4} \\
52 / 4000\end{array}$ \\
\hline - & $\begin{array}{l}190.00 \mathrm{~F} \\
\mathrm{~F}\end{array}$ & $\begin{array}{c}16.00 \mathrm{LF} \\
16+\cdots+\cdots+*\end{array}$ & \\
\hline 8700.00 & 74.00 & $2.10 \mathrm{U}$ & 500.00 \\
\hline 2300.00 & & & 400.00 \\
\hline 6200.00 & 290.00 & 2.100 & 500,00 \\
\hline & $\begin{array}{r}47.00 \\
100.00\end{array}$ & $\begin{array}{l}2.10 \mathrm{U} \\
2.10 \mathrm{U}\end{array}$ & \\
\hline 7700.00 & $\frac{80.00}{75.00}$ & $\begin{array}{l}2.100 \\
2.10 \mathrm{U}\end{array}$ & 700.00 \\
\hline 91000,000 & 70.00 & $2.10 \mathrm{t}$ & 600,00 \\
\hline
\end{tabular}

\begin{tabular}{|c|c|c|}
\hline Well & $\begin{array}{c}\text { Collection } \\
\text { Dete }\end{array}$ & $\begin{array}{l}\text { sonple } \\
\text { number }\end{array}$ \\
\hline $\begin{array}{l}199-\mathrm{HB}-2 \mathrm{C} \\
199-\mathrm{H4}-12 \mathrm{~A} \\
199-\mathrm{H}-12 \mathrm{C} \\
199-\mathrm{H4}-18 \\
199-\mathrm{H4}-3 \\
199-\mathrm{H4}-4 \\
199 \cdot \mathrm{H4}-6 \\
199-\mathrm{H4}-\mathrm{g}\end{array}$ & $\begin{array}{l}9 / 19 / 95 \\
9 / 20 / 95 \\
9 / 20 / 95 \\
9 / 20 / 95 \\
9 / 19 / 95 \\
9 / 20 / 95 \\
9 / 19 / 95 \\
9 / 20 / 95\end{array}$ & 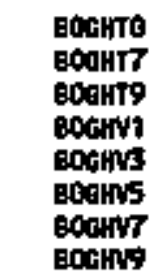 \\
\hline
\end{tabular}

\begin{tabular}{|c|c|}
\hline $\begin{array}{c}\text { FIRDN } \\
34 / \mathrm{pOH}^{2} \\
\text { B.9/3008 }\end{array}$ & $\begin{array}{c}\text { FUACHES } \\
34 / P Q 6 \\
26 / .\end{array}$ \\
\hline $\begin{array}{l}28.00 \mathrm{~L} \\
18.00 \mathrm{~L} \\
14.00 \mathrm{~L} \\
13.00 \mathrm{~L} \\
28.00 \mathrm{~L} \\
9.00 \mathrm{~L} \\
13.00 \mathrm{~L} \\
40.00\end{array}$ & $\begin{array}{c}30000.00 \mathrm{~F} \\
9000.00 \\
10000.00 \\
20009.00 \\
12000.00 \\
8500.00 \\
14009.00 \\
13000.00\end{array}$ \\
\hline
\end{tabular}

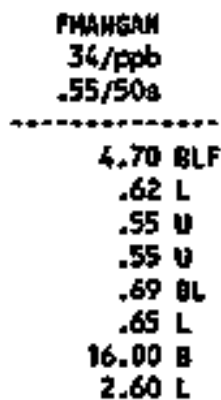

Fiscti
$34 / \mathrm{ppb}$
$11 / 100$
$43.00 \mathrm{~F}$
$11.00 \mathrm{v}$
$11.00 \mathrm{v}$
$71.00 \mathrm{v}$
$\$ 1.00 \mathrm{v}$
$11.00 \mathrm{v}$
$11.00 \mathrm{v}$
$11.00 \mathrm{v}$


Table 3-4. Constituents with at Least One Detected Value for the 183-H Solar Evaporation Basins Data for Reporting Period July 1 through September 30, 1995.

\begin{tabular}{|c|c|c|}
\hline Uell & $\begin{array}{c}\text { Collettion } \\
\text { Date }\end{array}$ & $\begin{array}{l}\text { Sarple } \\
\text { inniber }\end{array}$ \\
\hline $\begin{array}{l}-24 \\
-12 \lambda \\
-12 \lambda \\
-124 \\
-12 c \\
-18 \\
-18 \\
-3 \\
-4 \\
-4 \\
-6 \\
-9\end{array}$ & $\begin{array}{l}9 / 19 / 95 \\
9 / 20 / 95 \\
9 / 20 / 95 \\
9 / 20 / 95 \\
9 / 20 / \% 5 \\
9 / 20 / \% 5 \\
9 / 20 / 95 \\
9 / 19 / 95 \\
9 / 20 / 95 \\
9 / 20 / 95 \\
9 / 19 / 95 \\
9 / 20 / 95 \\
9 / 20 / 95\end{array}$ & 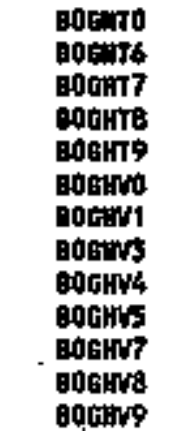 \\
\hline
\end{tabular}
(sheet 2 of 3 )

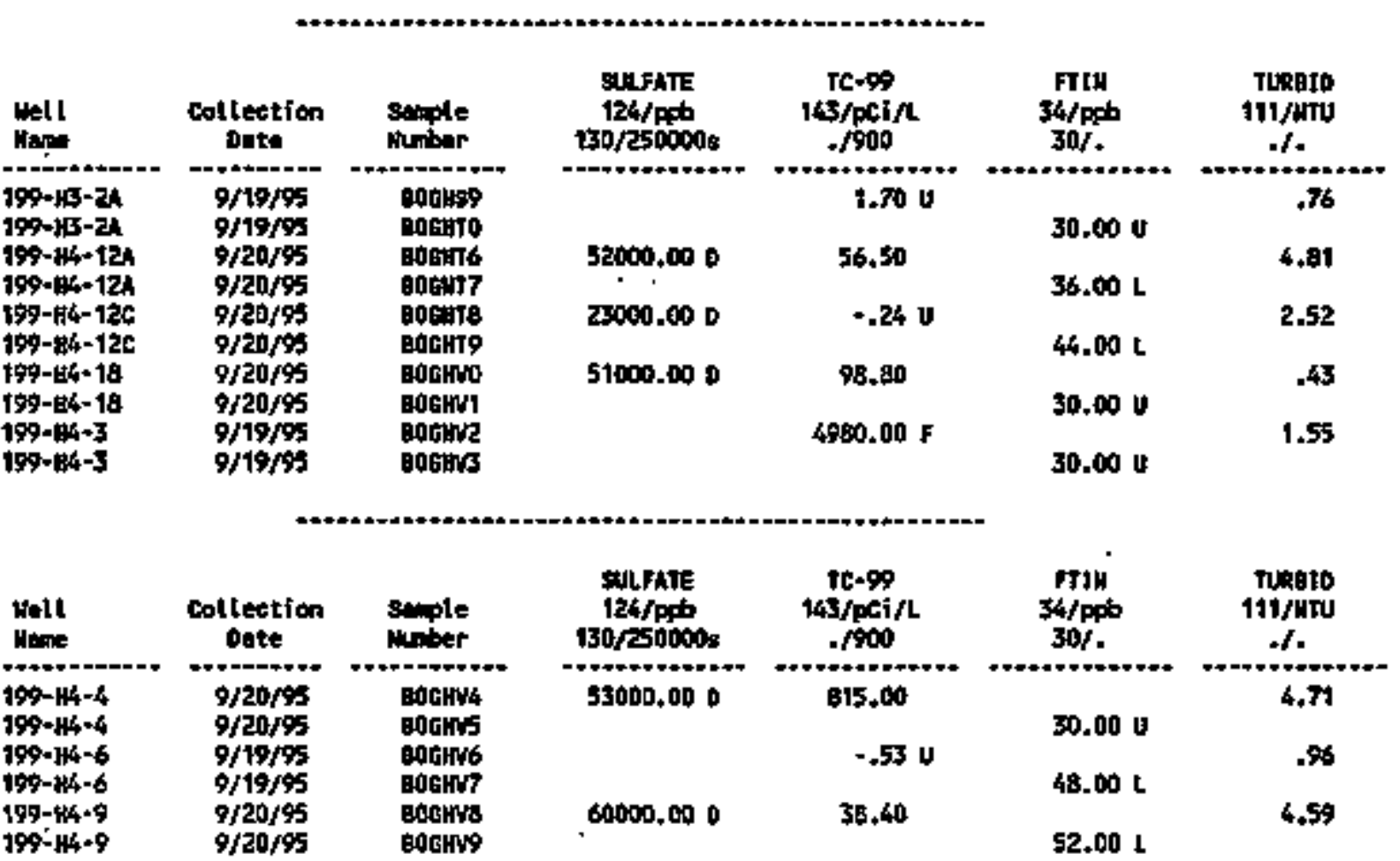


Tab]e 3-4. Constituents with at Least One Detected Yalue for the 183-H Solar Evaporation Basins Data for Reporting Period July I through September 30, 1995. (sheet 3 of 3)

\begin{tabular}{|c|c|c|c|c|c|}
\hline Wem & $\begin{array}{c}\text { collection } \\
\text { bote }\end{array}$ & $\begin{array}{l}\text { Sariple } \\
\text { Muntoper }\end{array}$ & $\begin{array}{c}\text { URANILI } \\
145 / \mathrm{ppb} \\
. / 4\end{array}$ & $\begin{array}{l}\text { Paanob1 } \\
36 / p p b \\
1.97:\end{array}$ & $\begin{array}{c}\text { FzJwc } \\
34 / \mathrm{ppb}^{\circ} \\
3.7 / 5000 \mathrm{~s}\end{array}$ \\
\hline $\begin{array}{l}199-113-2 A \\
199-135-2 A\end{array}$ & $\begin{array}{l}9 / 19 / 45 \\
9 / 19 / 95\end{array}$ & $\begin{array}{l}\text { BOEHSS } \\
\text { BOCHTO }\end{array}$ & 3.37 & $1.90 \mathrm{U}$ & $6.90 \mathrm{~L}$ \\
\hline $196-14-12 n$ & praorss & Boctit6 & 7.48 & & \\
\hline $\begin{array}{l}196-144-12 \lambda \\
199-134-12 c\end{array}$ & $\begin{array}{l}9 / 20 / 58 \\
9 / 20 / 95\end{array}$ & $\begin{array}{l}\text { BDEHT? } \\
\text { BDEHT: }\end{array}$ & 1.75 & $1.90 \mathrm{U}$ & 5.801 \\
\hline & $\begin{array}{l}9 / 20 / \% 5 \\
9 / 20 / 95\end{array}$ & $\begin{array}{l}\text { BOEHHS } \\
\text { BOEHWO }\end{array}$ & 9.94 & 24.00 & $3.70 \mathrm{~V}$ \\
\hline $\begin{array}{l}199-14 k-18 \\
199-\mu 4-3\end{array}$ & $9 / 20 / 95$ & Boofivi & $73 \mathrm{mo}$ & $5.30 \mathrm{~L}$ & $4.00 \mathrm{~L}$ \\
\hline $199-144-3$ & 9/19/4s & Bopers & Crs.0 & $6.70 \mathrm{~L}$ & $3.70 \mathrm{t}$ \\
\hline $\begin{array}{l}799-114-6 \\
195-184-6\end{array}$ & $\begin{array}{l}9 / 20 / 95 \\
9 / 19 / 95\end{array}$ & $\begin{array}{l}\text { gogws } \\
\text { gogsws }\end{array}$ & 5.17 & $3.40 \mathrm{~L}$ & $3.70 \mathrm{U}$ \\
\hline $\begin{array}{l}799-134-6 \\
199-144-6\end{array}$ & $\begin{array}{l}9 / 19 / 55 \\
9 / 20 / 95\end{array}$ & $\begin{array}{l}\text { Boswh7 } \\
\text { Bogews }\end{array}$ & 6.07 & $5.40 \mathrm{~L}$ & $6.80 \mathrm{~L}$ \\
\hline & $9 / 20 / 95$ & O00S\$49 & & $6.20 \mathrm{~L}$ & $6.60 \mathrm{~L}$ \\
\hline
\end{tabular}

For exptanation of this toble, get swetion $t .4$ of repert.

Table 3-5. Contamination Indicator Parameters for the 183-H Solar Evaporation Basins Data for Reporting Period July I through September 30, 1995.

\begin{tabular}{|c|c|c|c|c|c|c|c|c|}
\hline Well & $\begin{array}{l}\text { Collection } \\
\text { Date }\end{array}$ & 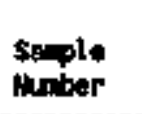 & $\begin{array}{c}\text { CONb FIELD } \\
\text { tho } \\
\text { I/. }\end{array}$ & $\begin{array}{c}\text { Cond LB } \\
\text { W1/20 } \\
1 \%\end{array}$ & $\begin{array}{l}\text { PH FJEL } \\
.01 / 6.5-9.58\end{array}$ & $\begin{array}{c}\text { PH LAB } \\
.01 / 6.5-8.58\end{array}$ & $\underset{* / .}{\operatorname{TOC}}$ & $\begin{array}{l}\operatorname{Tox} \\
\mathrm{ppb} \\
+/ .\end{array}$ \\
\hline 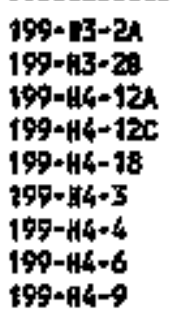 & $\begin{array}{l}9 / 19 / 95 \\
6 / 15 / 95 \\
9 / 20 / 95 \\
9 / 20 / 95 \\
9 / 20 / 95 \\
9 / 19 / 95 \\
9 / 20 / 95 \\
9 / 19 / 95 \\
9 / 20 / 95\end{array}$ & 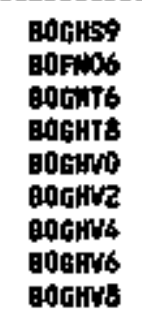 & $\begin{array}{l}461 \\
471 \\
472 \\
263 \\
470 \\
2590 \\
755 \\
519 \\
576\end{array}$ & & $\begin{array}{l}7.92 \\
7.88 \\
7.87 \\
8.05 \\
7.77 \\
7.58 \\
7.94 \\
6.23 \\
7.80\end{array}$ & & & 10.0 \\
\hline
\end{tabular}

- Dotection Ifaits for The and Tox vary depending on the perforelng laboratory. For explanation of this table, see section 1.4 of report. 
Table 3-6. Drinking Water Standards Exceeded for the 183-H Basins, Third Quarter, 1995.

\begin{tabular}{|c|c|}
\hline Constituent (concentration 1 init $\mathrm{t}^{\mathrm{a}}$ ) & $\begin{array}{l}\text { Nells exceeding } \\
\text { concentration limit }\end{array}$ \\
\hline Chromium, filtered samples (122 ppb) & $H 3-2 A, H 4-12 C$. \\
\hline Nitrate $(45,000 \mathrm{ppb})$ & H4-4, H4-18, H4-9. \\
\hline Technetium-99 (900 pCt/L) & $144-3^{c}$ \\
\hline
\end{tabular}

'Concentration liait is DWS or background (Hartalan and Chou 1995).

Anion data not yet recejved for wells $\mathrm{H}_{4-2 A} \mathrm{H4-3}$, and H4-6.

'Data are in question. Requests for Analytical Data Evaluation were submitted.

Table 3-7. Requests for Analytical Data Evaluation for the 183-H Solar Evaporation Basins, 3rd Quarter, 1995.

\begin{tabular}{|l|l|l|l|}
\hline We11 Ho. & Sample Date & \multicolumn{1}{|c|}{ Constituent } & \multicolumn{1}{|c|}{ Reasons } \\
\hline H3-2A & $9 / 19 / 95$ & $\begin{array}{l}\text { Fi1tered metals } \\
\text { (barium, calcium, } \\
\text { chromium, copper, } \\
\text { magnesium, manganese, } \\
\text { nicke1, potassium, } \\
\text { sodiun, strontium) }\end{array}$ & $\begin{array}{l}\text { Higher than historical } \\
\text { trend; does not match } \\
\text { conductivity. }\end{array}$ \\
\hline H4-3 & $9 / 19 / 95$ & Technet 1um-99 & $\begin{array}{l}\text { Higher than historical } \\
\text { trend. }\end{array}$ \\
\hline H4-3 & $9 / 19 / 95$ & Field conductivity & $\begin{array}{l}\text { Higher than historical } \\
\text { trend; does not match } \\
\text { cations. }\end{array}$ \\
\hline
\end{tabular}


$D 0 E / R L-95 \div 69-3$

CONTETS

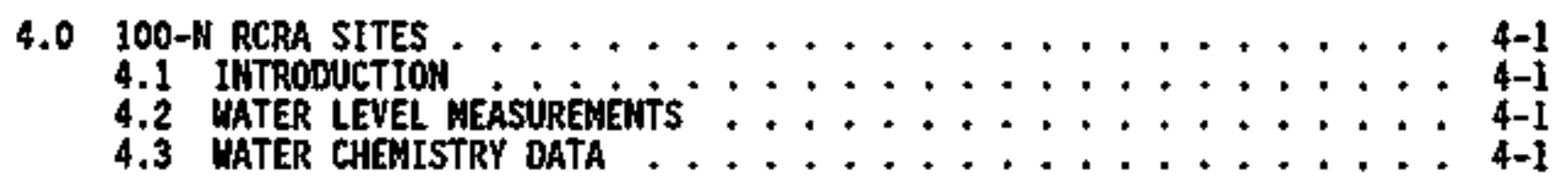




\section{LIST OF FIEURES}

4-1 Mell Location Map for the 1301-N, 1324-N/NA,

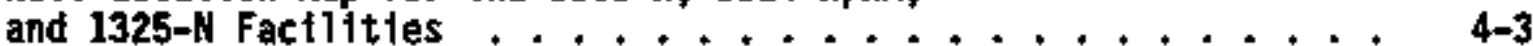

\section{LIST OF TABLES}

4-I Monitoring Me11 Purpose and Sampling Schedule for the 100-N Area . 4-4

4-2 RCRA Water Level Heasurement Report. 100-N Area,

Third Quarter $1995 \ldots . . . . . . . . . .4$ 4-5

4-3 Const ttuent List and Summary of Results for 100-M RCRA Sites

Data for Reporting Perfod July 1 through Septenber 30, 1995 . . . 4-7

4-4 Constituents with at Least one Detected value for the 100-N RCRA Sites Data for Reporting Period July 1 through September 30, $1995 \ldots \ldots$. . . . . . . . . 40

4-5 Contamination Indicator Parameters for the 100-N RCRA Sites Data for Reporting Period July 1 through September $30,1995 \ldots \ldots$ 4-27

4-6 Drinking water Standards Exceeded for 100-N Area RCRA sites :. 4-29

4-7 Requests for Analytical Data Evaluation for 100-N RCRA Sites, Thi rd Quarter, 1995. ................ 4-29 


\title{
4.0 100-N RCA SITES
}

\author{
A. J. Hartwan \\ Nestinghouse Hantord Company
}

\subsection{Introouction}

Four interiw-status RCRA disposs? units are located in the 100-h Area of the Hanford Site: the 1301-N Liquid Waste Disposal Facility (LWDF), the 1324-H Surface Impoundment, the 1324-NA Percolation Pond, and the 1325-N LWDF. These units and their associated well locations are shown in Figure 4-1. Sampling networks are 1isted in Table 4-1. The 1324-N and 1324-HA units are monitored as a single site. RCRA monitoring began in 1ate 1987. The 1324-W/M site is menitored under a groundwater quality assessment program because of elevated levels of TOX (40 CFR 265.93). The 1301-N and 1325-N LWDF are monitored under indicator parameter evaluation programs (40 CFR 265.92).

The 1301-N and 1325-N LWDFs were. used for disposal of liquid effluent from the $N$ Reactor and associated facilities. Primary waste constituents were radionuclides, including strontium-90, tritiun, and cobalt-60. Minor amounts of hazardous waste also were present in the waste streams (e.g., hydrazine). The 1324-H/HA units received corrosive waste. The 100-H RCRA units are currently not in use.

\subsection{HATER LEYEL MEASURENENTS} Table 4-2.

Water levels were measured before sample collection. Data are listed in

\subsection{MATER CTEHISTRY DATA}

Al1 of the 100-N RCRA we11s were sampled as scheduled last quarter. Downgradient wel1s for 1301-H and 1325-N did not exceed critical mean values for pH, conductivity, TOC, or TOX.

Two of four ToX sample bottles for well N-3 were broken. Because of sampler errors, quadruplicate $\mathrm{pH}$ and conductivity readings were not made at we]1 $\mathrm{N}-32$ and no field readings were made at weit $\mathrm{N}-75$, which is a pumping well for a ${ }^{90} \mathrm{Sr}$ pump-and-treat system.

Grotendwater chemistry data received before the cut-off date are 1 tsted in Tables 4-3 through 4-5. Data qual if ters and data flags are discussed in Sections 1.2 and 1.4 , respectively. DiSs were exceeded for the constituents listed in Table 4-6. RADEs submitted last quarter are listed in Table 4-7.

Sampling and analysis were conducted in coordination with the 100-MR-2 and sitewide survefllance programs to avoid duplication. For this reason, Tables 4-3 through 4-5 include data not requested specifically for the RCRA program (e.g., unfiltered meta]s, filtered anions, radionuclides, etc.). Hote that one of the methods of $\mathrm{pH}$ testing 1 tsted in Table 4-3 is "filtered pH." 


\section{DOE/RL-95-69-3}

These results are actually field readings from unfiltered groundwater that were input to the database under sample numbers associated with filtered samples (e.g. metals). 


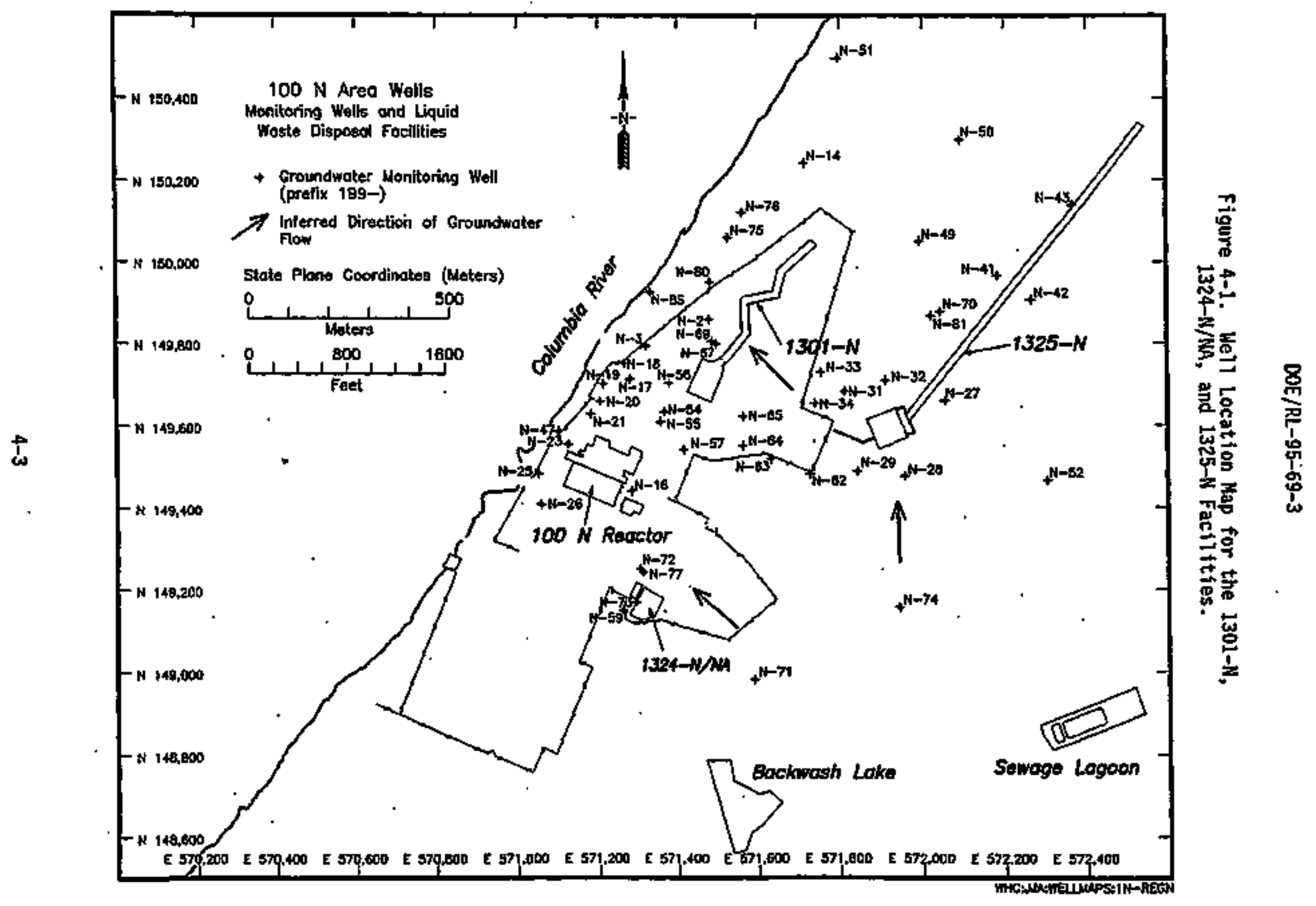


Table 4-I. Monitoring Well Purpose and Sampling Schedute for the 100-N Area.

\begin{tabular}{|c|c|c|c|c|}
\hline $\begin{array}{l}\text { Well no. } \\
(199-)\end{array}$ & RCRA site & Relative position & $\begin{array}{c}\text { Sarple } \\
\text { frequency }\end{array}$ & $\begin{array}{l}\text { Sample date, } \\
\text { 3rd Qtr 1995 }\end{array}$ \\
\hline $\mathrm{H}-2$ & $1301-\mathrm{N}$ & Downgradient & Semfannually & $9 / 8 / 95$ \\
\hline$M-3$ & $1301-\mathrm{N}$ & Downgradient & Semiannually & $9 / 15 / 95$ \\
\hline $\mathrm{N}-14$ & $1301-\mathrm{N}$ & Doimgradient & Semfannual1y & $8 / 30 / 95$ \\
\hline$N-27$ & $1325-N$ & Downgradient & Semiannualiy & $8 / 23 / 95$ \\
\hline $\mathrm{N}-32$ & $1325-N$ & Downgradient & Semianntualiy & $9 / 7 / 95$ \\
\hline $\mathrm{N}-34$ & $1301-n$ & Upgradient & Semiannual]y & $8 / 23 / 95$ \\
\hline$N-41$ & $1325-N$ & Downgradient & Semiannual]y & $8 / 23 / 95$ \\
\hline$N-43$ & $1325-N$ & Downgradjent & Semiannually & $8 / 28 / 95$ \\
\hline$N-57$ & $1301-N$ & Upgradtent & Semiannual1y & $8 / 25 / 95$ \\
\hline$N-59$ & $1324-N / N A$ & Downgradient & QuarterTy & $8 / 25 / 95$ \\
\hline $\mathrm{N}-67$ & $1301-H$ & Downgradient. & Semfannually & $9 / 7 / 95$ \\
\hline$N-69$ & $1301-N$ & $\begin{array}{l}\text { Doingradient; } \\
\text { bottom of aquifer }\end{array}$ & Semiannually & $9 / 8 / 95$ \\
\hline $\mathrm{N}-70$ & $1325-N$ & $\begin{array}{l}\text { Downgradjent; } \\
\text { bottom of aquifer }\end{array}$ & Semi annualiy & $9 / 18 / 95$ \\
\hline$N-71$ & 1324-N/NA & Upgradient & Quąrterly & $8 / 28 / 95$ \\
\hline$N-72$ & $1324-N 1 / \mathrm{WA}$ & Downgradjent & Quarterly & $8 / 28 / 95$ \\
\hline $\mathrm{N}-73$ & 1324-N/MA & Downgradient & Quarteriy & $8 / 28 / 95$ \\
\hline $\mathrm{N}-74$ & $1325-N$ & Upgradient & Seriannualiy & $8 / 28 / 95$ \\
\hline$N-75^{\circ}$ & $1301-N$ & Downgradient & Semiannually & $9 / 12 / 95$ \\
\hline$N-76$ & $1301-N$ & Downgradient & Seniannually & $9 / 5 / 95$ \\
\hline $\mathrm{N}-77$ & $1324-N / N A$ & $\begin{array}{l}\text { Downgradient; } \\
\text { bottom of aquifer }\end{array}$ & Quarter]y & $9 / 15 / 95$ \\
\hline$N-81$ & $1325-4$ & Downgradient & Semi annua $11 y$ & $8 / 30 / 95$ \\
\hline
\end{tabular}

Note: The hydrogeologic unit monitored is the unconfined aquifer, which comprises sands and gravels of the Ringold Formation.

RCRA = Resource Conservation and Recovery Act of 1976 .

Extraction well for ${ }^{90} \mathrm{Sr}$ pump-and-treat system, began operating in August 1995. 
Table 4-2. RCRA Mater Level Heasurement Report 100-N Area, Third Quarter 1995.

(sheet 1 of ' 2 )

\begin{tabular}{|c|c|c|c|c|}
\hline WeII & Date & $\begin{array}{l}\text { Depth to } \\
\text { water (ft) }\end{array}$ & $\begin{array}{l}\text { Wate } \\
\text { elevatiol } \\
\text { (ft) }\end{array}$ & $\begin{array}{l}\text { Tever } \\
\text { above msl } \\
\text { (m) }\end{array}$ \\
\hline \multicolumn{5}{|c|}{ Welis Monitoring the Top of the Unconfined Aquifer } \\
\hline $199-N-14$ & $8 / 30 / 95$ & $68.63^{*}$ & 384.52 & 117.20 \\
\hline $199-14-2$ & $9 / 08 / 95$ & $74.68 *$ & 385.15 & 117.39 \\
\hline $199-\mathrm{N}-27$ & $8 / 23 / 95$ & $61.81 *$ & 387.27 & 118.04 \\
\hline $199-N-3$ & $9 / 15 / 95$ & $75.30^{*}$ & 384.15 & 117.09 \\
\hline $199-N-32$ & $9 / 07 / 95$ & $75.29 *$ & 386.79 & 117.89 \\
\hline 199-N-34 & $8 / 23 / 95$ & $72.88 *$ & 386.75 & 117.88 \\
\hline $199-N-41$ & $8 / 23 / 95$ & $72.37 *$ & 385.22 & 117.42 \\
\hline $199-N-43$ & $8 / 28 / 95$ & $64.25 *$ & 384.76 & 117.27 \\
\hline $199-N-57$ & $8 / 25 / 95$ & $70.70 *$ & 387.06 & 117.98 \\
\hline $199-N-59$ & $8 / 25 / 95$ & $72.43 *$ & 387.10 & 117.99 \\
\hline $199-N-67$ & $9 / 07 / 95$ & $73.48 *$ & 384.98 & 117.34 \\
\hline 199-Щ-71 & $8 / 28 / 95$ & $74.08 *$ & 388.96 & 118.56 \\
\hline 199-ㅅ- -72 & $8 / 28 / 95$ & $71.55^{\star}$ & 387.44 & 118.09 \\
\hline $199-N-73$ & $8 / 28 / 95$ & $75.52^{*}$ & 387.76 & 118.19 \\
\hline $199-N-74$ & $8 / 28 / 95$ & $68.70 *$ & 388.96 & 118.56 \\
\hline 199-N-81 & $6 / 30 / 95$ & $75.97 *$ & 386.74 & 117.88 \\
\hline
\end{tabular}


Table 4-2. RCRA Water Level Measurement Report 100-N Area, Third Quarter 1995. (sheet 2 of 2)

We11 Date $\begin{gathered}\text { Depth to } \\ \text { water (ft) }\end{gathered} \quad \begin{gathered}\text { elevation above msl } \\ \text { (ft) }\end{gathered}$

Wells Monitoring the Bottom of the Unconfined Aquifer

199- $-1+69$

$9 / 08 / 95$

$73.82 *$

385.02

117.35

199-N-70

$9 / 18 / 95$

$68.47 *$

385.74

117.57

MOTES: 1. Water level elevations are calculated by subtracting the measured depth-to-water from the surveyed elevation for the well.

2. Depth-to-water values are transcribed from field records.

3. Measurements marked with an ' $*$ ' were taken at the time of sampling.

4. Measurements marked with $a^{\prime}+{ }^{\prime}$ are outsjde of the expected range, and are suspected of error. 


\section{Table 4-3. Constituent List and Sumary of Results} for 100-N RCRA Sites Data for Reporting Period July 1 through September 30, 1995.

(sheet 1 of 3)

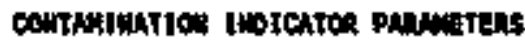

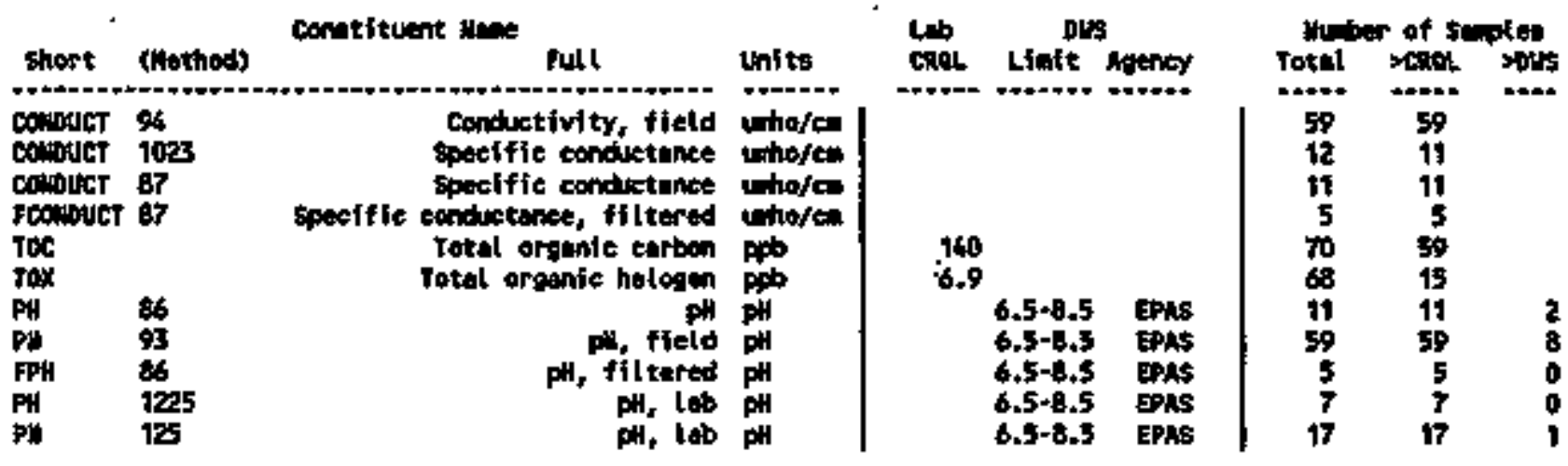

DRtMKJHG WTER PARNETERS

\begin{tabular}{|c|c|c|c|c|c|c|c|c|c|}
\hline & & Constituent ithe & & Ln' ${ }^{\circ}$ & Du & & Mun & of so & ples \\
\hline Shart & (Whothod) & Fuld & Unfte & CROL & Lìnit & Agency & Total & >CROL & OHS \\
\hline anturn & 1139 & Earita & $p$ & & 2000 & EPA & 12 & 2 & \\
\hline Franjuy & 1139 & Borlug, tiltered & $\operatorname{lab}_{\boldsymbol{b}}$ & & 2000 & EPA & 7 & 7 & \\
\hline FaARiul & 34 & Barita, filtered & b & 1.4 & 2000 & eta & 21 & 21 & \\
\hline conowlts & 1139 & cectrita & ppb & & & EPA & 12 & 0 & \\
\hline Fclints & 1139 & Codsiun, filtered & pob & & & EPA & 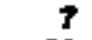 & 8 & \\
\hline FCLDHEU & 34 & Cedbiun, filtered & mb & \% & 5 & EPR & 21 & 4 & 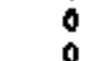 \\
\hline 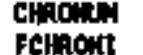 & $\begin{array}{l}1139 \\
1139\end{array}$ & Chreativa filtered & mb & & 100 & $\begin{array}{l}\text { EPA } \\
\text { EPA }\end{array}$ & $\frac{12}{7}$ & 8 & \\
\hline Ferinout & 34 & chromiun, filt & pb & 3.7 & 100 & EPA & 21 & 12 & \\
\hline Fltorid & 1093 & Fluo & ab & & 4000 & EPA & 12 & 9 & \\
\hline $\begin{array}{l}\text { Fluogid } \\
\text { FFllogid }\end{array}$ & 126 & Ftuaride. filterted & pob & 52 & $\begin{array}{l}4000 \\
4000\end{array}$ & EPA & 17 & 17 & \\
\hline Alpht & 267 & $\begin{array}{l}\text { Flcaride, tilterted } \\
\text { Grogs alpht }\end{array}$ & PCi/l. & & & & 4 & t & \\
\hline ALPHA & 135 & Croses ulpho & $\mathrm{pCI} / \mathrm{L}$ & & & & 17 & 9 & \\
\hline $\begin{array}{l}\text { NJTRATE } \\
\text { FUOS-N }\end{array}$ & & Mitrate & ppo & 120 & 45000 & & 17 & 17 & \\
\hline $\begin{array}{l}\text { Fillos-n } \\
\operatorname{lng}=n\end{array}$ & & $\begin{array}{l}\text { Witrogen in witrate, filtered } \\
\text { mitrogen in nitrate }\end{array}$ & Ppo & & 10000 & & 52 & $\begin{array}{r}7 \\
12\end{array}$ & - \\
\hline
\end{tabular}

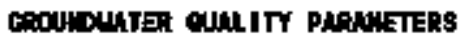

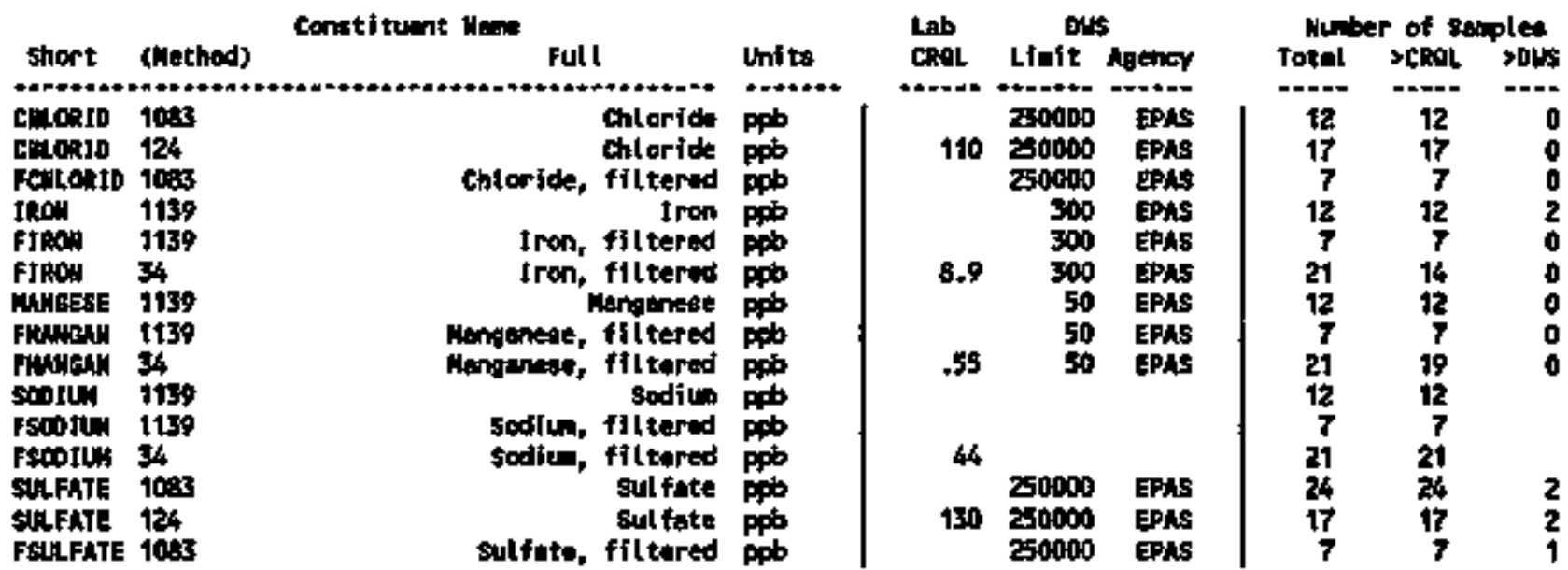


Table 4-3. Const1tuent List and Summary of Results for 100-H RCRA Sites Data for Reporting Period July 1 through September 30, 1995.

(sheet 2 of 3)

SIJE SPECTFIC MO OTRER DOWSTITUENTS

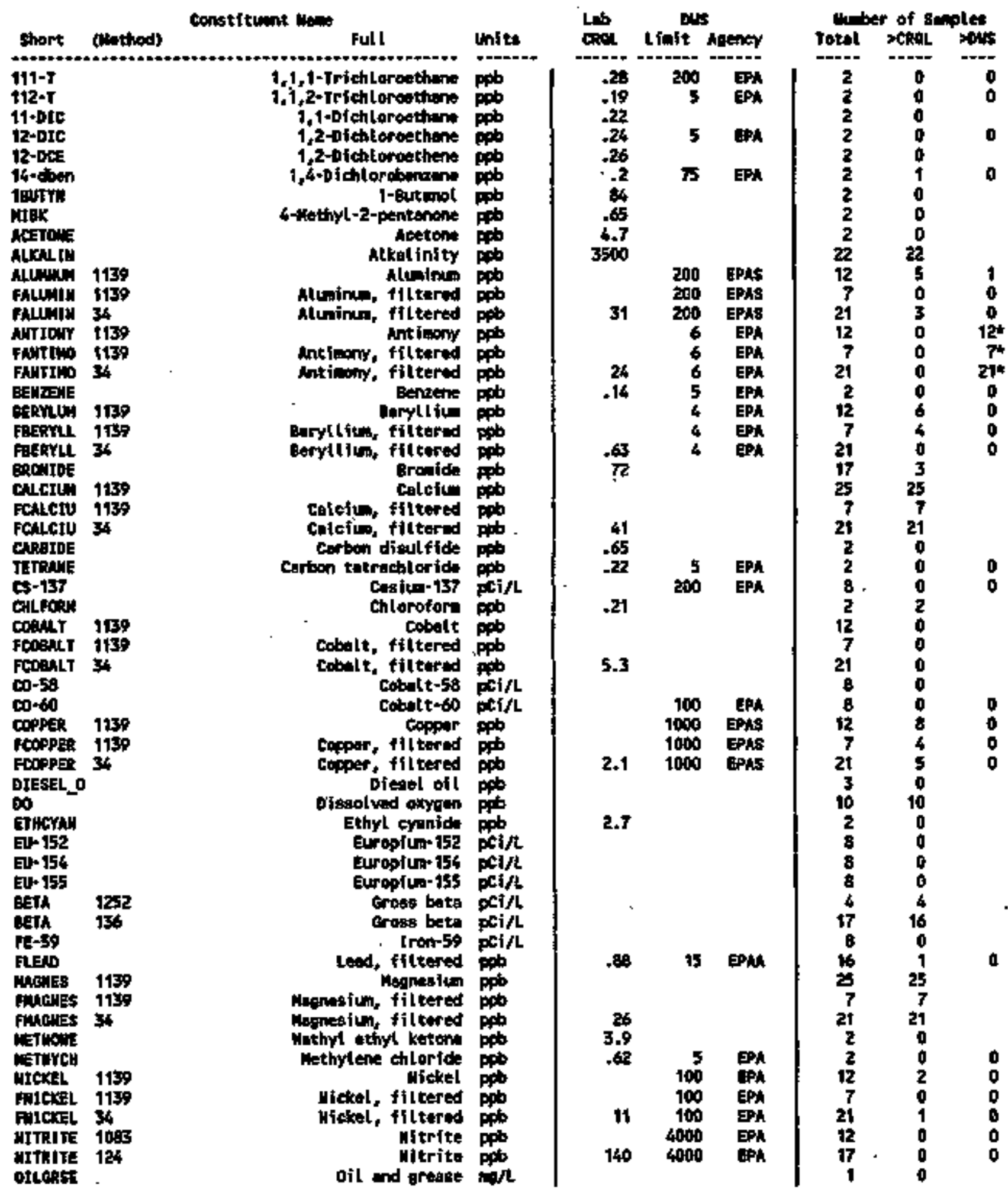


Table 4-3. Constituent List and Summary of Results for 100-N RCRA Sites Data for Reporting Period July 1 through September 30, 1995. (sheet 3 of 3 )

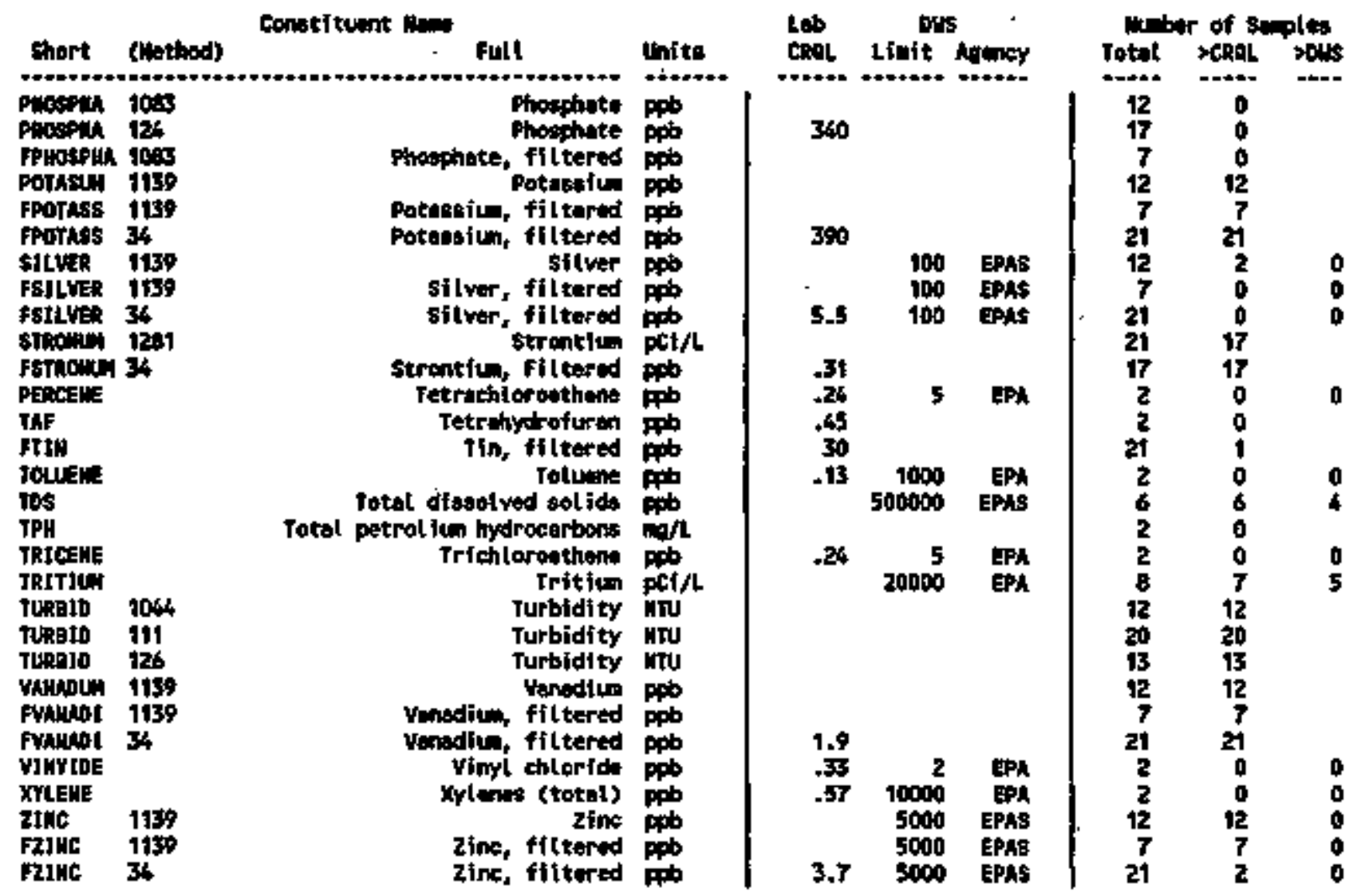

For axplanation of this takie, see section 1.4 of report. 
Tabie 4-4. Constituents with at Least One Detected Value for the 100-H RCRA Sites Data for Reporting Period July I through September 30, 1995.

(sheet 1 of 17)

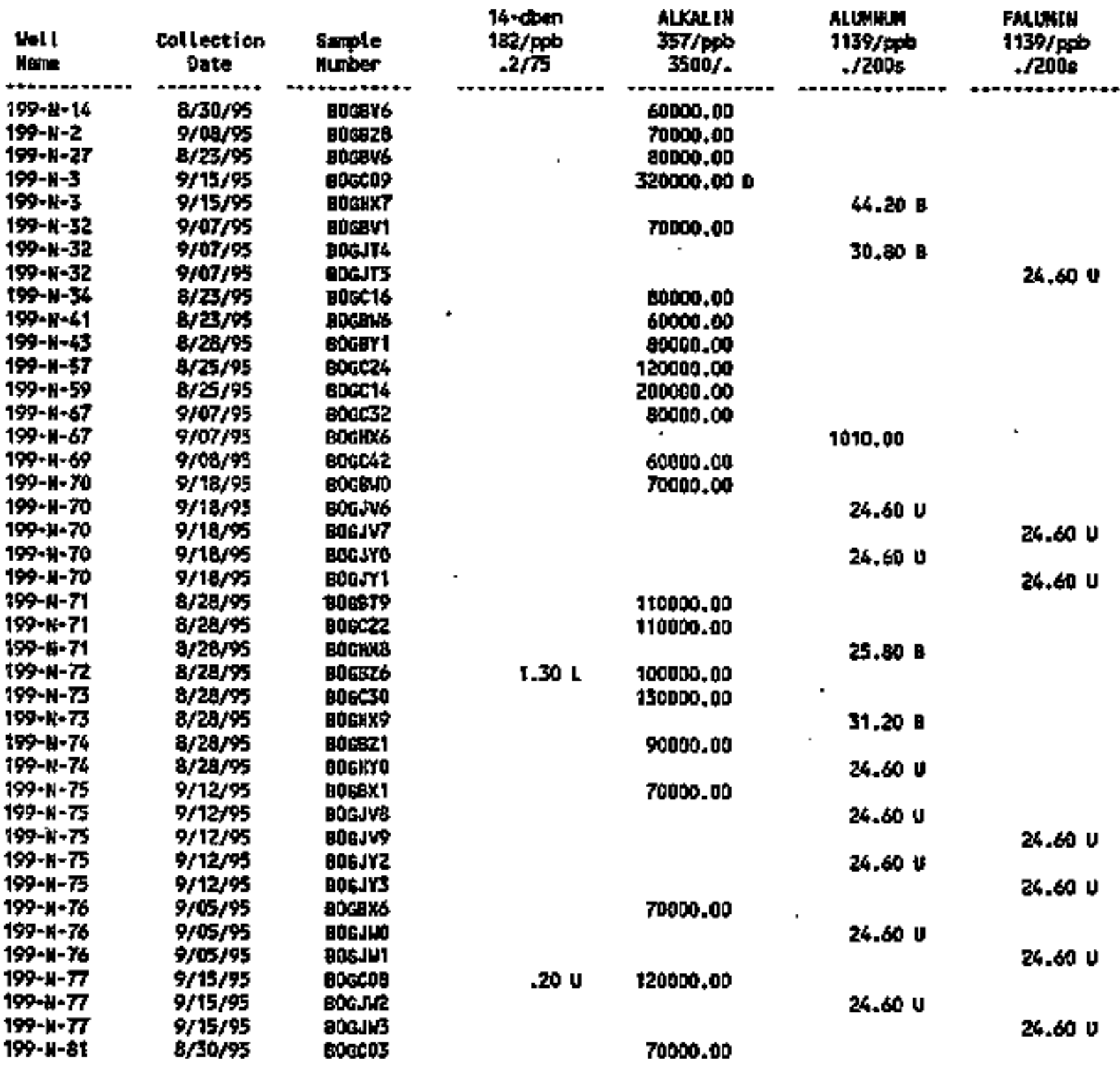


Table 4-4. Constftuents with at least One Detected Value for the 100-N RCRA Sttes Data for Reporting Period July 1 through September 30, 1995.

(sheet 2 of 17)

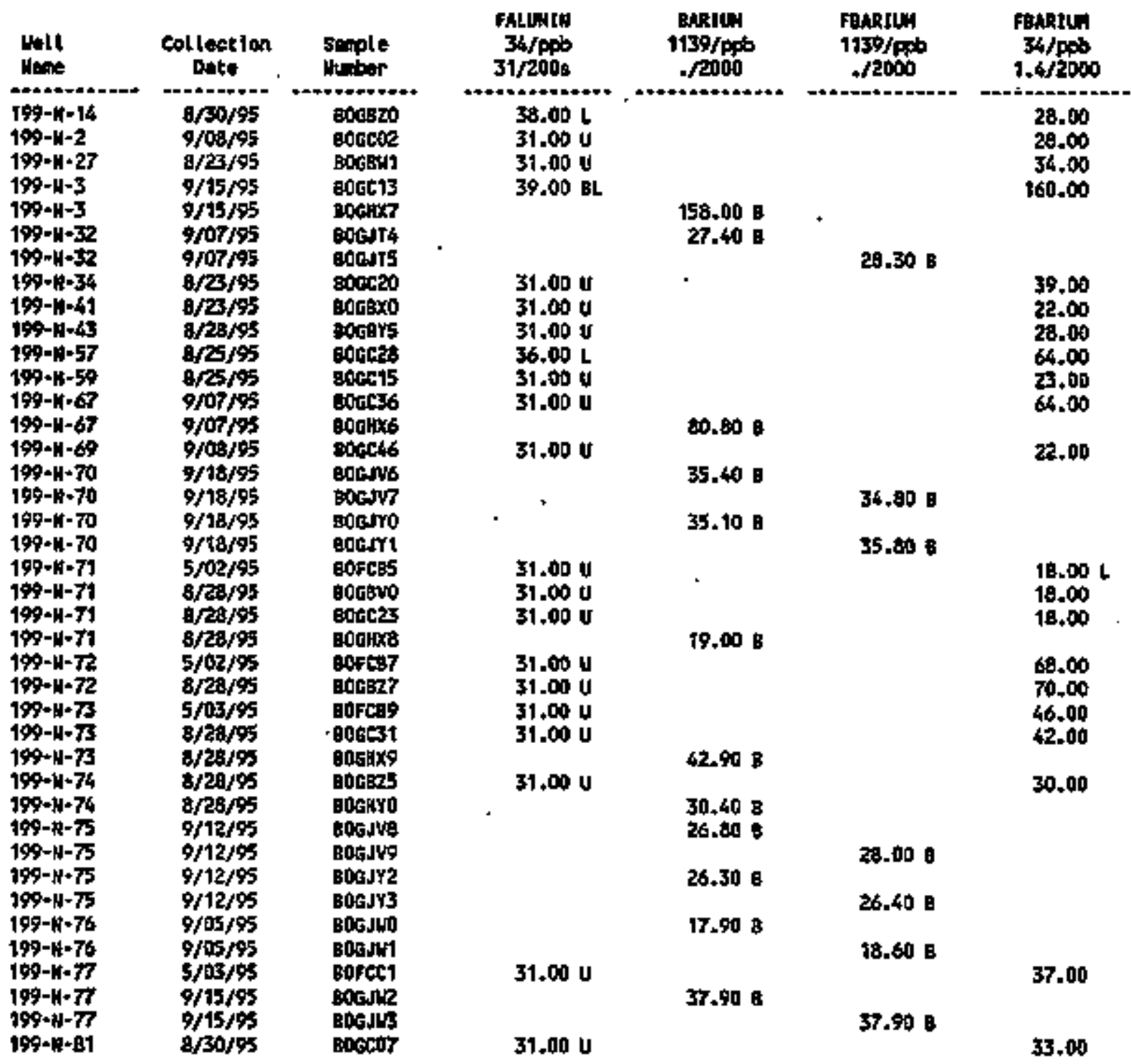


Table 4-4, Constituents with at Least One Detected Value for the 100-H RCRA S1tes Data for Reporting Period Juty 1 through September 30, 1995. (sheet 3 of 17)

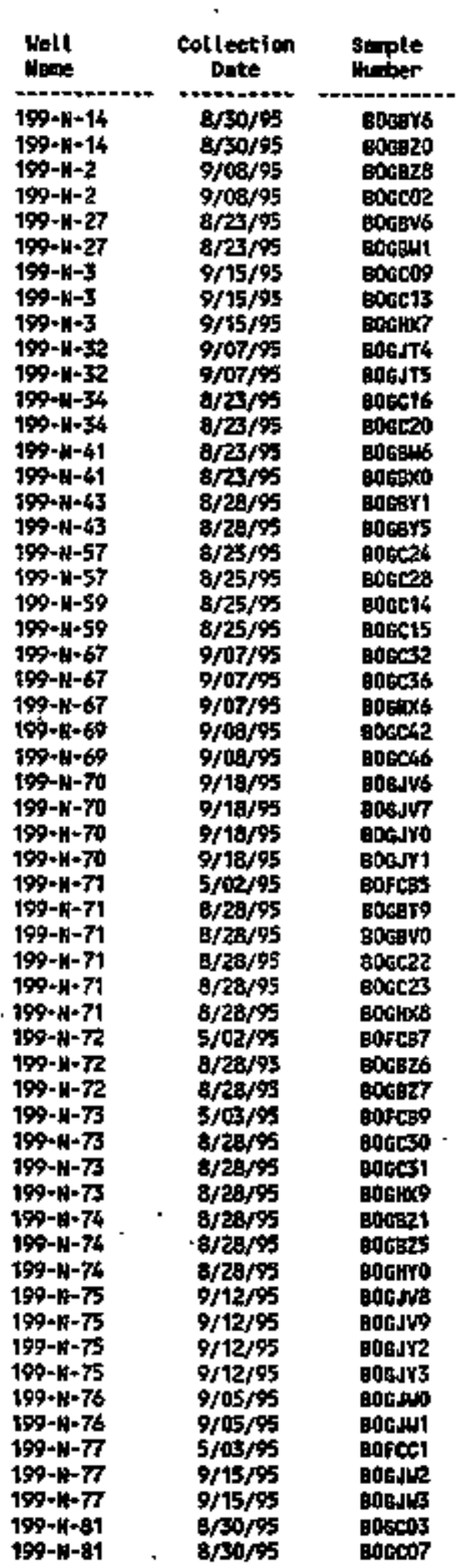

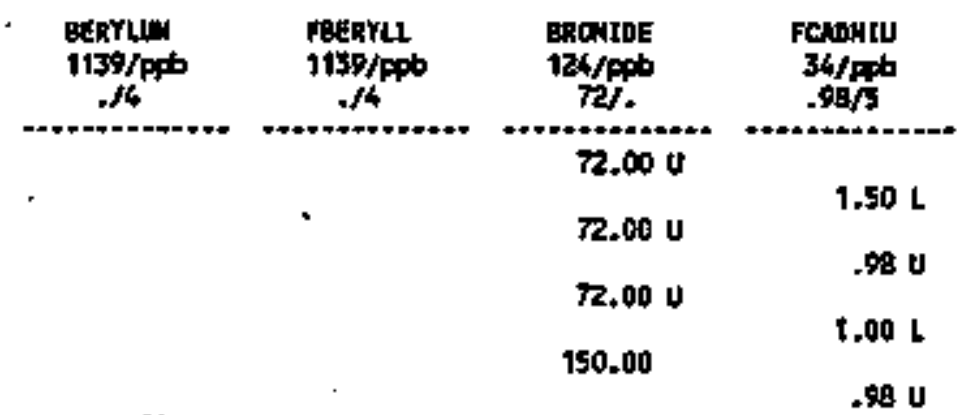

$.84 \mathrm{~B}$

.30 t

U

$\mathbf{7 2 . 0 0 u}$

72.00 u

, 究 U

72,00 v

.980

$100.00 \mathrm{~L}$

.98 v

200.00

$1.50 \mathrm{t}$

$.48 \mathrm{~V}$

.

$.50 \mathrm{U}$

72,00 th

72.00 4

$.98 \mathrm{v}$

st $\mathrm{B}$

.51 E

$.50 \mathrm{U}$

.598

$.98 \mathrm{U}$

$72.00 \mathrm{U}$

$.98 \mathrm{U}$

R.00 V

1,101

.500

.98

$72.00 \mathrm{v}$

$.98 \mathrm{U}$

$.98 \mathrm{U}$

$\pi, \infty 0$

$.98 \mathrm{v}$

$.50 \mathrm{t}$

$72.00 \mathrm{v}$

.99 บ

.500

$.69 \mathrm{O}$

$.68 \mathrm{~B}$

$.50 \mathrm{~V}$

.928

$.92 \mathrm{~B}$

$.67 \mathrm{~B}$

ע.98

$.66 \mathrm{~B}$

$.50 \mathrm{U}$
$72,00 \mathrm{~V}$ 
Table 4-4. Constituents with at Least One Detected VaTue for the 100-H RCRA Sites Data for Reporting Period July 1 through September 30, 1995. (sheet 4 of 17)

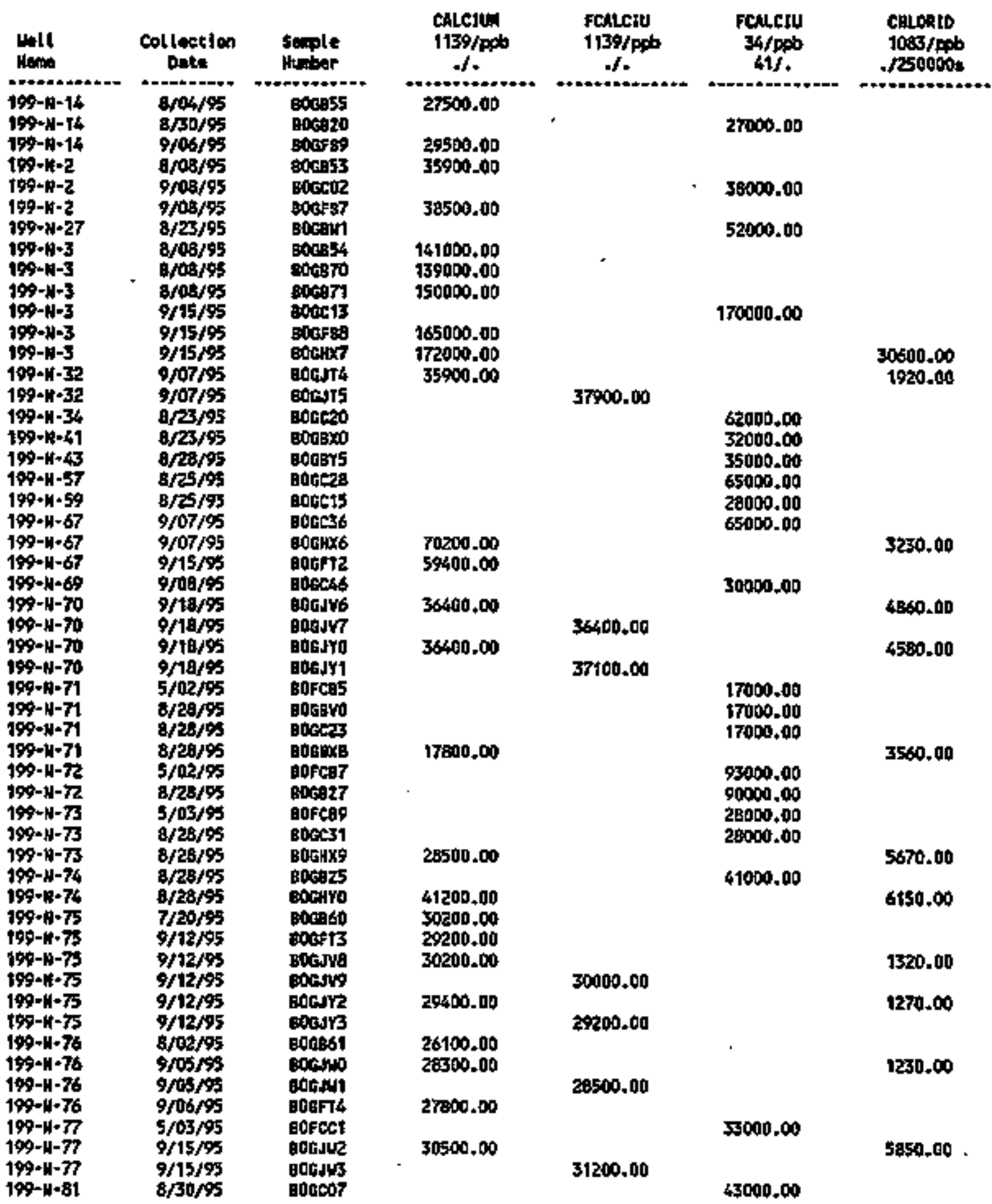


Tab]e 4-4. Constituents with at Least One Detected Value for the 100-W RCRA Sites Data for Reporting Period July 1 through September 30, 1995. (sheet 5 of 17 )

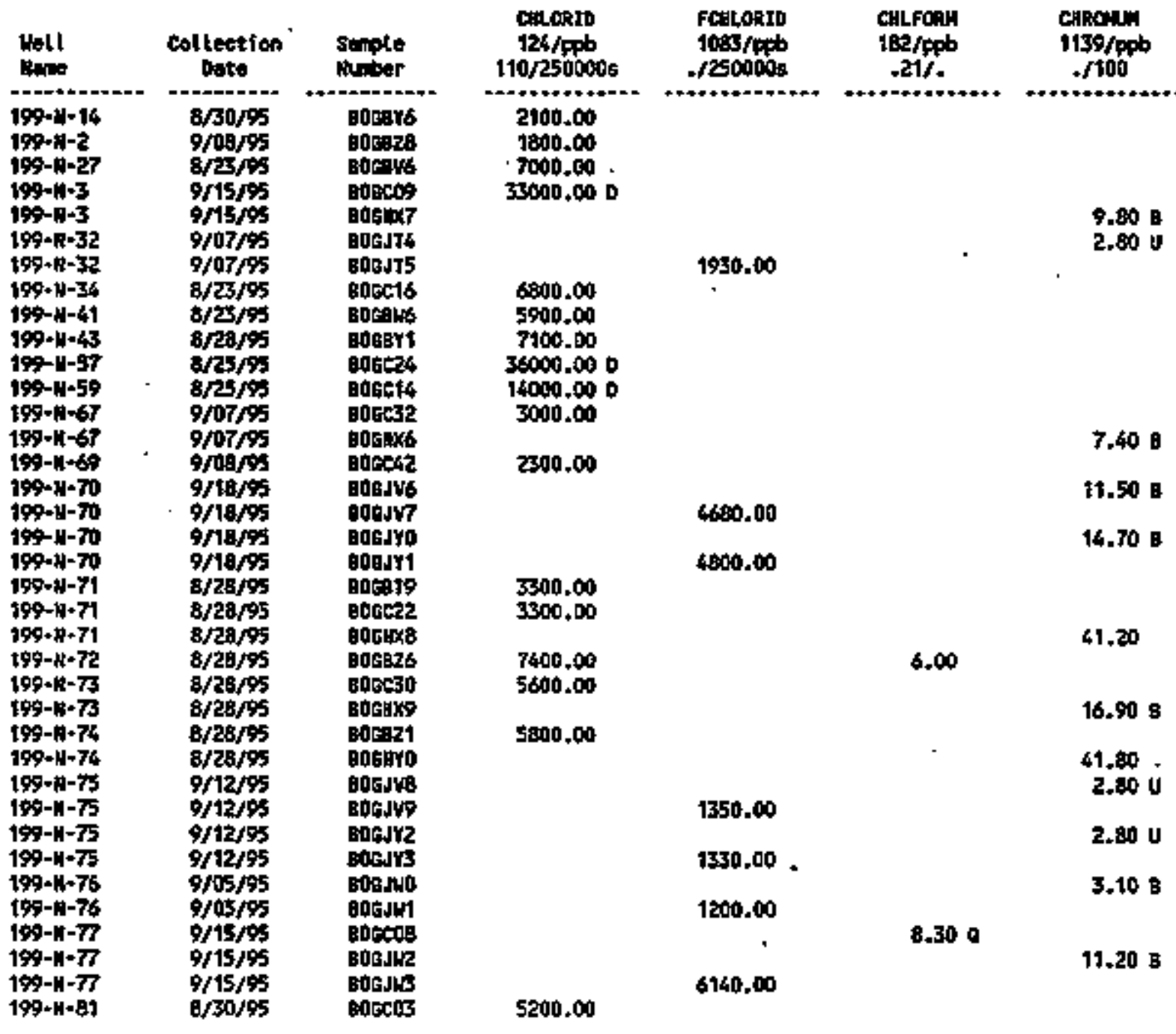

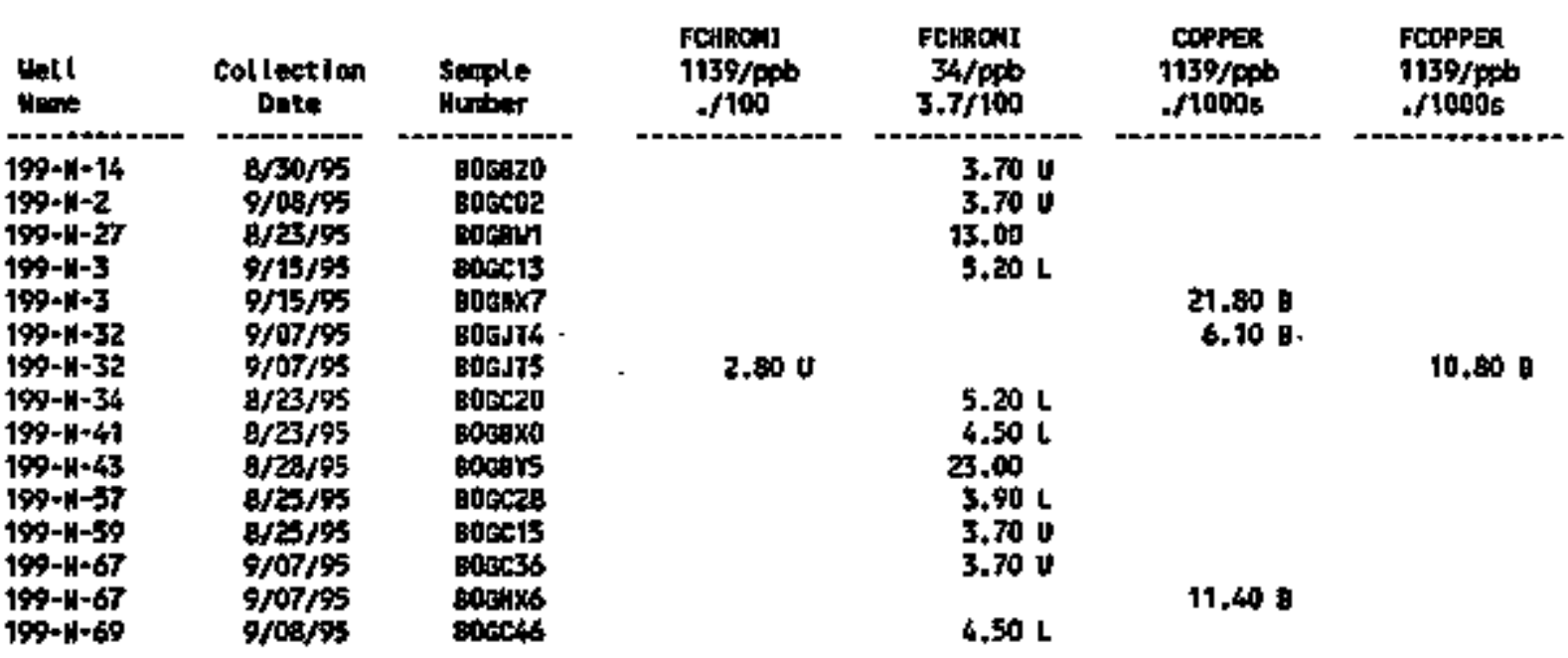


Table 4-4. Constituents with at Least One Detected VaTue for the 100-N RCRA Sites Data for Reporting Period Juily 1 through September 30, 1995.

(sheet 6 of 17 )

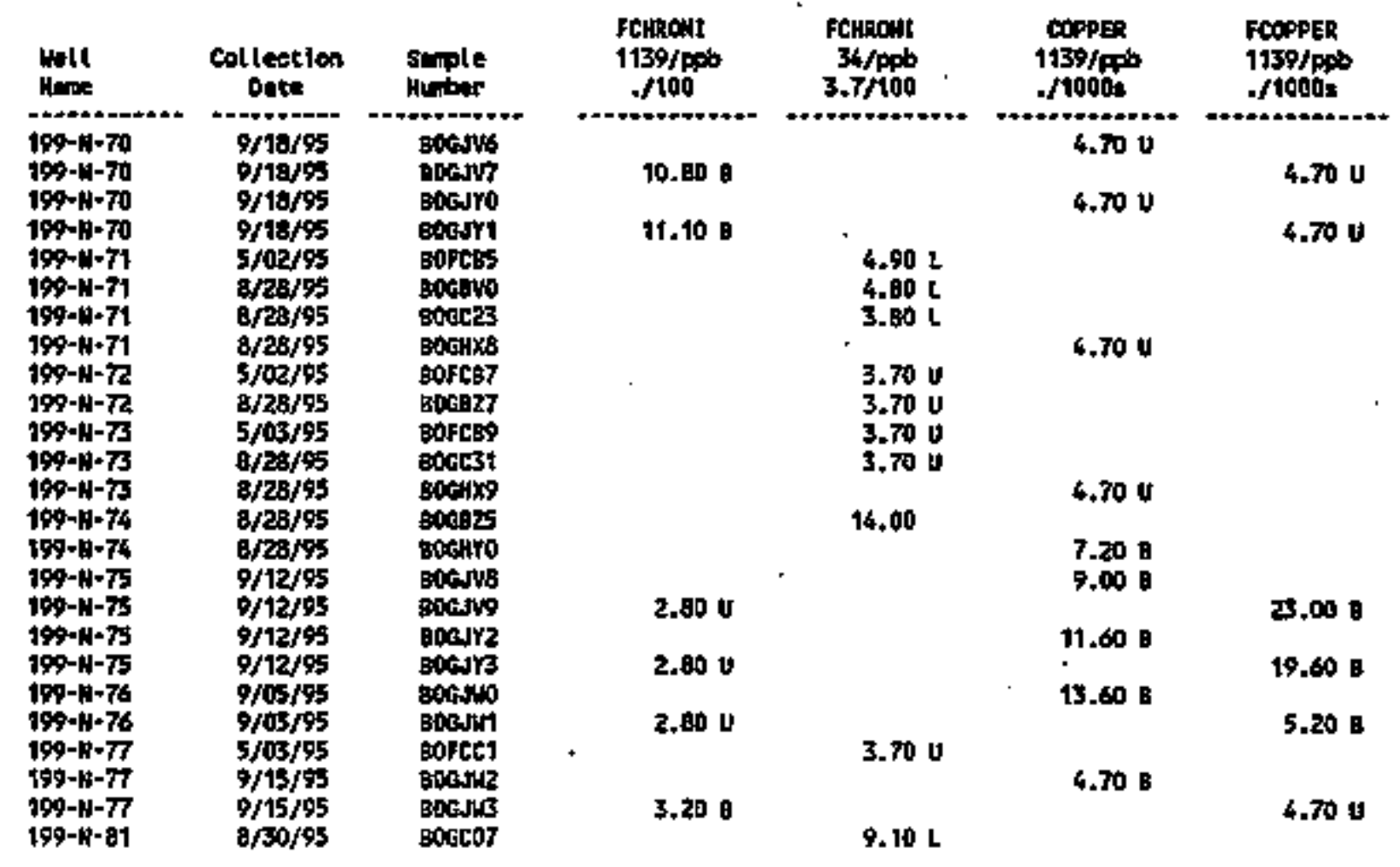

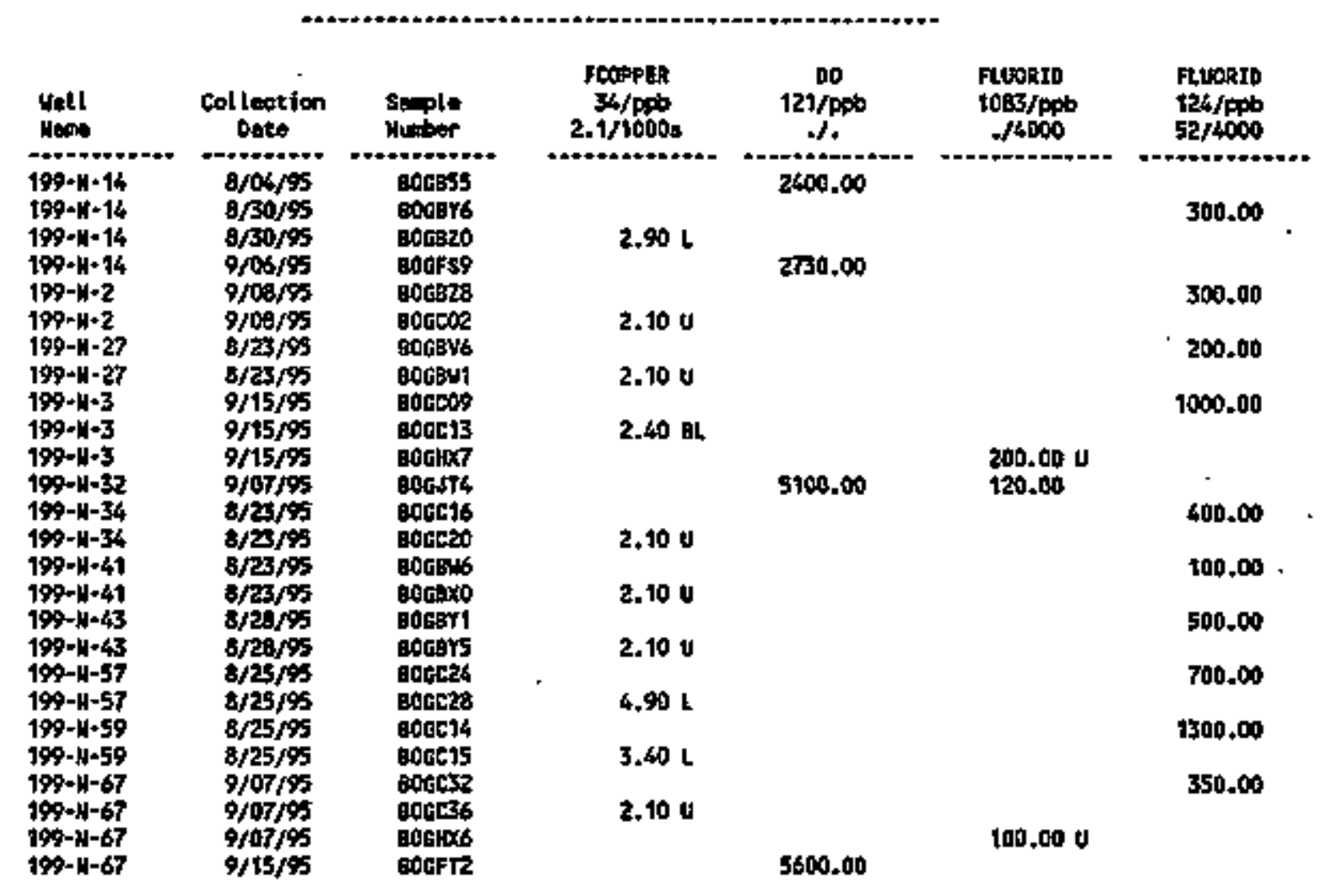


Table 4-4. Constituents with at Least One Detected Va]ue for the 100-H RCRA Sjtes Data for Reporting Period July 1 through September 30, 1995. (sheet 7 of 17 )

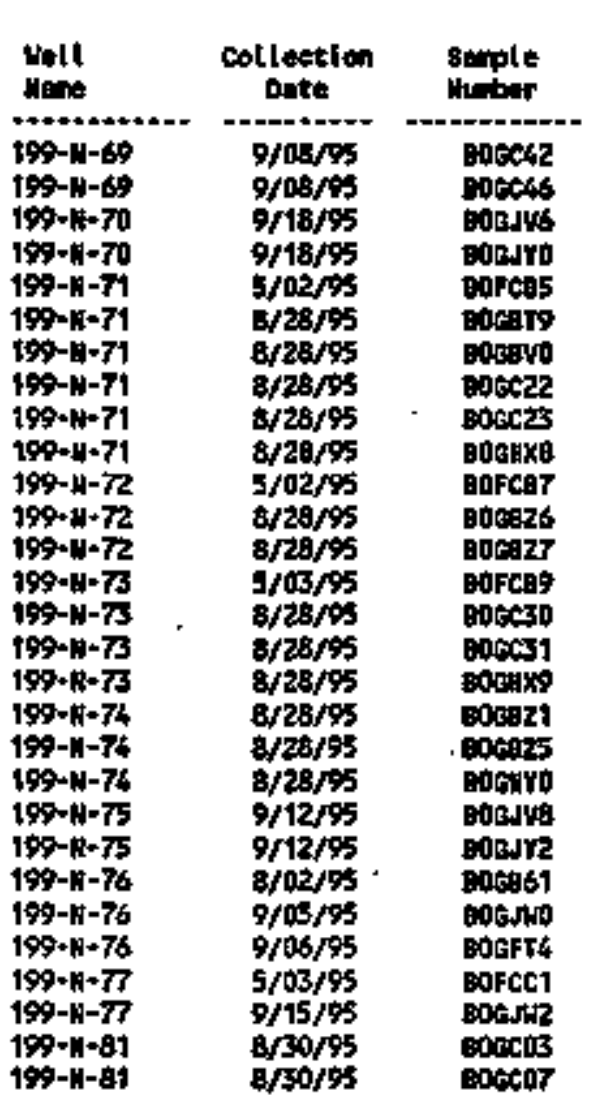

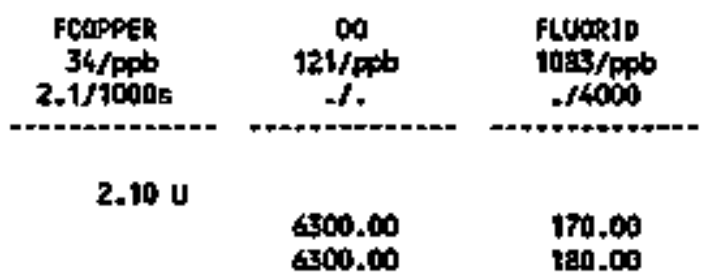

2.t0 $u$

$2.10 \mathrm{U}$

$2.10 \mathrm{U}$

3.20 L

2.10 U

$2.10 \mathrm{U}$

$2.10 \mathrm{U}$

2.100

570.00

280.00

140.00

150.00

5010.00

5500.00

$2.10 \mathrm{v}$

5100.00

8650,00

$2.10 \mathrm{U}$

.350 .00
$100.00 \mathrm{U}$

670.00

300.00
600.00

600.00

1200,00

1400.00

500.00

$52 / 4900$

250.00

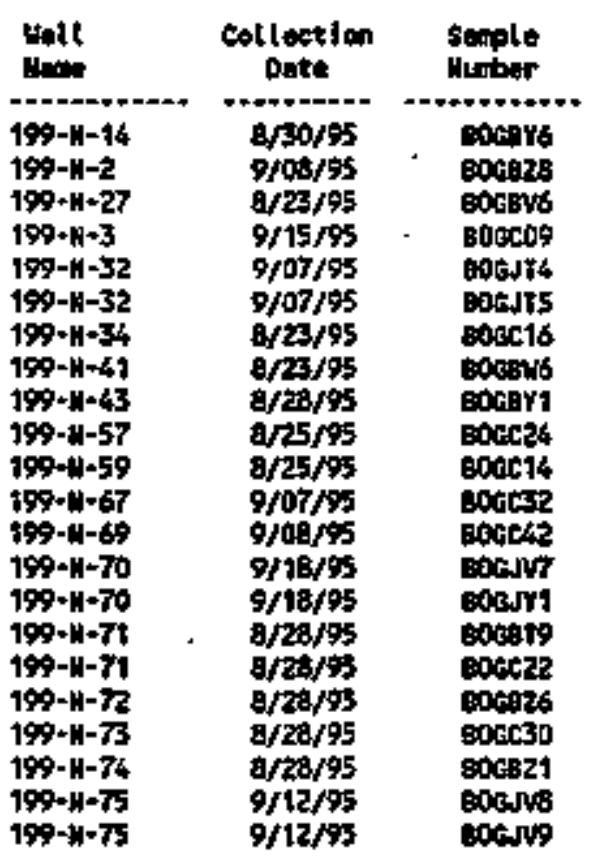

EDTh

$1252 / \mathrm{pCt} / \mathrm{L}$ . .

$.01 \mathrm{U}$

$.19 \mathrm{U}$

$\{.45$

9.13

1.59 小 13.70
130.00

$$
\begin{aligned}
& 2.64 \\
& .41 \mathrm{u} \\
& 1.59 \\
& 1.72 \mathrm{U} \\
& 2.78 \\
& 1.09 \\
& .56 \mathrm{U}
\end{aligned}
$$

180_06

190.06

1.28

$.40 \mathrm{U}$

.95 U

2.38 Ur

.30 t

160.00
2.11

1390.00 
Table 4-4. Constituents with at Least One Detected Value for the I00-N RCRA Sites Data for Reporting Period July 1 through September 30, 1995.

(sheet 8 of 17)

\begin{tabular}{|c|c|c|c|c|c|c|}
\hline Woll & $\begin{array}{c}\text { Col lection } \\
\text { Detet }\end{array}$ & $\begin{array}{l}\text { sample } \\
\text { unoper }\end{array}$ & $\begin{array}{c}\text { EFLUIDRAD } \\
1083 / \text { ppb } \\
. / 40: 90\end{array}$ & 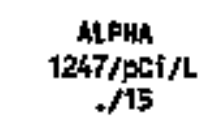 & $\begin{array}{c}\text { ALPHA } \\
\text { I35/PCi/L } \\
. / 15\end{array}$ & $\begin{array}{c}\text { BETh } \\
\text { Ia\$2/PCi/L } \\
, / .\end{array}$ \\
\hline $\begin{array}{l}199-n-75 \\
199-n-75 \\
199-1-76 \\
199-n-76 \\
199-11-77 \\
199-14-81\end{array}$ & $\begin{array}{l}9 / 12 / \% 5 \\
0 / 12 / 95 \\
\$ / 05 / 45 \\
\$ / 05 / 55 \\
9 / 45 / 95 \\
8 / \$ 0 / 45\end{array}$ & 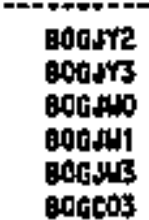 & $\begin{array}{l}160.00 \\
110.00 \\
660.00\end{array}$ & $\begin{array}{l}.60 \mathrm{U} \\
0.00 \mathrm{v}\end{array}$ & 1.45 & $\begin{array}{l}1380,00 \\
185.00\end{array}$ \\
\hline
\end{tabular}

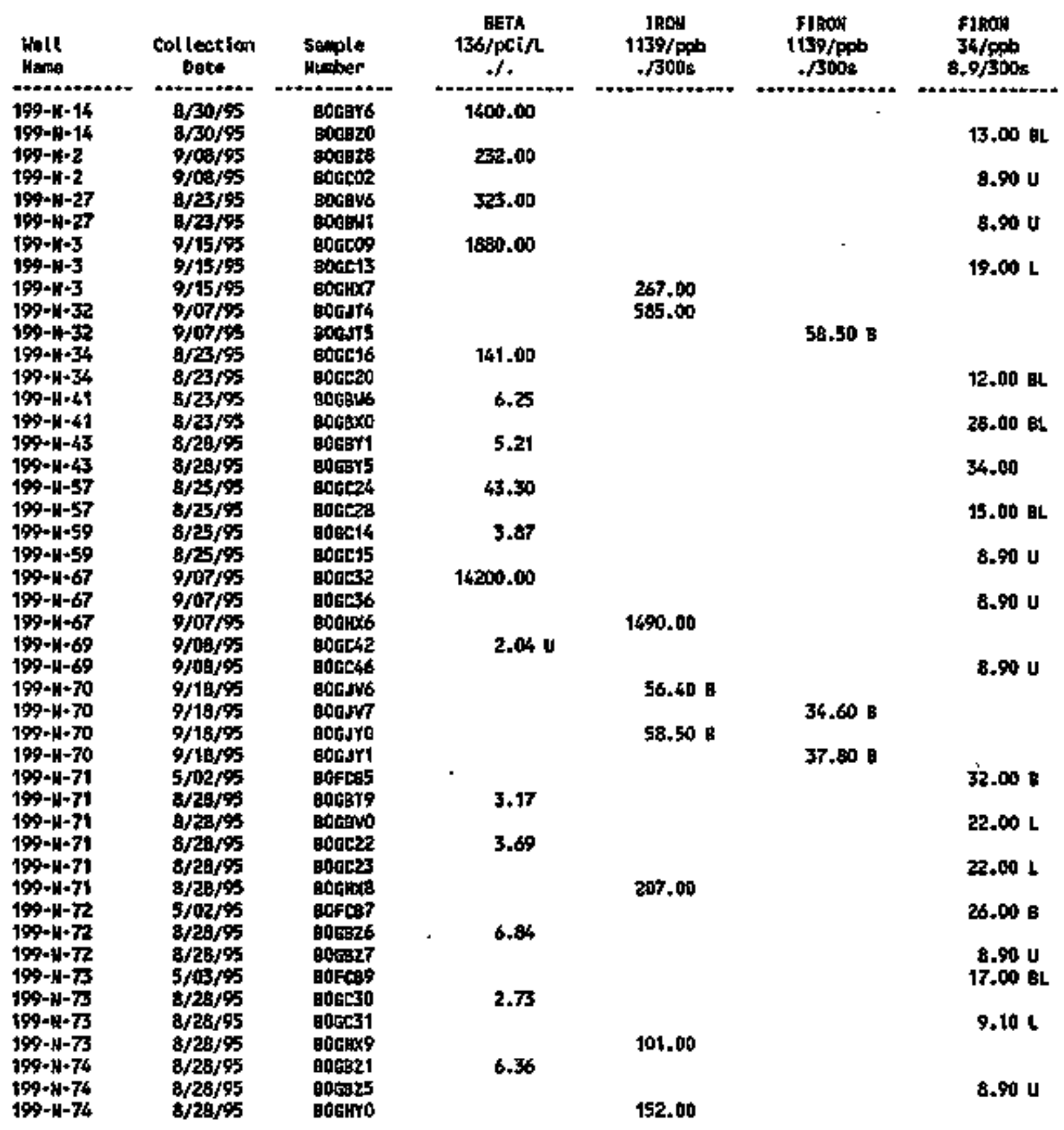


Table 4-4. Constituents with at Least One Detected Value for the 100-N RCRA Sites Data for Reporting Period July 1 through September 30, 1995. (sheet 9 of 17)

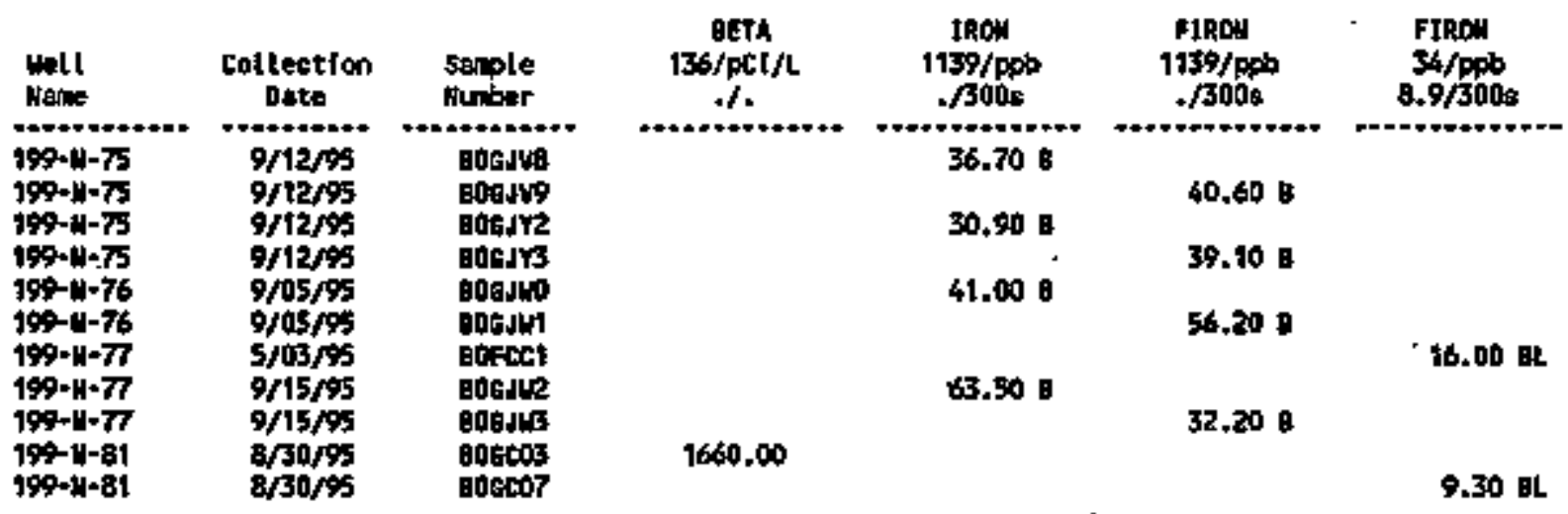

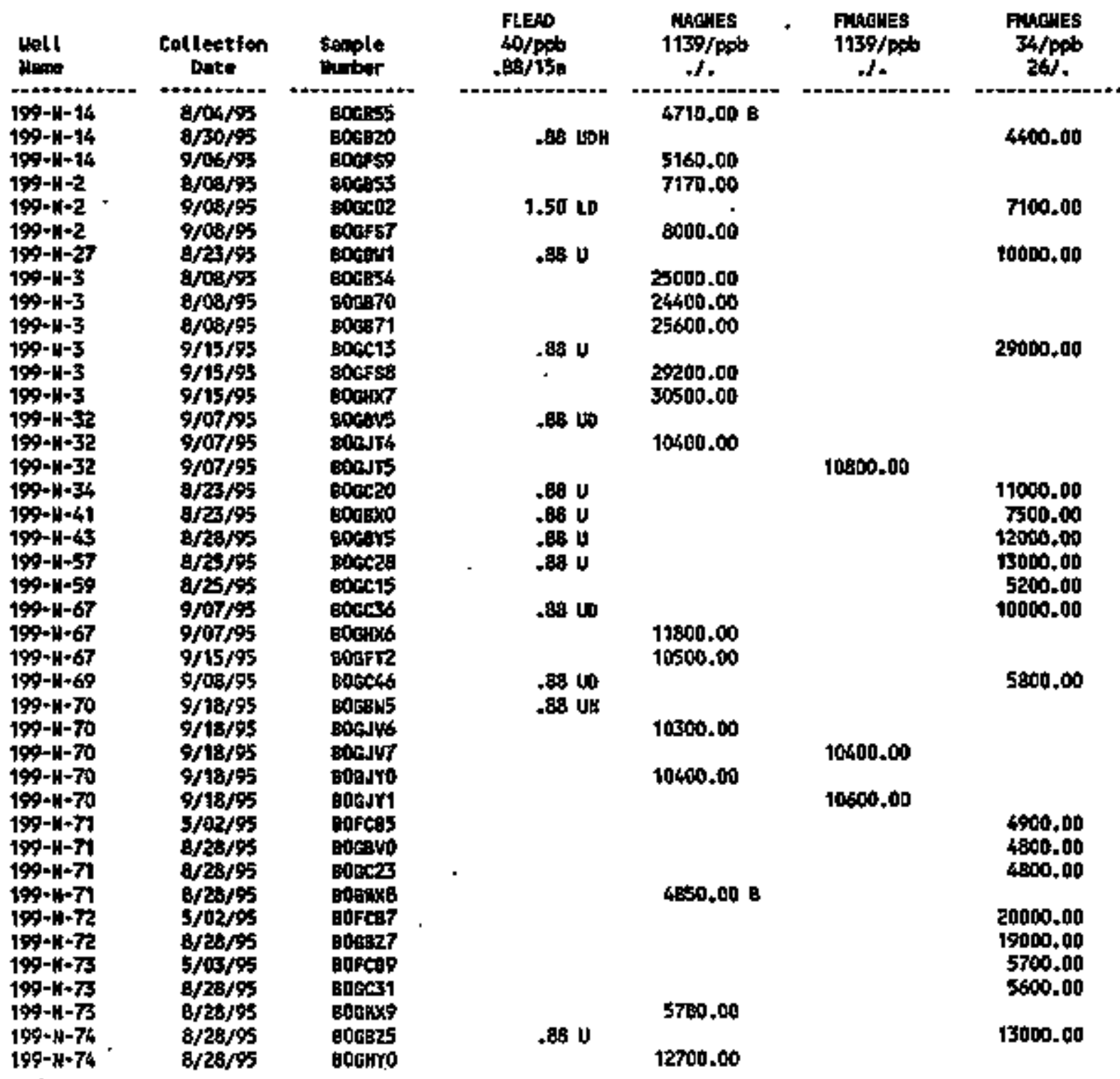


Table 4-4. Constituents with at Least One Detected Value for the 100-N RCRA Sites Data for Reporting Period July 1 through September 30, 1995. (sheet 10 of 17 )

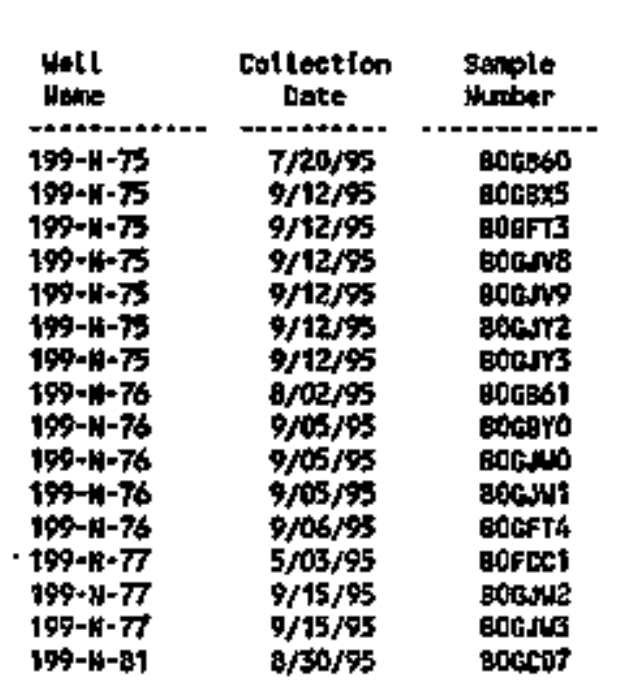

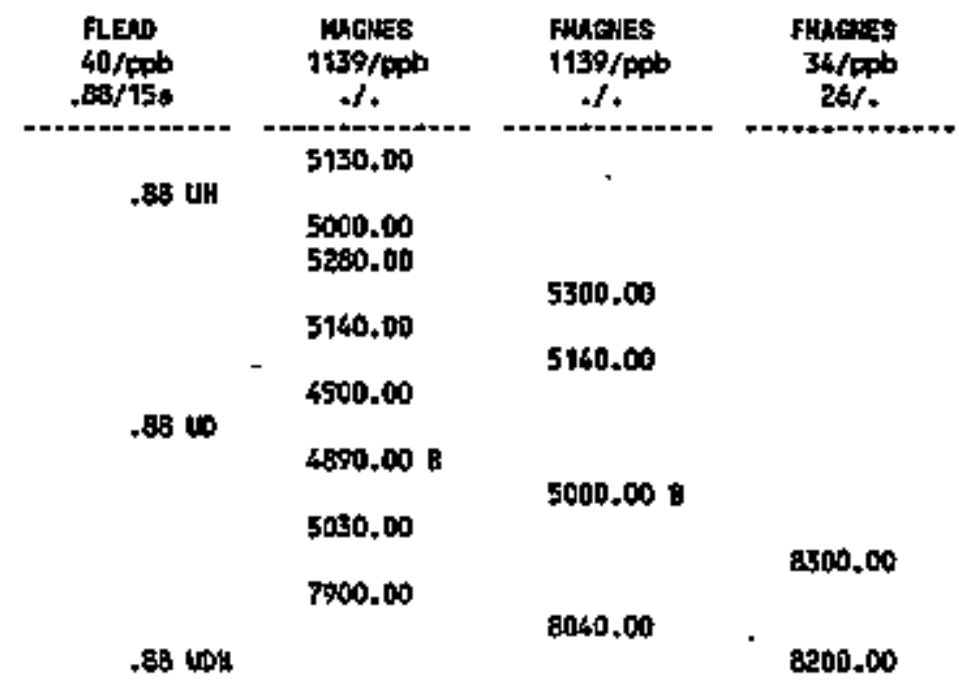

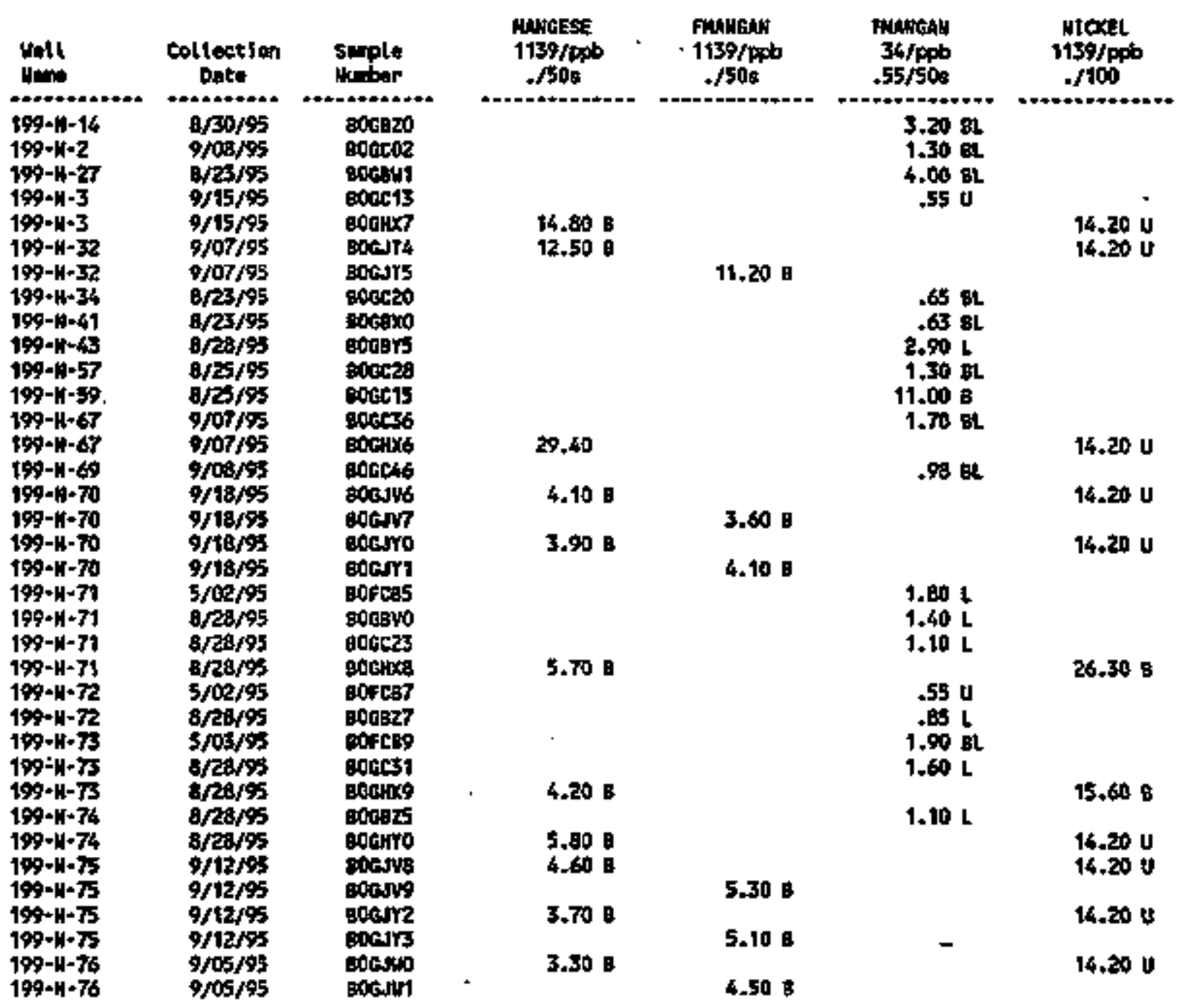


Table 4-4. Constituents with at Least one Oetected Value for the 100-N RCRA Sites Data for Reporting Period July 1 through September 30, 1995.

(sheet 11 of 17)

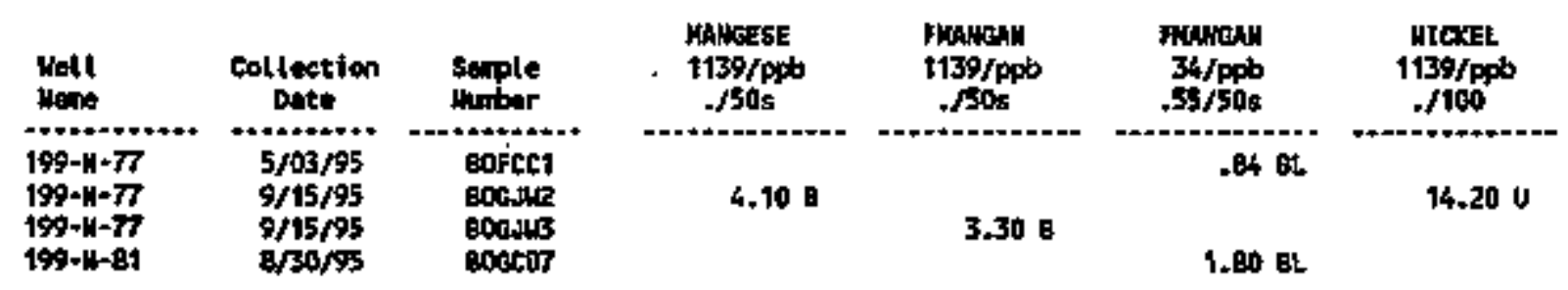

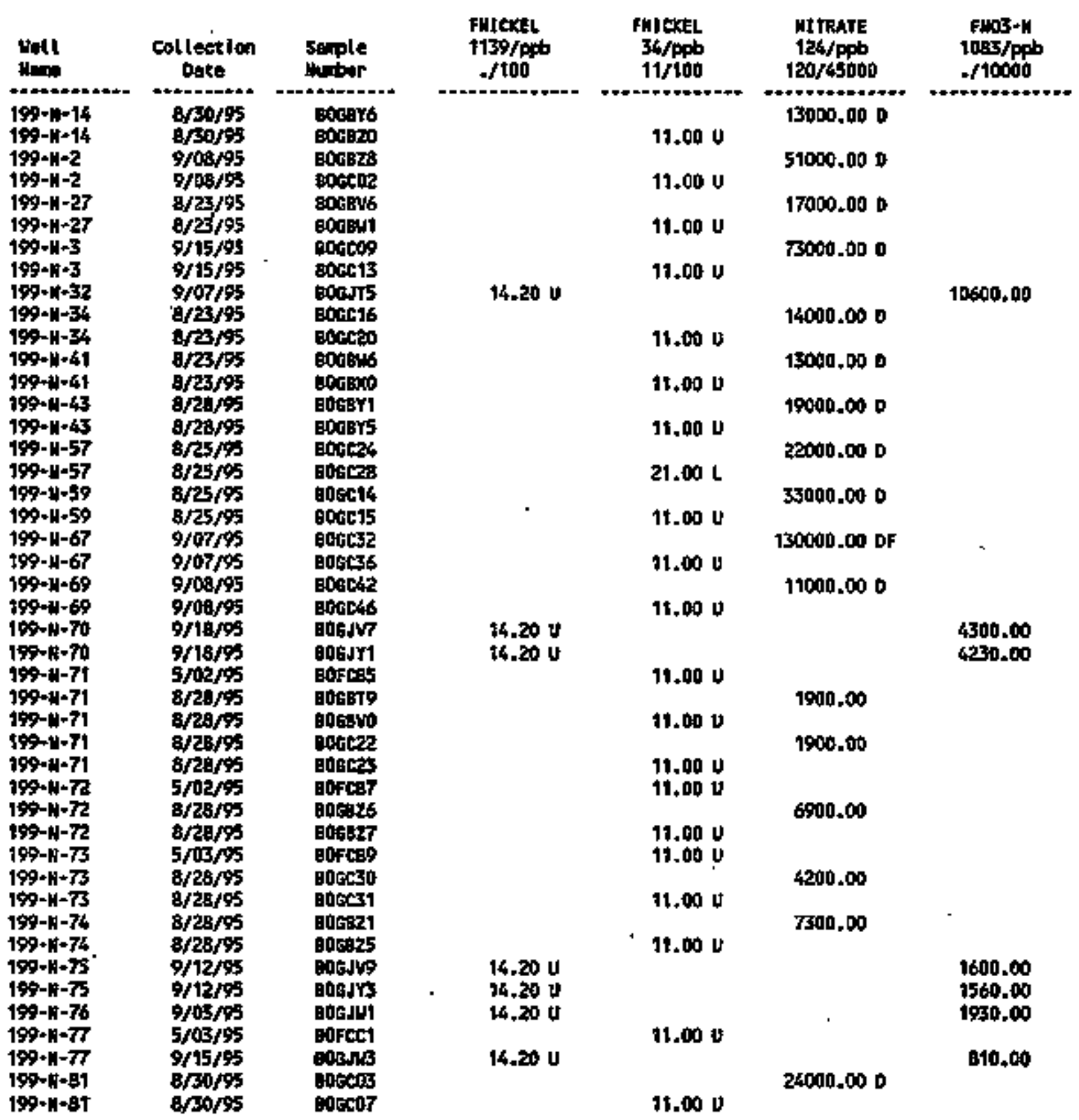


Table 4-4. Constituents with at Least One Detected Value for the 100-N RCRA 51tes Data for Reporting Period July 1 through September 30, 1995.

(sheet 12 of 17)

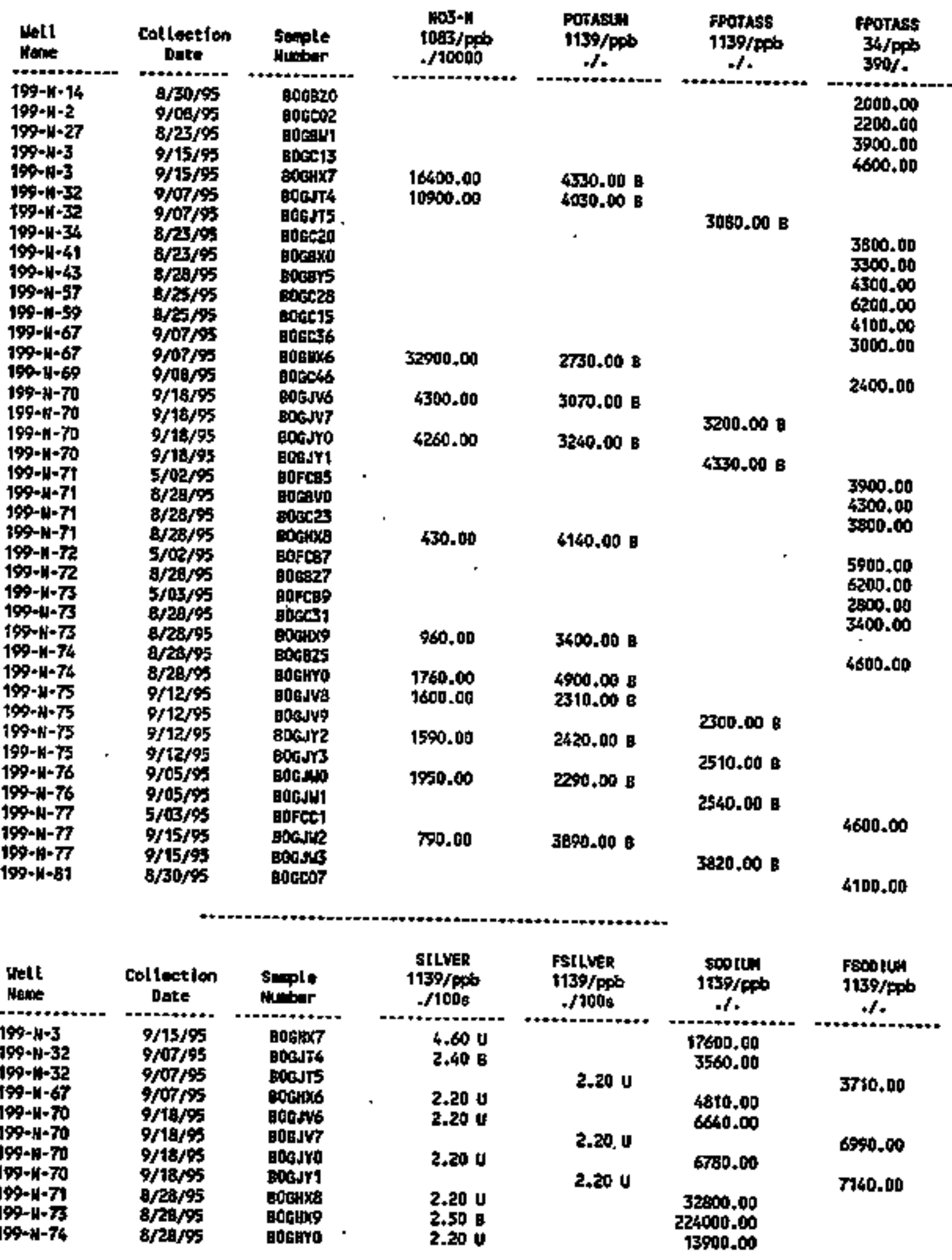


Table 4-4. Constituents with at Least One Detected Va]ue for the 100- $H$ RCRA Sites Data for Reporting Period July 1 through Septenber 30, 1995. (sheet 13 of 17)

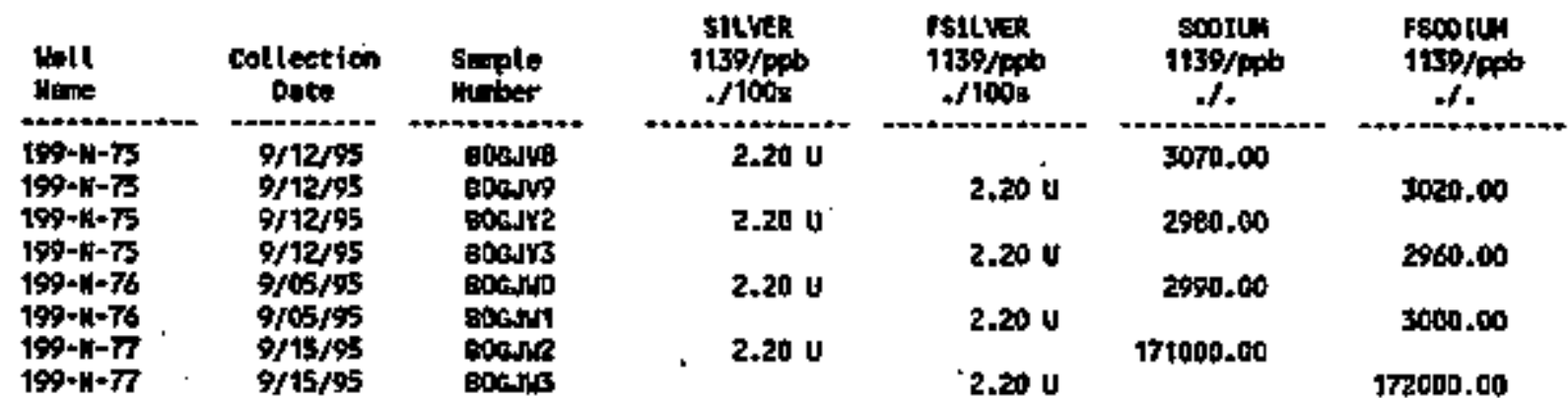

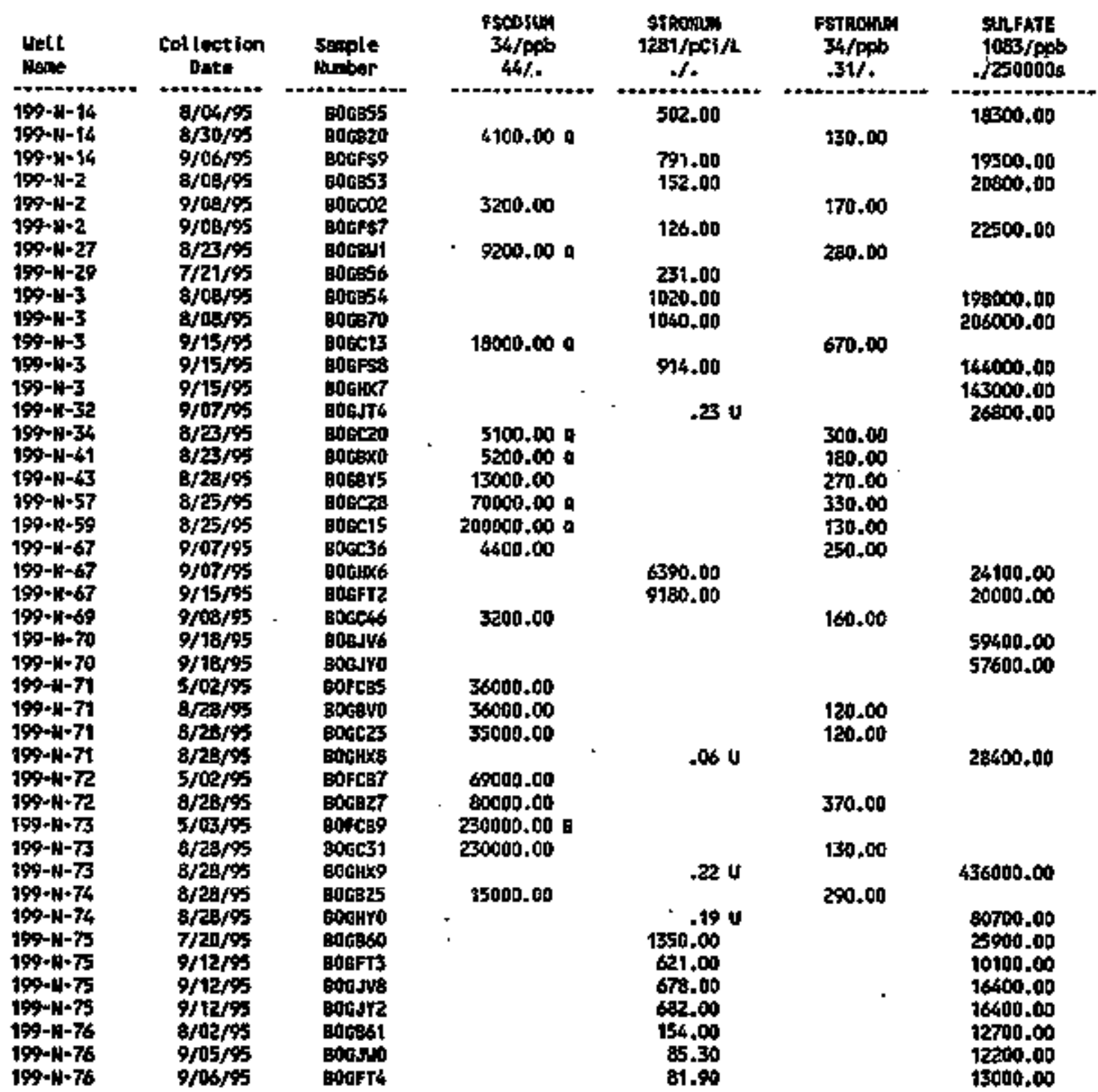


Table 4-4. Constituents with at Least One Detected Value for the 100-N RCRA Sites Data for Reparting Period July 1 through September 30, 1995. (sheet 14 of 17)

\begin{tabular}{|c|c|c|c|c|c|c|}
\hline Well & $\begin{array}{c}\text { collection } \\
\text { Oute }\end{array}$ & suplot & $\begin{array}{c}\text { Fsoolum } \\
\text { 34/peb } \\
44 \%\end{array}$ & $\begin{array}{c}\text { STROMN } \\
1281 / \mathrm{pCi} / \mathrm{L} \\
. / .\end{array}$ & 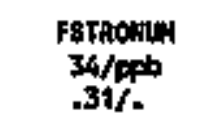 & $\begin{array}{l}\text { SULFATE } \\
1003 / p p b \\
. / 250000 \%\end{array}$ \\
\hline $\begin{array}{l}199-n-77 \\
190-k-77 \\
199-k-81\end{array}$ & $\begin{array}{l}5 / 03 / \% 5 \\
9 / 15 / 95 \\
6 / 50 / \% 5\end{array}$ & 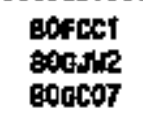 & $\begin{array}{r}180000.00 \mathrm{~B} \\
6000.00 \mathrm{O}\end{array}$ & & 230.00 & 3400010.00 \\
\hline
\end{tabular}

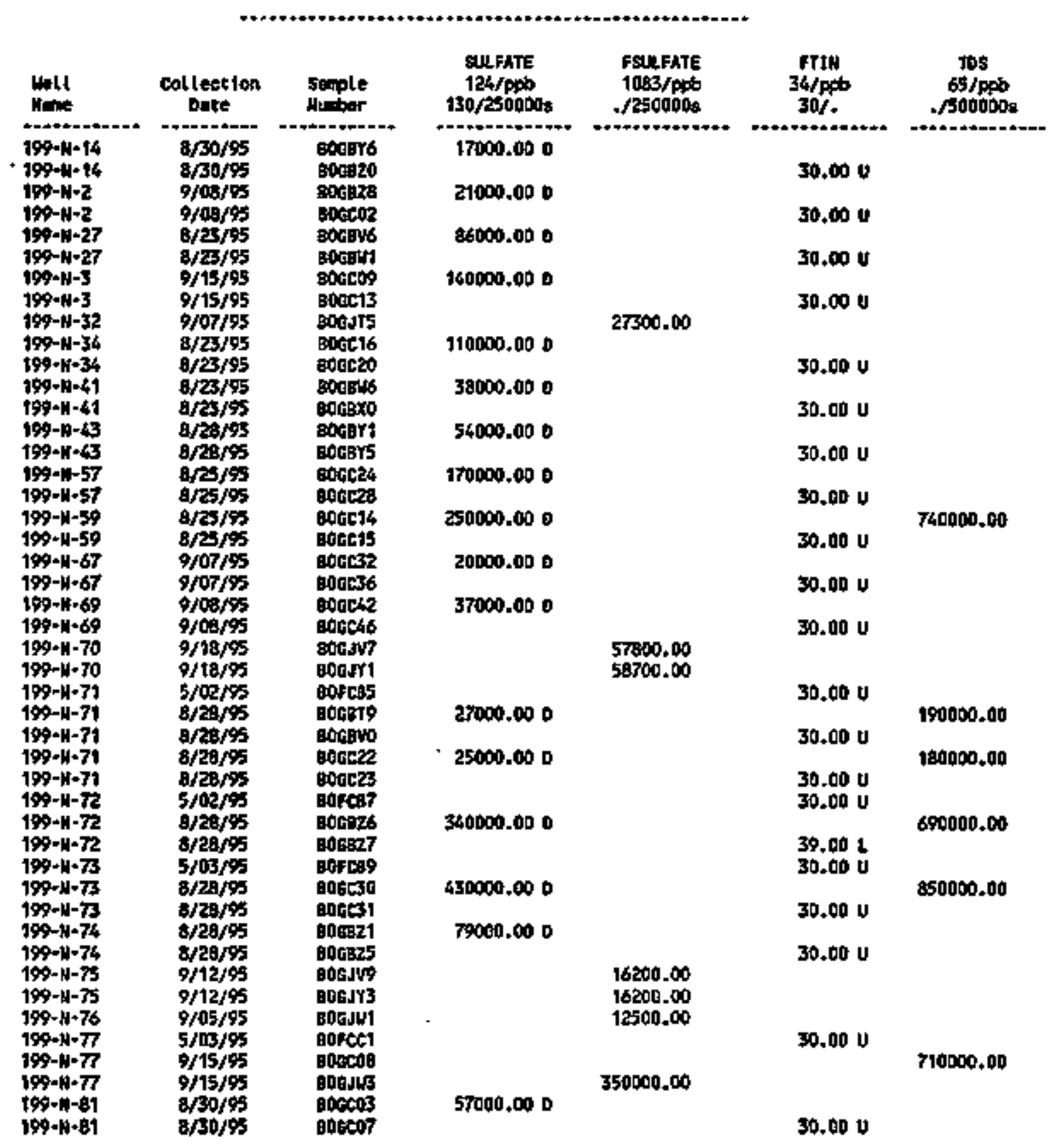


Table 4-4. Constituents with at Least One Detected Value for the 100-N RCRA Sttes Data for Reporting Period July 1 through September 30, 1995. (sheet 15 of 17)

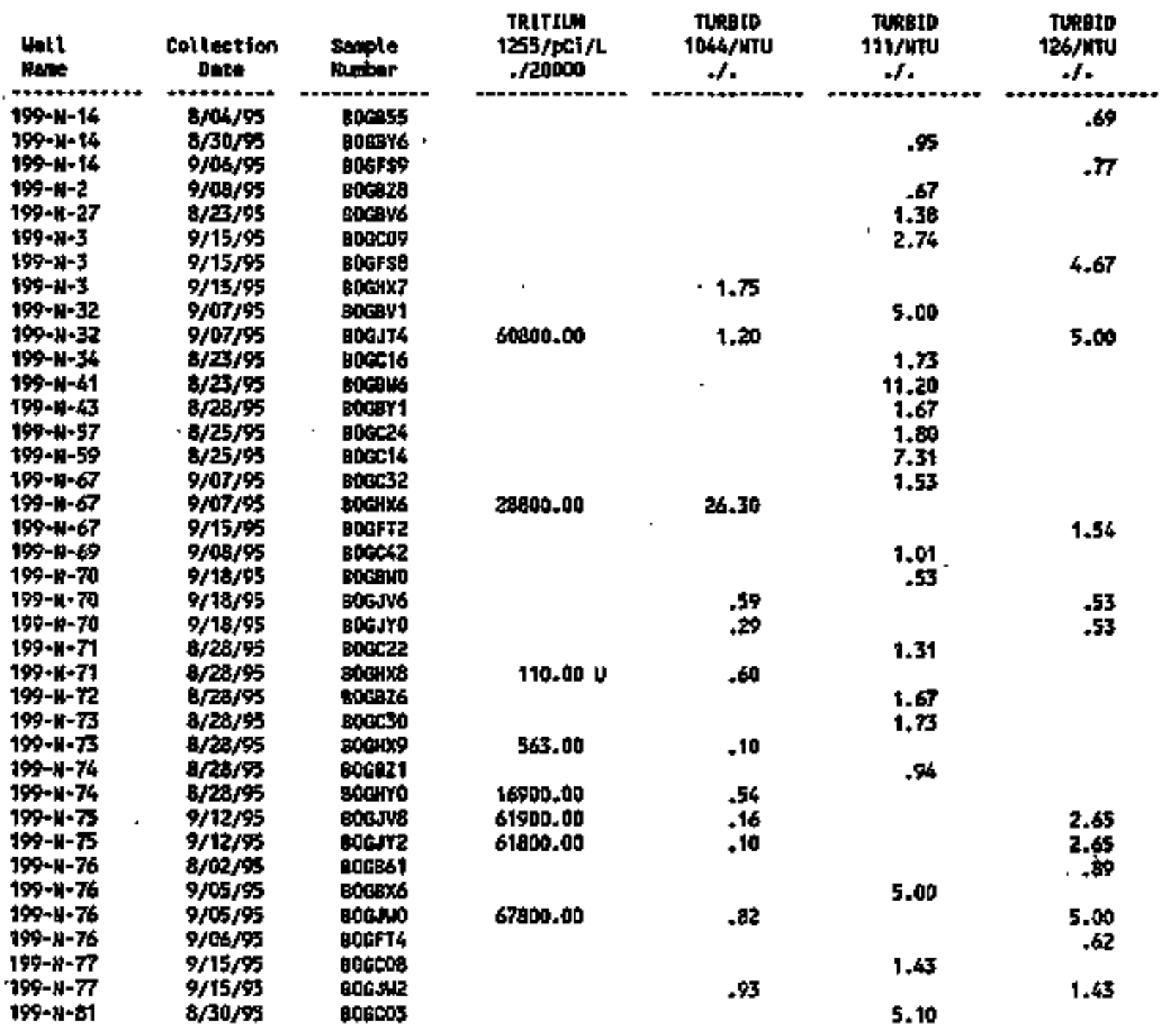

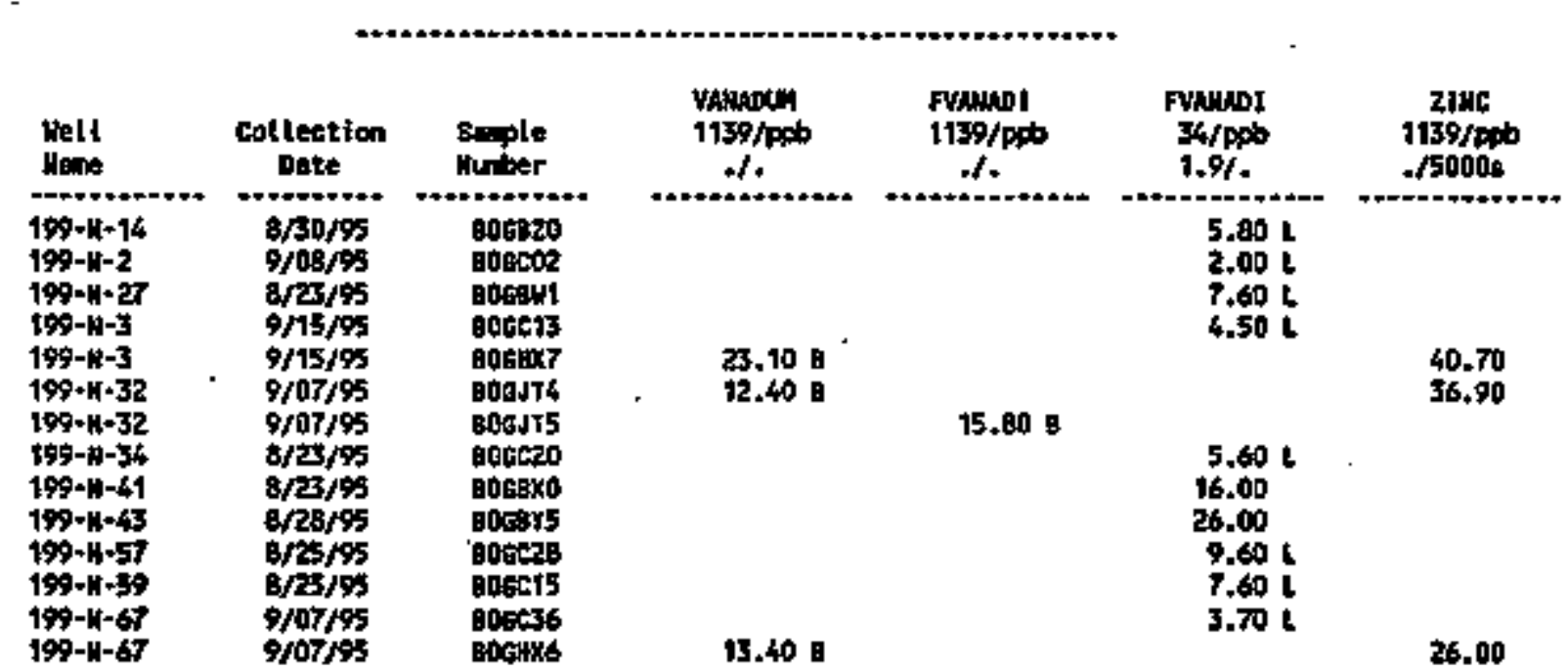


Table 4-4. Constituents with at Least One Detected Value for the 100-N RCRA Sites Data for Reporting Period July 1 through September 30, 1995.

(sheet 16 of 17 )

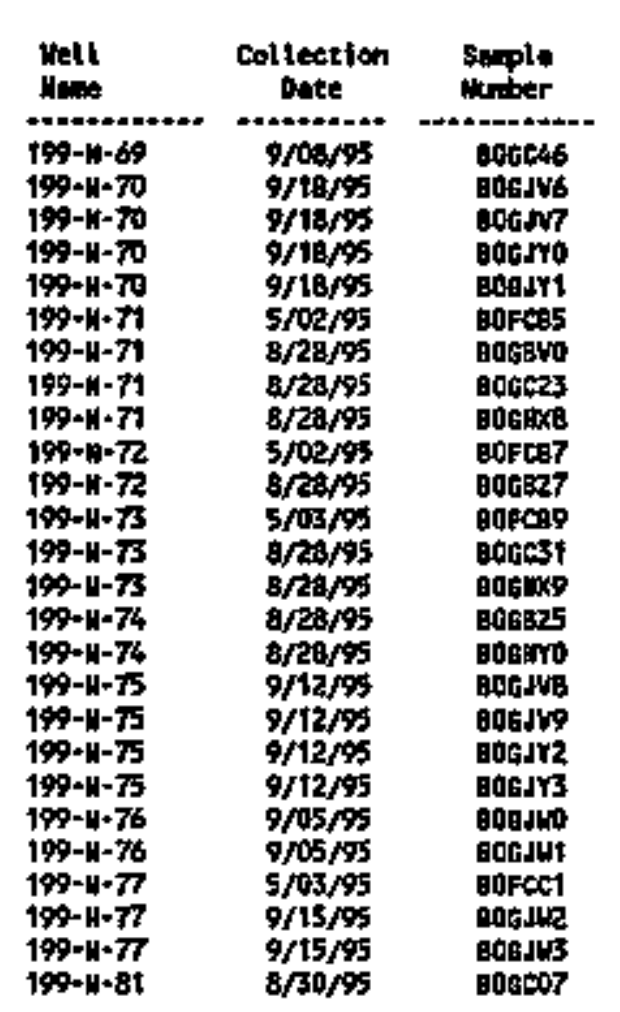

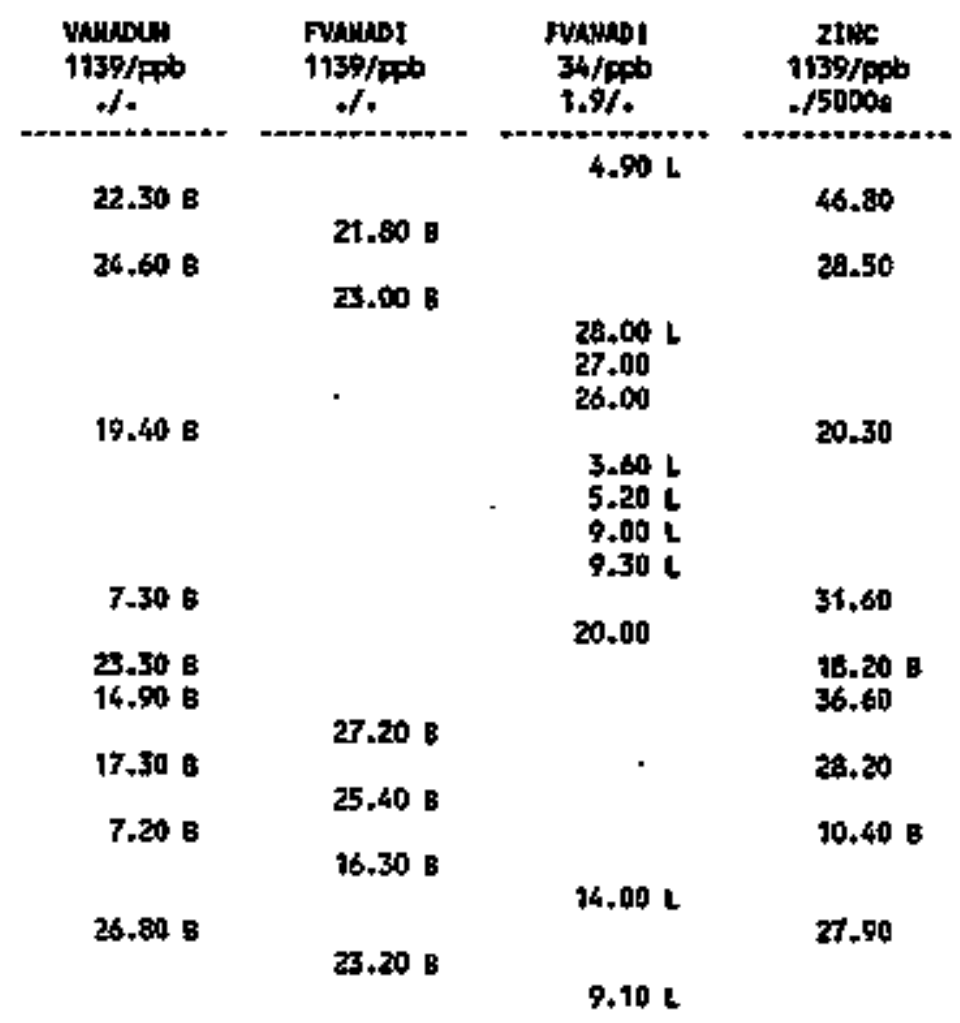

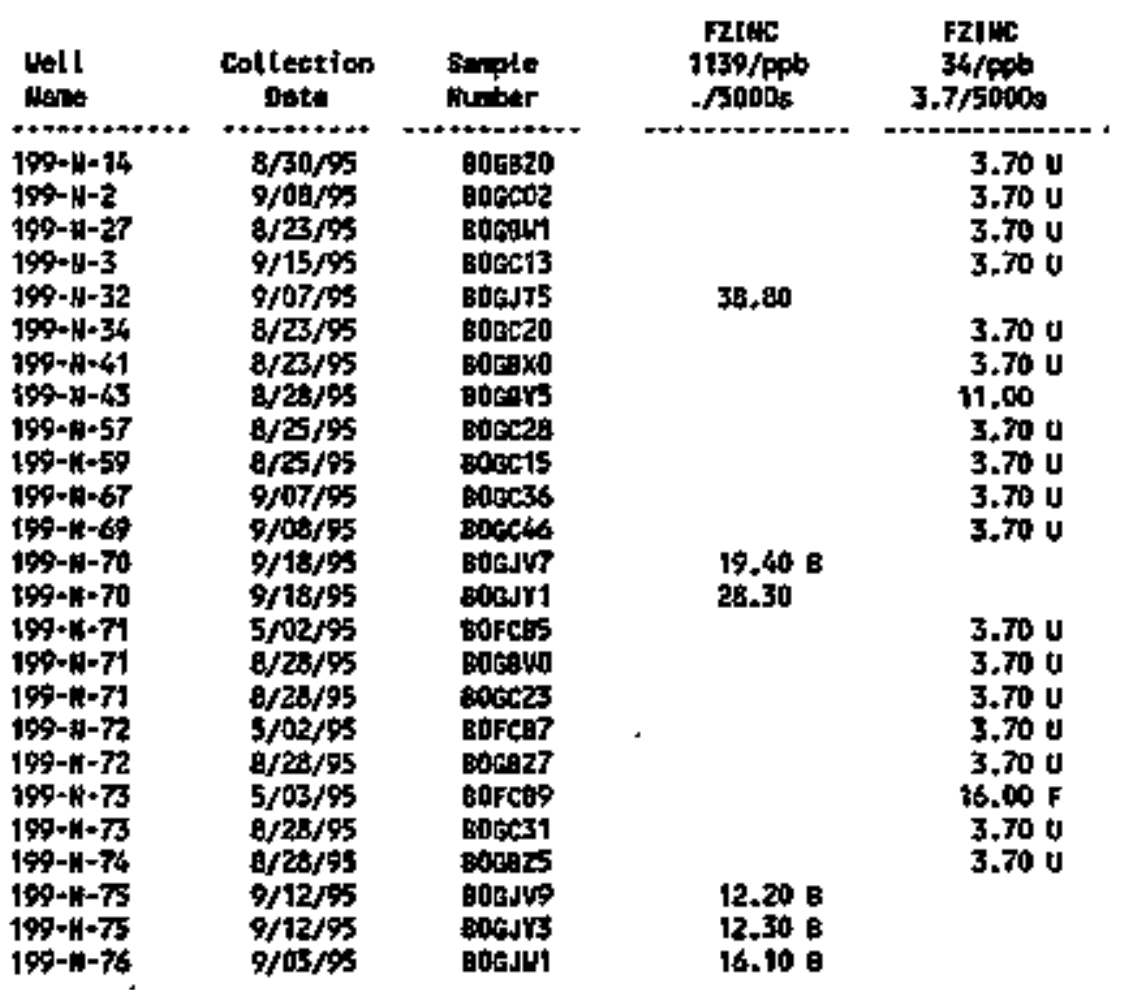


Tab7e 4-4. Constituents with at Least One Detected Valus for the 100-N RCRA Sites Data for Reporting Period July 1 through September 30, 1995. (sheet 17 of 17)

\begin{tabular}{|c|c|c|c|c|}
\hline Well & $\begin{array}{l}\text { Collection } \\
\text { Date }\end{array}$ & $\begin{array}{l}\text { sarpole } \\
\text { Mlututer }\end{array}$ & $\begin{array}{l}\text { Finc } \\
1 \mathrm{t3} / 1 \mathrm{ppb} \\
-/ 5100 \mathrm{x}\end{array}$ & $\begin{array}{c}\text { 12110 } \\
34 / \mathrm{pab}^{2} \\
3.7 / 5000 \mathrm{~s}\end{array}$ \\
\hline $\begin{array}{l}190-11-77 \\
190-11-77 \\
199-11-81\end{array}$ & $\begin{array}{l}5 / 03 / 95 \\
9 / 15 / 95 \\
6 / 30 / 95\end{array}$ & 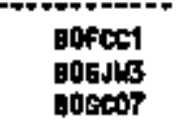 & 44.40 & $\begin{array}{l}3.70 \text { o } \\
3.70 \text { if }\end{array}$ \\
\hline
\end{tabular}

For explandion of this tobte, ato section $t .4$ of report. 
Table 4-5. Contamination Indicator Parameters for the 100-N RCRA Sites Data for Reporting Period July 1 through September 30, 1995.

(sheet 1 of 2)

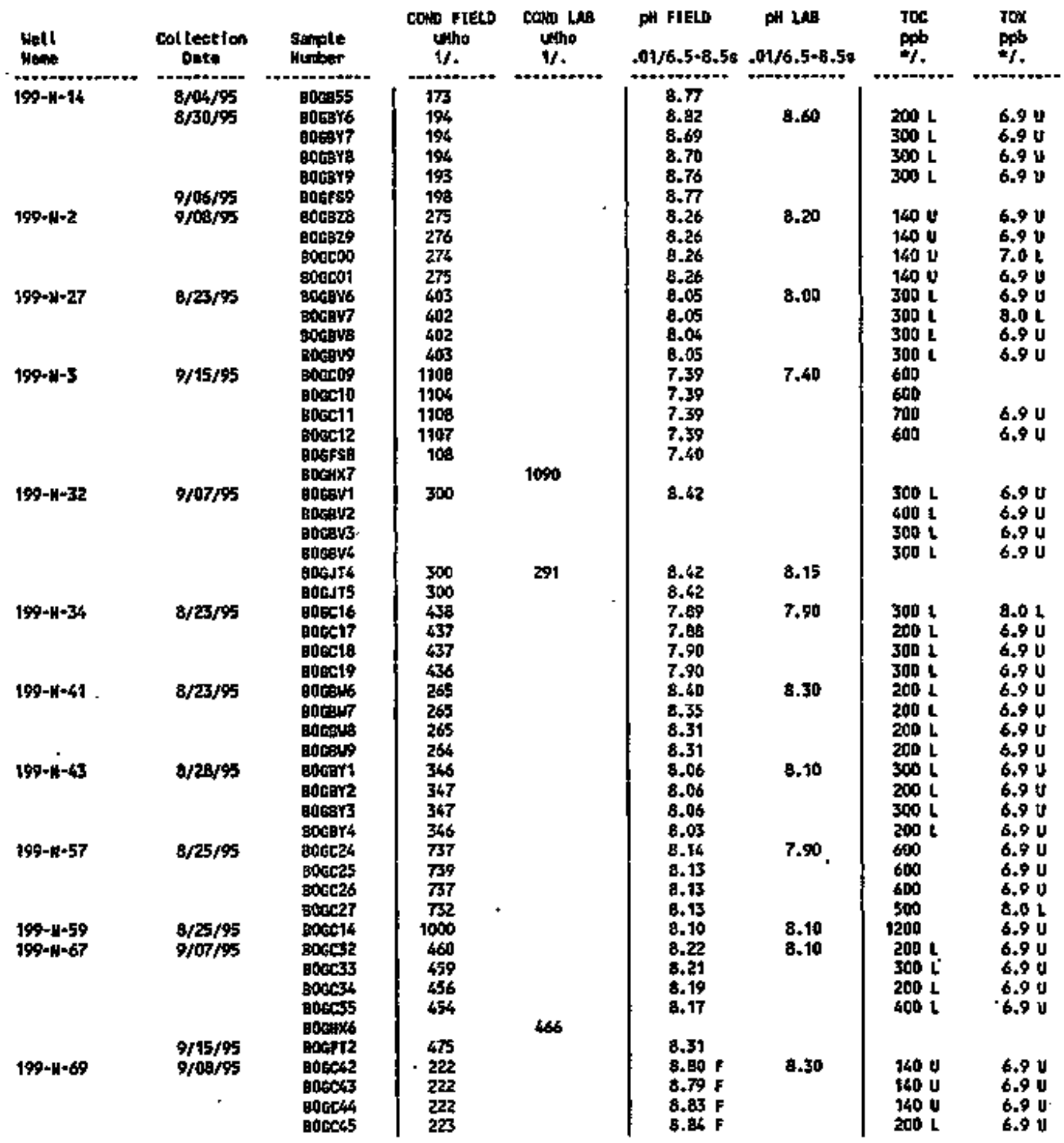


Table 4-5. Contamination Indicator Parameters for the 100-N RCRA Sites Data for Reporting Period July 1 through September 30, 1995.

(sheet 2 of 2)

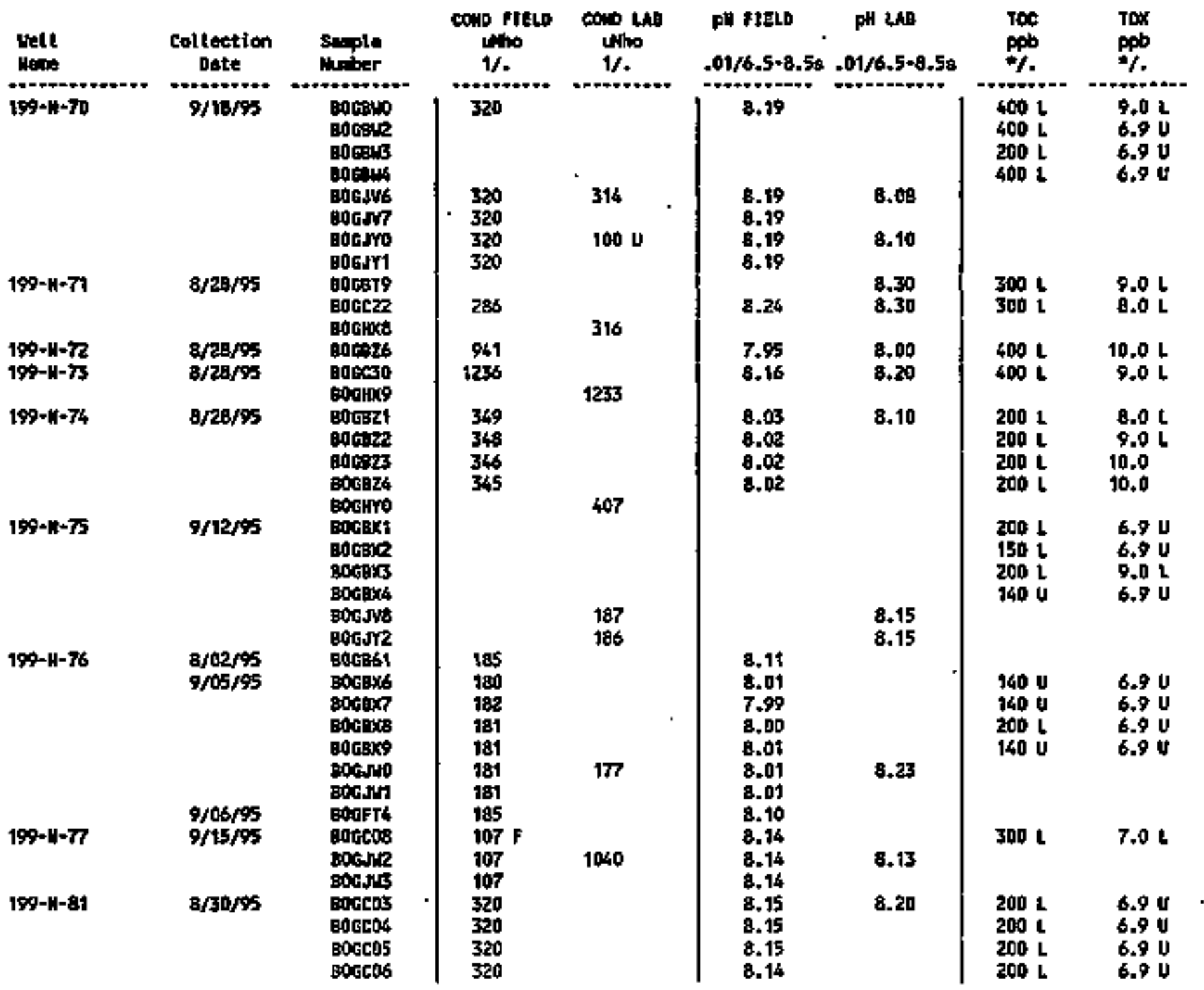

" Detection livits for TOS and rouk vary depending on the perforping laboratory. For explanstion of this table, gec section 1.4 of report. 
Tab7e 4-6. Drinking Nater Standards Exceeded for 100-N Area RCRA Sites.

\begin{tabular}{|l|l|}
\hline \multicolumn{1}{|c|}{ Constituent (ONS) } & \multicolumn{1}{c|}{$\begin{array}{c}\text { Wells exceeding DWS } \\
\text { (number of samples) }\end{array}$} \\
\hline Field pH (6.5-8.5) & $1301-\mathrm{N}: \mathrm{N}-14(4), \mathrm{N}-69(4)^{\mathrm{c}}$. \\
\hline Laboratory pH $(6.5-8.5)$ & $1301-\mathrm{N}: \mathrm{N}-14$. \\
\hline Nitrate $(45,000 \mathrm{ppb})$ & $1301-\mathrm{N}: \mathrm{N}-2, \mathrm{~N}-3, \mathrm{~N}-67^{\mathrm{e}}$ \\
\hline Sul fate $(250,000 \mathrm{ppb})$ & $1324-\mathrm{H} / \mathrm{NA}: \mathrm{N}-72, \mathrm{~N}-73$. \\
\hline Total dissolved solids (500 ppb) & $1324-\mathrm{N} / \mathrm{NA}: \mathrm{N}-59, \mathrm{~N}-72, \mathrm{~N}-73, \mathrm{~N}-77$. \\
\hline
\end{tabular}

"Data are in question. Request for Analytical Data Evaluation was submitted.

- BurS = drinking water standard.

Table 4-7. Requests for Analytical Data Evaluation for 100-N RCRA Sites, Third Quarter, 1995.

\begin{tabular}{|l|l|l|l|}
\hline He]1 No. & Sample Date & Constituent & Reasons \\
\hline $\mathrm{N}-67$ & $9 / 7 / 95$ & Nitrate & $\begin{array}{l}\text { Higher than historical } \\
\text { trend. }\end{array}$ \\
\hline $\mathrm{N}-77$ & $9 / 15 / 95$ & $\begin{array}{l}\text { Fjeld } \\
\text { conductivity }\end{array}$ & $\begin{array}{l}\text { Lower than historical } \\
\text { trend. }\end{array}$ \\
\hline $\mathrm{N}-69$ & $9 / 8 / 95$ & Field $\mathrm{pH}$ & $\begin{array}{l}\text { Higher than historical } \\
\text { trend; higher than lab pH. }\end{array}$ \\
\hline
\end{tabular}


$D O E / R L-95-69-3$

This page intentionally left b]ank. 
DOE/RL-95-69-1

\section{CONTEMS}

$5.0 \quad 216-B-3$ POND SYSTEH $\ldots \ldots \ldots \ldots \ldots \ldots \ldots \ldots \ldots \ldots \ldots \ldots$

5.1 INTRODUCTION $\ldots \ldots \ldots \ldots \ldots \ldots \ldots$

5.2 WATER LEVEL DATA $: \ldots \ldots \ldots \ldots \ldots \ldots$

5.3 WATER CHEMISTRY DATA $\ldots \ldots \ldots \ldots \ldots \ldots$ 
DOE/RL-95-69-1

\section{LIST OF FIEURES}

5-1 216-8-3 Pond Groundwater Honftoring Hetwork ......... 5-3

\section{LIST OF TABLES}

5-1 Monitoring Well Purpose and Sampling Schedule

for the 2I6-B-3 Pond Systen Hetwork ............... 5-4

5-2 RCRA Mater Level Measurement Report

216-B-3 Pond Systen, Third Quarter 1995 . . . . . . . . 5-5

5-3 Constituent List and Sunmary of Results

for the 216-B-3 Pond Data for Reporting Perjod

July 1 through September $30,1995 \ldots \ldots$ 5-7

5-4 Constituents with at least One Detected Value for the 216-B-3 Pond Data for Reporting Perjod July I through Septenber $30,1995 \ldots \ldots$ 5-10.

5-5 Contamination Indicator Parameters for the 216-B-3 Pond Data for Reporting Period

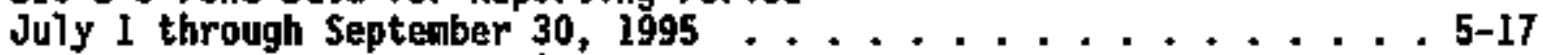




\title{
5.0 216-8-3 POND SYSTE
}

\author{
D. B. Barnett \\ Hest inghouse Hanford Company
}

\subsection{IMTROOUCTION}

The 216-B-3 Pond System is located east of the 200 East Area and originally consisted of a main pond and three expansion ponds for waste water disposal. Several ditches leading to the ponds and the main pond itself are no Tonger in use and are backfilled (Figures 1-1 and 5-1). These surface impoundiments cover approximately 41.3 hectares (102 acres). Only the $3 \mathrm{C}$ expansion pend now receives waste water. Table 5-1 lists the groundwater wells and their monitoring status.

The 216-B-3 Pond System groundwater monjtoring wel] locations are shown In Figure 5-1. Detection monitoring began at the 216-8-3 Pond System in November 1988 and contínued through June 1990, when assessment monitoring was scheduled to begin because of elevated levels of TOX in two downgradfent monitoring wells. Assessment monitoring actually began in June 1991 when groundwater sampling on the hanford Site resumed following a hiatus, as described in the Groundwater Quality Assessment Plan for the 216-B-3 Pond System (Harris 1990). Thus far, no specific constituents have been demonstrabTy linked to the elevated rox results.

\subsection{MATER LEVEL DATA}

Nater levels in 8 Pond wells were measured quarterly, and once during sample events during the July through September 1995 period. Table 5-2 1ists the results of the water level measurements. The results for weils 299-E18-1 and 699-43-42J are outside of the expected range of water levels for these wells, and may be in error.

\subsection{WATER CHEMISTRY DATA}

AlI wells scheduled for the July through September 1995 sampling were sampled during July, except upgradient well 299-E18-1, which was sampled in September. Any results for the period that are unavailable as of the writing of this report will be reported in future quarterly reports.

The 1 ist of constituents for which data were received and the summary of results avallable are given in rable 5-3. The results of the available analyses, for constituents with at least one value above the MOL, are reported in Table 5-4. Resutts of analyses for CIPs are reported in Table 5-5. Explanations of data flags and qualifiers are given in Section 1.4.

The constituent jist for this quarter includes those constituents required by regulations and compounds under investigation for potential links 
to elevated Tox results. Groundwater monitoring requirements and strategy for the 216-8-3 Pond System are described in Sweeney (1995).

Beginning with the July-through-September sampling event, the monitoring well network was reduced by 11 downgradient we]1s. This action was taken to reduce redundancy in the $B$ Pond network, and to reassign administrat ion of the three wells near the TEDF to that facility. Table 5-1 reflects these changes. Water levels from all wells of the original network, including TEDF wells, witl still be reported in this document.

Nell 699-40-40A produced a result for manganese in filtered samples (64 ppb) s1ightiy exceeding the drinking water standard (50 ppb). Well 699-43-41E produced an average $\mathrm{pH}$ of 8.52 , slightly over the upper limit for drinking water standards (8.50). Most results for filtered samples of calcium, sodium, and zinc are associated with suspect QC data. Vanadius results from filtered samples were assoctated with a contaninated biank sample. 
Figure 5-1. 216-8-3 Pond Groundwater Monitoring Network.

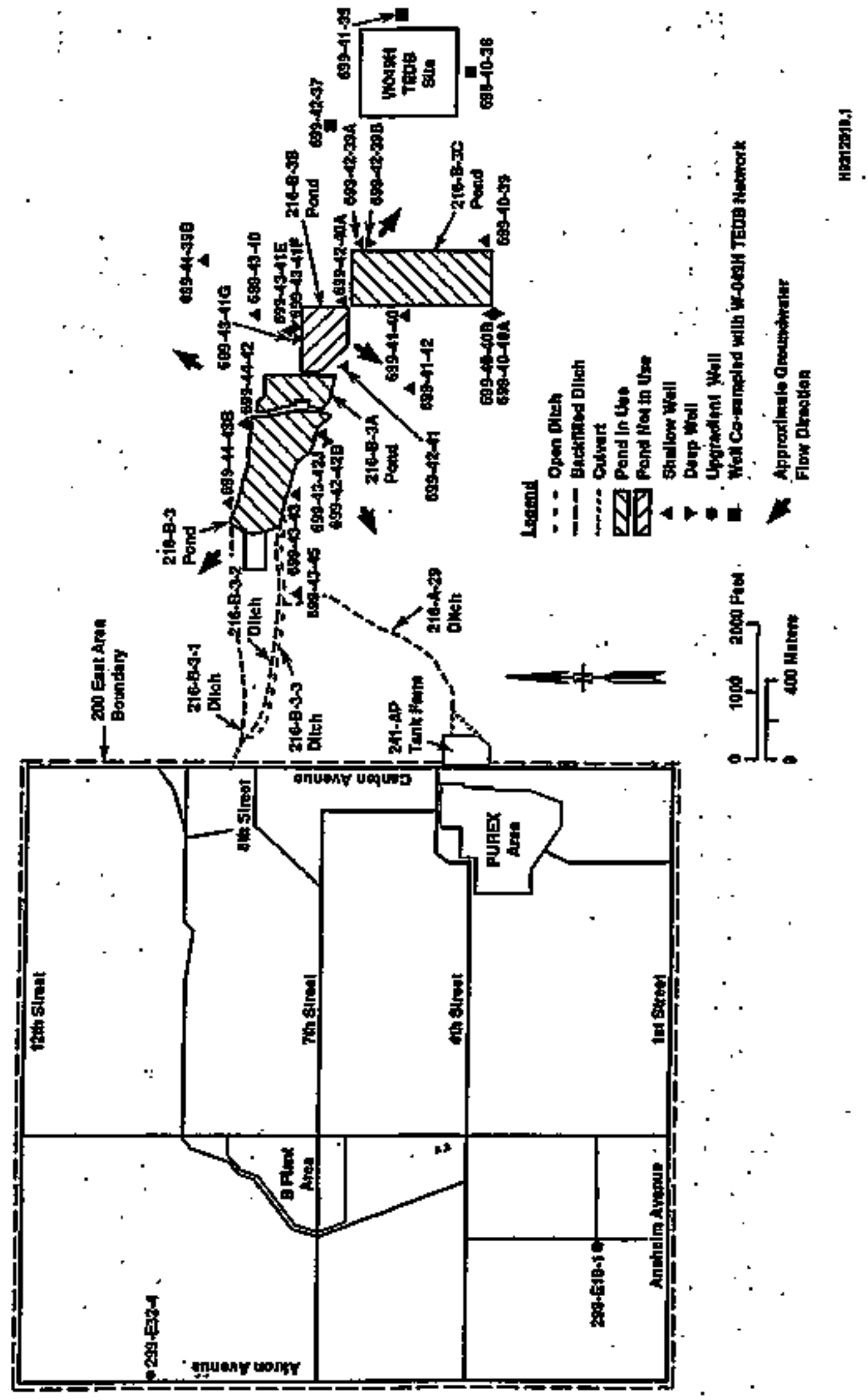


Table' 5-1. Monitoring He1] Purpose and Sampling Schedule for the 216-B-3 Pond System Hetwork.

\begin{tabular}{|c|c|c|c|c|}
\hline Nell no. & $\begin{array}{l}\text { Relative } \\
\text { position }\end{array}$ & Hydrogeologic unit & $\begin{array}{c}\text { Sample } \\
\text { frequancy }\end{array}$ & $\begin{array}{l}\text { Sample date, } \\
\text { 4th Qtr } 1994\end{array}$ \\
\hline 299-E18-1 & Upgradtent & Hanford: Watęr Tabie & Quarterly & $9 / 5 / 95$ \\
\hline $299-E 32-4^{-}$ & Upgradient & Hanford: Water Table & Quarterly & $7 / 18 / 95$ \\
\hline $699-40-40 A$ & Downgradient & Ringold: Lower Confined & QuarterTy & $7 / 21 / 95$ \\
\hline $699-41-40$ & Downgradient. & Ringold: Upper Confined & Quarter]y & $7 / 24 / 95$ \\
\hline $699-41-42$ & Downgradient: & Ringold: Top of Confined & Quarterly & $7 / 24 / 95$ \\
\hline $699-42-398$ & Doumgradient & Ringold: Lower Confined & Quarterly & $7 / 21 / 95$ \\
\hline $699-42-41$ & Oowngradient & Ringold: Water Table & Quarterly & $7 / 21 / 95$ \\
\hline $699-42-428$ & Downgradient. & Ringold: Lower Unconfined & Quarterly & $7 / 20 / 95$ \\
\hline $699-43-40$ & Downgradient & Ringold: Water Table & Quarterly & $7 / 19 / 95$ \\
\hline $699-43-41 E$ & Downgradient & Ringo]d: Upper Confined & Quarterly & $7 / 20 / 95$ \\
\hline 699-43-416 & Downgradient & Ringold: $\begin{array}{l}\text { Bottom of } \\
\text { Confined }\end{array}$ & Quarterly & $7 / 20 / 95$ \\
\hline $699-43-45^{b}$ & Downgradient & Hanford/Ringold: Water Table & Quarterly & $7 / 06 / 95$ \\
\hline $699-44-398$ & Downgradient & Rtngold: Hater Table & Quarterly & $7 / 20 / 95$ \\
\hline $699-44-438$ & Downgradjent & Ringold: Water Table & Quarterly & $7 / 20 / 95$ \\
\hline
\end{tabular}

We1l shared with the Low-Level Waste Burjal Grotuds network.

We11 shared with the 216-A-29 Ditch network. 
Table 5-2. RCRA Water Level Measurement Report 216-B-3 Pond Syster, Third Quarter 1995. (sheet 1 of 2)

\begin{tabular}{|c|c|c|c|c|}
\hline Wel] & Date & $\begin{array}{l}\text { Depth to } \\
\text { water (ft) }\end{array}$ & $\begin{array}{l}\text { Water } \\
\text { elevation } \\
\text { (ft) }\end{array}$ & $\begin{array}{l}\text { Tevel } \\
\text { above mst } \\
\text { (a) }\end{array}$ \\
\hline 299-E18-I & $\begin{array}{l}9 / 05 / 95 \\
9 / 14 / 95\end{array}$ & $\begin{array}{l}319.36^{\star}+ \\
318.71\end{array}$ & $\begin{array}{l}400.88 \\
401.53\end{array}$ & $\begin{array}{l}122.19 \\
122.39\end{array}$ \\
\hline $299-E 32-4$ & $\begin{array}{l}7 / 18 / 95 \\
9 / 20 / 95\end{array}$ & $\begin{array}{l}284.92^{*} \\
285.04\end{array}$ & $\begin{array}{l}401.19 \\
401.07\end{array}$ & $\begin{array}{l}122.28 \\
122.25\end{array}$ \\
\hline $699-40-36$ & $\begin{array}{l}7 / 10 / 95 \\
7 / 31 / 95 \\
8 / 18 / 95 \\
9 / 26 / 95\end{array}$ & $\begin{array}{l}118.69 * \\
118.16 \\
118.16 \\
118.17\end{array}$ & $\begin{array}{l}410.23 \\
410.76 \\
410.76 \\
410.75\end{array}$ & $\begin{array}{l}125.04 \\
125.20 \\
125.20 \\
125.20\end{array}$ \\
\hline $699-40-39$ & $\begin{array}{l}7 / 21 / 95 \\
7 / 31 / 95 \\
8 / 18 / 95 \\
9 / 26 / 95\end{array}$ & $\begin{array}{l}129.10^{\star} \\
129.29 \\
129.51 \\
129.58\end{array}$ & $\begin{array}{l}412.74 \\
412.55 \\
412.33 \\
412.26\end{array}$ & $\begin{array}{l}125.89 \\
125.75 \\
125.68 \\
125.66\end{array}$ \\
\hline $699-40-40 A$ & $\begin{array}{l}7 / 21 / 95 \\
9 / 26 / 95\end{array}$ & $\begin{array}{l}129.68 * \\
130.27\end{array}$ & $\begin{array}{l}411.53 \\
410.94\end{array}$ & $\begin{array}{l}125.43 \\
125.25\end{array}$ \\
\hline $699-40-40 B$ & $9 / 26 / 95$ & 130.90 & 411.28 & 125.36 \\
\hline $699-41-35$ & $\begin{array}{l}7 / 10 / 95 \\
7 / 31 / 95 \\
8 / 18 / 95 \\
9 / 26 / 95\end{array}$ & $\begin{array}{l}108.50^{\star} \\
108.59 \\
108.57 \\
108.45\end{array}$ & $\begin{array}{l}411.88 \\
411.79 \\
411.81 \\
411.93\end{array}$ & $\begin{array}{l}125.54 \\
125.51 \\
125.52 \\
125.56\end{array}$ \\
\hline $699-41-40$ & $\begin{array}{l}7 / 24 / 95 \\
9 / 26 / 95\end{array}$ & $\begin{array}{l}130.26^{*} \\
130.90\end{array}$ & $\begin{array}{l}415.68 \\
415.04\end{array}$ & $\begin{array}{l}126.70 \\
126.50\end{array}$ \\
\hline $699-41-42$ & $\begin{array}{l}7 / 24 / 95 \\
9 / 26 / 95\end{array}$ & $\begin{array}{l}232.40^{*} \\
233.25\end{array}$ & $\begin{array}{l}411.51 \\
410.66\end{array}$ & $\begin{array}{l}125.43 \\
125.17\end{array}$ \\
\hline $699-42-37$ & $\begin{array}{l}7 / 10 / 95 \\
7 / 31 / 95 \\
8 / 18 / 95 \\
9 / 26 / 95\end{array}$ & $\begin{array}{l}105.00^{\star} \\
104.46 \\
104.58 \\
104.36\end{array}$ & $\begin{array}{l}414.40 \\
414.94 \\
414.82 \\
415.04\end{array}$ & $\begin{array}{l}126.31 \\
125.47 \\
126.44 \\
126.50\end{array}$ \\
\hline $699-42-39 A$ & $9 / 26 / 95$ & 139.20 & 418.94 & 127.69 \\
\hline $699-42-39 B$ & $\begin{array}{l}7 / 21 / 95 \\
9 / 26 / 95\end{array}$ & $\begin{array}{l}138.85 * \\
139.62\end{array}$ & $\begin{array}{l}419.47 \\
418.70\end{array}$ & $\begin{array}{l}127.85 \\
127.62\end{array}$ \\
\hline $699-42-40 A$ & $9 / 26 / 95$ & 124.33 & 421.20 & 128.38 \\
\hline
\end{tabular}


Table 5-2. RCRA Hater Level Heasurement Report 216-8-3 Pond System, Thtrd Quarter 1995. (sheet 2 of 2)

\begin{tabular}{|c|c|c|c|c|}
\hline Well & Date & $\begin{array}{l}\text { Depth to } \\
\text { water (ft) }\end{array}$ & $\begin{array}{l}\text { Water } \\
\text { elevation } \\
\text { (ft) }\end{array}$ & $\begin{array}{l}\text { Tevel } \\
\text { above ms1 } \\
\qquad(\mathrm{m})\end{array}$ \\
\hline $699-42-41$ & $\begin{array}{l}7 / 21 / 95 \\
9 / 26 / 95\end{array}$ & $\begin{array}{l}146.56^{*} \\
147.36\end{array}$ & $\begin{array}{l}420.74 \\
419.94\end{array}$ & $\begin{array}{l}128.24 \\
128.00\end{array}$ \\
\hline $699-42-428$ & $\begin{array}{l}\text { 7/20/95 } \\
9 / 26 / 95\end{array}$ & $\begin{array}{l}166.68^{*} \\
167.74\end{array}$ & $\begin{array}{l}416.55 \\
415.49\end{array}$ & $\begin{array}{l}126.96 \\
126.64\end{array}$ \\
\hline $699-43-40$ & $\begin{array}{l}7 / 19 / 95 \\
9 / 26 / 95\end{array}$ & $\begin{array}{l}121.15^{*} \\
122.29\end{array}$ & $\begin{array}{l}421.05 \\
419.91\end{array}$ & $\begin{array}{l}128.34 \\
127.99\end{array}$ \\
\hline $699-43-41 E$ & $\begin{array}{l}7 / 20 / 95 \\
9 / 26 / 95\end{array}$ & $\begin{array}{l}129.87 * \\
130.68\end{array}$ & $\begin{array}{l}420.99 \\
420.18\end{array}$ & $\begin{array}{l}128.32 \\
128.07\end{array}$ \\
\hline $699-43-41 F$ & $9 / 26 / 95$ & 130.77 & 420.24 & 128.09 \\
\hline $699-43-416$ & $\begin{array}{l}7 / 20 / 95 \\
9 / 26 / 95\end{array}$ & $\begin{array}{l}134.20 \star \\
135.13\end{array}$ & $\begin{array}{l}417.14 \\
416.21\end{array}$ & $\begin{array}{l}127.14 \\
126.86\end{array}$ \\
\hline $699-43-42 \mathrm{~J}$ & $9 / 26 / 95$ & $124.73+$ & 456.95 & 139.28 \\
\hline $699-43-43$ & $\begin{array}{l}7 / 06 / 95 \\
9 / 26 / 95\end{array}$ & $\begin{array}{l}165.75^{*} \\
166.41\end{array}$ & $\begin{array}{l}413.62 \\
412.96\end{array}$ & $\begin{array}{l}126.07 \\
125.87\end{array}$ \\
\hline $699-43-45$ & $\begin{array}{l}7 / 06 / 95 \\
9 / 25 / 95\end{array}$ & $\begin{array}{l}195.96^{\star} \\
195.62\end{array}$ & $\begin{array}{l}401.72 \\
402.06\end{array}$ & $\begin{array}{l}122.44 \\
122.55\end{array}$ \\
\hline $699-44-398$ & $\begin{array}{l}7 / 20 / 95 \\
9 / 26 / 95\end{array}$ & $\begin{array}{l}92.26 * \\
93.41\end{array}$ & $\begin{array}{l}421.14 \\
419.99\end{array}$ & $\begin{array}{l}128.36 \\
128.01\end{array}$ \\
\hline $699-44-42$ & $9 / 26 / 95$ & 160.42 & 418.80 & 127.65 \\
\hline $699-44-438$ & $\begin{array}{l}7 / 20 / 95 \\
9 / 26 / 95\end{array}$ & $\begin{array}{l}166.20^{*} \\
167.23\end{array}$ & $\begin{array}{l}413.92 \\
412.89\end{array}$ & $\begin{array}{l}126.16 \\
125.85\end{array}$ \\
\hline
\end{tabular}

NOTES: 1. Mater level elevations are calculated by subtracting the measured depth-to-water from the surveyed elevation for the well.

2. Depth-to-water values are transcribed from field records.

3. Heasurements marked with an ' $*$ ' were taken at the time of sappling.

4. Measurements marked with $a^{\prime}+{ }^{\prime}$ are outside of the expected range, and are suspected of error. 
Table 5-3. Constituent List and Sumary of Results for the 216-B-3 Pond Data for Reporting Period July 1 through September 30, 1995.

(sheet I of 3)

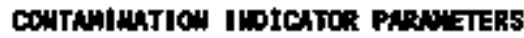

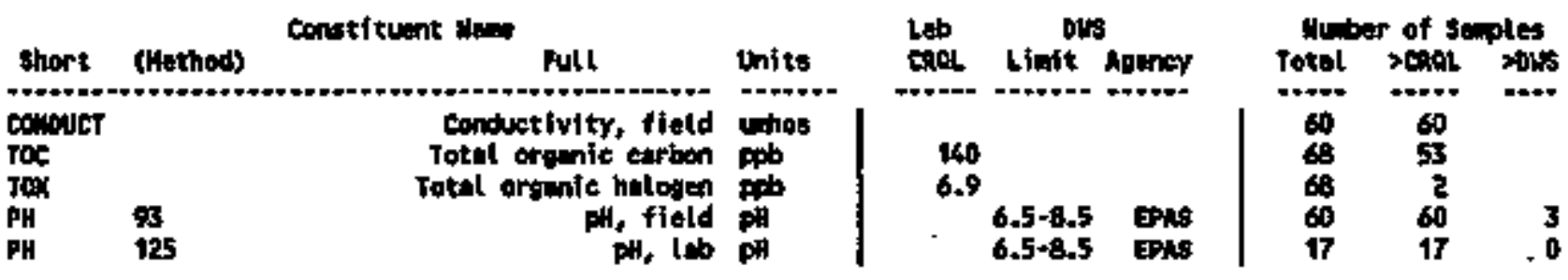

DRTMKING HATER PURMETERS

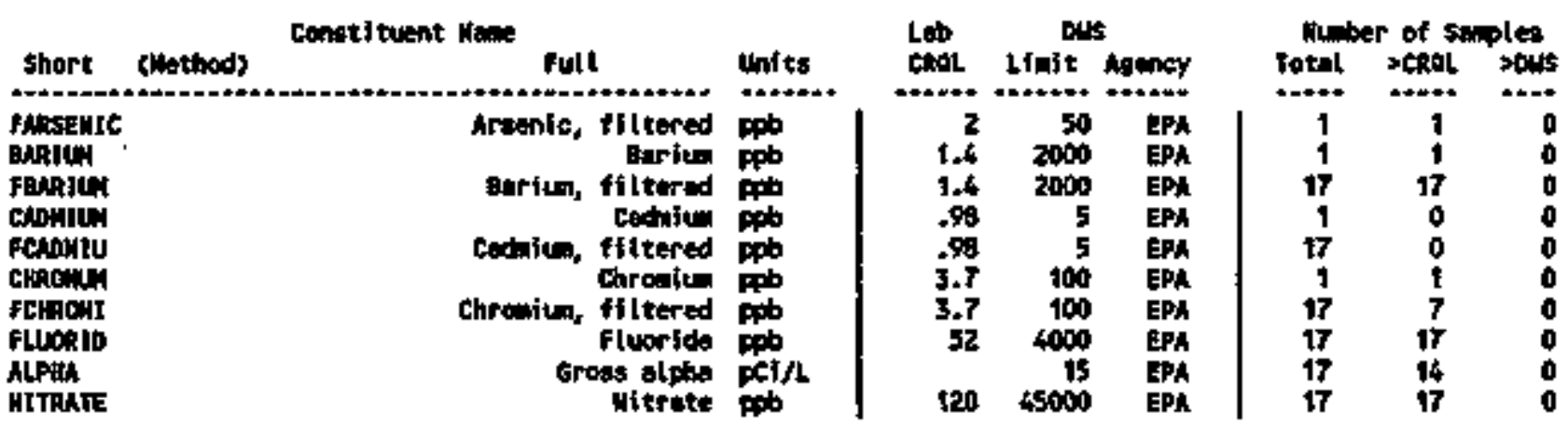

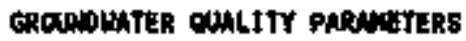

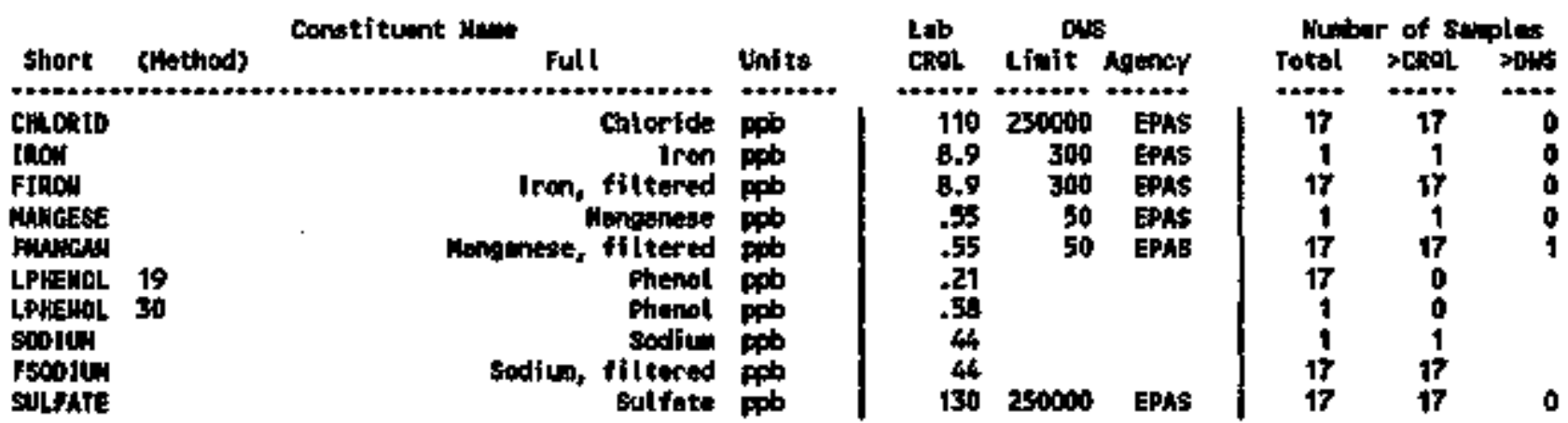


Table 5-3. Constituent List and Summary of Results for the 216-B-3 Pond Data for Reporting Perjod July 1 through September 30, 1995.

(sheet 2 of 3 )

SITE BECLFIC AD OTRER COHSIITUENTS

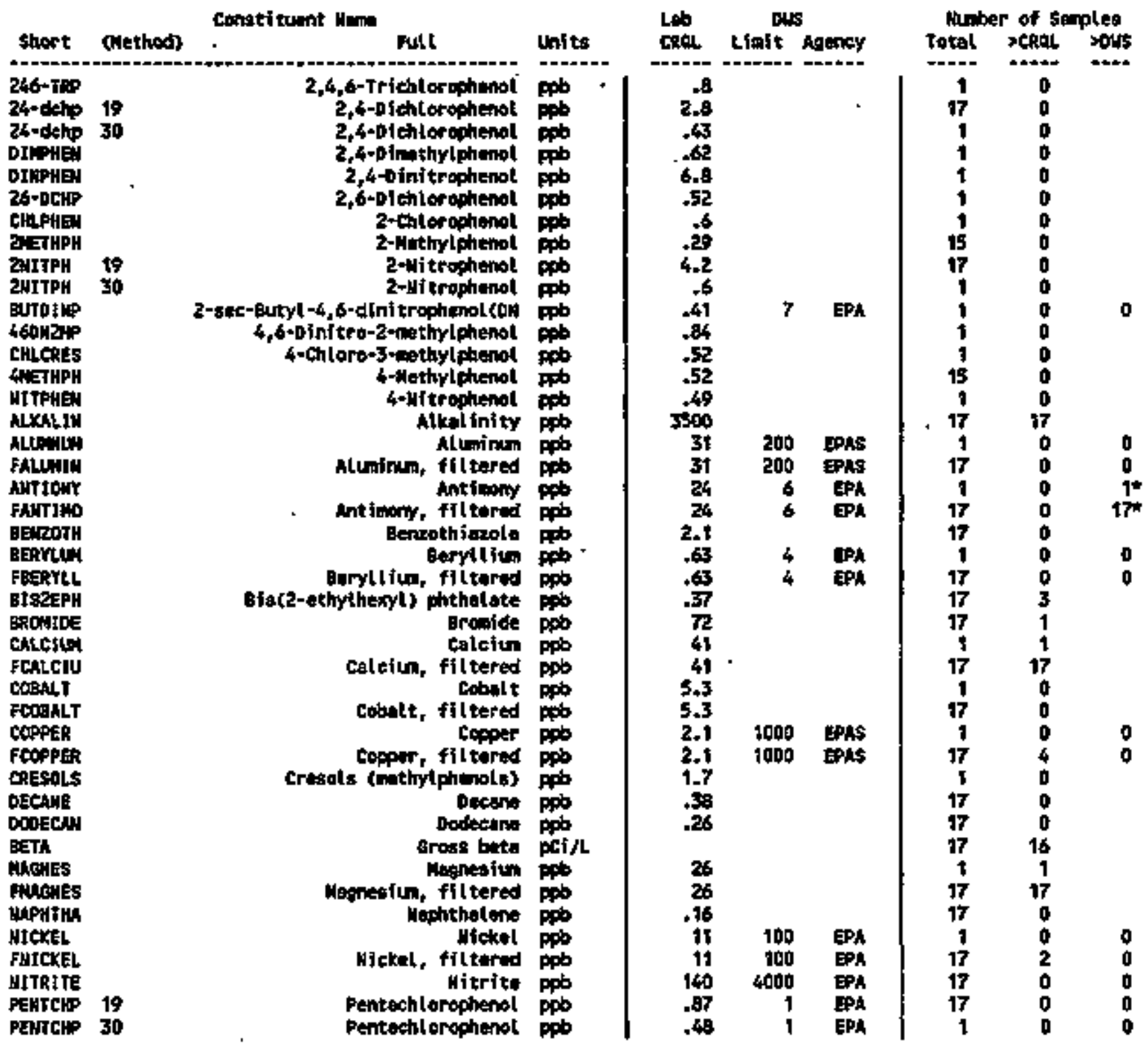


Tabie 5-3. Constituent List and Summary of Results

for the 216-B-3 Pond Data for Reporting Period July 1 through September 30, 1995.

(sheet 3 of 3)

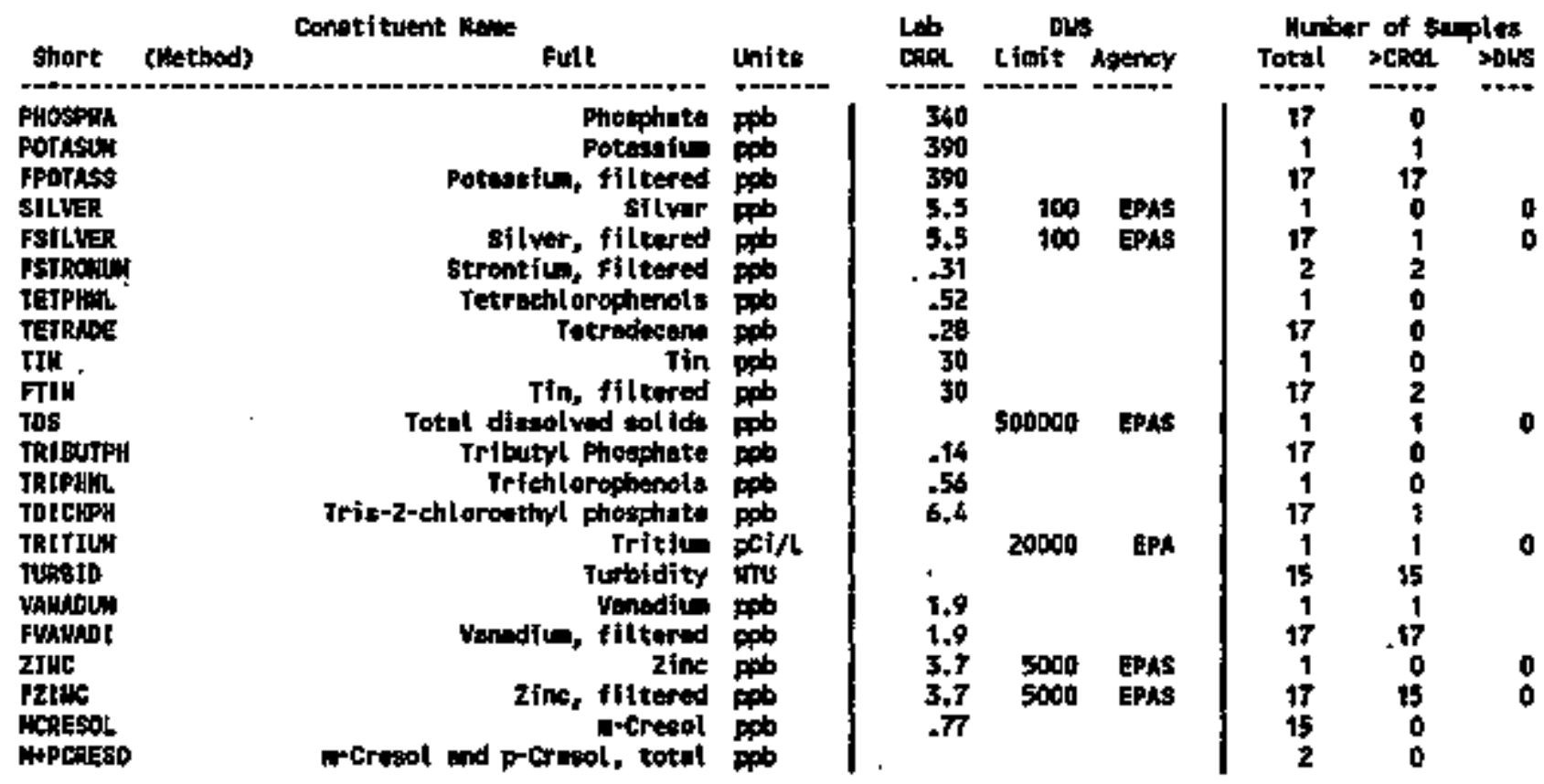

For aplention of this table, ses section 1.4 of report. 
Table 5-4. Constituents with at Least One Detected Value for the 216-8-3 Pond Data for Reporting Period July 1 through September 30, 1995. (sheet 1 of 7 )

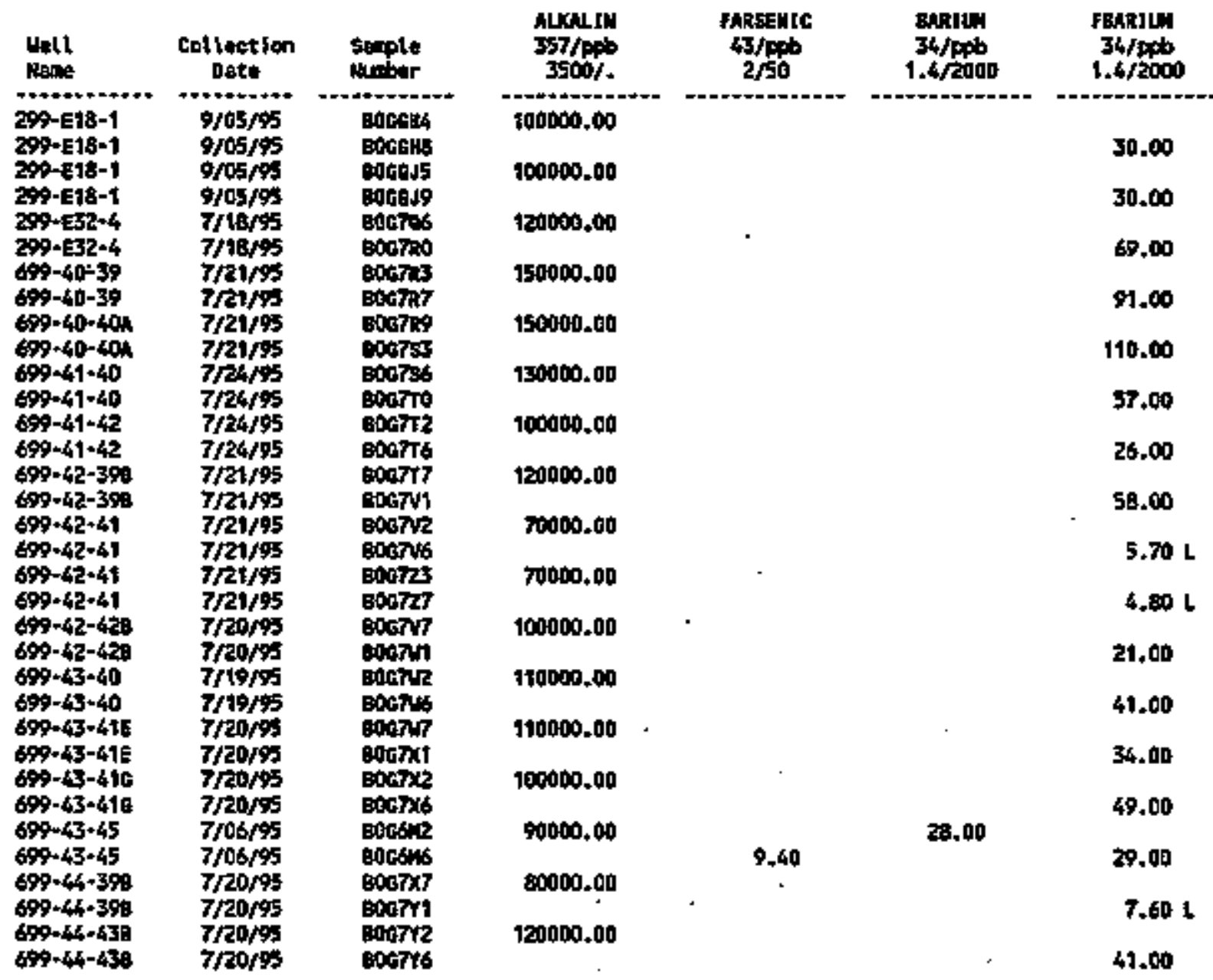

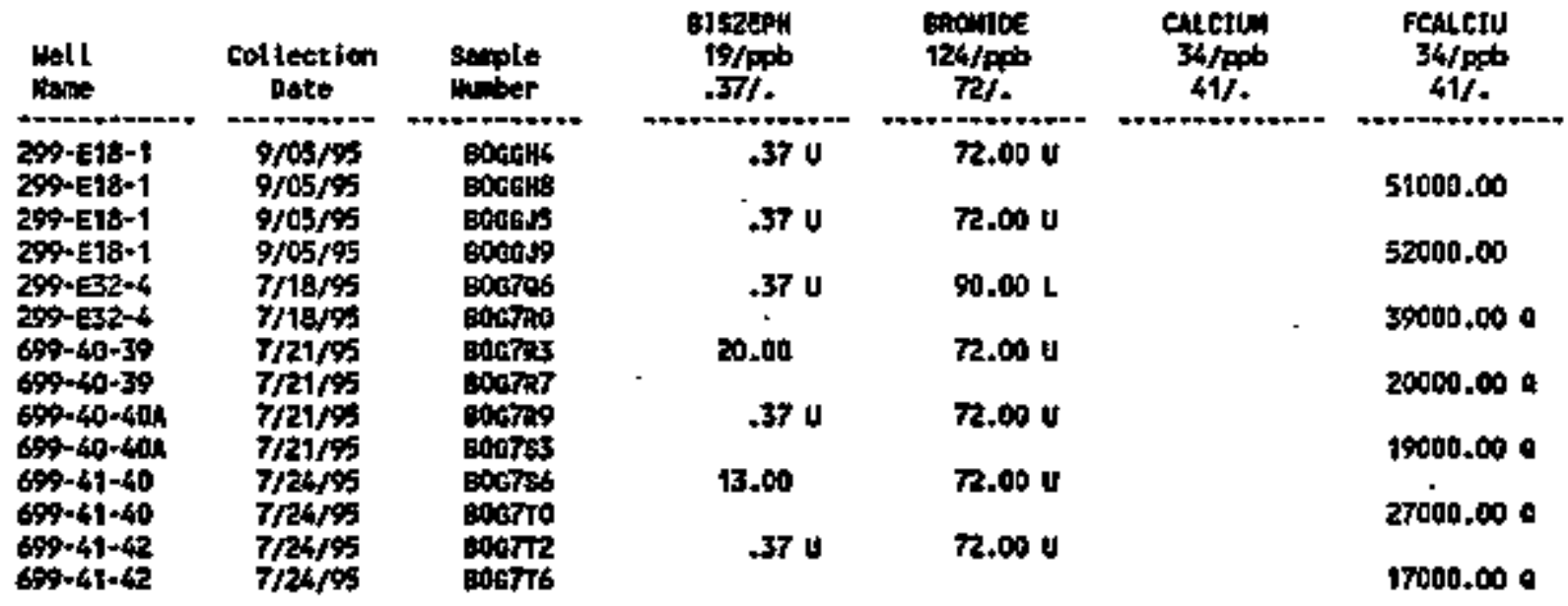


Table 5-4. Constituents with at Least One Detected Value for the 216-B-3 Pond Data for Reporting Period July 1 through Septenber 30, 1995.

(sheet 2 of 7 )

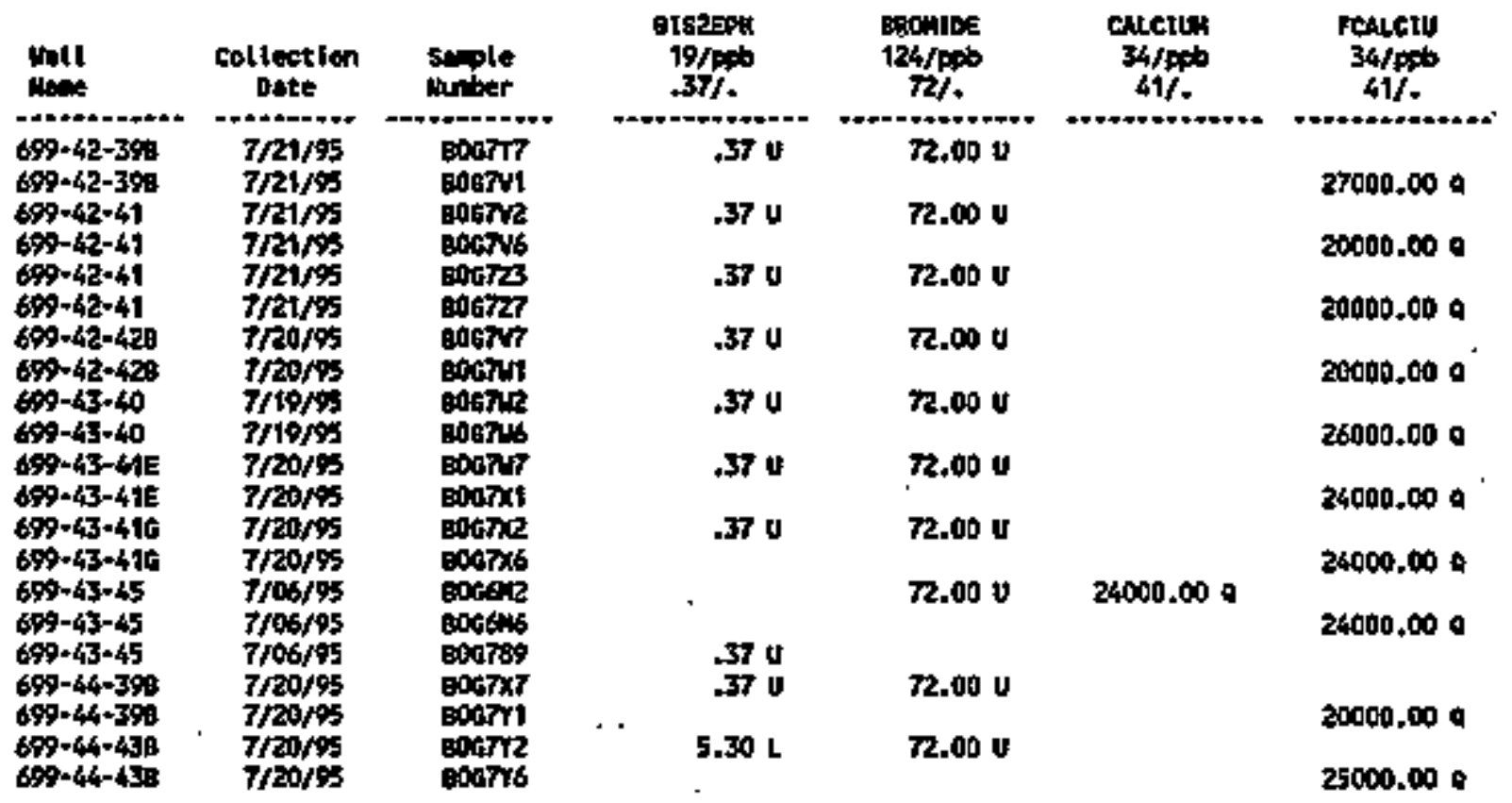

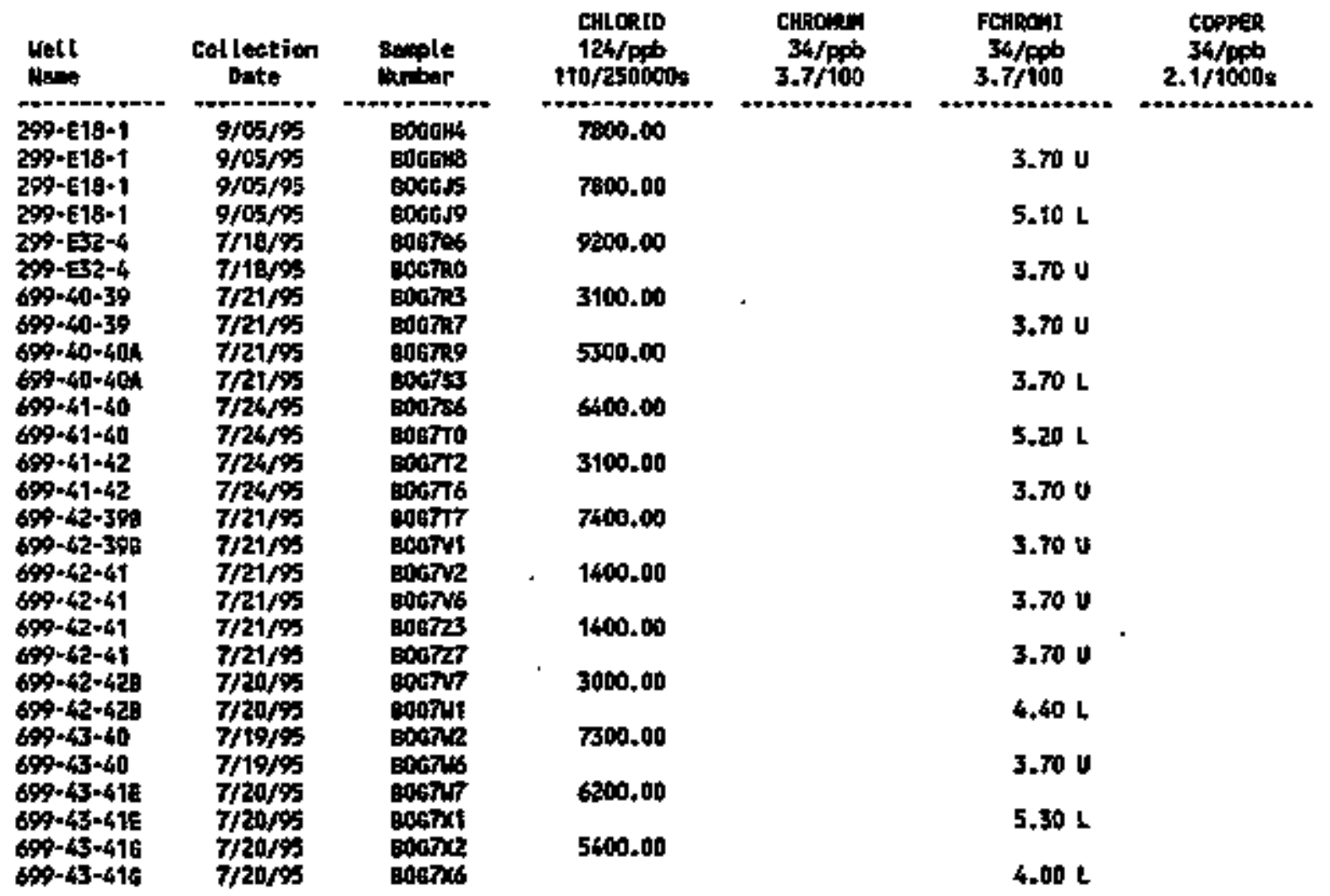


Table 5-4. . Constituents with at Least One Detected Value for the 216-8-3 Pond Dat for Reporting Period July 1 through September 30, 1995.

(sheet 3 of 7 )

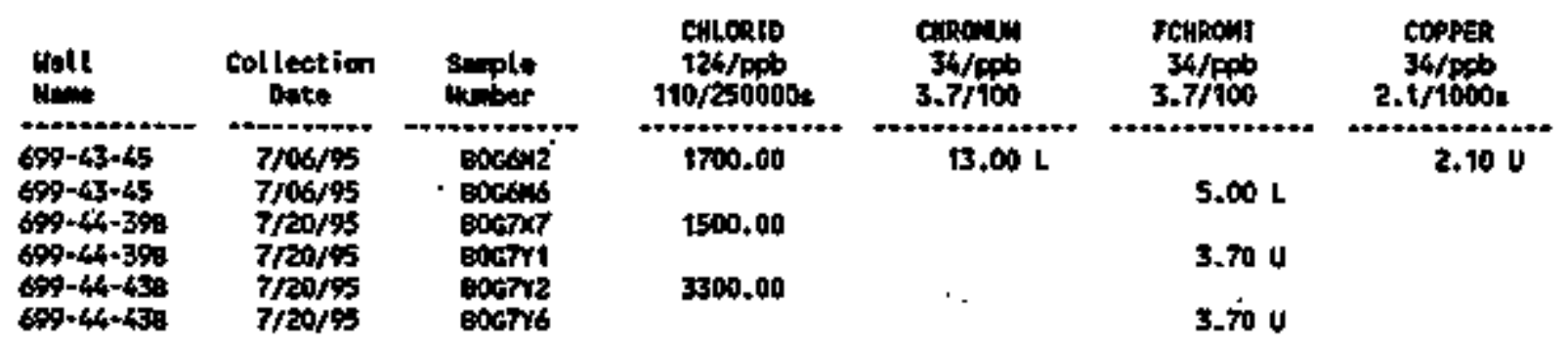

\begin{tabular}{|c|c|c|c|c|c|c|}
\hline $\begin{array}{l}\text { Welt } \\
\text { mos: }\end{array}$ & $\begin{array}{c}\text { Coll leetion } \\
\text { Dote }\end{array}$ & smple & $\begin{array}{c}\text { FCoppes } \\
34 / \mathrm{ppt}^{2} \\
2,1 / 1000 \mathrm{~s}\end{array}$ & $\begin{array}{l}\text { FLUORJd } \\
\$ 24 / \mathrm{ppb} \\
\$ 2 / 40090\end{array}$ & $\begin{array}{c}\text { ALPRK } \\
135 / \mathrm{PCI}^{\mathrm{Cl} / L} \\
. / 15\end{array}$ & $\begin{array}{c}\text { BETh } \\
136 / \propto<1 / L \\
. / .\end{array}$ \\
\hline 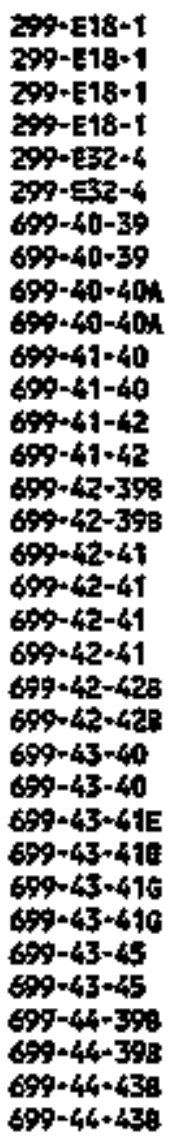 & $\begin{array}{l}9 / 05 / 95 \\
9 / 05 / 95 \\
9 / 05 / 95 \\
9 / 05 / 95 \\
7 / 18 / 95 \\
7 / 18 / 95 \\
7 / 21 / 95 \\
7 / 21 / 95 \\
7 / 21 / 99 \\
7 / 21 / 96 \\
7 / 24 / 99 \\
7 / 24 / 95 \\
7 / 24 / 95 \\
7 / 24 / 95 \\
7 / 21 / 95 \\
7 / 21 / 95 \\
7 / 21 / 95 \\
7 / 71 / 95 \\
7 / 21 / 95 \\
7 / 21 / 95 \\
7 / 20 / 95 \\
7 / 20 / 95 \\
7 / 19 / 95 \\
7 / 19 / 95 \\
7 / 20 / 95 \\
7 / 20 / 95 \\
7 / 20 / 95 \\
7 / 20 / 95 \\
7 / 106 / 95 \\
7 / 06 / 95 \\
7 / 20 / 95 \\
7 / 20 / 95 \\
7 / 20 / 95 \\
7 / 20 / 95\end{array}$ & 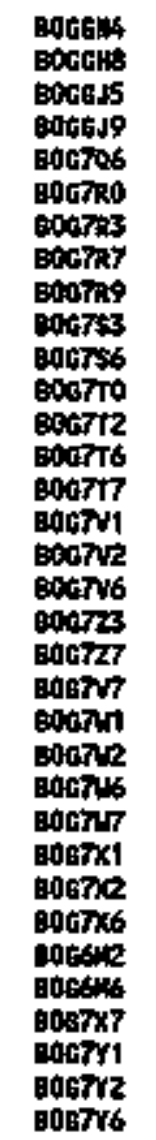 & $\begin{array}{l}2.10 \mathrm{U} \\
2.10 \mathrm{~V} \\
2.10 \mathrm{U} \\
2.10 \mathrm{U} \\
2.40 \mathrm{~L} \\
9.60 \mathrm{U} \\
2.10 \mathrm{U} \\
2.10 \mathrm{U} \\
2.40 \mathrm{~L} \\
2.10 \mathrm{U} \\
2.10 \mathrm{U} \\
2.10 \mathrm{U} \\
2.10 \mathrm{U} \\
2.40 \mathrm{~L} \\
2.10 \mathrm{U} \\
2.10 \mathrm{U} \\
2.10 \mathrm{~V}\end{array}$ & $\begin{array}{l}800.00 \\
700.00 \\
800.00 \\
9000.00 \\
1000.00 \\
1000.00 \\
800.00 \\
900.00 \\
200.00 \\
200.00 \\
700.00 \\
500.00 \\
700.00 \\
800.00 \\
300.00 \\
200.00 \\
700.00\end{array}$ & $\begin{array}{l}6.31 \\
5.03 \\
3.74 \\
6.69 \\
3.99 \\
3.80 \\
.840 \\
3.43 \\
.360 \\
.080 \\
2.25 \\
3.75 \\
1.82 \\
1.57 \\
1.09 \\
1.23 \\
2.49\end{array}$ & $\begin{array}{l}7.29 \\
8.11 \\
20.60 \\
8.15 \\
7.43 \\
7.95 \\
2.64 \\
6.42 \\
4.62 \\
3.65 \\
2.210 \\
6.15 \\
4.76 \\
5.61 \\
5.27 \\
2.86 \\
3.76\end{array}$ \\
\hline
\end{tabular}


Table 5-4. Constituents with at Least One Detected Value for the 216-8-3 Pond Data for Reporting Period July 1 through Septenber 30, 1995.

(sheet 4 of 7 )

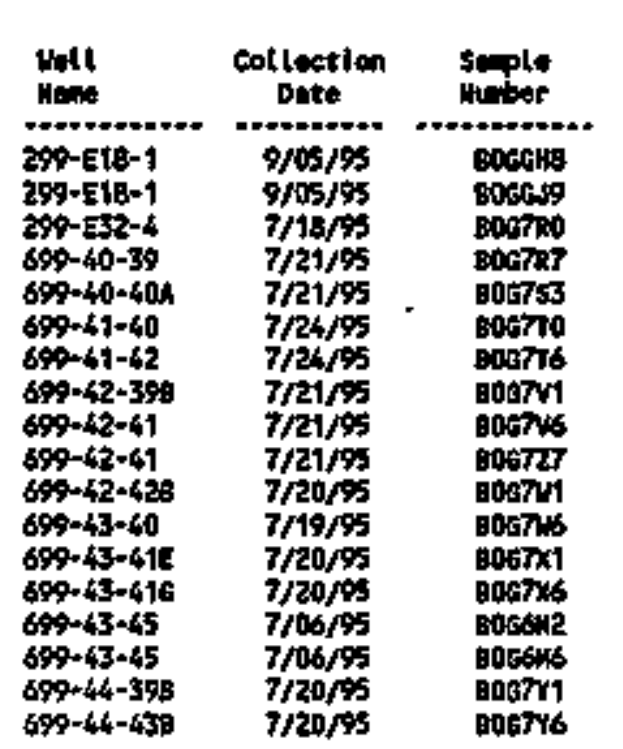

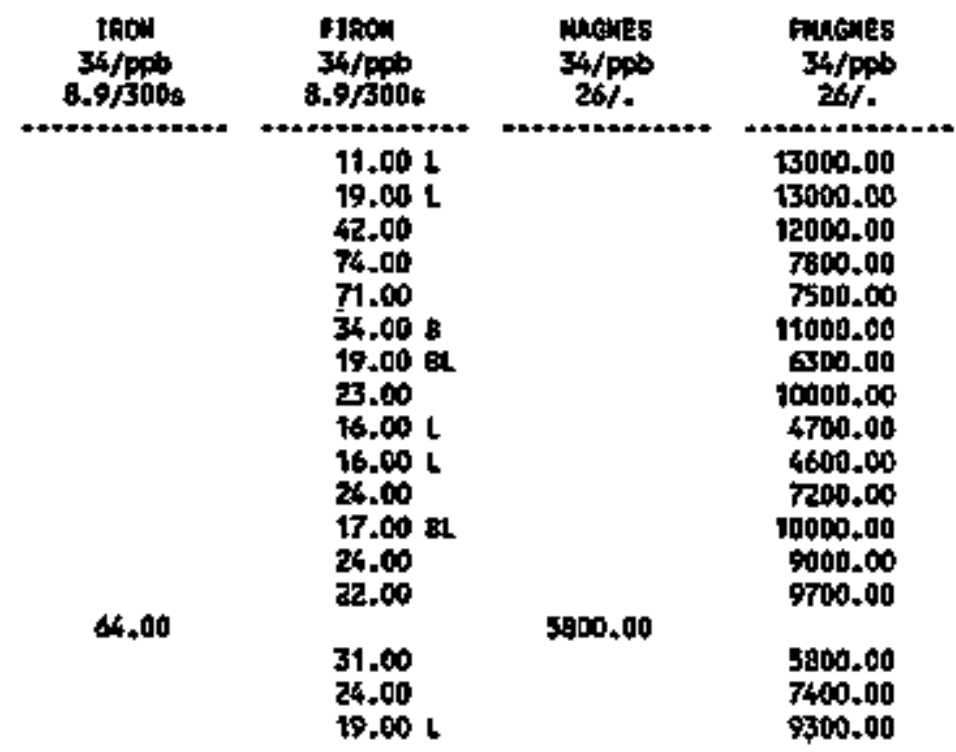

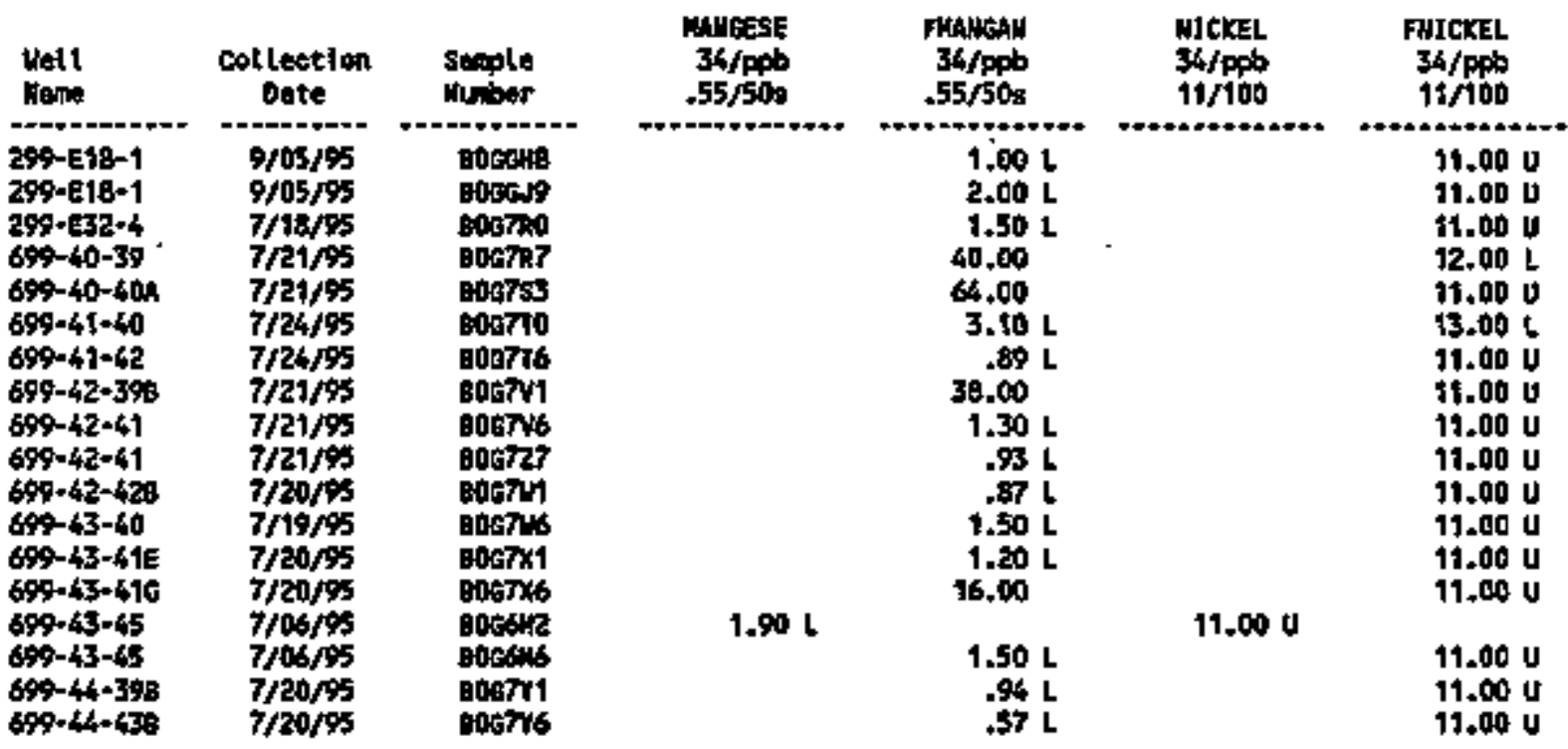


Table 5-4. Constituents with at Least One Detected Value for the 216-8-3 Pond Data for Reporting Perlod JuTy 1 through September 30, 1995. (sheet 5 of 7 )

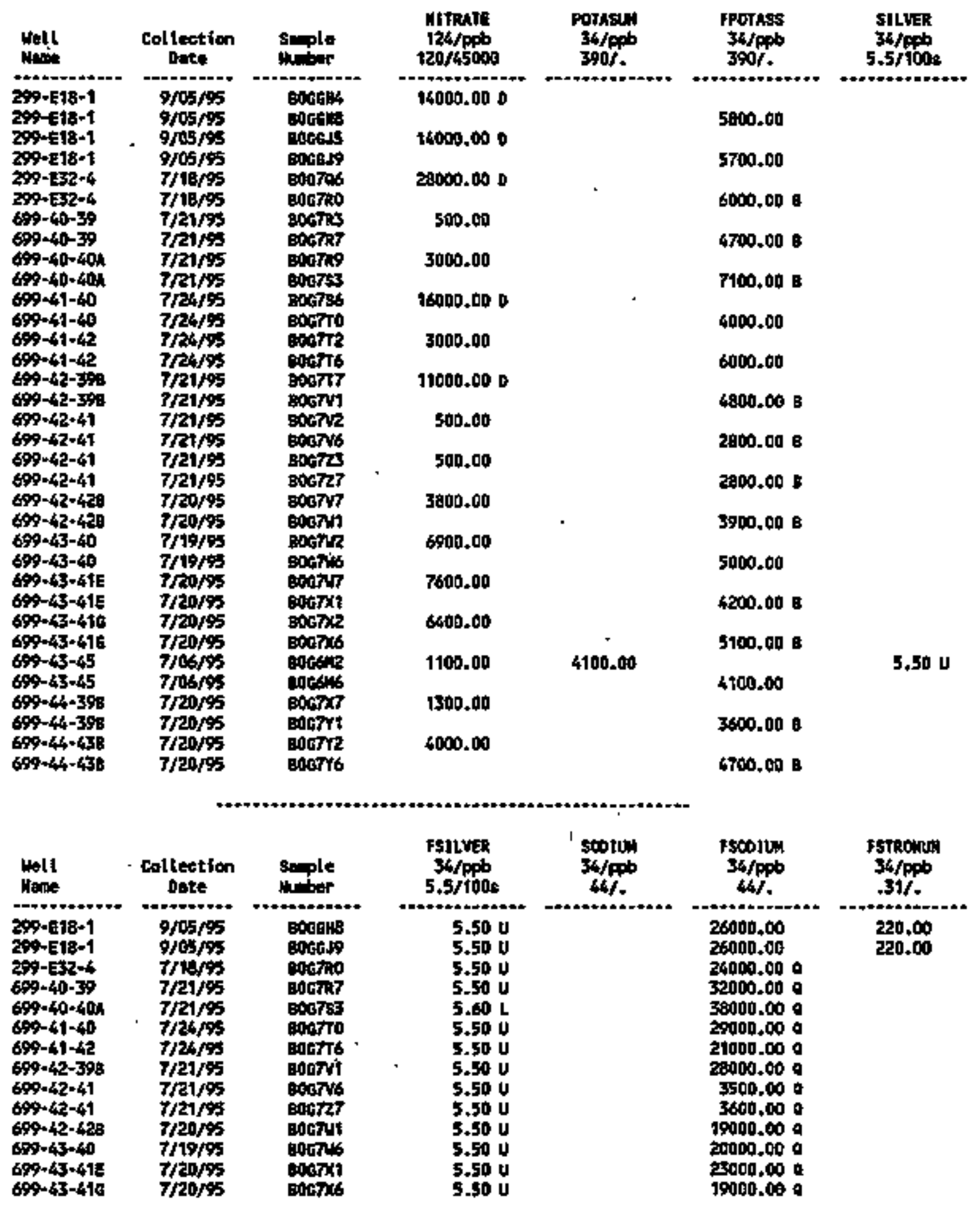


Table 5-4. Constituents with at Least One Detected Value for the 216-B-3 Pond Data for Reporting Perjod July 1 through September 30, 1995.

\begin{tabular}{|c|c|c|c|c|c|c|}
\hline inti & $\begin{array}{c}\text { Collection } \\
\text { Date }\end{array}$ & $\begin{array}{l}\text { strple } \\
\text { number }\end{array}$ & $\begin{array}{c}\text { F8ILVER } \\
34 / 00 b \\
5.5 / 100 s\end{array}$ & $\begin{array}{c}\text { soorus } \\
34 / \mathrm{ph} \\
44 / .\end{array}$ & $\begin{array}{c}\text { FscoILN } \\
34 / 506 \\
44 / 0\end{array}$ & $\begin{array}{l}\text { Fsreowin } \\
34 / \text { pot } \\
31 /\end{array}$ \\
\hline $\begin{array}{l}699-43-45 \\
697-43-45 \\
699-44-399 \\
699-44-43 B\end{array}$ & $\begin{array}{l}7 / 06 / 95 \\
7 / 06 / 95 \\
7 / 20 / 95 \\
7 / 20 / 95\end{array}$ & $\begin{array}{l}\text { gotesine } \\
\text { bocsins } \\
\text { gotrift } \\
\text { noterys }\end{array}$ & $\begin{array}{l}5.50 \mathrm{U} \\
5.50 \mathrm{U} \\
5.50 \mathrm{U}\end{array}$ & $7900.00 *$ & $\begin{array}{r}7900.000 \\
5300.000 \\
20000.000\end{array}$ & \\
\hline
\end{tabular}

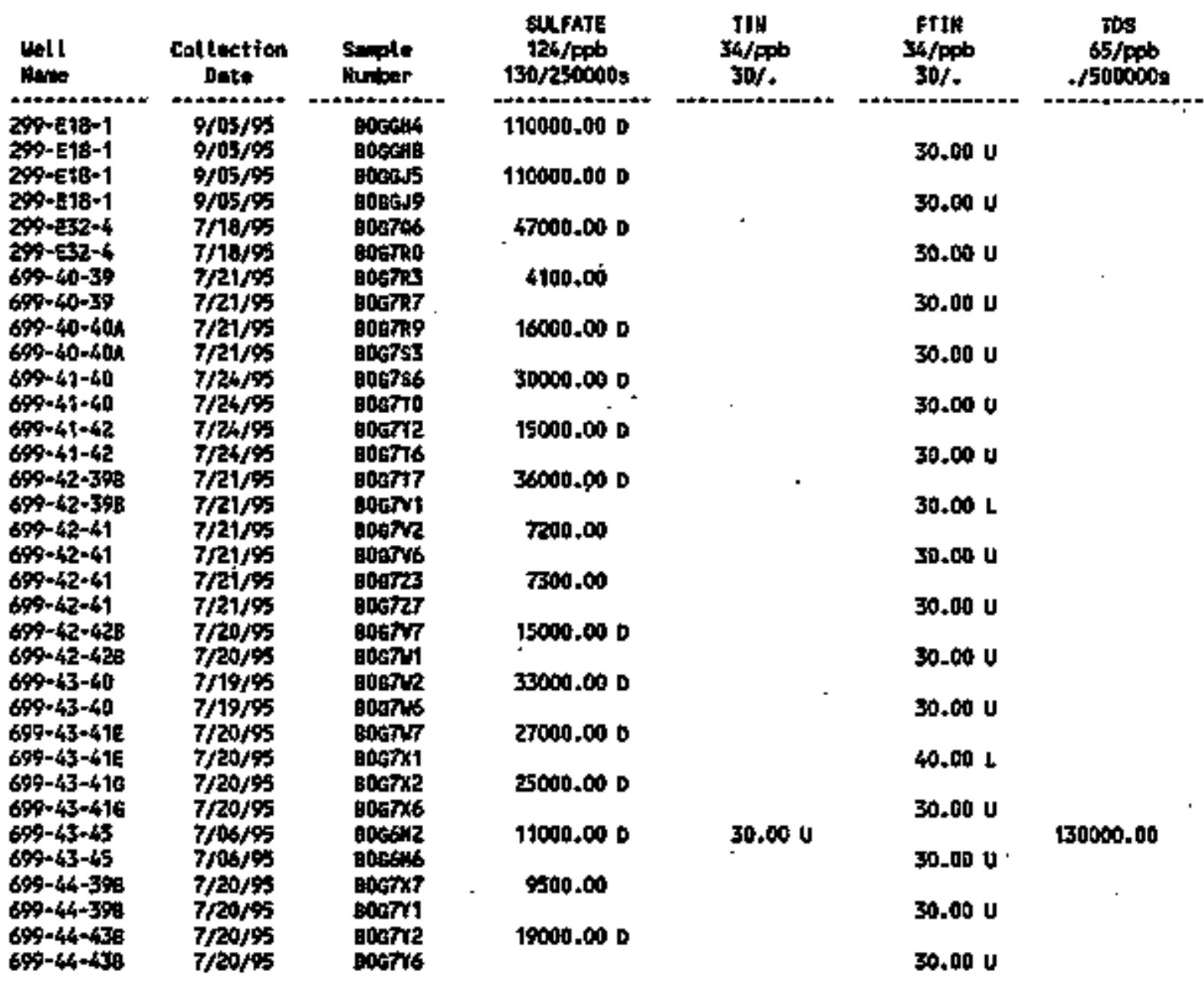


Table 5-4. Constituents with at Least. One Detected Value for the 216-B-3 Pond Data for Reporting Period July 1 through September 30, 1995.

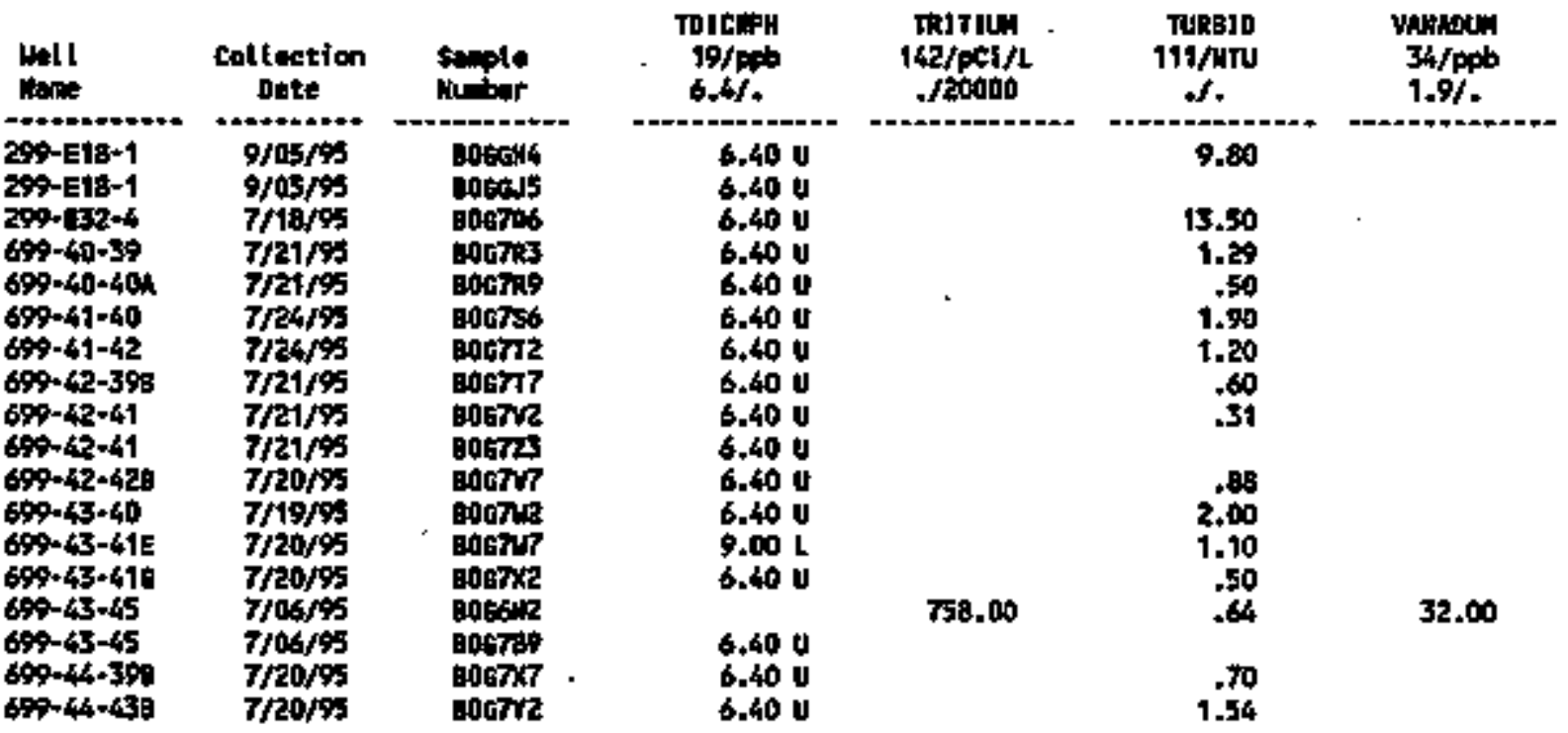

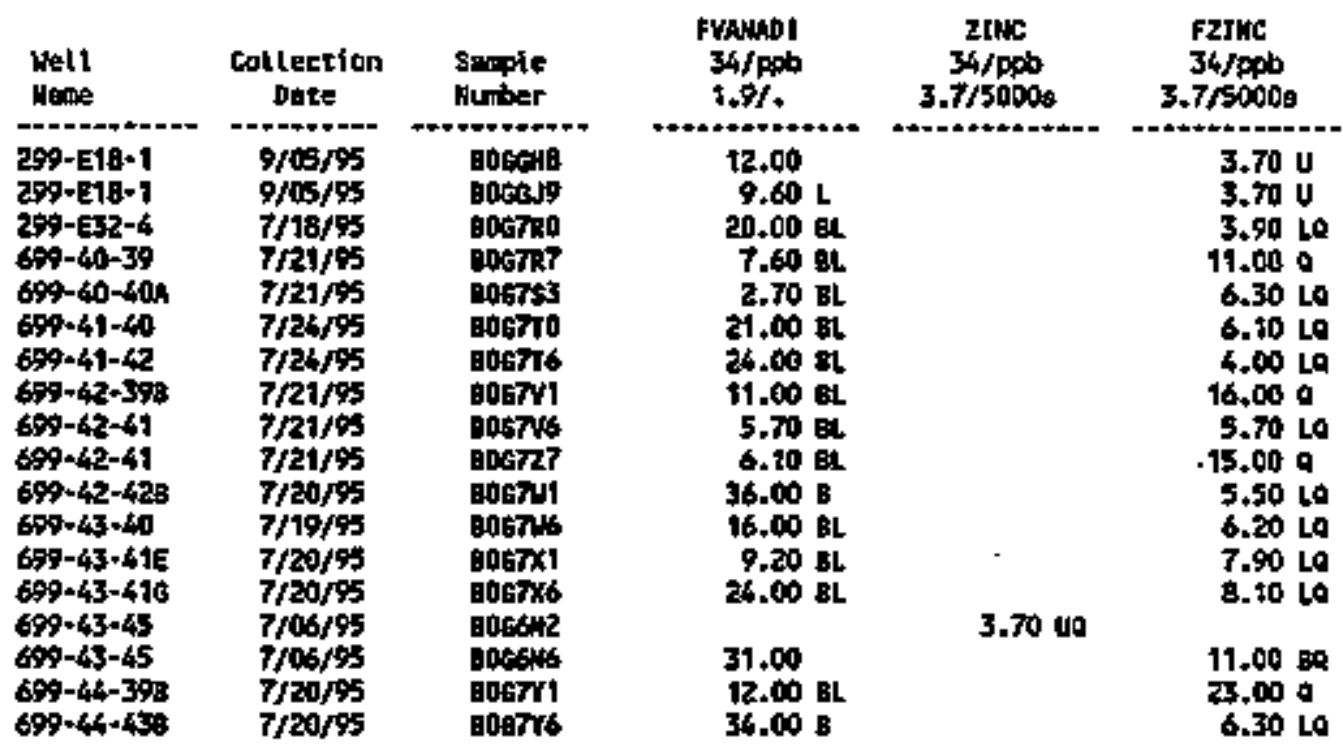

\footnotetext{
For explention of this table, was sectiei 1,4 of report.
} 
Table 5-5. Contamination indicator Parameters for the 216-B-3 Pond Data for Reporting Period

July 1 through September 30, 1995.

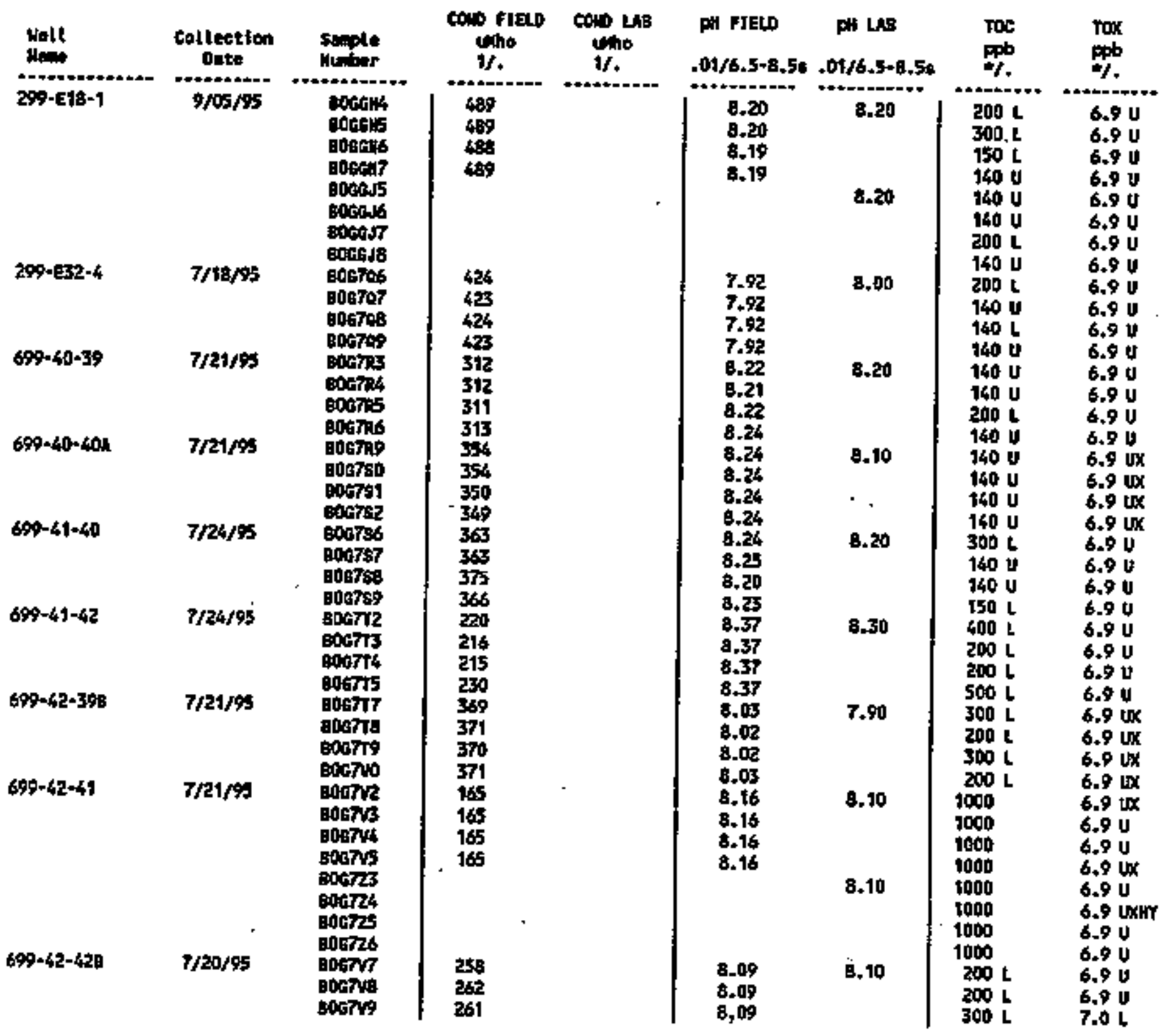


Table 5-5. Contamination Indicator Parameters for the 216-B-3 Pond Data for Reporting Period

July 1 through Septenber 30, 1995.

(sheet 2 of 2)

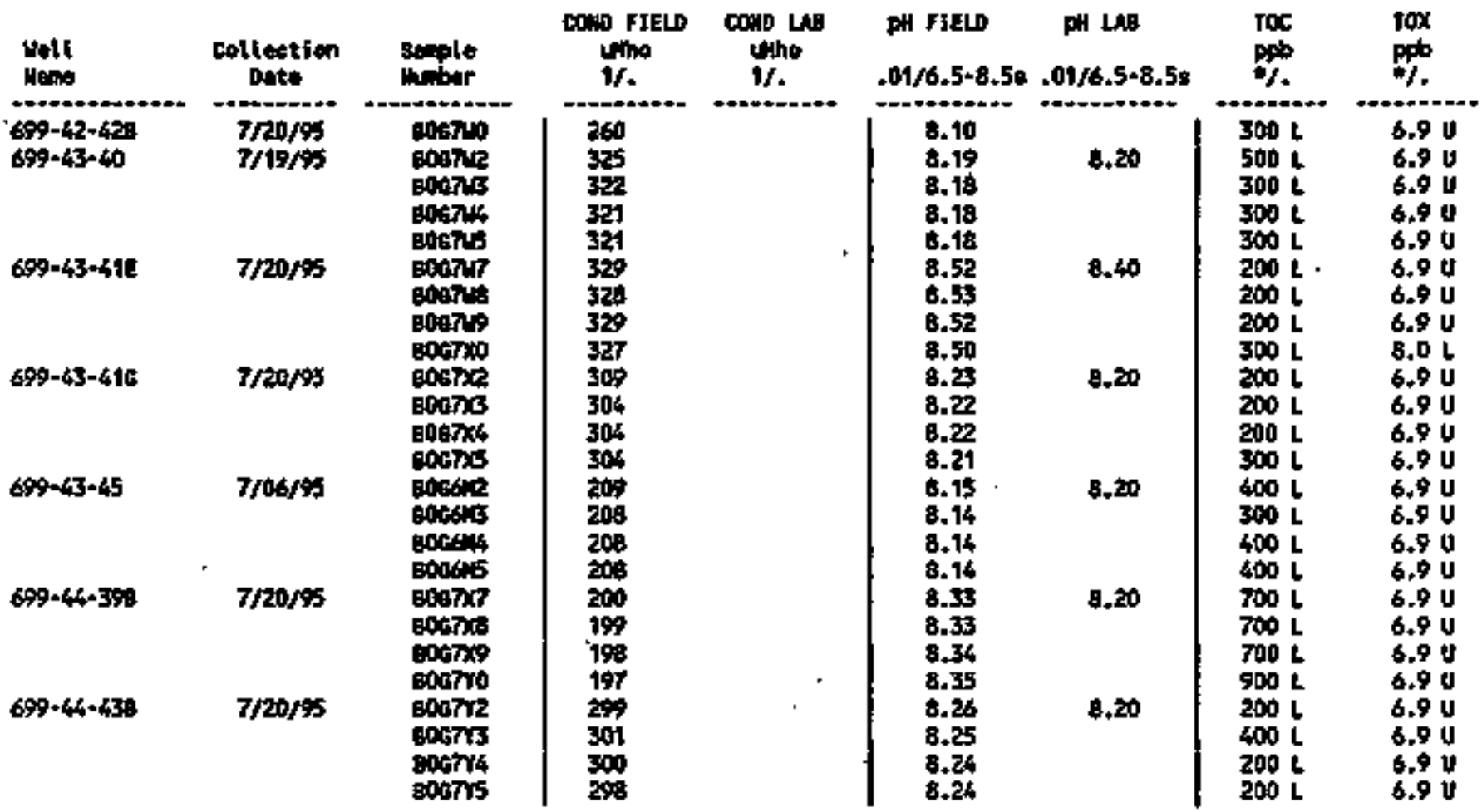

\footnotetext{
* Detection Itinits for Tox and Tox vary depending on the performing laboratory.
} for explenstion of this teble, sea sestion 1.4 of report. 
DOE/RL-95-69-3

\section{contants}

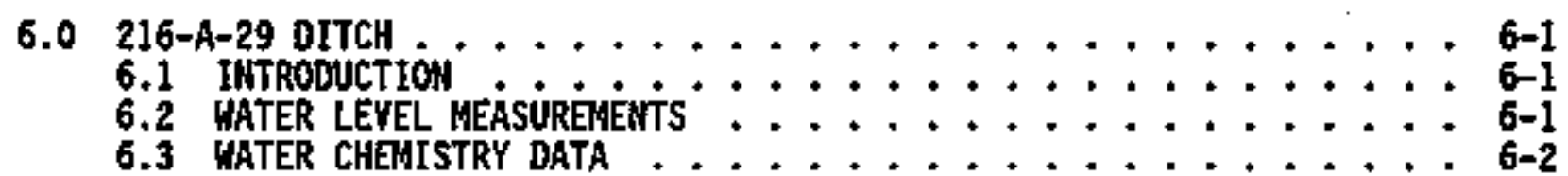


DOE/RL-95-69-3

\section{LIST OF FIGURES}

6-1 Well Location Map for the 216-A-29 Ditch . . . . . . . . . . 6-3

\section{LIST OF TABLES}

6-1 Konitoring Well Purpose and Sampling Schedule for the 216-A-29 Ditch ..................... 6-4

6-2 RCRA Water Leve] Measurement Report 216-A-29 Ditch, Third Quarter 1995 ... . . . . . . . . 6-6

6-3 Constituent L1st and Summary of Resu]ts for the 216-A-29 Ditch Data for Reporting Period July I through Septenter 30, $1995 \ldots$. . . . . . . . . . 6-9

6-4 Constituents with at least One Detected Value for the 216-A-29 Ditch Data for Reporting Period July 1 through Septenber $30,1995 \ldots \ldots$. . . . . . . . . 6-11

6-5 Contamination Indicator Parameters for the 216-A-29 Ditch Data for Reporting Period July 1 through September 30, $1995 \ldots \ldots$. . . . . . . . . . . 61

6-6 Drinking Water Standards Exceeded in the 216-A-29 Ditch Groundwater Honitoring Network .................. 6-22 


\title{
6.0 216-A-29 DITCH
}

\author{
J. H. Yotava \\ Hestinghouse Hanford Company
}

\subsection{IHTRODUCTIOH}

The 216-A-29 Ditch (A-29 Ditch) is located east of the 200 East Area in the central portion of the Hanford Site (Figure 6-1). From 1955 to July 1991, the unlined ditch received effluent from the Plutoniun-Uranium Extraction (PUREX) Plant chemical sewer line and conducted it to the 216-B-3-3 Ditch and the B Pond Syster. After July 15, 1991, the effluent to the A-29 Ditch was rerouted to the PUREX cooling water line. The djtch was backfilled and the site was graded and stabilized to meet Mitestone M-17-10 of the Hanford Federal Facilfty Agreement and Consent Order (Tri-Party Agreement) (Ecology et al. 1994).

Groundwater beneath the A-29 Ditch has been monitored by a RCRA groundwater monitoring network since 1988 because of earlier discharges of caustic and potentialiy hazardous chemicals to the ditch. The A-29 Ditch is currentiy in an interin-status groundwater qua]ity assessment monitoring program because of elevated specific conductance levels in one monitoring well (299-E25-35) (Chou et a], 1990).

The RCRA groundwater monitoring network consists of three upgradfent and 10 downgradient wells. Eleven additional wells in the surrounding area are used to provide supplementary data for the groundwater quality assessment investigation. Figure 6-1 shows the locations of the wells used to monitor groundwater quality for the A-29 Ditch. Table 6-1 presents pertinent data about these wel1s.

\subsection{MATER LEVEL MEASUREMENTS}

Water levels are measured monthly in the 13 wells that make up the A-29 0itch monitoring network and are measured quarterly in additional wells that provide supplemental data for the groundwater quality assessment investigation. Water level measurements are also recorded when the wells are sampled. Water level measurements obtained during this quarter are presented in Table 6-2. 


\subsection{WATER CHEISTRY DATA}

Groundwater beneath the A-29 Ditch is sampled quarterly in accordance with the groundwater quality assessment plan (Chou et al. 1990). The constituent 1 ist and summary of results are provided in Tab7e 6-3. Reported analytical data are included in Tables 6-4 and 6-5. Table 6-4 lists the wells and analytical results for chemica] constituents with at least one detected value above the MDL. Results of analyses for CIP are reported in Tab]e 6-5. Some of the data from the second quarter sampling were missing from the April through June quarterly report because of laboratory reporting delays. These data have not been reported yet.

Eight wells in the A-29 Ditch detection network had individual constituent results that are higher than the historical trends for filtered chroaium, fiitered iron, filtered nickel, unfiltered nickel, and trit fum. Data are flagged in Tables 6-4 and 6-5 according to the explanation found in Sections 1.2 and 1.4 .

Const tuents reported in concentrations that exced the DWS for the A-29 Ditch network and supplewental wells include unfiltered chromium, nitrate, filtered iron, filtered nickel, unfiltered nickel, and tritius (Table 6-6).

A groundwater assessment report for the 216-A-29 Ditch was released in October -1995 (Votava 1995). The report presents findings of the groundwater qual ity assessment program for the 216-A-29 Ditch. These findings are the result of a study to determine if effluent discharged to the 216-A-29 Ditch is the source of elevated speciffc conductance in well 299-E25-35. The study determined that the ditch is contributing to elevated specific conductance and the source is nonhazardous. The report describes the current monitoring status of the 216-A-29 Ditch and groundwater chemical data interpretation, and recommends refinstating an indicator-evaluation monitoring program in accordance with 40 CFR 265.93(d)(6) starting in October 1995. 
Figure 6-1. Well Location Map for the 216-A-29 Ditch.

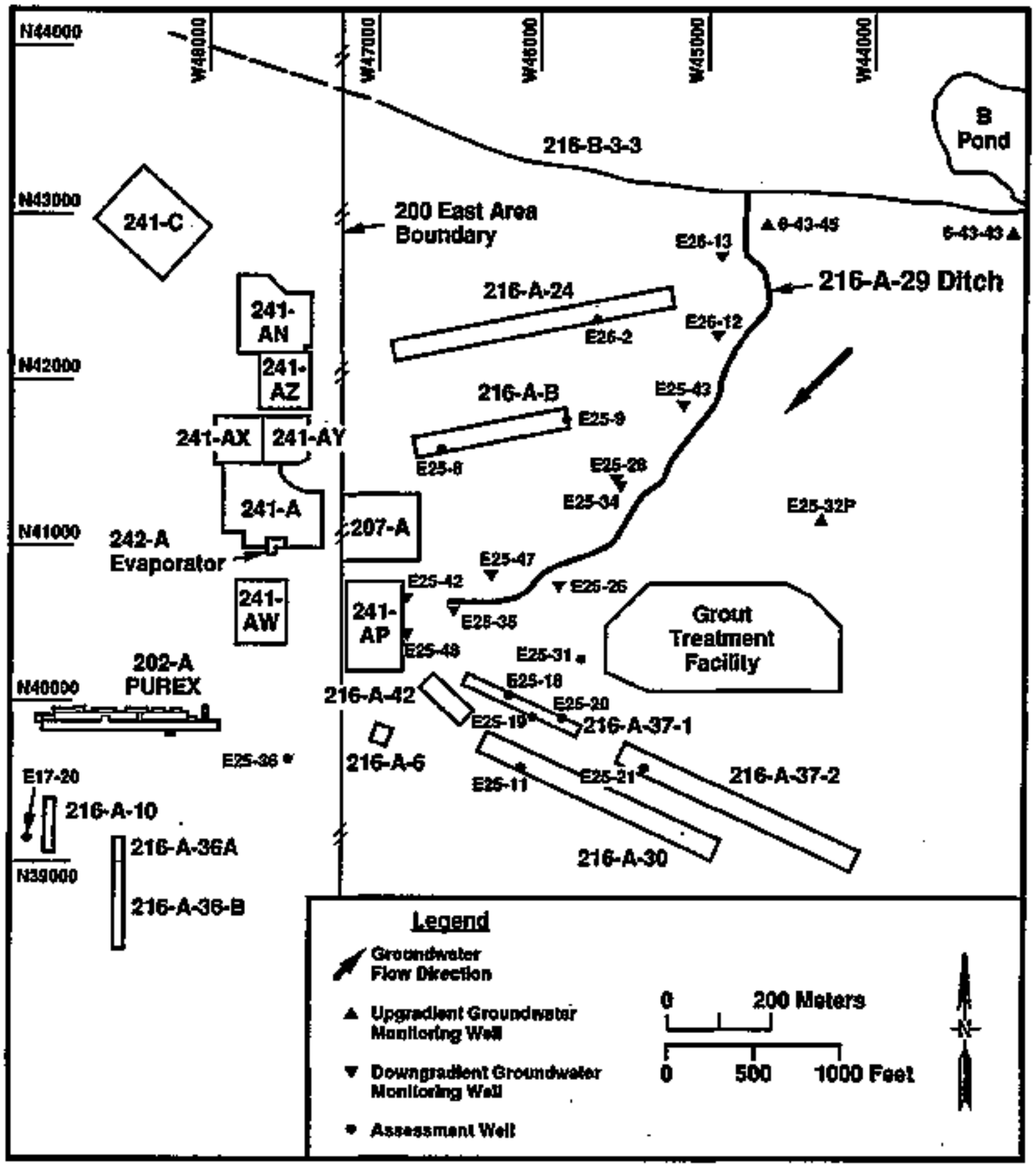


Table 6-1. Monitoring Well Purpose and Sampling Schedule for the 216-A-29 Ditch. (2 sheets)

\begin{tabular}{|c|c|c|c|c|}
\hline Mell no. & $\begin{array}{l}\text { Relative } \\
\text { position }\end{array}$ & $\begin{array}{l}\text { Hydrogeofogic } \\
\text { unit }\end{array}$ & $\begin{array}{c}\text { Sample } \\
\text { frequancy }\end{array}$ & $\begin{array}{l}\text { Sample date, } \\
\text { 3rd Qtr } 1995\end{array}$ \\
\hline $699-43-43^{b}$ & Upgradient & $\begin{array}{l}\text { Hanford: Water } \\
\text { Table }\end{array}$ & Quarterly & $07 / 06 / 95$ \\
\hline $699-43-45^{b}$ & Upgradient & $\begin{array}{l}\text { Hanford: Water } \\
\text { Table }\end{array}$ & Quarteriy & . $\quad 07 / 06 / 95$ \\
\hline 299-E25-32P & Downgradient & $\begin{array}{l}\text { Hanford: Water } \\
\text { Table }\end{array}$ & Quarter]y & $07 / 06 / 95$ \\
\hline $299-E 25-26$ & Downgradient & $\begin{array}{l}\text { Hanford: } \\
\text { Hid-Depth }\end{array}$ & Quarter]y & $07 / 17 / 95$ \\
\hline 299-E25-28 & Downgradient & Ringold: Deep & Quarter]y & $07 / 17 / 95$ \\
\hline 299-E25-34 & Downgradient & $\begin{array}{l}\text { Hanford: Water } \\
\text { TabTe }\end{array}$ & Quarterly & $07 / 17 / 95$ \\
\hline $299-E 25-35$ & Downgradient & $\begin{array}{l}\text { Hanford: Water } \\
\text { TabTe }\end{array}$ & Quarterly & $07 / 14 / 95$ \\
\hline 299-E25-42 & Downgradient & $\begin{array}{l}\text { Hanford: Water } \\
\text { Table }\end{array}$ & Quarterly & $07 / 14 / 95$ \\
\hline $299-E 25-43$ & Downgradient & $\begin{array}{l}\text { Hanford: Water } \\
\text { Table }\end{array}$ & Quarterly & $07 / 18 / 95$ \\
\hline $299-E 25-47$ & Doungradient & $\begin{array}{l}\text { Hanford: Water } \\
\text { Table }\end{array}$ & Quarterty & $07 / 14 / 95$ \\
\hline $299-E 25-48$ & Downgradient & $\begin{array}{l}\text { Hanford: Water } \\
\text { Table }\end{array}$ & Quarteriy & $07 / 14 / 95$ \\
\hline $299-E 26-12$ & Doumgradient & $\begin{array}{l}\text { Hanford: Water } \\
\text { Table }\end{array}$ & Quarter]y & $07 / 17 / 95$ \\
\hline $299-E 26-13$ & Doungradtent & $\begin{array}{l}\text { Hanford: Water } \\
\text { Table }\end{array}$ & Quarter]y & $07 / 17 / 95$ \\
\hline $299-\mathrm{E} 17-20^{\mathrm{d}, \mathrm{e}}$ & Downgradient & $\begin{array}{l}\text { Ringold: Water } \\
\text { Table }\end{array}$ & Qtrarter]y & $07 / 14 / 95$ \\
\hline $299-E 25-11^{e}$ & Doimgradient & $\begin{array}{l}\text { Undetermined: } \\
\text { Mid-Depth }\end{array}$ & Quarter]y & $07 / 18 / 95$ \\
\hline $299-E 25-18^{*}$ & Domgradient & $\begin{array}{l}\text { Undetermined: } \\
\text { Mid-Depth }\end{array}$ & Quarter]y & $07 / 11 / 95$ \\
\hline $299-E 25-19^{\circ}$ & Downgradient & $\begin{array}{l}\text { Undetermined: } \\
\text { Mid-Depth }\end{array}$ & Quarterly & $07 / 11 / 95$ \\
\hline $299-E 25-20^{\circ}$ & Doingradlent & $\begin{array}{l}\text { Undeterwined: } \\
\text { Mid-Depth }\end{array}$ & Quarterly & $07 / 11 / 95$ \\
\hline
\end{tabular}


Table 6-1. Monitoring Well Purpose and Sampling Schedule for the 216-A-29 Ditch. (2 sheets)

\begin{tabular}{|c|l|l|c|c|}
\hline Well no. & $\begin{array}{c}\text { Relative } \\
\text { position }\end{array}$ & \multicolumn{1}{|c|}{$\begin{array}{c}\text { Hydrogeologic } \\
\text { unit }^{\text {e }}\end{array}$} & $\begin{array}{c}\text { Sample } \\
\text { frequency }\end{array}$ & $\begin{array}{c}\text { Sample date, } \\
\text { 3rd Qtr 1995 }\end{array}$ \\
\hline 299-E25-21* & Downgradient & $\begin{array}{l}\text { Hanford: Water } \\
\text { Table }\end{array}$ & Quarterly & $07 / 11 / 95$ \\
\hline 299-E25-31* & Downgradient & $\begin{array}{l}\text { Hanford: Water } \\
\text { Table }\end{array}$ & Quarterly & $07 / 11 / 95$ \\
\hline 299-E25-36,,d,e & Downgradient & $\begin{array}{l}\text { Ringold: Water } \\
\text { Table }\end{array}$ & Quarterly & $07 / 17 / 95$ \\
\hline
\end{tabular}

Hydrogeologic units include the sandy gravels of the Hanford formation and the silty sands of the Ringold Formation.

Wells shared with the 216-B-3 Pond monitoring network.

We17s shared with the 216-A-36B nonitoring network.

WWe17s shared with the 216-A-10 monitoring network.

"Wells used for supplemental groundwater quality. 
Table 6-2. RCRA Water Level Measurement Report 216-A-29 Ditch, Third Quarter 1995.

(sheet 1 of 3)

\begin{tabular}{|c|c|c|c|c|}
\hline WeTl & Date & $\begin{array}{l}\text { Depth to } \\
\text { water (ft) }\end{array}$ & $\begin{array}{l}\text { Wate } \\
\text { elevatio } \\
\text { (ft) }\end{array}$ & $\begin{array}{l}\text { level } \\
\text { above ms1 } \\
\text { (m) }\end{array}$ \\
\hline \multicolumn{5}{|c|}{ Wells in the Monitoring System } \\
\hline $299-E 25-26$ & $\begin{array}{r}7 / 17 / 95 \\
.7 / 20 / 95 \\
8 / 18 / 95 \\
9 / 07 / 95\end{array}$ & $\begin{array}{l}266.60^{*} \\
266.94 \\
266.97 \\
267.06\end{array}$ & $\begin{array}{l}401.92 \\
401.58 \\
401.55 \\
401.46\end{array}$ & $\begin{array}{l}122.51 \\
122.40 \\
122.39 \\
122.37\end{array}$ \\
\hline 299-E25-28 & $\begin{array}{r}7 / 17 / 95 \\
7 / 20 / 95 \\
8 / 18 / 95 \\
9 / 07 / 95\end{array}$ & $\begin{array}{l}260.84 * \\
260.51 \\
260.57 \\
260.68\end{array}$ & $\begin{array}{l}401.60 \\
401.93 \\
401.87 \\
401.76\end{array}$ & $\begin{array}{l}122.41 \\
122.51 \\
122.49 \\
122.46\end{array}$ \\
\hline 299-E25-32P & $\begin{array}{r}7 / 06 / 95 \\
7 / 20 / 95 \\
8 / 18 / 95 \\
9 / 07 / 95\end{array}$ & $\begin{array}{l}268.01 * \\
268.19 \\
268.34 \\
268.44\end{array}$ & $\begin{array}{l}401.18 \\
401.00 \\
400.85 \\
400.75\end{array}$ & $\begin{array}{l}122.28 \\
122.22 \\
122.18 \\
122.15\end{array}$ \\
\hline 299-E25-34 & $\begin{array}{l}7 / 17 / 95 \\
7 / 20 / 95 \\
8 / 18 / 95 \\
9 / 07 / 95\end{array}$ & $\begin{array}{l}261.08 \star \\
261.41 \\
261.73 \\
261.32\end{array}$ & $\begin{array}{l}401.79 \\
401.46 \\
401.14 \\
401.55\end{array}$ & $\begin{array}{l}122.47 \\
122.37 \\
122.27 \\
122.39\end{array}$ \\
\hline $299-E 25-35$ & $\begin{array}{l}7 / 14 / 95 \\
7 / 20 / 95 \\
8 / 18 / 95 \\
9 / 07 / 95\end{array}$ & $\begin{array}{l}273.12^{\star} \\
272.85 \\
272.87 \\
272.89\end{array}$ & $\begin{array}{l}401.27 \\
401.54 \\
401.52 \\
401.50\end{array}$ & $\begin{array}{l}122.31 \\
122.39 \\
122.38 \\
122.38\end{array}$ \\
\hline $299-E 25-42$ & $\begin{array}{l}7 / 14 / 95 \\
7 / 20 / 95 \\
8 / 18 / 95 \\
9 / 07 / 95\end{array}$ & $\begin{array}{l}292.70 *+ \\
281.67 \\
281.68 \\
281.70\end{array}$ & $\begin{array}{l}390.59 \\
401.62 \\
401.61 \\
401.59\end{array}$ & $\begin{array}{l}119.05 \\
122.41 \\
122.41 \\
122.40\end{array}$ \\
\hline 299-E25-43 & $\begin{array}{l}7 / 18 / 95 \\
7 / 20 / 95 \\
9 / 07 / 95\end{array}$ & $\begin{array}{l}247.93^{*} \\
247.89 \\
248.07\end{array}$ & $\begin{array}{l}401.95 \\
402.00 \\
401.82\end{array}$ & $\begin{array}{l}122.52 \\
122.53 \\
122.47\end{array}$ \\
\hline 299-E25-47 & $\begin{array}{l}7 / 14 / 95 \\
7 / 20 / 95 \\
8 / 18 / 95 \\
9 / 07 / 95\end{array}$ & $\begin{array}{l}271.80 \pm \\
271.94 \\
271.94 \\
272.00\end{array}$ & $\begin{array}{l}401.97 \\
401.83 \\
401.83 \\
401.77\end{array}$ & $\begin{array}{l}122.52 \\
122.48 \\
122.48 \\
122.46\end{array}$ \\
\hline
\end{tabular}


Table 6-2. RCRA Water Level Measurement Report 216-A-29 Diteh - 3rd Quarter 1995.

(sheet 2 of 3 )

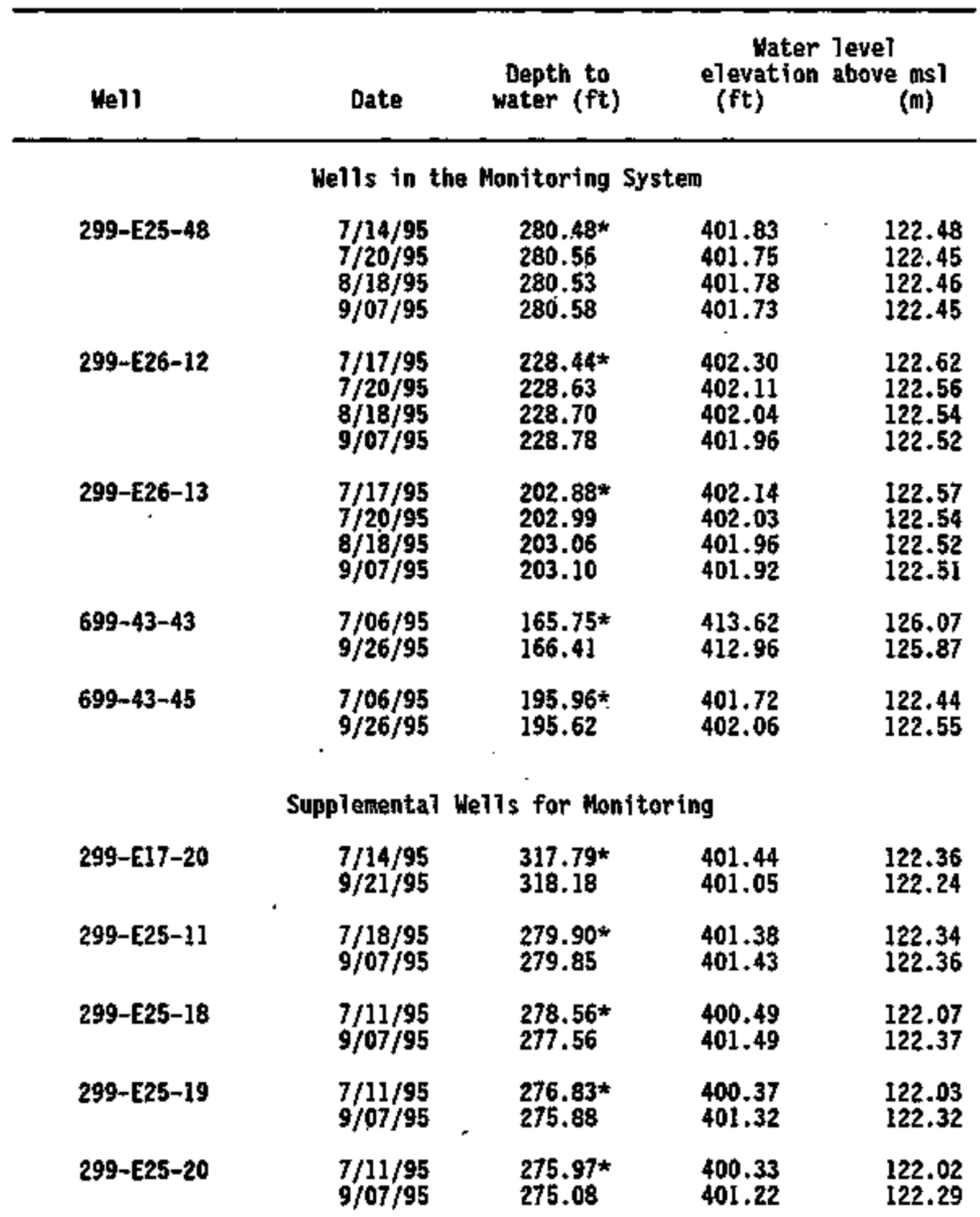


Table 6-2. RCRA Mater Level Measurement Report 216-A-29 Ditch - 3rd Quarter 1995.

(sheet 3 of 3 )

\begin{tabular}{|c|c|c|c|c|}
\hline We!̣ I & Date & $\begin{array}{l}\text { Depth to } \\
\text { water (ft) }\end{array}$ & $\begin{array}{l}\text { Water } \\
\text { elevation } \\
\text { (ft) }\end{array}$ & $\begin{array}{l}\text { level } \\
\text { above ms] } \\
\text { (m) }\end{array}$ \\
\hline \multicolumn{5}{|c|}{ Supplemental Wells for Monitoring } \\
\hline 299-E25-21 & $9 / 07 / 95$ & 275.63 & 401.64 & 122.42 \\
\hline $299-E 25-31$ & $\begin{array}{l}7 / 17 / 95 \\
9 / 07 / 95\end{array}$ & $\begin{array}{l}274.81 \\
274.95\end{array}$ & $\begin{array}{l}401.94 \\
401.80\end{array}$ & $\begin{array}{l}122.51 \\
122.47\end{array}$ \\
\hline 299-E25-36 & $\begin{array}{l}7 / 17 / 95 \\
9 / 21 / 95\end{array}$ & $\begin{array}{l}306.30 * \\
306.29\end{array}$ & $\begin{array}{l}401.09 \\
401.10\end{array}$ & $\begin{array}{l}122.25 \\
122.26\end{array}$ \\
\hline 299-E25-6 & $9 / 07 / 95$ & 259.51 & 398.80 & 121.55 \\
\hline $299-E 25-9$ & $9 / 07 / 95$ & 255.60 & 399.26 & 121.69 \\
\hline $299-E 26-2$ & $9 / 07 / 95$ & 233.73 & 401.57 & 122.40 \\
\hline
\end{tabular}

NOTES: 1. Water level elevations are calculated by subtracting the measured depth-to-water from the surveyed elevation for the well.

2. Depth-to-water values are transeribed from field records.

3. Measurements marked with an '\$' were taken at the time of sampling.

4. Measurements marked with a ' + ' are outside of the expected range, and are suspected of error. 


\section{Tab]e 6-3, Constituent List and Summary of Results \\ for the 216-A-29 Ditch Data for Reporting Perjod July 1 through September 30, 1995. \\ (sheet 1 of 2)}

\section{CONTAHIMTION JWDICATOR PARAYITERS}

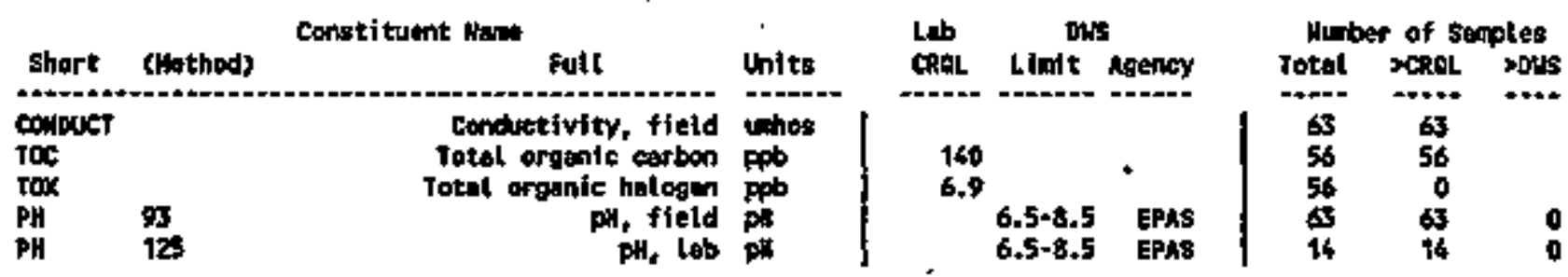

DRIAKIDU WhTER PARANETERS

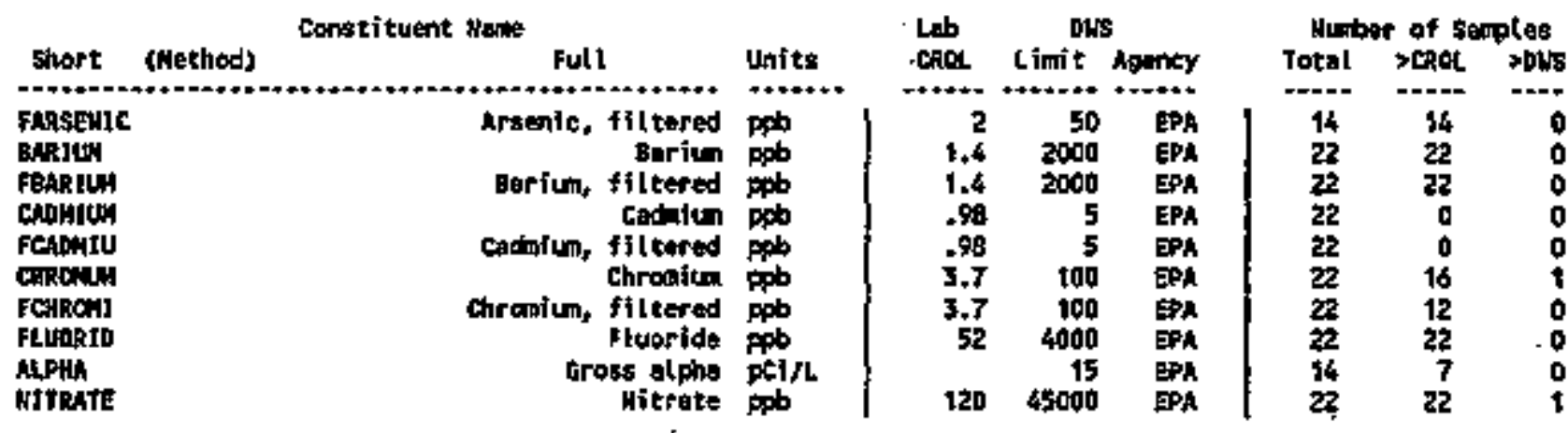

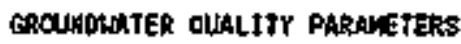

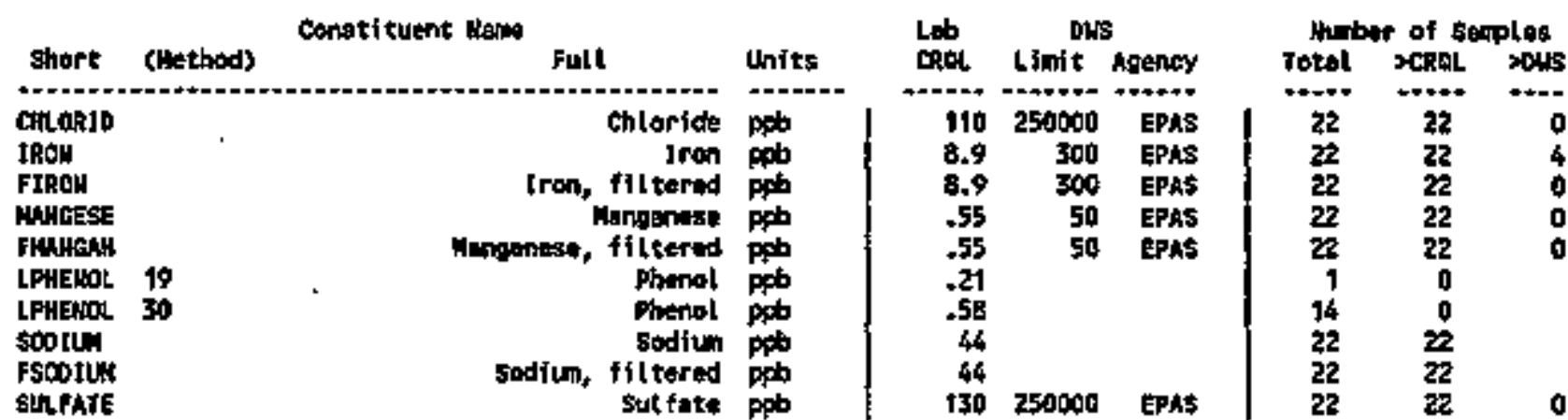

SITE SPECSFIC AHD OTYER COHSTI IUEKTS

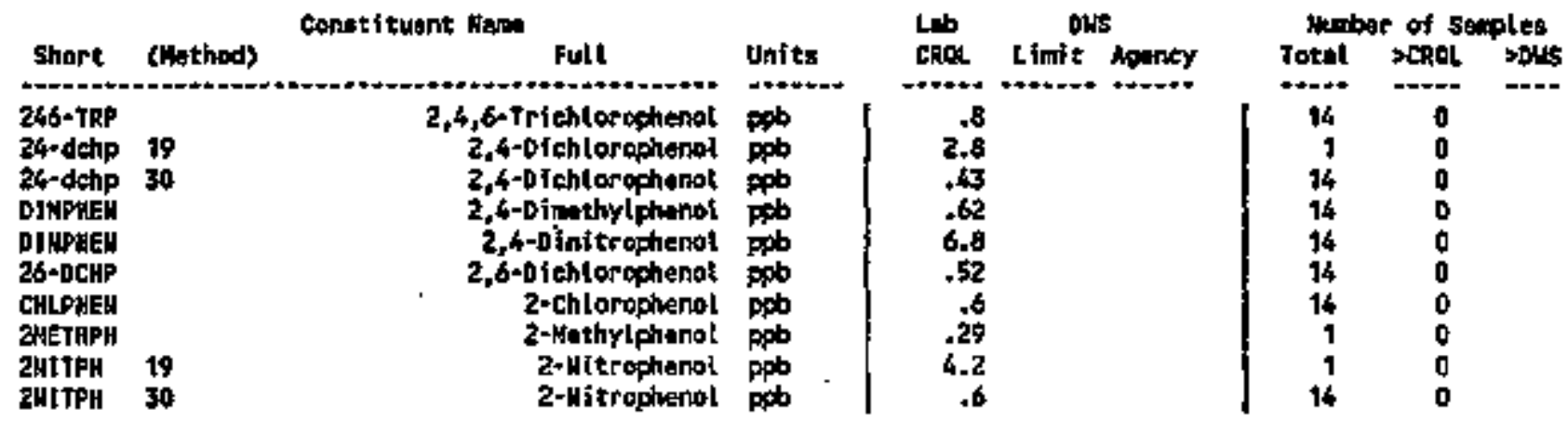


Table 6-3. Constituent List and Summary of Results

for the 216-A-29 Ditch Data for Reporting Period

July I through September 30, 1995.

(sheet 2 of 2)

SITE SPECIFIC NID OTHER COHSTITUENTS

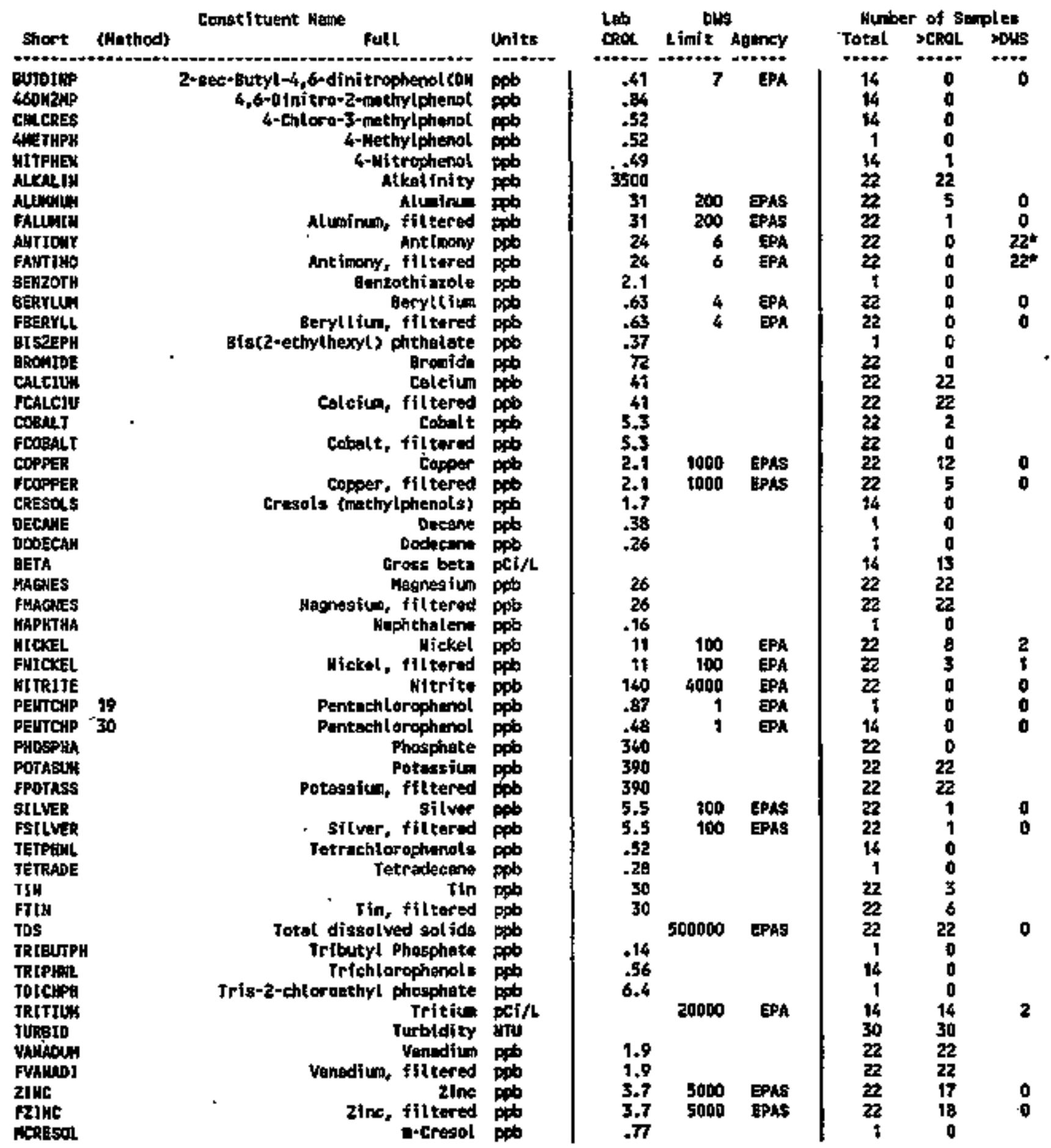

For explandion of this table, see section 1.4 of raport. 
Table 6-4. Constituents with at Least One Detected Value for the 216-A-29 Dftch Data for Reporting Period JuTy I through September 30, 1995.

(sheet 1 of 10)

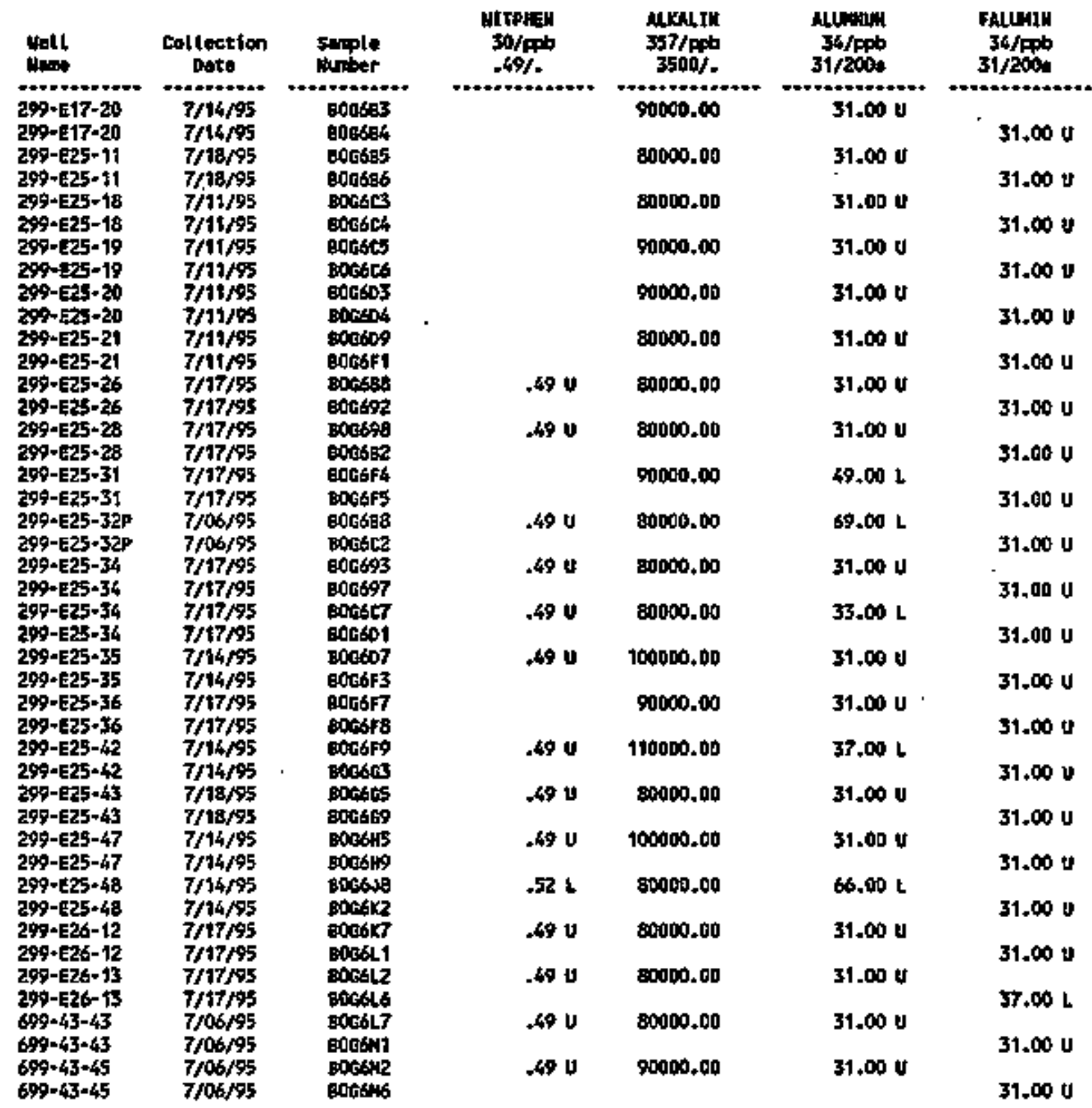


Table 6-4. Constituents with at Least One Betected Value for the 216-A-29 Ditch Data for Reporting Period July I through September 30, 1995.

(sheet 2 of 10)

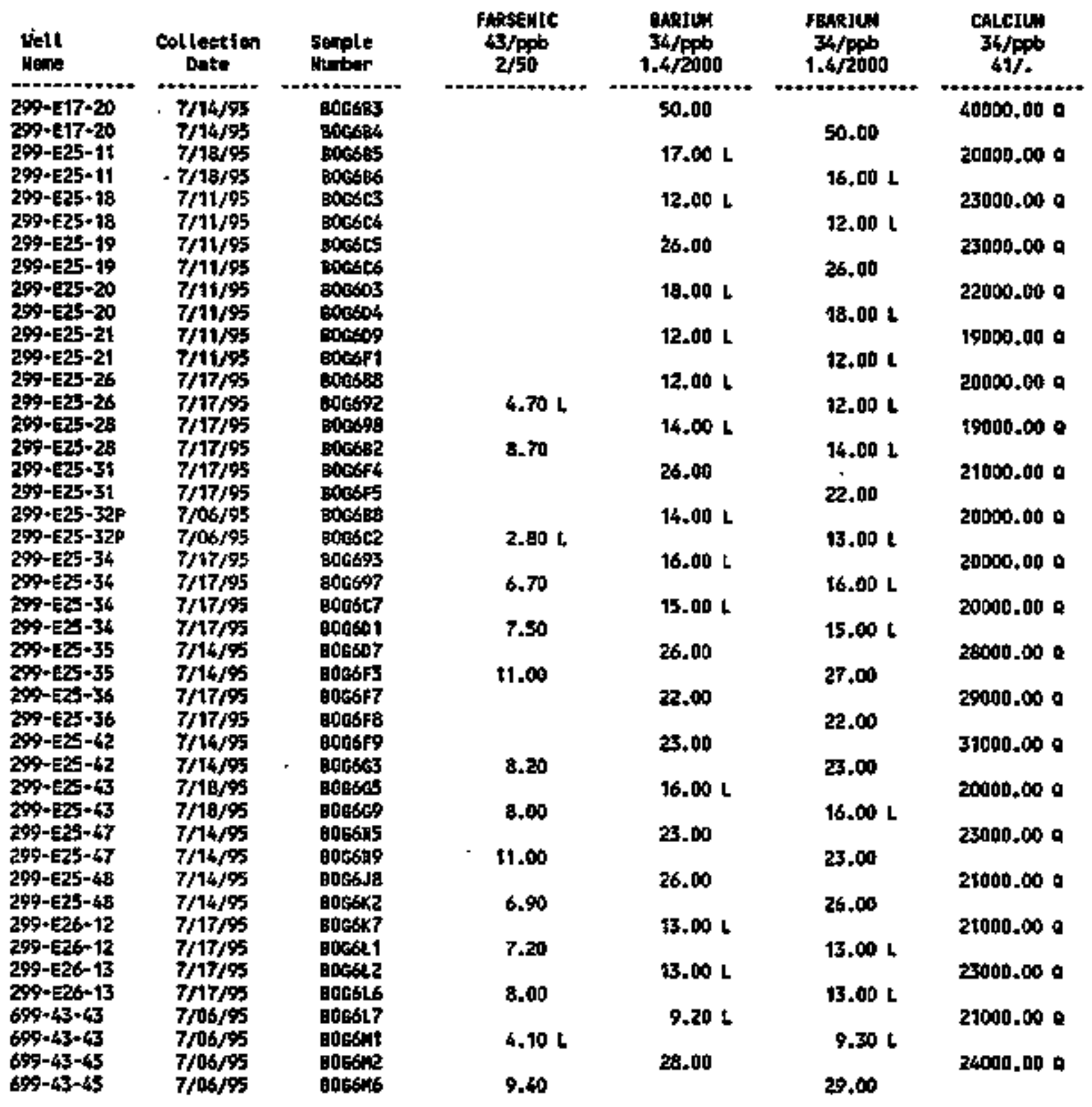


Table 6-4. Constituents with at Least One Detected Vajue for the 216-A-29 Ditch Data for Reporting Period July 1 through September 30, 1995.

(sheet 3 of 10 )

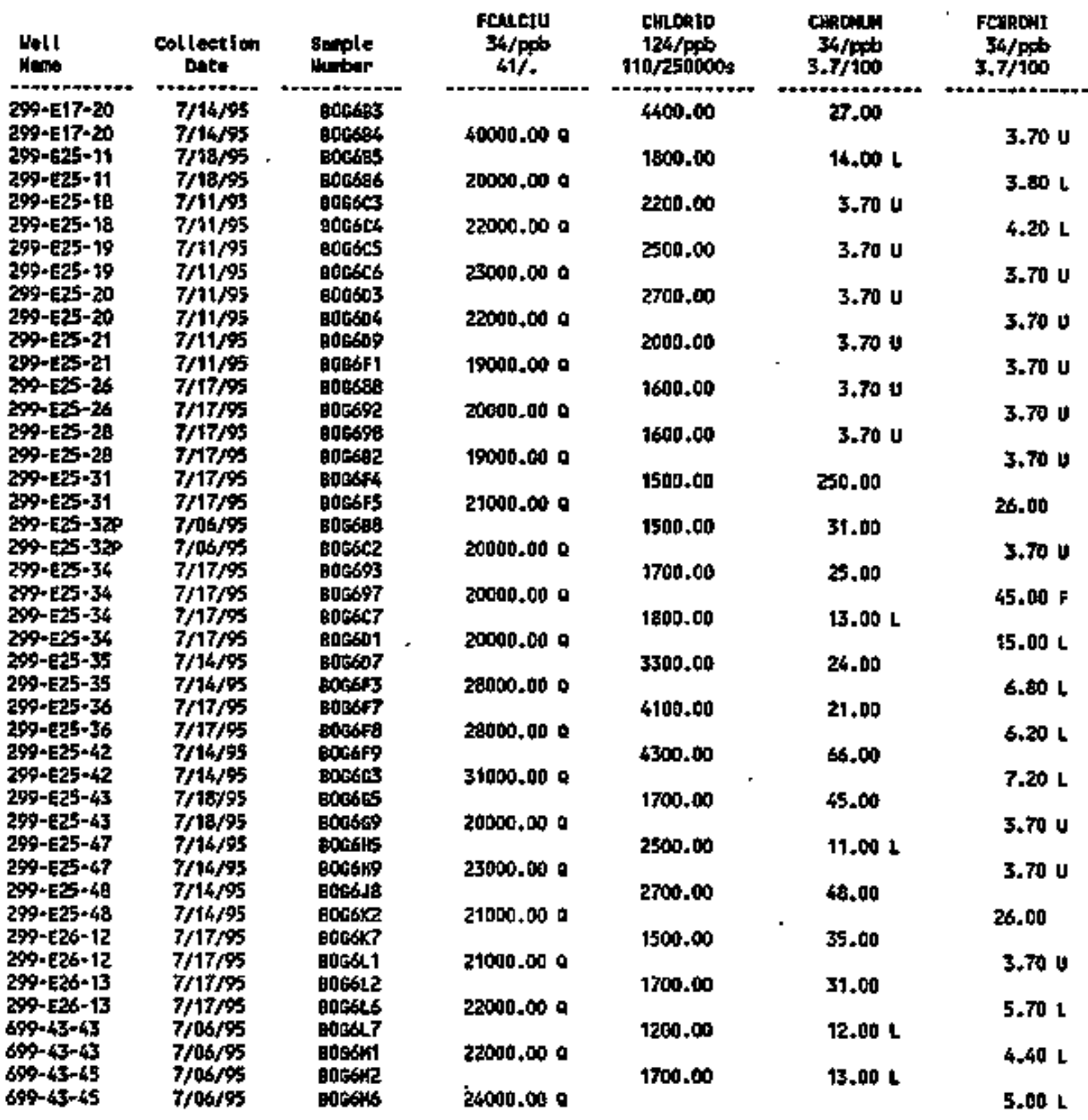


Table 6-4. Constituents with at Least One Detected Value for the 216-A-29 Ditch Data for Reporting Period Juty 1 through September 30, 1995.

(sheet 4 of 10)

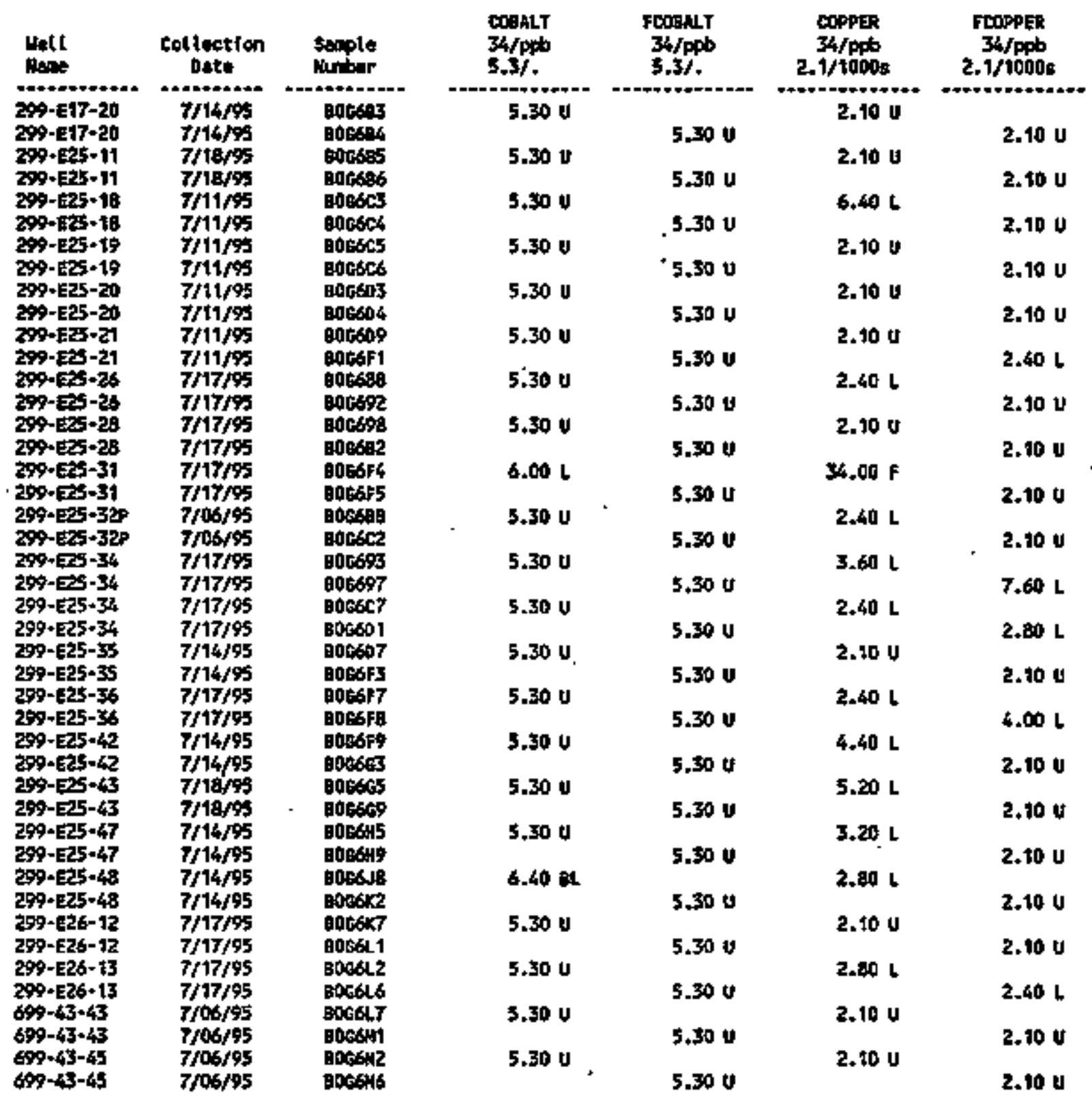


Table 6-4. Constituents with at Least One Detected Value for the 216-A-29 Ditch Data for Reporting Period July it through September 30, 1995.

(sheet 5 of 10)

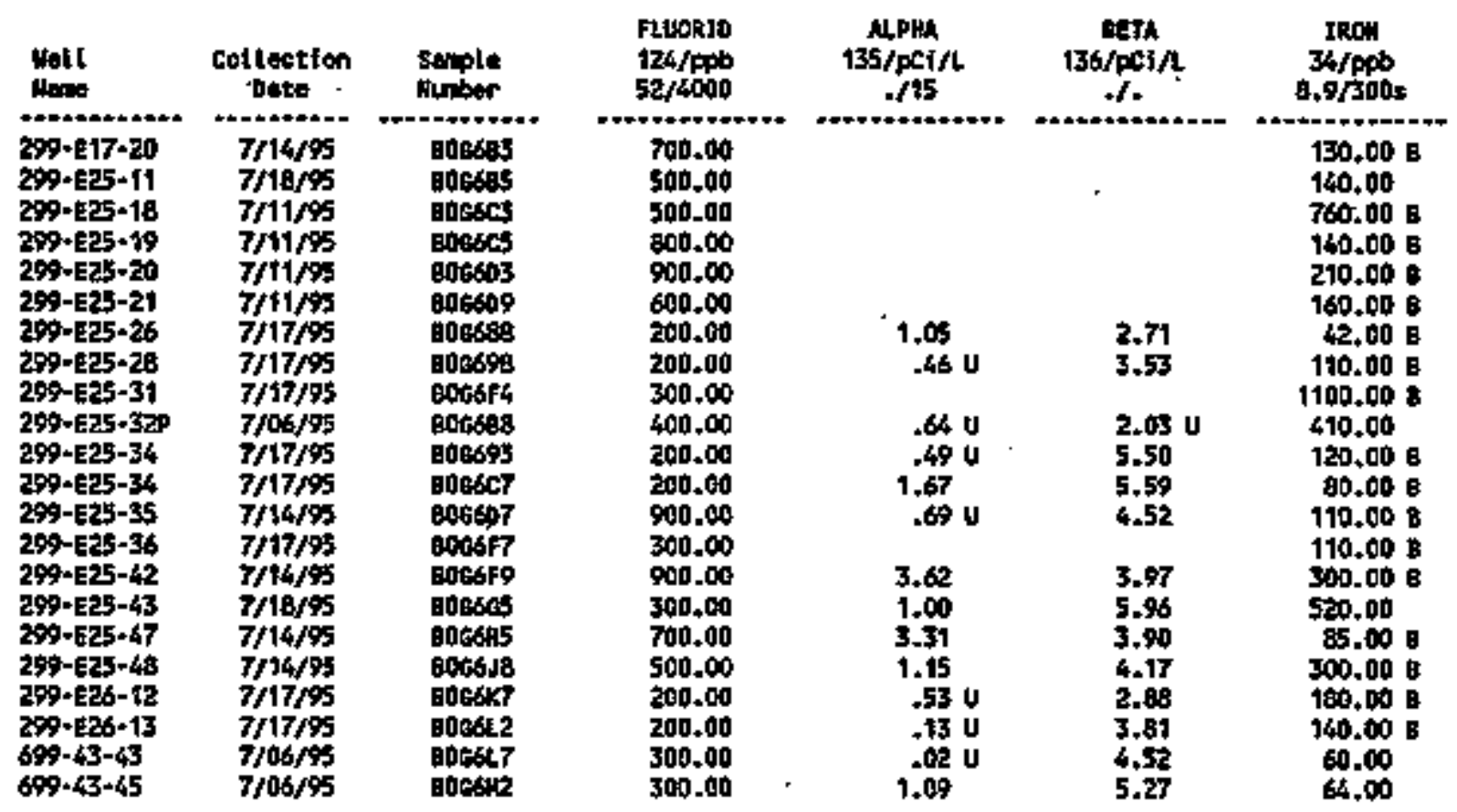

\begin{tabular}{|c|c|c|c|c|c|c|}
\hline $\begin{array}{l}\text { Hell } \\
\text { Hente }\end{array}$ & $\begin{array}{l}\text { follection } \\
\text { Oate }\end{array}$ & $\begin{array}{l}\text { Sienple } \\
\text { lubber }\end{array}$ & $\begin{array}{c}\text { Ftrow } \\
34 / \mathrm{ppb} \\
8.9 / 500,8\end{array}$ & $\begin{array}{c}\text { YHANES } \\
34 / \mathrm{ppb} \\
26 / .\end{array}$ & $\begin{array}{l}\text { FMAGHES } \\
36 / \text { pph } \\
26 \%\end{array}$ & $\begin{array}{l}\text { MGHGEsE } \\
34 / \text { gpb } \\
.55 / 503\end{array}$ \\
\hline 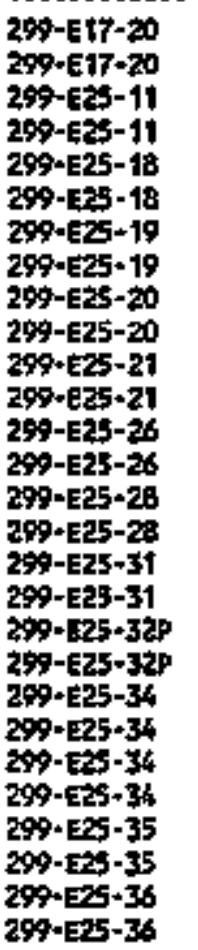 & 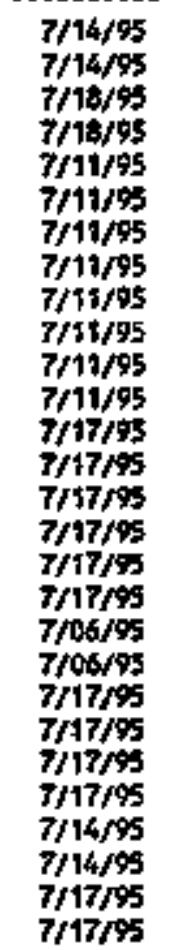 & 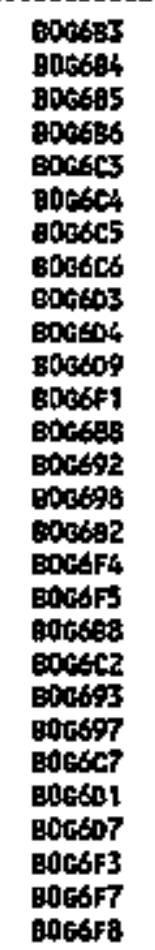 & $\begin{array}{l}25.00 \mathrm{~B} \\
20.00 \\
33.00 \mathrm{~B} \\
43.00 \mathrm{~B} \\
25.00 \mathrm{~B} \\
32.00 \mathrm{~B} \\
16.00 \mathrm{BL} \\
15.00 \mathrm{BL} \\
35.00 \mathrm{~B} \\
25.00 \\
220.00 \mathrm{BF} \\
72.00 \mathrm{~B} \\
24.00 \mathrm{~B} \\
41.00 \mathrm{~B}\end{array}$ & $\begin{array}{l}73000.00 \\
5500.00 \\
6300.00 \\
6000.00 \\
5800.00 \\
5300.00 \\
5500.00 \\
5700.00 \\
6000.00 \\
5600.00 \\
5600.00 \\
5600.00 \\
8100.00 \\
7900.00\end{array}$ & $\begin{array}{l}13000.00 \\
5400.00 \\
5900.00 \\
5900.00 \\
5800.00 \\
5500.00 \\
5400.00 \\
5600.00 \\
5900.00 \\
5600.00 \\
5600.00 \\
5500.00 \\
5200.00 \\
7700.00\end{array}$ & $\begin{array}{c}3.00 \mathrm{~L} \\
5.40 \mathrm{~L} \\
12.00 \\
11.00 \\
5.20 \mathrm{~L} \\
4.00 \mathrm{~L} \\
1.10 \mathrm{~L} \\
.59 \mathrm{~L} \\
15.00 \\
8.90 \mathrm{~L} \\
2.90 \mathrm{~L} \\
2.00 \mathrm{~L} \\
2.50 \mathrm{~L} \\
2.90 \mathrm{~L}\end{array}$ \\
\hline
\end{tabular}


Table 6-4. Constituents with at Least One Betected Vaiue for the 216-A-29 Ditch Data for Reporting Period July 1 through Septenber 30, 1995.

(sheet 6 of 10)

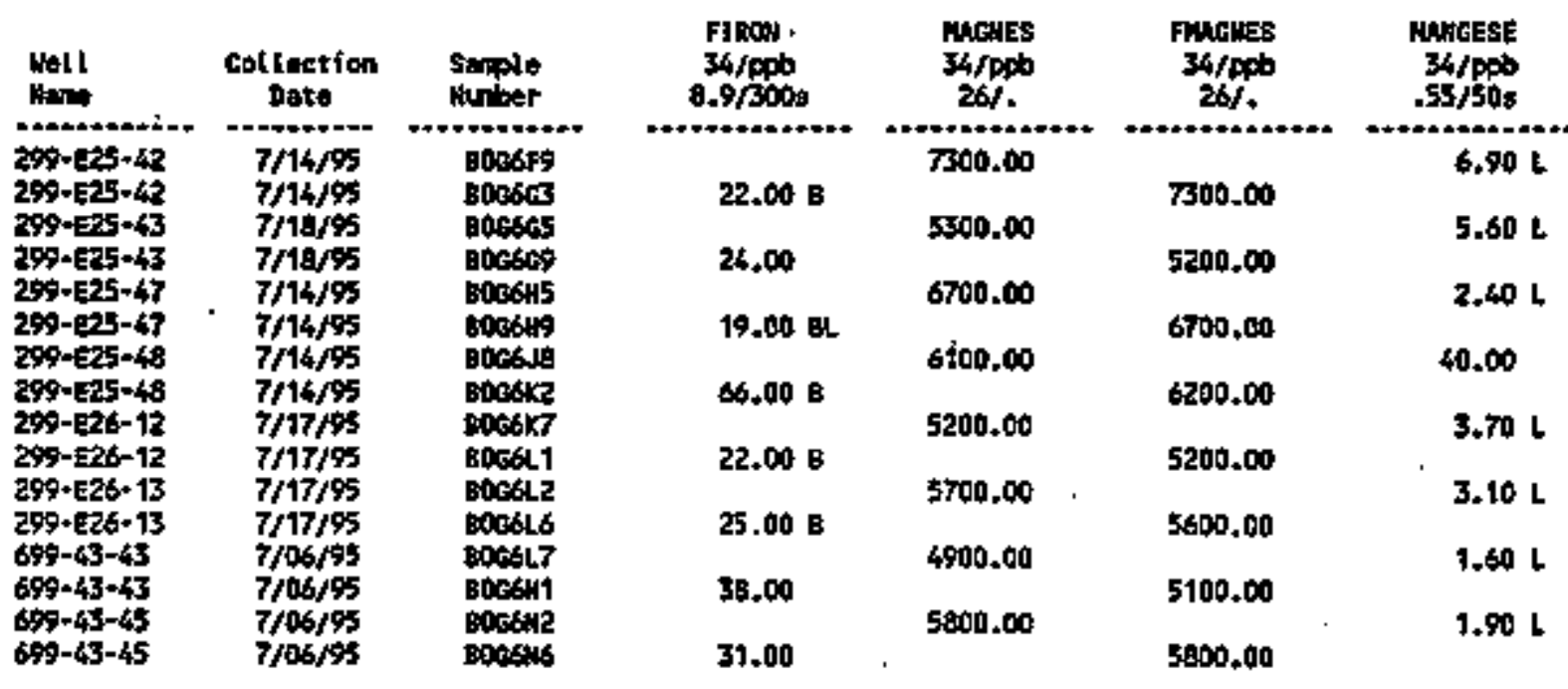

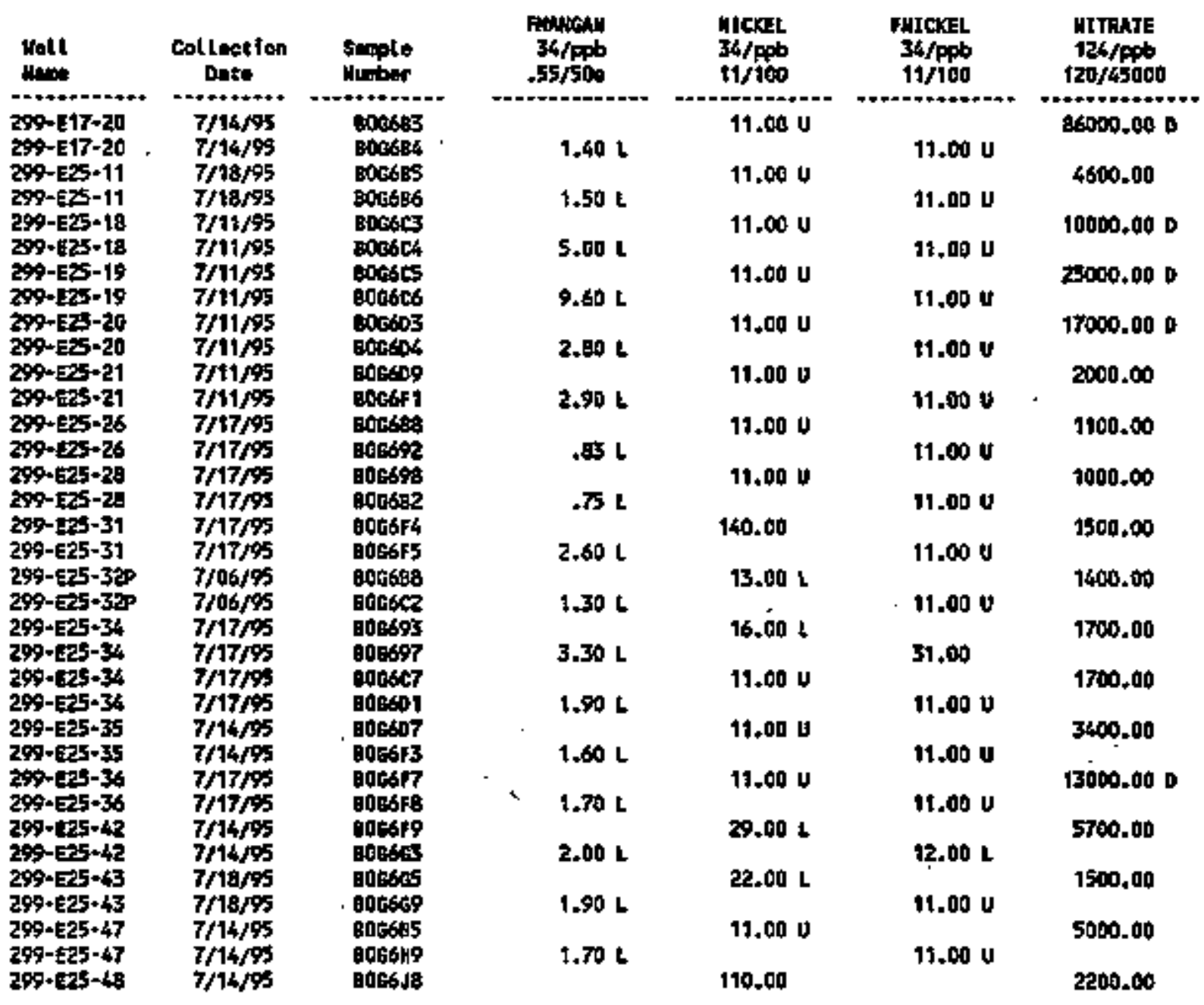


Table 6-4. Constituents with at Least One Detected Value for the 216-A-29 Ditch Data for Reporting Period July 1 through September 30, 1995.

(sheet 7 of 10 )

\begin{tabular}{|c|c|c|c|c|c|c|}
\hline Nell & $\begin{array}{c}\text { Cotlection } \\
\text { nete }\end{array}$ & $\begin{array}{l}\text { Sample } \\
\text { Hunber. }\end{array}$ & $\begin{array}{l}\text { FHapent } \\
34 / \mathrm{ppb} \\
+55 / 50 \mathrm{~s}\end{array}$ & $\begin{array}{l}\text { utckel } \\
34 / \mathrm{ppb} \\
11 / 100\end{array}$ & $\begin{array}{c}\text { FAICKEL } \\
34 / \mathrm{pob} \\
1 \% / 100\end{array}$ & $\begin{array}{l}\text { NITRATE } \\
124 / p p b \\
120 / 45000\end{array}$ \\
\hline $\begin{array}{l}99-E z-40 \\
99-E 26-12 \\
99-E 26-12 \\
99-126-13 \\
999-E 26-13 \\
999-63-43 \\
599-43-43 \\
599-43-45 \\
599-43-45\end{array}$ & $\begin{array}{l}7 / 14 / 95 \\
7 / 17 / 45 \\
7 / 17 / 95 \\
7 / 17 / 95 \\
7 / 17 / 95 \\
7 / 06 / 95 \\
7 / 06 / 95 \\
7 / 06 / 95 \\
7 / 06 / 95\end{array}$ & 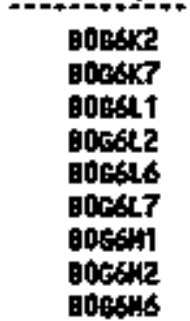 & $\begin{array}{l}40.00 \\
1.10 \mathrm{~L} \\
1.80 \mathrm{~L} \\
1.20 \mathrm{~L} \\
1.50 \mathrm{~L}\end{array}$ & $\begin{array}{l}14.00 \mathrm{~L} \\
12.00 \mathrm{~L} \\
11.00 \mathrm{U} \\
11.00 \mathrm{U}\end{array}$ & $\begin{array}{r}110.00 \mathrm{~F} \\
-11.00 \mathrm{U} \\
11.00 \mathrm{U} \\
11.00 \mathrm{U} \\
11.00 \mathrm{U}\end{array}$ & $\begin{array}{l}1200.00 \\
1500.00 \mathrm{~F} \\
700.00 \\
1100.00\end{array}$ \\
\hline
\end{tabular}

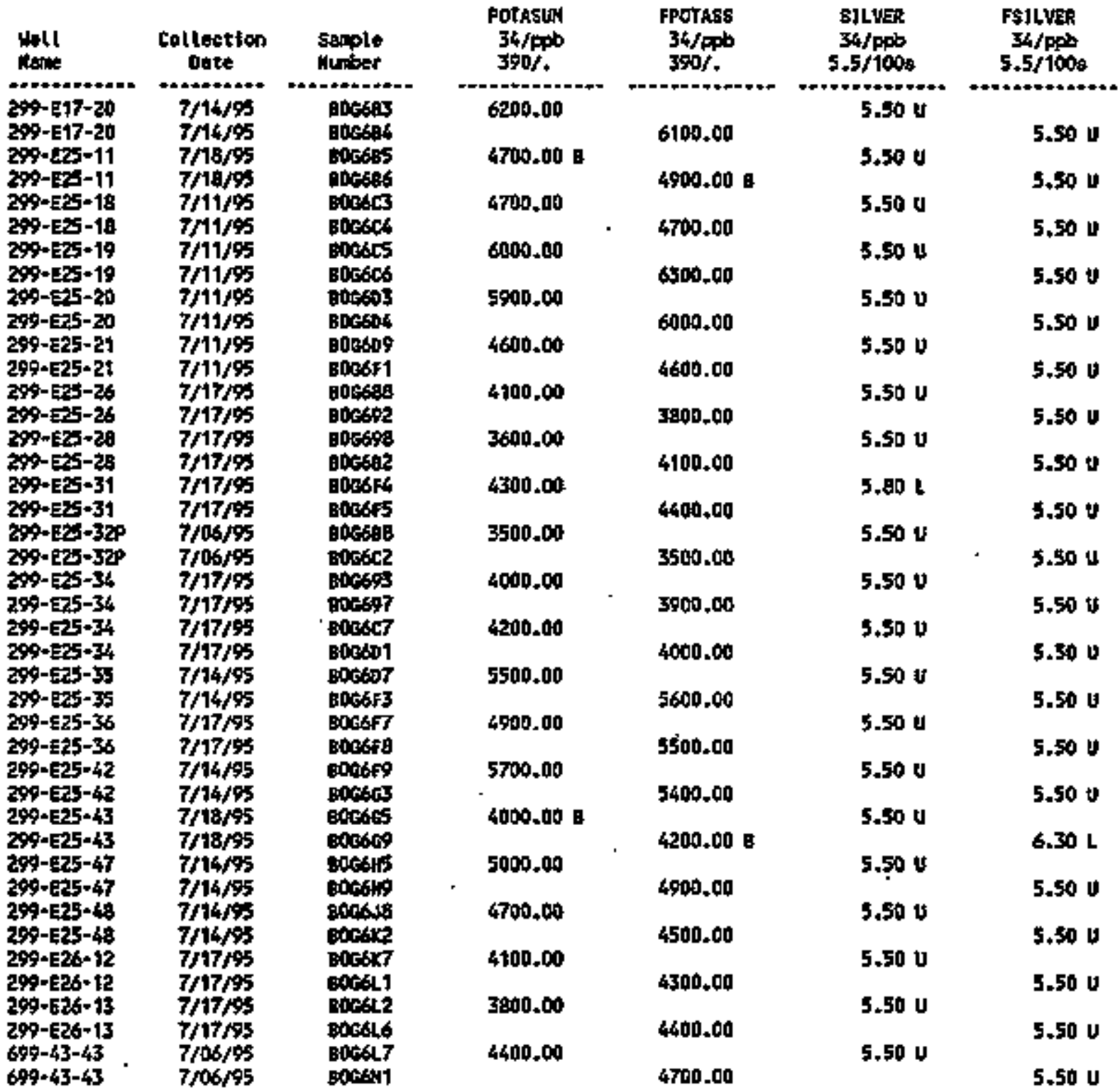


Table 6-4. Constituents with at Least One Detected Value for the 216-A-29 Ditch Data for Reporting Perjod July 1 through September 30, 1995.

(sheet 8 of 10)

\begin{tabular}{|c|c|c|c|c|c|c|}
\hline $\begin{array}{l}\text { Mell } \\
\text { Nase: }\end{array}$ & $\begin{array}{c}\text { Colluction } \\
\text { Dite }\end{array}$ & sanpli & $\begin{array}{l}\text { POTASAM } \\
34 / \mathrm{PpD} \\
390 / .\end{array}$ & $\begin{array}{c}\text { FoTAS5 } \\
\text { 34/PPD } \\
390 \% .\end{array}$ & $\begin{array}{c}\text { SILVER } \\
\text { 34/pph } \\
5.5 / 100 \mathrm{~s}\end{array}$ & $\begin{array}{l}\text { FSItVER } \\
34 / \mathrm{ppb} \\
5.5 / 100 \mathrm{~s}\end{array}$ \\
\hline $\begin{array}{l}699-43-45 \\
699-43-45\end{array}$ & $\begin{array}{l}7 / 06 / 95 \\
7 / 06 / 95\end{array}$ & $\begin{array}{l}\text { Docsine } \\
\text { botsins }\end{array}$ & . $\quad 4100.00$ & 4100.00 & $5.50 \mathrm{~V}$ & $3.50 \mathrm{U}$ \\
\hline
\end{tabular}

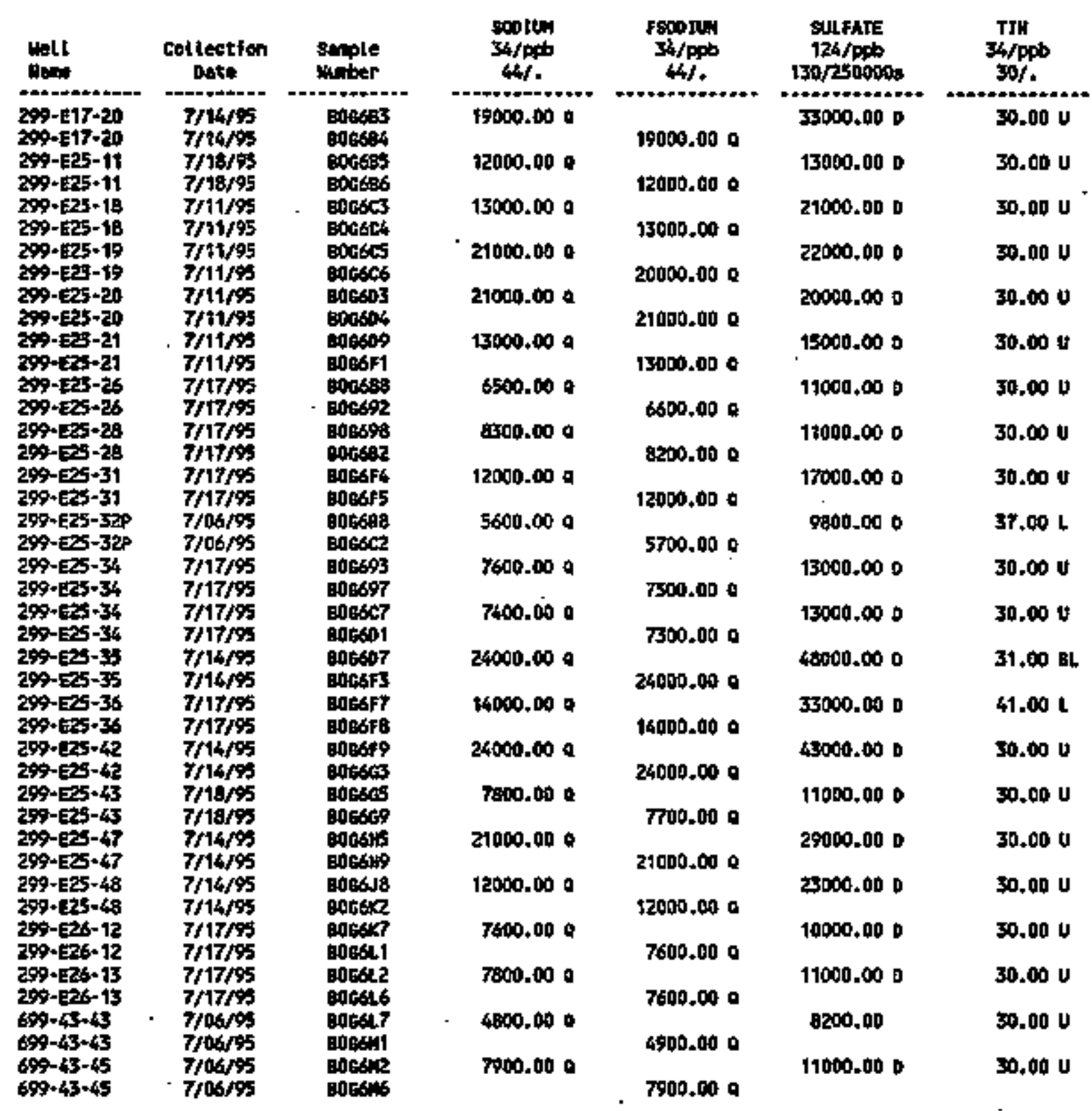


Table 6-4. Constituents with at Least One Detected Value for the 216-A-29 Ditch Data for Reporting Period July 1 through September 30, 1995.

(sheet 9 of 10)

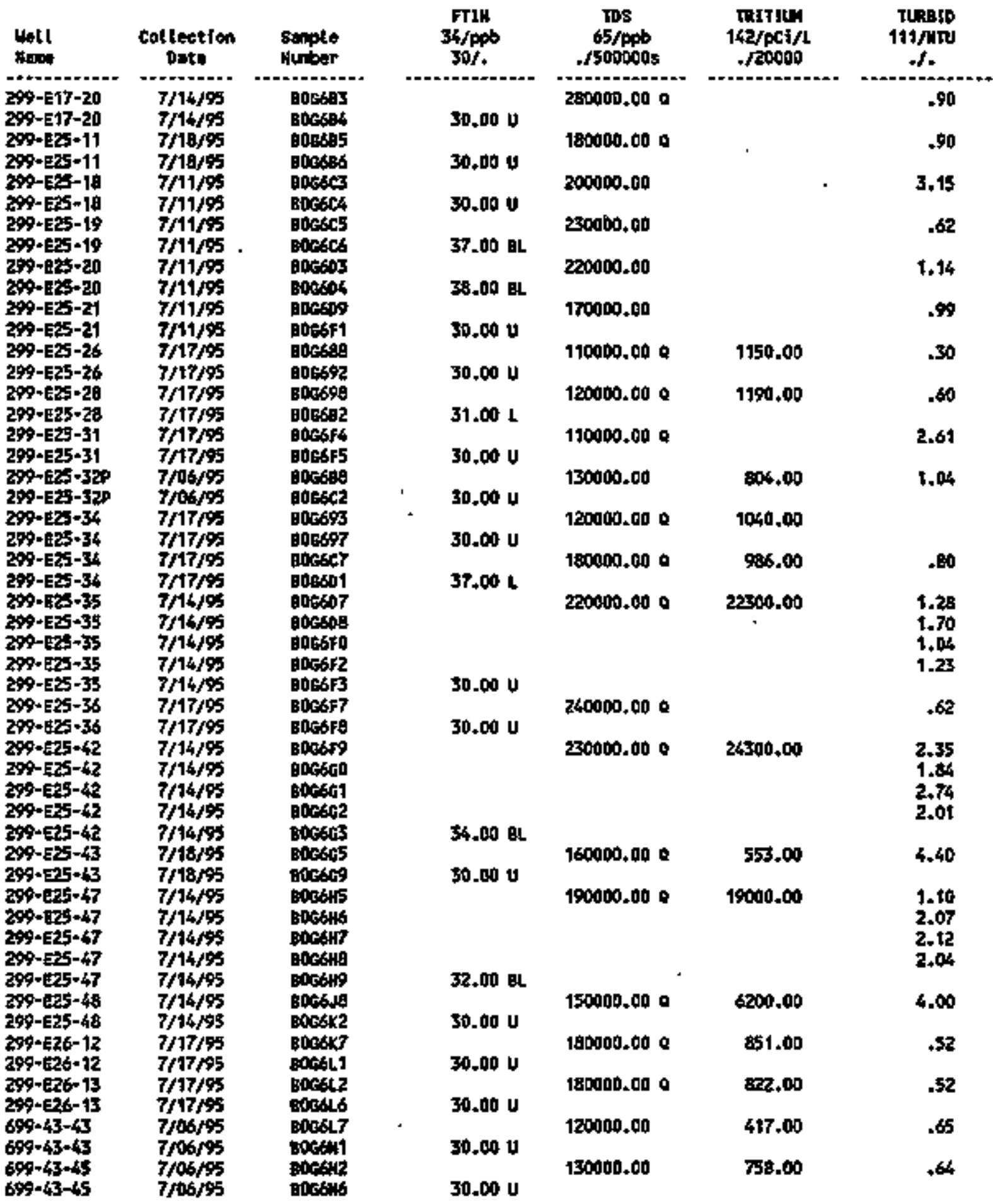


Table 6-4. Constituents with at Least One Detected Value for the 216-A-29 Ditch Data for Reporting Period July 1 through September 30, 1995.

(sheet 10 of 10 )

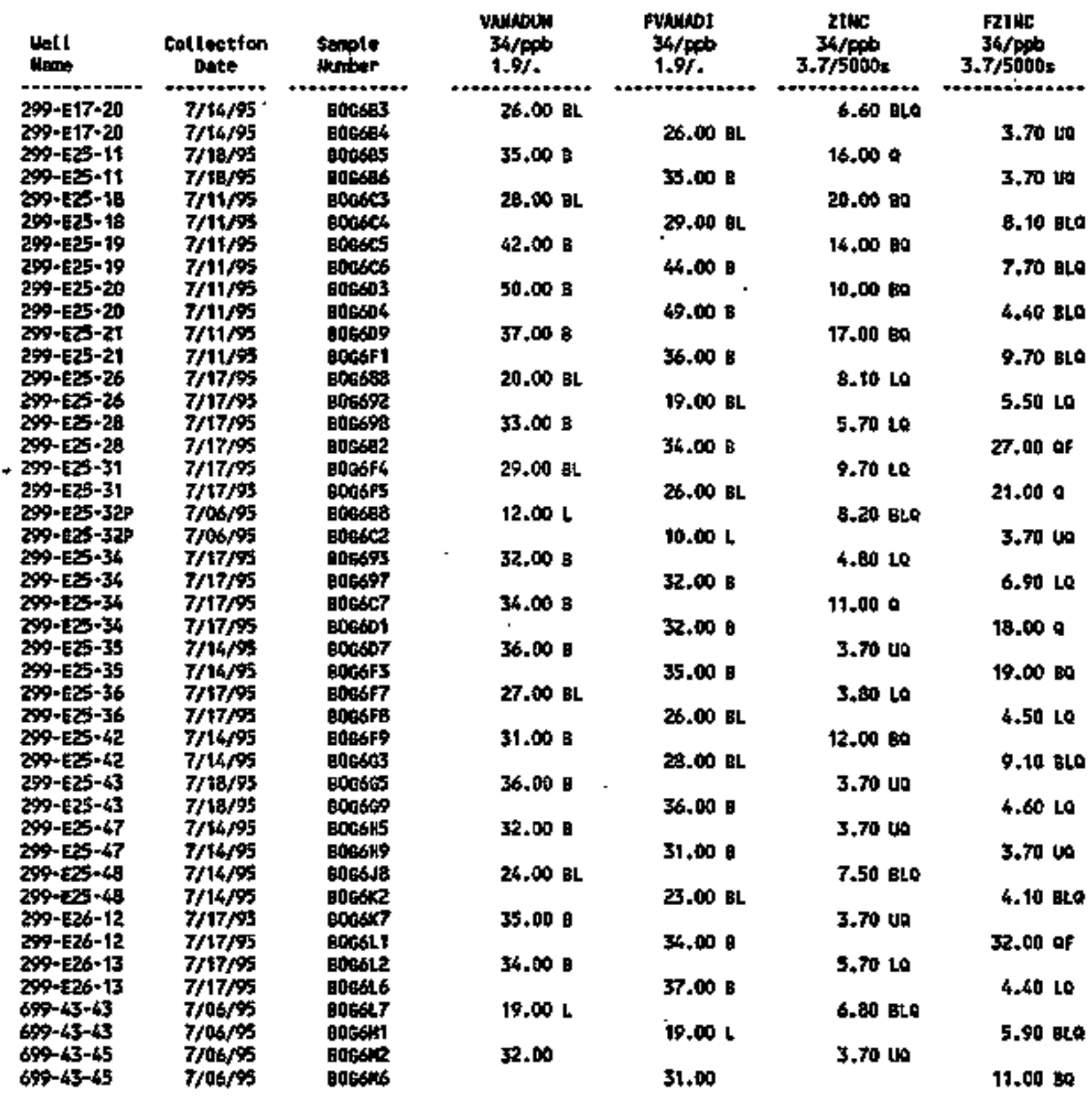

For explenstion of the toble, sectection $t .4$ of repert. 
Table 6-5. Contanination Indicator Parameters for the 216-A-29 Ditch Data for Reporting Period July 1 through September 30, 1995.

(sheet 1 of 2)

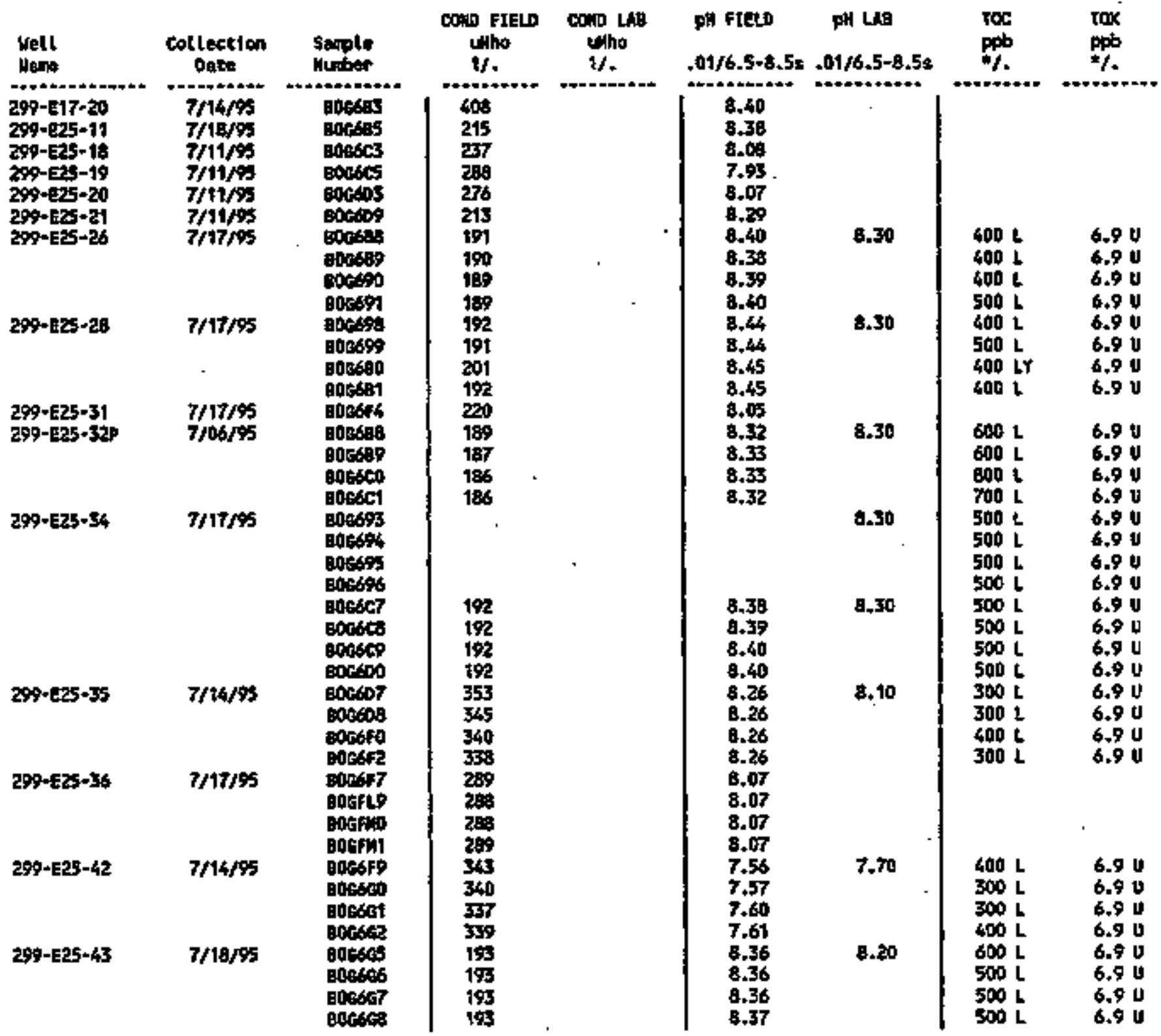


Tabie 6-5. Contarination Indicator Parameters for the 216-A-29 Ditch Data for Reporting Periad July 1 through September 30, 1995.

(sheet 2 of 2)

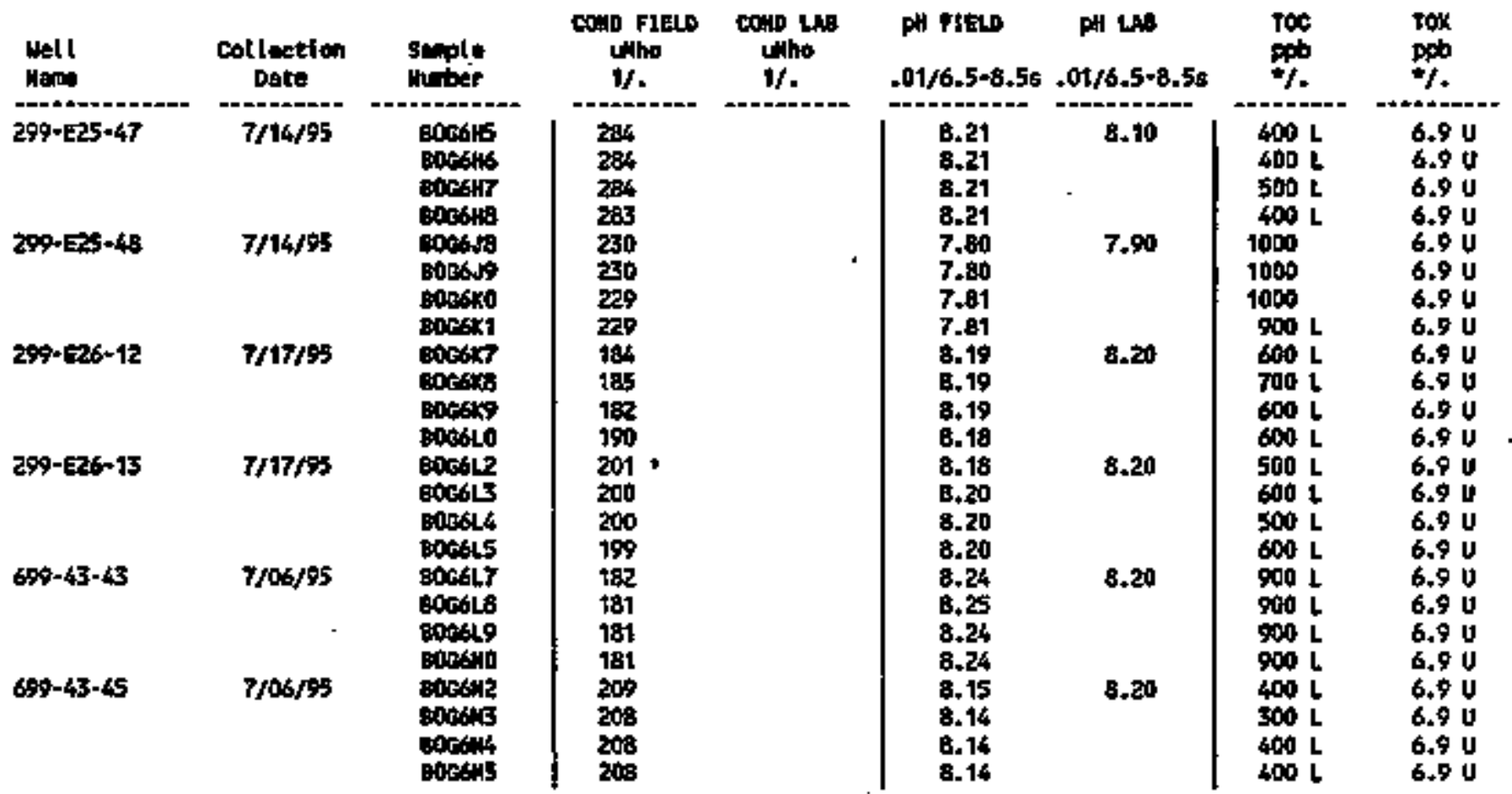

\footnotetext{
- Detection limits for Toc and Tox viry deynding on the parfoming laboratery.
} For explanation of this teble, ste section 1.4 of ressort.

Table 6-6. Drinking Water Standards Exceeded in the 216-A-29 Ditch Groundwater Monjtoring Hetwork.

\begin{tabular}{|l|l|}
\hline \multicolumn{1}{|c|}{ Constituent (DWS) } & \multicolumn{1}{c|}{ We11s exceedting OWS } \\
\hline Chromiun, unfiltored (100 ppb) & $299-E 25-31^{\circ}$ \\
\hline Nitrate (45000 ppb) & $299-E 17-20^{\circ}$ \\
\hline Iron, unfi1tered (300 ppb) & $\begin{array}{l}299-E 25-18^{\circ}, 299-E 25-31^{\circ}, 299-E 25-32 P \\
299-E 25-43\end{array}$ \\
\hline Hickel, unfiltered (100 ppb) & $299-E 25-31^{\circ}, 299-E 25-48$ \\
\hline Nickel, fi]tered (100 ppb) & $299-E 25-48$ \\
\hline Tritiun (20000 ppb) & $299-E 25-35,299-E 25-42$ \\
\hline
\end{tabular}

"We17 in supplemental network.

ois - Drinking Nater Standard. 
DOE/RL-95்-69-3

Conterts

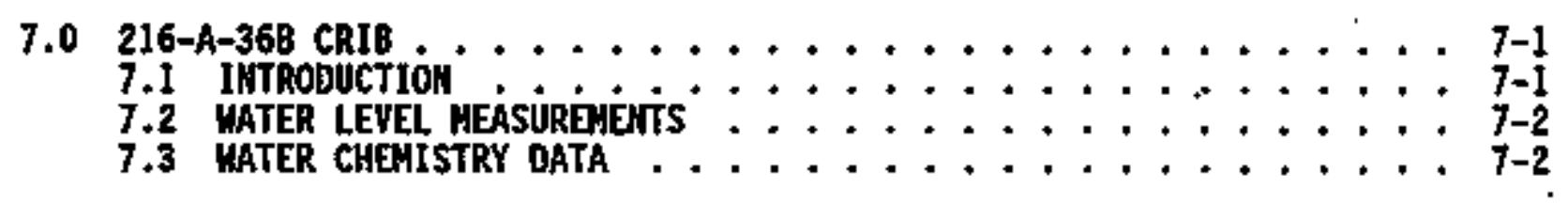


DOE/RL-95-69-3

LIST OF FIGURES

7-1 We11 Location Map for the 216-A-36B Crib . . . . . . . . 7-3

\section{LIST OF TABLES}

7-1 He]l Identification and Sampling Scheduie

for the 216-A-36B Crib Groundwater Monitoring Network . . . . 7-4

7-2 RCRA Water Leve1 Weasurement Report 216-A-36B Crib, Third Quarter 1995 ............ 7-5

7-3 Constituent List and Summary of Results for the 216-A-36B Crib Data for Reporting Period JuTy 1 through September $30,1995 \ldots \ldots$ 7-6

7-4 Constituents with at Least one Detected Value for the 216-A-36B Crib Data for Reporting Period July 1 through September $30,1995 \ldots \ldots \ldots$ 7-8

7-5 Contamination Indicator Parameters for the 216-A-36B Crib Data for Reporting Period July 1 through September 30,1995 


\title{
7.0 216-A-36B CRIB
}

\author{
ง. K. Votava \\ Westinghouse Hanford Company
}

\subsection{INTRODLCTION}

The 216-A-36B Crib (A-36B Crib) is a retired liquid waste disposal facility for the PUREX Plant. The crib is located in the 200 East Area approximately $365 \mathrm{~m}(1,200 \mathrm{ft})$ south of the PUREX P1 ant (Figures $1-1$ and $7-1)$. The A-36B Crib is the southernmost $152 \mathrm{~m}$ (500 ft) of a crib origina11y known as the 216-A-36 $\mathrm{Cr} 1 \mathrm{~b}$. The ammonia scrubber disti1late waste stream that resulted from PUREX aperations was discharged to the crib to percolate through the soil colum. The originat crib received liquid effituent from September 1965 to Harch 1966. Because a substantlal inventory of radionuclides was disposed of in the crib and was assumed to have infiltrated the sediments near the inlet of the crib, a grout curtain was constructed to partition the crib into two sections, known as $A$ and $B$. Discharge to the A-368 Crib section resumed in March 1966 and continued until 1972 when the crib was temporarily removed fron service. The crib was placed back in service in November 1982 and operated until it was retired in October 1987.

Ammonia scrubber distill late effluent disposed of in the A-36B Crib was the condensate from the fuel-rod decladding operation. In this process, the zircalloy cladding was removed from the irradtated fuel by dissolving the cladding in a boling solution of antuonium fluoride and ampionitum nitrate. Waste stream constituents are known to have included radionuclides (tritium, strontium-90, cesium-137, ruthenitum-106, cobalt-60, and uranium) and hazardous substances (atmonitum, fluoride, and nitrate).

In accordance with the Tri-Party Agreement (Ecology et al, 1994), Milestone M-20-33, a RCRA closure/post-closure plan for the A-36B Crib is to be submitted to Ecology and the EPA in June 1998. An interim-status detection-level RCRA groundwater monitoring network has been established at. the A-36B Crib since May 1988.

The RCRA groundwater monitoring system consists of four RCRA-compliant downgradient wells and three upgradient wells, as listed in Table 7-1. The two upgradient we1]s fron the 216-A-10 Crib nonitoring network, 299-E24-18 and 299-E25-36, have been added as upgradient wells for the A-36B Crib. Well 299-E17-5 is ne longer sampled and is used for water level information only.

Well locations for the A-36B Crib nonitoring network are shown in Ffgure 7-1. CIP critical means were reestablished using four quarters of data from upgradient wells (see Table 7-1) during Septenber 1988 to October 1989. As shown in Tabie 7-1, data from we17 299-E25-36 are also used by the 216-A-29 Ditch groundwater qua7ity assessient. 


\subsection{MRTER LEVEL MEASURENEMTS}

Water level measurewents are ade quarterly and during semiannual RCRA sample collection. Water level data collected during the July 1 through Septexaber 30, 1995 period are reported in Table 7-2.

\subsection{MATER CHEGISTRY DATA}

Wells in the A-36B Crib groundwater monitoring network are sampled semiannually. The network was not scheduled for sampling during the July through September 1995 period. Some of the data from the prevtous quarter were aissing because of laboratory reporting delays. These data will be reported when they are available. Well 299-E25-36 was sampled during the report period for the $216-A-29$ Ditch groundwater qual ity assessment. The analytical results are presented in chapter 6. 
Figure 7-1. Well Location Map

for the 216-A-36B Crib.

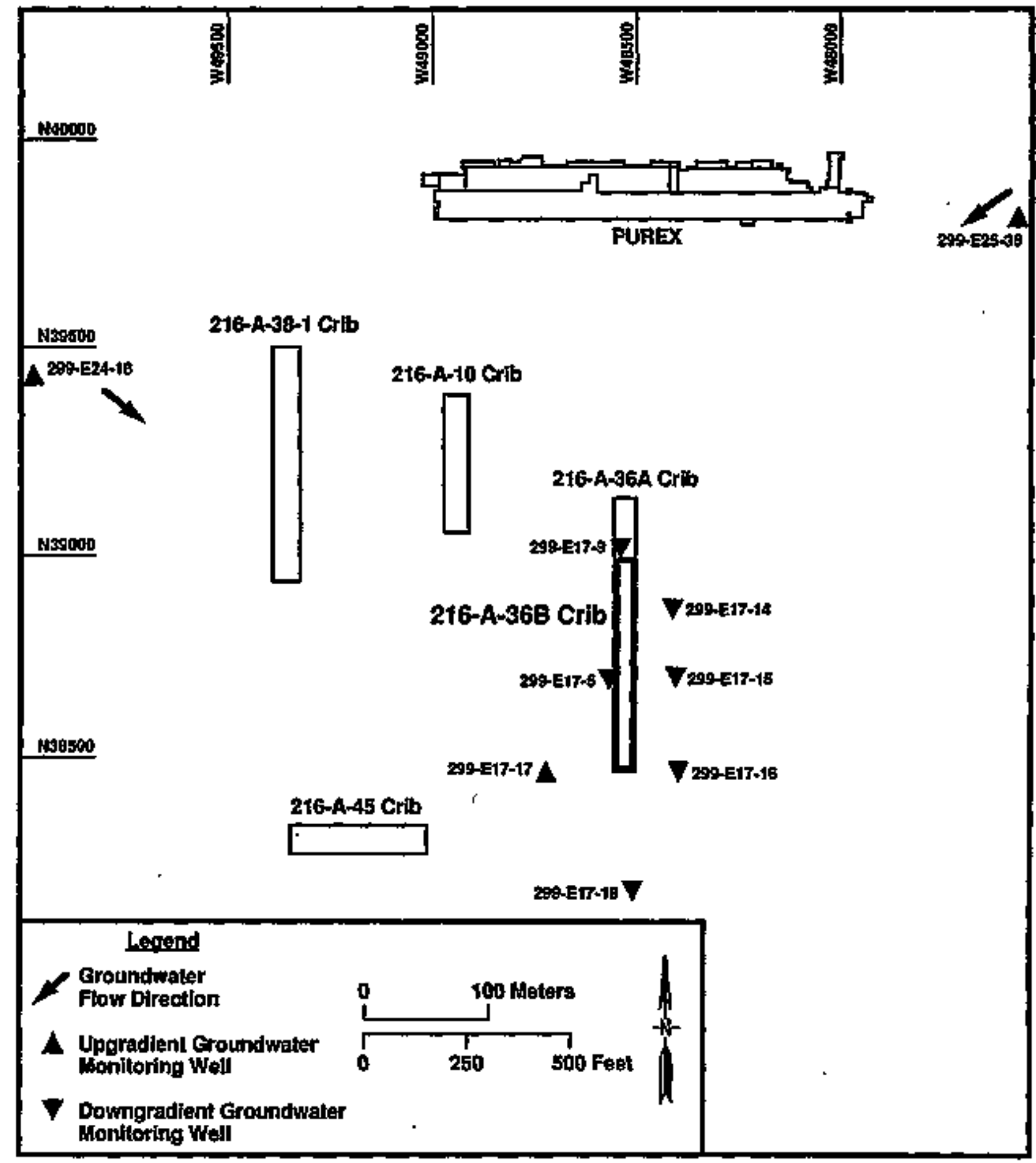

Fo11010.12 
Table 7-1. Well Identification and Sampling Schedule for the 216-A-36B Crib Groundwater Monitoring Network.

\begin{tabular}{|c|c|c|c|c|}
\hline $\begin{array}{c}\text { Hell } \\
\text { no. } \\
\text { (299-) }\end{array}$ & $\begin{array}{l}\text { Relative } \\
\text { position }\end{array}$ & Hydrogeologic unit & $\begin{array}{c}\text { Sample } \\
\text { frequency }\end{array}$ & $\begin{array}{l}\text { Sample date, } \\
\text { 3rd Qtr } 1995\end{array}$ \\
\hline $\mathrm{E} 17-17$ & Upgradient & Ringold: Water Table & Semiannual1y & $\begin{array}{c}\text { Not } \\
\text { Scheduled }\end{array}$ \\
\hline$E 24-18^{9}$ & Upgradient & Ringold: Water Table & Sem lannuał1y & $\begin{array}{c}\text { Not } \\
\text { Scheduled }\end{array}$ \\
\hline$E 25-36^{b}$ & Upgradient & Ringold: Water Table & Senjannua]ly & $7 / 17 / 95$ \\
\hline$E 17-5^{c}$ & Downgradient & Ringold: Water Table & $\begin{array}{c}\text { Water Level } \\
\text { Only }\end{array}$ & - \\
\hline$E 17-9^{\circ}$ & Downgradient & Ringold: Water Table & Seniannua $11 y$ & $\begin{array}{c}\text { Not } \\
\text { Scheduled }\end{array}$ \\
\hline E17-14 & Oowngradient & Ringold: Water Table & Segiannually & $\begin{array}{c}\text { Not } \\
\text { Scheduled }\end{array}$ \\
\hline E17-15 & Downgradient & Ringold: Water Table & Semiannual1y & $\begin{array}{c}\text { Not } \\
\text { Scheduled }\end{array}$ \\
\hline El7-16 & Downgradient & Ringold: Water Table & Semtannual1y & $\begin{array}{c}\text { Not } \\
\text { Scheduled }\end{array}$ \\
\hline E17-18 & Downgradient & Ringold: Water Table & Seriannually & $\begin{array}{c}\text { Not } \\
\text { Scheduled }\end{array}$ \\
\hline
\end{tabular}

Yell shared with the 216-A-10 Crib network.

Wie]] shared with the 216-A-29 01tch network.

Well is not used for statistical evaluation. 
Table 7-2. RCRA Water Level Measurement Report 216-A-36B Crib, Third Quarter 1995.

\begin{tabular}{lcccc}
\hline We17 & Date & $\begin{array}{c}\text { Water leve] } \\
\text { Nater (ft) }\end{array}$ & $\begin{array}{c}\text { (fevation above ns) } \\
\text { (ft) }\end{array}$ & (m) \\
\hline $299-E 17-14$ & $9 / 21 / 95$ & 321.15 & 401.03 & 122.23 \\
$299-E 17-15$ & $9 / 21 / 95$ & 321.61 & 400.17 & 121.97 \\
$299-E 17-16$ & $9 / 21 / 95$ & 319.76 & 400.82 & 122.17 \\
$299-E 17-17$ & $9 / 21 / 95$ & 319.11 & 400.81 & 122.17 \\
$299-E 17-18$ & $9 / 21 / 95$ & 319.73 & 400.92 & 122.20 \\
$299-E 17-5$ & $9 / 21 / 95$ & 318.13 & 400.56 & 122.09 \\
$299-E 17-9$ & $9 / 21 / 95$ & 316.84 & 400.80 & 122.16 \\
$299-E 24-18$ & $9 / 21 / 95$ & 318.25 & 401.03 & 122.23 \\
$299-E 25-36$ & $7 / 17 / 95$ & $306.30^{*}$ & 401.09 & 122.25 \\
& $9 / 21 / 95$ & 306.29 & 401.10 & 122.26 \\
\hline
\end{tabular}

NOTES: 1. Water level elevations are calculated by subtracting the measured depth-to-water from the surveyed eTevation for the well.

2. Depth-to-water values are transcribed from field records.

3. Measurements marked with an $1 * 1$ were taken at the time of sampling.

4. Measurements marked $w i$ th a ' $t$ ' are outside of the expected range, and are suspected of error. 
Table 7-3. Constituent List and Summary of Results

for the 216-A-36B Crib Data for Reporting Perjod

July 1 through September 30, 1995.

(sheet 1 of 2)

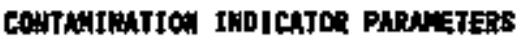

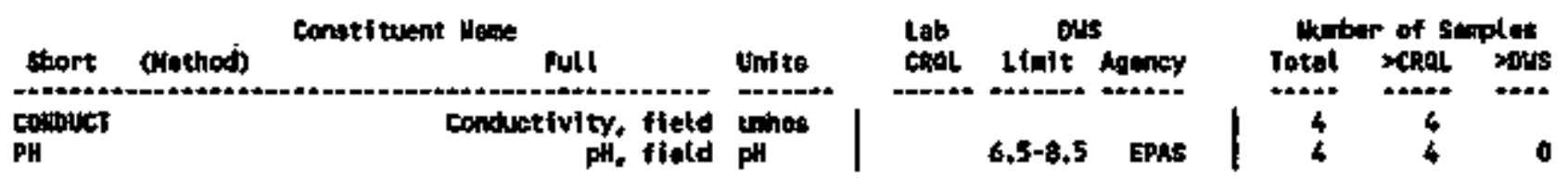

DRINTHG WTER PARNAETERS

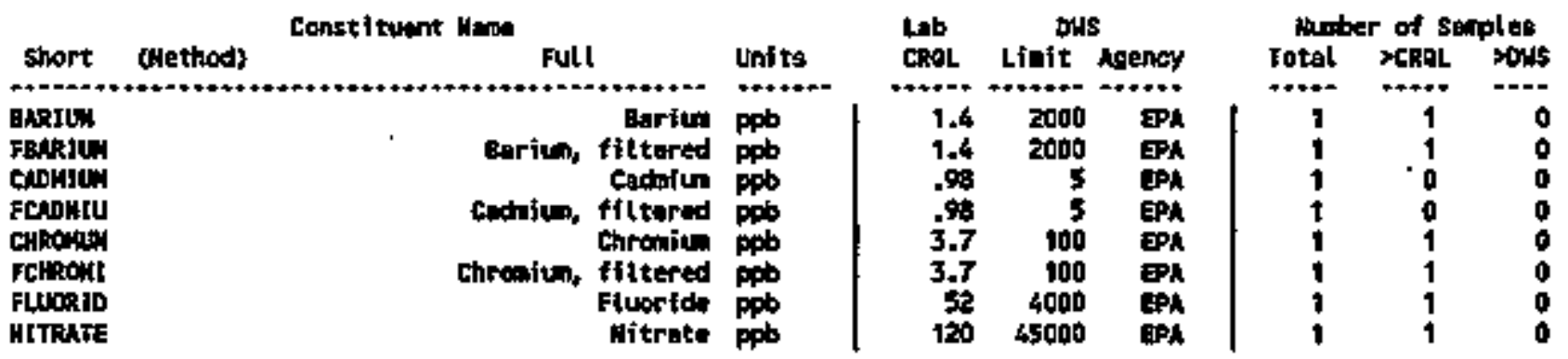

GROANDLATER DimL1TY PARUIETEAS

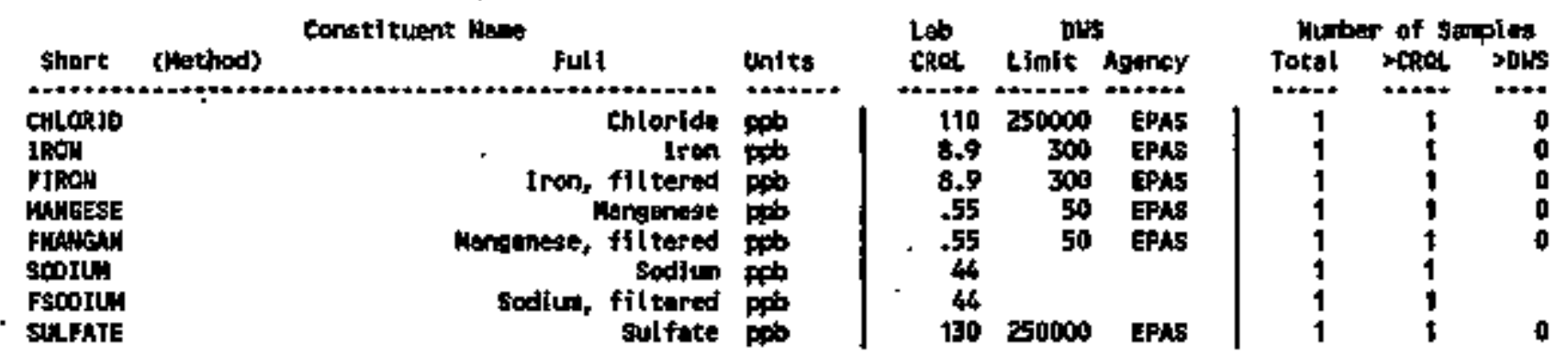

SIJE SPECIFIC AW OTHER CONSTITUEYTS

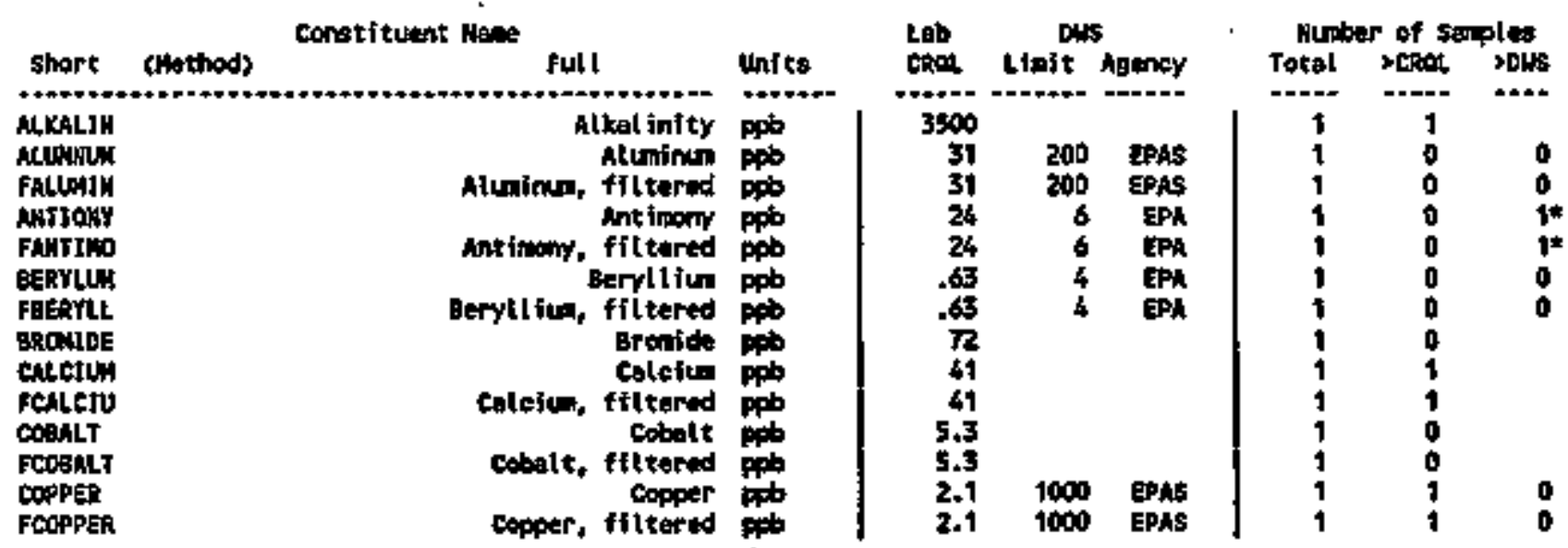


Table 7-3. Constituent List and Stmmary of Resutts for the 216-A-36B Crib Data for Reporting Period July 1 through September 30, 1995.

(sheet 2 of 2)

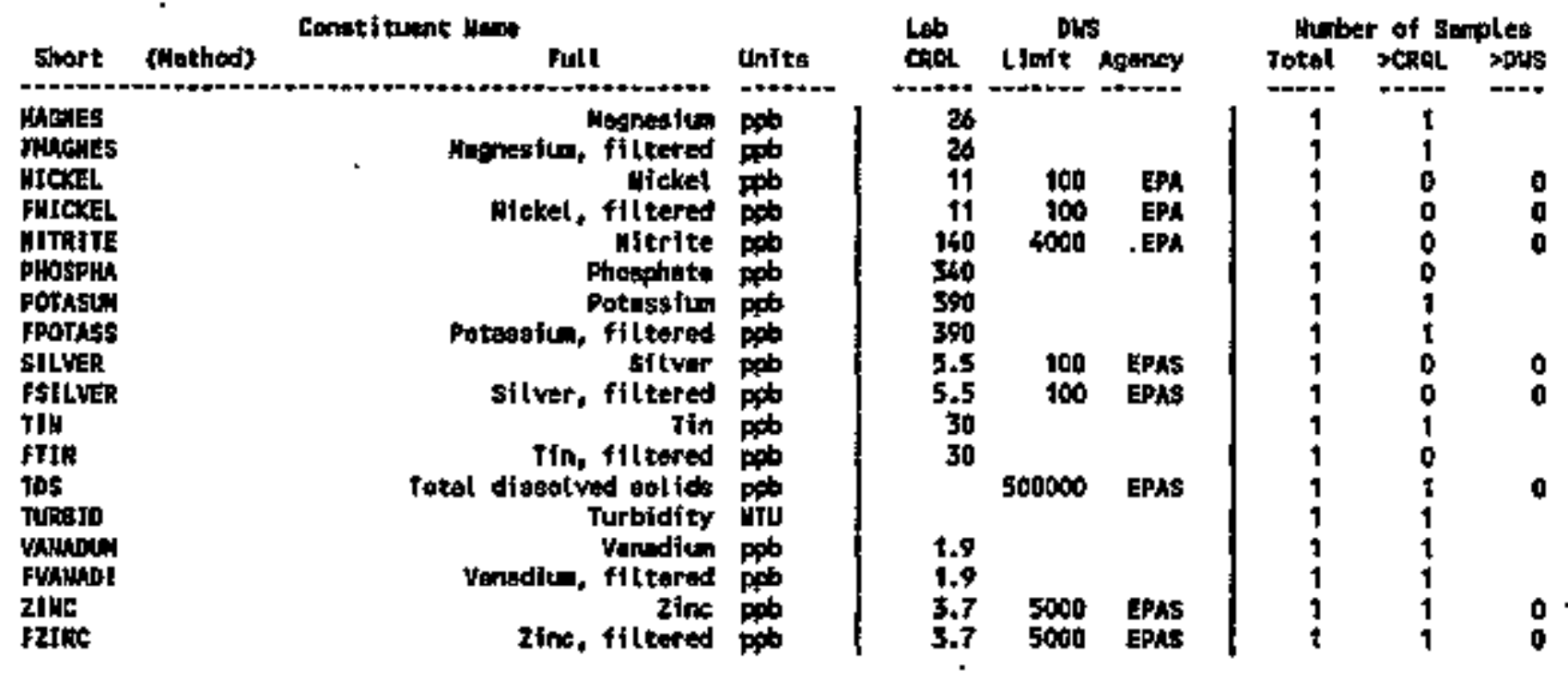

For expianstion of this thite, set section 1,4 of roport. 
Table 7-4. Constituents with at Least One Detected Value for the 2]6-A-368 Crib Data for Reporting Period July 1 through Septenber 30, 1995.

(sheet 1 of 2)

\begin{tabular}{|c|c|c|c|c|c|c|}
\hline Wall & $\begin{array}{c}\text { Collection } \\
\text { Dote }\end{array}$ & $\begin{array}{l}\text { Semple } \\
\text { Murber }\end{array}$ & $\begin{array}{c}\text { ALKAL IH } \\
357 / p p b 0 \\
3500 / .\end{array}$ & $\begin{array}{c}\text { Barjew } \\
34 / \mathrm{ppb} \\
1.4 / \mathrm{apod}\end{array}$ & $\begin{array}{l}\text { PENRIUM } \\
34 / \mathrm{ppb} \\
1,4 / 2000\end{array}$ & $\begin{array}{c}\text { CALCIUW } \\
34 / \mathrm{ppb} \\
41 / .\end{array}$ \\
\hline $\begin{array}{l}299-\mathrm{E} 25-36 \\
299-\mathrm{Ez} 5-36\end{array}$ & $\begin{array}{l}7 / 17 / 99 \\
7 / 17 / 95\end{array}$ & $\begin{array}{l}\text { exesp7 } \\
\text { B066f8 }\end{array}$ & 90000.00 & 20.00 & & 29000.000 \\
\hline
\end{tabular}

\begin{tabular}{|c|c|c|c|c|c|c|}
\hline Litl & $\begin{array}{c}\text { collection } \\
\text { botion }\end{array}$ & $\begin{array}{l}\text { Semple } \\
\text { Munber }\end{array}$ & $\begin{array}{c}\text { reatciu } \\
34 / \mathrm{ppb} \\
41 / .\end{array}$ & $\begin{array}{c}\text { chLorto } \\
124 / p 006 \\
190 / 2500000\end{array}$ & $\begin{array}{l}\text { ClRawm } \\
34 / \mathrm{ppb} \\
3.7 / 100\end{array}$ & $\begin{array}{l}\text { PCHRCOI } \\
36 / \mathrm{ppb} \\
3.7 / 100\end{array}$ \\
\hline $\begin{array}{l}5-36 \\
5-36\end{array}$ & $\begin{array}{l}7 / 17 / 95 \\
7 / 17 / 65\end{array}$ & $\begin{array}{l}\text { B0G6F7 } \\
300698\end{array}$ & & 4100.00 & 21.00 & \\
\hline
\end{tabular}

\begin{tabular}{|c|c|c|c|c|c|c|}
\hline $\begin{array}{l}\text { Melt } \\
\text { Mame }\end{array}$ & $\begin{array}{l}\text { Cotlection } \\
\text { Dete }\end{array}$ & $\begin{array}{l}\text { Somple } \\
\text { Inumber }\end{array}$ & $\begin{array}{c}\text { COPPER } \\
\text { 34/ppo } \\
2.1 / 1001 / 3\end{array}$ & $\begin{array}{c}\text { FCOOPER } \\
3 \notin / \mathrm{ppb} \\
2.1 / 1000 \mathrm{~s}\end{array}$ & $\begin{array}{l}\text { Fluopio } \\
124 / \mathrm{pat} \\
52 / 4000\end{array}$ & $\begin{array}{c}180 \mathrm{y} \\
34 / \mathrm{ppb} \\
8.9 / 5000\end{array}$ \\
\hline $\begin{array}{l}299-E 25-36 \\
299-E 25-36\end{array}$ & $\begin{array}{l}7 / 17 / 95 \\
7 / 17 / \% 5\end{array}$ & $\begin{array}{l}\text { BDC6FY } \\
\text { BOGG68 }\end{array}$ & 2.401 & $4.00 \mathrm{~L}$ & 300,00 & $1+0.000$ \\
\hline
\end{tabular}

\begin{tabular}{|c|c|c|c|c|c|c|}
\hline $\begin{array}{l}\text { Well } \\
\text { Mame }\end{array}$ & $\begin{array}{c}\text { Collection } \\
\text { Deze }\end{array}$ & $\begin{array}{l}\text { Sompte } \\
\text { Muibur }\end{array}$ & $\begin{array}{c}\text { VIRON } \\
34 / \mathrm{ppob}^{\circ} \\
8 . \$ / 500 \mathrm{~s}\end{array}$ & $\begin{array}{c}\text { MUGNES } \\
34 / \mathrm{Fpb} \\
26 / .\end{array}$ & $\begin{array}{c}\text { FWAGKES } \\
\text { 34/Dpb } \\
26 / .\end{array}$ & $\begin{array}{l}\text { MANEESE } \\
34 / 1 p^{2} \\
.55 / 508\end{array}$ \\
\hline $\begin{array}{l}299-E 25-36 \\
299-625-36\end{array}$ & $\begin{array}{l}7 / 17 / 45 \\
7 / 17 / 95\end{array}$ & $\begin{array}{l}\text { 8006r77 } \\
\text { B056F }\end{array}$ & $\$ 1.008$ & 7900.00 & & 2 \\
\hline
\end{tabular}

\begin{tabular}{|c|c|c|c|c|c|c|}
\hline $\begin{array}{l}\text { Untl } \\
\text { Whene }\end{array}$ & $\begin{array}{c}\text { Collection } \\
\text { Dute }\end{array}$ & $\begin{array}{l}\text { Seqplo } \\
\text { homber }\end{array}$ & $\begin{array}{l}\text { munotw } \\
34 / \mathrm{ppb}^{2} \\
+3+50 \mathrm{~s}\end{array}$ & $\begin{array}{c}\text { NE TR/re } \\
\text { t24/ppb } \\
120 / 45000\end{array}$ & $\begin{array}{l}\text { Potaslin } \\
\text { 34/ppb } \\
\text { 390/. }\end{array}$ & $\begin{array}{c}\text { FPOTASs } \\
34 / \mathrm{ppb} \\
300 \% .\end{array}$ \\
\hline $\begin{array}{l}299-E 25-36 \\
299-E-25-36\end{array}$ & $\begin{array}{l}7 / 17 / 95 \\
7 / 17 / 95\end{array}$ & $\begin{array}{l}\text { ExGSF7 } \\
\text { 10GSF: }\end{array}$ & $1.70 \mathrm{C}$ & 23090.000 & 4940,00 & $5 \$ 000.00$ \\
\hline
\end{tabular}

\begin{tabular}{|c|c|c|c|c|c|c|}
\hline $\begin{array}{l}\text { Welt } \\
\text { Wane }\end{array}$ & $\begin{array}{c}\text { Col lection } \\
\text { Date }\end{array}$ & $\begin{array}{l}\text { Strple } \\
\text { Mtrober }\end{array}$ & 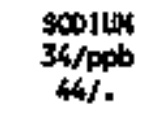 & $\begin{array}{c}\text { Fscotllor } \\
36 / \mathrm{ppb}^{2} \\
46 / .\end{array}$ & $\begin{array}{c}\text { SulfaTe } \\
\text { 124/ppb } \\
130 / 250000 \mathrm{~s}\end{array}$ & $\begin{array}{c}\text { Tid } \\
34 / \mathrm{ppb} \\
30 / .\end{array}$ \\
\hline $\begin{array}{l}299-E 25-36 \\
299- \pm 25-36\end{array}$ & $\begin{array}{l}7 / 17 / 95 \\
7 / 17 / 95\end{array}$ & $\begin{array}{l}\text { BOCGF } 7 \\
\text { O0C6FB }\end{array}$ & 14000.00 & & 33000.000 & 41.00 \\
\hline
\end{tabular}

\begin{tabular}{|c|c|c|c|c|c|c|}
\hline Hoil & $\begin{array}{l}\text { Collection } \\
\text { Date }\end{array}$ & $\begin{array}{l}\text { semple } \\
\text { Mutber }\end{array}$ & $\begin{array}{c}\text { Tr1W } \\
34 / p p b \\
30 \%\end{array}$ & $\begin{array}{c}105 \\
65 / p^{b} \\
.5000000\end{array}$ & $\begin{array}{c}\text { TURBID } \\
111 / / 4 U \mathrm{UU} \\
. / .\end{array}$ & $\begin{array}{l}\text { venubur } \\
34 / \mathrm{ppb}^{\circ} \\
1.9 \%\end{array}$ \\
\hline $\begin{array}{l}299-125-36 \\
299-E 25-36\end{array}$ & $\begin{array}{l}7 / 17 / 95 \\
7 / 17 / 95\end{array}$ & $\begin{array}{l}8066 \% 7 \\
\text { B066:8 }\end{array}$ & 30.00 & 240000.000 & .62 & $27,00 \mathrm{BL}$ \\
\hline
\end{tabular}


Table 7-4. Constituents with at Least One Detected Value for the 216-A-36B Crib Data for Reporting Period July 1 through Septealber 30, 1995.

(sheet 2 of 2)

\begin{tabular}{|c|c|c|c|c|c|}
\hline $\begin{array}{l}\text { Well } \\
\text { faile }\end{array}$ & $\begin{array}{l}\text { Col lattion } \\
\text { Oate }\end{array}$ & $\begin{array}{l}\text { Stiple } \\
\text { Humber }\end{array}$ & $\begin{array}{l}\text { FWANRI ' } \\
\text { 34/ppts } \\
1.9 / .\end{array}$ & $\begin{array}{c}\text { Z1Hc } \\
\text { 34/ppo } \\
3.7 / 5004 \mathrm{~s}\end{array}$ & $\begin{array}{c}\text { f2twe } \\
34 / 1 \mathrm{pb} \\
3.7 / 500 \mathrm{bo}\end{array}$ \\
\hline $\begin{array}{l}299-E 25-36 \\
299-125-36\end{array}$ & $\begin{array}{l}7 / 17 / 95 \\
7 / 17 / 95\end{array}$ & $\begin{array}{l}\text { Buckst } \\
\text { bot668 }\end{array}$ & $26.00 \mathrm{BL}$ & Lo & $4.50 \mathrm{la}$ \\
\hline
\end{tabular}

Far explanution of this tuble, sese stetion 1.4 of report.

Table 7-5. Contamination Indicator Parameters for the 216-A-36B Crib Data for Reporting Period July I through September 30, 1995.

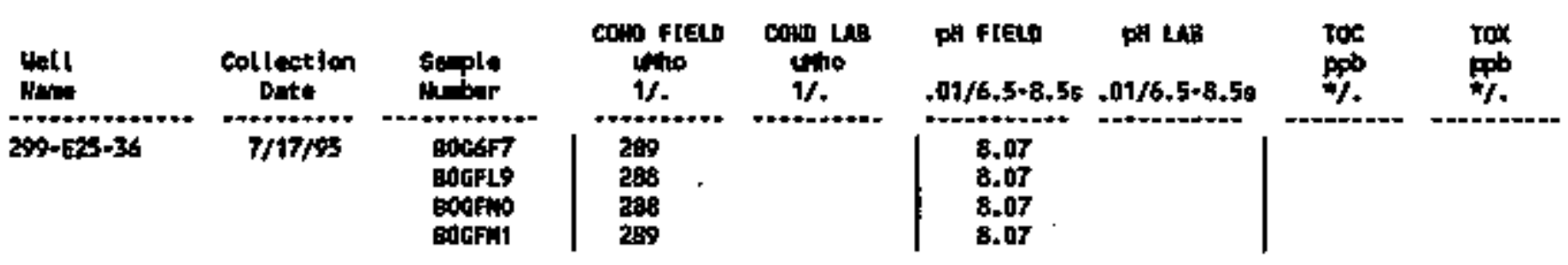

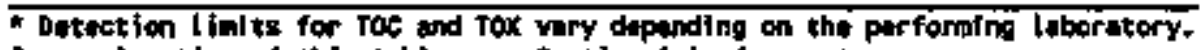

For explanetion of this teble, ete section 1.4 of report. 
DOE/RL-95-69-3

This page intentionally left blank. 
DOE/RL-95-69-3

cortents

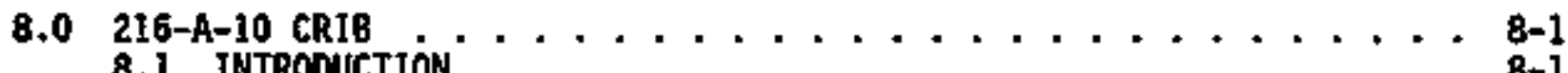

8.1 INTRODUCTION .....................

8.2 WATER LEVEL MEASUREHENTS $\ldots \ldots \ldots$. . . . . . . . .

8.3 WATER CHEMISTRY DATA $\ldots \ldots \ldots \ldots . \ldots . \ldots . \ldots$ 
DOE/RL-95-69-3

LIST OF FIGURES

8-1 We11 Location Map for the 216-A-10 Crib ........... 8-3

\section{LIST OF TABLES}

8-1 Well Identification and Sampling Schedule for the 216-A-10 Crib Groundwater Monitoring Hetwork . . . . . . . 8-4

8-2 RCRA Hater Level Measurement Report 216-A-10 Crib, Third quarter 1995 ........... 8-5

8-3 Constituent List and Summary of Results for the 216-A-10 Crib Data for Reporting Period July 1 through September 30, 1995 ............ 8-6

8-4 Constituents with at Least One Detected value for the 216-A-10 Crib Data for Reporting Period July 1 through September $30,1995 \ldots \ldots$ 8-8

8-5 Contamination Indicator parameters for the 216-A-10 Crib Data for Reporting Period July 1 through September $30,1995 \ldots \ldots$. . . . . . . . . . . 


\title{
8.0 216-A-10 CRIB
}

\author{
J. h. Votava \\ Nestinghouse Hanford Company
}

\subsection{IKTRODUCTIOH}

The 216-A-10 crib (A-10 Crib) is a retired liquid waste disposal facjlity that was used during the operation of the Plutonium-Uranium Extraction (PUREX) Plant. The crib is located in the 200 East Area approximately $120 \mathrm{~m}$ (400 ft) south of the PUREX P1ant (Figures 1-1 and 8-1).

Severai waste streans, collectively referred to as the process dtstillate discharge, were disposed of in the A-10 Crib for soil-column disposal during PUREX PI ant operations. Process distill ate discharge waste was first disposed of in the crib for 4 months during the 1956 PUREX startup. In 1961, the A-10 Crib replaced the 216-A-5 Crib and received PUREX effluent continuously unti1 1973. Periodic discharges were received in 1977, 1978, and 1981. From 1982 to 1987 , continuous effluent discharges to the A-10 Crib were resumed. Effiuent volumes from 1981 to 1986 averaged $1 \times 10^{8} \mathrm{~L}$ (2.6 $\times 10^{6}$ gat) per year. In 1987 , the A-10 Crib was removed from service and replaced by the $216-A-45 \mathrm{Crib}$.

The process distillate discharge waste stream sent to the A-10 Crib was characteristically acidic and contained concentrated salts. Waste strean constituents inciuded al jphatic hydrocarbon compounds, organic complexants, and radiontcl ides (plutonium, uranium, strontjum-90, cobait-60, cesium-134 and -137 , ruthen itum-103 and -106, and tritium).

In accordance with the Tri-Party Agreenent (Ecology et a]. 1994), Milestone $\mathrm{H}-20-33$, a RCRA closure/post-closure plan for the A-10 Crib is to be submitted to Ecology and EPA in June 1998. An interim-status detection-leve] RCRA graundwater nonitoring network has been operational since Hovember 1988.

The RCRA groundwater monitoring systen consists of six downgradient wells and two upgradient wells, as Tisted in Table 8-1. Two wells in the 216-A-10 network, 216-E15-20 and 216-E25-36, are shared with the groundwater monltoring network for the A-29 Ditch faciljty. WelTs 216-E24-18 and 216-E25-36 are shared with the 216-A-36B $\mathrm{Cr}$ ib. Nell locations are shown in Figure 8-1. Background monitoring was completed in the fourth quarter of 1989 , following the first four quarters of groundwater sampling.

\subsection{WATER LEVEL MEASURENEUTS}

Water level measurements are made quarterly and during semtannual RCRA sample collection. Water level data collected from July through September 1995 are reported in Table 8-2. 


\subsection{WATER CHEXISTRY DATA}

Mells in the A-10 Crib groundwater monitoring network are sampled semiannually. The network was not scheduled for sampling during the July through Septeaber 1995 period. Soale of the data from the previous quarter sampling were missing because of laboratory reporting delays. These data will be reported when they are avallable. Chemistry data that were avajlable are reported in Tables 8-3 through 8-5. Nells 299-E17-20 and 299-E25-36 were sampled during the report period for the $216-A-29$ Ditch groundwater quality assessment. The analytical results are presented in Chapter 6. 
Figure 8-1. Well Location Map for the 216-A-10 Crib.

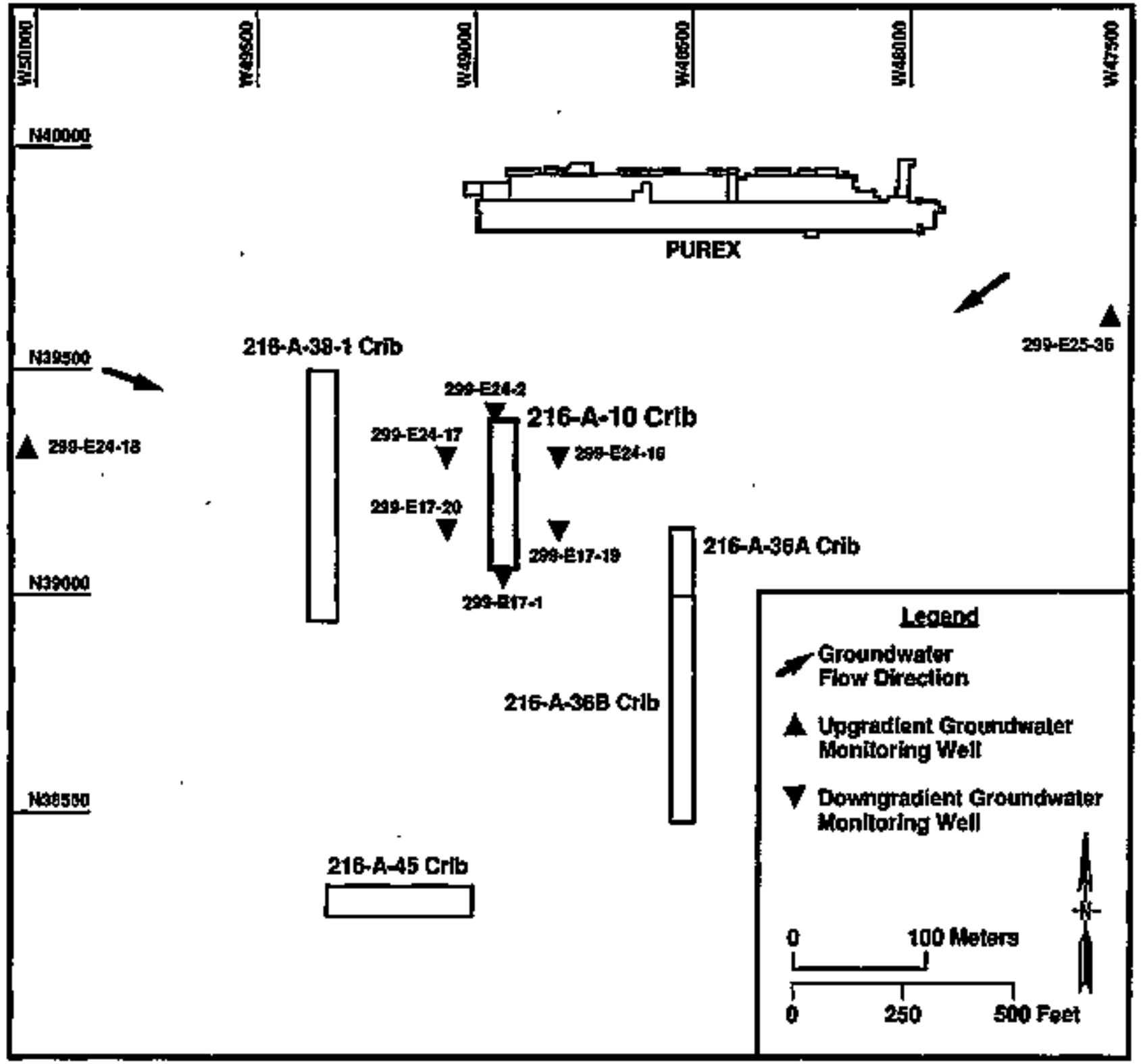

Ho41 10t0.135 
TabTe 8-1. Me11 Identification and Sampling Schedule for the 216-A-10 Crlb Groundwater Monttoring Network.

\begin{tabular}{|c|c|c|c|c|}
\hline $\begin{array}{c}\text { Nell } \\
\text { no. } \\
(299-)\end{array}$ & $\begin{array}{l}\text { Relative } \\
\text { position }\end{array}$ & Hydrogeologic unit & $\begin{array}{l}\text { Sample } \\
\text { frequency }\end{array}$ & $\begin{array}{l}\text { Sample date, } \\
\text { 3rd Qtr } 1995\end{array}$ \\
\hline$E 24-18^{\circ}$ & Upgradient & $\begin{array}{l}\text { Ringold: Water } \\
\text { Table }\end{array}$ & Semi annua11y & $\begin{array}{c}\text { Not } \\
\text { scheduled }\end{array}$ \\
\hline$E 25-36^{b}$ & Upgradient & $\begin{array}{l}\text { Ringold: Water } \\
\text { Table }\end{array}$ & Semlannually & $7 / 17 / 95$ \\
\hline E17-1 & Downgradient & $\begin{array}{l}\text { Ringold: Water } \\
\text { Table }\end{array}$ & Semiannual1y & $\begin{array}{c}\text { Not: } \\
\text { scheduled }\end{array}$ \\
\hline E17-19 & Downgradient & $\begin{array}{l}\text { Ringold: Water } \\
\text { Table. }\end{array}$ & Semiannual1y & $\begin{array}{c}\text { Hot } \\
\text { scheduled }\end{array}$ \\
\hline$E 17-20^{b}$ & Downgradient & $\begin{array}{l}\text { Ringold: Water } \\
\text { Table }\end{array}$ & Sentannually & $7 / 14 / 95$ \\
\hline$E 24-2$ & Downgradient & $\begin{array}{l}\text { Ringold: Water } \\
\text { Tabie }\end{array}$ & $\begin{array}{l}\text { Water Level } \\
\text { Oniy }\end{array}$ & - \\
\hline E24-16 & Downgradient & $\begin{array}{l}\text { Ringold: Water } \\
\text { Table }\end{array}$ & Sem1 annua11y & $\begin{array}{c}\text { Not } \\
\text { scheduled }\end{array}$ \\
\hline E24-17 & Downgradient & $\begin{array}{c}\text { Ringold: Water } \\
\text { Table }\end{array}$ & Semiannua $1 \mathrm{y}$ & $\begin{array}{c}\text { Not } \\
\text { schefuled }\end{array}$ \\
\hline
\end{tabular}

Well sampled during period for 216-A-36B Crib network.

We11 sampled during period for 216-A-29 Ditch network.

'Well is not used for statistical evaluation. 
Table 8-2. RCRA Hater Level Measurement Report 216-A-10 Crib, Third Quarter 1995.

\begin{tabular}{|c|c|c|c|c|}
\hline Well & Date & $\begin{array}{l}\text { Depth to } \\
\text { water (ft) }\end{array}$ & $\begin{array}{l}\text { Water } \\
\text { elevation } \\
\text { (ft) }\end{array}$ & $\begin{array}{l}\text { level } \\
\text { above ms1 } \\
\text { (ii) }\end{array}$ \\
\hline 299-E17-1 & $9 / 21 / 95$ & 318.38 & 400.79 & 122.16 \\
\hline $299-\varepsilon 17-19$ & $9 / 21 / 95$ & 318.85 & 400.48 & 122.07 \\
\hline 299-E17-20 & $\begin{array}{l}7 / 14 / 95 \\
9 / 21 / 95\end{array}$ & $\begin{array}{l}317.79 * \\
318.18\end{array}$ & $\begin{array}{l}401.44 \\
401.05\end{array}$ & $\begin{array}{l}122.36 \\
122.24\end{array}$ \\
\hline 299-E24-I6 & $9 / 21 / 95$ & 317.14 & 401.13 & 122.26 \\
\hline 299-E24-17 & $9 / 21 / 95$ & 318.57 & 400.12 & 121.96 \\
\hline 299-E24-18 & $9 / 21 / 95$ & 318.25 & 401.03 & 122.23 \\
\hline 299-E24-2 & $9 / 21 / 95$ & 316.35 & 401.12 & 122.26 \\
\hline $299-E 25-36$ & $\begin{array}{l}7 / 17 / 95 \\
9 / 21 / 95\end{array}$ & $\begin{array}{l}306.30 * \\
306.29\end{array}$ & $\begin{array}{l}401.09 \\
401.10\end{array}$ & $\begin{array}{l}122.25 \\
122.26\end{array}$ \\
\hline
\end{tabular}

NOTES: 1. Water level elevations are calculated by subtracting the maasured depth-to-water from the surveyed elevation for the well.

2. Depth-to-water values are transcribed from field records.

3. Measurements marked with an ' $*$ ' were taken at the time of sampling.

4. Measurements marked with a ' + ' are outside of the expected range, and are suspected of error. 
Table 8-3. Constituent List and Summary of Results for the 216-A-10 Crib Data for Reporting Period July 1 through September 30, 1995.

(sheet 1 of 2)

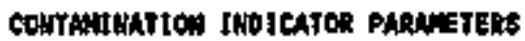

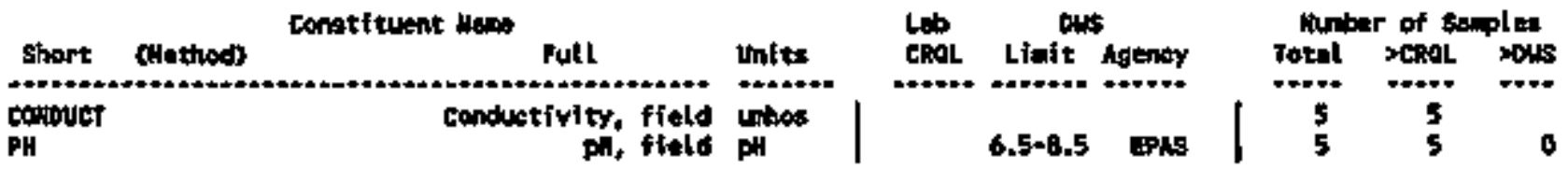

ORINKIIS WhTER PhPanetems

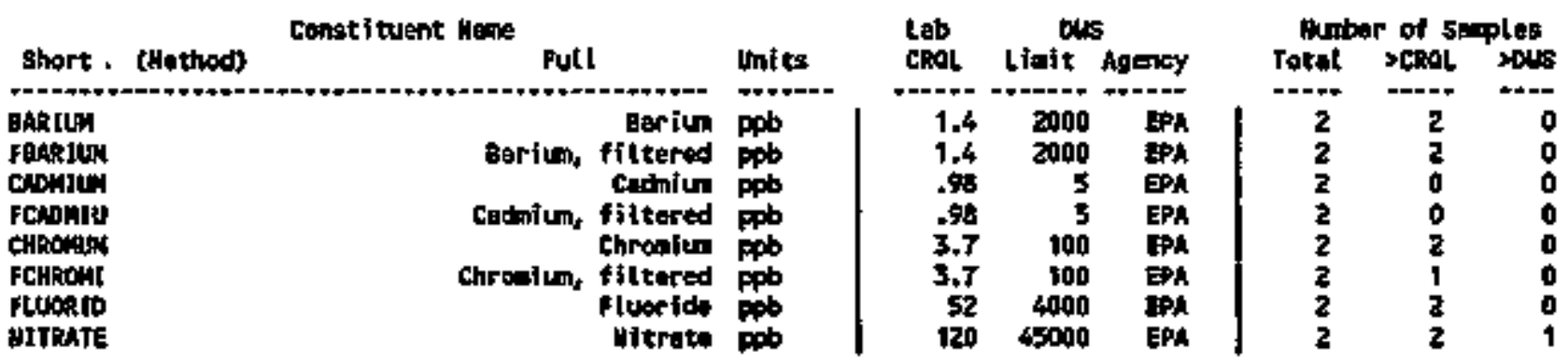

-EROFUWUTER OULITY PRRUETERS

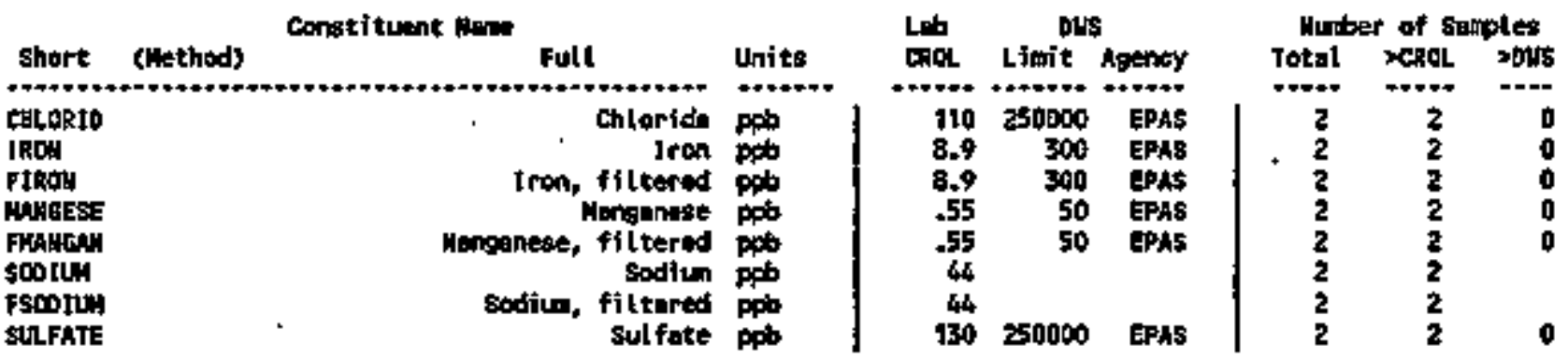

SJTE SPECIFIC ND OTHER CONSTITAENTS

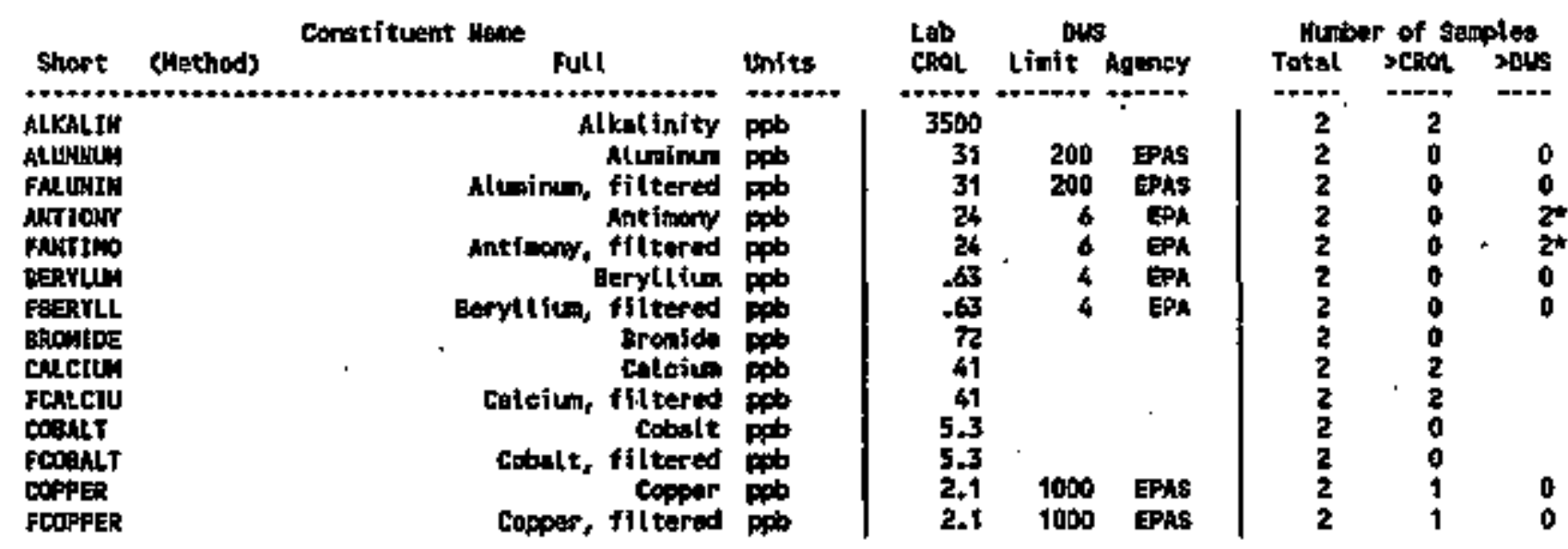


Table 8-3. Constttuent List and Summary of Results for the 216-A-10 Crib Data for Reporting Period July 1 through Septenber 30, 1995.

(sheet 2 of 2)

\begin{tabular}{|c|c|c|c|c|c|c|c|c|c|}
\hline & & Lonstipunt Hane & & tab & Dus & & nut & of $\mathrm{se}$ & ples \\
\hline short & (Hathod) & Full & Units & crent & Limit & Agency & Total & PCROt & $>0 \mathrm{WS}$ \\
\hline & & & & & & & & & - \\
\hline mains & & Negnestur & pob & 26 & & & 2 & 2 & \\
\hline Funtives & & Muanesium, filitured & ppb & 28 & & & & & \\
\hline NTCKat & & Miekal & pob & 11 & 100 & EPA & 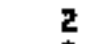 & 0 & 0 \\
\hline Fulciel & & wicket, filtared & Pptb & 11 & 100 & EPA & & 0 & 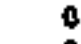 \\
\hline HITRITE & & Witrite & Ppts & 140 & 4000 & EPh & 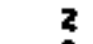 & 0 & 0 \\
\hline Pordisin & & Ploophate - & $\mathrm{ppb}$ & 340 & & & & 0 & \\
\hline FPOTAS\$ & & potaksing, filtered & $\begin{array}{l}\text { pop } \\
\text { pot }\end{array}$ & 390 & & & 2 & $z$ & \\
\hline SILIER & & stlvar & $p p b$ & 5.5 & 100 & EPAS & 2 & 0 & 0 \\
\hline PSILVER & & sitver, fittered & ppo & 5.5 & 100 & EPAS & 2 & 0 & 0 \\
\hline Ttk & & Tin & Ppo & 30 & & & 2 & 1 & \\
\hline ToS & & Fotal dissplived soltds & $p_{p p o}$ & & 500000 & EPA5 & 2 & 2 & 0 \\
\hline TURB ID & & $\begin{array}{l}\text { Turbidity } \\
\text { The }\end{array}$ & int & & & & 2 & 2 & \\
\hline VALADLM & & Vantilin & pph & 1.9 & & & 2 & 2 & \\
\hline PWALBDI & & Vanediun, filtared & $p$ & 1. & & & & & \\
\hline $\begin{array}{l}\text { zuLE } \\
\text { PzItic }\end{array}$ & & zine, filtered & $\begin{array}{l}\text { ppl } \\
p p b\end{array}$ & $\begin{array}{l}3.7 \\
3.7\end{array}$ & $\begin{array}{l}5000 \\
5000\end{array}$ & $\begin{array}{l}\text { Ephs } \\
\text { EPAS }\end{array}$ & 2 & $i$ & 0 \\
\hline
\end{tabular}

For explenation of this table, set seation $t .4$ of report. 
Table 8-4. Constituents with at Laast One Datected Value for the 216-A-10 Crib Data for Reporting Period Juty 1 through September 30, 1995.

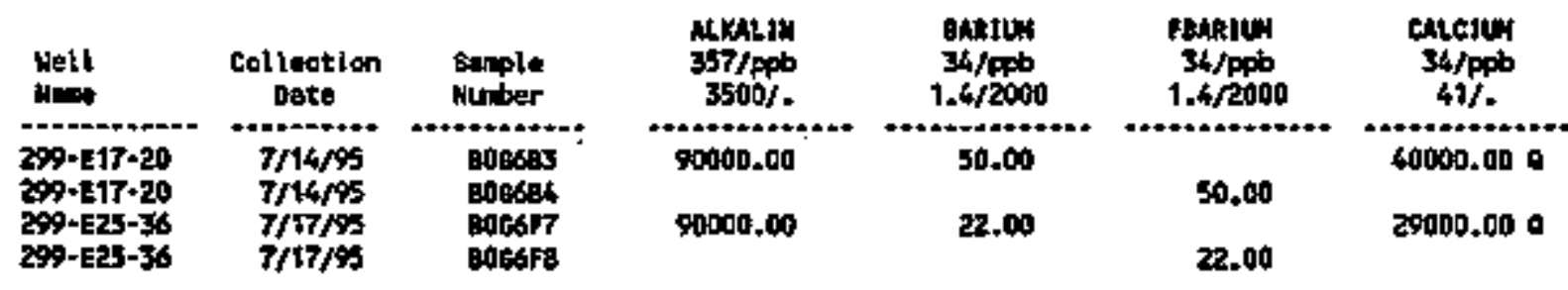

\begin{tabular}{|c|c|c|}
\hline $\begin{array}{l}\text { Nedl } \\
\text { Neses }\end{array}$ & $\begin{array}{c}\text { Collection } \\
\text { Dote }\end{array}$ & suple \\
\hline $\begin{array}{l}299-E 17-20 \\
299-E 17-20 \\
299-E 25+36 \\
299-E 25-36\end{array}$ & $\begin{array}{l}7 / 14 / 95 \\
7 / 14 / 95 \\
7 / 17 / 95 \\
7 / 17 / 45\end{array}$ & $\begin{array}{l}\text { Botses } \\
\text { E00684 } \\
\text { e0t56F7 } \\
\text { Bog6F8 }\end{array}$ \\
\hline
\end{tabular}

\begin{tabular}{|c|c|}
\hline $\begin{array}{c}\text { FChLCJU } \\
\text { 34/ppb } \\
41 / .\end{array}$ & $\begin{array}{c}\text { CKRORID } \\
124 / \mathrm{pph} \\
110 / 250000 \mathrm{x}\end{array}$ \\
\hline $40000.00 a$ & $\begin{array}{l}4400.00 \\
4100.00\end{array}$ \\
\hline
\end{tabular}

Chrowan
$34 / 90 \mathrm{~b}$
$3.7 / 100$
27.00
21.00

FCHFOHI
$34 / \mathrm{ppb}$
$3.7 / \mathrm{tab}$
$3.70 \mathrm{U}$
$6.20 \mathrm{~L}$

Well
Iane
$299-E 17-20$
$299-E 17-20$
$299-E 25-36$
$299-E 25-36$

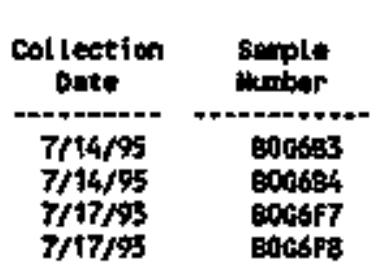

Coppes
$34 f p p b$
$2.1 / 10000$
$2.90 \mathrm{~V}$
$2.40 \mathrm{~L}$

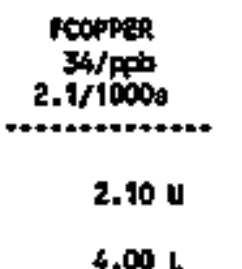

7Lokso
$124 / \mathrm{ppb}^{\mathrm{b}}$
$52 / 4000$
700.00
300.00

$\frac{1 k 0 \mathrm{~m}}{34 / \mathrm{ppb}}$
$0.9 / 300 \mathrm{~s}$
$130.00 \mathrm{~B}$
$110.00 \mathrm{~B}$

yatt

\begin{tabular}{|c|c|c|c|}
\hline $\begin{array}{c}\text { col loctien } \\
\text { Date }\end{array}$ & $\begin{array}{l}\text { seaple } \\
\text { htroer }\end{array}$ & $\begin{array}{l}\text { Ftkan } \\
\text { 34/pod } \\
\text { B.9/300s }\end{array}$ & $\begin{array}{c}\text { moliss } \\
34 / \text { pith } \\
26 / .\end{array}$ \\
\hline $\begin{array}{l}7 / 14 / 95 \\
7 / 44 / 95 \\
7 / 17 / 75 \\
7 / 17 / 95\end{array}$ & $\begin{array}{l}\text { Bocks3 } \\
\text { o0c686 } \\
\text { opest? } \\
\text { B00678 }\end{array}$ & $\begin{array}{l}25.00 \mathrm{~B} \\
41.00 \mathrm{~B}\end{array}$ & $\begin{array}{l}13000.00 \\
7900.00\end{array}$ \\
\hline
\end{tabular}

FHAGHES
$34 / \mathrm{ppb}$
267
130000.00
7700,00

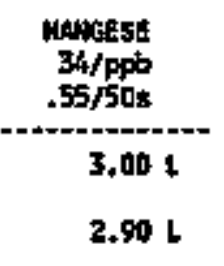

$299-17-20$

$209-617-20$

299-E25-36

299-E25-36

\begin{tabular}{|c|c|c|}
\hline Well & $\begin{array}{c}\text { col lection } \\
\text { bate }\end{array}$ & $\begin{array}{l}\text { 5enple } \\
\text { Inthar }\end{array}$ \\
\hline $\begin{array}{l}7-20 \\
7-20 \\
5-36\end{array}$ & $\begin{array}{l}7 / 14 / 95 \\
7 / 14 / 95 \\
7 / 17 / 95 \\
7 / 47 / 95\end{array}$ & 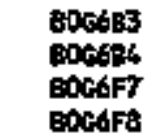 \\
\hline
\end{tabular}

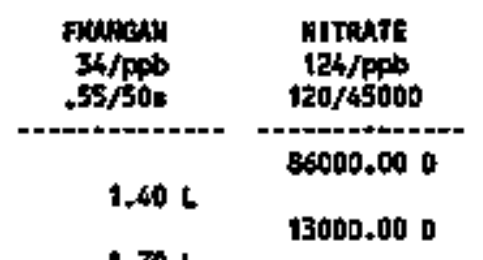

pothsin
$34 / p \times b$
$390 \%$
6200.00
4900.00

motass

36/pab $390 /$.

6100,00

$1.70 \mathrm{~L}$

5500.00 
Table 8-4. Constituents with at Least One Detected Value for the 216-A-10 Crib Data for Reporting Period July 1 through Septenber 30, 1995.

(sheet 2 of 2)

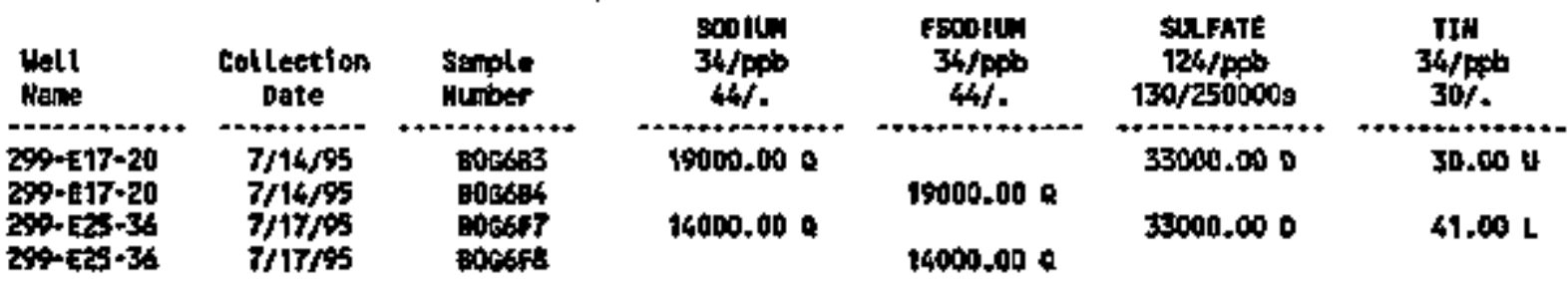

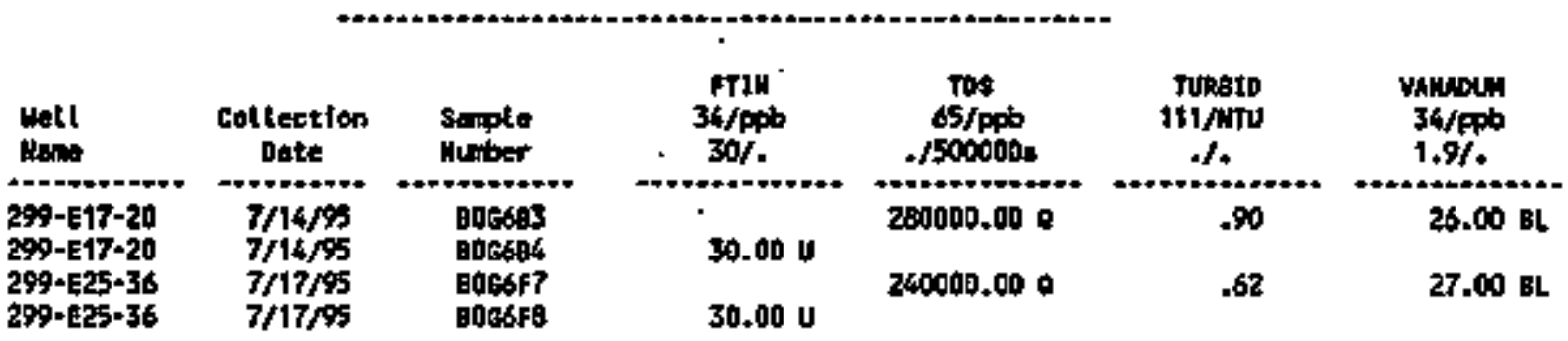

\begin{tabular}{|c|c|c|c|c|c|}
\hline $\begin{array}{l}\text { Wakl } \\
\text { Wense }\end{array}$ & $\begin{array}{c}\text { collestion } \\
\text { Dett. }\end{array}$ & $\begin{array}{l}\text { Semple } \\
\text { iturier }\end{array}$ & $\begin{array}{l}\text { Frownd } \\
34 / 2 \ldots b \\
1.9 / .\end{array}$ & $\begin{array}{c}21 \mathrm{kC} \\
34 / 40 \mathrm{~b} \\
3.7 / 5000 \mathrm{~s}\end{array}$ & $\begin{array}{c}\text { FZIAS } \\
34 / \mathrm{ppb} \\
3.7 / 5000 \mathrm{~s}\end{array}$ \\
\hline $\begin{array}{l}299-E 17-20 \\
299-E 17-20 \\
259-E 25-36 \\
299-E 24-36\end{array}$ & $\begin{array}{l}\text { 7/4/ss } \\
7 / 14 / 95 \\
7 / 17 / 45 \\
717 / 45\end{array}$ & $\begin{array}{l}\text { Bowsgs } \\
\text { Boese4 } \\
\text { Boesf7 } \\
\text { Buesfe }\end{array}$ & $\begin{array}{l}26.0081 \\
26.008\end{array}$ & $\begin{array}{l}5.60 \mathrm{640} \\
3.60 \mathrm{ce}\end{array}$ & $3.70 \mathrm{Lg}$ \\
\hline
\end{tabular}

For explanation of this teble, see stetion 1.4 of report. 
Table 8-5. Contamination Indicator Parameters for the 216-A-10 Crib Data for Reporting Period

July 1 through Septenber 30, 1995.

\begin{tabular}{|c|c|c|c|c|c|c|c|c|}
\hline $\begin{array}{l}\text { thet } \\
\text { hrome }\end{array}$ & $\begin{array}{c}\text { Collettion } \\
\text { Dote }\end{array}$ & swiper & 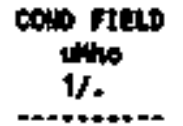 & $\begin{array}{c}\text { cold th } \\
\text { this } \\
1 / .\end{array}$ & $\begin{array}{l}\text { pH telo } \\
\text {.0t/6.5+8.5s }\end{array}$ & $\begin{array}{c}\text { pil Lis } \\
.01 / 6.5-8.50\end{array}$ & toc & $\begin{array}{l}70 x \\
\text { ppb } \\
1 .\end{array}$ \\
\hline $\begin{array}{l}299-117-20 \\
759-275-36\end{array}$ & $\begin{array}{l}7 / 14 / 95 \\
7 / 17 / 95\end{array}$ & $\begin{array}{l}\text { Bopda3 } \\
\text { gocse7 } \\
\text { Bogfis } \\
\text { Bosfit } \\
\text { Dosfin }\end{array}$ & $\begin{array}{l}408 \\
269 \\
283 \\
285 \\
289\end{array}$ & & $\begin{array}{l}8.40 \\
8.07 \\
8.07 \\
8.07 \\
8.07\end{array}$ & & & \\
\hline
\end{tabular}

\footnotetext{
- Dotection Ifints for TDC and TOX vary depending on the poffoming laboratory-
} For explention of this table, see section 1.4 of report. 
DOE/RL-95-69-3

\section{CONTEUTS}

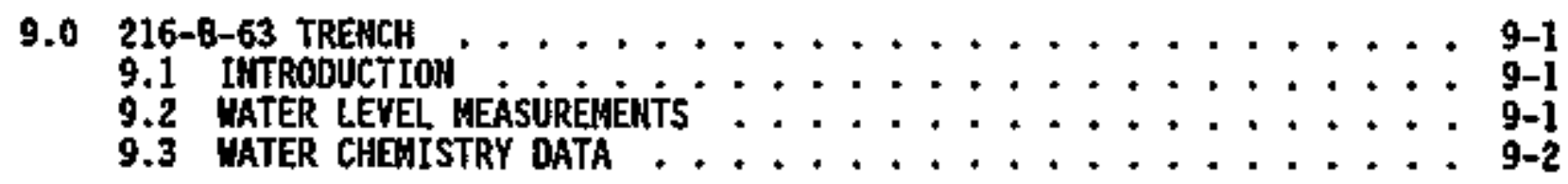

9-i 
DOE/RL-95-69-3

\section{LIST OF FIGURES}

9-1 Well Location Map for the 216-8-63 Trench. . . . . . . . . . 9-3

\section{LIST OF TABLES}

9-1 Monitoring Weit Purpose and Sampting Schedute for the 216-B-63 Trench Network ............. 9-4

9-2 RCRA Water Level Measurenent Report 216-8-63 Trench, Third Quarter 1995 ............. 9-5

9-3 Constituent List and Sumnary of Results for the 216-B-63 Trench Data for Reporting Perjod July 1 through Septenber 30 , I995 $\ldots \ldots$ 9-6

9-4 Constituents with at Least one Detected value for the 216-8-63 Trench Data for Reporting Period July 1 through Septeatber $30,1995 \ldots . \ldots$. . . . . . . . .

9-5 Contamination Indjcator Parameters for the 216-B-63 Trench Data. for Reporting Period July 1 through September $30,1995 \ldots \ldots$ 9-8 
DOE/RL-95-69-3

\title{
$9.0 \quad 216-8-63$ TREHCH
}

\author{
k. D. Sireeney \\ Wostinghouse Hanford Cowpany
}

\section{I Immonaction}

A RCRA interiu-status detection-level groundwater monitoring network has been established for the 216-B-63 Trench. The 216-B-63 Trench received wastewater contajning hazardous waste and radjoactive naterials from the B Plant located in the 200 East Area (Figures 1-1 and 9-1). Liquid effiuent discharge to the 216-8-63 Trench ceased in February 1992.

The 216-B-63 Trench was an open, unlined earthen trench that was in service from Warch 1970 until February 1992. The trench received $4.0 \times 10^{5}$ to $1.5 \times 10^{\circ} \mathrm{L} / \mathrm{day}(100,000$ to $400,000 \mathrm{gal} / \mathrm{day})$ of 1iquid effluent from the $B$ Plant chemical sewer, none of which is now designated as dangerous waste. The effluent was a mixture of stean condensate and raw water. Doctulented hazardous discharges occurred from 1970 to October 1985 and consisted of aqueous sulfuric acid and sodiun hydroxide solutions exceeding the ph 1 inits of 2.0 and 12.5, respectively. Radioactive soils were dredged from the trench in August 1970. In 1985, physical controls, radiation monitors, and operating procedures were modified to avoid inadvertent discharge of chemicals or radjoactive substances to the wastewater stream. The trench has since been backfilled and stablized and the diversion box leading to it has been fllled with concrete.

Four quarters of groundwater background sampling were completed for all the wells in the network in April 1993. Background sampling is done to establish background water quality, in accordance with the requirenents outlined in 40 CFR 265 Interin-Status Indicator-Evaluation Groundwater Honitoring Plan for the 216-8-63 Trench (PNL 1989). The 12 wells in the network are now sampled semiannualiy. Based on the present groundwater flow direction, seven of the we]ls are downgradient and five are upgradient of the trench (see Figure 9-1). The purpese of the wells, their sampiling schedule, and the dates sampled are sumarized in Tab7e 9-1. As indicated in Table 9-1, some wells in the 216-B-63 Trench network are shared with the low-level burial grounds (LLBG) and single-she11 tanks (SST) groundwater monitoring networks.

\subsection{MATER LEVEL MEASUREMETS}

Water level measurements obtained during the July 1 through Septenber 30 , 1995, period are presented in Table 9-2. These measurements are obtained during sampling and as part of a sentiannual water table evaluation. Water measurements in well 299-E33-36 indjcate water levels lower than in nearby velis in the network. Reasons for the apparent discrepancy are still under investigation. 


\subsection{WATER CHEHISTRY DATA}

Chemistry data received before the cutoff date for this report are reported in Tables 9-3 through 9-5. The 216-B-63 Trench is sampled semiannually and was not scheduled for sampling this quarter. Sample results in the tables include both data from previous samping periods and data from wells sampled for 5ST B-BX-BY. An explanation of data flags can be found in section 1.4 . 


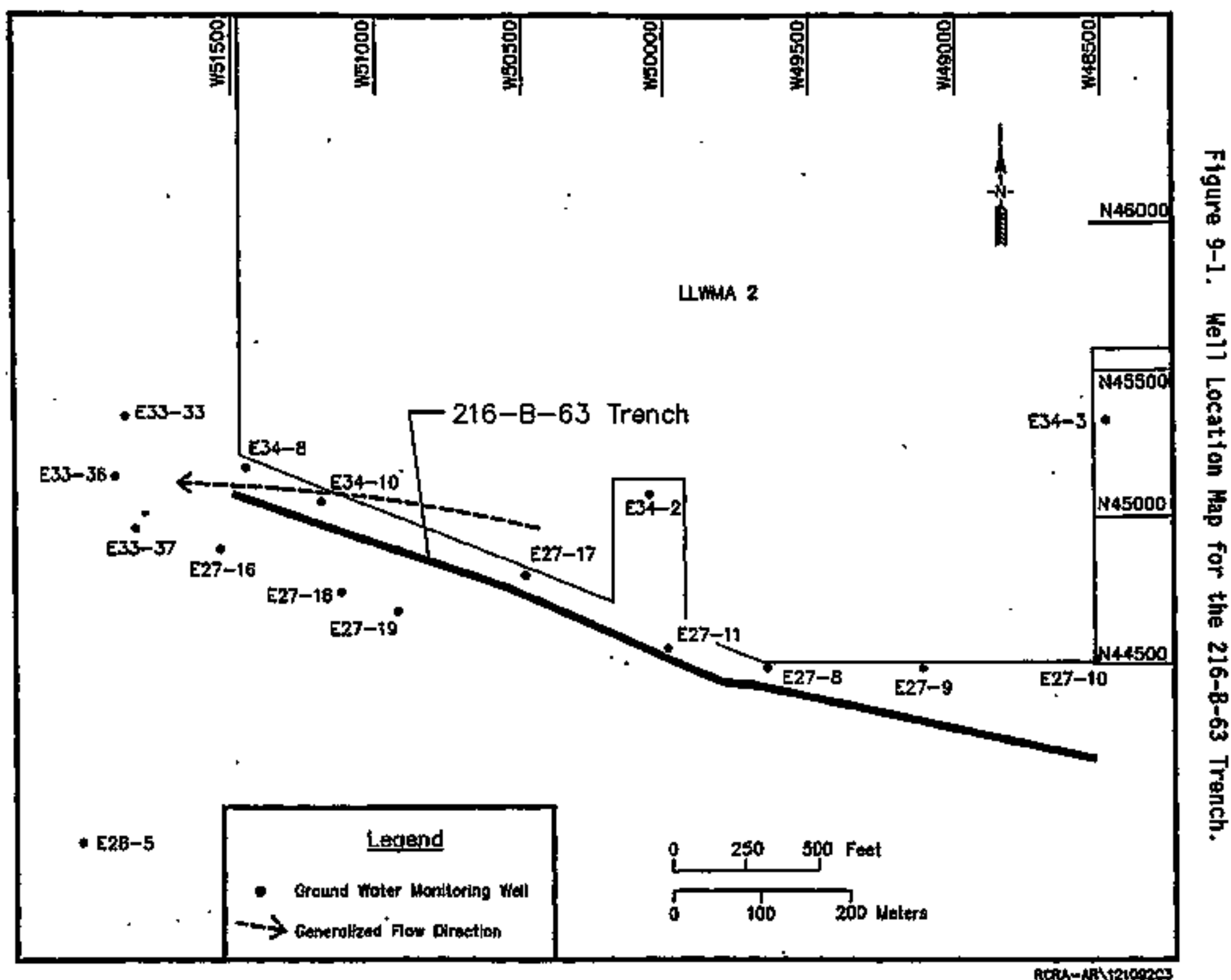


Table 9-1. Monitoring Well Purpose and Sampling Schedute for the 216-8-63 Trench Hetwork.

\begin{tabular}{|c|c|c|c|c|}
\hline $\begin{array}{c}\text { Hell } \\
\text { no. } \\
\text { (299-) }\end{array}$ & $\begin{array}{l}\text { Relative } \\
\text { position }\end{array}$ & Hydrogeologic unit & $\begin{array}{c}\text { Sample } \\
\text { frequency }\end{array}$ & $\begin{array}{c}\text { Sample } \\
\text { date, 3rd } \\
\text { Qtr } 1995\end{array}$ \\
\hline$E 27-8^{\circ}$ & Upgradient & Hanford: Hater Tab]e & Semiannuatiy & $\begin{array}{c}\text { Not } \\
\text { Sampled }\end{array}$ \\
\hline$E 27-9^{6}$ & Upgradient & Hanford: Water Table & Sentannually & $\begin{array}{c}\text { Not } \\
\text { Sampled }\end{array}$ \\
\hline E27-11" & Upgradient & Hanford: Water Table & Semlannually & $\begin{array}{c}\text { Not } \\
\text { Sampled }\end{array}$ \\
\hline E27-16 & Downgradient & Hanford: Water Table & Semiannuatiy & $\underset{\text { Nampled }}{\text { Nampled }}$ \\
\hline$E 27-17^{\circ}$ & Upgradient & Hanford: Water Table & Semtannually & $\begin{array}{c}\text { Not } \\
\text { Sampled }\end{array}$ \\
\hline E27-18 & Downgradient & Hanford: Water Table & Semiannuatiy & $\begin{array}{c}\text { Not } \\
\text { Sampled }\end{array}$ \\
\hline$E 27-19$ & Downgradient & Hanford: Water Table & Semiannually & $\begin{array}{c}\text { Not } \\
\text { Sampled }\end{array}$ \\
\hline$E 33-33^{\circ}$ & Downgradient & Hanford: Water Table & Semtannually & $08 / 01 / 95$ \\
\hline$E 33-36^{b}$ & Downgradient & Hanford: Water Table & Semiannually & $08 / 01 / 95$ \\
\hline E33-37 & Downgradient & Hanford: Water Table & Semiannually & $\begin{array}{c}\text { Not } \\
\text { Sampled }\end{array}$ \\
\hline E34-8 & Downgradient & Hanford: Water Table & Semfannually & $\begin{array}{c}\text { Not } \\
\text { Sampled }\end{array}$ \\
\hline$E 34-10^{\circ}$ & Upgradient & Hanford: Water Table & Semiannually & $\begin{array}{c}\text { Not } \\
\text { Sampled }\end{array}$ \\
\hline
\end{tabular}

-WeJ] shared with the Low-Level Burial Grounds facility network.

Well shared with the single-sheil tanks facility network. 
Table 9-2. RCRA Water Leve1 Heasurement Report 216-B-63 Trench, Third Quarter 1995.

\begin{tabular}{|c|c|c|c|c|}
\hline Mell & Date & $\begin{array}{l}\text { Depth to } \\
\text { water (ft) }\end{array}$ & $\begin{array}{l}\text { Water } 1 \\
\text { elevation } \\
\text { (ft) }\end{array}$ & $\begin{array}{l}\text { level } \\
\text { above ms1 } \\
\text { (\$) }\end{array}$ \\
\hline \multicolumn{5}{|c|}{ Wells Monitoring the Top of the Unconfined Aquifer } \\
\hline 299-E27-11 & $9 / 20 / 95$ & 242.22 & 401.07 & 122.25 \\
\hline 299-E27-16 & $9 / 08 / 95$ & 251.88 & 400.25 & 122.00 \\
\hline 299-E27-17 & $9 / 20 / 95$ & 233.36 & 401.36 & 122.33 \\
\hline $299-E 27-18$ & $9 / 08 / 95$ & 248.79 & 401.36 & 122.33 \\
\hline 299-E27-19 & $9 / 08 / 95$ & 249.58 & 401.30 & 122.32 \\
\hline 299-E27-8 & $9 / 20 / 95$ & 236.64 & 401.19 & 122.28 \\
\hline 299-E27-9 & $9 / 20 / 95$ & 227.95 & 401.26 & 122.30 \\
\hline 299-E,33-33 & $\begin{array}{l}7 / 25 / 95 \\
8 / 01 / 95 \\
8 / 16 / 95 \\
9 / 13 / 95\end{array}$ & $\begin{array}{l}238.92 \\
238.69 * \\
238.94 \\
238.94\end{array}$ & $\begin{array}{l}401.47 \\
401.70 \\
401.45 \\
401.45\end{array}$ & $\begin{array}{l}122.37 \\
122.44 \\
122.36 \\
122.36\end{array}$ \\
\hline $299-E 33-36$ & $\begin{array}{l}8 / 01 / 95 \\
9 / 08 / 95\end{array}$ & $\begin{array}{l}246.96 * \\
247.72\end{array}$ & $\begin{array}{l}399.71 \\
398.95\end{array}$ & $\begin{array}{l}121.83 \\
121.60\end{array}$ \\
\hline 299-E33-37 & $9 / 08 / 95$ & 251.77 & 401.24 & 122.30 \\
\hline 299-E34-10 & $9 / 20 / 95$ & 238.37 & 401.40 & 122.35 \\
\hline $299-\mathrm{E} 34-8$ & $9 / 08 / 95$ & 241.37 & 399.15 & 121.66 \\
\hline
\end{tabular}

NOTES: 1. Water level elevations are calculated by subtracting the measured depth-to-water from the surveyed elevation for the well.

2. Depth-to-water values are transeribed from field records.

3. Measurements marked with an ${ }^{1 *}$ ' were taken at the time of sampling.

4. Measurements 'narked with a ' + ' are outsjde of the expected -range, and are suspected of error. 
Table 9-3. Constituent List and Summary of Results for the 216-B-63 Trench Data for Reporting Period July 1 through Septewber 30, 1995.

CORTNMIMATJON JEDICKTAR PARANETLRS

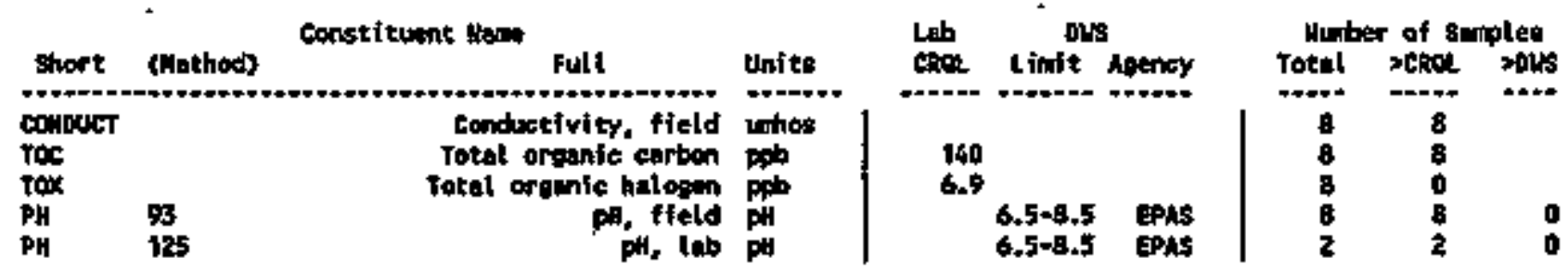

DRINGHO WhER PARHeTERS

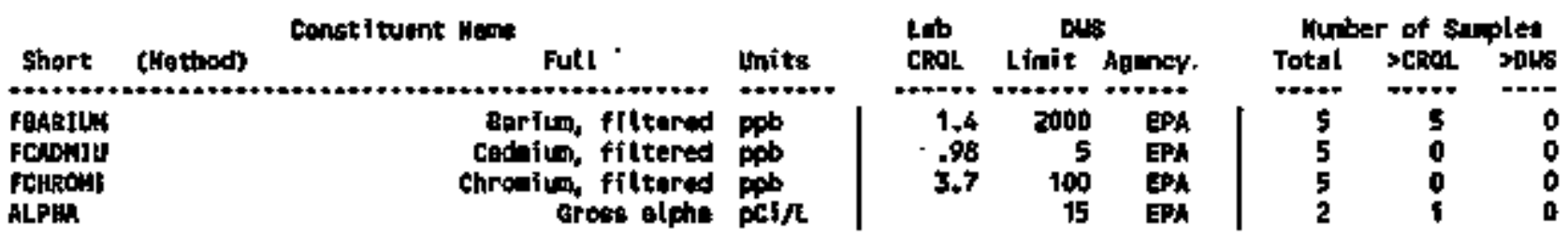

GROUDURTER GUALITY PARNETERS

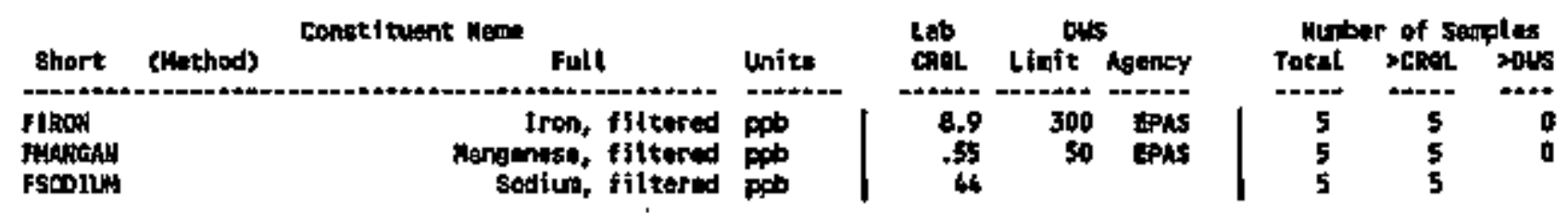

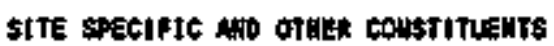

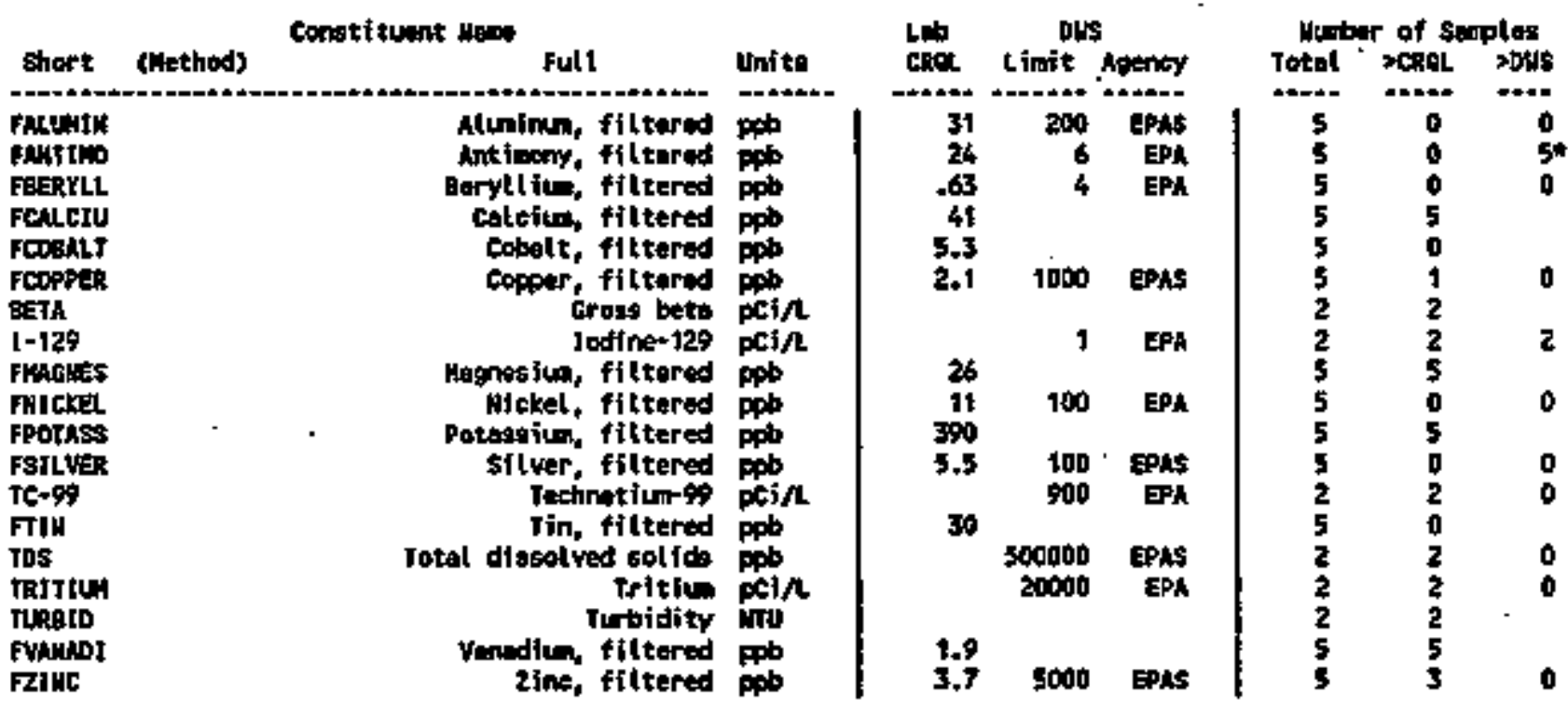

For explanation of this tabie, sie siction 1,4 of raport. 
Table 9-4. Constituents with at Least One Detected Value for the 216-B-63 Trench Data for Reporting Period

- July 1 through September 30, 1995.

Nell
$299-E 27-11$
$299-E 27-17$
$299-E 27-8$
$299-E 27-9$
$269-E 33-33$
$299-[13-36$
$299-E 36-10$

\begin{tabular}{|c|c|}
\hline $\begin{array}{c}\text { Col lection } \\
\text { Date }\end{array}$ & $\begin{array}{l}\text { serple } \\
\text { Nupber }\end{array}$ \\
\hline $\begin{array}{l}5 / 03 / 95 \\
5 / 03 / 95 \\
5 / 04 / 95 \\
5 / 63 / 95 \\
8 / 04 / 95 \\
\text { 1/0t/9s } \\
5 / 03 / 95\end{array}$ & 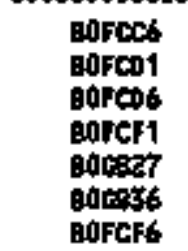 \\
\hline
\end{tabular}
(sheet 1 of 2)

Fanitu
$34 / \mathrm{ppb}$
$1.4 / 2000$
36.00
32.00
2.00
22.00
35.00

FCNLCIU
$36 /$ ppb
$61 /$.
35000.00
39000.000
34000.00
35000.00

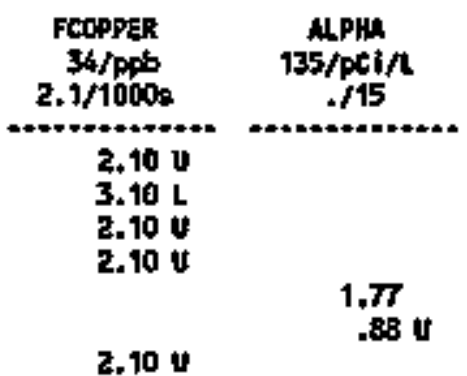

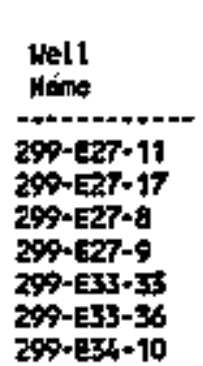

\begin{tabular}{|c|c|}
\hline $\begin{array}{c}\text { Col lection } \\
\text { bute }\end{array}$ & suple \\
\hline $\begin{array}{l}5 / 03 / 95 \\
5 / 03 / 95 \\
5 / 04 / 95 \\
5 / 03 / 45 \\
8 / 01 / 95 \\
5 / 01 / 95 \\
5 / 05 / 95\end{array}$ & 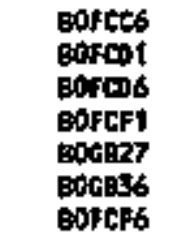 \\
\hline
\end{tabular}
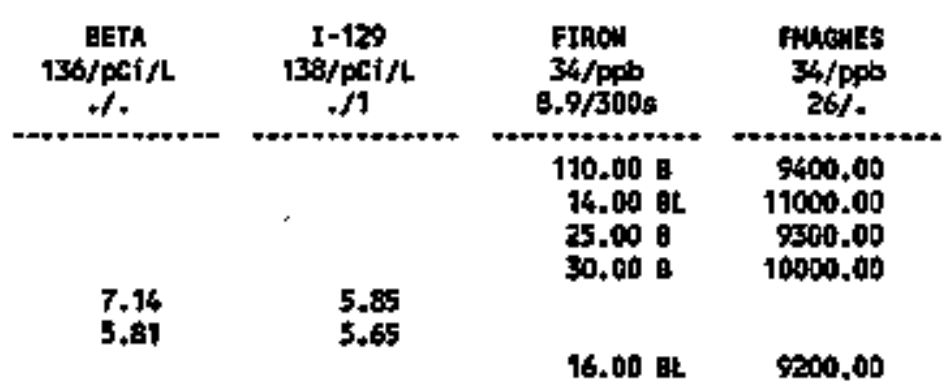

well
Heme
$299-627-11$
$297-627-17$
$299-627-8$
$299-627-9$
$297-633-33$
$297-E 33-36$
$297-634-10$

\begin{tabular}{|c|c|}
\hline $\begin{array}{c}\text { Col lect ion } \\
\text { Date }\end{array}$ & $\begin{array}{l}\text { Sprpla } \\
\text { Phrber }\end{array}$ \\
\hline $\begin{array}{l}5 / 03 / 95 \\
5 / 05 / 95 \\
5 / 04 / 95 \\
5 / 03 / 95 \\
8 / 01 / 95 \\
8 / 0 t / 95 \\
5 / 03 / 95\end{array}$ & 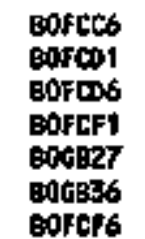 \\
\hline
\end{tabular}

\begin{tabular}{|c|c|}
\hline 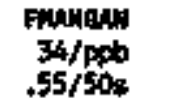 & $\begin{array}{l}\text { Feriss } \\
34 / \mathrm{ppb} \\
390 / .\end{array}$ \\
\hline $\begin{array}{l}2.30 \mathrm{BL} \\
1.70 \mathrm{BL} \\
1.80 \mathrm{EL} \\
1.00 \mathrm{BL}\end{array}$ & $\begin{array}{r}6000.00 \\
6700.00 \\
6400.00 \\
.5900 .00\end{array}$ \\
\hline & " \\
\hline 3.20 日L & 6200.00 \\
\hline
\end{tabular}

\begin{tabular}{|c|c|}
\hline $\begin{array}{c}\text { F80010 } \\
\text { 34/pips } \\
44 / .\end{array}$ & $\begin{array}{c}\text { Te-7t } \\
143 / \mathrm{pct} / \mathrm{t} \\
-/ 900\end{array}$ \\
\hline $\begin{array}{l}13000.00 \mathrm{~B} \\
16000.00 \mathrm{~B} \\
13000.00 \mathrm{~B} \\
+2000.09 \mathrm{~B}\end{array}$ & \\
\hline $14000.00 \mathrm{~B}$ & $\begin{array}{l}3,01 \\
4,32\end{array}$ \\
\hline
\end{tabular}

\begin{tabular}{|c|c|c|c|c|c|c|}
\hline $\begin{array}{l}\text { Noll } \\
\text { Hane }\end{array}$ & $\begin{array}{l}\text { Collection } \\
\text { Dute }\end{array}$ & seaple & $\begin{array}{c}\text { TDS } \\
65 / p \infty b \\
-A_{0000}\end{array}$ & $\begin{array}{c}\text { IRITIUH } \\
\text { 142/pct/R } \\
. / 20000\end{array}$ & $\begin{array}{c}\text { TUR日ELD } \\
111 / \pi \pi U \\
. / .\end{array}$ & $\begin{array}{c}\text { FVMUNDI } \\
34 / \mathrm{peb} \\
1,9 \% .\end{array}$ \\
\hline $\begin{array}{l}299-E 27-11 \\
299-E 27-17 \\
299-E 27-8 \\
299-E 27-9 \\
209-E 33-33 \\
299-E 33-36 \\
299-E 34-10\end{array}$ & $\begin{array}{l}5 / 03 / 95 \\
5 / 03 / 95 \\
5 / 04 / 95 \\
5 / 03 / 95 \\
8 / 0 t / 95 \\
8 / 01 / 95 \\
5 / 03 / 95\end{array}$ & 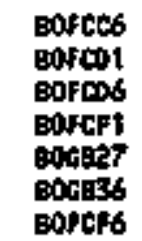 & $\begin{array}{l}150000.00 \\
170000.00\end{array}$ & $\begin{array}{l}3370.00 \\
\mathbf{2 0 5 0 . 0 0}\end{array}$ & $\begin{array}{r}.62 \\
9.32\end{array}$ & $\begin{array}{l}\mathbf{2} .00 \mathrm{~L} \\
21.00 \mathrm{~L} \\
24.001 \\
27.00 \mathrm{~L}\end{array}$ \\
\hline
\end{tabular}


Table 9-4. Const Ituents with at Least One Detected Value for the 216-8-63 Trench Data for Reporting Period July 1 through September 30, 1995.

\begin{tabular}{|c|c|c|c|}
\hline $\begin{array}{l}\text { Nell } \\
\text { Warke }\end{array}$ & $\begin{array}{l}\text { Collection } \\
\text { Date }\end{array}$ & sonple & $\begin{array}{c}\text { f2twe } \\
34 / 9 \mathrm{pb} \\
3.7 / 5000 \mathrm{~s}\end{array}$ \\
\hline $\begin{array}{l}279-E 27-11 \\
299-E 27-17 \\
299-E 27-8 \\
279-E 27-9 \\
299-E 4-10\end{array}$ & $\begin{array}{l}5 / 03 / 45 \\
5 / 03 / 95 \\
5 / 04 / 55 \\
5 / 03 / 95 \\
5 / 05 / 45\end{array}$ & 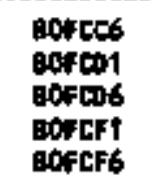 & $\begin{array}{l}6.80 \mathrm{~L} \\
6.00 \mathrm{~L} \\
9.70 \mathrm{~L} \\
3.70 \mathrm{U} \\
3.70 \mathrm{U}\end{array}$ \\
\hline
\end{tabular}

For axplonstion of this tablit, 904 section 1.4 af report.

Table 9-5. Contamfnation Indicator Parameters for the 216-B-63 Trench Data for Reporting Period July 1 through September 30, 1995.

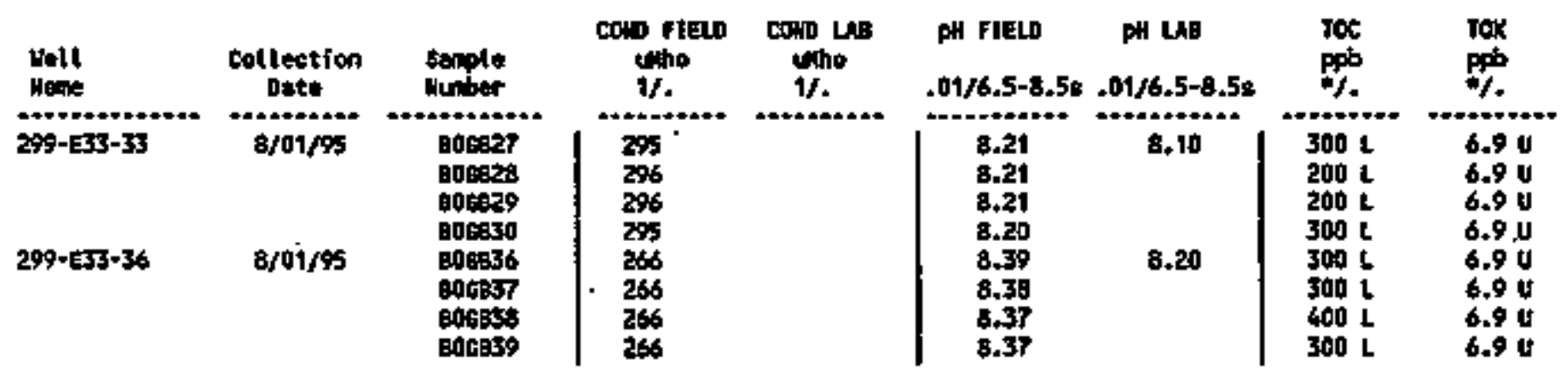

\footnotetext{
Detection liate for TOC and TOX vary depending on the performing laberotory.
} for explanetion of this teble, see section 1.4 of report. 
DOE/RL-95-69-3

COHTEHTS

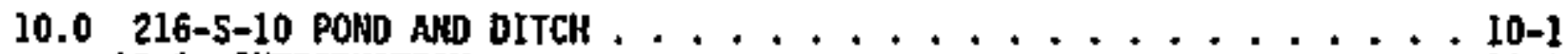

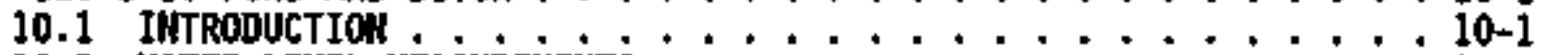

10.2 MATER LEYEL HEASUREMEHTS . . . . . . . . . . 10-2

10.3 MATER CHEHISTRY DATA . . ................ 
DOE/RL-95-69-3

\section{LIST OF FIEURES}

10-1 Weit Location Map for the 216-S-10 Faci7ity . . . . . . . . 10-3

\section{LIST OF TABLES}

10-1 Monitoring Mell Purpose. and Sampling Schedule for the 216-5-10 Facility . . . . . . . . . . . . 10-4

10-2 RCRA Water Level Measurement Report 216-5-10 Facility, Third Quarter 1995 ........... 10-5

10-3 Const ttuent List and Summary of Results for the 216-5-10 Facility Data for Reporting Period Ju7y 1 through September 30, $1995 \ldots \ldots$ 10-6

10-4. Contamination Indicator Parameters for the 216-5-10 Facility Data for Reporting Period July 1 through September 30, 1995 
DOE/RL-95-69-3

\title{
10.0 216-5-10 POHD WiD BITCH
}

\author{
J. H. Lindberg \\ West inghouse Henford coupany
}

\subsection{ISTRODUCTIOA}

The 216-S-10 Pond and Ditch (S-10 Facility) is located south-southwest of the $\mathbf{2 0 0}$ West Area, directly outside the perineter fence. The S-10 Facility recelved wastewater from the Reduction-0xidation (REDOX) P7 ant (202-S Building) located in the 200 West Area. In the past, wastevater disposed of at the S-10 Facility has contajned hazardous waste and radioactive materials. Since 1985, physical controls and operating procedures have been nodified to avold inadvertent discharge of hazardous and radioactive substances to the wastewater stream. The facility has been monitored under Interim status since August 1991. In October 1991, the efficent waste stream, including the water source, to the 216-S-10 Ditch was deactivated. Because the S-10 Factlity is not expected to receive additional hazardous and radjoactive substances, the factlity will be closed in accordance with WAC 173-303-610 (final status). The closure/post-closure plan is due in 2003.

The S-10 Facility initially comprised an open, unlined ditch $1.8 \mathrm{~m}$ (6 ft) deep, $1.2 \mathrm{~m}(4 \mathrm{ft})$ wide at the bottom, and $686 \mathrm{~m}(2,250 \mathrm{ft})$ long. In addition, an open, unlined percolation pond, approximately 2 hectares (5 acres) in area, was constructed at the southwest end of the ditch, and was active during part of the tine the ditch was receiving waste.

The 216-\$-10 01tch began receiving wastewater from the REOOX Plant in August 1951, and the 216-S-10 Pond was dug and placed in service in February 1954. In October 1985, the 216-\$-10 Pond and portions of the 216-S-10 Ditch were deconmissioned, backfilled, and stabilized. The rentaining portion of the 216-S-10 Ditch (approximately $594 \mathrm{n}[1,950 \mathrm{ft}]$ ) received nonhazardous, nonregulated waste fron the 202-S Building chemical sewer until deactivation in october, 1991 . This waste strean comprised cooling water, steap condensate, wateristower overflow, and drain effluent. These source streans had been routed so that they would not. cone into contact with hazardous materials.

Releases of hazardous materials and constituents to the S-10 Factlity are poorly documented. Radjoactive waste was reportedly disposed of to the S-10 Facility as a result of contaminated floor and sewer drains at the REDOX Plant. Chemical releases were documented in 1954 and 1983, and known constf tuents are $\mathrm{Al}_{(}\left(\mathrm{MO}_{3}\right)_{3}, \mathrm{NaNO}_{3}, \mathrm{NaOH}, \mathrm{Na}_{3} \mathrm{PO}_{4}, \mathrm{NaF}$, $\mathrm{MaCl}$, and $\mathbf{K}_{2} \mathrm{Cr}_{2} \mathrm{O}_{7}$.

During 1991 the anntal volume of effluent discharged to the 216-S-10 Ditch was between $1.9 \times 10^{8}$ and $5.7 \times 10^{8} \mathrm{~L}\left(5.0 \times 10^{7}\right.$ and $\left.1.5 \times 10^{\circ} \mathrm{gal}\right)$. Annual discharges of these volumes created a localized recharge mound and an associated perched water table directly below the recelving (north) end of the ditch. This recharge mound has since dissipated, and the perched water zone has been dry since shortly after the ditch was deactivated. 
Groundwater beneath the S-10 Facility is sampled semiannually in accordance with the Interia-status Groundwater Monitoring Plan for the 216-5-10 Pond and Ditch (WHC 1990a). The site is currently in a detectionlevel indicator parameters evaluation progran.

The monitoring network currently consists of two upgradient wells, three dormgradient wells, and one dongradient we11 that monitors the base of the unconfined aquifer (Figure 10-1). Table 10-1 1ists the wells and their samp ing status. The first four quarters of groundwater sampling and analysis in the detection-level progran were completed in 1992. The results of the background statistical calculations for the upgradient monitoring wells (based on the first four quarters of analytical data) were presented in the Annual Report for RCRA Groundwater Monitoring Projects at Hanford Site Facllities for 1993 (DOE-RL 1994).

\subsection{MATER LEVEL MEASUREMETS}

Water levels were measured at five S-10 Facility wells during the third quarter. They included wells 299-W26-7, 299-426-8, 299-W26-9, 299-w26-10, and 299-W26-12 (Table 10-2). The sixth wel1 (299-W27-2) was not measured during the third quarter because it is measure only at the time of sarpling. Like the other wells in the well network, it is on a semiannual sampling schedule and was not sampled during the third quarter (see Section 10.3).

\subsection{MATER CHEHISTRY DATh}

Because the sampling schedule for all six S-10 facility wells is seniannual, groundwater samples were not collected during the third quarter of 1995. These wells will be sampled again in the fourth quarter. Tables 10-3 and 10-4 are previously unreported data. 


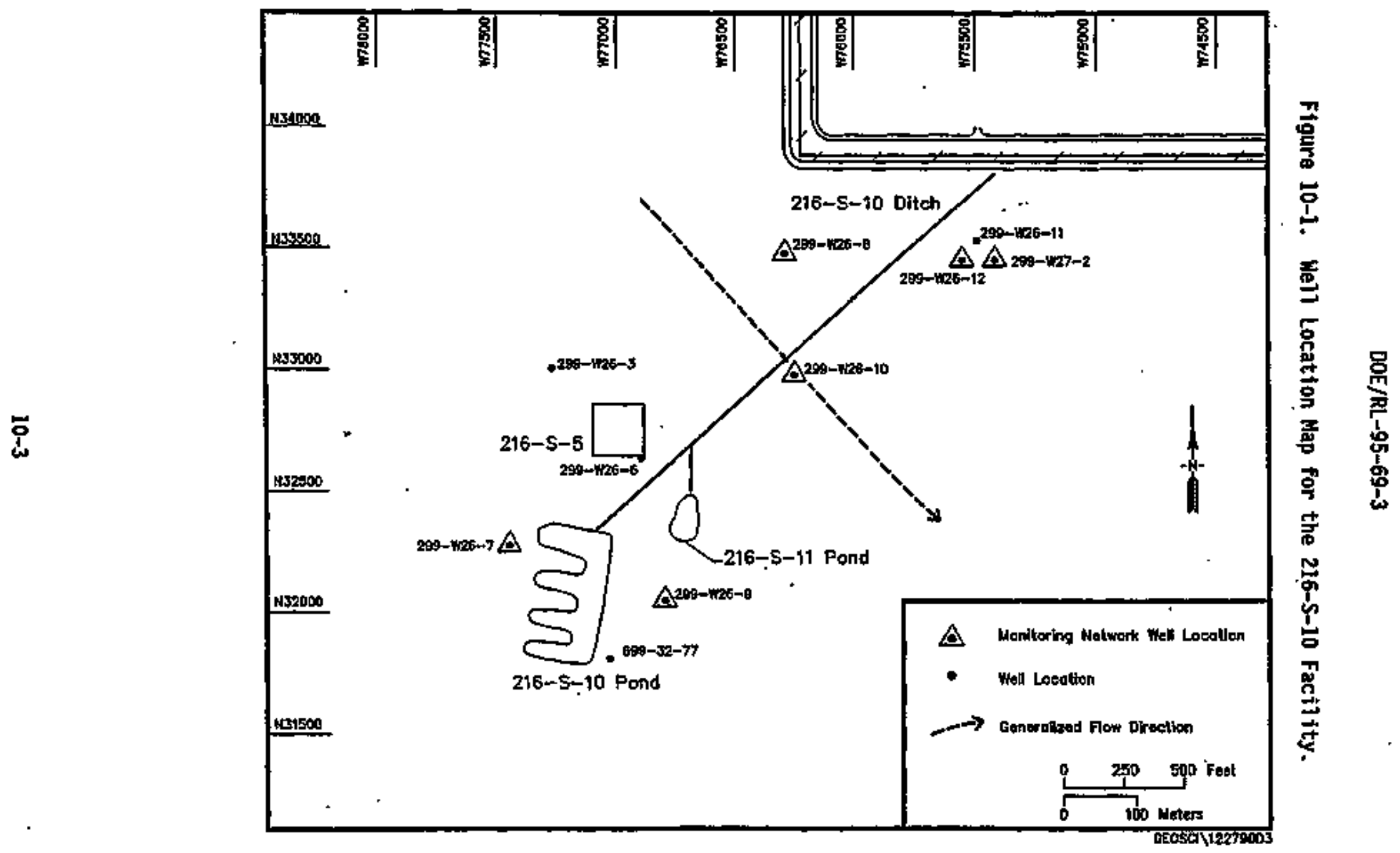


Table 10-1. Monftoring Well Purpose and Sampling Schedule for the 216-\$-10 Facility.

\begin{tabular}{|c|c|c|c|c|}
\hline $\begin{array}{l}\text { nell } \\
\text { no. } \\
(299-)\end{array}$ & $\begin{array}{l}\text { Relative } \\
\text { position }\end{array}$ & Hydrogeologic unit & $\begin{array}{l}\text { Sampling } \\
\text { frequency }\end{array}$ & $\begin{array}{l}\text { Last } \\
\text { sampling } \\
\text { date }\end{array}$ \\
\hline W26-7 & Upgradient & Ringold: Hater Table & Semtannually & $06 / 13 / 95$ \\
\hline W26-8 & Upgradient & Ringold: Water Table & Semiannually & $06 / 14 / 95$ \\
\hline 426-9 & Downgradient & Ringold: Water Table & Semiannually & $06 / 13 / 95$ \\
\hline W26-10 & Dewngradient & Ringold: Nater Table & Semiannually & $06 / 10 / 95$ \\
\hline W26-12 & Domgradient & Ringold: Water Table & Semfannually & $06 / 14 / 95$ \\
\hline W27-2 & Downgradient & $\begin{array}{l}\text { Ringold: Base of } \\
\text { Unconfined Aquifer }\end{array}$ & Semiannually & $/ 95$ \\
\hline
\end{tabular}


Tab]e 10-2. RCRA Water Level Heasurement Repart 216-5-10 Faciltty, Third Quarter 1995.

We11 Date $\quad \begin{gathered}\text { Depth to } \\ \text { water (ft) }\end{gathered} \quad \begin{gathered}\begin{array}{c}\text { Water leve] } \\ \text { elevation above ms } \\ \text { (ft) }\end{array} \\ \text { (m) }\end{gathered}$

Mells Honitoring the Top of the Unconfined Aquifer

$\begin{array}{lllll}299-W 26-10 & 9 / 08 / 95 & 216.85 & 454.02 & 138.39 \\ 299-W 26-12 & 9 / 08 / 95 & 221.39 & 454.30 & 138.47 \\ 299-W 26-7 & 9 / 08 / 95 & 195.98 & 456.01 & 138.99 \\ 299-W 26-8 & 9 / 08 / 95 & 211.63 & 454.94 & 138.67 \\ 299-\text { W26-9 } & 9 / 08 / 95 & 199.70 & 454.72 & 138.60\end{array}$

MOTES: 1. Water level elevations are calculated by subtracting the measured depth-to-water from the surveyed elevation for the well.

2. Depth-to-water values are transcribed from field records.

3. Measurements marked with an $1 * 1$ were taken at the time of sampling.

4. Heasurements marked with a ' + ' are outside of the expected range, and are suspected of error. 
Tabie 10-3. Constituent List and Summary of Results for the 2I6-S-10 Facility Data for Reporting Period July 1 through September 30, 1995.

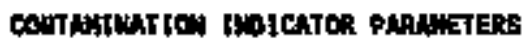

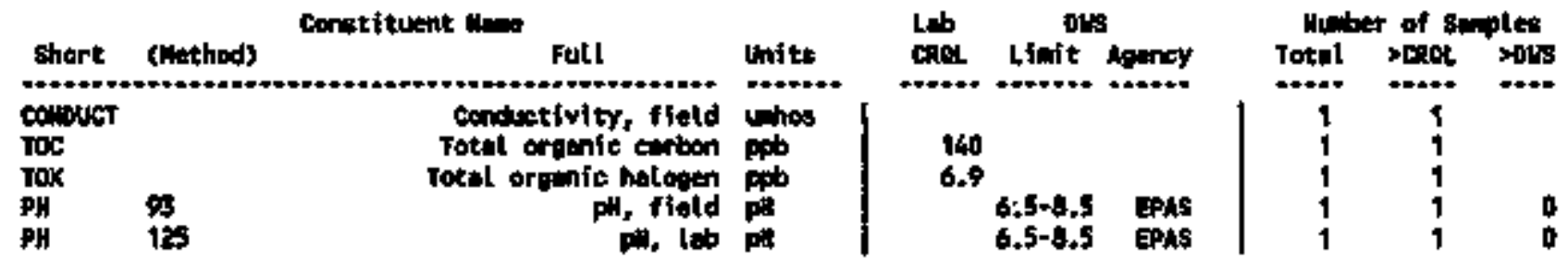

For explenstion of this tebile, see section 1.4 of repart. 
Table 10-4. Contamination Indicator Parameters for the 216-S-10 Facility. Data for Reporting Period July 1 through September 30, 1995.

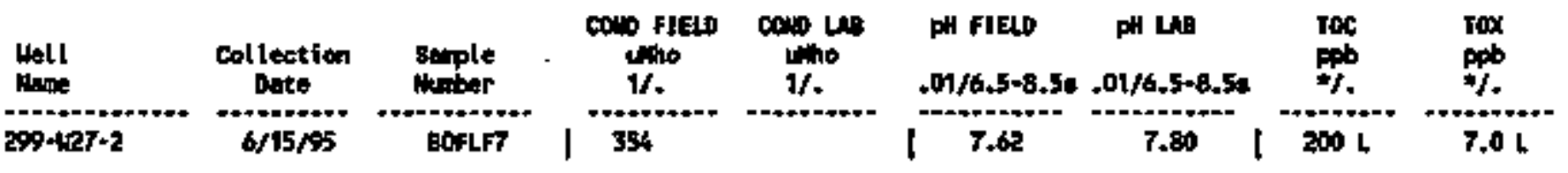

\footnotetext{
Detaction IIfits for toc and Teit vary depending on the perforing leboratory.
} for explaretion of thita table, see section 1.4 of report. 
DOE/RL-95-69-3

This page intentionally left blank.

$10-8$ 


\section{DOE/RL-95-69-3}

\section{CONTENTS}

11.0 216-U-12 CRIB . . . . . . . . . . . . . . . 11-1

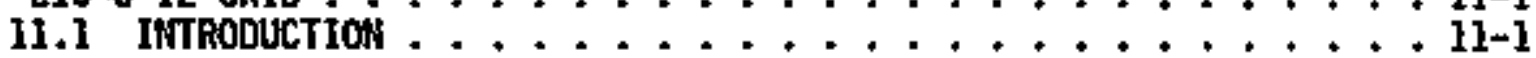

11.2 WATER LEVEL MEASUREMENTS ............... II-2

11.3 WATER CHEMISTRY DATA ...................... 
DOE/RL-95-69-3

\section{LIST OF FIGURES}

11-1 Well Location Map for 216-U-12 Crib . . . . . . . . . . . 11-3

\section{LIST OF TABLES}

11-1 Monftoring Well Purpose and Sampling Schedule for the

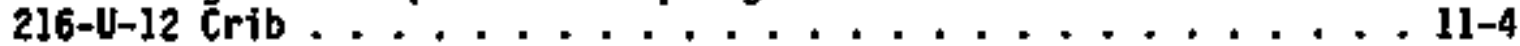

11-2 RCRA Water Leve1 Measurement Report 216-U-12 Crib, Third Quarter 1995 . . . . . . . . . 11-5

11-3 Constituent List and Summary of Resuits for the 216-U-12 Crib Data for Reporting Period July 1 through September $30,1995 \ldots \ldots$ 11-6

11-4 Constituents with at Least one Detected vaive for the 216-0-12 Crib Data for Reporting Period Juty 1 through Septenber 30 , 1995 ... . . . . . . . . 11-9

11-5 Contamination Indicator Parameters for the 216-U-12 Crib Data for Reporting Period July 1 through September $30,1995 \ldots \ldots$. . . . . . . . 11-12 
COE /RL-95-69-3

\title{
11.0 216-U-12 CRIB
}

\author{
B. A. Willians \\ Westinghouse Hanford Company
}

\subsection{INTRODHCTION}

The 216-U-12 Crib (U-12 Crib) is located approximately $609 \mathrm{fm}(1,998 \mathrm{ft}$ ) south of the $U$ Plant in the 200 West Area (see Figures 1-1 and 11-1). The crib is an unlined, gravel-bottow, percolation crib that is $3 \mathrm{~m}$ by $30.5 \mathrm{~m}$ (10 ft by $100 \mathrm{ft}$ ) and $4 \mathrm{E}(13 \mathrm{ft}$ ) deep. The crib has a plastic barrier cover and is backfilled with the original excavated soil. A vitrified clay distribution pipe burjed in the gravel was used to disperse the effluent. across the bottom of the crib.

The U-12 Crib received wastewater from the U Plant in the 200 West Area from April 1960 until February 1988, when it was replaced by the 216-U-17 Crib. The closure of the U-12 Crib will be coordinated with the CERCLA Past-Practice activity (200-UP-2 Operabie Unit cleanup). The 216-U-12 Crib is scheduled to be included in the Hanford RCRA Pernit (Ecology 1994) as a TSD unit undergoing closure through the permit modification process in 1997 .

The wastewater disposed of in the U-12 Crib consisted of effluent fron the U Plant and included 291-U-1 Stack drainage and process condensate from the 224-U Butlding. The U-12 Crib received this waste stream from April 1960 unt 17 1972, when it was deactivated. The U-12 Crib was reactivated in November 1981 and received U Plant waste until it was permanently retired in february 1980. An average of over 1,000,000 L (264,200 gal) of effluent was disposed of in the crib during each year of its active liffe. This waste contained hazardous waste and radioactive materials. The $\mathrm{j}-12 \mathrm{Cr}$ ib received low-level radioactive waste that is known to have included chenicals such as nitric acid in addition to plutonium, strontium, ruthenium, and uranium. In 1985, physical controls and operating procedures were modified to avoid inadvertent discharge of hazardous chemicals to the wastewater stream. In 1983, hydrogeologic evaluation of the crib revealed radioactive contamination to a depth of a least $43 \mathrm{~m}$ (140 ft). The depth to water betow the crib is approximately $72.5 \mathrm{~m}$ (237 ft).

The RCRA Interin-Status Groundwater Honitoring Plan for the 216-U-12 Crib (WHC 1990b) presents the progran used to estabi ish the background levels for the CIPs. The document sumarizes the available data for the disposal facility. In accordance with the plan, four RCRA groundwater monitoring wel1s were installed in 1990 . The nonitoring network consisted of one upgradient we11 and three doimgradient weils (Figure 11-1). A RCRA interin-status assessient-1eve1 groundwater monitorling network was established for the U-12 Crib in 1993 (wit 1993a) because of elevated levels of specific conductivity in two downgradient wells.

The sample results for the third quarter of 1995 represent the 17th quarter of RCRA data collected at the U-12 Crib. Data collected during the third quarter of 1992 from downgradient we11s 299-422-41 and 299-W22-42 showed elevated specific conductivity. Veriftcation sampling results and data 
obtained in subsequent quarters corroborated these findings. Currently, the U-12 Crib groundwater monitoring we]] network ts sampled quarterly.

The current network consists of five we]ls-one upgradient wel1 (299-422-43) and four downgradient weils (299-W22-40, -41, -42 , and 699-36-70A). Two old wells (299-W22-22 and 299-1/22-23) initialiy sampled during assessment monitoring are no longer sampled because of 7ess-thanoptimal well conditions, high turbjdity, and casing corrosion products. TabTe 11-1 11sts the network wells and thetr sampling status during the reporting period.

\subsection{MATER LEVEL MEASURENENTS}

Hater levels in each network wel] are measured at least quarterly and When water samples are collected. Hater level measurements obtained during the third quarter-are presented in Table 11-2.

\subsection{WATER CHEMISTRY DATA}

Groundwater samples were collected September 21-25, 1995 from al1 network wells. All data currently available from this and previous unreported sampling periods are shown in Tables 11-3 through 11-5. Table 11-3 includes the constituent list and a summiary of results. Tables 11-4 and 11-5 show results for specific constituents. Analytical data unavailable at the time this report was prepared witl be included in future quarterly reports.

Specific conductivity results in wells 699-36-70A, 299-W22-41 and 299-W22-42 continue to exceed the critical mean of 437 amho/cm. Results for nitrate (BWS $=45,000 \mathrm{ppb}$ ), one of the principal constituents suspected of causing elevated conductivity at the crib are 150,000,150,000, and $550,000 \mathrm{ppb}$, respectiveTy, for the wells with elevated specjfic conductance. Technetium-9g (beta indicators are elevated also) is also elevated above the Hanford Sitewide background values in these same wells (consistent with previous downgradjent well data). Iodine-129 continues to be elevated above Hanford Sitewide background values in wells 699-36-70A and 299-H22-42 and tritium in well 699-36-70A is $324,000 \mathrm{pCi} / \mathrm{L}$, above the $20,000 \mathrm{pCi} / \mathrm{L}$ DWS. An explanation of any data flags can be found in Sectjons 1.2 and 1.4 .

Analytical results flagged with an ' $F$ ' indicate that a RADE has been issued because the results did not follow past trends. 


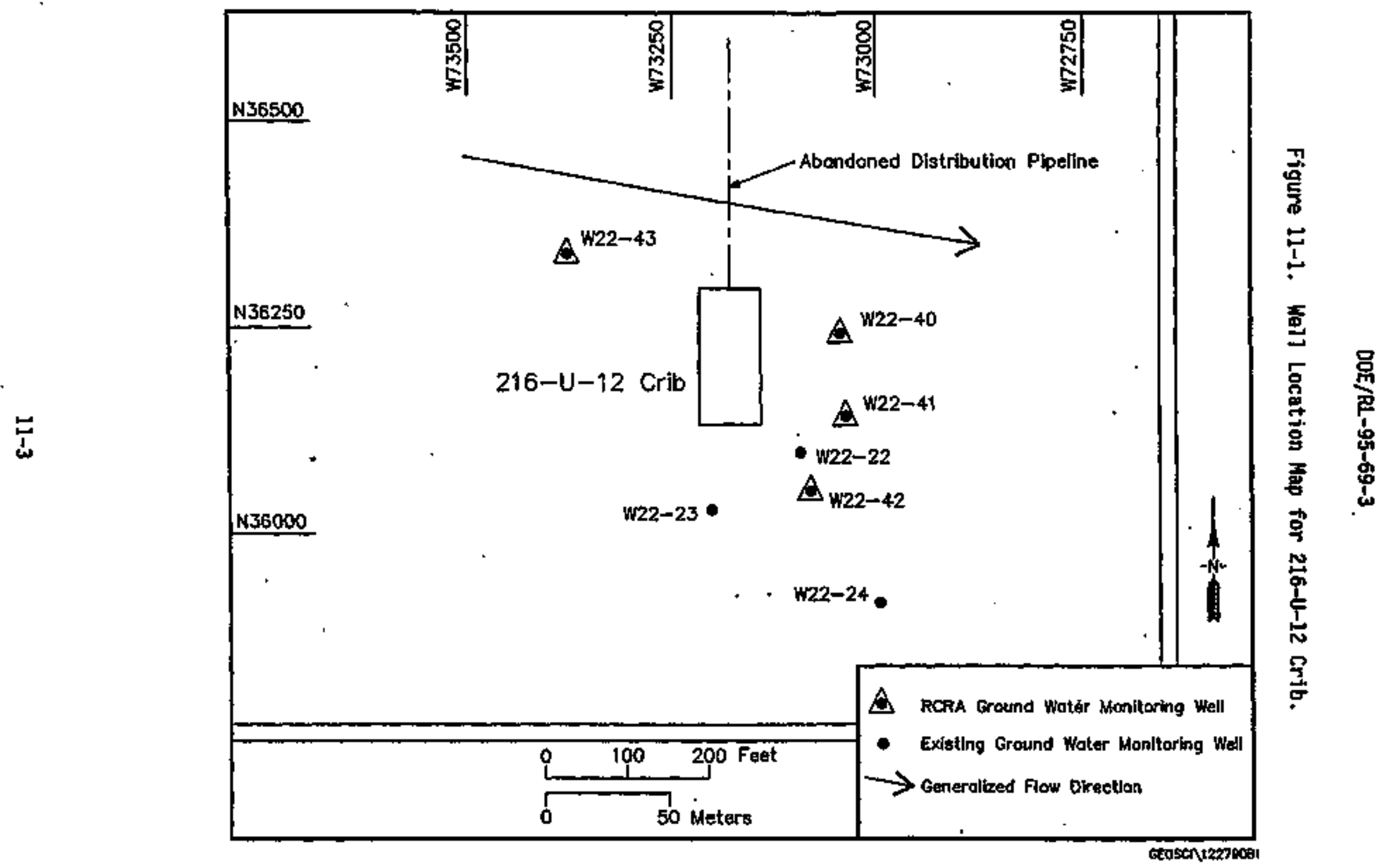


Table 11-1. Monitoring Well Purpose and Sampling Schedule for the 216-u-12 "Crib.

\begin{tabular}{|l|l|l|l|l|}
\hline $\begin{array}{c}\text { Hell no. } \\
(229-)\end{array}$ & $\begin{array}{c}\text { Relative } \\
\text { position }\end{array}$ & Hydrogeologic unjt & $\begin{array}{c}\text { Sample } \\
\text { frequency }\end{array}$ & $\begin{array}{c}\text { Sample } \\
\text { date, } \\
\text { 3rd Qtr } \\
1995\end{array}$ \\
\hline W22-43 & Upgradient & $\begin{array}{l}\text { Ringo]d: Water } \\
\text { Table }\end{array}$ & Quarterly & $09 / 21 / 95$ \\
\hline W22-40 & Downgradient & $\begin{array}{l}\text { Ringo]d: Water } \\
\text { Tabie }\end{array}$ & Quarterly & $09 / 21 / 95$ \\
\hline W22-41 & Downgradient & $\begin{array}{l}\text { Ringold: Water } \\
\text { Table }\end{array}$ & Quarterly & $09 / 22 / 95$ \\
\hline W22-42 & Downgradient & $\begin{array}{l}\text { Ringo]d: Water } \\
\text { Table }\end{array}$ & Quarterly & $09 / 22 / 95$ \\
\hline $699-36-70 A$ & Downgradient & $\begin{array}{l}\text { Ringold: Water } \\
\text { Table }\end{array}$ & Quarterly & $09 / 25 / 95$ \\
\hline
\end{tabular}

Wote: Hydrogeologic units include the sandy gravel of the Hanford formation and the Ringold Formation. 
Table 11-2. RCRA Water Level Measurement Report 216-U-12 Crib, Third Quarter 1995.

\begin{tabular}{|c|c|c|c|c|}
\hline Wel1 & Date & $\begin{array}{l}\text { Depth to } \\
\text { water (ft) }\end{array}$ & $\begin{array}{l}\text { Hater } \\
\text { elevation } \\
\text { (ft) }\end{array}$ & $\begin{array}{l}\text { level } \\
\text { above ms1 } \\
\qquad(m)\end{array}$ \\
\hline \multicolumn{5}{|c|}{ Wells Monitoring the Top of the Unconfined Aquifer } \\
\hline $299-W 22-40$ & $9 / 21 / 95$ & $239.88 \star$ & 452.35 & 137.88 \\
\hline 299-W22-41 & $\begin{array}{l}9 / 08 / 95 \\
9 / 22 / 95\end{array}$ & $\begin{array}{l}238.93 \\
239.10^{\star}\end{array}$ & $\begin{array}{l}453.07 \\
452.90\end{array}$ & $\begin{array}{l}138.10 \\
138.04\end{array}$ \\
\hline 299-W22-42 & $\begin{array}{l}9 / 08 / 95 \\
9 / 22 / 95\end{array}$ & $\begin{array}{l}238.41 \\
.238 .70^{\star t}\end{array}$ & $\begin{array}{l}453.01 \\
452.72\end{array}$ & $\begin{array}{l}138.08 \\
137.99\end{array}$ \\
\hline 299-W22-43 & $\begin{array}{l}9 / 08 / 95 \\
9 / 21 / 95\end{array}$ & $\begin{array}{l}237.74 \\
238.80^{\star}\end{array}$ & $\begin{array}{l}453.87 \\
452.81\end{array}$ & $\begin{array}{l}138.34 \\
138.02\end{array}$ \\
\hline $699-36-70 A$ & $\begin{array}{l}9 / 08 / 95 \\
9 / 25 / 95\end{array}$ & $\begin{array}{l}258.40 \\
258.33^{*}\end{array}$ & $\begin{array}{l}447.03 \\
447.10\end{array}$ & $\begin{array}{l}136.25 \\
136.28\end{array}$ \\
\hline
\end{tabular}

NOTES: 1. Water level elevations are calculated by subtracting the measured depth-to-water fron the surveyed elevation for the weil.

2. Depth-to-water values are transcribed from field records.

3. Measurements marked with an ' $*$ ' were taken at the time of sampiing.

4. Keasurements marked with a ' + ' are outside of the expected range, and are suspected of error. 
Table 11-3. Constituent List and Sumary of Results for the 216-J-12 Crib Data for Reporting Period July 1 through Septenter 30, 1995.

$$
\text { (sheet } 1 \text { of } 3 \text { ) }
$$

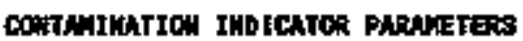

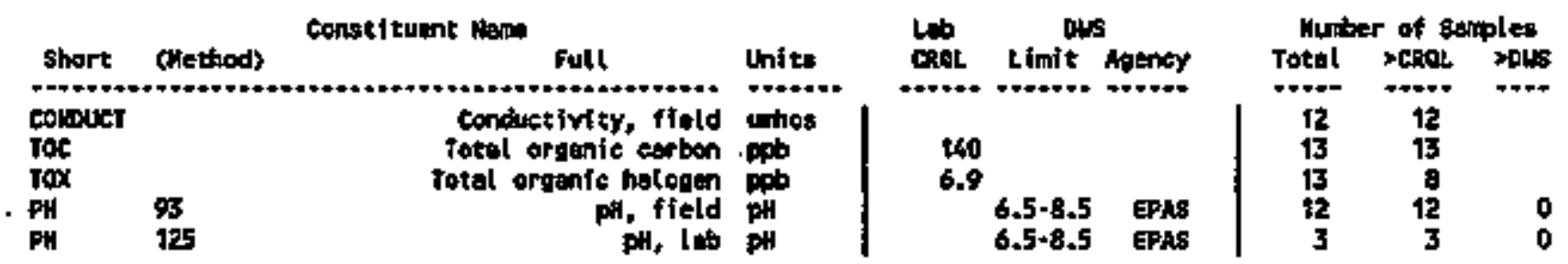

DRTLKIMB WhTER PARUETERS

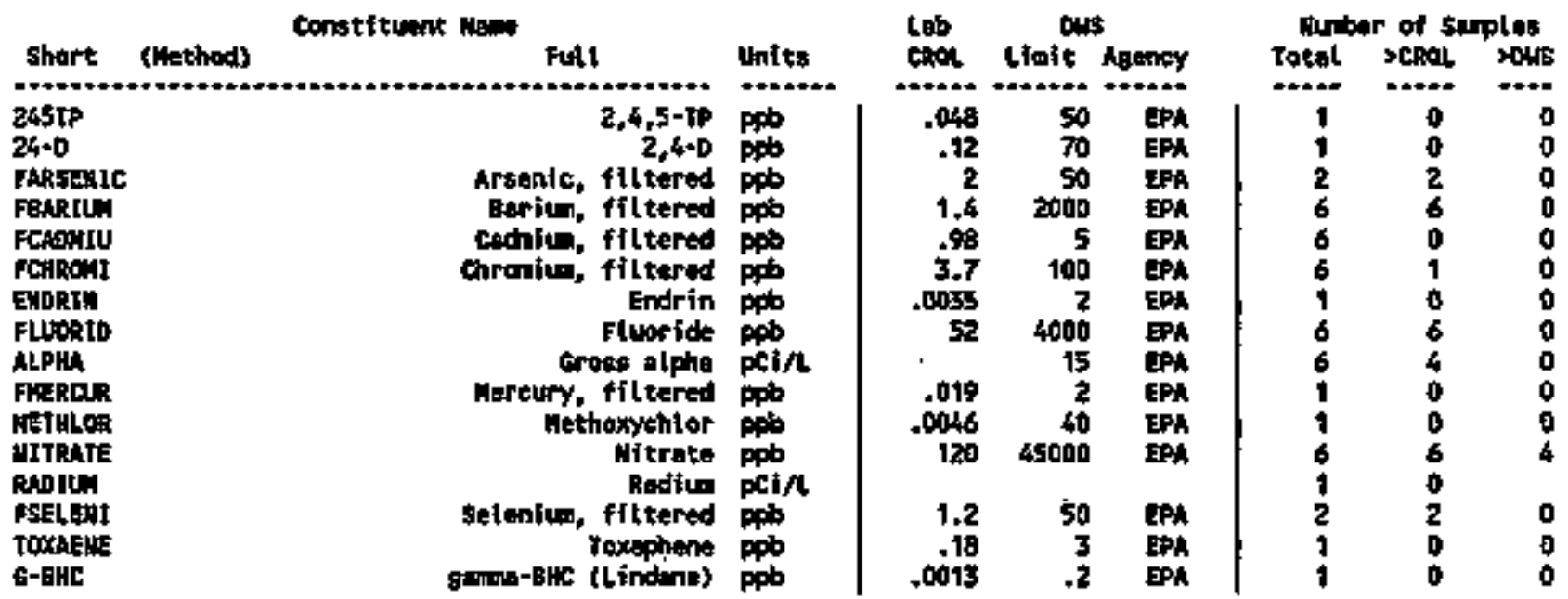

GROMOHATER GULITY PARNAETERS

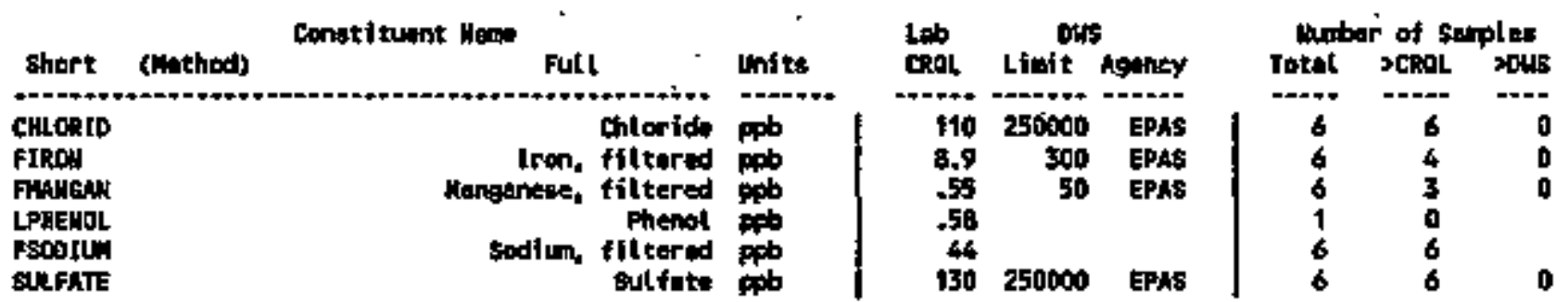


Table 11-3. Constituent List and Sumbary of Resthts for the 216-U-12 Crib Data for Reporting Period July 1 through September 30, 1995.

- (sheet 2 of 3 )

SITE SPECIFIC MD OTHEK COUSTITUCNTS

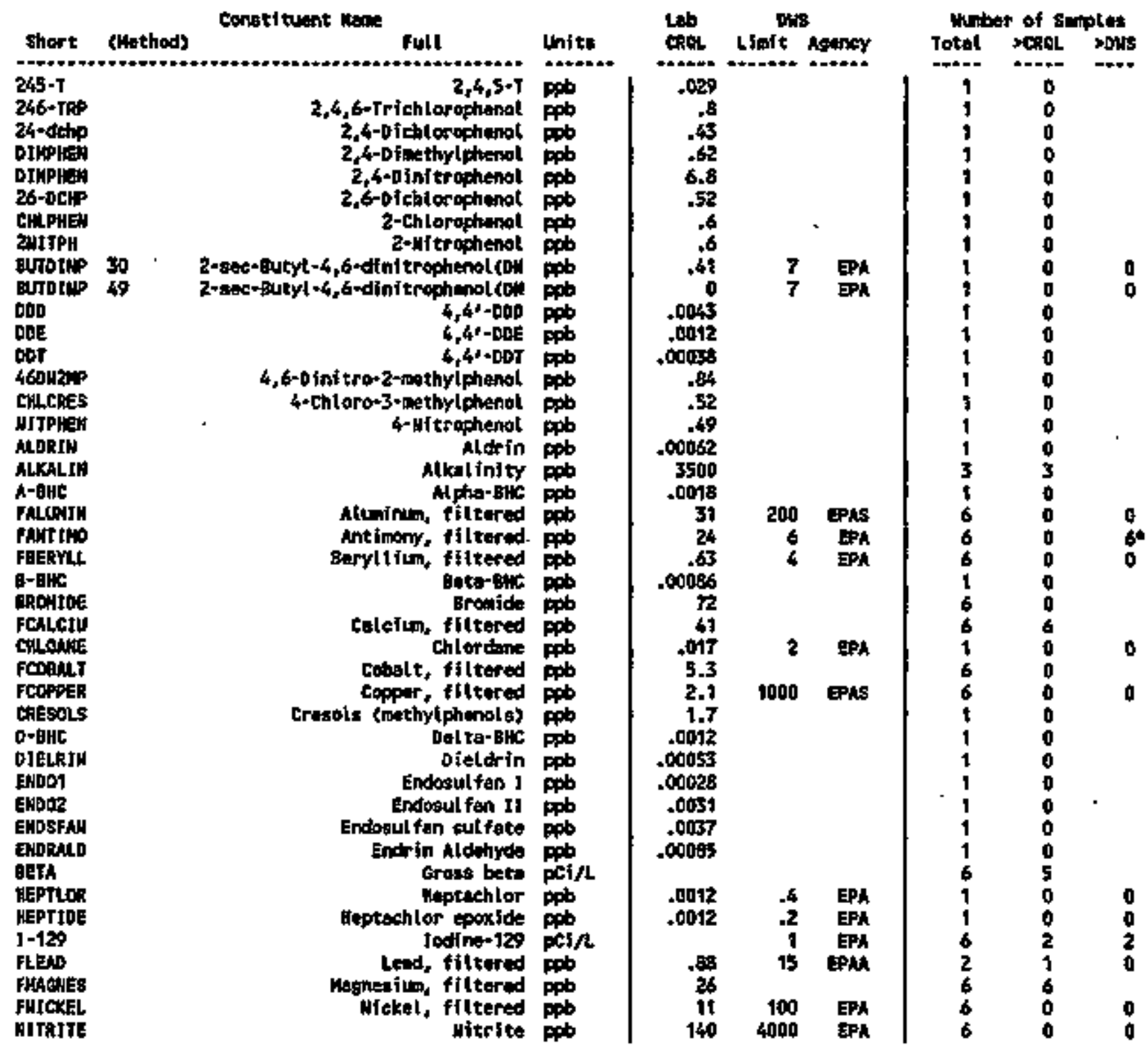


Table 11-3. Constituent List and Summary of Results for the 216-U-12 Crib Data for Reporting Period JuTy I through September 30, 1995.

(sheet 3 of 3 )

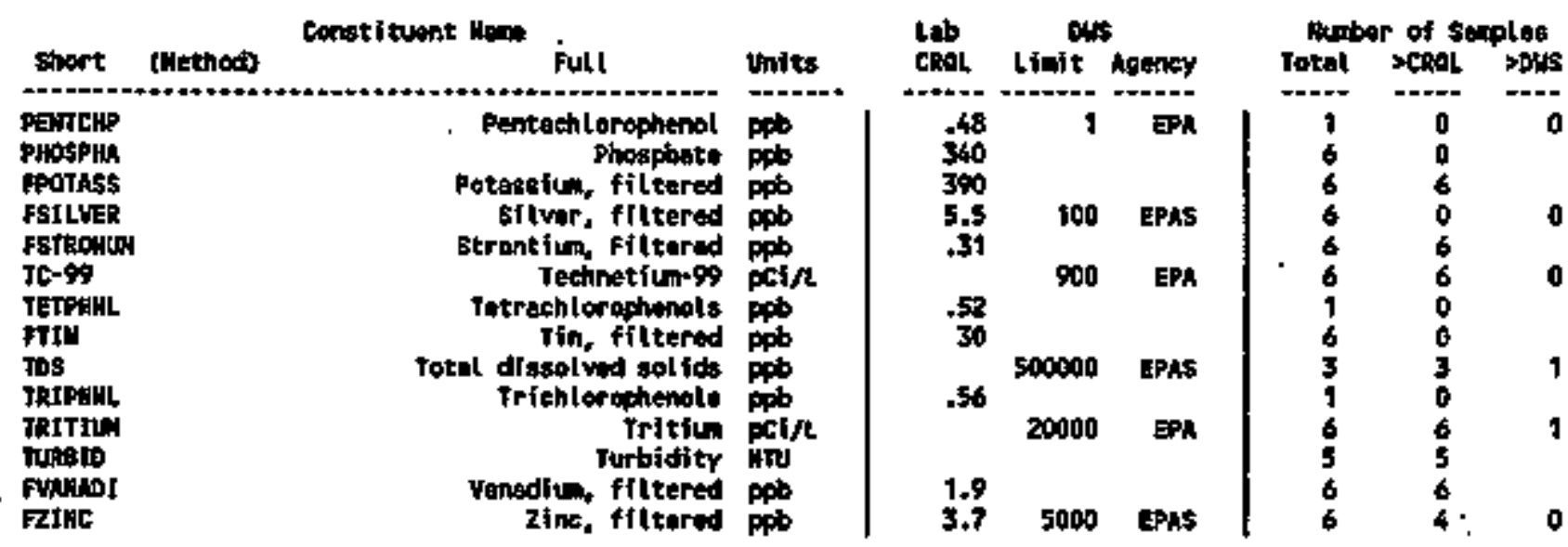

For explenstion of this table, see section 1.4 of repoft. 
Table 11-4. Constituents with at Least One Detected Value for the 216-4-12 Crib Data for Reporting Period July 1 through September 30, 1995.'

(sheet 1 of 3 )

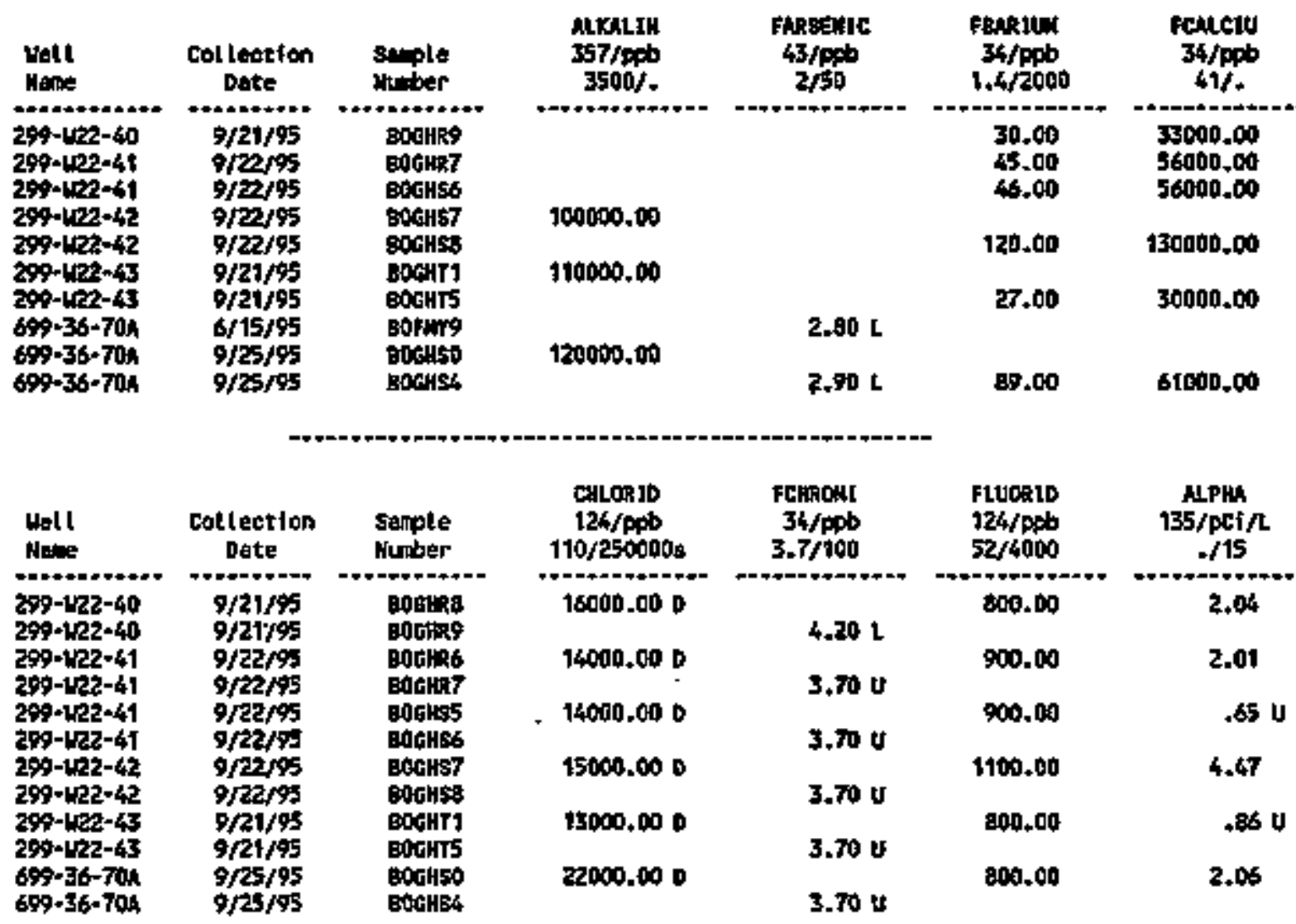

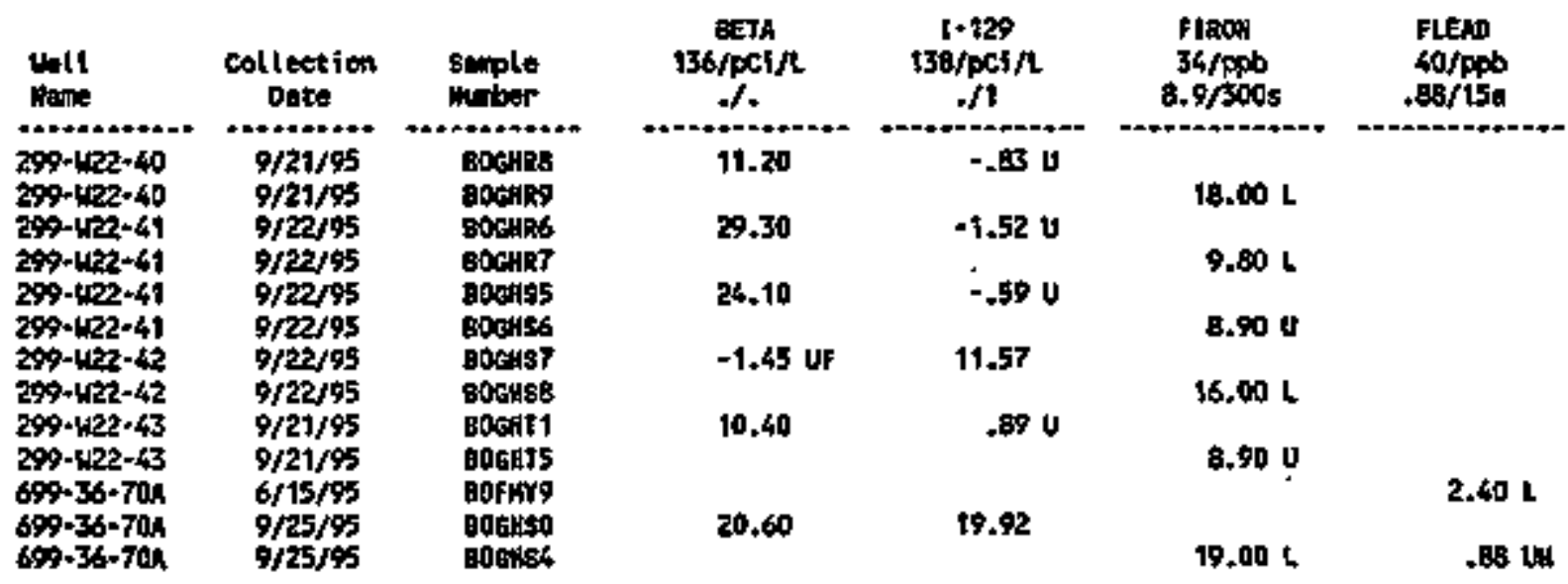


Table 11-4. Constituents with at Least One Detected Value for the 216-U-12 Crib Data for Reporting Period Ju7y 1 through Septerber 30, 1995.

(sheet 2 of 3)

\begin{tabular}{|c|c|c|c|c|c|c|}
\hline Well & $\begin{array}{c}\text { Collection } \\
\text { Date }\end{array}$ & $\begin{array}{l}\text { somple } \\
\text { Whiber }\end{array}$ & $\begin{array}{c}\text { Ewhenss } \\
34 / \mathrm{ppo} \\
26 /\end{array}$ & $\begin{array}{l}\text { runesh } \\
34 / \mathrm{ppb} \\
+55 / 50 \mathrm{i}\end{array}$ & $\begin{array}{c}\text { MITRATE } \\
124 / \mathrm{ppb} \\
120 / 45000\end{array}$ & $\begin{array}{l}\text { FFothss } \\
34 / \text { ppb } \\
390 / .\end{array}$ \\
\hline $\begin{array}{l}299-122-40 \\
259-122-40 \\
297-122-41 \\
299-122-41 \\
299-122-41 \\
293-122-41 \\
24-122-42 \\
299-122-42 \\
299-122-43 \\
297-12^{2}-43 \\
699-36-704 \\
699-36-704\end{array}$ & $\begin{array}{l}9 / 21 / 95 \\
9 / 21 / 95 \\
9 / 22 / 95 \\
9 / 22 / 95 \\
9 / 22 / 55 \\
9 / 22 / 95 \\
9 / 22 / 55 \\
9 / 22 / 95 \\
9 / 21 / 45 \\
9 / 21 / 95 \\
9 / 2 / 45 \\
9 / 21 / 45\end{array}$ & 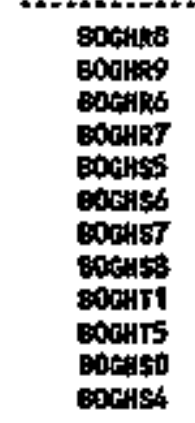 & $\begin{array}{l}12000.00 \\
10000.00 \\
19000.00 \\
44000.00 \\
11000.00 \\
22000.00\end{array}$ & $\begin{array}{l}.75 \mathrm{~L} \\
.55 \mathrm{U} \\
.55 \mathrm{U} \\
.55 \mathrm{U} \\
.65 \mathrm{~L} \\
1.20 \mathrm{~L}\end{array}$ & $\begin{array}{l}25000.000 \\
150000.000 \\
140000.000 \\
550000.000 \\
17000.00 \mathrm{D} \\
250000.00 \mathrm{D}\end{array}$ & $\begin{array}{l}3800.00 \\
3500.00 \\
3900.00 \\
6500.00 \\
3500.00 \\
5700.00\end{array}$ \\
\hline Well & $\begin{array}{l}\text { Cotlertion } \\
\text { Dett }\end{array}$ & 8Eple & $\begin{array}{l}\text { F8ELEN1 } \\
\text { 68/pph. } \\
1.2 / 54\end{array}$ & $\begin{array}{c}\text { Fecostin } \\
34 / \mathrm{pow} \\
44 /\end{array}$ & $\begin{array}{l}\text { FBTRQHUH } \\
34 / \text { PAs } \\
.31 / 4\end{array}$ & $\begin{array}{l}\text { sulfate } \\
124 / \mathrm{pos} \\
130 / 250001 \mathrm{~s}\end{array}$ \\
\hline 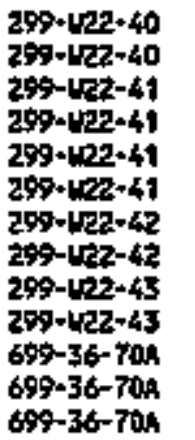 & $\begin{array}{l}9 / 21 / 95 \\
9 / 21 / 95 \\
9 / 22 / 95 \\
9 / 22 / 55 \\
9 / 2 / 95 \\
9 / 22 / 95 \\
9 / 22 / 95 \\
9 / 22 / 95 \\
9 / 21 / 95 \\
9 / 21 / 95 \\
6 / 15 / 95 \\
9 / 25 / 95 \\
9 / 25 / 55\end{array}$ & 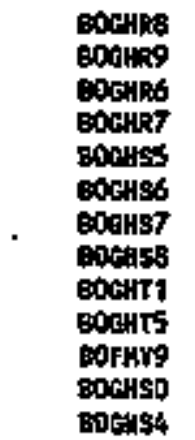 & $\begin{array}{l}1.30 \mathrm{~L} \\
4.80 \mathrm{LH}\end{array}$ & $\begin{array}{l}20000.000 \\
22000.000 \\
25000.000 \\
35000.000 \\
21000.000\end{array}$ & $\begin{array}{l}190.00 \text { K } \\
330.00 \text { K } \\
340.00 \text { K } \\
790.00 \text { K } \\
170.00 \text { H }\end{array}$ & $\begin{array}{l}31000.000 \\
29000.00 \mathrm{D} \\
29000.00 \mathrm{D} \\
31000.00 \mathrm{~b} \\
29000.00 \mathrm{D}\end{array}$ \\
\hline
\end{tabular}

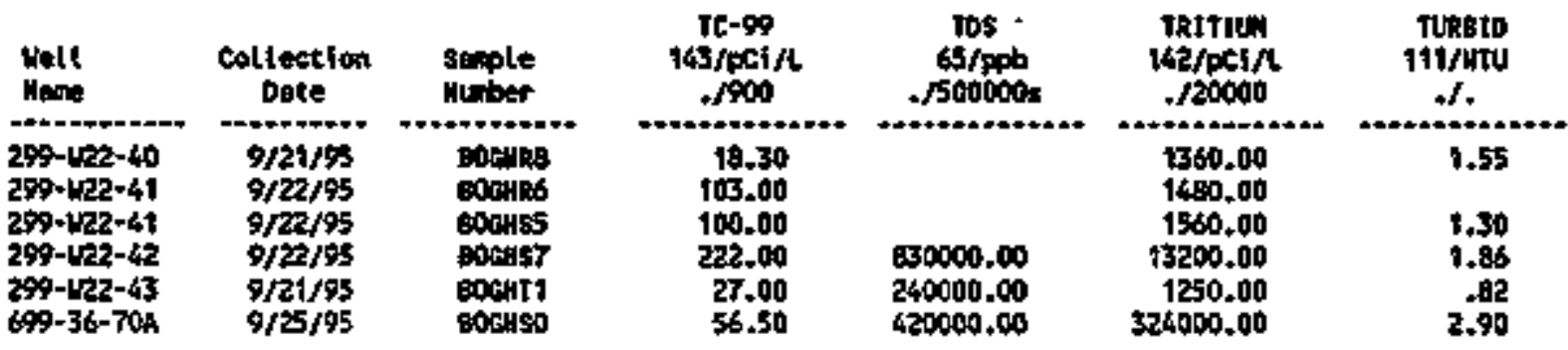


Table 11-4. Constituents with at Least One Detected Vajue for the 216-U-12 Crtb Data for Reporting Perfod July 1 through September 30, 1995.

(sheet 3 of 3 )

\begin{tabular}{|c|c|c|c|c|}
\hline Well & $\begin{array}{c}\text { Col lection } \\
\text { Date }\end{array}$ & semple & $\begin{array}{c}\text { Finud } \\
34 / \mathrm{pot} \\
1.9 /\end{array}$ & $\begin{array}{c}\text { r2tuc } \\
34 / \mathrm{ppb} \\
3.7 / 500 \mathrm{ss}\end{array}$ \\
\hline $\begin{array}{l}99-422-40 \\
99-1422-41 \\
99-422-41 \\
99-422-42 \\
9-422-43 \\
9-36-701\end{array}$ & $\begin{array}{l}9 / 21 / 45 \\
9 / 22 / 45 \\
9 / 22 / 95 \\
9 / 22 / 95 \\
9 / 21 / 45 \\
9 / 25 / \% 5\end{array}$ & 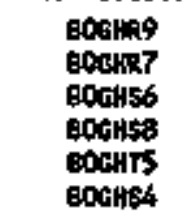 & $\begin{array}{l}28.00 \\
26.00 \\
25.00 \\
17.00 \\
27.00 \\
25.00\end{array}$ & $\begin{array}{c}6.00 \mathrm{~L} \\
4.30 \mathrm{~L} \\
28.00 \mathrm{~V} \\
3.70 \mathrm{~V} \\
3.70 \mathrm{~V} \\
11.00 \mathrm{~V}\end{array}$ \\
\hline
\end{tabular}

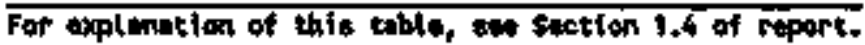


Table 11-5. Contamination Indicator Parameters for the 216-U-12 Crib Data for Reporting Period

July 1 through September 30, 1995.

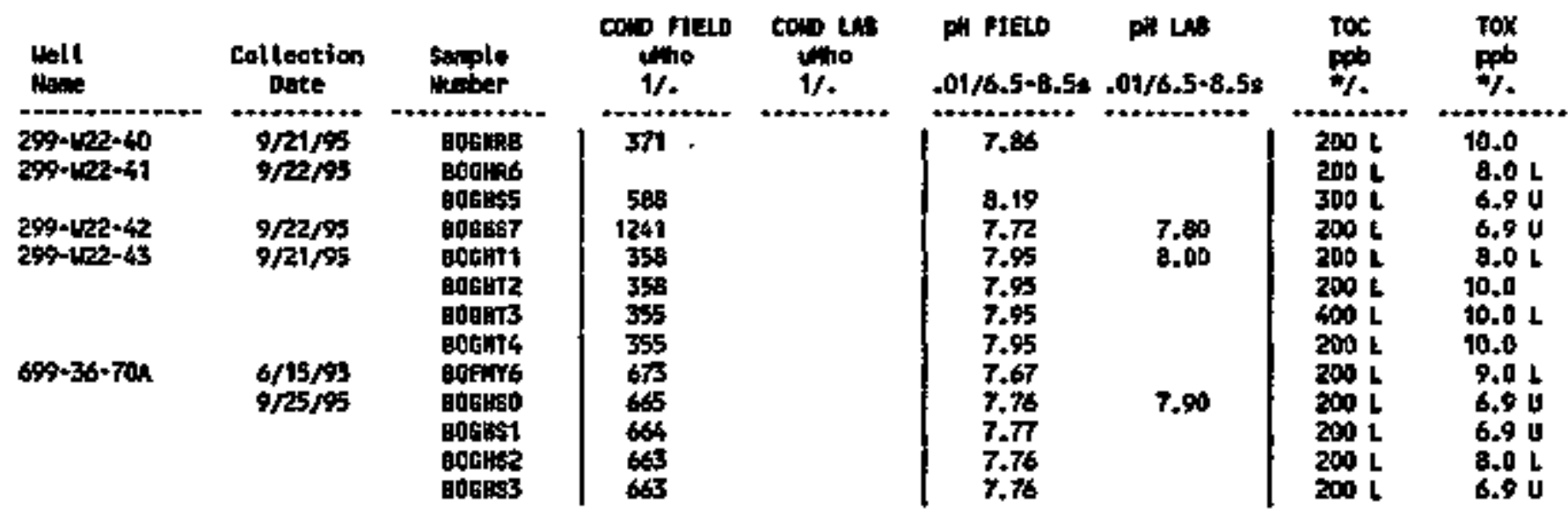

\footnotetext{
- Detection livitg for TOC und Tox vary depending on the perfoming laboratory. For explanation of this table, oee setetion 1.4 of report.
} 
DOE/RL-95-69-3

CONTENTS

12.0 LIQUID EFFLUENT RETENTION FACILITY . . . . . . . . . 12-1

12.1 INTRODUCTION $\ldots \ldots \ldots \ldots \ldots \ldots \ldots . \ldots \ldots 12-1$

12.2 WATER LEVEL MEASUREMENTS $\ldots \ldots \ldots \ldots \ldots \ldots \ldots \ldots \ldots$ I2-1

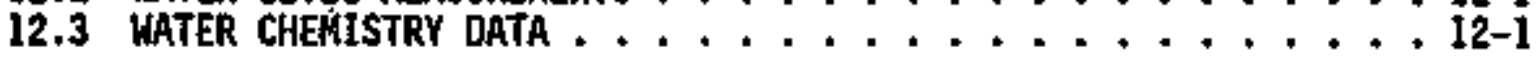


DOE/RL-95-69-3

\section{LIST OF FIGURES}

12-1 He11 Location Nap for the Llquid Effluent Retention facility ................ 12-2

\section{LIST OF TABLES}

12-1 Monitoring Well Purpose and Sampling Schedu1e for the Liquid Effluent Retention Facility Network . . . . . 12-3

12-2 RCRA Water Leve1 Measurement Report LERF, Third Quarter 1995 . . . . . . . . . . . 12-4

12-3 Constituent List and Sumarary of Results for the Liquid EffTuent Retention Facility Data for Reporting Period July 1 through September 30, $1995 \ldots \ldots$. . . 12-5

12-4 Constituents with at Least One Detected Value for the Ltquid Effiuent Retention Facility Data for Reporting Period July 1 through September 30, $1995 \ldots \ldots$. . . . 12-6

12-5 Contamination Indicator Parameters for the Liquid Effiuent Retention Facjlity Data for Reporting Period July 1 through September $30,1995 \ldots \ldots$ 32-7 
DOE/RL-95-69-3

\title{
12.0 LIQUID EFFLUET RETERIOH FACILITY
}

\author{
H. D. Sweeney \\ Nestinghouse thanford Company
}

\subsection{INTRODUCTIOA}

The Liquid Effluent Retention Facility (LERF) was constructed to provide interim storage of 242-A Evaporator process condensate effluent suspected of containing listed and dangerous (ammonia) waste constituents. Construction of the LERF began in May 1990. The LERF, classifled as a surface impoundment for mixed waste storage, will be permitted as a RCRA TSO facility. The evaporator was shut down in 1989 and restarted on April 14, 1994. The LERF basins currently serve as temporary storage for evaporator process condensate unt 1 the Effluent Treatment Facility is operational.

The facility is classified as a surface impoundment and was origtnally planned to have four basins arranged side by side. Four excavations were made; only three of the four basins are lined and are currently scheduled for use. The basins are located imediately east of the northeast corner of the 200 East Area. The liquid efflusent may contain nonhalogenated 5olvents (F003 and F005, WAC 173-303-9004, "Dangerous Waste Source List"), namely acetone, methyl ethyl ketone, and methyl isobutyl ketone. The aturonia content in the 1 jquid effiuent may a]so justify handling the effluent as a dangerous waste.

An interin-status, detection-level groundwater monjtoring network was installed around the LERF in 1990 in accordance with the Interin Status Groundwater Honitoring Plan for the 200 East Area Liquid Effluent Retention Facility (WHC 1991). Four quarters of background groundwater sampling and analysis were completed in Apri1 1992. Three downgradient wel\}s and one upgradient we11 constitute the monitoring network. Figure 12-1 shows the well locations. Table 12-1 provides the last sampling date and frequency and identifies the upgradient and downgradient groundwater monitoring wells. Table 12-2 shows current water table elevations for three of the four monitoring wells.

\subsection{MATER LEYEL MEASUREMEMTS}

Water level measurements obtained during the July 1 through Septenber 30, 1995, period are prosented in Table 12-2. These measurements are obtained during sampling and as part of a semiannual water tablo evaluation.

\subsection{WATER CHEMISTRY DATA}

Chemtstry data received before the cutoff date for this report are reported th Tables 12-3 through 12-5. Mo excerdances were noted for this quarter. An explanation of data flags can be found in Section 1.4. 


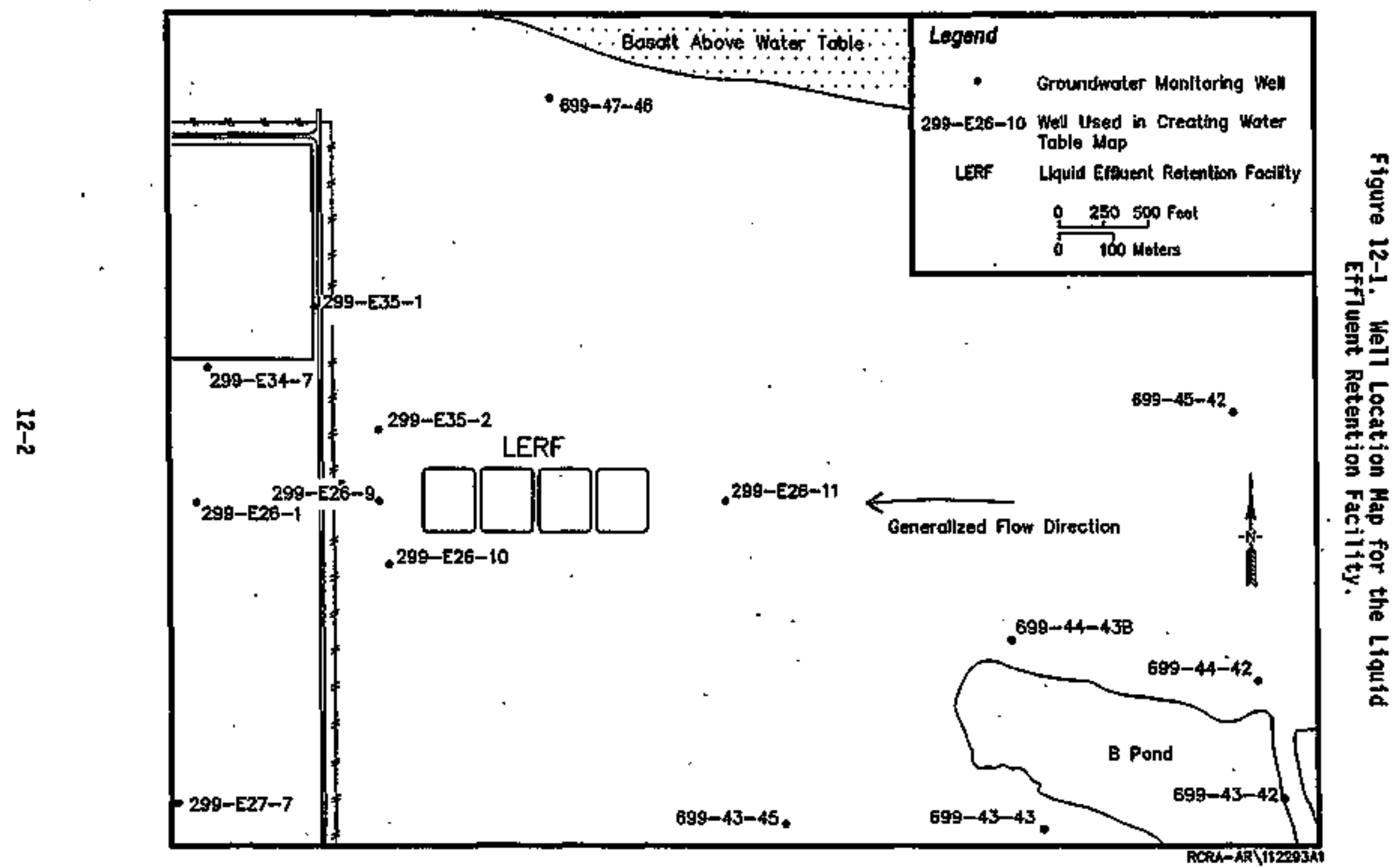


Table 12-1. Monitoring Well Purpose and Sampling Schedule for the Liquid Effiuent Retention Facility Network.

\begin{tabular}{|c|l|l|l|l|}
\hline $\begin{array}{c}\text { We]1 } \\
\text { no. } \\
(299-)\end{array}$ & $\begin{array}{c}\text { Relative } \\
\text { position }\end{array}$ & Hydrogeologic unit & $\begin{array}{c}\text { Sample } \\
\text { frequency }\end{array}$ & $\begin{array}{c}\text { Sample } \\
\text { date, 3rd } \\
\text { Qtr 1995 }\end{array}$ \\
\hline E26-9 & Downgradient & Hanford: Hater Table & Semiannually & $07 / 18 / 95$ \\
\hline E26-10 & Downgradtent & Hanford: Water Table & Semiannua11y & $07 / 18 / 95$ \\
\hline E26-11 & Upgradient & Hanford: Water Table & Semiannually & $07 / 18 / 95$ \\
\hline E35-2 & Downgradient & Hanford: Water Table & Semiannually & $07 / 18 / 95$ \\
\hline
\end{tabular}


Table 12-2. RCRA Water Level Heasurement Report LERF, Third Quarter 1995.

\begin{tabular}{|c|c|c|c|c|}
\hline We17 & Date & $\begin{array}{l}\text { Depth to } \\
\text { water (ft) }\end{array}$ & $\begin{array}{l}\text { Water } \\
\text { elevation } \\
\text { (ft) }\end{array}$ & $\begin{array}{c}\text { level } \\
\text { above msl } \\
\text { (m) }\end{array}$ \\
\hline $299-E 26-10$ & $\begin{array}{l}7 / 18 / 95 \\
9 / 08 / 95\end{array}$ & $\begin{array}{l}199.62 * \\
199.78\end{array}$ & $\begin{array}{l}401.85 \\
401.69\end{array}$ & $\begin{array}{l}122.48 \\
122.44\end{array}$ \\
\hline 299-E26-11 & $\begin{array}{l}7 / 18 / 95 \\
9 / 08 / 95\end{array}$ & $\begin{array}{l}194.18^{*} \\
194.37\end{array}$ & $\begin{array}{l}405.50 \\
405.31\end{array}$ & $\begin{array}{l}123.60 \\
123.54\end{array}$ \\
\hline $299-£ 26-9$ & $\begin{array}{l}7 / 19 / 95 \\
9 / 08 / 95\end{array}$ & $\begin{array}{l}200.98^{\star} \\
201.18\end{array}$ & $\begin{array}{l}401.91 \\
401.71\end{array}$ & $\begin{array}{r}122.50 \\
122.44\end{array}$ \\
\hline $299- \pm 35-2$ & $\begin{array}{l}7 / 18 / 95 \\
9 / 08 / 95\end{array}$ & $\begin{array}{l}200.28 \\
200.39\end{array}$ & $\begin{array}{l}402.08 \\
401.97\end{array}$ & $\begin{array}{l}122.55 \\
122.52\end{array}$ \\
\hline
\end{tabular}

NOTES: 1. Water level elevations are calculated by subtracting the measured depth-to-water from the surveyed elevation for the well.

2. Depth-to-water values are transcribed from field records.

3. Measurements marked with an ' $*$ ' were taken at the time of sampling.

4. Measurements marked with a ' + ' are outside of the expected range, and are suspected of error. 


\section{Table 12-3. Constituent List and Summary of Results \\ for the Liquid Effluent Retention Facility Data for Reporting Period July 1 \\ through Septenber 30, 1995.}

COATANIMAYION IODICATOR PARANETERS

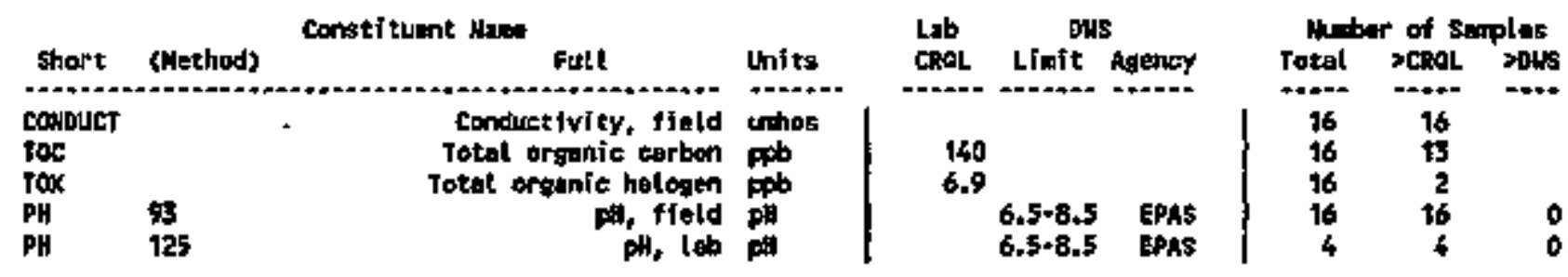

DRINKIRB WATER PARNETERS

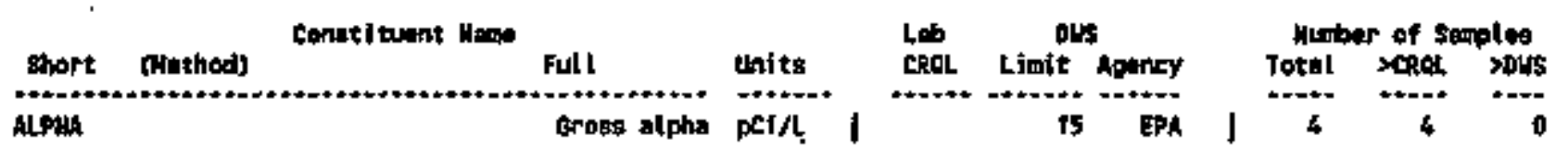

SIJE SPEC1FtE AIO OTHER COMSTIJUEMT

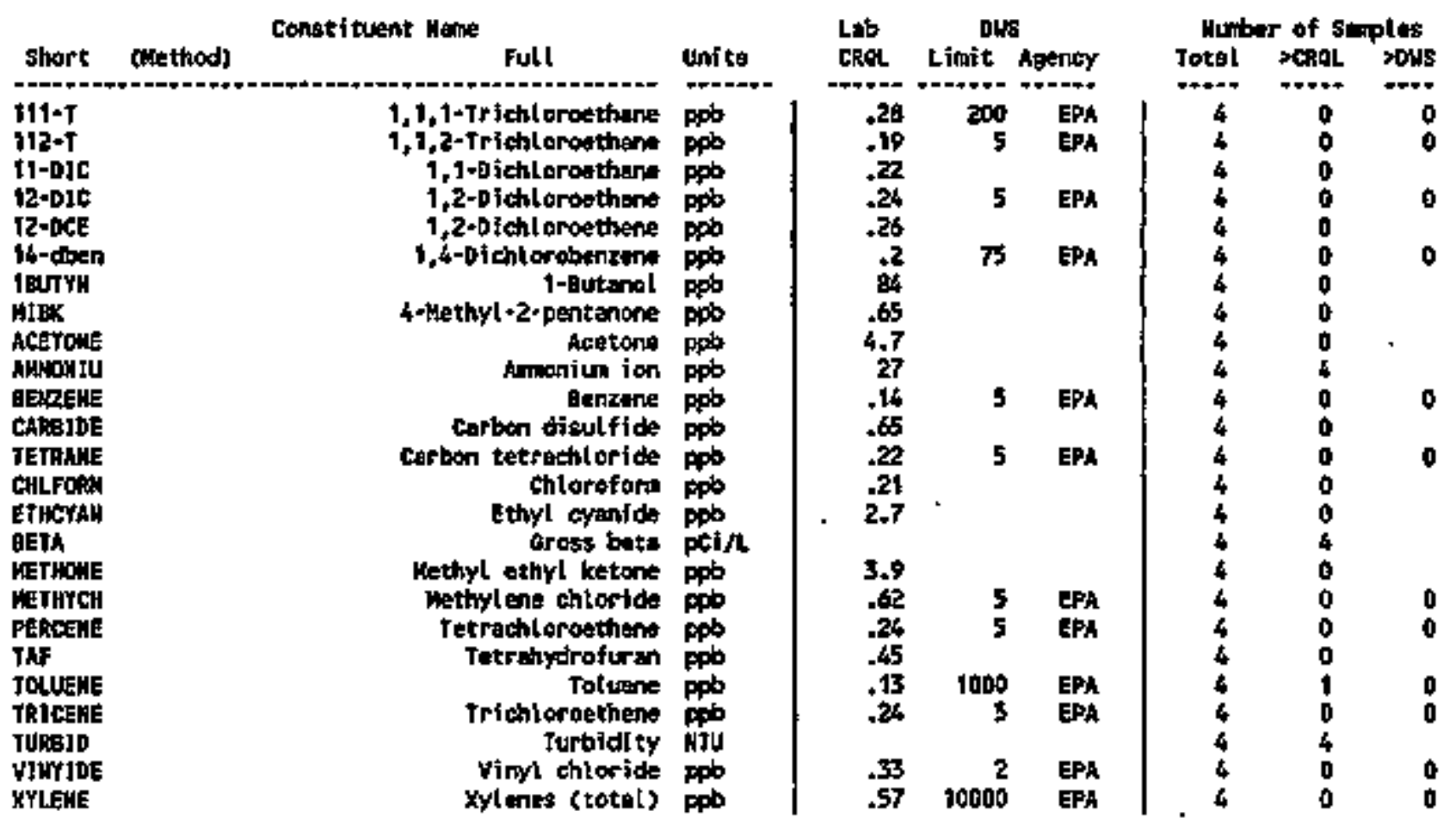


Table 12-4. Constituents with at Least One Detected Value for the Liquid Effluent Retention Fact]ity Data for Reporting Period July 1 through September 30, 1995.

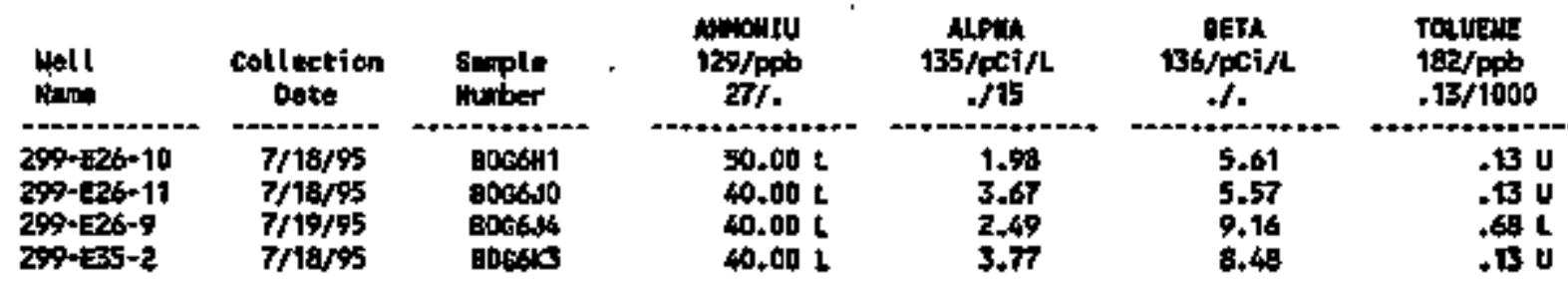

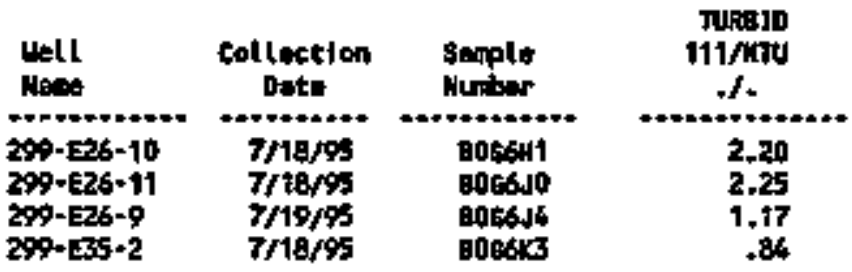

For explarstion of this teble, see section 1.4 of report. 
Table 12-5. Contamination Indicator Parameters for the Liquid Effluent Retention Facility Data for

Reporting Period July 1 through

Septenber 30, 1995.

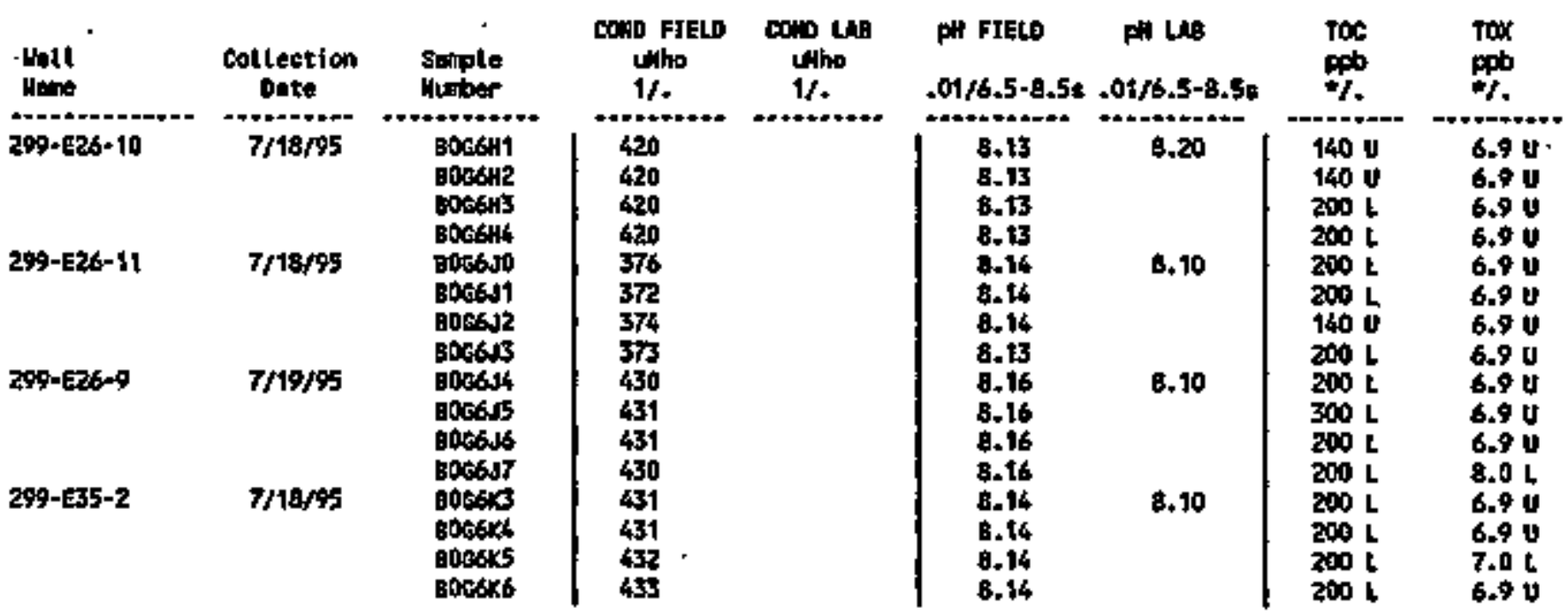

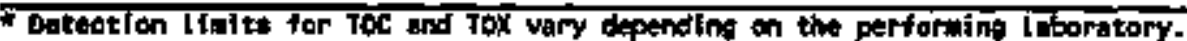

For explenation of this table, sete setion 1.4 of report. 
DOE/RL-95-69-3

This page Intentionally left blank. 
DOE/RL-95-69-3

\section{COHTENTS}

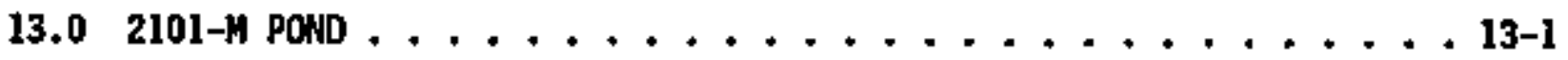


DOE/RL-95-69-3

This page intentionally left blank. 


\subsection{1-\# POND}

\section{E. C. Thornton \\ Mestinghouse Hanford Company}

Washington State Department of Ecology indicated acceptance of a clean closure certification for the 2101-H Pond on October 26, 1995. The last groundwater sampling activities for this facility occurred in June 1995 and all associated data were reported in the RCRA quarterly report for the perlod Apri1 1 through June 30, 1995. A section will not be included for the 2101-K Pond in future quarteriy reports. 


\section{DOE/RL-95-69-3}

This page intentionally Teft blank. 
DOE/RL-95-69-3

Coitans

14.0 LOH-LEVEL BURIAL GROUNDS $\ldots \ldots \ldots \ldots \ldots \ldots \ldots \ldots$

14.1 INTROOUCTION . ...................... 14-1

14.2 WATER LEVEL MEASUREHENTS ................ 14-1

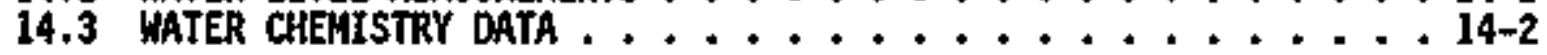




\section{LIST OF FIGURES}

14-1 He11 Location Map for Low-Leve] Waste Management Area 1..... 14-3

14-2 We11 Location Map for Low-Level Waste Management Area $2 . . . .$. 14-4

14-3 We11 Location Hap for Lou-Leve] Waste Managenent Area 3...... 14-5

14-4 We1] Location Map for Low-Level Waste Managentent Area $4 \ldots . . .$. 14-6

14-5 We11 Location Hap for Low-Leve] Waste Management Area $5 . . .$. 14-7 
DOE/RL-95-69-3

\section{LIST OF TABLES}

14-1 Low-Level Burjał Grounds . . . . . . . . . . . . . 14-8

14-2 Monitoring Well Purpose and Sampling Schedule for Low-Levei Waste Managenent Area $1 \ldots \ldots$ 14-9

14-3 Monitoring Hel\} Purpose and Sampling Schedule for Low-Level Waste Management Area 2 . . . . . . . . . 14-10

14-4 Monitoring We17 Purpose and Sampling Schedule for Low-Level Waste thanagenent Area $3 \ldots$... . . . . . . 14-11

14-5 Monttoring Weli Purpose and Sampling Schedule for Low-Leve] Waste Management Area $4 \ldots \ldots$ 14-12

14-6 Honitoring Well Purpose and Sampling Schedule for Low-Level Waste Management Area $5 \ldots+\ldots$. . . . . . . 14-13

14-7 RCRA Water Leve1 Measurentent Report LLWMA 1, Third Quarter 1995 ................ 14-14

14-8 RCRA Water Leve] Measurement Report LLWMA 2, Third Quarter 1995 ................. 14-16

14-9 RCRA Water Leve] Heasurement Report LLWFA 3, Third Quarter 1995 .............. 14-17

14-10 RCRA Water Levei Heasurement Report LLWHA 4, Third Quarter 1995 ................ 14-19

14-11 RCRA Water Leve1 Measurement Report LLWMA 5, Third Quarter 1995 .................. 14-21

14-12 Constituent List and Summary of Results for the Low-Level Haste Management Area 1 Data for Reporting Period July 1 through September 30, 1995 . . . . 14-23

14-13 Constituents with at Least One Detected Value for the

Low-Level Hasto Management Area 1 Data for Reporting

Perlod July 1 through September 30, 1995. . . . . . . . . . . 14-25

14-14 Contamination Indicator Parameters for the Low-Leve] Waste Management Area 1 Bata for Reporting Period July 1 through September 30, $1995 \ldots$ 14-26

14-15 Constituent ist and Sumary of Results for the Low-Leve] Haste Hanagenent Area 2 Data for Reporting Period July 1 through September 30, 1995 . . . . . . . . 14-27

14-16 Constituents with at Least One Detected Value for the Low-Leve7 Waste Managenent Area 2 Data for Reporting Period July 1 through September 30, 1995 . . . . . . . . . 14-28

14-17 Contamination Indicator Parameters for the Low-Leve1 Waste Management Area 2 Data for Reporting Perlod July 1 through September 30, 1995 . . . . . . . . . . 14-29

14-18 Constituent List and Summary of Resuits for the Low-Leve] Naste Hanagement Area 3 Data for Reporting Period July 1 through September 30, 1995.......... 14-30

14-19 Constituents with at Least One Detected Value for the Low-Leve 1 Maste Management Area 3 Data for Reporting Period July 1 through September 30, 1995 .......... 14-32

14-20 Contamination Indicator Parameters for the Low-Level Waste Management Area 3 Data for Reporting Period July 1 through September 30, 1995 . . . . . . . . . . . 14-42

14-21 Constituent List and Summary of Results for the Low-Leve1 Waste Hanagement Area 4 Data for Reporting Period July 1 through September 30, 1995 
14-22 Constituents with at Least One Detected Value for the Low-Leve1 Waste Management Area 4 Data for Reporting Pertod July 1 through September 30, 1995 . . . . . . . . 14-45

14-23 Contawination Ind cator Paraweters for the Low-Leve1 Waste Management Area 4 Data for Reporting Period July 1 through Septenfer 30, 1995 ...... 14-45

14-24 Constituent List and Summary of Results for the Low-Level Waste Management Area 5 Data for Reporting Period July 1 through September 30, 1995 .......... 14-46

14-25 Constituents with at Least One Detected Value for the Low-Level Waste Management Area 5 Data for Reporting Period July 1 through September 30, 1995 . . . . . . 14-48

14-26 Contamination Indicator Parameters for the Low-Level Waste Management Area 5 Data for Reporting Period July I through September 30, 1995 ........... 14-56

14-27 Water Quality Standards Exceeded at Low-Level Waste Management Area 3............ 14-57

14-28 Water Quality Standards Exceeded at Low-Level Waste Management Area 5. ......... 14-57 
DOE/RL-95-69-3

\title{
14.0 LON-LEVEL BURIAL GROUNBS
}

\author{
R. B. Hercer \\ Vestinghouse Hanford Company
}

\subsection{INTRODUCTION}

The 200 Areas LLBG consist of five Lor-Level Waste Management Areas (LLWMA). LLWMA 1 and LLEMA 2 are located in the 200 East Area and ILWMA 3, LLBMA 4, and LLNHA 5 are located in the 200 West Area (Figures 14-1 through 14-5). Table 14-1 describes the waste contajned in each burjat ground.

The groundwater monitoring program for the LLBG began with the installation of 35 monitoring wells in 1987 . Additional wells have been. installed in subsequent years. The present monitoring networks are listed in Tables 14-2 through 14-6. Semiannual detectionglevel sampling is being conducted at ali five burial grounds. Additional samples are being coilected at the upgradient LLHA-5 water table wells to estabijish a critical mean for TOX.

The carbon tetrachlorjde pump-and-treat demonstration project is continuing near LLWAMA 4. Groundwater is being extracted from well 299-W18-1 and injected into well 299-W18-4 after treatment. Groundwater leve1s are being monitored with transducers in the extraction and injection wells and in 299-W18-2, 299-W18-5, and 299-W18-24. Vell 299-W18-24 is an upgradient monitoring wei] for LLWMA 4 . No impacts on the LLWNA 4 monitoring network have been noted to date. Significant impacts are expected when the full pumpand-treat program is started in early 1996.

\subsection{MRTER LEVEL. MEASURENENTS}

In adition to measurenents taken at the time of sampling, water levels were measured qtarterly at the LLBG. Water level measurements are presented in Tables 14-7 through 14-11. Addjtional water level measurements are reported for some weils if they are monitored for other projects. Suspected errors in the water level measurements occurred at several wells this quarter, as indicated in the tables. Water level measurements were not taken at well 299-W6-5 because of an obstructed casing; the casing in we]] 299-W6-5 was crimped during Instaliation. Although remediat action enlarged the casing to permit the insta]lation of a pump, the well does not have sufficient space to wasure water Jeve1s, and additional well rewedlation nay not be possible without danaging the casing. Well 299-E34-4 has been dry since it was constructed. Water levels are no longer measured in LLMi 2 wells 299-E34-6 and 299-E35-1 because they have fallen below the botton of the screens. Al? three of these wells were completed to the top of basalt. The LLWIA 2 monitoring network continues to meet the requitrewent of at least one upgradient and three downgradient monitoring wells. 


\subsection{WATER CHEMISTRY DATA}

Groundwater samples were collected from all scheduted wells. Additional samples were collected at well 299-E32-4 (LLWMA 1) for 216-B-3 Pond. Samples were analyzed for CIPs, interim primary drinking water parameters, water qual ity parameters, and site-specific parameters. Results of analyses are 1isted in Tables 14-12 through 14-26. These tables also contain any previousiy unreported data and data that are being rereported because of changes in the data qualifier flags.

Samples were collected at Some LLWAA 3 and LLMMA 5 groundwater manitoring we17s for iodine-129, strontium-90, technetium-99, and total dissolved solids. These samples were collected in support of groundwater quality assessment at the T and TX-TY SST farms. AnaTytical resuits from these samples are reported in the data tables in this section.

Field pH measurements from upgradient we11 299-\$6-4 and downgradient well 299-45-5 exceeded the critical range estabilshed for LLWMA 5. Laboratory measurements for these wells were wfthin acceptable jimfts. RADEs have been filed of the field pH data. Resampling for field $\mathrm{pH}$ has been requested for we11 299-1/6-5. Other indicator parameter averages did not exceed the critical means at LLWHA 3 or LLwA 5 this quarter.

Analytical results flagged with a ' $Q$ ' are associated with suspect QC data. Data qual iffers and data flags are discussed in more detail in Sections 1.2 and 1.4, respectively. Result's are flagged with an ' $F$ ' if a RADE has been issued because the results did not follow the past trends. Tables 14-27 and 14-28 1 ist the water quality standards that were exceeded for the data reported thts quarter. 
Figure 14-1. Wel1 Location Hap for Low-Leve] Vaste Wanagement Area 1.

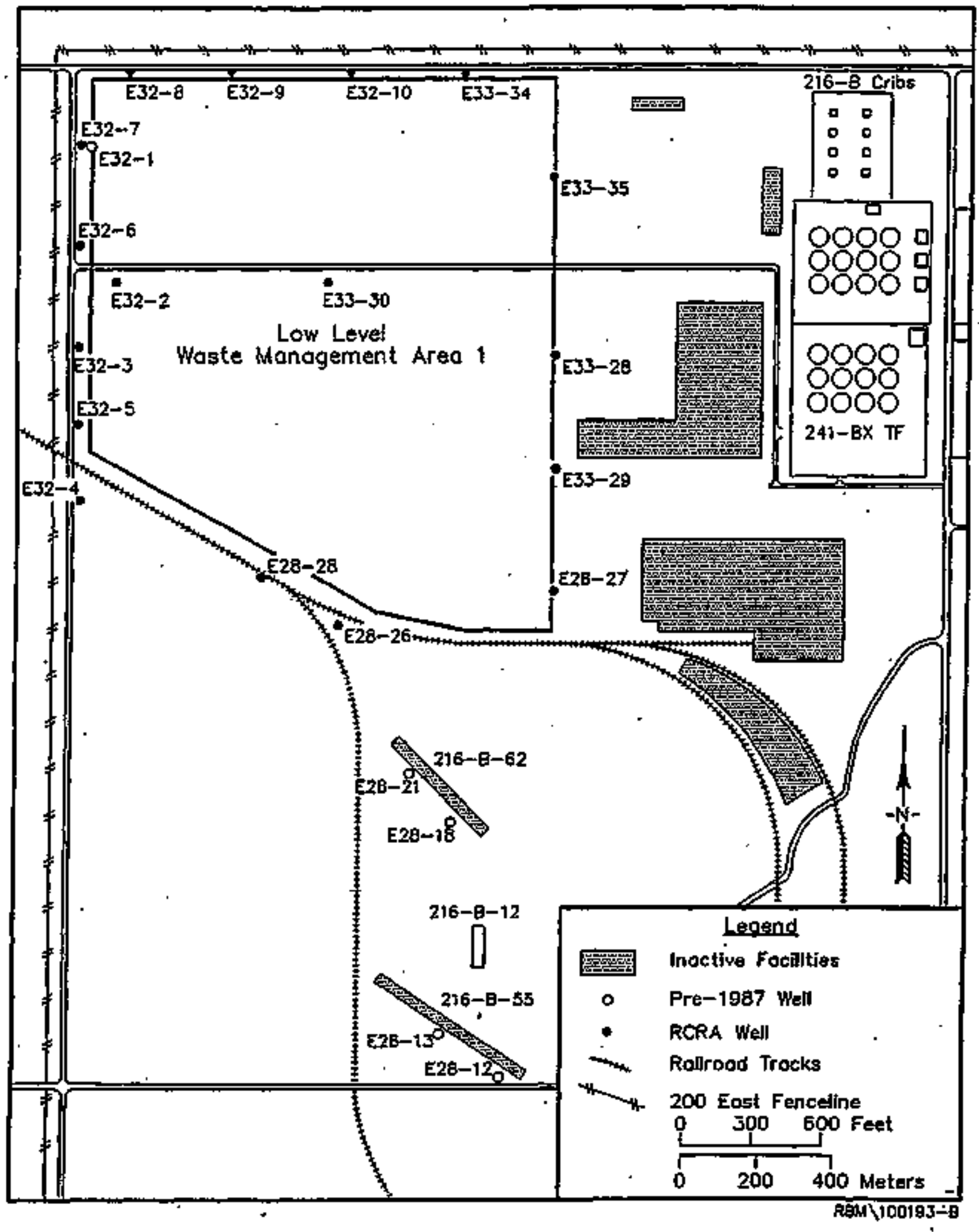


Figure 14-2. We1] Lecation Map for Low-Level Waste Hanagement Area 2.

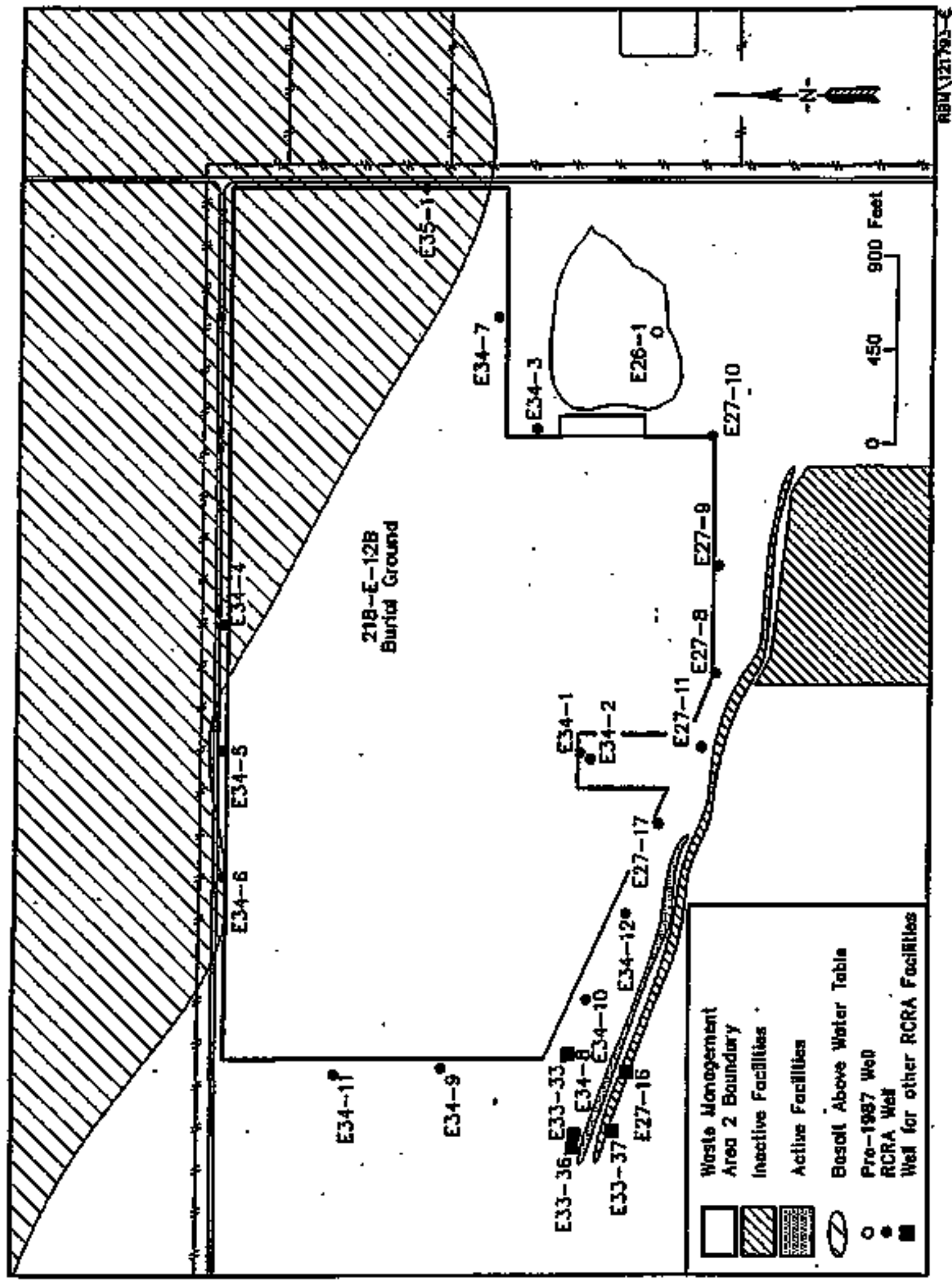


Figure 14-3. Well Location Map for Low-Level Waste Management Area 3.

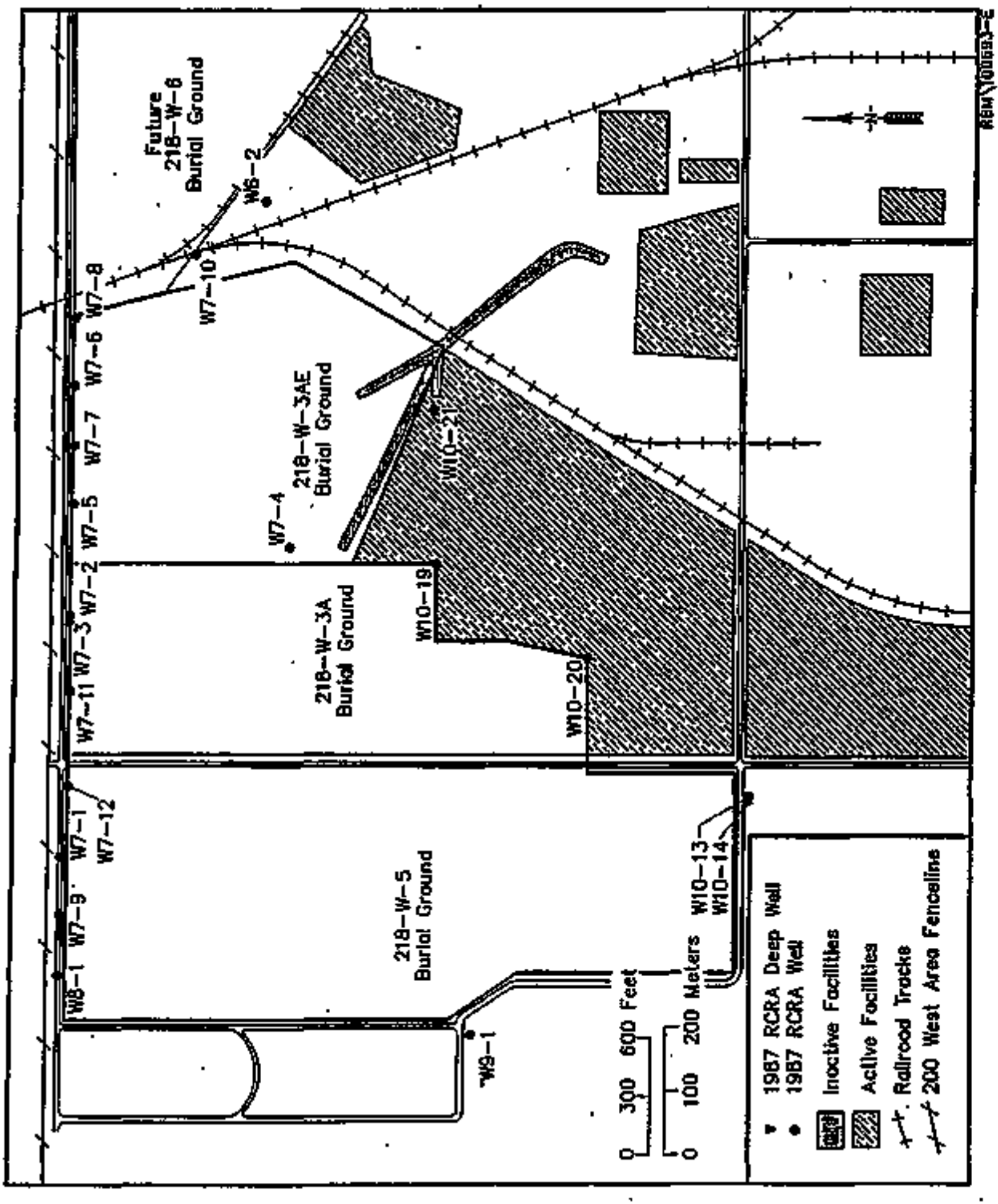


Figure 14-4. We11 Location Map for Low-Level Waste Management Area 4.

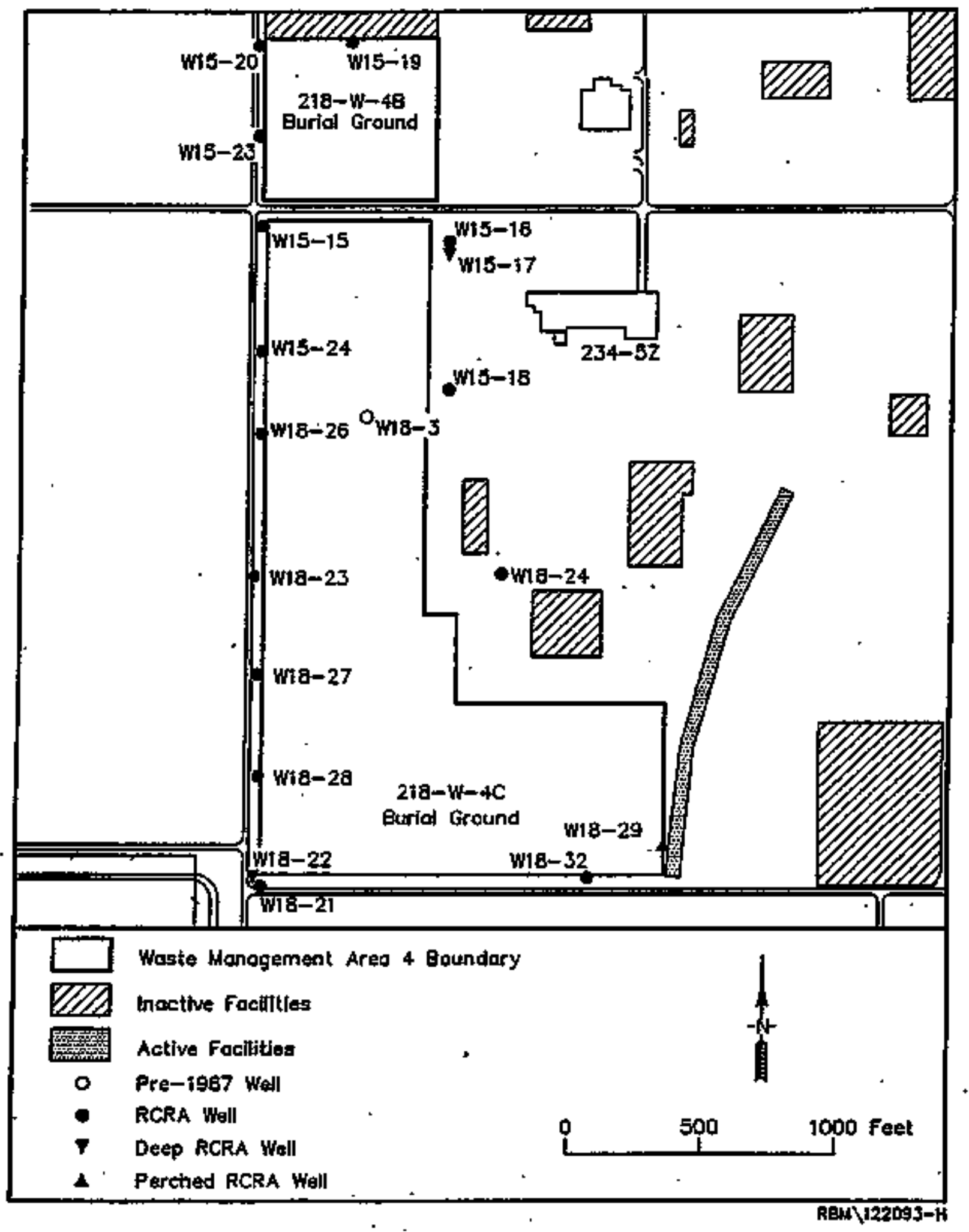




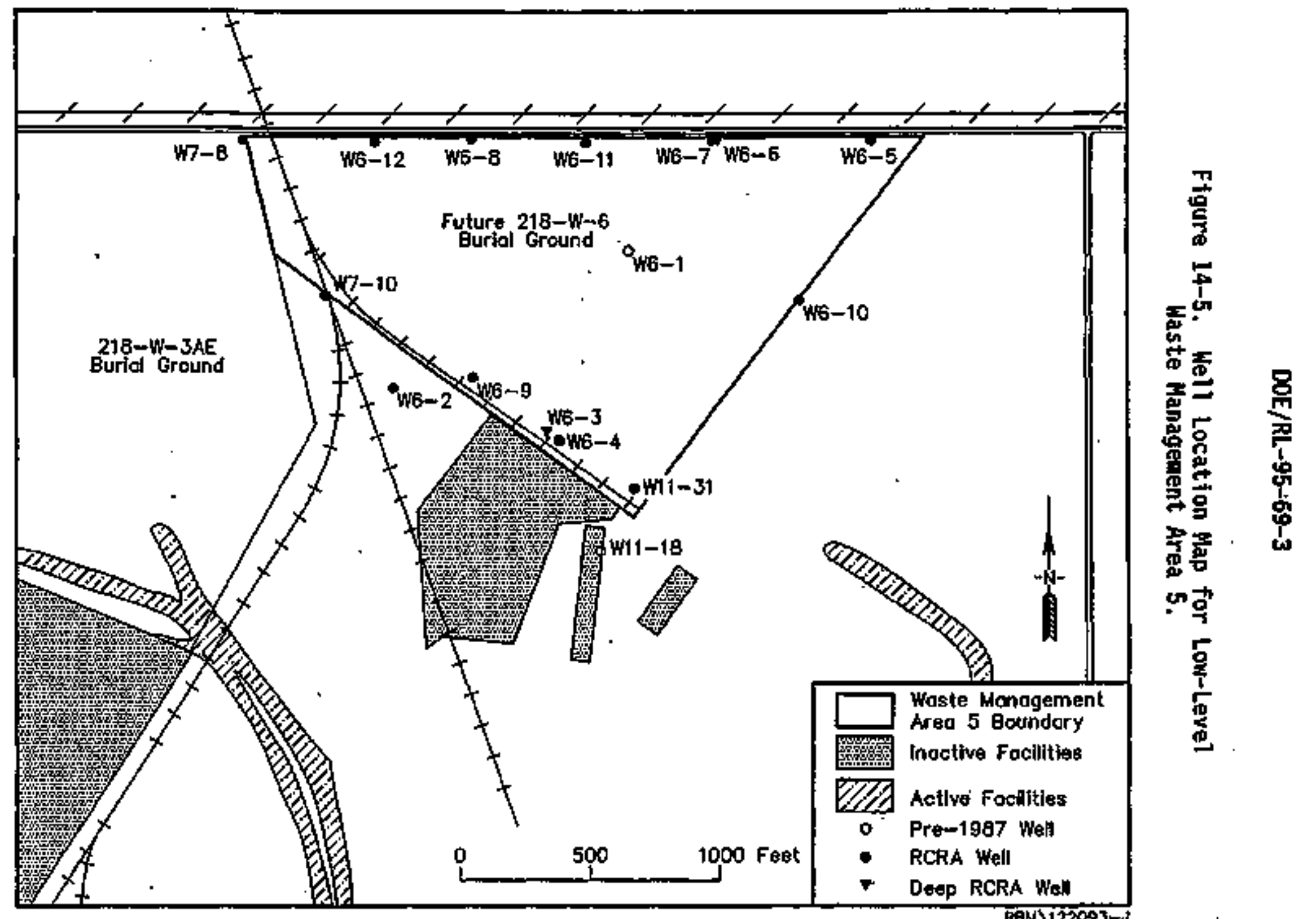


Table 14-1, Low-Level Burial Grounds.

\begin{tabular}{|c|c|c|c|}
\hline LLMANA & Burial ground & First use & Contents \\
\hline 1 & $218-E-10$ & 1960 & $\begin{array}{l}\text { Dragoff material } \\
\text { Faifed equipment } \\
\text { Mixed waste }\end{array}$ \\
\hline 2 & 218-E-12B & 1968 & $\begin{array}{l}\text { Miscellaneous dry waste } \\
\text { Transuranic waste } \\
\text { Submarine reactor compartments }\end{array}$ \\
\hline \multirow[t]{3}{*}{3} & $21 B-W-3 A$ & 1970 & $\begin{array}{l}\text { Ion exchange resins } \\
\text { failed equipment }\end{array}$ \\
\hline & 218-W-3AE & 1981 & $\begin{array}{l}\text { Low-level waste } \\
\text { Lon-level mixed waste }\end{array}$ \\
\hline & $218-W-5$ & 1986 & $\begin{array}{l}\text { Low-7evel waste } \\
\text { Low-level mixed waste }\end{array}$ \\
\hline \multirow[t]{2}{*}{4} & $218-W-4 B$ & 1968 & $\begin{array}{l}\text { Mixed waste } \\
\text { Retrievable transuranjc waste. }\end{array}$ \\
\hline & $218-1 W-4 C$ & 1978 & $\begin{array}{l}\text { Transuranic waste } \\
\text { Mixed waste } \\
\text { Low-level waste }\end{array}$ \\
\hline 5 & $218-4-6$ & $\mathrm{~N} / \mathrm{A}$ & Hone to date \\
\hline
\end{tabular}

$\mathrm{N} / \mathrm{A}=$ not applicable. 
Table 14-2. Monitoring Well Purpose and Sampling Schedule for Low-Level Waste Management Area 1.

\begin{tabular}{|c|c|c|c|c|}
\hline $\begin{array}{l}\text { We11 no. } \\
\text { (299-) }\end{array}$ & $\begin{array}{l}\text { Relative } \\
\text { position }\end{array}$ & Hydrologic unit & $\begin{array}{l}\text { Sample } \\
\text { interval }\end{array}$ & $\begin{array}{l}\text { Sample date: } \\
\text { 3rd Qtr } 1995\end{array}$ \\
\hline E28-26 ${ }^{87}$ & Upgradfent & Hanford: Water Table & Sewiannually & NS \\
\hline$E 28-27^{87}$ & Upgradient & Hanford: Water Table & Semi annual jy & HS \\
\hline$E 28-28^{90}$ & Upgradtent. & Hanford: Water Table & Semiannual7y & N5 \\
\hline$E 32-2^{87}$ & Downgrad lent & Hanford: Water Table & Sext anntaa $7 y$ & NS \\
\hline$E 32-3^{97}$ & Downgradtent & Hanford: Water Table & Semiannual ly & NS \\
\hline$E 32-4^{87}$ & Upgradient. & Hanford: Water Table & Semiannual]y & $07 / 18 / 95 *$ \\
\hline$E 32-5^{89}$ & Downgradient & Hanford: Water Table & Semiannually & NS \\
\hline$E 32-6^{91}$ & Downgradient & Hanford: Hater Table & Semiannually & HS \\
\hline$E 32-7^{91}$ & Downgradient & Hanford: Water Table & Semiannua]7y & NS \\
\hline$E 32-8^{91}$ & Downgradtent & Hanford: Water Table & Semiannually & NS \\
\hline E32-9 91 & Downgradient & Hanford: Water Table & Semiannua?7y & N5 \\
\hline E32-10\%2 & Downgradient & Hanford: Hater Tab7e & Semiannually & MS \\
\hline$E 33-28^{97}$ & Upgradient & Hanford: Water Table & Semtannual 1y & MS \\
\hline$E .33-29^{87}$ & Upgradient & Hanford: Water Table & Semíannually & NS \\
\hline$E 33-30^{87}$ & Bowngradient & Hanford: Yater Table & Semt annual ly & NS \\
\hline$E 33-34^{90}$ & Downgradient & Hanford: Water Table & Semf annual 1y & NS \\
\hline $633-35^{90}$ & Upgradient & Hanford: Water Table & Semfannually & NS \\
\hline
\end{tabular}

Notes: Notes: * - Sampled for B-Pond

NS - Not Scheduled

Superscript following well number indicates year of instal tation. 
Table 14-3. Monitoring Well Purpose and Sampling ScheduTe for Low-Level Waste Nanagement Area 2.

\begin{tabular}{|c|c|c|c|c|}
\hline $\begin{array}{c}\text { Well no. } \\
(299-)\end{array}$ & $\begin{array}{l}\text { Relative } \\
\text { position }\end{array}$ & Hydrologic unft & $\begin{array}{c}\text { Sample } \\
\text { interval }\end{array}$ & $\begin{array}{l}\text { Sample date: } \\
\text { 3rd Qtr } 1995\end{array}$ \\
\hline$E 27-B^{87}$ & Downgradient & Hanford: Water Table & Semiannual1y & NS \\
\hline$E 27-9^{87}$ & Downgradient & Hanford: Water Table & Semiannually & NS \\
\hline$E 27-10^{87}$ & Upgradient & Hanford: Water Table & Semiannual1y & NS \\
\hline$E 27-11^{89}$ & Downgradient & Hanford: Water Tabie & Semiannualiy & MS \\
\hline$\left[27-17^{91}\right.$ & Downgradient & Hanford: Water Table & Semiannual iy & HS \\
\hline$E 34-2^{87}$ & Downgradient & Hanford: Water Table & Semiannua] ]y & NS \\
\hline$E 34-3^{87}$ & Upgradient & Hanford: Water Table & Semiannua ily & MS \\
\hline$E 34-4^{67}$ & Upgradient & Hanford: Water Table & NA & Dry \\
\hline$E 34-5^{87}$ & Upgradient & Hanford: Water Table & Septiantua] $7 y$ & WS \\
\hline$E 34-6^{87}$ & Upgradient & Hanford: Water Table & HA & Dry \\
\hline$\left[34-7^{89}\right.$ & Upgradient & Hanford: Water Table & Semi annuai Iy & HS \\
\hline $\mathrm{E3}^{4}-9^{91}$ & Downgradient & Hanford: Water Table & Semiannualiy & NS \\
\hline$E 34-10^{91}$ & Downgradient & Hanford: Water Table & Semiannual Iy & HS \\
\hline$E 34-11^{92}$ & Downgradient & Hanford: Water Table & Semiannualiy & NS \\
\hline$E 34-12^{92}$ & Downgradient & Hanford: Hater Tab]e & Semiannua 11y & NS \\
\hline$E 35-1^{\text {क9 }}$ & Upgradient & Hanford: Water Table & NA & Ory \\
\hline
\end{tabular}

Notes: NS - Not Scheduled

Superscript following well number indicates year of installation. 
Table 14-4. Monttoring Wel\} Purpose and Sampling Schedule for Low-Leve1 Waste Management Area 3.

\begin{tabular}{|c|c|c|c|c|}
\hline $\begin{array}{c}\text { Nel1 no. } \\
\text { (299-) }\end{array}$ & $\begin{array}{l}\text { Relative } \\
\text { position }\end{array}$ & Hydrologic unit & $\begin{array}{l}\text { Sample } \\
\text { interval }\end{array}$ & $\begin{array}{l}\text { Sample date: } \\
\text { 3rd Qtr } 1995\end{array}$ \\
\hline $46-2^{87 \pi}$ & Downgradient & Ringold: Water Table & Semiannual $7 y$ & $09 / 11 / 95$ \\
\hline $47-1^{87}$ & Downgradient & Ringold: Water Table & Semiannully & $09 / 11 / 95$ \\
\hline $47-2^{87}$ & Downgradient & Ringold: Water Table & Seriannually & $09 / 11 / 95$ \\
\hline $47-3^{87}$ & Downgradient & Ringold: Deep & Semfannual1y & $09 / 12 / 95$ \\
\hline $17-4^{87}$ & Bowngradient & Ringold: Nater Table & SemiannuaTty & $09 / 12 / 95$ \\
\hline W7-5 & Downgradient & Ringold: Water Table & Semiannually & $09 / 12 / 95$ \\
\hline $47-6^{87}$ & Downgradient & Ringold: Water Table & Semtannually & $09 / 12 / 95$ \\
\hline $47-7^{59}$ & Downgradient & Ringold: Water Tabte & Semiannually & $09 / 12 / 95$ \\
\hline$W 7-8^{59}$ & Downgradient & Ringold: Water Table & Semiannually & $09 / 14 / 95$ \\
\hline$W 7-9^{90}$ & Downgradient & Ringold: Water Table & Semiannually & $09 / 13 / 95$ \\
\hline$W 7-10^{90 n}$ & Downgradient & Ringold: Water Table & Semiannual1y & $09 / 11 / 95$ \\
\hline$W 7=11^{91}$ & Downgradient & Ringold: Water Table & Semfannually & $09 / 11 / 95$ \\
\hline$W 7-12^{51}$ & Downgradient & Ringold: Water Table & Semiannually & $09 / 12 / 95$ \\
\hline$W 8-1^{B 7}$ & Downgradient & Ringold: Water Table & Semiannually & $09 / 13 / 95$ \\
\hline $19-1^{87}$ & Upgradient & Ringold: Water Table & Semiannually & $09 / 12 / 95$ \\
\hline $110-13^{87}$ & Upgradient & Ringold: Water Table & Semiannually & $09 / 05 / 95$ \\
\hline $110-14^{87}$ & Upgradient & Ringold: Deep & Semiannually & $09 / 05 / 95$ \\
\hline $110-19^{92}$ & Upgradient & Ringold: Water Table & Semiannuatiy & $09 / 06 / 95$ \\
\hline $110-20^{93}$ & Upgradient & Ringold: Water Table & Semiannually & $09 / 06 / 95$ \\
\hline $110-21^{93}$ & Upgradient & Ringo]d: Water Table & Semianntually & $09 / 11 / 95$ \\
\hline
\end{tabular}

Superscript following well number indicates year of installạtion.

Well monitors multiple LLHitAs.

Deep indicates we11 monitors botton of the unconfined aquifer. 
Table 14-5. Honitoring Mel1 Purpose and Sampling Schedule for Lou-Leve] Waste Management Area 4.

\begin{tabular}{|c|c|c|c|c|}
\hline $\begin{array}{l}\text { Wel1 no. } \\
\text { (299-) }\end{array}$ & $\begin{array}{l}\text { Relative } \\
\text { position }\end{array}$ & Hydrologic unit & $\begin{array}{c}\text { Sample } \\
\text { interval }\end{array}$ & $\begin{array}{l}\text { Sample date: } \\
\text { 3rd Qtr } 1995\end{array}$ \\
\hline$W 15-15^{87}$ & Downgradient & Ringold: Nater Table & Semiannual1y & HS \\
\hline W15-16 $6^{87}$ & Upgradient & Ringold: Water Table & Semiannually & HS \\
\hline $115-17^{87}$ & Upgradient & Ringold: Deep & Semiannualiy & MS \\
\hline W15-18 8 & Upgradient & Ringold: Nater Table & Semiannually & WS \\
\hline$W 15-19^{89}$ & Downgradtent & Ringold: Water Table & Semiannually & HS \\
\hline $415-20^{89}$ & Downgradient & Ringold: Watèr Table & Semiannually & HS \\
\hline W15-23 & Downgradient & Ringold: Nater Table & Semiannual1y & HS \\
\hline $\mathrm{H} 15-24^{\mathrm{g} 9}$ & Downgradient & Ringold: Hater Table & Semiannually & HS \\
\hline $418-21^{87}$ & Downgradient & Ringold: Water Table & Semiannually & NS \\
\hline$W 18-22^{6 t}$ & Downgradient & Ringold: Deep & Semia annual17y & MS \\
\hline W18-23 & Downgradient & Ringold: Water Table & Semfannually & NS \\
\hline$W 18-24^{87}$ & Upgradient & Ringold: Water Table & Semiannualiy & HS \\
\hline$W 18-26^{89}$ & Downgradient & Ringold: Water Table & Semiannua]ly & MS \\
\hline W18-27 & Downgradient & Ringold: Water Table & Semianntsa17y & NS \\
\hline$W 18-28^{91}$ & Downgradient & Ringold: Water Tabie & Semf annual $1 y$ & HS \\
\hline$W 18-29^{91}$ & Upgradient & Hanford: Perched & Semiannually & HS \\
\hline $418-32^{92}$ & Upgradient & Ringold: Water Tabje & Semiannua $11 y$ & HS \\
\hline
\end{tabular}

Notes: IS - Not Scheduled.

Superscript following well nuber indicates year of installation.

Deep indicates well monitors bottom of the unconfined aquifer. 
Table 14-6. Monitoring Well Purpose and Sampling ScheduTe for Low-Leve1 Waste Management Area 5.

\begin{tabular}{|c|c|c|c|c|}
\hline $\begin{array}{l}\text { Well no. } \\
\text { (299-) }\end{array}$ & $\begin{array}{l}\text { Relative } \\
\text { position }\end{array}$ & Hydrologic unit & $\begin{array}{c}\text { Sample } \\
\text { interval }\end{array}$ & $\begin{array}{l}\text { Sample date: } \\
\text { 3rd Qtr } 1995\end{array}$ \\
\hline H6-2 $2^{877}$ & Upgradient & Ringold: Water Tabie & Semiannually & $09 / 11 / 95$ \\
\hline $46-3^{91}$ & Upgradient & Ringold: Deep & Semiannua11y & $09 / 14 / 95$ \\
\hline $166-4^{n 1}$ & Upgradient & Ringold: Mater Table & Seni annually & $09 / 14 / 95$ \\
\hline $166-5^{91}$ & Downgradient & Ringold: Nater Table & Semiannually & $09 / 15 / 95$ \\
\hline $46-6^{91}$ & Downgradient & Ringold: Deep & Seni annual ly & $09 / 18 / 95$ \\
\hline $16-7^{91}$ & Downgradient & Ringold: Nater Table & Semiannually & $09 / 18 / 95$ \\
\hline$w 6-8^{9 t}$ & Downgradient & Ringold: Nater Table & Senjannually & $09 / 21 / 95$ \\
\hline$W 6-9^{92}$ & Upgradient & Ringold: Nater Table & Senjannually & $09 / 14 / 95$ \\
\hline $46-10^{92}$ & Upgradient & Ringold: Mater Table & Sentannually & $09 / 13 / 95$ \\
\hline $46-11^{92}$ & Downgradient & Ringold: Nater Table & Sentanntaliy & $09 / 15 / 95$ \\
\hline W6-12 & Downgradient & Ringold: Water Table & Segiannually & $09 / 15 / 95$ \\
\hline $67-10^{90+n}$ & Upgradient & Ringold: Nater Table & Semi annual1y & $09 / 11 / 95$ \\
\hline $411-31^{92}$ & Upgradient & Ringold: Hater Table & Seatiannually & $09 / 13 / 95$ \\
\hline
\end{tabular}

Superscrtpt following well number indicates year of installation.

- Well monitors multiple LLWMAs.

Deep indicates weill monitors bottom of the unconfined aquifer. 
Table 14-7. RCRA Nater Leve] Measurement Report LLwWA 1, Third Quartier 1995.

(sheet 1 of 2)

\begin{tabular}{|c|c|c|c|}
\hline Wett & Date & $\begin{array}{l}\text { Depth to } \\
\text { water (ft) }\end{array}$ & $\begin{array}{l}\text { Water level } \\
\text { elevation above msl } \\
\text { (ft) }\end{array}$ \\
\hline
\end{tabular}

LLwist-1 Wells Monitoring the Top of the Unconfined Aquifer

299-E28-26

$9 / 20 / 95$

286.14

401.12

122.26

299-E28-27

$9 / 20 / 95$

279.29

401.29

122.31

299-E28-28

$9 / 20 / 95$

285.58

401.17

122.28

299-E32-10

$9 / 20 / 95$

236.68

401.20

122.29

299-E32-2

$9 / 20 / 95$

269.08

401.17

122.28

299-E32-3

9/20/95

277.02

399.70

121.83

299-E32-4

$7 / 18 / 95$

284. 92*

401.19

122.28

$9 / 20 / 95$

285.04

401.07

122.25

299-E32-5

$9 / 20 / 95$

281.14

401.21

122.29

299-E32-6

9/20/95

266.16

401.29

122.31

299-E32-7

9/20/95

257.17

401.20

122.29

299-E32-8

$9 / 20 / 95$

244.45

401.14

122.27

299-E32-9

$9 / 20 / 95$

242.10

401.23

122.29

299-E33-28

9/20/95

263.09

401.36

122.33

299-E33-29

$9 / 20 / 95$

272.67

401.31

122.32

299-E33-30

$9 / 20 / 95$

262.58

401.12

122.26 
Table 14-7. RCRA Water Level Measurement Report

LLWFA 1, Third Quarter 1995.

(sheet 2 of 2)

\begin{tabular}{|c|c|c|c|c|}
\hline Well & Date & $\begin{array}{l}\text { Depth to } \\
\text { water (ft) }\end{array}$ & $\begin{array}{l}\text { Mate } \\
\text { elevatio } \\
\text { (ft) }\end{array}$ & $\begin{array}{l}\text { level } \\
\text { above msl } \\
\text { (m) }\end{array}$ \\
\hline \multicolumn{5}{|c|}{ LLWMA-1 Wells Monitoring the Top of the Unconfined Aquifer } \\
\hline 299-E33-34 & $9 / 20 / 95$ & 232.34 & 401.23 & 122.29 \\
\hline 299-E33-35 & $9 / 20 / 95$ & 242.04 & 401.18 & 122.28 \\
\hline
\end{tabular}

NOTES: 1. Hater level elevations are calculated by subtracting the measured depth-to-water from the surveyed elevation for the well.

2. Depth-to-water values are transcribed from field records.

3. Meastrements marked with an ' $* 1$ were taken at the time of sampling.

4. Measurements marked with a ' + ' are outside of the expected range, and are suspected of error. 
Table 14-8. RCRA Water Level Measurement Report LLWHA 2, Third Quarter 1995.

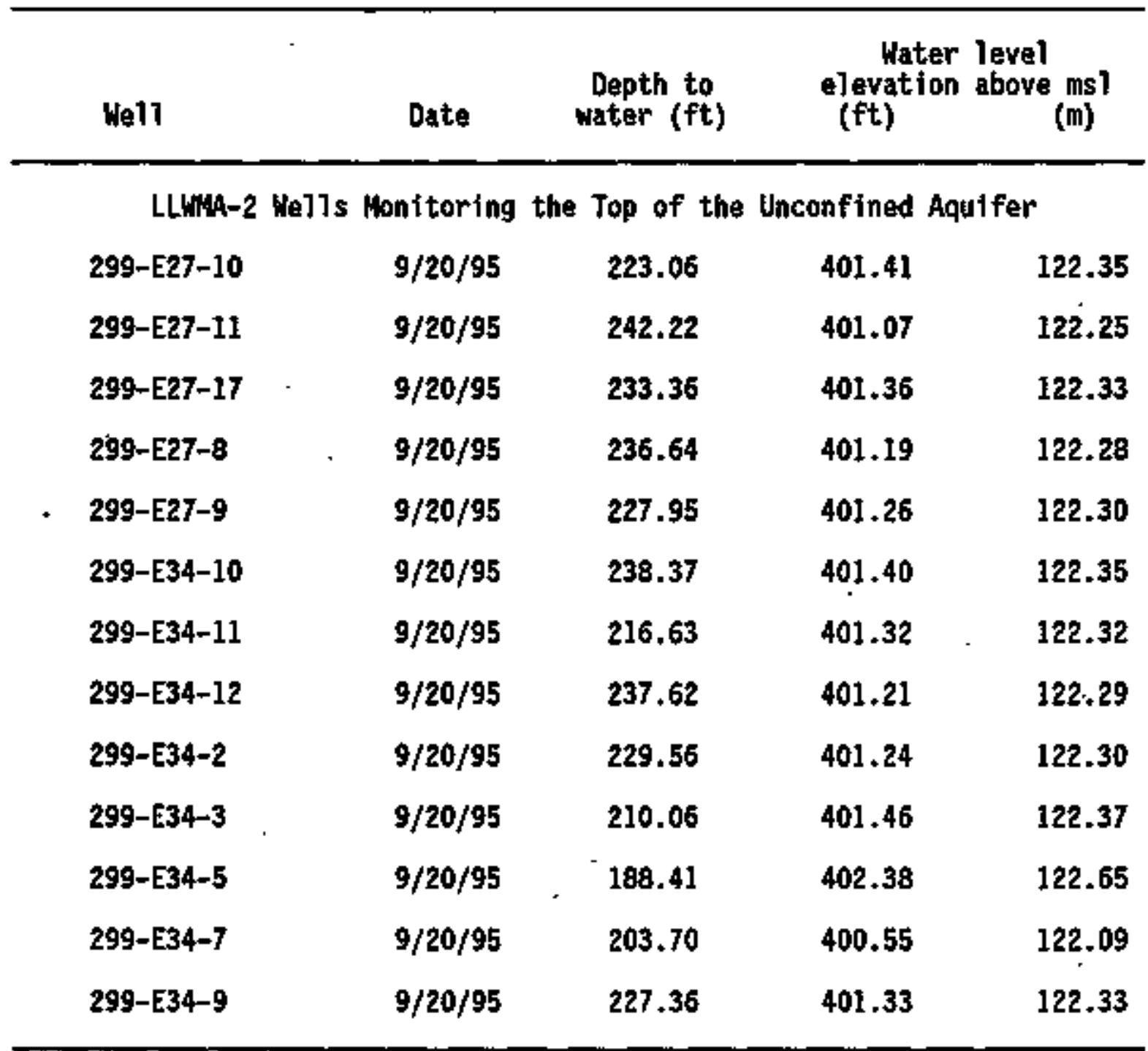

NOTES: 1. Water level elevations are calculated by subtracting the measured depth-to-water from the surveyed elevation for the well.

2. Depth-to-water values are transcribed fron fjeld records.

3. Measurements marked with an '*' were taken at the time of sampling.

4. Heasurements marked with a ' + ' are outsjde of the expected range, and are suspected of error. 
Table 14-9. RCRA Hater Level Measurement Report. LLIMA 3, Third Quarter 1995. (sheet 1 of 2)

\begin{tabular}{|c|c|c|c|c|}
\hline We] & Date & $\begin{array}{l}\text { Depth to } \\
\text { water (ft) }\end{array}$ & $\begin{array}{l}\text { Wat } \\
\text { elevati } \\
\text { (ft) }\end{array}$ & $\begin{array}{l}\text { level } \\
\text { obove ms } 1 \\
\text { (m) }\end{array}$ \\
\hline \multicolumn{5}{|c|}{ LLIAA-3 Wells Monitoring the Top of the Unconfined Aqutfer } \\
\hline $299-110-13$ & $\begin{array}{l}9 / 05 / 95 \\
9 / 19 / 95\end{array}$ & $\begin{array}{l}239.82^{*} \\
239.49\end{array}$ & $\begin{array}{l}459.22 \\
459.55\end{array}$ & $\begin{array}{l}139.97 \\
140.07\end{array}$ \\
\hline $299-1110-19$ & $\begin{array}{l}9 / 06 / 95 \\
9 / 19 / 95\end{array}$ & $\begin{array}{l}224.98^{*} \\
224.74\end{array}$ & $\begin{array}{l}458.01 \\
458.25\end{array}$ & $\begin{array}{l}139.60 \\
139.67\end{array}$ \\
\hline $299-1410-20$ & $\begin{array}{l}9 / 06 / 95 \\
9 / 19 / 95\end{array}$ & $\begin{array}{l}227.91^{*} \\
228.71\end{array}$ & $\begin{array}{l}459.63 \\
458.83\end{array}$ & $\begin{array}{l}140.10 \\
139.85\end{array}$ \\
\hline $299-110-21$ & $\begin{array}{l}9 / 11 / 95 \\
9 / 19 / 95\end{array}$ & $\begin{array}{l}217.16^{\star} \\
216.95\end{array}$ & $\begin{array}{l}456.90 \\
457.11\end{array}$ & $\begin{array}{l}139.26 \\
139.33\end{array}$ \\
\hline $299-46-2$ & $\begin{array}{l}9 / 11 / 95 \\
9 / 18 / 95\end{array}$ & $\begin{array}{l}237.52^{*} \\
237.19\end{array}$ & $\begin{array}{l}454.93 \\
455.26\end{array}$ & $\begin{array}{l}138.66 \\
138.76\end{array}$ \\
\hline $299-47-1$ & $\begin{array}{l}9 / 11 / 95 \\
9 / 19 / 95\end{array}$ & $\begin{array}{l}234.25^{*} \\
234.35\end{array}$ & $\begin{array}{l}456.46 \\
456.36\end{array}$ & $\begin{array}{l}139.13 \\
139.10\end{array}$ \\
\hline $299-17-10$ & $\begin{array}{l}9 / 11 / 95 \\
9 / 18 / 95\end{array}$ & $\begin{array}{l}235.22^{*} \\
234.94\end{array}$ & $\begin{array}{l}454.44 \\
454.72\end{array}$ & $\begin{array}{l}138.51 \\
138.60\end{array}$ \\
\hline $299-177-11$ & $\begin{array}{l}9 / 11 / 95 \\
9 / 18 / 95\end{array}$ & $\begin{array}{l}225.62^{\star} \\
225.34\end{array}$ & $\begin{array}{l}455.83 \\
456.11\end{array}$ & $\begin{array}{l}138.94 \\
139.02\end{array}$ \\
\hline $299-47-12$ & $\begin{array}{l}9 / 12 / 95 \\
9 / 18 / 95\end{array}$ & $\begin{array}{l}231.83^{\star} \\
231.52\end{array}$ & $\begin{array}{l}456.10 \\
456.41\end{array}$ & $\begin{array}{l}139.02 \\
139.11\end{array}$ \\
\hline $299-47-2$ & $\begin{array}{l}9 / 11 / 95 \\
9 / 18 / 95\end{array}$ & $\begin{array}{l}220.28^{*} \\
220.30\end{array}$ & $\begin{array}{l}455.31 \\
455.29\end{array}$ & $\begin{array}{l}138.78 \\
138.77\end{array}$ \\
\hline $299-147-4$ & $\begin{array}{l}9 / 12 / 95 \\
9 / 19 / 95\end{array}$ & $\begin{array}{l}215.10^{*} \\
214.85\end{array}$ & $\begin{array}{l}456.82 \\
457.07\end{array}$ & $\begin{array}{l}139.24 \\
139.31\end{array}$ \\
\hline $299-47-5$ & $\begin{array}{l}9 / 12 / 95 \\
9 / 18 / 95\end{array}$ & $\begin{array}{l}218.40 * \\
218.25\end{array}$ & $\begin{array}{l}454.65 \\
454.80\end{array}$ & $\begin{array}{l}138.58 \\
138.62\end{array}$ \\
\hline $299-47-6$ & $\begin{array}{l}9 / 13 / 95 \\
9 / 18 / 95\end{array}$ & $\begin{array}{l}224.18 * \\
224.02\end{array}$ & $\begin{array}{l}454.46 \\
454.62\end{array}$ & $\begin{array}{l}138.52 \\
138.57\end{array}$ \\
\hline
\end{tabular}


Table 14-9. RCRA Water Level Measurement Report L1HHA 3, Third Quarter 1995.

(sheet 2 of 2)

\begin{tabular}{|c|c|c|c|c|}
\hline Well & Date & $\begin{array}{l}\text { Depth to } \\
\text { water (ft) }\end{array}$ & $\begin{array}{l}\text { Wate } \\
\text { elevatiol } \\
\text { (ft) }\end{array}$ & $\begin{array}{l}\text { leveT } \\
\text { above ons] } \\
\text { (il) }\end{array}$ \\
\hline \multicolumn{5}{|c|}{ LLWith-3 Nel]s Monitoring the Top of the Unconfined Aquifer } \\
\hline $299-17-7$ & $\begin{array}{l}9 / 12 / 95 \\
9 / 18 / 95\end{array}$ & $\begin{array}{l}220.41 * \\
220.15\end{array}$ & $\begin{array}{l}454,53 \\
454,79\end{array}$ & $\begin{array}{l}138.54 \\
138.62\end{array}$ \\
\hline $299-1 / 7-8$ & $\begin{array}{l}9 / 14 / 95 \\
9 / 18 / 95\end{array}$ & $\begin{array}{l}233.88^{\star} \\
235.28\end{array}$ & $\begin{array}{l}453.47 \\
452.07\end{array}$ & $\begin{array}{l}138.22 \\
137.79\end{array}$ \\
\hline $299-W 7-9$ & $\begin{array}{l}9 / 13 / 95 \\
9 / 19 / 95\end{array}$ & $\begin{array}{l}236.50 * t \\
235.30\end{array}$ & $\begin{array}{l}455.59 \\
456.79\end{array}$ & $\begin{array}{l}138.86 \\
139.23\end{array}$ \\
\hline 299-W8-1 & $\begin{array}{l}9 / 13 / 95 \\
9 / 19 / 95\end{array}$ & $\begin{array}{l}244.80^{*} \\
244.53\end{array}$ & $\begin{array}{l}456.53 \\
456.80\end{array}$ & $\begin{array}{l}139.15 \\
139.23\end{array}$ \\
\hline 299-W9-1 & $\begin{array}{l}9 / 12 / 95 \\
9 / 19 / 95\end{array}$ & $\begin{array}{l}278.99 \pi \\
278.83\end{array}$ & $\begin{array}{l}458.74 \\
458.90\end{array}$ & $\begin{array}{l}139.82 \\
139.87\end{array}$ \\
\hline \multicolumn{5}{|c|}{ LLHMA-3 Mells Monitoring the Bottom of the Unconfined Aquifer } \\
\hline 299-W10-14 & $\begin{array}{l}9 / 05 / 95 \\
9 / 19 / 95\end{array}$ & $\begin{array}{l}240.66^{*} \\
240.31\end{array}$ & $\begin{array}{l}458.77 \\
459.12\end{array}$ & $\begin{array}{l}139.83 \\
139.94\end{array}$ \\
\hline $299-17-3$ & $\begin{array}{l}9 / 12 / 95 \\
9 / 18 / 95\end{array}$ & $\begin{array}{l}222.05 * \\
221.91\end{array}$ & $\begin{array}{l}454.09 \\
454.23\end{array}$ & $\begin{array}{l}138.41 \\
138.45\end{array}$ \\
\hline
\end{tabular}

NOTES: 1. Water level elevations are calculated by subtracting the measured depth-to-water from the surveyed elevation for the vell.

2. Depth-to-water va]ues are transcribed from field records.

3. Heasurements marked with an ' $*$ ' were taken at the time of sampling.

4. Heasurements marked w1th a ' + ' are outside of the expected range, and are suspected of error. 
Table 14-10. RCRA Water Leve1 Measurement Report LLMHA 4, Third Quarter 1995. (sheet 1 of 2)

\begin{tabular}{|c|c|c|c|c|}
\hline We11 & Date & $\begin{array}{l}\text { Depth to } \\
\text { water (ft) }\end{array}$ & $\begin{array}{l}\text { Water } \\
\text { elevation } \\
\text { (ft) }\end{array}$ & $\begin{array}{l}\text { level } \\
\text { above ms1 } \\
\quad \text { (m) }\end{array}$ \\
\hline \multicolumn{5}{|c|}{ LLWMA-4 Wells Menitoring the Top of the Unconfined Aquifer } \\
\hline $299-W 15-15$ & $9 / 19 / 95$ & $237.8 B$ & 460.08 & 140.23 \\
\hline $299-W 15-16$ & $9 / 19 / 95$ & 224.45 & 460.44 & 140.34 \\
\hline 299-W15-18 & $9 / 19 / 95$ & 225.74 & 459.97 & 140.20 \\
\hline 299-W15-19 & $9 / 19 / 95$ & 231.40 & 460.20 & 140.27 \\
\hline $299-W 15-20$ & $9 / 19 / 95$ & 238.14 & 460.22 & 140.28 \\
\hline $299-115-23$ & $9 / 19 / 95$ & 239.26 & 460.23 & 140.28 \\
\hline $299-W 15-24$ & $9 / 19 / 95$ & 239.16 & 460.21 & 140.27 \\
\hline $299-W 18-21$ & $9 / 19 / 95$ & 208.35 & 460.27 & 140.29 \\
\hline $299-W 18-23$ & $9 / 19 / 95$ & 236.61 & 460.20 & 140.27 \\
\hline 299-W19-24 & 9/19/95 & 224.03 & 460.32 & 140.31 \\
\hline $299-W 18-26$ & 9/19/95 & 238.69 & 460.36 & 140.32 \\
\hline $299-418-27$ & $9 / 19 / 95$ & 229.98 & 460.27 & 140.29 \\
\hline $299-118-28$ & $9 / 19 / 95$ & 219.54 & 460.45 & 140.35 \\
\hline $299-w 18-32$ & $\begin{array}{l}7 / 31 / 95 \\
9 / 19 / 95\end{array}$ & $\begin{array}{l}214.61 *_{+} \\
216.58\end{array}$ & $\begin{array}{l}462.04 \\
460.07\end{array}$ & $\begin{array}{l}140.83 \\
140.23\end{array}$ \\
\hline
\end{tabular}


Table 14-10. RCRA Water Level Measurement Report LLWHA 4, Third Quarter 1995. (sheet 2 of 2)

\begin{tabular}{lcccc}
\hline Well & Date & $\begin{array}{c}\text { Depth to } \\
\text { water (ft) }\end{array}$ & $\begin{array}{c}\text { Water level } \\
\text { elevation above ms } \\
(\mathrm{ft})\end{array}$ & (m) \\
\hline LLWWA-4 Wells Monjtoring the Botton of the Unconfined Aqujfer \\
$299-$ W15-17 & $9 / 19 / 95$ & 224.42 & 460.22 & 140.28 \\
$299-$ W18-22 & $9 / 19 / 95$ & 208.69 & 459.80 & 140.15 \\
\hline
\end{tabular}

MOTES: 1. Water level elevations are calculated by subtracting the measured depth-to-water fron the surveyed elevation for the weil.

2. Depth-to-water values are transcribed from field records.

3. Heasurenents narked with an ' $*$ ' were taken at the time of sampling.

4. Heasurements marked with a ' + ' are outside of the expected range, and are suspected of error. 
Table 14-11. RCRA Water Level Heasurement Report LLWinA 5, Third Quarter 1995.

(sheet 1 of 2)

\begin{tabular}{|c|c|c|c|c|}
\hline Well & Date & $\begin{array}{l}\text { Depth to } \\
\text { water }(f t)\end{array}$ & $\begin{array}{l}\text { Wat } \\
\text { elevat } \\
\text { (ft) }\end{array}$ & $\begin{array}{l}\text { el } \\
\text { (m) ms] }\end{array}$ \\
\hline \multicolumn{5}{|c|}{ LEMWA-5 Wel1s Monitoring the Top of the Unconfined Aquifer } \\
\hline $299-411-31$ & $\begin{array}{l}9 / 13 / 95 \\
9 / 18 / 95\end{array}$ & $\begin{array}{l}252.06^{*} \\
251.76\end{array}$ & $\begin{array}{l}454.80 \\
455.10\end{array}$ & $\begin{array}{l}138.62 \\
138.71\end{array}$ \\
\hline $299-146-10$ & $\begin{array}{l}9 / 13 / 95 \\
9 / 18 / 95\end{array}$ & $\begin{array}{l}260.11 * \\
259.98\end{array}$ & $\begin{array}{l}452.37 \\
452.50\end{array}$ & $\begin{array}{l}137.88 \\
137.92\end{array}$ \\
\hline $299-16-11$ & $\begin{array}{l}9 / 15 / 95 \\
9 / 18 / 95\end{array}$ & $\begin{array}{l}250.11^{*} \\
250.15\end{array}$ & $\begin{array}{l}452.75 \\
452.71\end{array}$ & $\begin{array}{l}138.00 \\
137.99\end{array}$ \\
\hline $299-16-12$ & $\begin{array}{l}9 / 15 / 95 \\
9 / 18 / 95\end{array}$ & $\begin{array}{l}238.08^{\star+} \\
238.71\end{array}$ & $\begin{array}{l}454.43 \\
453.80\end{array}$ & $\begin{array}{l}138.51 \\
138.32\end{array}$ \\
\hline $299-1+6-2$ & $\begin{array}{l}9 / 11 / 95 \\
9 / 18 / 95\end{array}$ & $\begin{array}{l}237.52^{*} \\
237.19\end{array}$ & $\begin{array}{l}454.93 \\
455.26\end{array}$ & $\begin{array}{l}138.65 \\
138.76\end{array}$ \\
\hline $299-16-4$ & $\begin{array}{l}9 / 14 / 95 \\
9 / 18 / 95\end{array}$ & $\begin{array}{l}246.13^{\star} \\
246.11\end{array}$ & $\begin{array}{l}455.12 \\
455.14\end{array}$ & $\begin{array}{l}138.72 \\
138.73\end{array}$ \\
\hline $299-16-7$ & $\begin{array}{l}9 / 18 / 95 \\
9 / 18 / 95\end{array}$ & $\begin{array}{l}258.48 * \\
258.36\end{array}$ & $\begin{array}{l}451.80 \\
451.92\end{array}$ & $\begin{array}{l}137.71 \\
137.75\end{array}$ \\
\hline $299-116-8$ & $\begin{array}{l}9 / 18 / 95 \\
9 / 21 / 95\end{array}$ & $\begin{array}{l}241.44 \\
241.68 *\end{array}$ & $\begin{array}{r}453.29 \\
453.05\end{array}$ & $\begin{array}{l}138.16 \\
138.09\end{array}$ \\
\hline $299-146-9$ & $\begin{array}{l}9 / 14 / 95 \\
9 / 18 / 95\end{array}$ & $\begin{array}{l}240.95^{*} \\
240.95\end{array}$ & $\begin{array}{l}455.15 \\
455.15\end{array}$ & $\begin{array}{l}138.73 \\
138.73\end{array}$ \\
\hline $299-67-10$ & $\begin{array}{l}9 / 11 / 95 \\
9 / 18 / 95\end{array}$ & $\begin{array}{l}235.22^{*} \\
234.94\end{array}$ & $\begin{array}{l}454.44 \\
454.72\end{array}$ & $\begin{array}{l}138.51 \\
138.60\end{array}$ \\
\hline
\end{tabular}


Table 14-11. RCRA Water Level Measurement Report LLWHA 5, Third Quarter 1995.

(sheet 2 of 2)

\begin{tabular}{|c|c|c|c|c|}
\hline We11 & Date & $\begin{array}{l}\text { Depth to } \\
\text { water (ft) }\end{array}$ & $\begin{array}{r}\text { Hater } \\
\text { elevation } \\
\text { (ft) }\end{array}$ & $\begin{array}{l}\text { level } \\
\text { above ms1 } \\
\text { (m) }\end{array}$ \\
\hline \multicolumn{5}{|c|}{ LLWHA-5 Mells Honitoring the Bottom of the Unconfined Aquifer } \\
\hline $299-146-3$ & $\begin{array}{l}9 / 14 / 95 \\
9 / 18 / 95\end{array}$ & $\begin{array}{l}245.45^{\star} \\
245.32\end{array}$ & $\begin{array}{l}454.38 \\
454.51\end{array}$ & $\begin{array}{l}138.50 \\
138.53\end{array}$ \\
\hline $299-N 6-6$ & $\begin{array}{l}9 / 18 / 95 \\
9 / 18 / 95\end{array}$ & $\begin{array}{l}258.35 * \\
258.07\end{array}$ & $\begin{array}{l}451.64 \\
451.92\end{array}$ & $\begin{array}{l}137.66 \\
137.75\end{array}$ \\
\hline
\end{tabular}

NOTES: 1. Water level elevations are calculated by subtracting the measured depth-to-water from the surveyed elevation for the well.

2. Depth-to-water values are transcribed from field records.

3. Weasurements marked with an ' $\neq 1$ were taken at the time of sampling.

4. Measurements marked with $a$ ' $\neq$ ' are outside of the expected range, and are suspected of error. 
Table 14-12. Constituent List and Summary of Results for the Low-Level Waste Management Area 1 Data

for Reporting Period July 1

through September 30, 1995.

(sheet 1 of 2)

contandusion tibjCATOR PARAnetEas

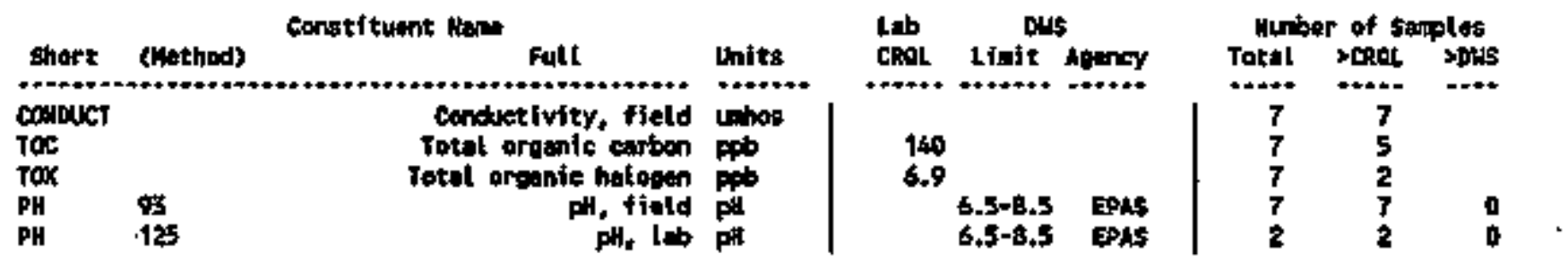

DRJWKIMG WNTE PARNoTERS

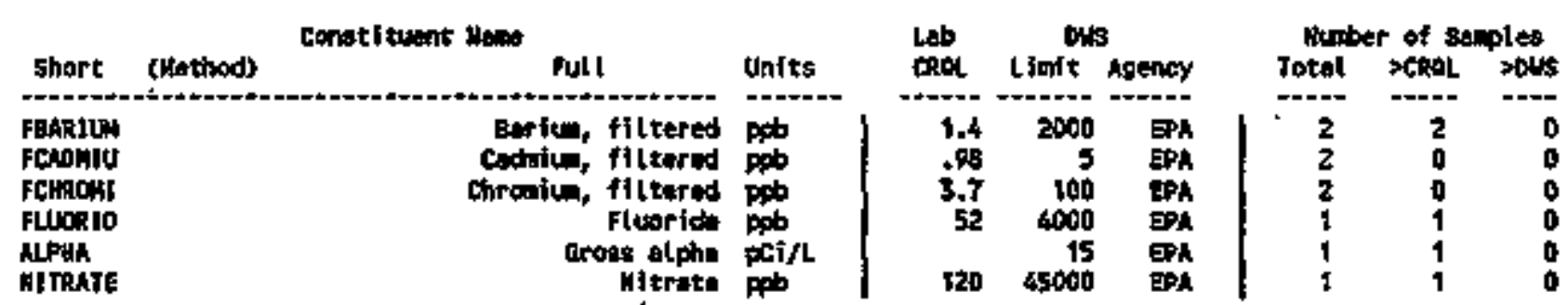

EROUHOLATER OUALITY PHANETERS

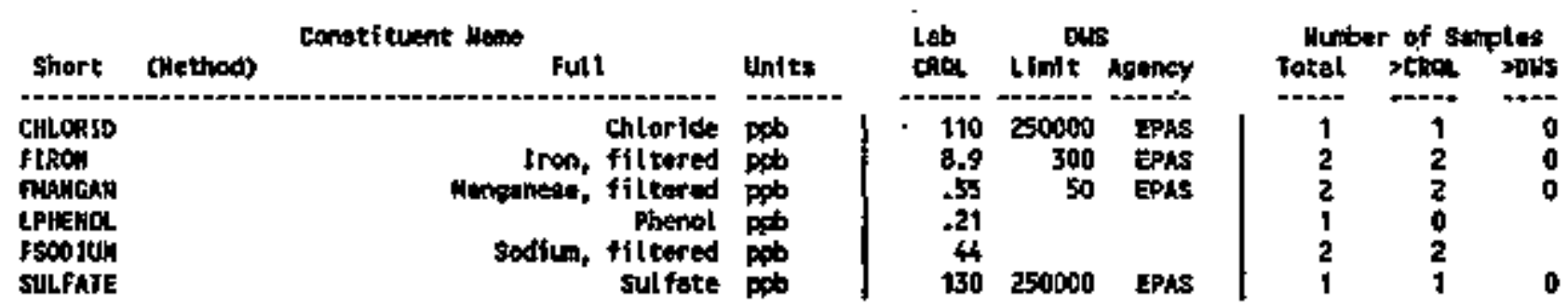

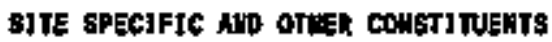

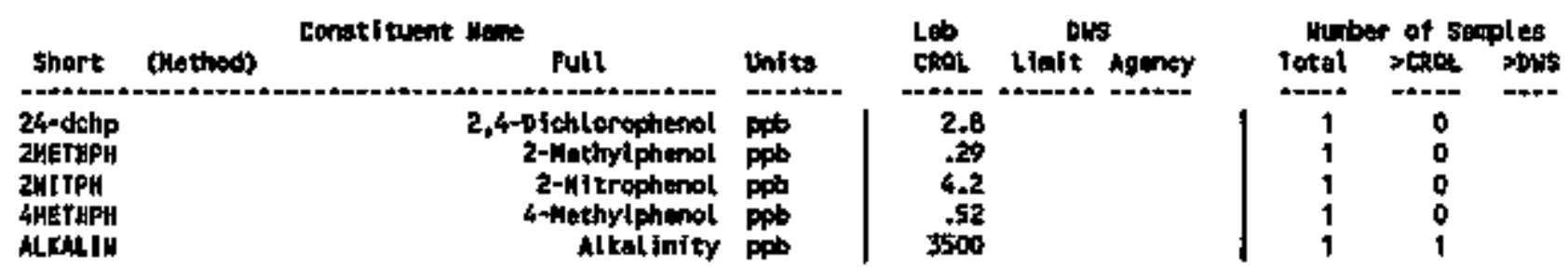


Table 14-12. Constituent List and Sunmary of Results for the Low-Level Waste Management Area 1 Data

for Reporting Period July 1

through September 30, 1995.

(sheet 2 of 2)

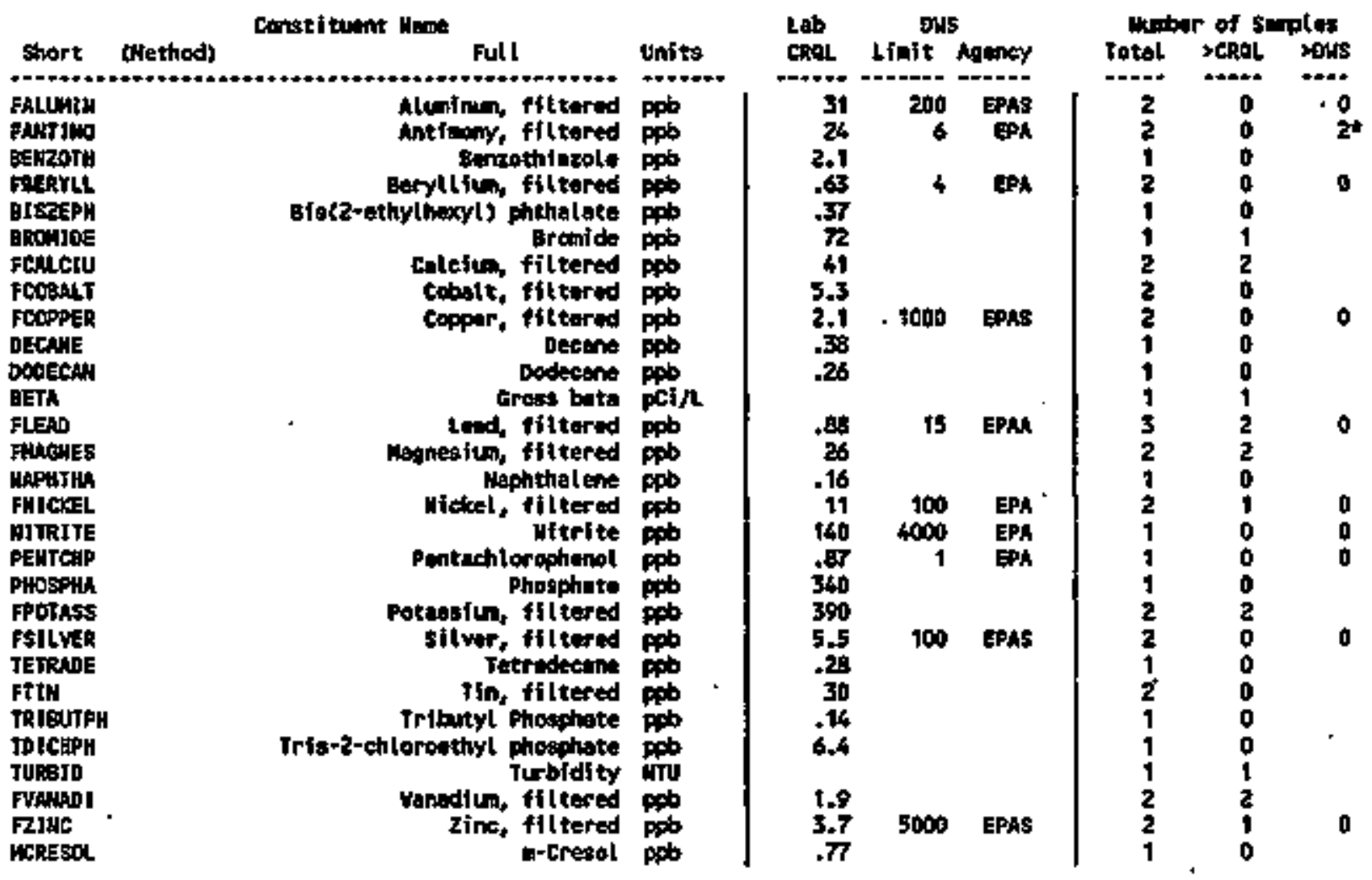

For explention of this table, bee section 1.4 of report. 
Table 14-13, Constituents with at Least One Detected Value for the Lol-Level Haste Management Area 1 Data for Reporting Period July 1 through Septerber 30, 1995.

\begin{tabular}{|c|c|c|c|c|c|c|}
\hline Nell & $\begin{array}{c}\text { Cotlection } \\
\text { Dute }\end{array}$ & Somple & - $\frac{\text { llkatid }}{357 / 00 b}$ & $\begin{array}{l}\text { EBARJtin } \\
\text { 34/pob } \\
1.4 / 2000\end{array}$ & 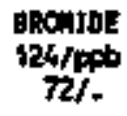 & $\begin{array}{c}\text { FEllcIU } \\
34 / f p^{3} \\
41 / .\end{array}$ \\
\hline $\begin{array}{l}299-E 3 z-4 \\
299-E 3 z-4 \\
299-E 33-35\end{array}$ & $\begin{array}{l}7 / 19 / 95 \\
7 / 18 / 95 \\
6 / 29 / 95\end{array}$ & 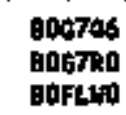 & 120000,00 & $\begin{array}{r}69.00 \\
34.00\end{array}$ & $\$ 0.00$ & $\begin{array}{l}3900 \\
3000\end{array}$ \\
\hline
\end{tabular}

\begin{tabular}{|c|c|c|c|c|c|c|}
\hline thoth & $\begin{array}{c}\text { Collection } \\
\text { Datt: }\end{array}$ & sapple & $\begin{array}{c}\text { CHLORID } \\
\text { 124/ppb } \\
110 / 2000 \text { os }\end{array}$ & $\begin{array}{l}\text { FUNoRLD } \\
124 / \mathrm{ppb} \\
52 / 4000\end{array}$ & $\begin{array}{c}\text { ALPHA } \\
\text { 135/pCif/L } \\
. / 15\end{array}$ & $\begin{array}{c}\text { BETh } \\
\text { I36/pci } / \mathrm{L} \\
. / .\end{array}$ \\
\hline $296-E 32-4$ & $7 / 18 / 95$ & Bostas & 9200.00 & 800.00 & 3.74 & 10.60 \\
\hline
\end{tabular}

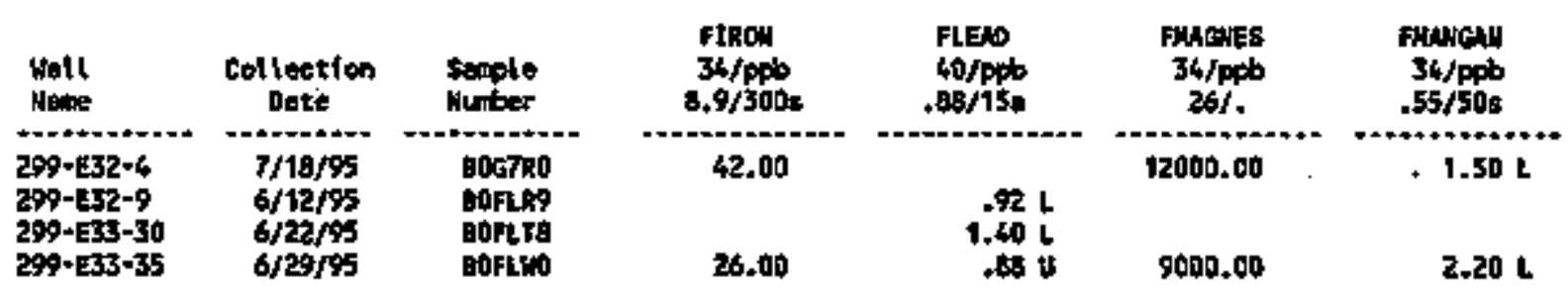

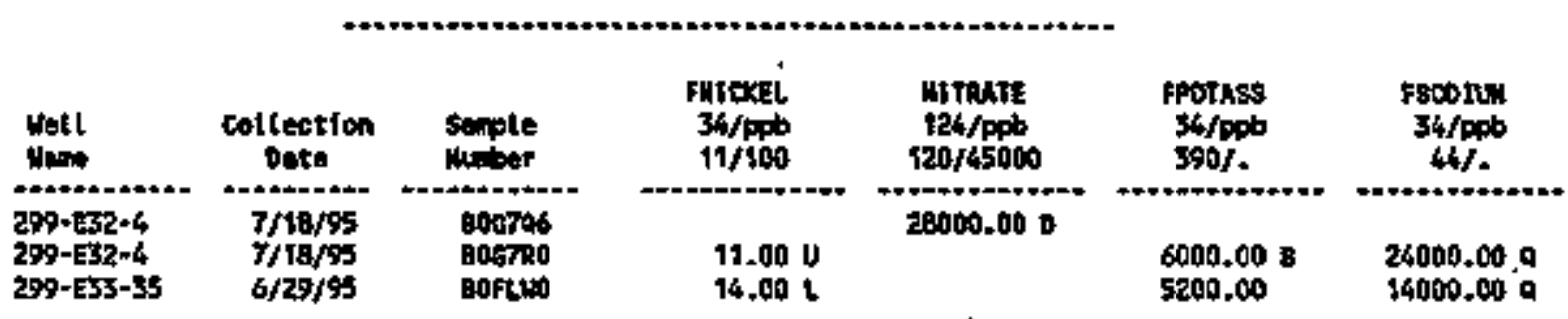

\begin{tabular}{|c|c|c|c|c|c|c|}
\hline Well & $\begin{array}{c}\text { Cotlection } \\
\text { nete }\end{array}$ & $\begin{array}{l}\text { sample } \\
\text { miber }\end{array}$ & $\begin{array}{c}\text { SULFATE } \\
\text { 124/ppb } \\
\text { 130/25000\%s }\end{array}$ & $\begin{array}{c}\text { Thasso } \\
111 / \mathrm{m} \text { d } \\
-/ .\end{array}$ & $\begin{array}{l}\text { Whupl } \\
34 / \mathrm{ppb} \\
1.9 \%\end{array}$ & $\begin{array}{c}\text { rtlkt } \\
34 / \mathrm{ppb} \\
3.7 / 5000 \mathrm{~s}\end{array}$ \\
\hline $\begin{array}{l}299-E 32-4 \\
299-E 32-4 \\
299-E 33-35\end{array}$ & $\begin{array}{l}7 / 14 / 45 \\
7 / 18 / 95 \\
6 / 29 / 95\end{array}$ & $\begin{array}{l}\text { Botres } \\
\text { Botrino } \\
\text { BofLito }\end{array}$ & $67000.60 \mathrm{D}$ & 13.50 & $\begin{array}{l}20.00 \mathrm{BL} \\
3.00 \mathrm{~L}\end{array}$ & $\begin{array}{l}3.50 \mathrm{la} \\
3.70 \mathrm{un}\end{array}$ \\
\hline
\end{tabular}

For explanstion of this tals, Bes Section 1,4 of report. 
Table 14-14. Contamination Indicator Parameters for the Low-Level Waste Management Area 1 Data for Reporting Perjod July 1 through Septerber 30, 1995.

\begin{tabular}{|c|c|c|c|c|c|c|c|c|}
\hline Holl & $\begin{array}{c}\text { Colleetion } \\
\text { Dute }\end{array}$ & $\begin{array}{l}\text { smple } \\
\text { Hinper. }\end{array}$ & $\begin{array}{c}\text { CON ELELD } \\
\text { Whe } \\
1 / .\end{array}$ & $\begin{array}{l}\text { Com Lo } \\
\text { utho } \\
1 / .\end{array}$ & $\begin{array}{l}\text { OH FTEW } \\
.01 / 6,5-8,5,\end{array}$ & $\begin{array}{c}\text { pt LB } \\
.01 / 6.5-8.59\end{array}$ & 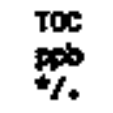 & $\begin{array}{l}\operatorname{Tox} \\
\text { pab } \\
t / .\end{array}$ \\
\hline $\begin{array}{l}299-E 2 \theta-2 B \\
299-E 32-4\end{array}$ & $\begin{array}{l}6 / 06 / \% 5 \\
7 / 18 / 45\end{array}$ & 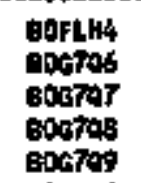 & $\begin{array}{l}413 \\
426 \\
423 \\
424 \\
423\end{array}$ & & $\begin{array}{l}7.75 \\
7.92 \\
7.92 \\
7.92 \\
7.92\end{array}$ & $\begin{array}{l}7.90 \\
8.00\end{array}$ & $\begin{array}{l}300 \mathrm{C} \\
200 \mathrm{C} \\
140 \mathrm{U} \\
140 \mathrm{~L} \\
140 \mathrm{U}\end{array}$ & $\begin{array}{l}6.9 \mathrm{U} \\
6.9 \mathrm{U} \\
6.9 \mathrm{U} \\
6.9 \mathrm{U} \\
6.9 \mathrm{U}\end{array}$ \\
\hline $\begin{array}{l}299-632-8 \\
299+E 32-9\end{array}$ & $\begin{array}{l}6 / 00 / 95 \\
6 / 12 / 95\end{array}$ & $\begin{array}{l}\text { BOFfR1 } \\
\text { gOFLR7 }\end{array}$ & $\begin{array}{l}351 \\
327\end{array}$ & - & $\begin{array}{l}8.07 \\
8.09\end{array}$ & & $\begin{array}{l}400 \mathrm{~L} \\
300 \mathrm{~L}\end{array}$ & $\begin{array}{l}7.0 \mathrm{~L} \\
8.0 \mathrm{~L}\end{array}$ \\
\hline
\end{tabular}

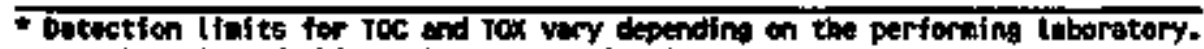
For explanation of this tuble, ine section 1.4 of raport. 
Tabie 14-15. Constituent List and Summary of Resuits for the Low-Lovel Maste Management Area 2 Data for Reporting

Period July 1 through September 30, 1995.

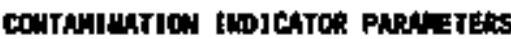

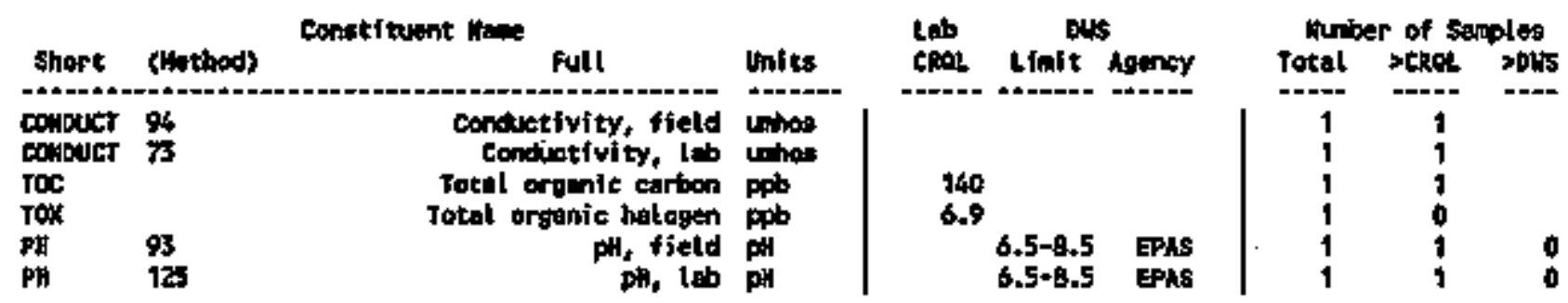

DAIEIMO WhTER PARNATERS

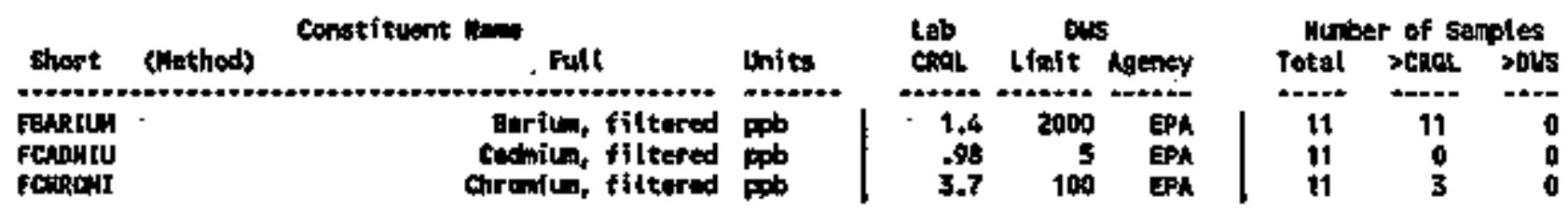

CROHOWHTER OUALITY PARUYETEAS

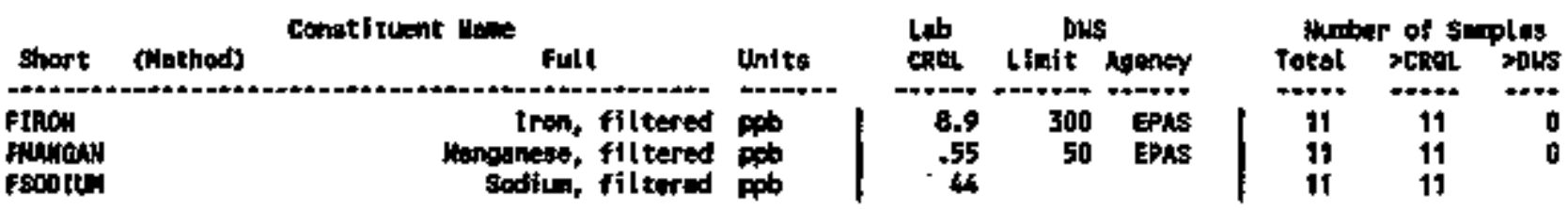

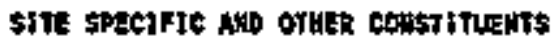

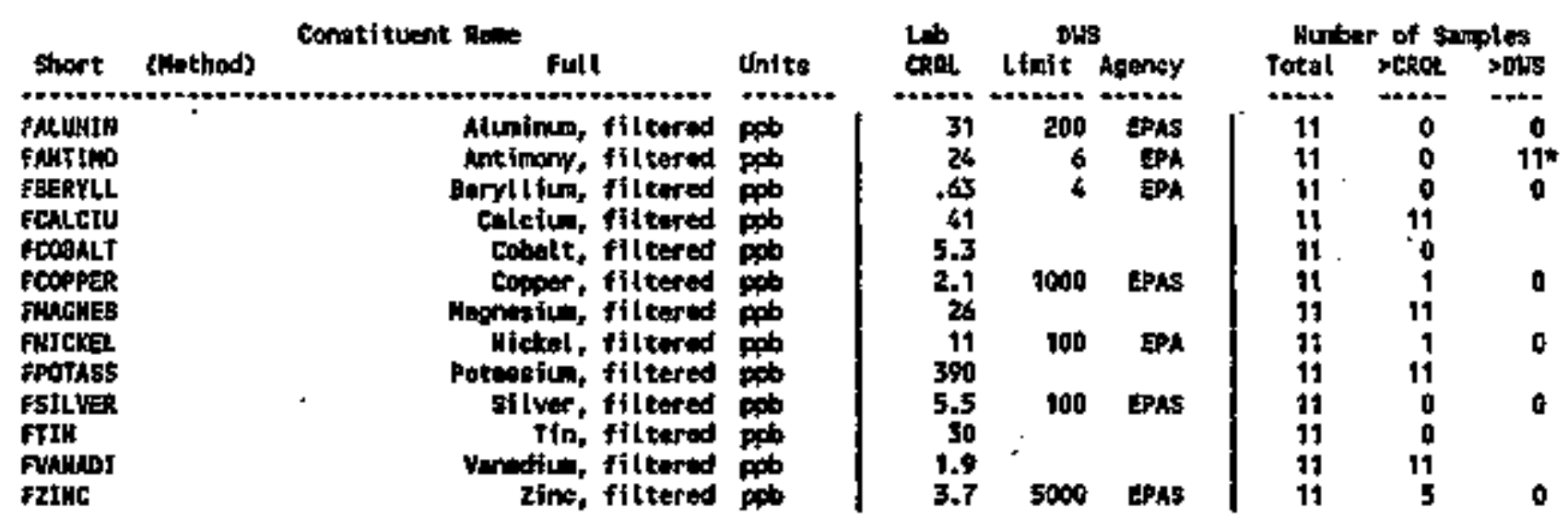

For explanation of this table, see section t. 4 of report. 
Table 14-16. Constituents with at Least One Detected Value for the Low-Level Waste Management Area 2 Data for Reporting Period July I through September 30, 1995.

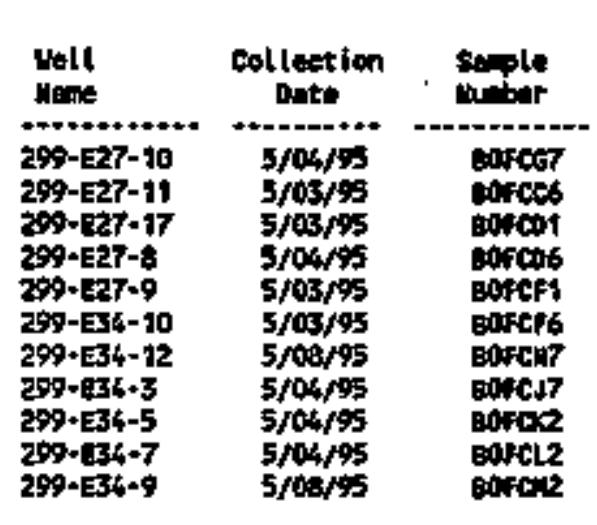

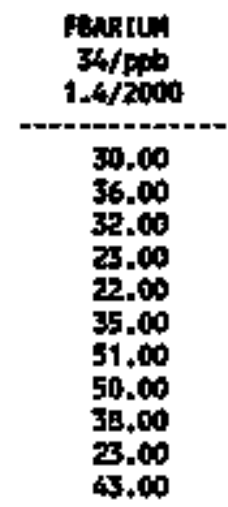

resuctu
$34 / 000$
$41 /$.
36000.00
35000.00
39000.00
34060.00
35400.00
35000.00
51000.00
41000.00
34000.00
40000.00
37000.00

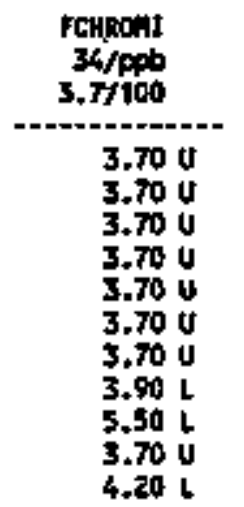

PCoper
$34 / 90 b$
$2.1 / 10005$
$2.10 \mathrm{U}$
$2.10 \mathrm{U}$
$3.10 \mathrm{~L}$
$2.10 \mathrm{U}$
$2.10 \mathrm{U}$
$2.10 \mathrm{U}$
$2.10 \mathrm{U}$
$2.10 \mathrm{U}$
$2.10 \mathrm{U}$
$2.10 \mathrm{U}$
$2.10 \mathrm{U}$

Wett

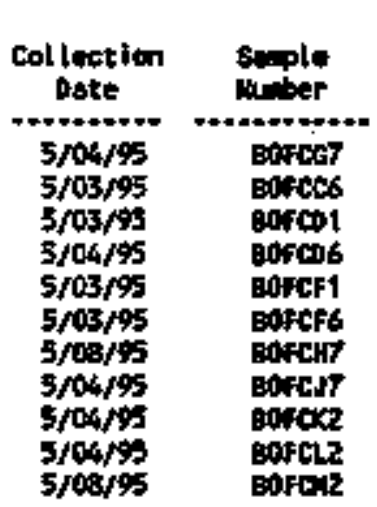

\begin{tabular}{|c|c|}
\hline $\begin{array}{c}\text { FIRas } \\
34 / \text { aw } \\
\text { 9.9/300s }\end{array}$ & $\begin{array}{l}\text { fincles } \\
34 / \text { pab } \\
26 / .\end{array}$ \\
\hline $\begin{array}{c}31.00 \mathrm{~B} \\
170.00 \mathrm{~B} \\
14.00 \mathrm{BL} \\
25.00 \mathrm{~B} \\
30.00 \mathrm{~B} \\
16.00 \mathrm{BL} \\
25.00 \mathrm{~B} \\
34.00 \mathrm{~B} \\
54.00 \mathrm{~B} \\
67.00 \mathrm{~B} \\
21.00 \mathrm{~B}\end{array}$ & $\begin{array}{r}9400.00 \\
\$ 400.00 \\
11000.00 \\
\$ 300.00 \\
10000.00 \\
\$ 200.00 \\
14000.00 \\
11000.00 \\
11000.00 \\
14000.00 \\
10000.00\end{array}$ \\
\hline
\end{tabular}

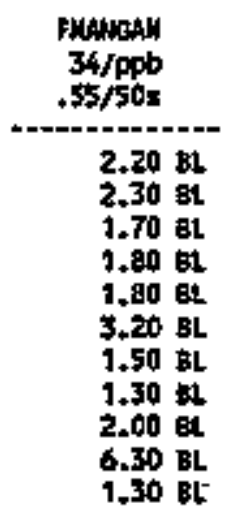

$7.1 \mathrm{CKEL}$
$34 / 0 \mathrm{pb}$
$11 / 100$
$11.00 \mathrm{U}$
$11.00 \mathrm{U}$
$11.00 \mathrm{U}$
$11.00 \mathrm{U}$
$11.00 \mathrm{U}$
$11.00 \mathrm{U}$
$11.00 \mathrm{U}$
$11.00 \mathrm{U}$
$11.00 \mathrm{U}$
$34.00 \mathrm{U}$
$11.00 \mathrm{U}$

$259-\mathbf{B} 4-7$

5rosis

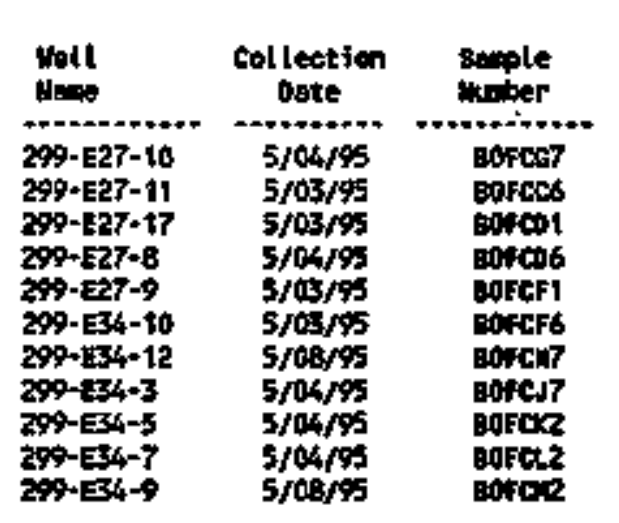

FPOTASS
$34 / p p+6$
$390 \%$.
5800.09
6000.00
6700.00
6400.00
5900.00
6200.00
7690.00
6000.00
7800.00
7200.00
6300.00

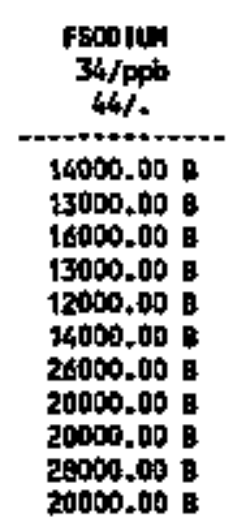

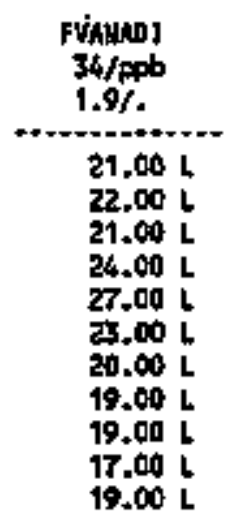

FInC

$34 / \mathrm{Ppb}$

$3.7 / 5000 \mathrm{~s}$

$8.30 \mathrm{t}$

$6.80 \mathrm{~L}$

$6.00 \mathrm{~L}$

$9.70 \mathrm{~L}$

$\mathbf{3 . 7 0} \mathrm{U}$

$3.70 \mathrm{~V}$

3.70 U

3.70 U

$3.70 \mathrm{U}$

$3.70 \mathrm{~V}$

$4.50 \mathrm{~L}$

For explenecion of this tabte, dee section 7.4 of raport. 
Tabie 14-17. Contaxination Indicator Parameters for the Low-Level Waste Management Area 2 Data for Reporting Period July 1 through September 30, 1995.

\begin{tabular}{|c|c|c|c|c|c|c|c|c|}
\hline Well & collection & $\begin{array}{l}\text { suple } \\
\text { Hupter }\end{array}$ & $\begin{array}{c}\text { Cond FIELD } \\
\text { itho } \\
1 / .\end{array}$ & $\begin{array}{l}\text { cons us } \\
\text { who } \\
1 / .\end{array}$ & $\begin{array}{l}\text { PH FIELD } \\
.01 / 6.5-8.58\end{array}$ & $\begin{array}{c}\text { pH } 14 \mathrm{~B} \\
.01 / 6.5-8.5 t\end{array}$ & $\begin{array}{l}\text { ras } \\
\text { pob } \\
\text { nt. }\end{array}$ & $\begin{array}{l}\text { Tox } \\
\text { epb } \\
+1 .\end{array}$ \\
\hline$-B 36-11$ & $5 / 0 \% / 95$ & eorecs & 443 & 440 & 7.96 & 8,00 & 201 & $6.9 \mathrm{~V}$ \\
\hline
\end{tabular}

- Detection linits for TCE and Tox viry eipending on the parforming laboratory. for explention of this toble, ate section $t .4$ of report. 
Table 14-18. Constttuent List and Summary of Results for the Low-Level Waste Hanagenent Area 3 Data for Reporting Pertod July 1 through Septenber 30, 1995.

(sheet 1 of 2)

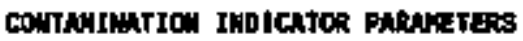

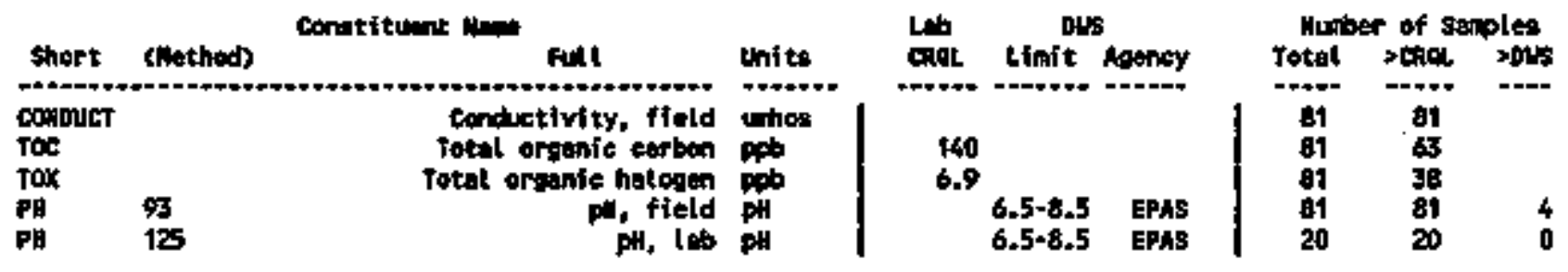

DRINKINE WHTER PARUVETERS

\begin{tabular}{|c|c|c|c|c|c|c|c|c|c|}
\hline & & Constituent Mas; & & Leb & ows & & Num & $r$ of $\mathrm{st}$ & Ples \\
\hline & (Winthod) & Fult & Units & catos & Lipst & Agency & Totel & $>5004$ & $>$ DUS \\
\hline 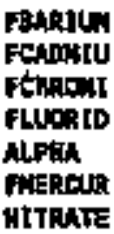 & & 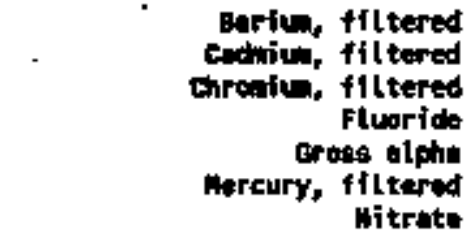 & 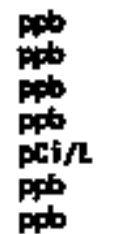 & $\begin{array}{r}1.4 \\
.08 \\
3.7 \\
52 \\
.019 \\
120\end{array}$ & $\begin{array}{r}2000 \\
5 \\
100 \\
4000 \\
15 \\
2 \\
45000\end{array}$ & $\begin{array}{l}\text { EPA } \\
\text { EPA } \\
\text { EPA } \\
\text { EPA } \\
\text { EPh } \\
\text { EPA } \\
\text { EPA }\end{array}$ & $\begin{array}{l}20 \\
20 \\
20 \\
20 \\
20 \\
20 \\
20\end{array}$ & $\begin{array}{r}20 \\
4 \\
19 \\
19 \\
6 \\
9 \\
20\end{array}$ & $\begin{array}{l}0 \\
0 \\
0 \\
0 \\
0 \\
0 \\
5\end{array}$ \\
\hline
\end{tabular}

BOUIPHHER CULLITY PARANETERS

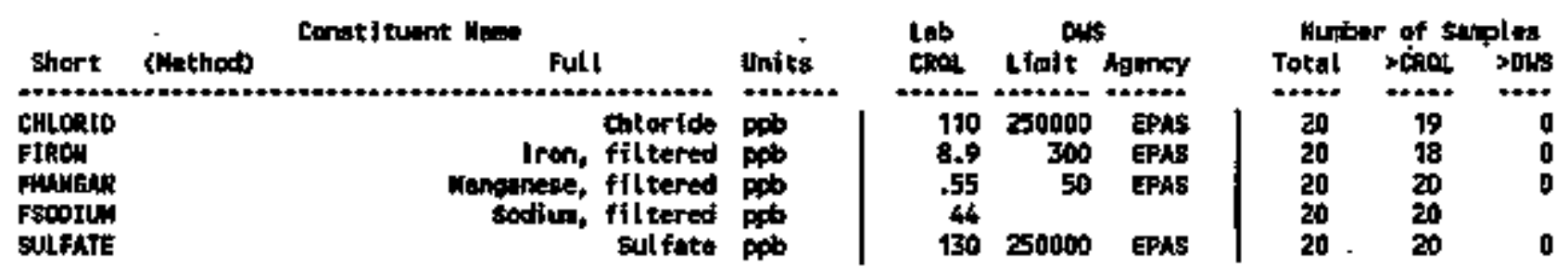

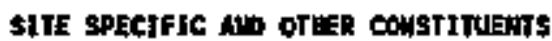

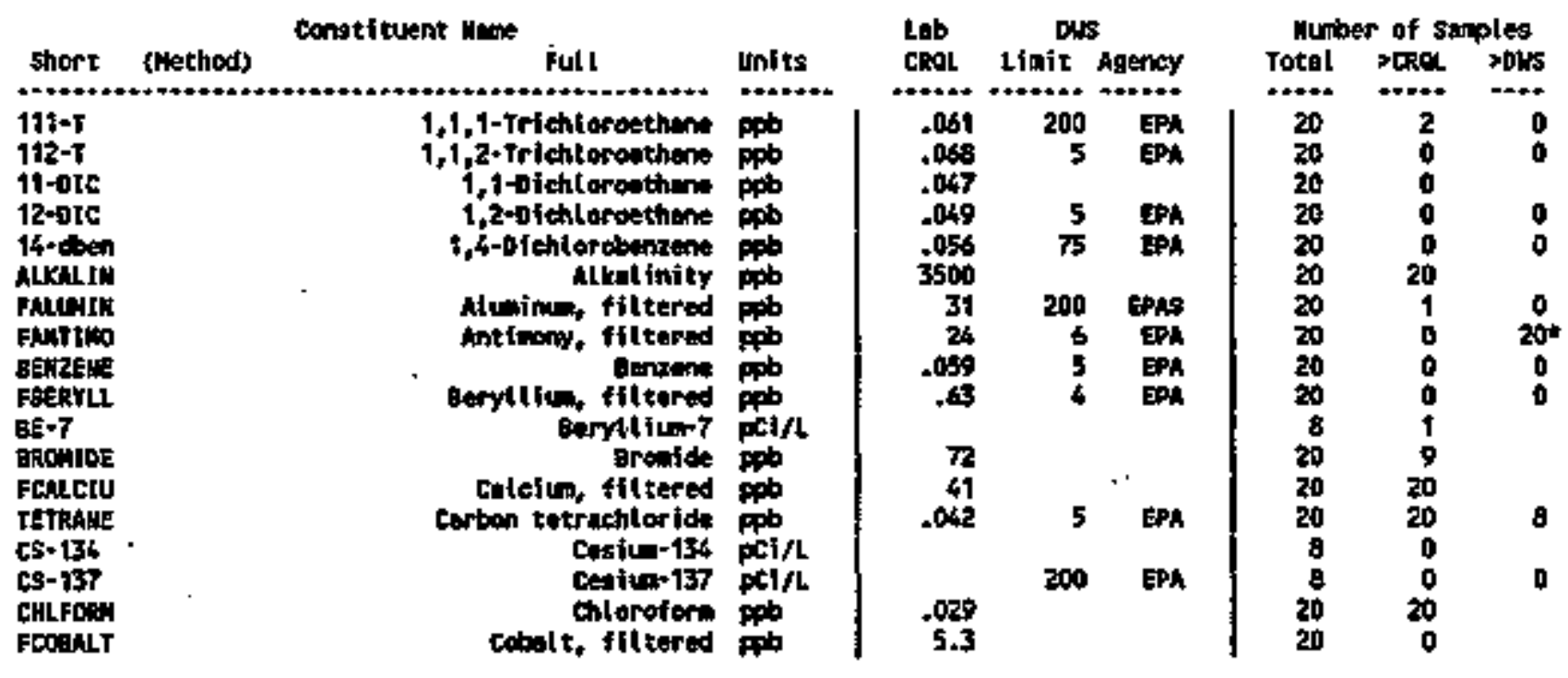


Tab7e 14-18. Constituent List and Swmary of Results for the Low-Level Waste Managenent Area 3 Data for Reporting

Period July 1 through Septewber 30, 1995.

(sheet 2 of 2)

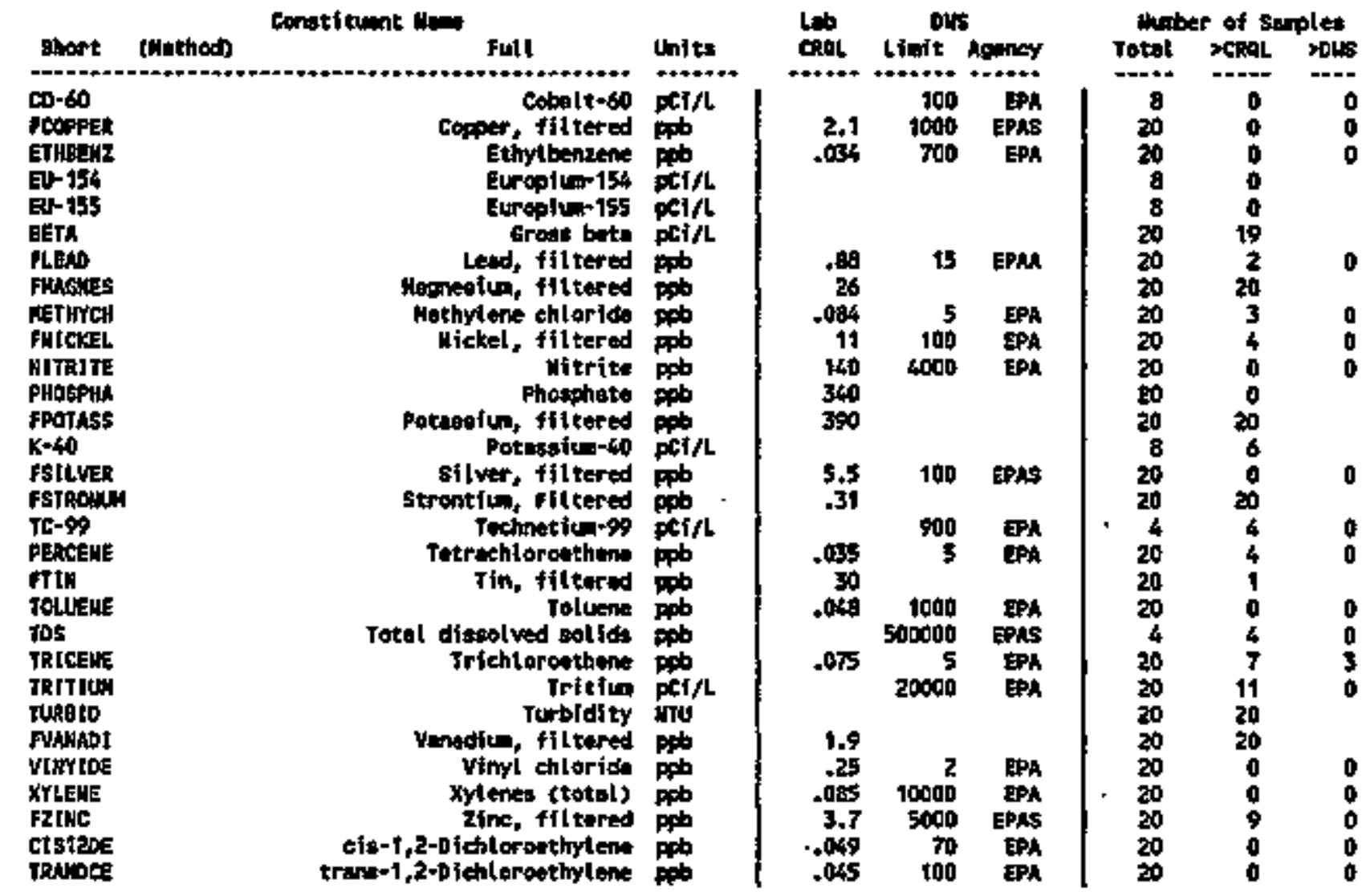

7ar explanation of this teble, ses section 1.4 of report. 
Table 14-19. Constituents with at Least one Detected Value for the Low-Level Waste Management Area 3 Data for Reporting

Period July 1 through September 30, 1995.

(sheet 1 of 10)

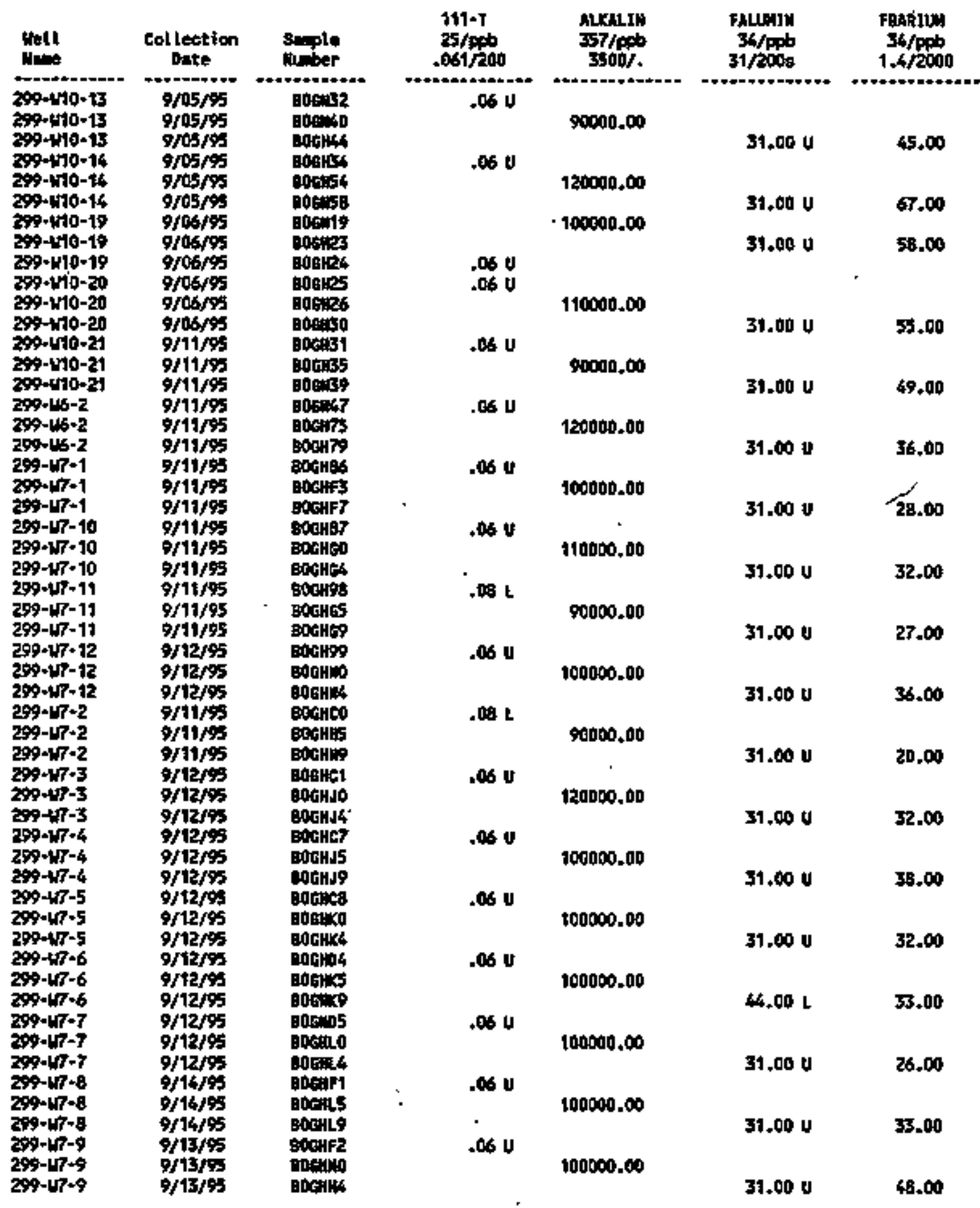


Tabie 14-19. Constituents with at Least One Detected Va]ue for the
Low-Level Waste Hanagement Area 3 Data for Report Ing Period JuTy I through September 30, 1995. ,

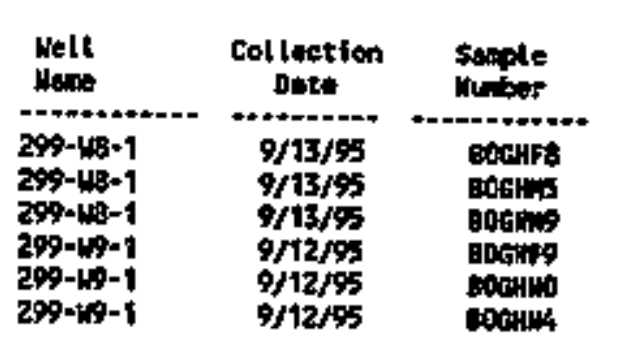
(sheet 2 of 10)

\begin{tabular}{|c|c|c|c|}
\hline $\begin{array}{c}111-T \\
25 / p p b \\
.051 / 200\end{array}$ & $\begin{array}{l}\text { 4tKaLIu } \\
357 / \mathrm{ppb} \\
3500 / .\end{array}$ & $\begin{array}{l}\text { Fatuith } \\
\text { 34/ppb } \\
31 / 200 \mathrm{~s}\end{array}$ & $\begin{array}{c}\text { PGaRLUM } \\
34 / \mathrm{ppb} \\
1.4 / 2000\end{array}$ \\
\hline $.06 \mathrm{U}$ & 100000.00 & & \\
\hline .064 & 90000.00 & $31.00 \mathrm{v}$ & 29.00 \\
\hline & & $34.00 \mathrm{v}$ & 32.00 \\
\hline
\end{tabular}

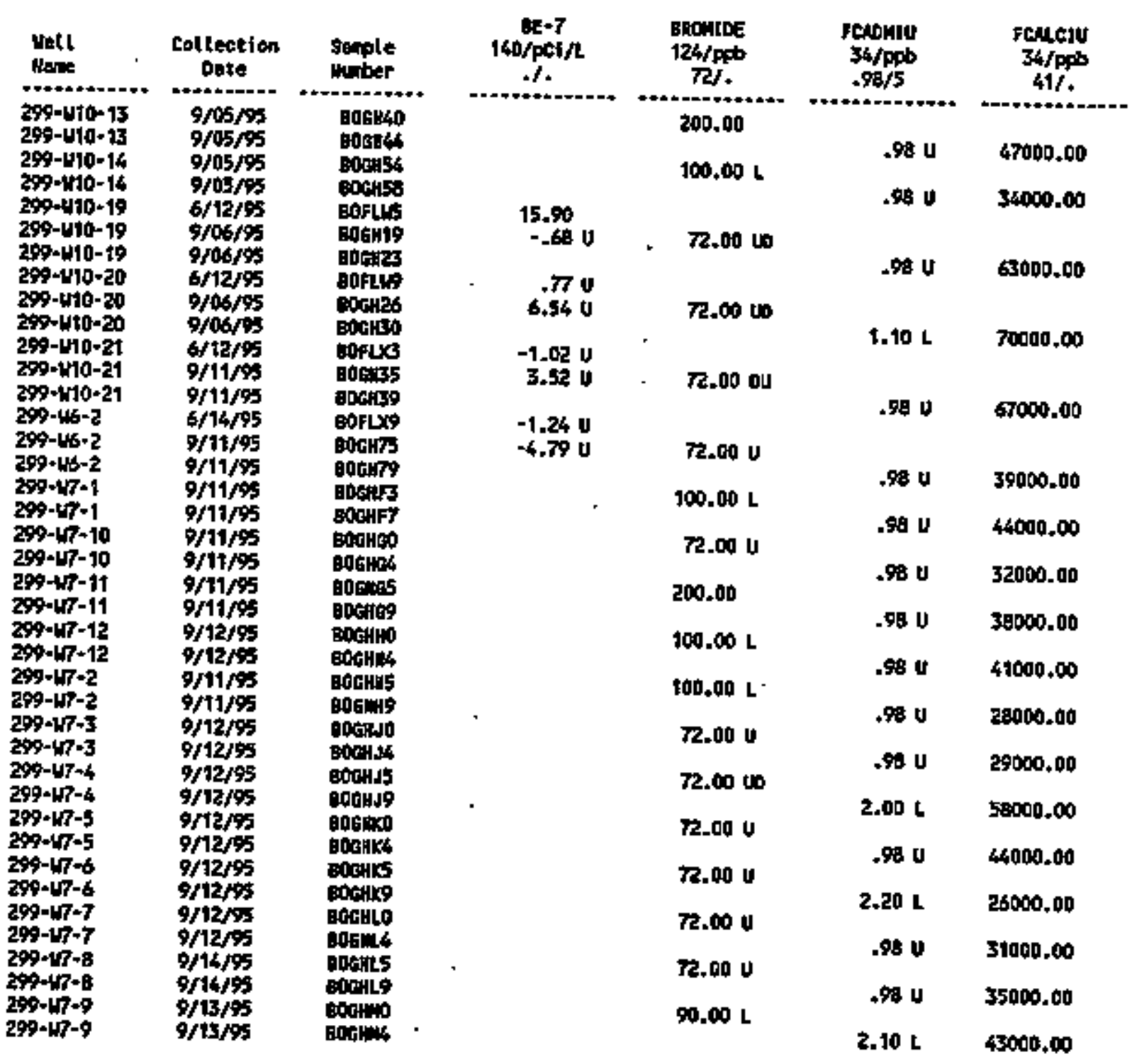


Table 14-19. Constituents with at Least One Detected $V_{a}$ ]ue for the Low-Level Waste Management Area 3 Data for Reporting

Period July 1 through September 30, 1995.

(sheet 3 of 10 )

\begin{tabular}{|c|c|c|}
\hline $\begin{array}{l}\text { Hetl } \\
\text { Hane }\end{array}$ & $\begin{array}{l}\text { Collection } \\
\text { Dete }\end{array}$ & Simpolt \\
\hline $\begin{array}{l}299-1+9-1 \\
20-1+9-1 \\
209-19-1 \\
299-19-1\end{array}$ & $\begin{array}{l}\% / 3 / \% \\
9 / 13 / 9 \\
9 / 2 / \% \\
9 / 2 / 95\end{array}$ & 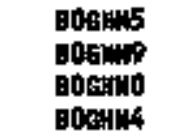 \\
\hline
\end{tabular}
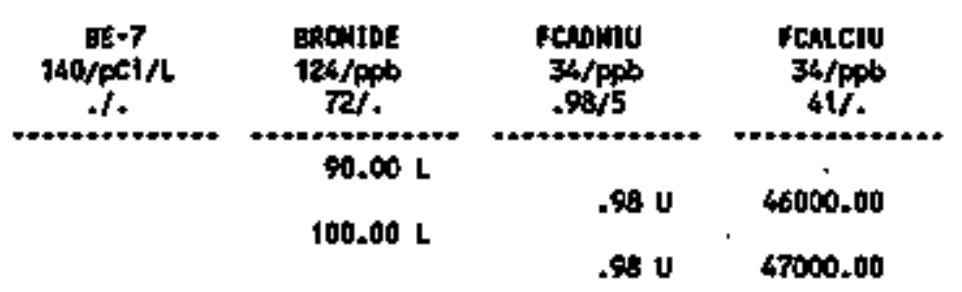

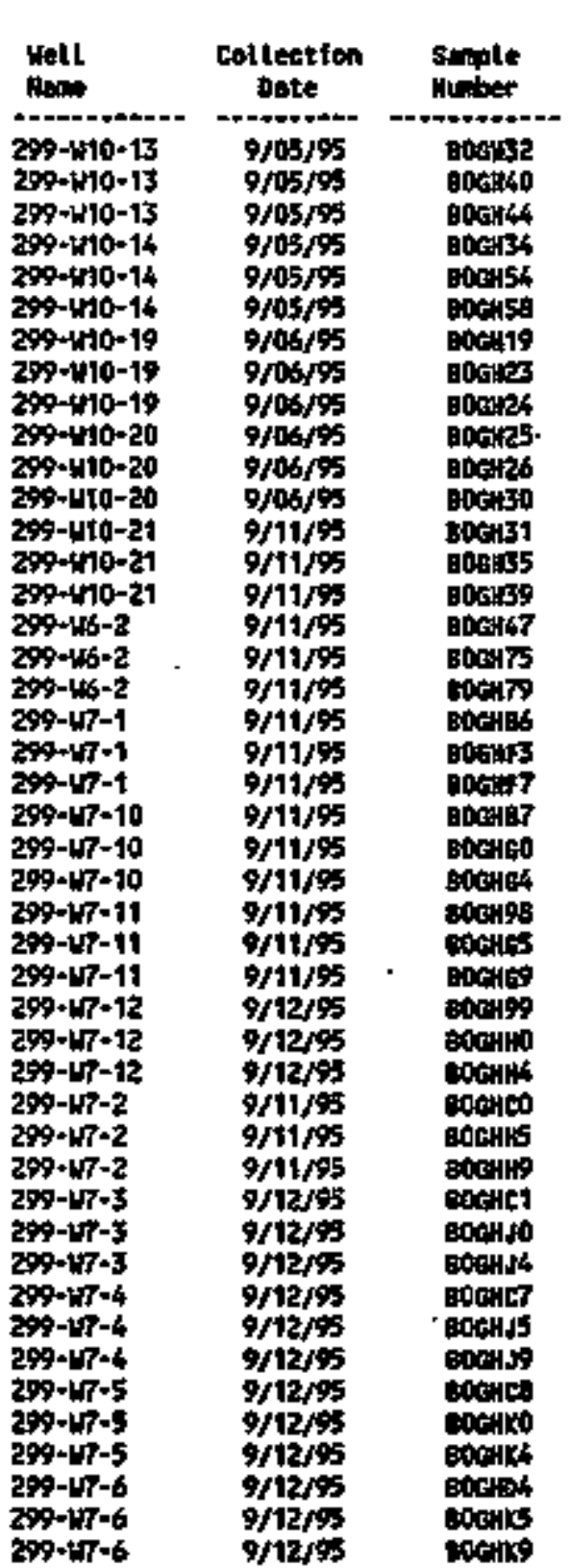

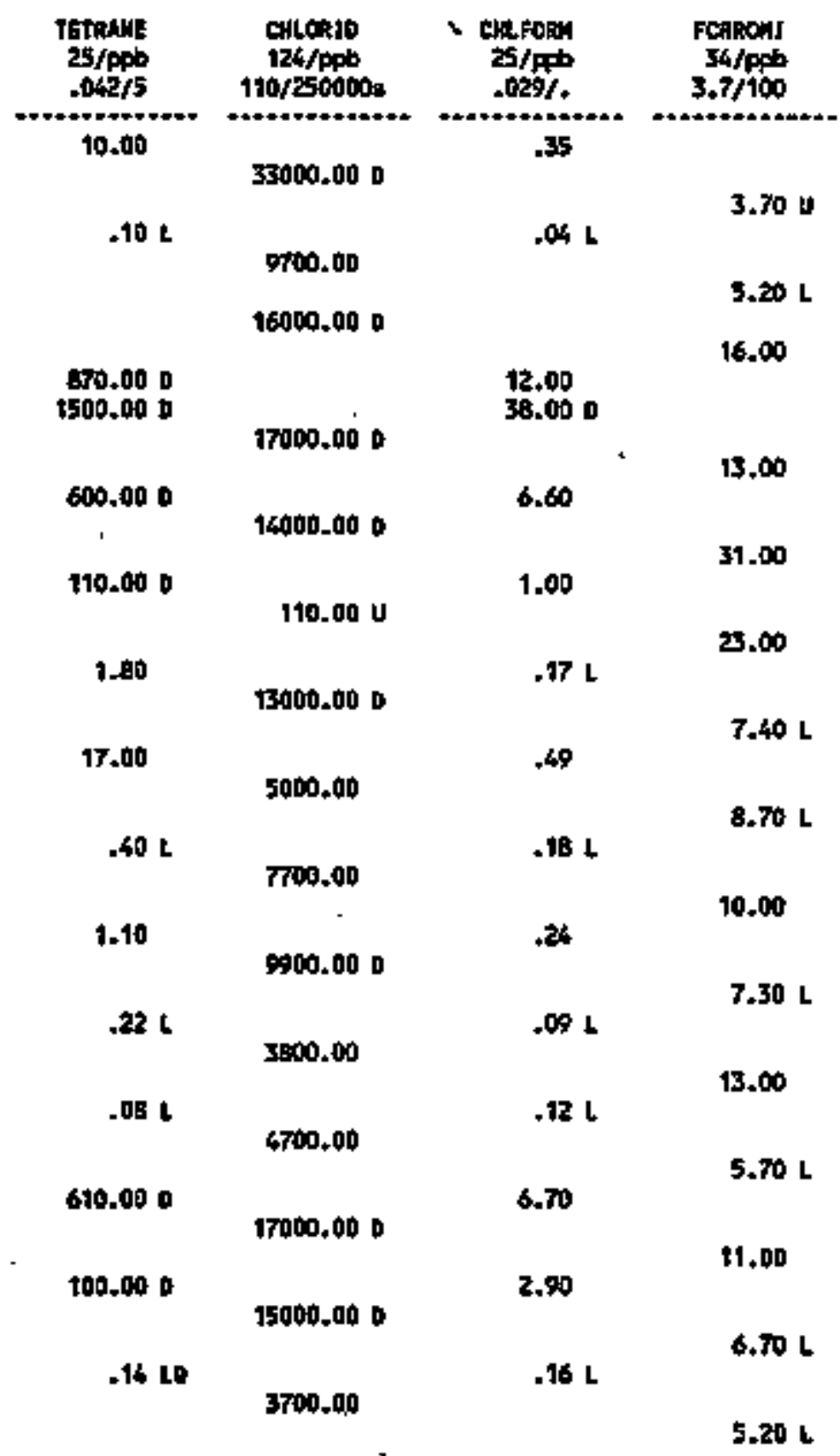


Table 14-19. Constituents with at Least One Detected Value for the Low-Level Waste Management Area 3 Data for Reporting

Period July 1 through September 30, 1995.

(sheet 4 of 10 )

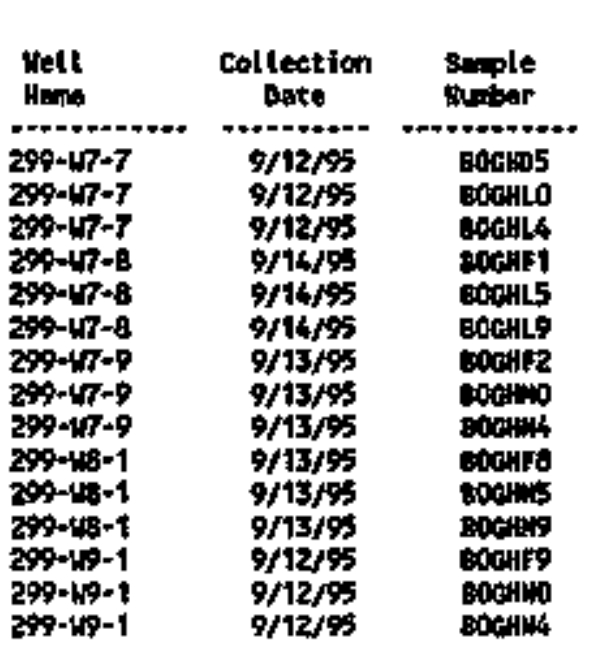

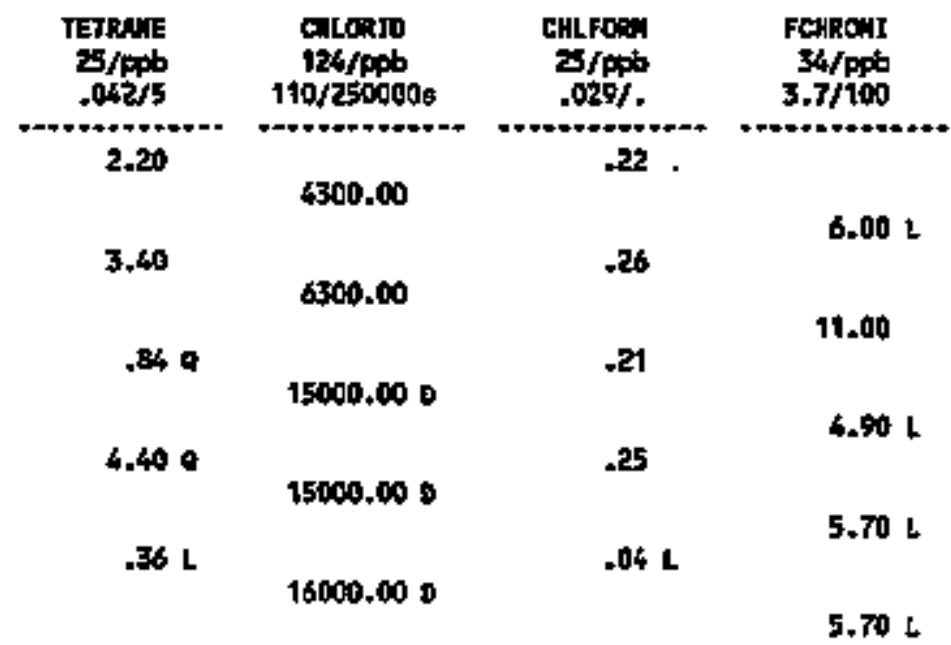

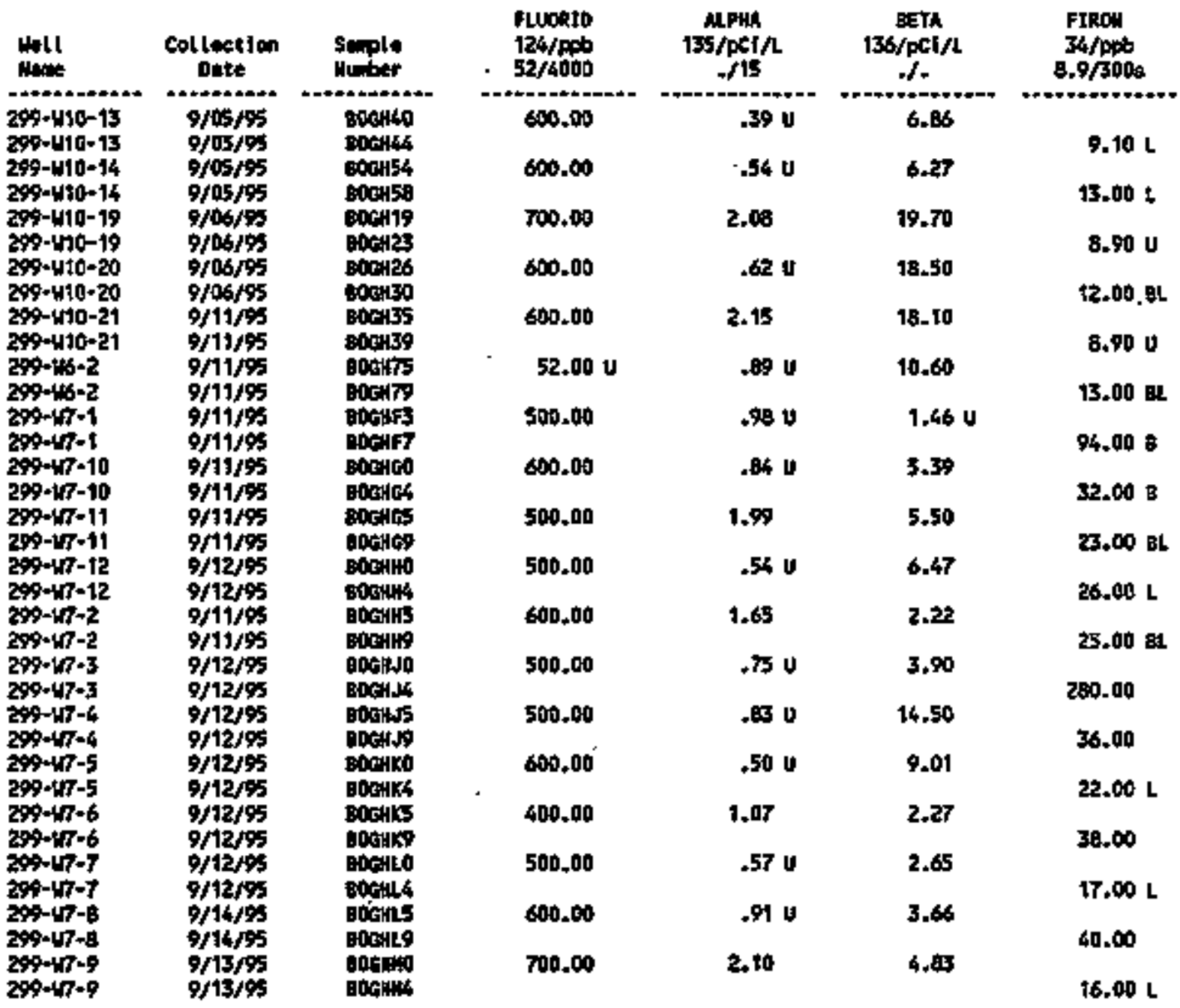




\section{Table 14-19. Constituents with at Least One Detected Value for the Low-Level Waste Management Area 3 Data for Reporting \\ Period July 1 through September 30, 1995. \\ (sheet 5 of 10 )}

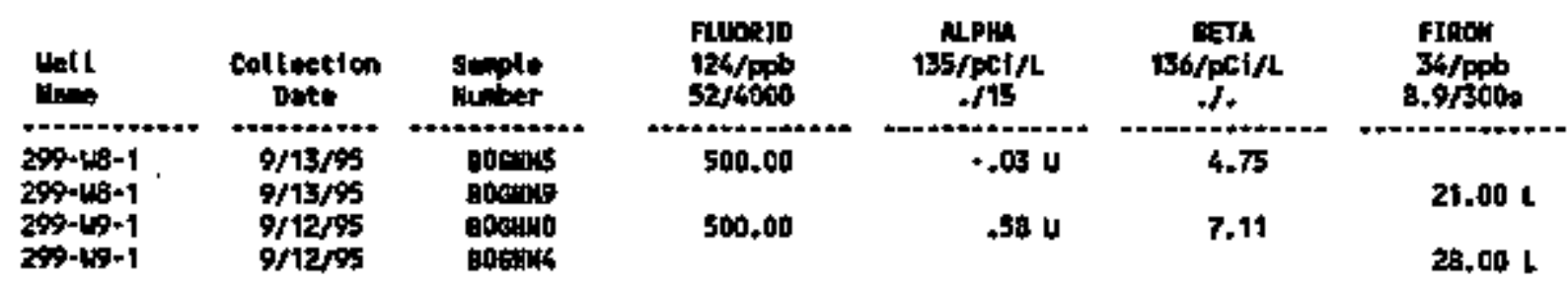

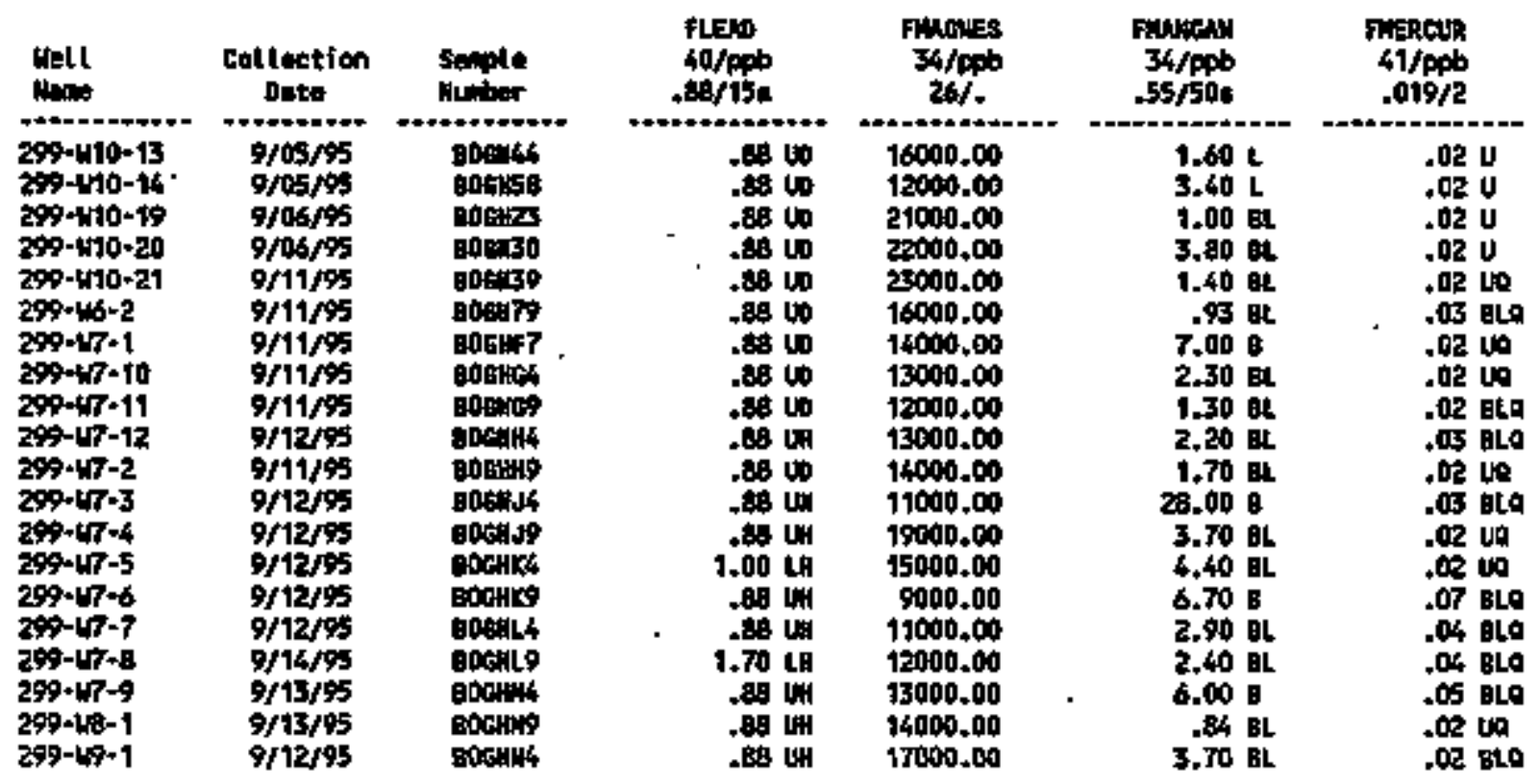

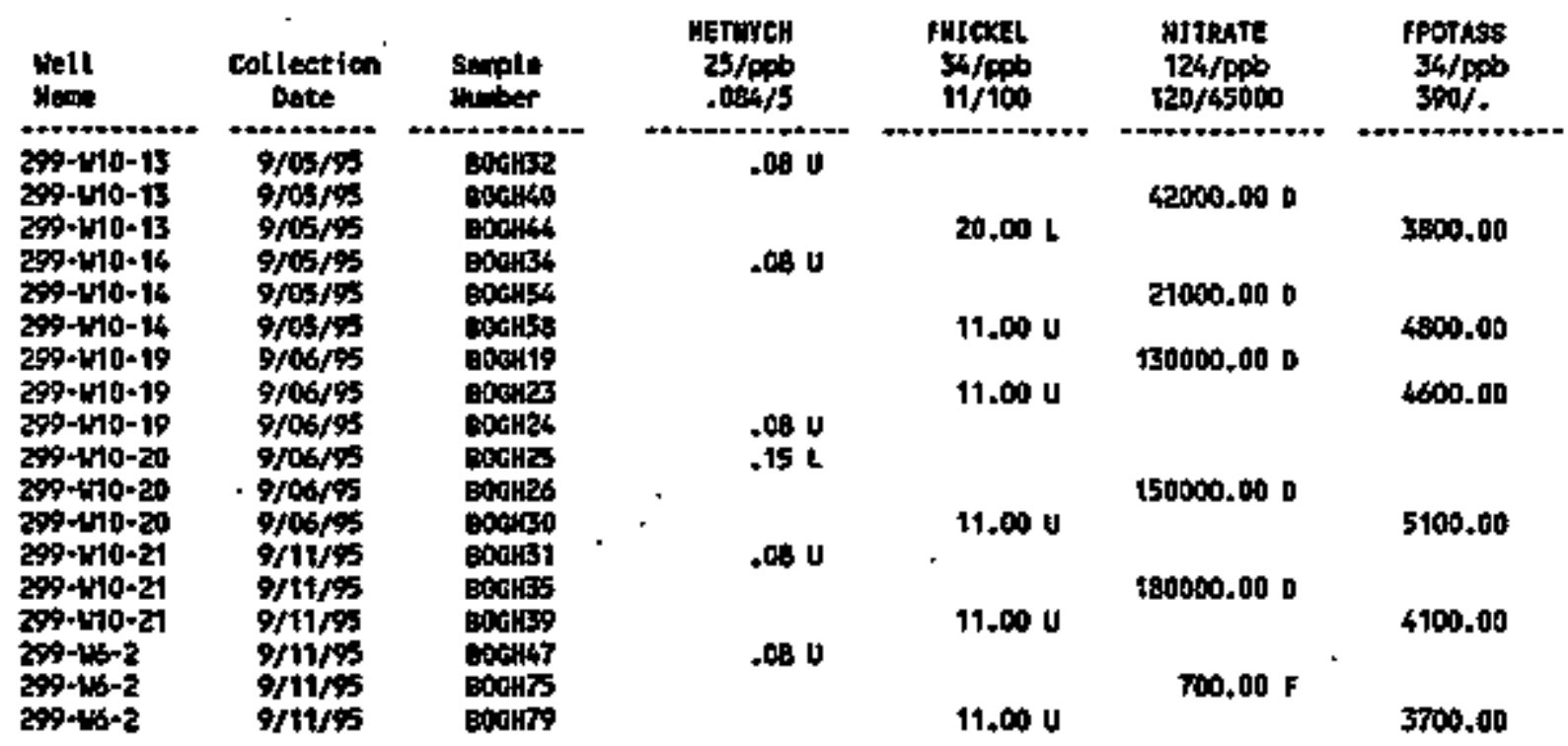


Table 14-19. Constituents with at Least One Detected Vajue for the Low-Level Waste Hanagenent Area 3 Data for Reporting

Perlod July 1 through September 30, 1995. (sheet 6 of 10)

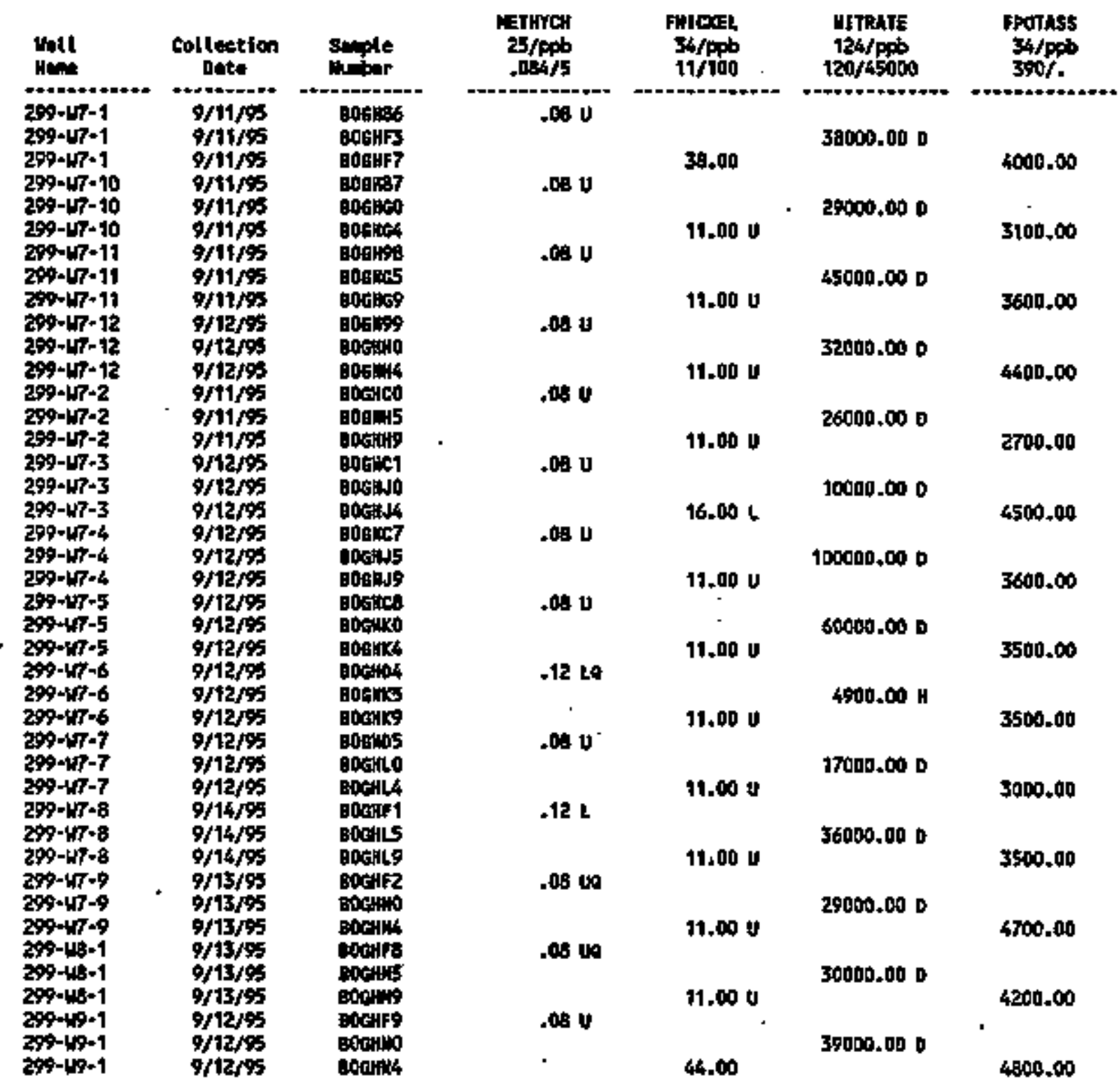


Table 14-19. Constituents with at Least One Detected Value for the Low-Level Waste Managenent Area 3 Data for Reporting

Period July 1 through September 30, 1995.

(sheet 7 of 10)

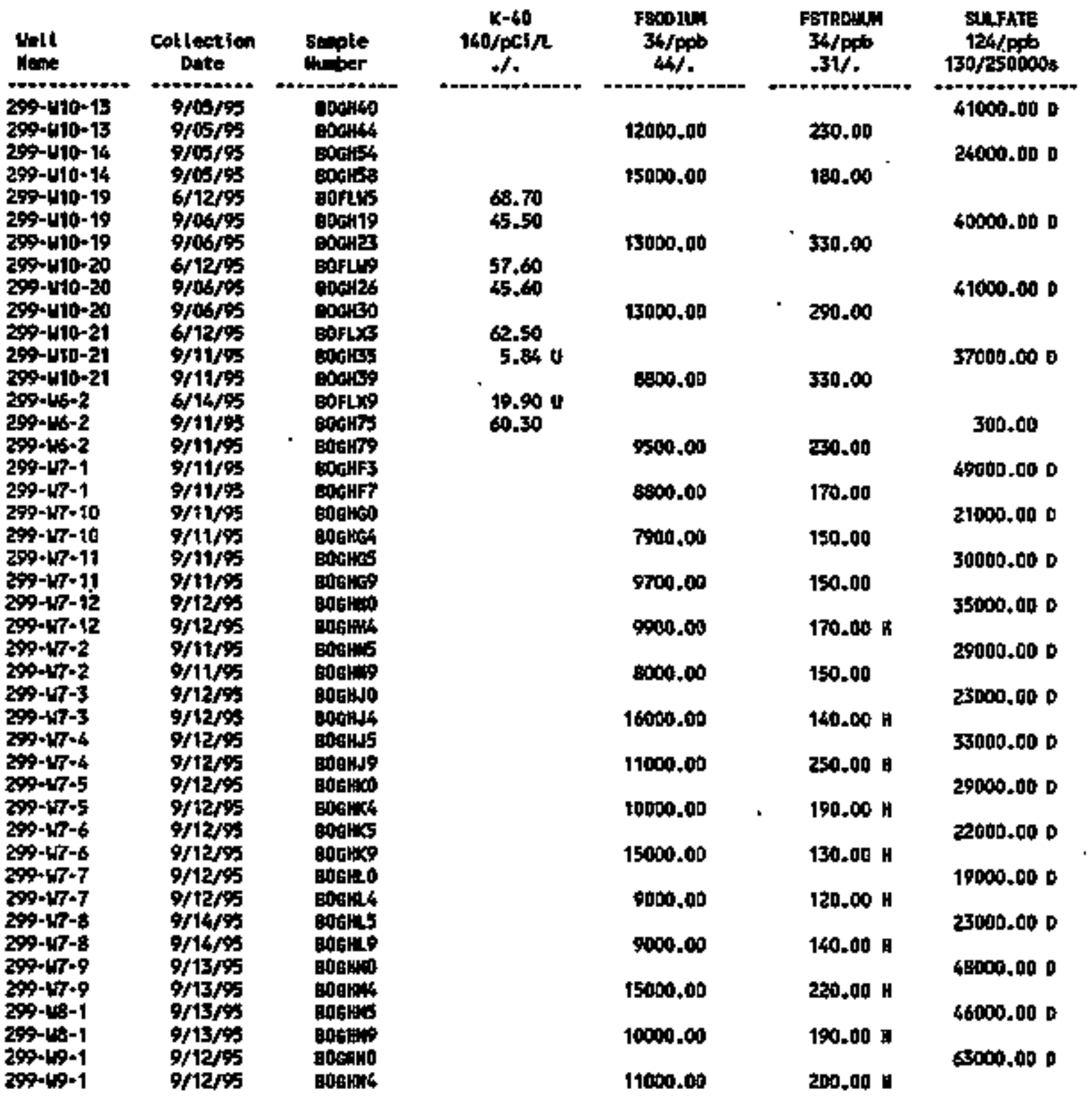


Table 14-19. Constjtuents with at Least One Detected Value for the Low-Level Waste Hanagenent Area 3 Data for Reporting

Period July 1 through September 30, 1995.

(sheet 8 of 10 )

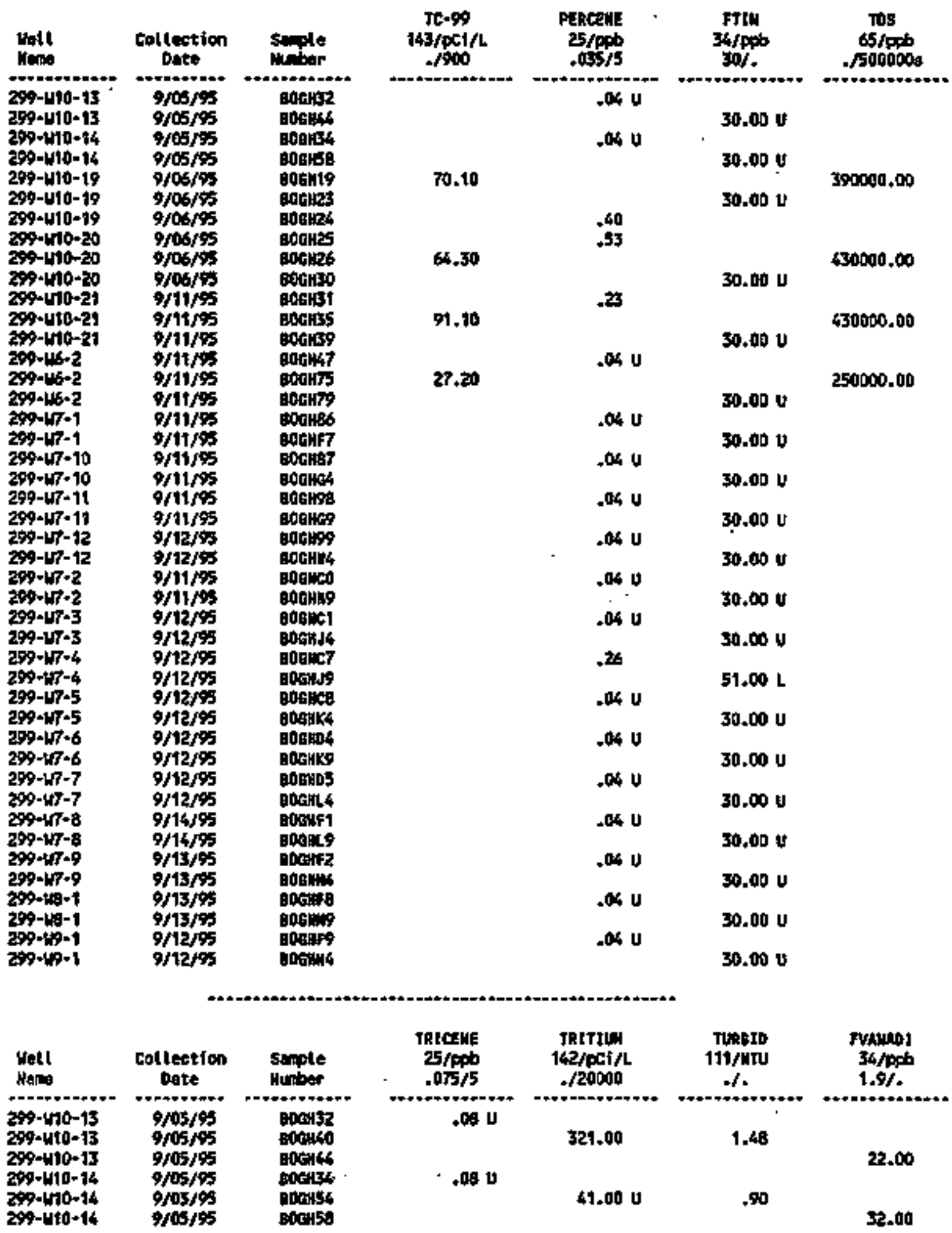


Table 14-19. Constituents with at Least One Detected Value for the Low-Level Waste Management Area 3 Data for Reporting

Period July 1 through September 30, 1995.

$$
\text { (sheet } 9 \text { of 10) }
$$

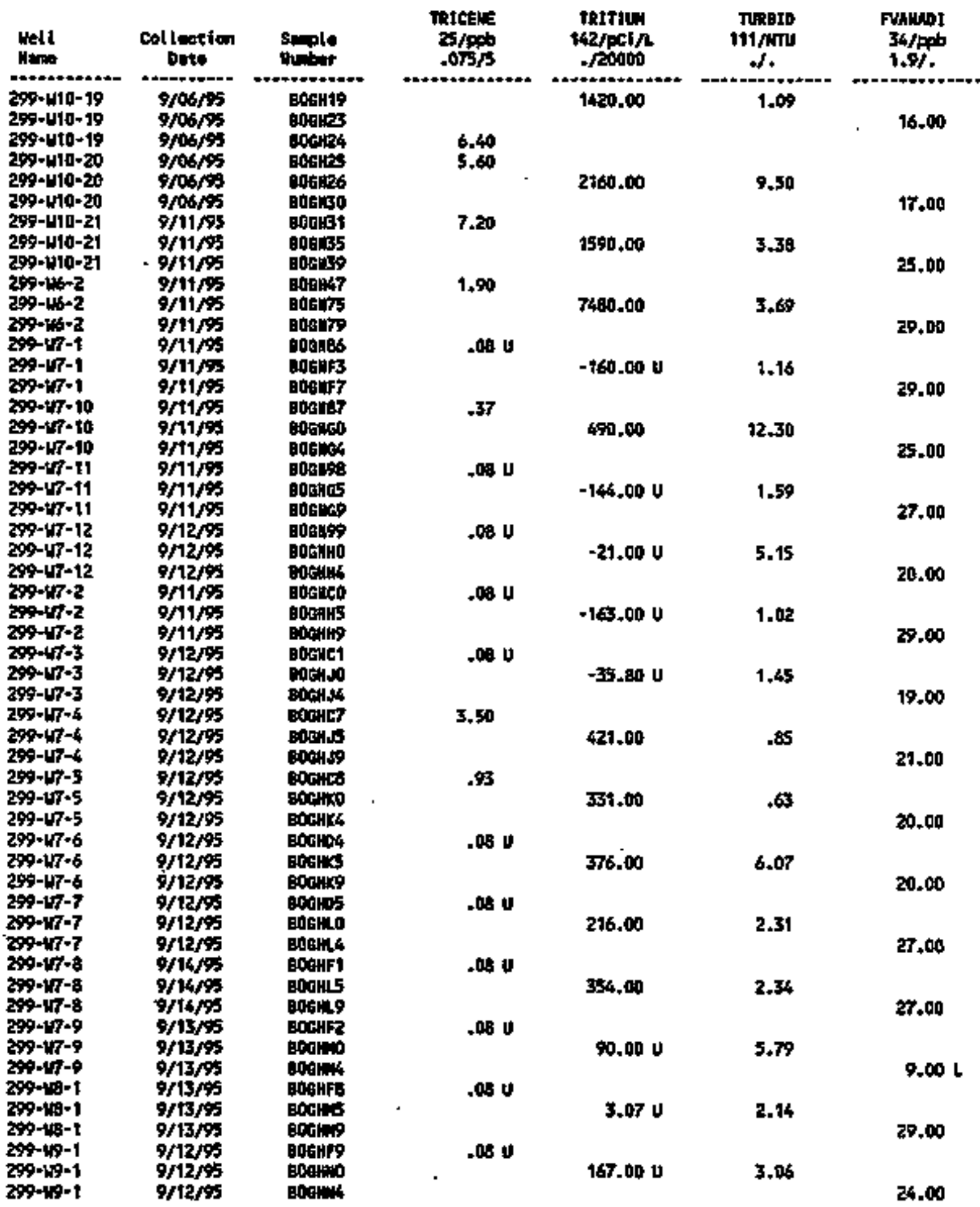


Table 14-19. Constituents with at Least One Detected Va]ue for the Low-Level Waste Management Area 3 Data for Reporting

Period July 1 through Septeaber 30, 1995.

(sheet 10 of 10 )

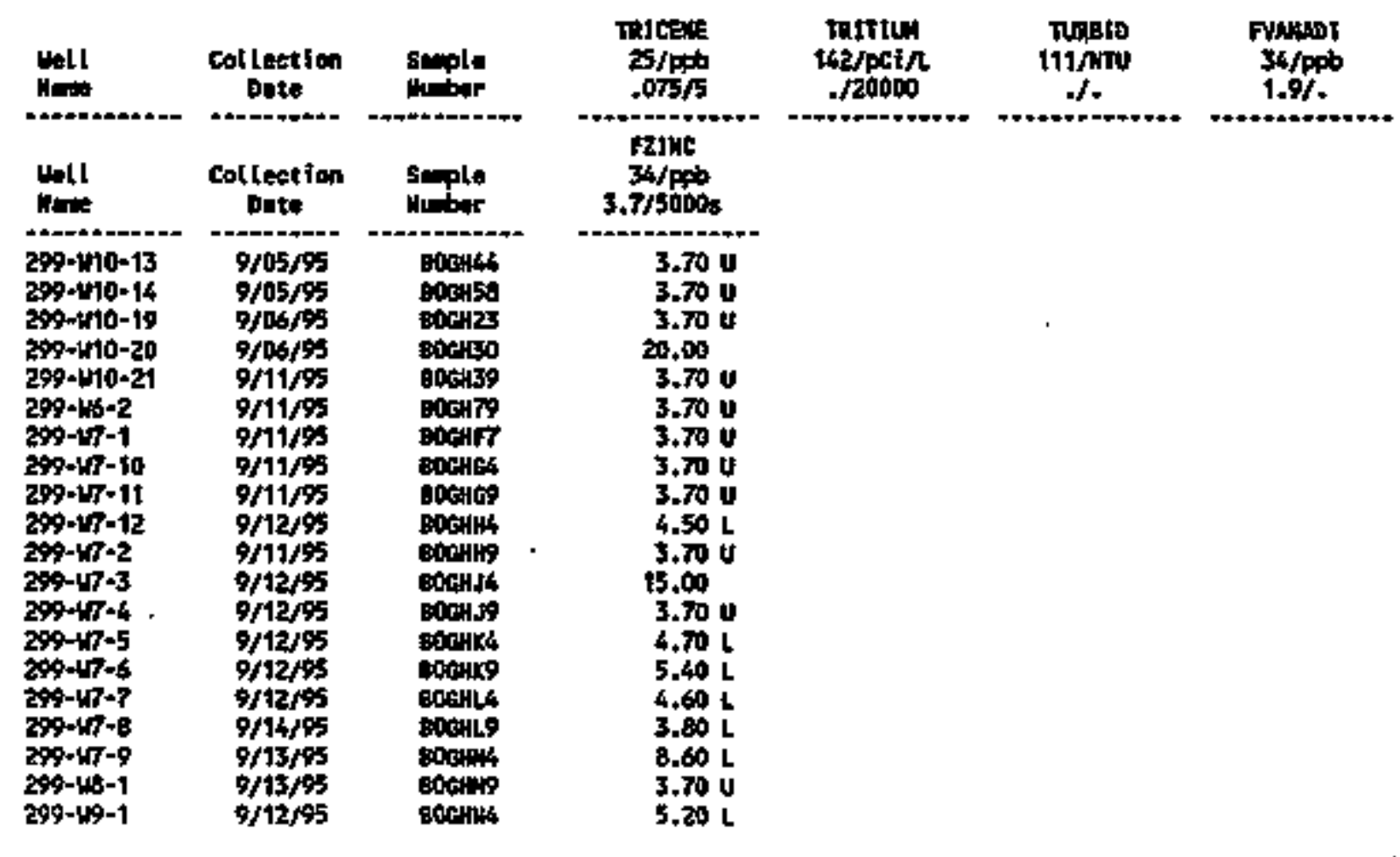

For explenstion of this taste, ses section 1.4 of repert. 
Tab]e 14-20. Contamfnation Indicator Parameters for the Low-Leve] Waste Management Area 3 Data for Reporting Period July 1 through September 30, 1995. (sheet 1 of 2)

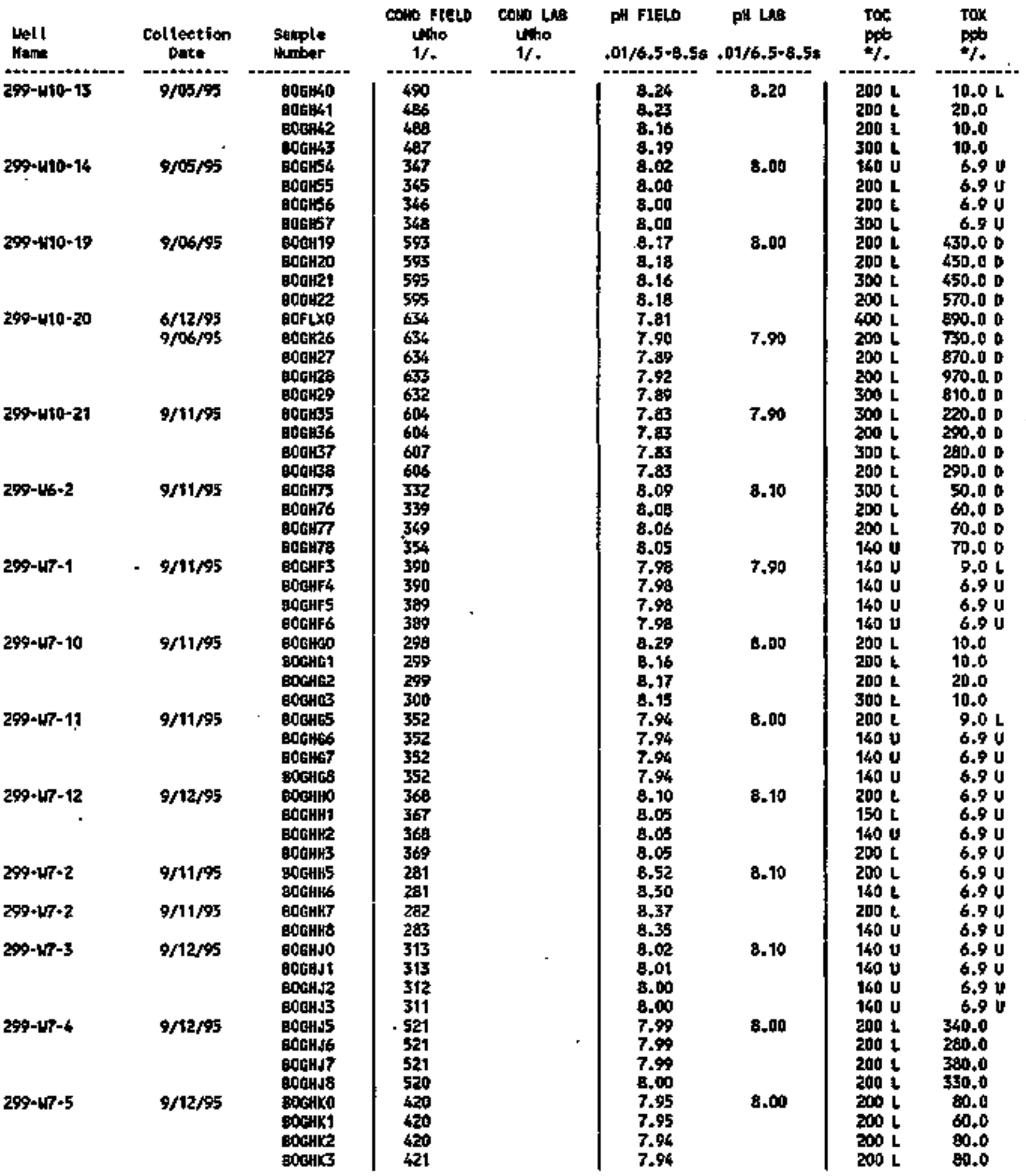


Tabie 14-20. Contanination Indicator Parameters for the Low-Level Waste Management Area 3 Data for Reporting Period

July I through Septenber 30, 1995. (sheet 2 of 2)

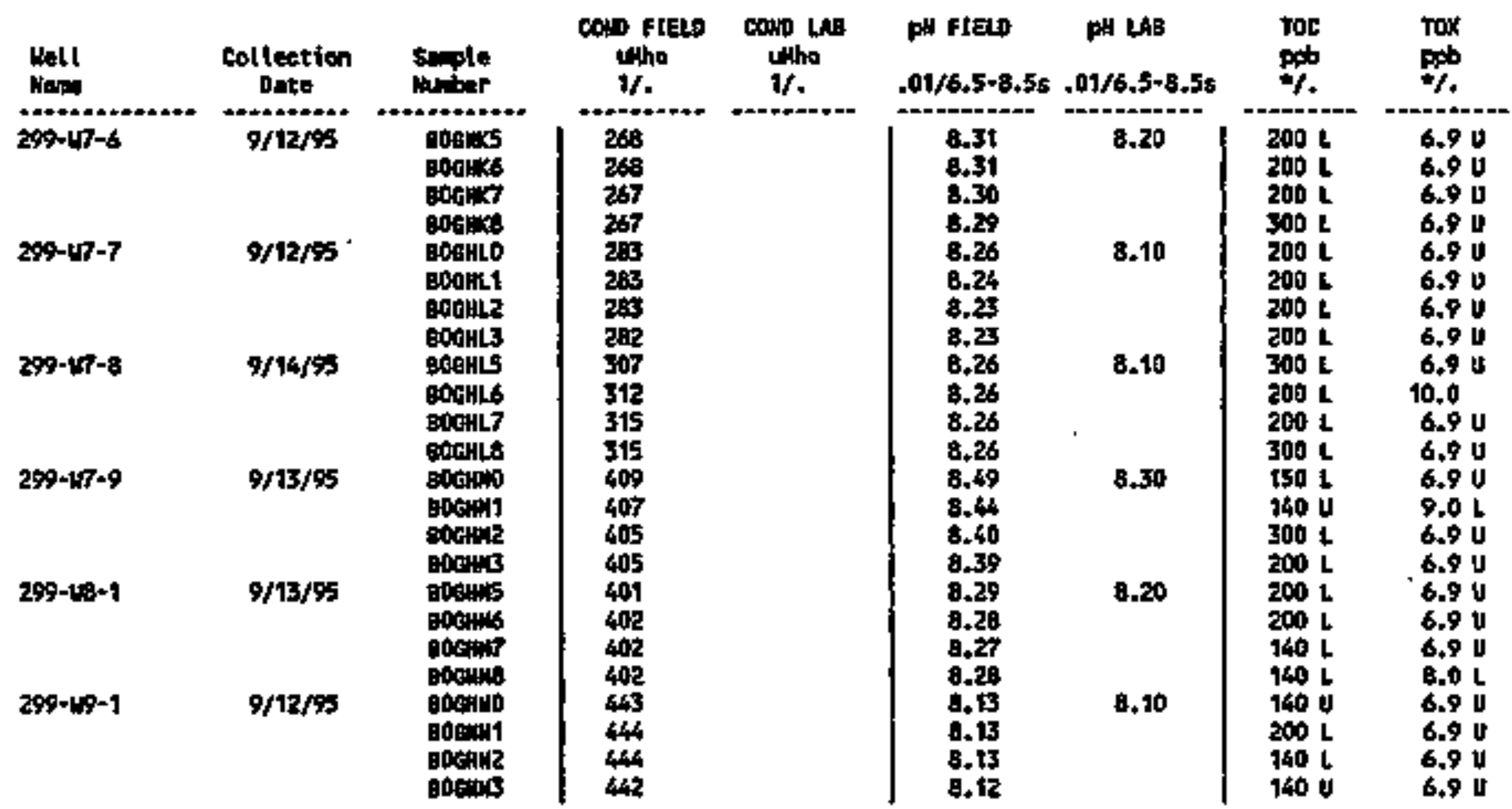

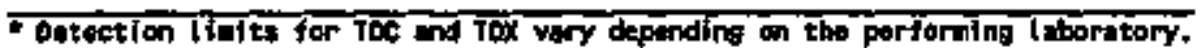
For erplentition of this toble, sete stetion 1.4 of report. 
Table 14-21. Constituent List and Sulmiary of Results for the Low-Level Waste Management Area 4 Data for Reporting Period July 1 through September 30, 1995.

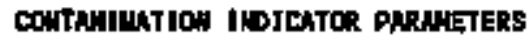

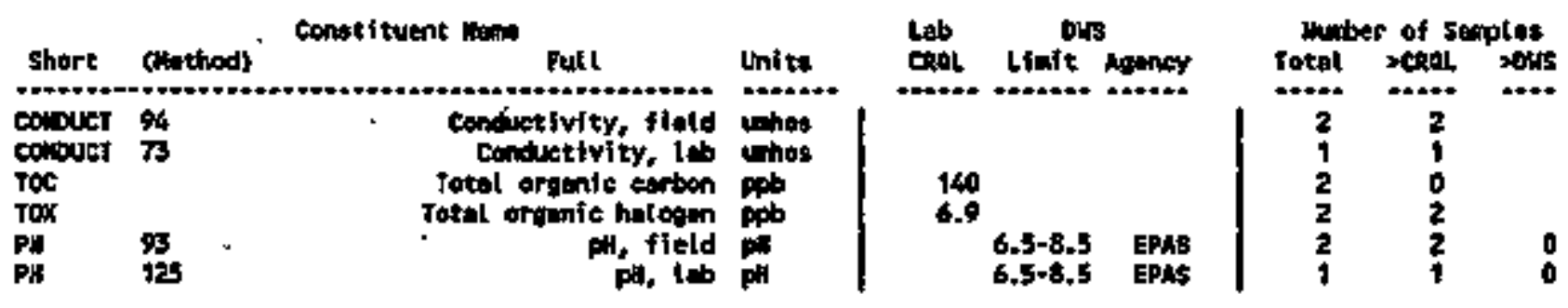

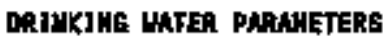

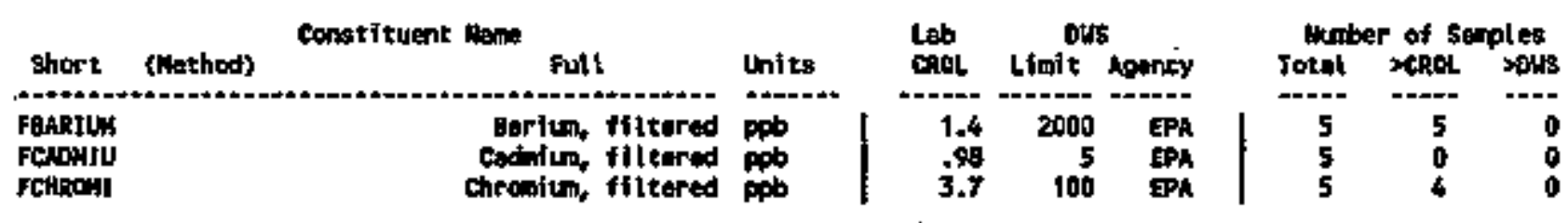

GROANOHTER OWhITY PHONETERS

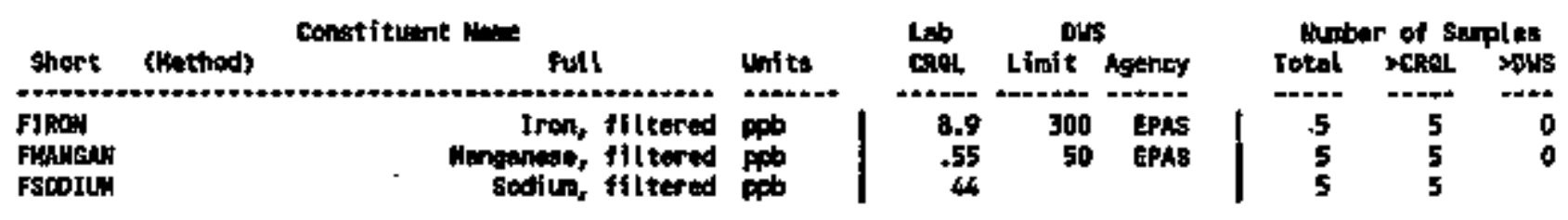

SITE SPECIFIC NHO OTHER CONSTITUEMS

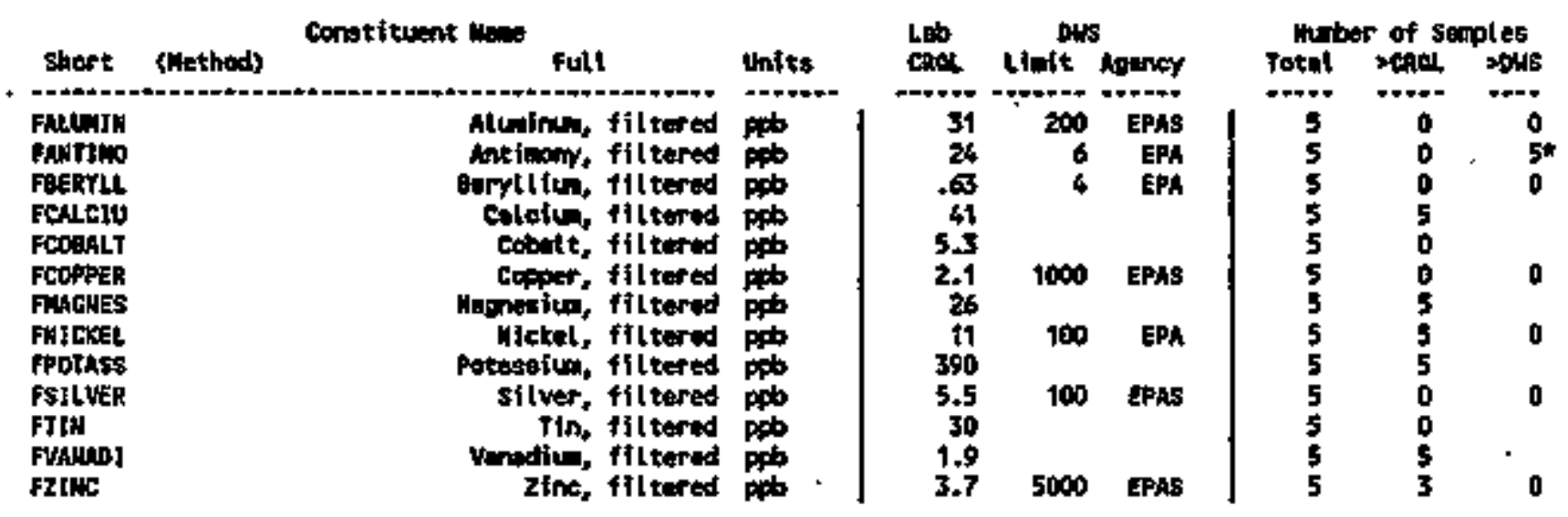

For explantion of thit table, set Stetion 1.6 of report. 
Table 14-22. Constituents with at Least One Detected Value for the Low-Leve 1 Waste Management Area 4 Data for Reporting

Period July 1 through September 30, 1995.

\begin{tabular}{|c|c|c|c|c|c|c|}
\hline $\operatorname{tint}_{\ln }$ & $\begin{array}{c}\text { Collection } \\
\text { Data }\end{array}$ & $\begin{array}{l}\text { sanple } \\
\text { Humber }\end{array}$ & $\begin{array}{l}\text { rankJum } \\
34 / \mathrm{ppb} \\
\text { t.4/2000 }\end{array}$ & $\begin{array}{c}\text { FChLeto } \\
\text { 34/ppb } \\
4 \%\end{array}$ & 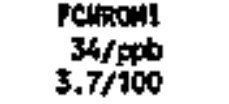 & $\begin{array}{c}\text { Fthow } \\
34 / / \mathrm{pb}^{\circ} \\
8.5 / 300 \mathrm{~s}\end{array}$ \\
\hline $\begin{array}{l}299-115-16 \\
299-115-18 \\
299+118-23 \\
299-118-26 \\
279-118-26\end{array}$ & $\begin{array}{l}5 / 08 / 95 \\
5 / 08 / 95 \\
5 / 15 / 95 \\
5 / 08 / 95 \\
5 / 15 / 95\end{array}$ & 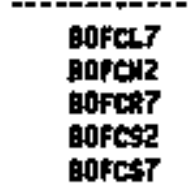 & $\begin{array}{r}75.00 \\
62.00 \\
25.00 \\
36.00 \\
55.00\end{array}$ & $\begin{array}{l}69000.00 \\
57000.00 \\
26000.00 \\
37000.00 \\
36009.00\end{array}$ & $\begin{array}{l}13.00 \mathrm{~L} \\
6.80 \mathrm{~L} \\
5.30 \mathrm{~L} \\
6.40 \mathrm{~L} \\
3.70 \mathrm{U}\end{array}$ & $\begin{array}{r}34.008 \\
32.008 \\
26.00 \mathrm{~B} \\
44.00 \mathrm{~B} \\
21.00 \mathrm{~B}\end{array}$ \\
\hline
\end{tabular}

\begin{tabular}{|c|c|c|c|c|c|c|}
\hline $\begin{array}{l}\text { Woll } \\
\text { fent }\end{array}$ & $\begin{array}{c}\text { collection } \\
\text { Date }\end{array}$ & rele & $\begin{array}{c}\text { FUGHES } \\
34 / \mu b 6 \\
26 / .\end{array}$ & $\begin{array}{l}\text { Fuswell } \\
34 / \mathrm{cpb} \\
.55 / \mathrm{sos}\end{array}$ & $\begin{array}{l}\text { FaIexta } \\
34 / \mathrm{pds} \\
11 / 100\end{array}$ & $\begin{array}{l}\text { FPorASs } \\
34 / \text { P中 } \\
390 / .\end{array}$ \\
\hline $\begin{array}{l}299-415-16 \\
299-415-18 \\
297-1418-23 \\
297-418-26 \\
299-418-26\end{array}$ & $\begin{array}{l}\text { 5/08/9s } \\
5 / 08 / 95 \\
5 / 15 / 95 \\
5 / 08 / 95 \\
5 / 15 / 95\end{array}$ & 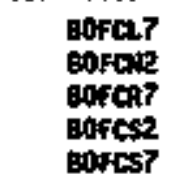 & $\begin{array}{r}19000.00 \\
17000.00 \\
7000.00 \\
9800.00 \\
9400.00\end{array}$ & $\begin{array}{l}2.00 \mathrm{Bt} \\
1.30 \mathrm{BL} \\
3.50 \mathrm{Bt} \\
1.40 \mathrm{Bt} \\
19.00 \mathrm{~B}\end{array}$ & $\begin{array}{l}16.00 \mathrm{~L} \\
51.00 \\
13.00 \mathrm{~L} \\
15.00 \mathrm{~L} \\
15.00 \mathrm{~L}\end{array}$ & $\begin{array}{l}5400,00 \\
4900.00 \\
3300.00 \\
3600.00 \\
4500,00\end{array}$ \\
\hline
\end{tabular}

\begin{tabular}{|c|c|c|c|c|c|}
\hline Wist & $\begin{array}{c}\text { Colleation } \\
\text { Datt }\end{array}$ & seple & $\begin{array}{c}\text { Frowilu } \\
34 / 0 p b \\
44 /\end{array}$ & $\begin{array}{l}\text { Funnd } \\
36 / 1 \mathrm{ppb} \\
1.9 \%\end{array}$ & $\begin{array}{c}12[14 \mathrm{C} \\
34 / \mathrm{ppb} \\
3.7 / 5000 \mathrm{~s}\end{array}$ \\
\hline $\begin{array}{l}209+115-16 \\
299-1115-18 \\
299-118-23 \\
799+118+26 \\
299-418-26\end{array}$ & $\begin{array}{l}5 / 08 / 95 \\
5 / 08 / 95 \\
5 / 15 / 95 \\
5 / 08 / 95 \\
5 / 15 / 95\end{array}$ & 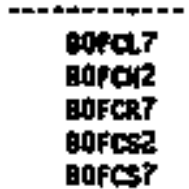 & $\begin{array}{l}40000.00 \mathrm{E} \\
27000.00 \mathrm{E} \\
15000.00 \mathrm{~B} \\
18000.00 \mathrm{~B} \\
17000.00 \mathrm{~B}\end{array}$ & $\begin{array}{l}28.00 \mathrm{~L} \\
24.00 \mathrm{~L} \\
24.00 \mathrm{~L} \\
28.00 \mathrm{~L} \\
5.20 \mathrm{C}\end{array}$ & $\begin{array}{c}22.00 \\
3.70 \mathrm{U} \\
3.70 \mathrm{U} \\
24.00 \\
6.90 \mathrm{~L}\end{array}$ \\
\hline
\end{tabular}

For explantion of this teble, Eat section 1.4 of report:

Table 14-23. Contanination Indicator Parameters for the Low-Leve] Haste Management Area 4 Data for Reporting Period July 1 through September 30, 1995.

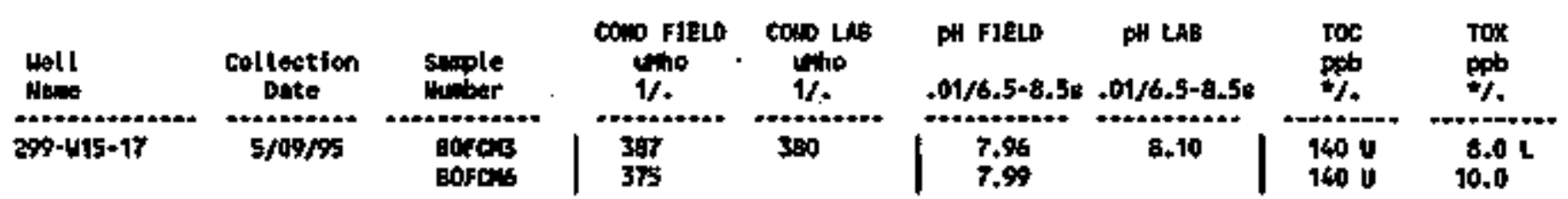

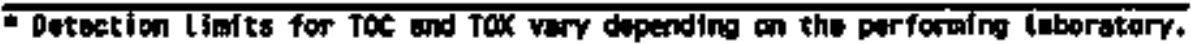
For explandition of this table, sese section 1.4 of report.
} 
Table 14-24. Constituent List and Summary of Results for the Low-Level Waste Managesent Area 5 Data for Reporting

Period July 1 through Septenber 30, 1995.

(sheet 1 of 2)

COMTAMLMTISW INDTCNTOR PMRMETERS

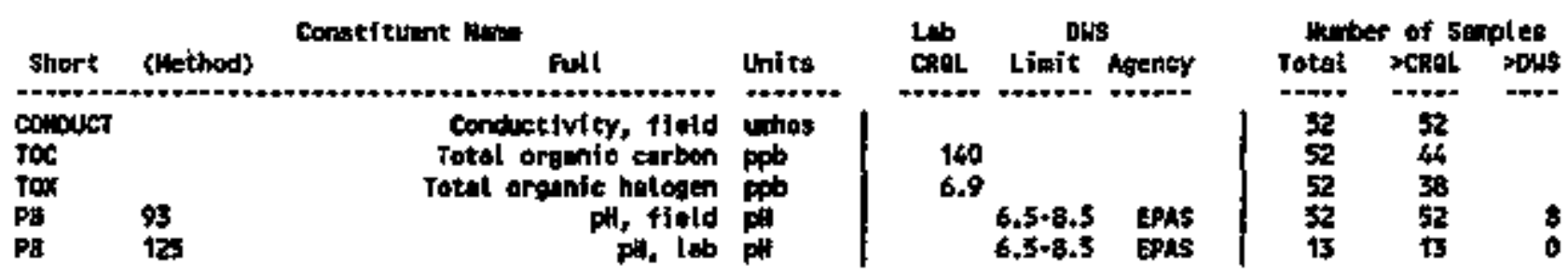

DAHUKIMS UKTER PARANETERS

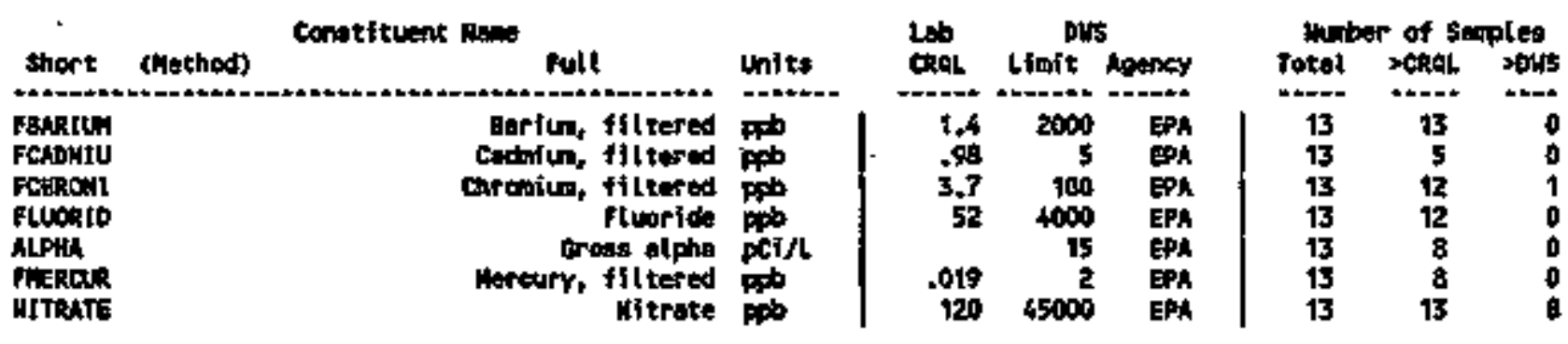

EROUVWWTER QUALITY PARNOTERS

\begin{tabular}{|c|c|c|c|c|c|c|c|c|c|c|}
\hline & & constiguent Wess & & & Lי & DH & & wh & of $\mathrm{Se}$ & ptes \\
\hline Stort & (Hethod) & Ful & & Unfte & cres & Liturit & hoency & Totel & $>\mathrm{CROCL}$ & $>$ ous \\
\hline 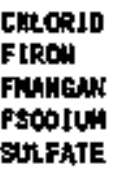 & . & $\begin{array}{r}\text { Iron, } \\
\text { Mangenese, } \\
\text { sodiun, }\end{array}$ & $\begin{array}{l}\text { Chloride } \\
\text { fllteres } \\
\text { filtered } \\
\text { filtered } \\
\text { sulfote }\end{array}$ & $\begin{array}{l}p+b \\
p p b \\
p p b\end{array}$ & $\begin{array}{r}210 \\
8.9 \\
.55 \\
44 \\
130\end{array}$ & $\begin{array}{r}250000 \\
300 \\
50 \\
250000\end{array}$ & $\begin{array}{l}\text { EPAS } \\
\text { EPAS } \\
\text { EPAS } \\
\text { EPAS }\end{array}$ & $\begin{array}{l}13 \\
13 \\
13 \\
13 \\
13\end{array}$ & $\begin{array}{l}12 \\
13 \\
12 \\
13 \\
13\end{array}$ & 0 \\
\hline
\end{tabular}

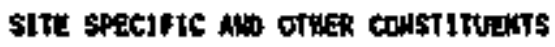

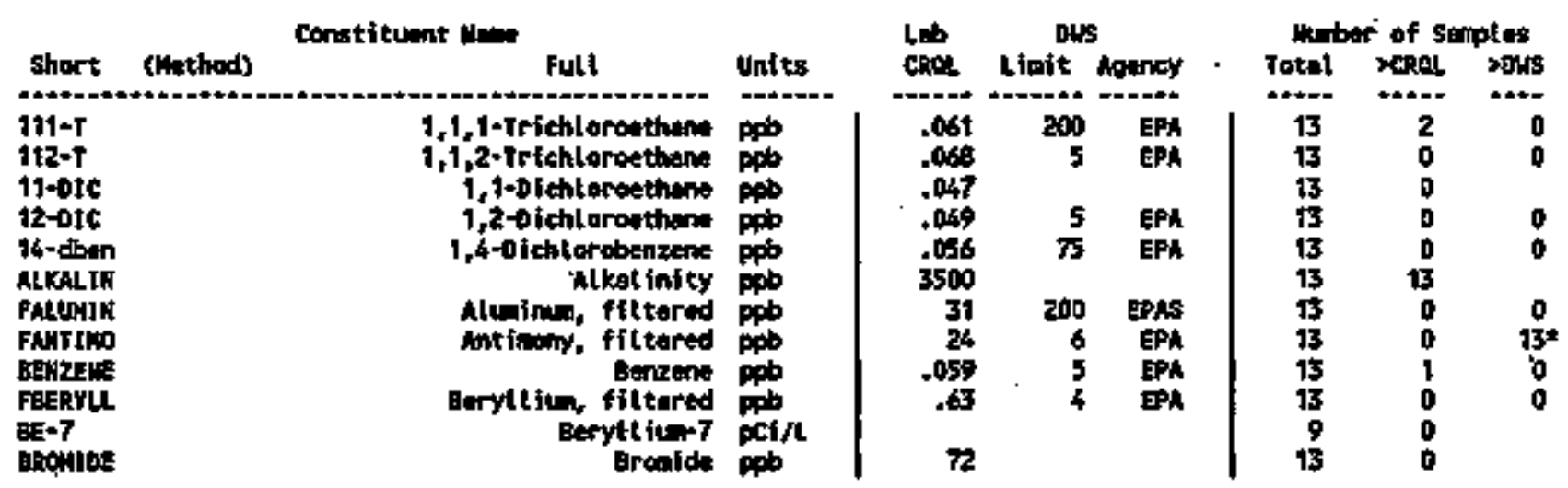


Table 14-24. Constituent List and Summary of Results for the Low-Level Waste Management Area 5 Data for Reporting

Period July 1 through September 30, 1995.

(sheet 2 of 2)

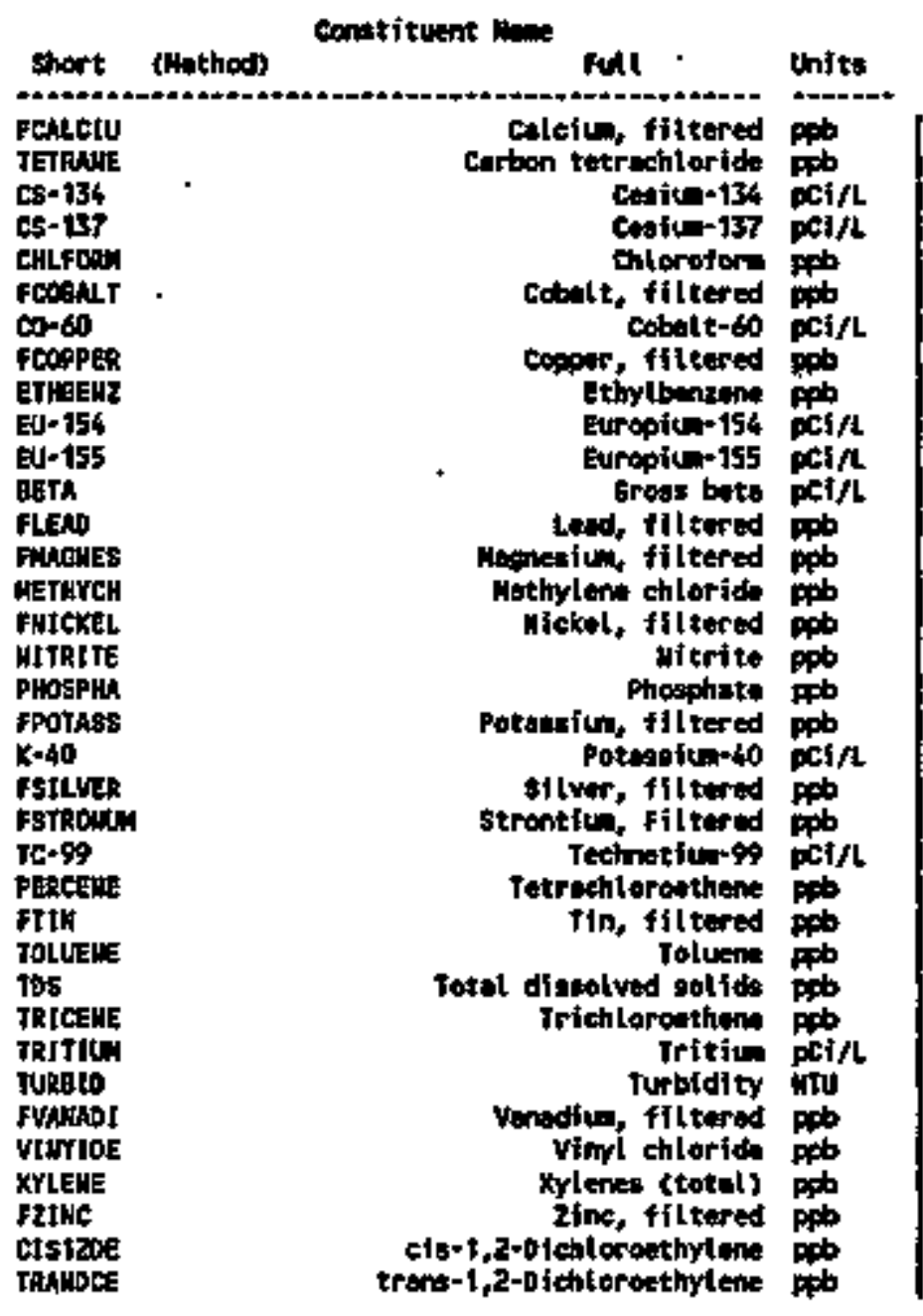

Lab Dis

GRoL Ligitt Agency

41

.042

200

3 EPK

$+079$

5.3

$2.1 \quad 100$ EPh

$+034700$

teph

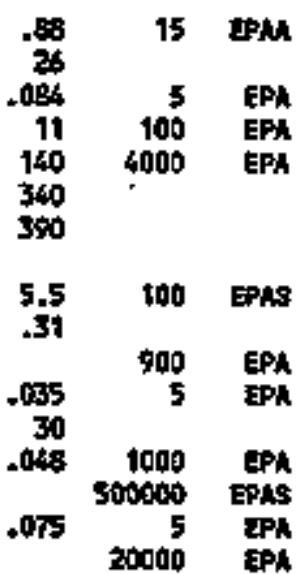

1.9

自施

.065

.049

.045

APh

$\begin{array}{rr}2 & \text { EPA } \\ 10000 & \text { EPA } \\ 5000 & \text { EPAs } \\ 70 & \text { EPh } \\ \text { tod } & \text { EPK }\end{array}$

Number of samples

Totol scal >olls

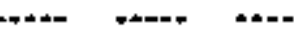

$\begin{array}{lll}13 & 13 \\ 13 & 13\end{array}$

$\begin{array}{lll}9 & 0 & 0\end{array}$

13

13

9

310

130

91

2313

$13 \quad 30$

13

;

13

13

13

13

1

5

73

5 
Table 14-25. Constituents with at Least One Detected Value for the Low-Level Waste Management Area 5 Data for Reporting Perfod July 1 through September 30, 1995. (sheet 1 of 8 )

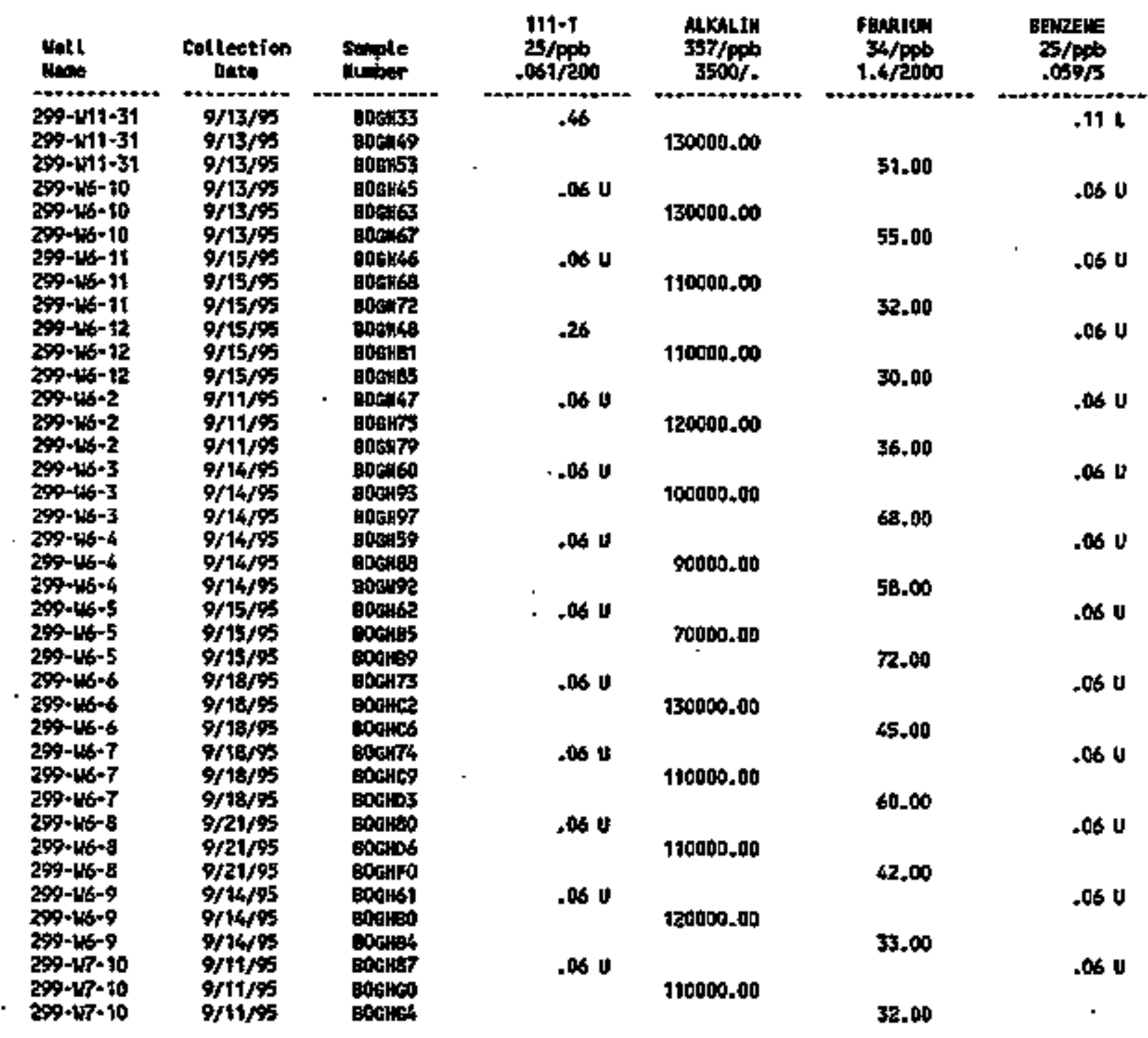


Table 14-25. Constituents with at Least one Detected Value for the Low-Level Waste Management Area 5 Bata for Reporting

Period July 1 through Septenter 30, 1995. (sheet 2 of 8 )

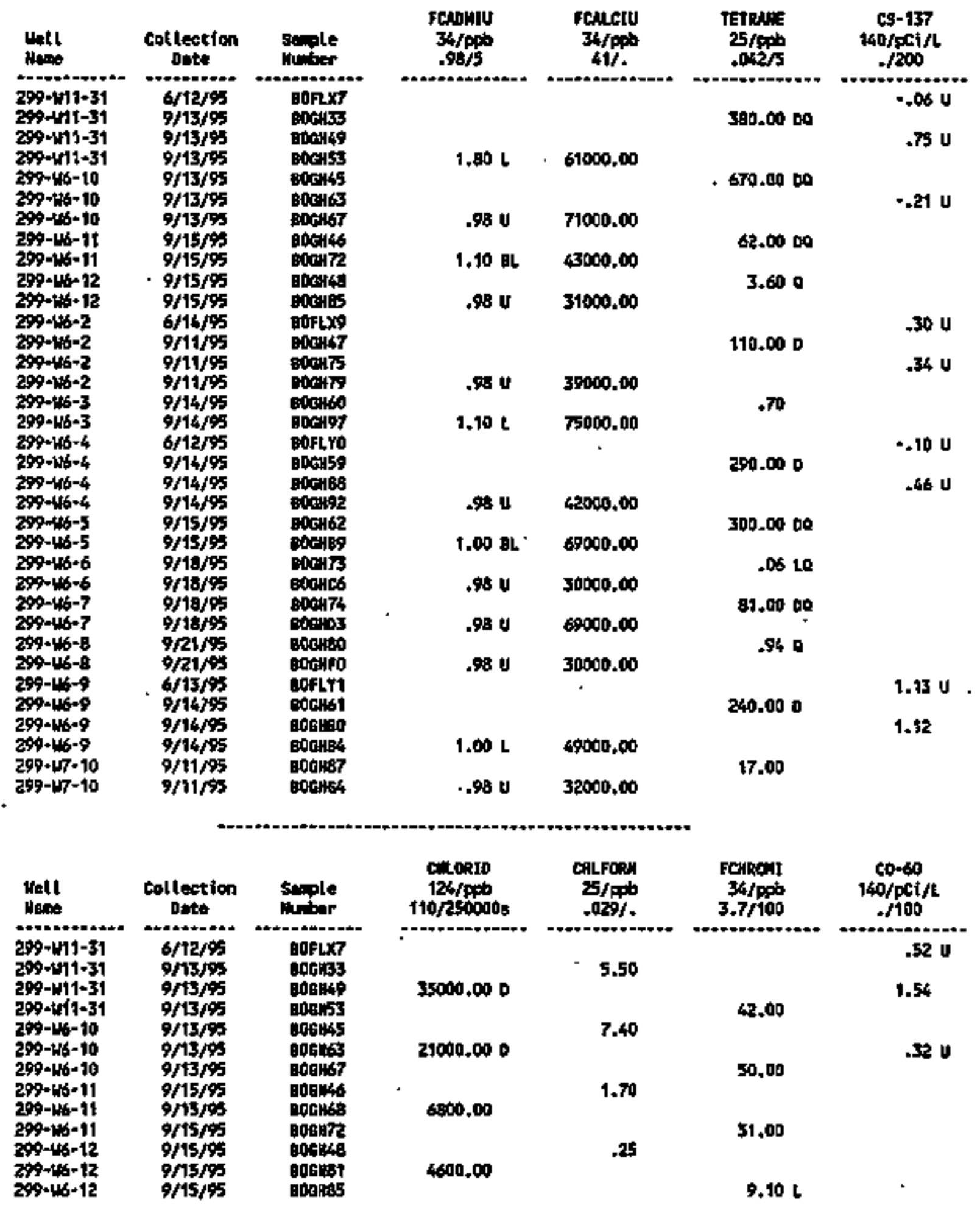


Tab7e 14-25. Constituents with at Least One Detected Va]ue for the Low-Level Waste Management Area 5 Data for Reporting Period July 1 through September 30, 1995. (sheet 3 of 8 )

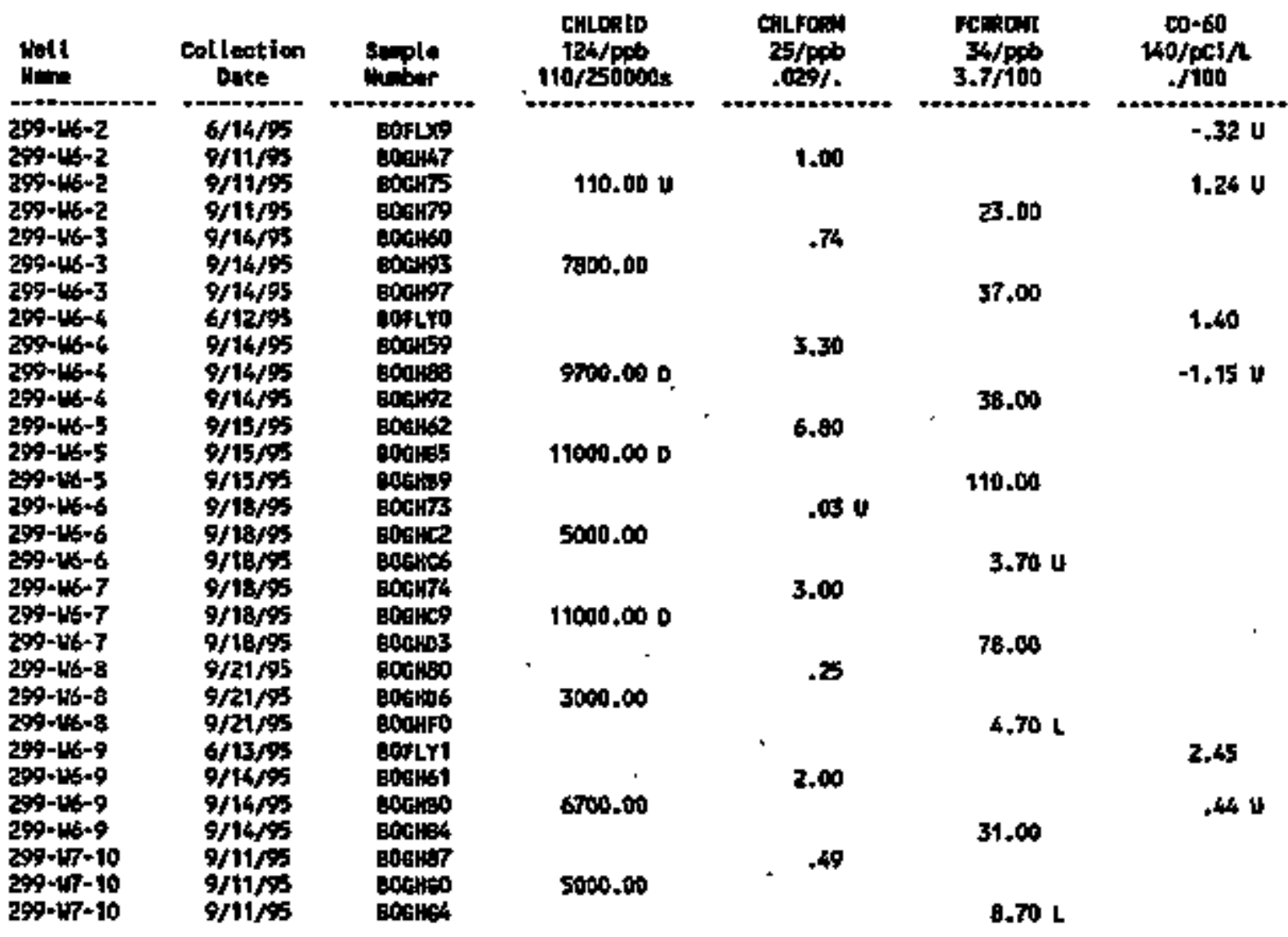


Table 14-25. Const 1 tuents with at Least One Detected Value for the Low-Level Waste Management Area 5 Data for Reporting

Period July 1 through September 30, 1995.

(sheet 4 of 8 )

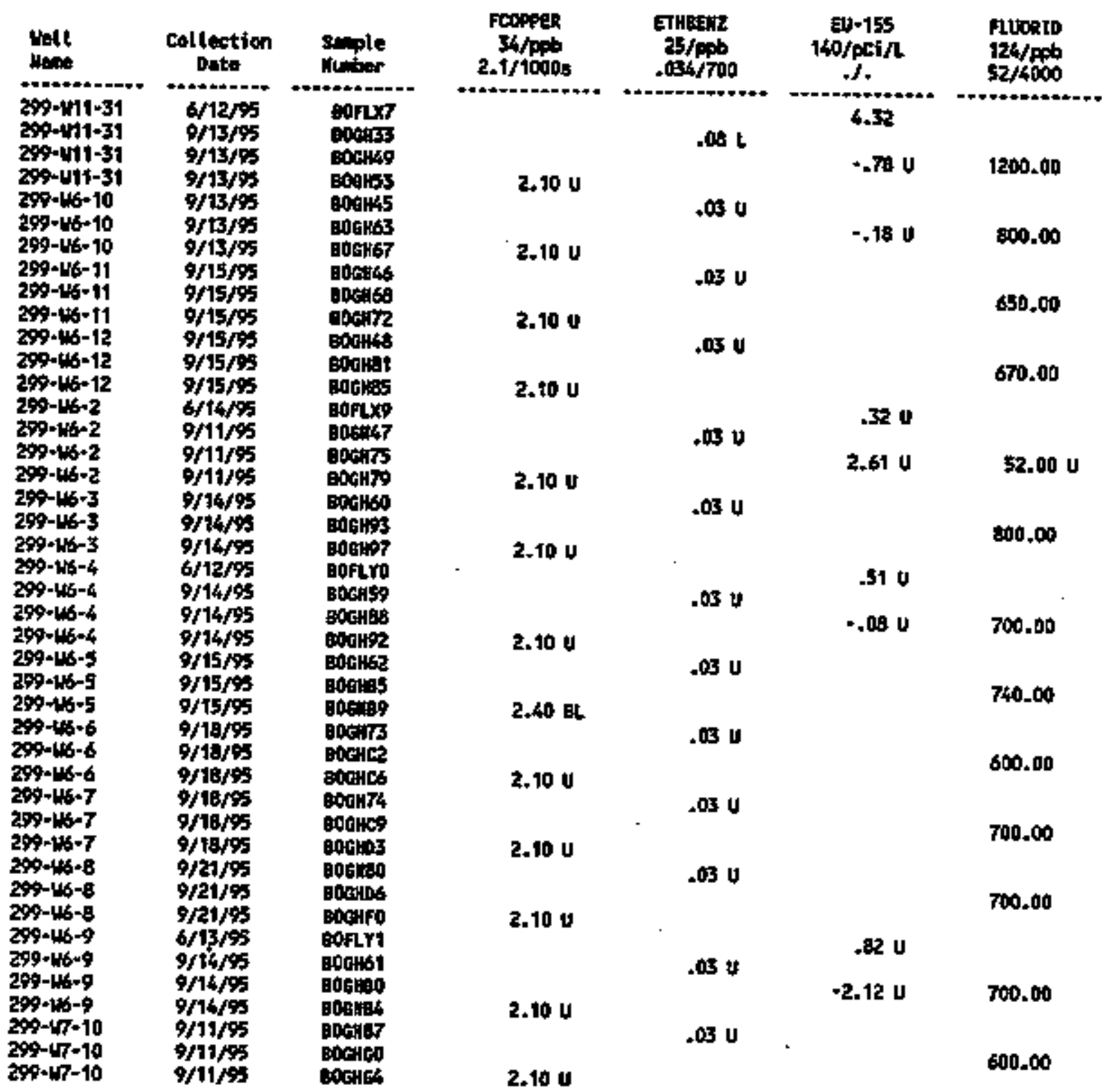


Table 14-25. Constituents with at Least One Detected Value for the Low-Leve] Waste Managenent Area 5 Data for Reporting

Period July 1 through September 30, 1995.

(sheet 5 of 8 )

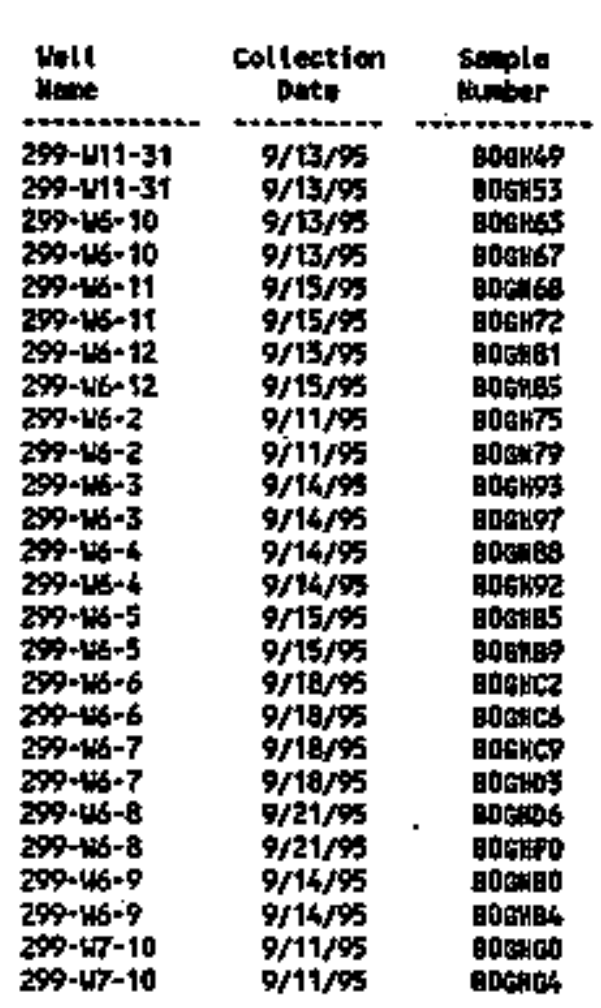

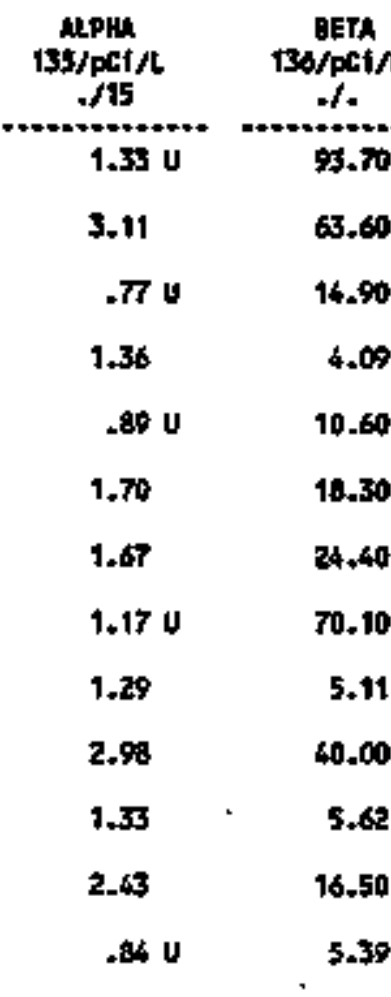

\begin{tabular}{|c|c|}
\hline $\begin{array}{l}\text { fteoll } \\
34 / 2 p b \\
8.9 / 500 \mathrm{~s}\end{array}$ & $\begin{array}{l}\text { FLEN } \\
\text { 40/ppb } \\
\text {.89/150 }\end{array}$ \\
\hline 36.00 & 1.20 내 \\
\hline 23.002 & .89 내 \\
\hline 13.001 & A U U \\
\hline 18,004 & .031 \\
\hline $23.00 \mathrm{EL}$ & סו \\
\hline 49.00 & .89 UA \\
\hline $\boldsymbol{7} .00$ & -68 Un \\
\hline 18.001 & $.8 B U$ \\
\hline $26.00 \mathrm{~L}$ & t.10 Lh \\
\hline 33.00 & . 8 d U \\
\hline $20.00 \mathrm{~L}$ & .8B 네 \\
\hline $24.00 \mathrm{~L}$ & .8 บн \\
\hline $32.00 \mathrm{a}$ & .8010 \\
\hline
\end{tabular}

\begin{tabular}{|c|c|c|c|c|c|c|}
\hline $\begin{array}{l}\text { Uitll } \\
\text { Hewn }\end{array}$ & $\begin{array}{c}\text { Col lestion } \\
\text { bate }\end{array}$ & Semplet & $\begin{array}{c}\text { Furdes } \\
3 / \mathrm{pr} \\
26 \mathrm{f}\end{array}$ & 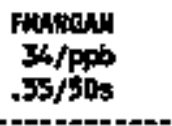 & $\begin{array}{l}\text { FUERAR } \\
41 / \mathrm{pb} \\
.019 / 2\end{array}$ & $\begin{array}{l}\text { Heturey } \\
25 / \mathrm{ppb} \\
.084 / 5\end{array}$ \\
\hline 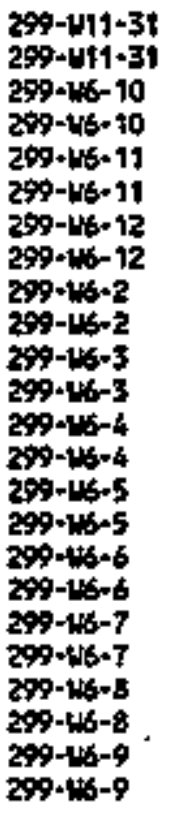 & $\begin{array}{l}9 / 13 / 95 \\
9 / 13 / 95 \\
9 / 13 / 95 \\
9 / 13 / 95 \\
9 / 15 / 95 \\
9 / 15 / 95 \\
9 / 15 / 95 \\
9 / 15 / \% 5 \\
9 / 11 / 95 \\
9 / 11 / \% 5 \\
9 / 14 / \% \\
9 / 14 / 95 \\
9 / 14 / 95 \\
9 / 14 / 95 \\
9 / 19 / 45 \\
9 / 15 / 95 \\
9 / 18 / 95 \\
9 / 15 / \% 5 \\
9 / 18 / 95 \\
9 / 18 / 95 \\
9 / 21 / 95 \\
9 / 21 / 95 \\
9 / 14 / 95 \\
9 / 14 / 95\end{array}$ & 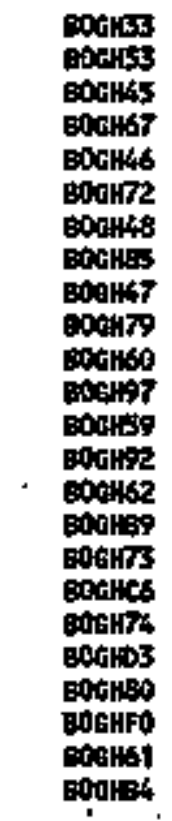 & $\begin{array}{l}2000.00 \\
25000.00 \\
15000.00 \\
12000.00 \\
16000.00 \\
28000.00 \\
25000.00 \\
35000.00 \\
12000.00 \\
30000.00 \\
11000.00 \\
18000.00\end{array}$ & $\begin{array}{r}15.00 \mathrm{~B} \\
55 \mathrm{~L} \\
.66 \mathrm{~L} \\
2.00 \mathrm{~L} \\
.93 \mathrm{EL} \\
1.09 \mathrm{QL} \\
1.30 \mathrm{EL} \\
6.30 \\
62.00 \mathrm{~K} \\
5.60 \mathrm{~L} \\
2.40 \mathrm{~L} \\
2.20 \mathrm{EL}\end{array}$ & 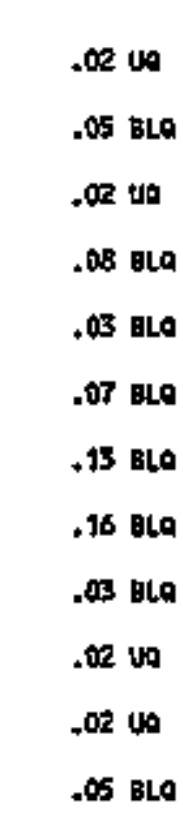 & 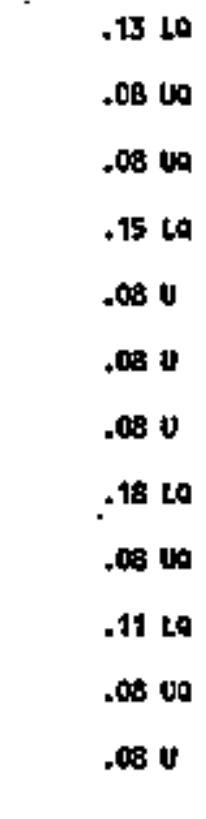 \\
\hline
\end{tabular}


Tabie 14-25. Constituents with at Least One Detected Value for the Low-Level Waste Management Area 5 Data for Reporting Period July 1 through Septenber 30, 1995. (sheet 6 of $B$ )

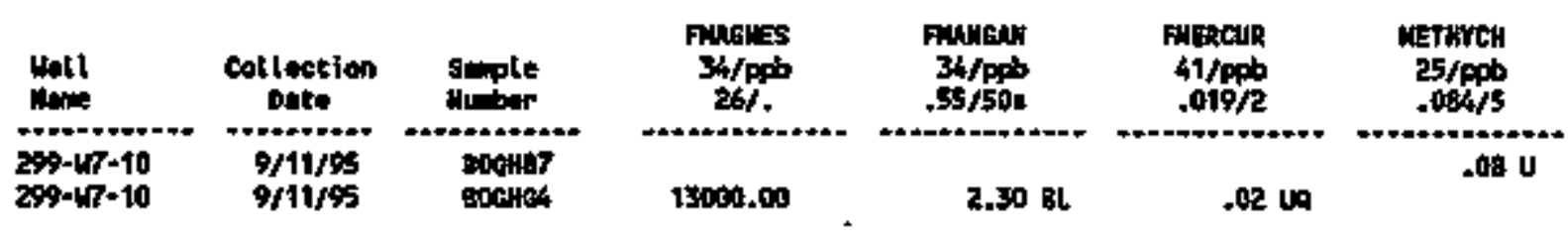

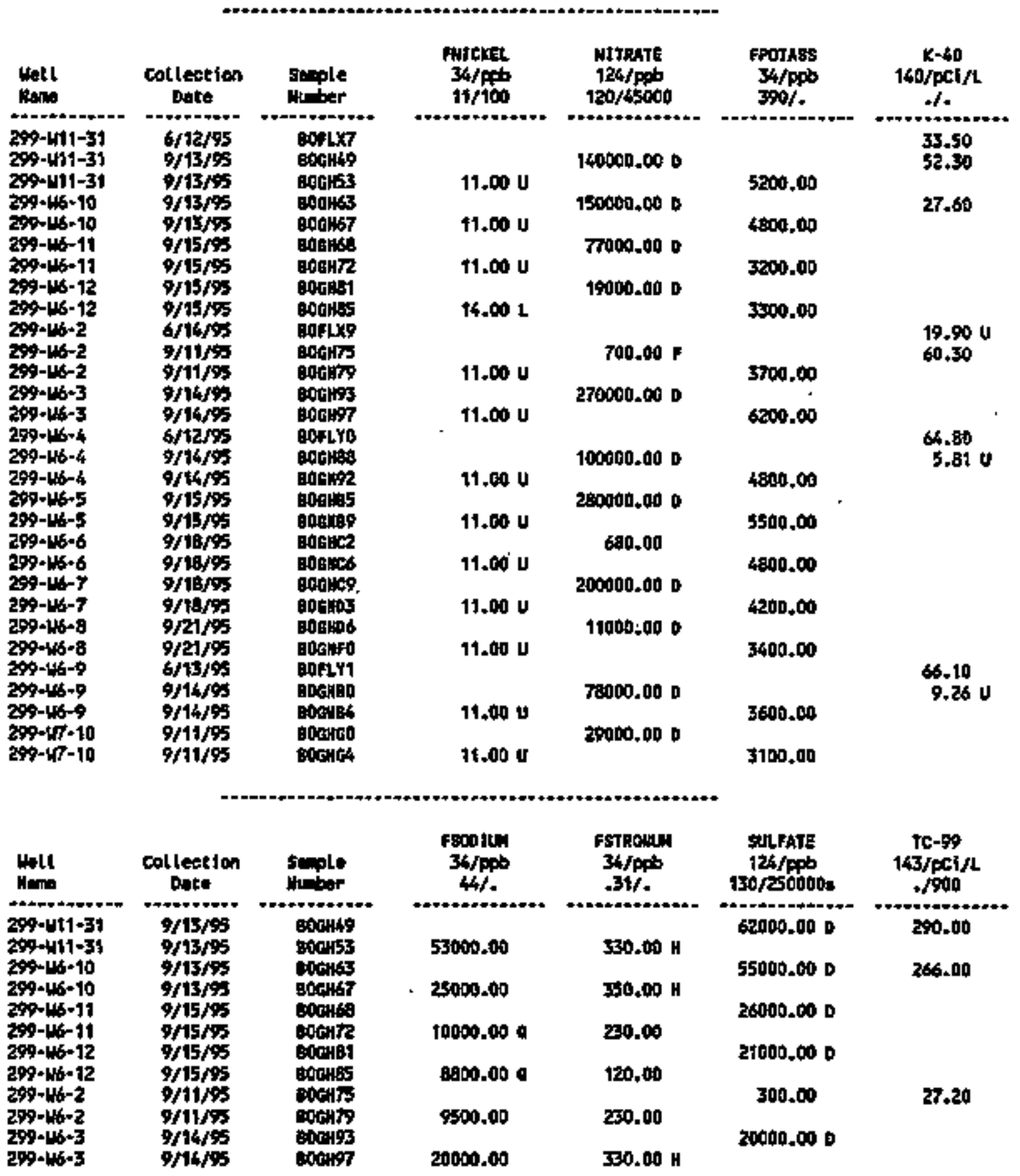


Table 14-25. Constituents with at Least One Detected Value for the Low-Level Maste Management Area 5 Data for Reporting

Period July 1 through September 30, 1995.

(sheet 7 of 8 )

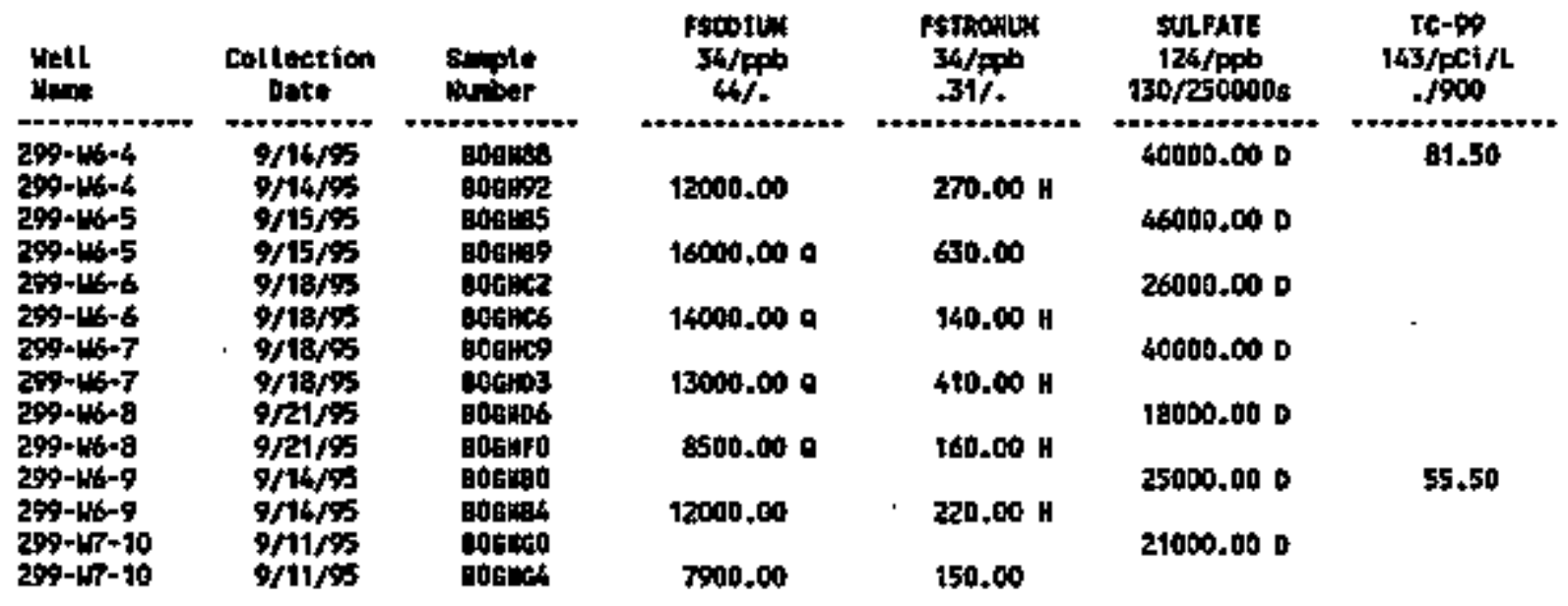

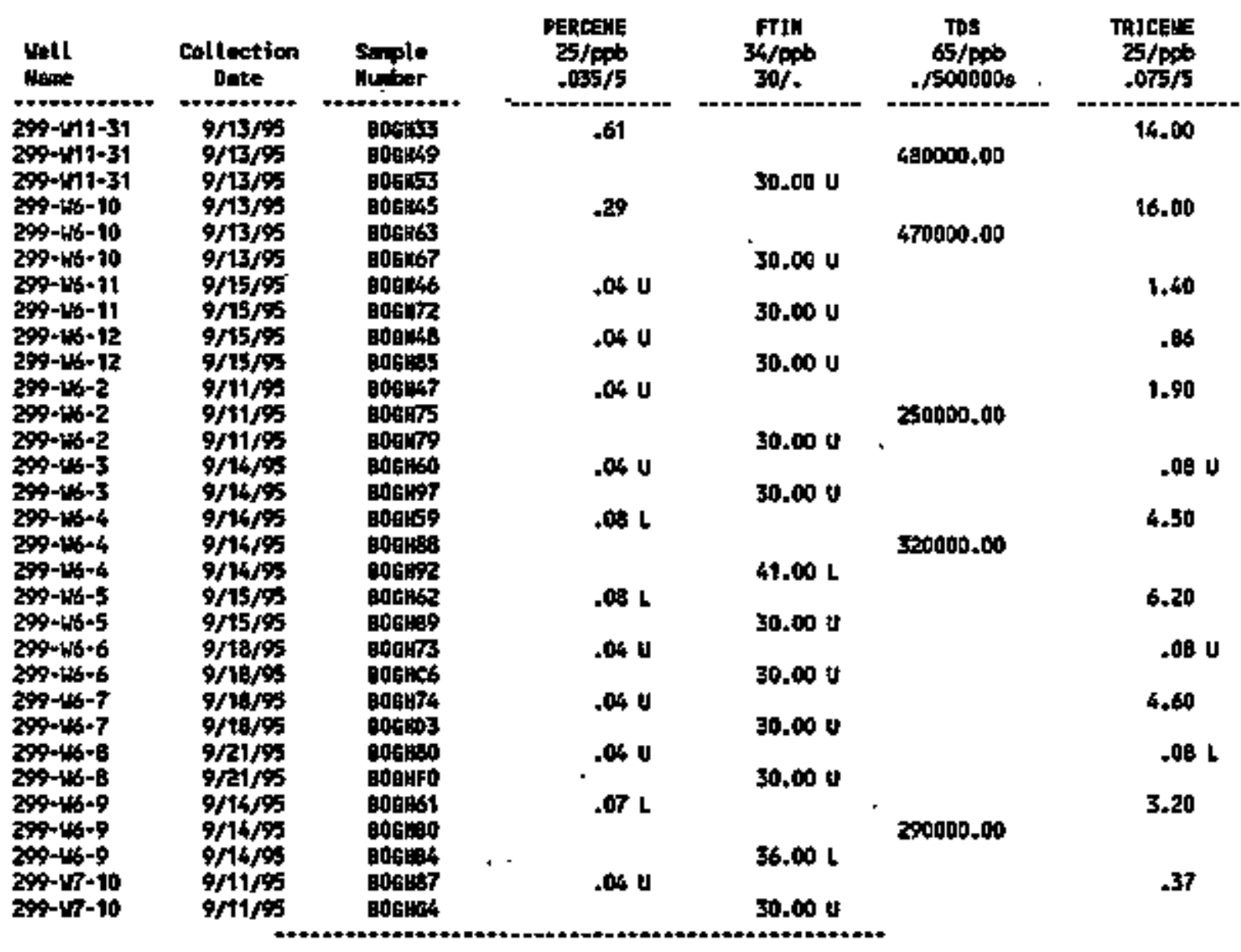


Table 14-25. Constituents with at Least 0ne Detected Va] ue for the Low-Level Waste Managenent Area 5 Data for Reporting

Period July 1 through September 30, 1995.

(sheet 8 of 8)

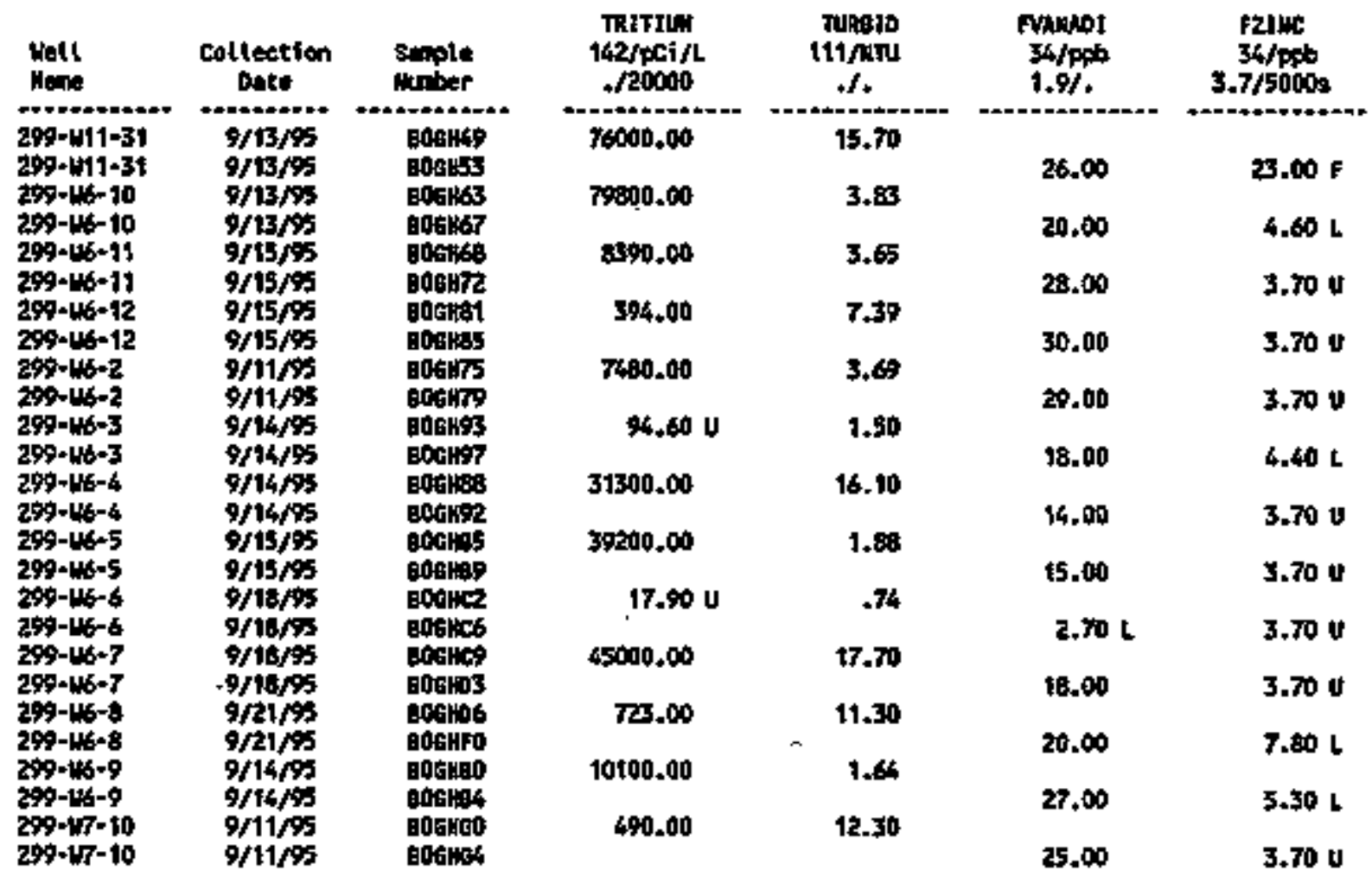

For explaration of this table, we section 1.4 af raport. 
Table 14-26. Contanination Indicator Parameters for the Low-LeveT Waste Management Area 5 Data for Reporting Period July 1 through September 30, 1995.

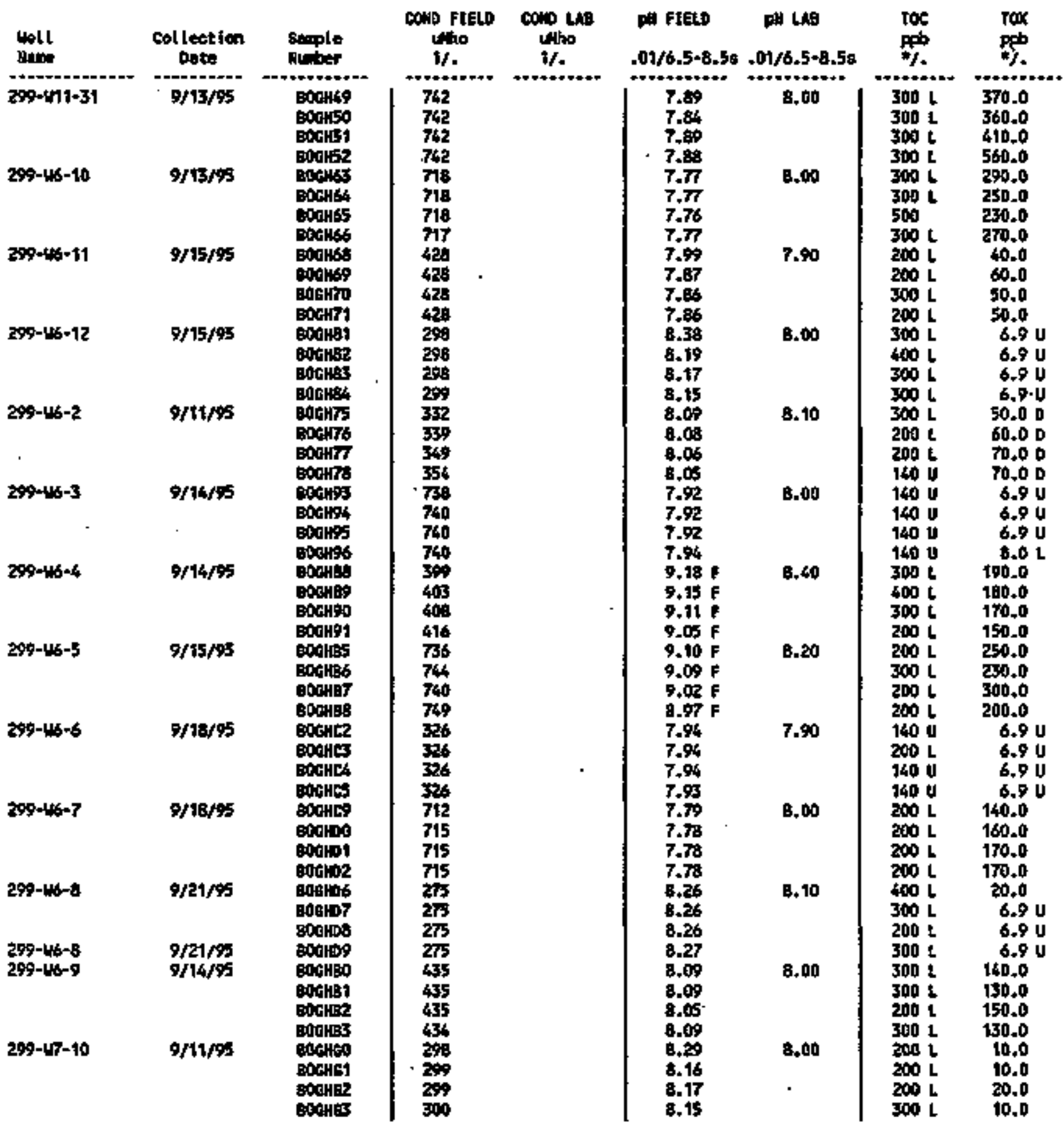

Detetion limits for Toc and Tox vary depending on the perforaing laboratory. For explention of this table, see settion 1.4 of repert. 
Table 14-27. Water Qualjty Standards Exceeded at Lou-Level Waste Managentent Area 3 .

\begin{tabular}{|c|c|c|c|}
\hline $\begin{array}{l}\text { Nitrate } \\
(45,000 \mathrm{ppb})\end{array}$ & $\begin{array}{l}299-w 7-1 \quad 299-w 7-4 \\
299-W 10-21\end{array}$ & 299-\$10-1 & $299-410-20$ \\
\hline $\begin{array}{l}\text { Carbon Tetrachloride } \\
(5 \mathrm{ppb})\end{array}$ & $\begin{array}{rr}299-46-2 & 299-47-4 \\
299-410-19 & 299\end{array}$ & $\begin{array}{l}299-W 7-5 \\
-W 10-20\end{array}$ & $\begin{array}{l}299-47-10 \quad 299-410-13 \\
299-W 10-21\end{array}$ \\
\hline $\begin{array}{l}\text { Trichloroethene } \\
(5 \mathrm{ppb})\end{array}$ & $299-1410-19$ & $-W 10-20$ & $299-410-21$ \\
\hline
\end{tabular}

Table 14-28. Kater Quality Standards Exceeded at Low-Level Waste Management Area 5.

\begin{tabular}{|c|c|}
\hline$\underset{(>8.5)}{\mathrm{pH}-\text { Field }}$ & $299-\$ 6-4 \dagger 299-46-5 t$ \\
\hline $\begin{array}{l}\text { Chrowium, fi]tered } \\
(100 \mathrm{ppb})\end{array}$ & $299-146-5$ \\
\hline $\begin{array}{l}\text { Nitrate } \\
(45,000 \mathrm{ppb})\end{array}$ & 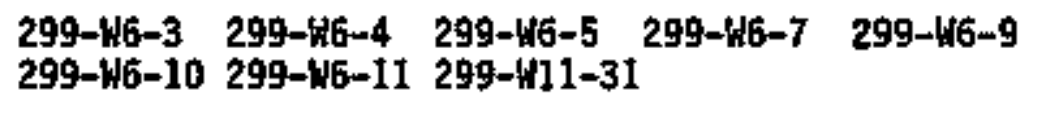 \\
\hline $\begin{array}{l}\text { Manganese, filtered } \\
(50 \mathrm{ppb})\end{array}$ & $299-N 6-6$ \\
\hline $\begin{array}{l}\text { Carbon Tetrachloride } \\
(5 \mathrm{ppb})\end{array}$ & $\begin{array}{llll}299-W 6-2 & 299-W 6-4 & 299-146-5 & 299-W 6-7 \\
299-16-10 & 299-\$ 6-11 & 299-W 7-10 & 299-W 11-31\end{array}$ \\
\hline $\begin{array}{l}\text { Trichloroethene } \\
(5 \mathrm{ppb})\end{array}$ & $299-W 6-5 \quad 299-W 6-10 \quad 299-W 11-31$ \\
\hline $\begin{array}{l}\text { Tritiun } \\
(20,000 \mathrm{pC} 1 / 1)\end{array}$ & $299-W 6-4 \quad 299-W 6-5 \quad 299-W 6-7 \quad 299-W 6-10 \quad 299-W 11-31$ \\
\hline
\end{tabular}

$t$ Suspect data, RADE has been filed. 
DOE/RL-95-69-3

This page intentionally left blank. 
CONTENTS

15.0 SIMGLE-SHELL TANKS $\ldots \ldots \ldots \ldots \ldots \ldots \ldots \ldots \ldots \ldots \ldots$

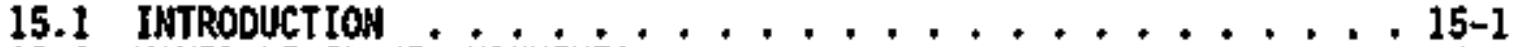

15.2 WATER LEVEL MEASUREMENTS $\ldots \ldots \ldots \ldots \ldots \ldots$

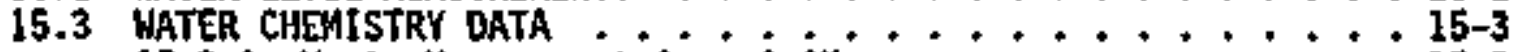

15.3.1 Waste Management Area A-AX . ........ 15-3

15.3.2 Waste Management Area B-BX-BY .......... 15-4

15.3.3 Waste Management Area $C \ldots \ldots \ldots . . . . . . . .15-4$

15.3.4 Waste Management Area S-SX ........... 15-4

15.3.5 Waste Management Area $\mathrm{T} \ldots \ldots \ldots \ldots . . . . . .15-4$

15.3.6 Waste Management Area $\mathrm{TX}-\mathrm{TY} . \ldots \ldots \ldots . . . . . . .15-5$

15.3.7 Waste Management Area $\mathrm{J} . . . . . . . . . .15-5$ 
DOE/RL-95-69-3

\section{LIST OF FICFURES}

15-1 Single-Shell Tank Waste Management Areas in the 200 East Area. . . 15-6 15-2 Single-Shell Tank Waste Management Areas in the 200 West Area. . . . . . . . . . . . 15-7

15-3 Wel] Location Hap for the Single-Shell Tank Waste Management Areas in the 200 East Area. . . . . . . . . . 15-8

15-4 We11 Location Map for the Singie-Shell Tank Waste Managenent Areas in the 200 Hest Area. . . . . . . . . 15-9 


\section{LIST OF TABLES}

15-1 Groundwater Monitoring Hells for the Single-Shell Tanks . . . 15-10

15-2 RCRA Water Level Measurement Report

SingTe-Shell Tanks, Third Quarter 1995 .......... 15-12

15-3 Constituent List and Summary of Results for the

Single-Shell Tank Waste Management Area A-AX Data for

Reporting Period July I through September 30, 1995 . . . . 15-20

15-4 Constituents with at Least one Detected Value for the

Single-Shell Tank Waste Management Area A-AX Data for

Reporting Period July 1 through September 30, $1995 \ldots . . . .15-21$

15-5 Contapination Indicator Parameters for the Single-Sheit

Tank Waste Management Area A-AX Data for Reporting

Period July 1 through September 30, 1995 ......... 15-22

15-6 Const ftuent List and Sumary of Results for the

Single-Shell Tank Waste Management Area B-BY-BX Data for

Reporting Period July 1 through September 30, $1995 \ldots . . . .15-23$

15-7 Const ftuents with at Least One Detected VaTue for the

Single-She11 Tank Waste Manageident Area B-BY-BX Data for

Reporting Period July 1 through September 30, 1995 . . . . . . 15-24

15-8 Contamination Indicator Parameters for the Single-Sheil

Tank Waste Management Area B-BY-BX Data for Reporting

Perlod July 1 through September 30, 1995 ........ 15-25

15-9 Constituent List and Sumbary of Results for the Single-Sheli

Tank Waste Management Area C Data for Reporting Period

July i through September 30, 1995 ................ 15-26

15-10 Constituents with at Least One Detected Value for the

Single-Shell Tank Maste Management Area C Data for

Reparting Period July 1 through September 30, 1995 .... 15-27

15-11 Contamination Indicator Parameters for the Single-sheil Tank

Waste Management Area C Data for Reporting

Period July 1 through September 30, 1995 . . . . . . . . 15-28

15-12 Constituent List and Summary of Resuits for the

Sfngle-Shell Tank Waste Management Area S-SX Data for

Reporting Poriod July 1 through September 30, 1995 ...... 15-29

15-13 Constituents with at Least one Detected Value for the

Stngle-Shell Tank Waste Management Area 5-SX Data for

Reporting Period July 1 through September 30, 1995 ...... 15-30

15-14 Contamination Indicator Parameters for the Single-Sheil

Tank Waste Management Area S-5X Data for Reporting

Period July 1 through September 30, $1995 \ldots . . . . . . . .15-31$

15-15 Constituent List and Summary of Results for the Single-Sheli

Tank Waste Management Area T Data for Reporting

Period July I through September 30, 1995 ........ 15-32

15-16 Constituents with at Least One Detected value for the

Singlo-Shell Tank Maste Management Area $T$ Data for Reporting

Period Juty 1 through September 30, 1995 ...... 15-34

15-17 Contamination Indicator Parameters for the Singlo-Shell

Tank Waste Management Area T Data for Reporting

Period July 1 through September 30, 1995 ........... 15-41

15-18 Constiturent List and Stumatary of Results for the Single-She]l

Tank Maste Management Area TX-TY Data for Reporting

Period July 1 through September 30, 1995 ......... 15-42 


\section{LIST OF TABLES (cont)}

15-19 Constituents with at Least One Detected Vaiue for the Single-Shell Tank Waste Management Area TX-TY Data for Reporting Perfod July 1 through September 30, 1995 . . . . . . 15-44

15-20 Contamination Indicator Parameters for the \$ingle-She7] Tank Waste Management Area TX-TY Data for Reporting Period Jaly 1 through September 30, 1995 . . . . . . . . 15-46

15-21 Constituent List and Summary of Results for the Single-Shell Tank Waste Wanagement Area U Data for Reporting Period July 1 through September 30, $1995 . \ldots$ 15-47

15-22 Constituents with at Least One Detected Value for the Single-She1] Tank Waste Management Area U Data for Reporting Period July 1 through Septewber 30, $1995 . \ldots$ 15-49

15-23 Contamination Indicator Paramiters for the Single-She11 Tank Waste Management Area U Data for Reporting Pertad Juty 1 through September 30, 1995 ........... 15-51 


\title{
15.0 SIHGLE-SHELL TAMS
}

\author{
J. A. Caggi ano \\ Westinghouse Hanford Company
}

\subsection{InTRODUCTIOA}

Although deconosisstoned in 1980, the SSTs are actively storing hazardous and radioactive waste and have been designated as RCRA facilities. The SSTs received metal and first-cycle waste from the chemical processing of uranium-bearing spent fuel rods. Spent fuel rods from reactors in the 100 Areas were processed using the bisath phosphate, REDDX, or PUREX process. Metal waste is that which was produced in the first step of the process of dissolving spent fuels, and contains about $90 \%$ of fission product radionuclides as we11 as uraniun and some piutonium. other waste strearus (e.g., coating, noncomplexed, and delonizing waste) also ware sent to SSTs at some time in their history. Anderson (1990), discusses types of waste sent to varlous SSTs from startup untij the tank was no longer used. Chemical and radiological contents of the various \$STs were summarized in wHC (1993c).

The 149 SSTs are located in 7 waste management areas (WMA) containting one or wore tank farms (Figures 15-1 and 15-2). Three WMAs are in the 200 East Area (A-AX, B-BX-BY, and C) and four are in the 200 West Area (S-SX, T, TX-TY, and U). Each tank farm contatns from 4 (AX Tank Farm) to 18 (TX Tank Farm) tanks, each of which is an underground, reinforced-concrete tank with a singte liner of carbon stool. The larger tanks have a diameter of $23 \mathrm{~m}(75 \mathrm{ft})$, vary in height, and are buried at least $2 \mathrm{~m}(6 \mathrm{ft})$ below the ground surface. The capacity of these tanks varies from $2,006,000$ to $3,785,000 \mathrm{~L}(530,000$ to $1,000,000 \mathrm{gal})$. There are sixteen 200 -sertes tanks, each of which holds $208,175 \mathrm{~L}(55,000 \mathrm{ga})$. Four 200-series tanks are jocated in each of the original four tank farms: 241-B, 241-C, 241-T, and 241-U. The origina] four tank farms were built in 1944; SSTs were built frow 1944 to 1964.

Interim-status, detection-Teve] groundwater monitoring was initiated at the SSTs in 1989 with the construction of 12 RCRA standard wells. Sampi ing was initiated in February 1990 at mas $A-A X, B-B X-B Y, C$, and $T$, but was suspended until July 1991 because of the absence of analytical Iaboratory support. Sampling resumed in 1991 and continued quarterly through early 1993. Most we11s have been sampled for more than four quarters. All WMAs, except $T$ and TX-TY, are now in detection-level indicator-parameter-evaluation status and are sampled semiannually. WAs $T$ and TX-TY continue to be sampled quarterly, as these sites are now in assessment-level monitoring status because of elevated specific conductance. A groundwater quality assessment monitoring plan (Caggiano and Chou 1993) for KuAs $T$ and TX-TY was submitted to Ecology in July 1993. The SST WA well locations are shown in Figures 15-3 and 15-4. Welis in the SST monitoring networks are listed in Table I5-1. Groundwater monitoring at the SSTs follows the groundwater monitoring plan (Jensen et aT. 1989) as revised (Caggiano and Goodwin 1991).

Numerous older weils in the vicinity of the tank farms are constructed of carbon steel casing that is perforated and open to different levels in the unconfined aquifer. These wells were part of an operational groundwater 
monitoring system at some point in their history and currently are used only for water level measurements or to collect samples for operational monitoring. Sofe are used for environental survelllance monitoring to check for conformance to BOE orders (e.g., Schmidt et al. 1992). These ofder wells do not comply with the construction specifications of UAC 173-160 and are not used for collecting RCRA samples. One exception is we11 2-E27-7, a well that was constructed In 1982 and has a carbon steel casing, an annular seal, and a stainless steel sereen below the carbon steel casing. There is no carbon steel in contact with groundwater in well 2-E27-7. Samples are collected from this well, which is upgradient of wa $C$.

\subsection{MATER LEVEL MEASHREMENTS}

Water levels measured in 62 wells for this quarter are given in Table 15-2. These data include routine water level measurements taken when sampling, as well as nonroutine monthly measurements made by Well Services personne1. Suspected errors in water jeve] measurements this quarter are flagged in Table 15-2.

The water table in the 200 East Area is nearly flat because of declining water levels and high transmissivity of the Hanford formation. The water table map for June 1995 (DOE-RL 1996, Fig 4.11-7) illustrates the very low slope to the water table.

A barrier may be constructed over the 216-BY cribs in operable unit 200-BP-1 located north of WNA B-BX-BY. A modified RCRA cover was the preferred alternative in the Record of Dectsion that is going out for public comment. After comments are addressed, the definitive design of the cover wt1l commence. However, it is anticipated that all wells in the area of the 216-BY cribs wt1] decommissioned to construction and to el iminate potential pathways through the cover. New groundwater enitoring wells beyond the new cover will be constructed. Nell 2-E33-38, along with nine older carbon steel wolls formerly used by the Operational Monitoring program to monitor the 216-BY cribs, will be decomissioned. Wells 2-E33-1 and 2-E33-5, along with wel] 2-E33-38, have been used to monitor water levels for the SST\$ in this area of very low water table gradient in the 200 East Area.

Contingency planning for the lacation and design of replacement wells has been initiated, but the project is on hold pending review of the Record of Decision by the Yakama Nation.

Water levels hava been declining steadily over the past several years at a]1 SST sites (see hydrographs of all WAS in DOE-RL [1995]). The rate of decl the appears to be accelerating after a slowing in 1994.

Water level data for this quarter at WA $U$ indicate that groundwater is flowing to the south-5outhwest, but the difference in head anong all wells is generally less than $0.5 \mathrm{ft}$. The borehole velocity flowmeter was run at three different levels within the screens of wells 2-W18-25, 2-W19-31 and 2-W19-32 in May. The direction of flow in 2-W19-31 and 2-W19-32 is north-northwest; the direction of flow measured in $2-W 18-25$ is east-northeast. The conflicting data on the direction of groundwater in an area of very low gradient indicate further work is needed to confirm the direction of groundwater flow to determine whether an additional well may be needed. At this time, WHA U 
appears to be situated above the apex of a local water table mound creating a situation of confounding head data that make the determination of true direction of groundwater flow enigmatic.

\subsection{WATER CHENISTRY DATh}

Data recelved fron analyses of samples collected during the third quarter of 1995 are provided in Tables 15-3 through 15-23. Host SST wells were sanpled this quarter. Quarterly sanpling is presently conducted in WHAs $T$ and TX-TY because these sites are in groundwater assessment monitoring under interin status (40 CFR 265). ATl other WMAs are sampled semiannually except for WMA U. Statistica] methods are provided in Appendix C of the 1995 RCRA Annual Report (DOE 1996).

Regulatory 1 imits for constituents in groundwater have been revised in Tables 15-3 through $15-32$ to reflect current limits in 40 CFR 141 and 40 CFR 143.

Four quarters of background data have been obtained from wells in all WhAs and the data have been statistically evaluated for indications of contanination through analyses of $T O C$, $\mathrm{pH}$, spectfic conductance and TOX for each site. Replicate average values from downgradient wells are compared to the critical means for-Indicator paraneters to determine whether the facijities in any SST WMAs may be affecting groundwater quality. The change in groundwater flow direction beneath WMA U will necessitate a new calculation of background for this site.

Several LLBG RCRA standard wells have been added to the expanded network for groundwater qua]ity assessment nonitoring at WAs $T$ and $T X-T Y$. We 115 299-W6-2, 299-W6-4, 299-W6-6, 299-W6-9, 299-410-19, 299-W10-20, 299-W10-21 and 299-W11-31 may be found on Figures 14-3 (for LLBG WiA 3) or Figure 14-5 (for LLBG WiA 5). Ten radionuclides fron the list of site-specific constituents for the SSTs have been added to the sampling list. for these $11 \mathrm{~s}$, all of whtch are located north of WMA $T$. Some data on radionuclides from these wells were received this quarter and may be found in Tables 15-19 and 15-25. These two MMAs are in groundwater quality assessment status because of elevated specific conductance.

\subsubsection{Haste Management Area A-AX}

A] wells were sampled this quarter, Data are included in Tables 15-3, 15-4, and 15-5. There were no exceedances of the critical mean for field specific conductance or pH. The four values for $\mathrm{pH}$ for well 2-E24-19 are well below the historical trend and have been flagged as suspect. The four values for field pH also dfffer stgnificantly from the pH of 7.50 determined in the laboratory for this sampling event. Data for TOC and TOX do not exceed the critica 1 mean for these parameters. 129 ranges between 3.26 and $7.65 \mathrm{pCi} / \mathrm{L}$ for the five welts, alt of which exceed the regulatory limit. Turbidity exceeded 1 NTU in all wells, with a high of 17.6 NTU in well 299-524-19. 


\subsubsection{Maste Managemant Area B-BX-BY}

All five wells were sampled this quarter. Data are reported in Tables 15-6 through 15-8. Turbidity exceeded 1 NTU in four samples this quarter, with a high of 3.51 NTU in well 299-E33-41. "Tc exceeded the regulatory 7 imit of $900 \mathrm{pCi} / \mathrm{L}$ in well 2-E33-41, but the data are suspect and are being investigated. Gross beta of $356 \mathrm{pCi} / \mathrm{L}$ reflects this value for ${ }^{99} \mathrm{Tc}$. ${ }^{129} \mathrm{I}$ exceeded the regulatory 1 imit in all wells this quarter and rarged between 3.61 and $7.59 \mathrm{pCi} / \mathrm{L}$.

There were no exceedances of the critical mean (or critical range) for the reported indicator parameters for this quarter.

\subsubsection{Haste Management Area C}

All five wells in $\mathrm{W}$. yere sampled this quarter. Data are reported in Tables 15-9 through 15-11. ${ }^{129} 1$ exceeded the regulatory linit ( $1 \mathrm{pC} / \mathrm{i}$ ) in al1 wells this quarter and ranged between 3.80 and $4.37 \mathrm{pCj} / \mathrm{L}$. Turbidity ranged from 0.49 to 3.16 NTU for all samples. There were no exceedances of the critical mean for field specific conductance, $\mathrm{pH}_{\text {, }}$ or TOC. Al1 TOX data are non-detects and do not exceed the upgradient/downgradient comparison value.

\subsubsection{Waste Management Area S-SX}

Data are reported for a11 wells in WMA S-SX in Tables 15-12 through 15-14. Turbidity ranged fron $1.59 \mathrm{NTU}$ to $7.45 \mathrm{NTU}$, with four wells having turbidity below 5 NTU. Trititum exceeded the 20,000 pCi/L DWS in weITs 2-W23-14 and 2-W23-15. 99 Tc (and gross beta) was elevated in well 2-W22-39, but did not exceed the $900 \mathrm{pCi} / \mathrm{L}$ DWS. There were no exceedances of the critical mean for field specific conductance, ph, or TOC. TOX data did not exceed the upgradient/downgradient comparison value.

\subsubsection{Waste Wanagement Area $T$}

All wells were sampled this quarter and the data are included in Tab7es 15-15 through 15-17. Fi]tered chromitum, iron, and nickel exceeded the DWS in well 2-W10-15, but the data are suspect ard are being tnvestigated. Fiuoride also exceeded the 4,000 ppb DWS in well 2-W10-15. Ft1 tered manganese exceeded the $50 \mathrm{ppb}$ DWS in well 2-W6-6, a supplementa] assessment well. Nitrate exceeded the $45,000 \mathrm{ppb}$ DWS in al\} wells but 2-W11-27, 2-W6-2, and 2-W6-6. Tritium exceeded the 20,000 pCi/L. DW5 in wells 2-W10-I5, 2-Wio-16, 2-W6-4, 2-W11-28, and 2-W11-31. TOS exceeded the 500-ppm DWS in a]1 these wel]s except 2-W11-31. Turbidity ranged between 0.74 and 16.1 NTU this quarter, with five weils exceeding 6 NTU.

Field specific conductance continues to be elovated in downgradient well 299-410-15 and is the parameter that triggered this site into groundwater quality assessment monitoring under interim-status regulations. Four values for field $\mathrm{pH}$ exceeded the 8.5 DUS in well 2-1/6-4, but these values depart significantly from the historical trend and an investigation has been inftiated. The 7aboratory $\mathrm{pH}$ for this sample was $\mathbf{8 . 4}$. 


\subsubsection{Waste Management Area TX-TY}

A77 wells in Wint TX-TY were sampled this quarter. Data are in Tables 15-18 through 15-20. Nitrate exceeded the DIS in all wells this quarter, with a maximum of $350,000 \mathrm{ppb}$ in we]1 299-H14-12. "9c continues to exceed the $900 \mathrm{pCi} / \mathrm{L}$. OAS in we11 2-H14-12. ${ }^{129} \mathrm{I}$, TDS and fi]tered chrowium are also elevated above the DNS in this we11. Tritium exceeds the 1 imit of $20,000 \mathrm{pCi} / \mathrm{L}$ in ail wells but 2-N10-18. Turbidity ranges from 2.5 to $30.7 \mathrm{kTU}$.

Field specific conductance continues to be elevated in downgradient wells 2-W14-12 and 2-W10-17 and is the reason WA TX-TY has been triggered into groundwater quality assessment monitoring under interim-status regulations.

\subsubsection{Waste Managenent Area U}

Samples were collected in all wells this quarter at WMA U. The data are reported in Tables 15-21 through 15-23. There were no exceedances of any DWS for any constituents monjtored this quarter. Turbidity ranged between 1.46 and 5.64 NTU this quarter, with all bat one value below 5 NTU.

There were no exceedances of the critical mean or critical range for data reported this quarter. The background levels for indicator parameters have been recalculated because of a change in groundwater flow direction beneath WA U. Quarterly sampling for JOX will be jnjtiated in 1996 to establish a background level for that parameter. 
Figure 15-1. Single-\$hell Tank Waste Management Areas in the 200 East Area.

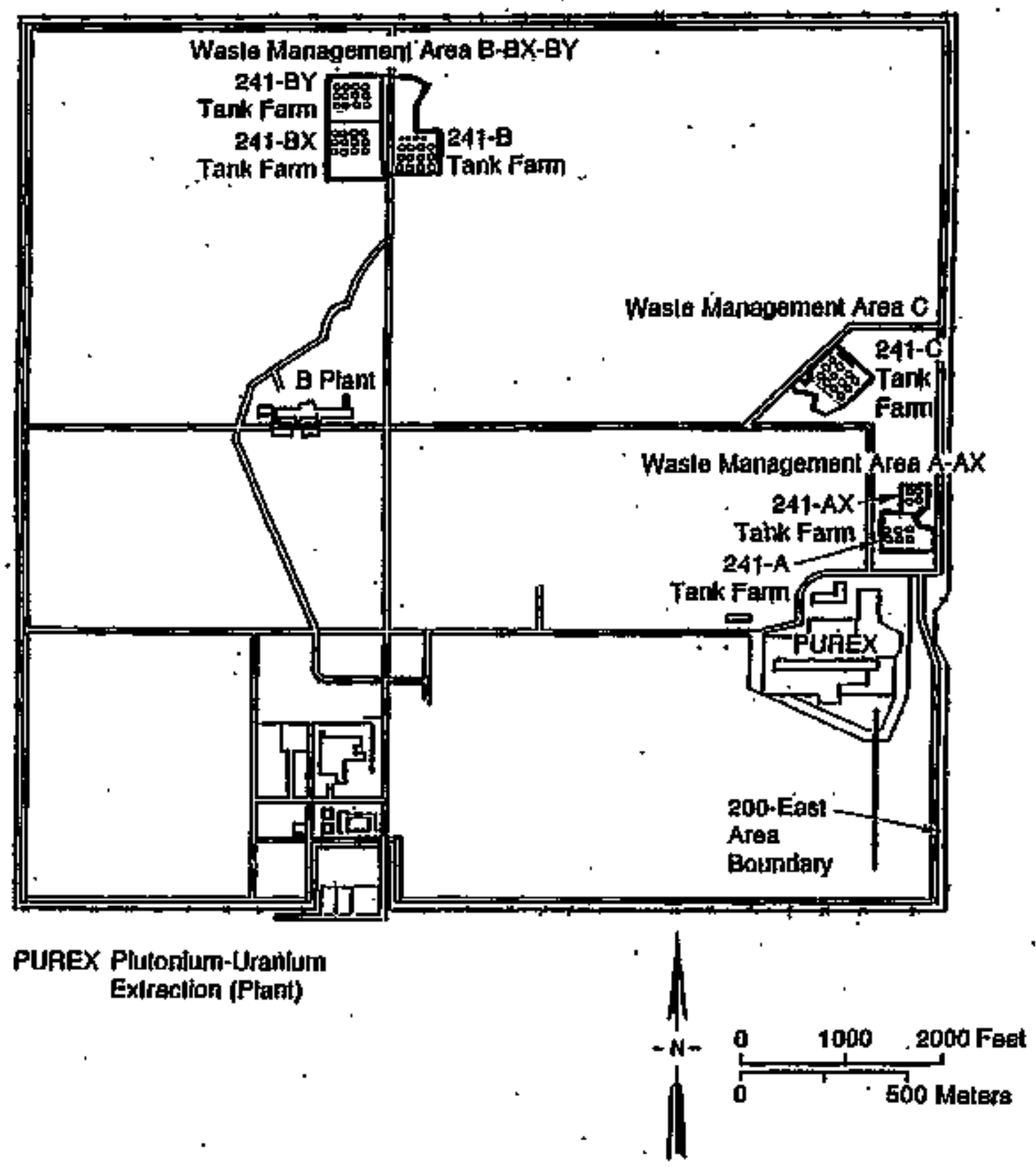

P8-90-71 
Figure 15-2. Single-She11 Tank Waste Management Areas in the 200 West Area.

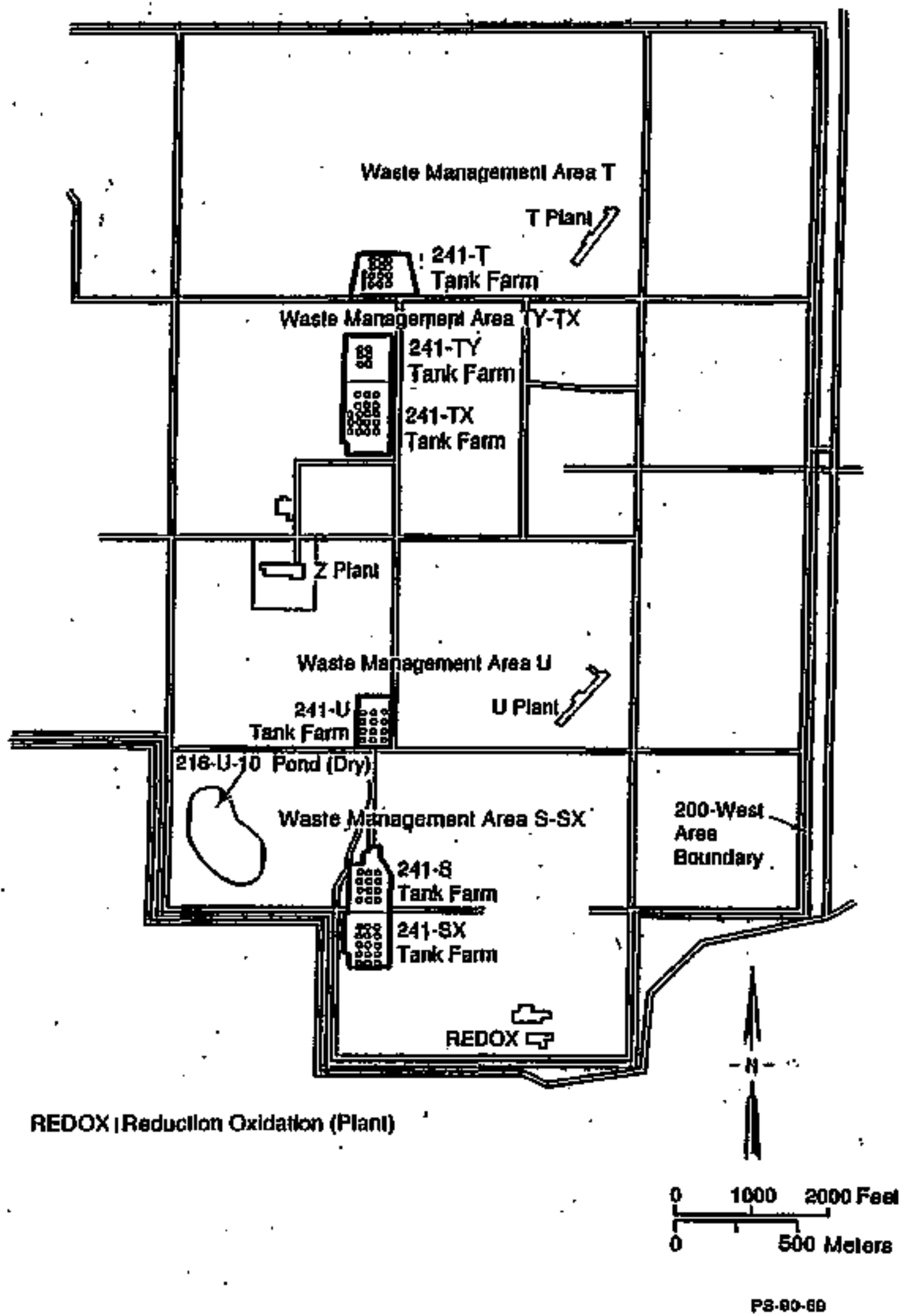




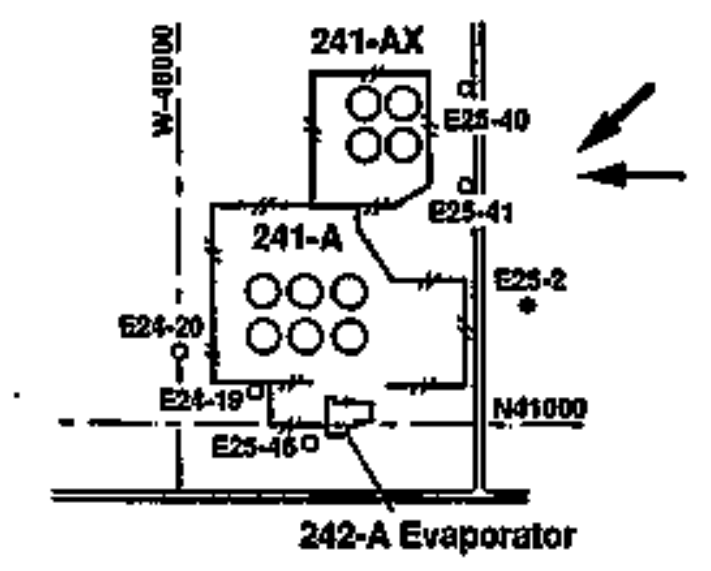

Waste Banogoment Area C

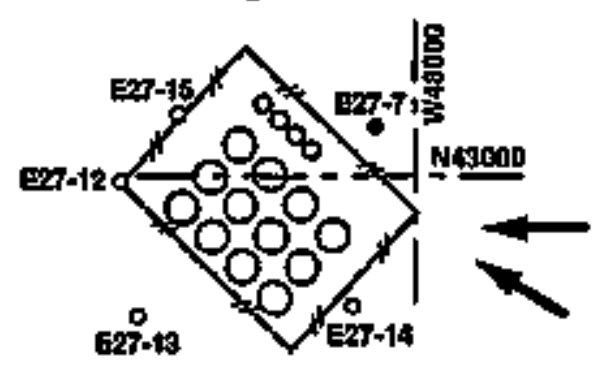

Monitoring Well Locatlons for the Single-Shel] Tank Waste Management Arees Wiknin the 200 East Area

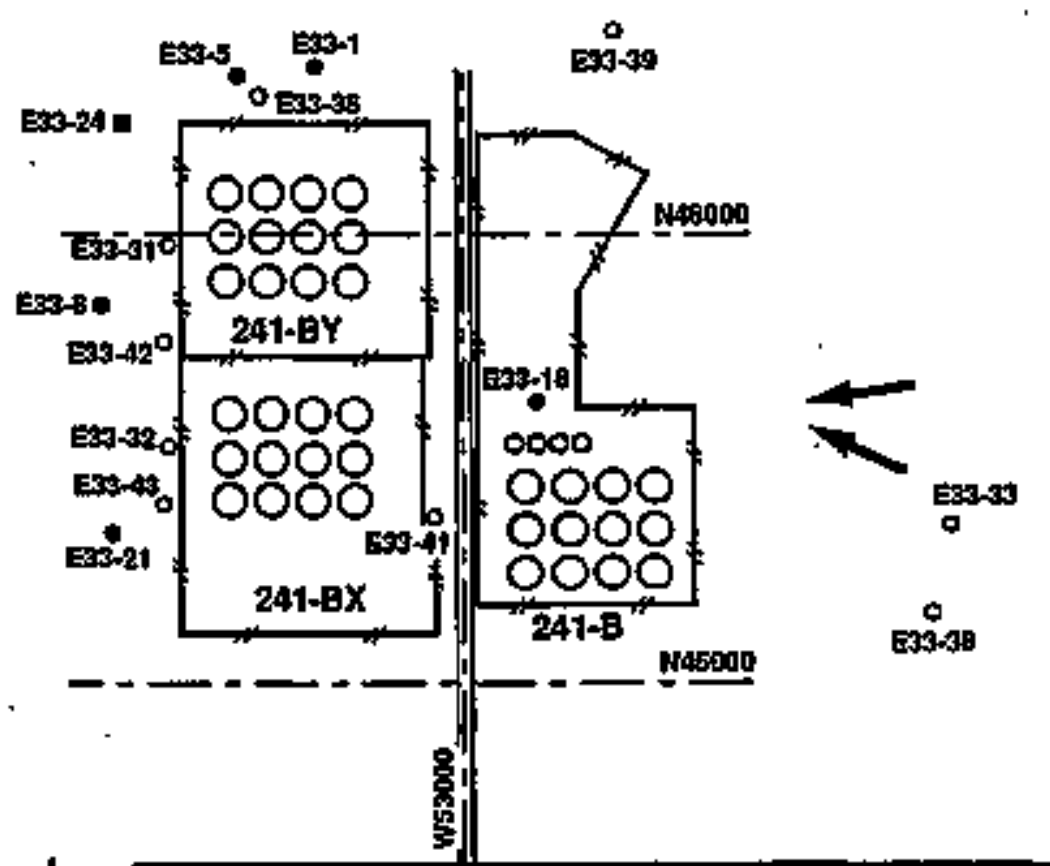

- Cabon Steal Ground Woter Wonltoring Weth

- gCproround Whater Ponltoring Wed

2 Dagommatatonted Ground Water Honllorlng Wall

Eas-1 Humbaring 8yalom tor Ground Water Honlloring Welle. All Wallo Protixed ity 20 .

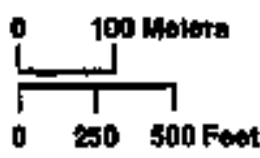

2005otion Singto-

Sheil that.

0 gingtashont tank

- Fanted

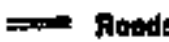

C cenemelked Dheotion of enound Wast Fiow

Haobo Fentord sivio coordinated

RCFA Hosautec Consesvation and Racovary Aal 


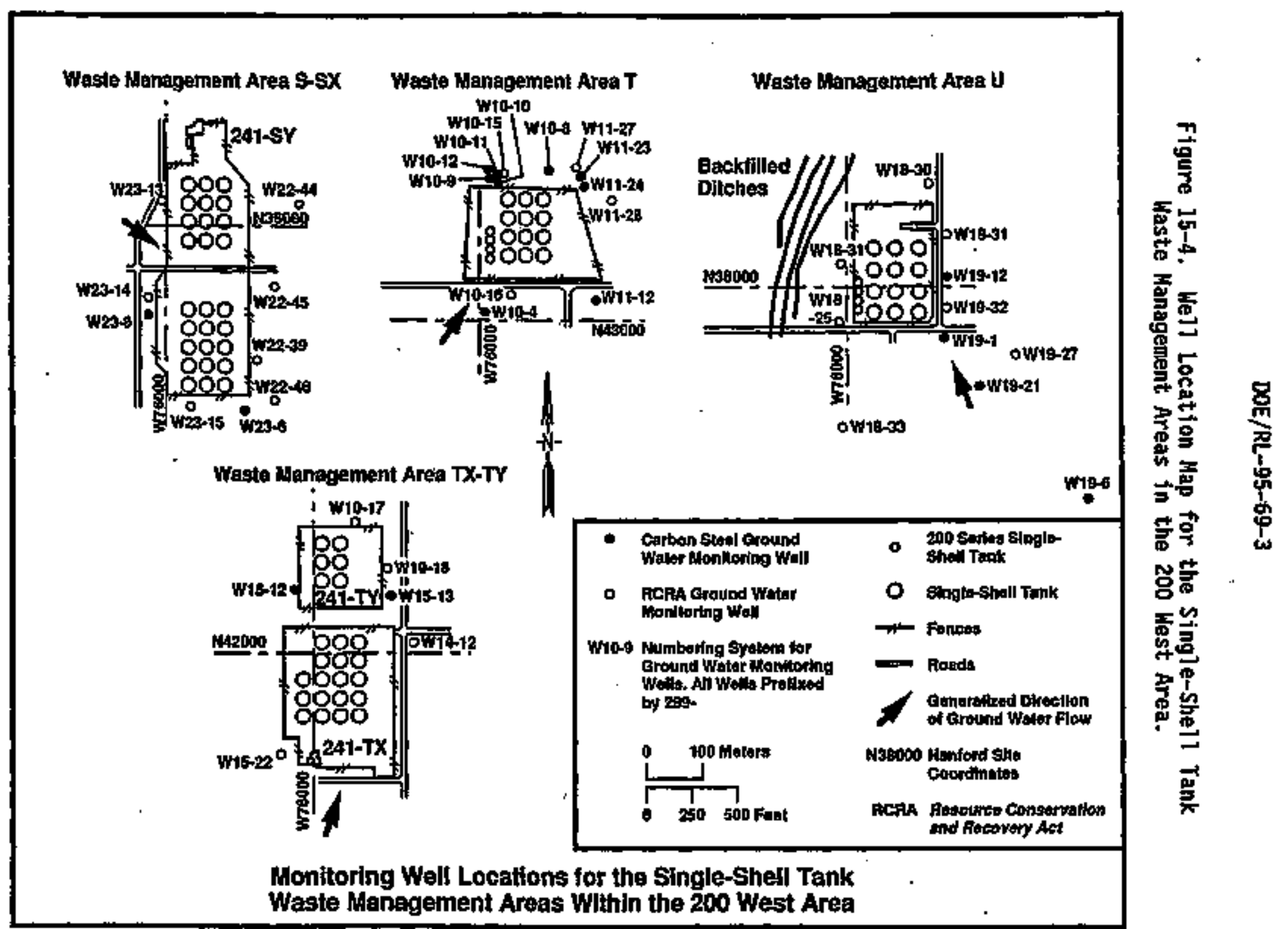

1411010.10 
Table 15-1. Groundwater Monitoring Wells for the Single-Shell Tanks. (sheet 1 of 2)

\begin{tabular}{|c|c|c|c|}
\hline $\begin{array}{c}\text { Well no. } \\
(299-)\end{array}$ & Aquifer" & $\begin{array}{c}\text { Sample } \\
\text { frequency }\end{array}$ & $\begin{array}{l}\text { Sample date, } \\
\text { 3rd Qtr } 1995\end{array}$ \\
\hline \multicolumn{4}{|c|}{ Single-Shell Tanks Waste Wanagement Area A-AX } \\
\hline$E 24-19^{39}$ & Ringold: Water Table & Semi annual 7y & $8 / 3 / 95$ \\
\hline$E 24-20^{91}$ & Ringold: Nater Table & Semiannually & $8 / 2 / 95$ \\
\hline$E 25+40^{\$ 2}$ & Ringold: Hater Table & Semantanually & $8 / 1 / 95$ \\
\hline $\mathrm{E} 25-41^{39}$ & Ringold: Water Table & Sematannta $11 y$ & $8 / 2 / 95$ \\
\hline$E 25-46^{92}$ & Ringold: Water Table & Semiannually & $8 / 1 / 95$ \\
\hline \multicolumn{4}{|c|}{ Single-She11 Tanks Waste Managenent Area B-BX-BY } \\
\hline$E 33^{3}-31^{86}$ & Hanford: Water Table & Semiannual1y & $8 / 4 / 95$ \\
\hline$E 33-32^{69}$ & Hanford: Water Table & Semtannual ly & $8 / 2 / 95$ \\
\hline $533-93^{6 / 2}$ & Hanford: Water Table & Semilannuat Ty & $8 / 1 / 95$ \\
\hline$E 33-36^{345}$ & Hamford: Water Table & Semfamuatiy & $8 / 1 / 95$ \\
\hline $\mathrm{E33-41^{913 }}$ & Hanford: Water Table & Semfannually & $8 / 4 / 95$ \\
\hline$E 33-38^{\text {gic }}$ & Hanford: Water Table & Semt arnually & HS \\
\hline$E 33-39^{91 c}$ & Hanford: Water Table & Semt annual ly & NS \\
\hline$E 33-42^{31}$ & Hanford: Water Table & Semiannually & $8 / 3 / 95$ \\
\hline$E 33-43^{91}$ & Hanford: Water Table & Semiannually & $8 / 3 / 95$ \\
\hline \multicolumn{4}{|c|}{ Single-Shell Tank Waste Management Area C } \\
\hline$E 27-12^{89}$ & Ringold: Water Table & Semtannually & $8 / 2 / 95$ \\
\hline$E 27-13^{\mathrm{Bg}}$ & Ringold: Water Table & SemiannuaTly & $8 / 3 / 95$ \\
\hline$E 27-14^{18-}$ & Ringold: Water Table & Semiannualiy & $8 / 2 / 95$ \\
\hline $527-15^{89}$ & Ringold: Water Table & Semiannually & $8 / 2 / 15$ \\
\hline$E 27-7^{82}$ & Ringold: Water Table & Semi๋ annuaity & $8 / 2 / 95$ \\
\hline \multicolumn{4}{|c|}{ Single-Shel] Tanks Waste Kanagement Area S-SX } \\
\hline W22-39 & Ringold: Water Table & Semiannua $11 y$ & $8 / 9 / 95$ \\
\hline W22-44 & Ringold: Water Table & Seviannua7ly & $8 / 9 / 95$ \\
\hline$W 22-45^{92}$ & Ringold: Water Table & Sermiannual7y & $8 / 9 / 95$ \\
\hline$W 22-46^{51}$ & Ringold: Water Table & Semiannua]ly & $8 / 9 / 95$ \\
\hline $123-13^{90}$ & Ringold: Water Table & Semtannually & $8 / 4 / 95$ \\
\hline
\end{tabular}


Table 15-1. Groundwater Honttoring Wells for the Stingle-Shell Tanks. (sheet 2 of 2)

\begin{tabular}{|c|c|c|c|}
\hline $\begin{array}{c}\text { We11 no. } \\
(299-)\end{array}$ & Aquifer" & $\begin{array}{c}\text { Sample } \\
\text { frequency }\end{array}$ & $\begin{array}{l}\text { Sawple date, } \\
\text { 3rd Qtr } 1995\end{array}$ \\
\hline $123-14^{900}$ & Ringold: Water Table & Sewianutat1y & $8 / 9 / 95$ \\
\hline $123-15^{9 i}$ & Ringold: Water Table & Sentannual1y & $8 / 9 / 95$ \\
\hline \multicolumn{4}{|c|}{ Single-Shel1 Tanks Waste Wanagement Area $T$} \\
\hline $410-15^{9}$ & Ringold: Water Table & Quarterly & $8 / 8 / 95$ \\
\hline $410-16^{35}$ & Rengold: Water Table & Quarterly & $8 / 7 / 95$ \\
\hline $110-19^{92}$ & Ringold: Water Table & Quarterly & $.9 / 6 / 95$ \\
\hline$M 10-20^{93}$ & Ringold: Water Table & Quarteriy & $9 / 6 / 95$ \\
\hline $410-21^{93}$ & Ringold: Water Table & Quarterly & $9 / 11 / 95$ \\
\hline$N I I-27^{4 I}$ & Ringold: Water Table & Quarterly & $8 / 8 / 95$ \\
\hline $411-28^{91}$ & Ringold: Water Table & Quarterly & $8 / 10 / 95$ \\
\hline $411-31^{92}$ & Ringold: Mater Table & Quarterly & $9 / 13 / 95$ \\
\hline \multicolumn{4}{|c|}{ Single-She11 Tanks Waste Hanagement Area TX-TY } \\
\hline $410-17^{91}$ & Rtngold: Hater Table & Quartarly & $8 / 7 / 95$ \\
\hline $\mathrm{W10-18^{ \textrm {g } 0 }}$ & Rtngold: Water Table & Quarterly & $8 / 7 / 95$ \\
\hline $114-12^{51}$ & Ringold: Water Table & Quarterly & $8 / 8 / 95$ \\
\hline $115-22^{51}$ & Ringold: Nater Table & Quarterty & $8 / 24 / 95$ \\
\hline \multicolumn{4}{|c|}{ Single-\$he11 Tanks Waste Management Area U } \\
\hline$W 18-25^{9 / 4}$ & Ringold: Water Table & Semiannually & $7 / 31 / 95$ \\
\hline $\mathbf{W 1 8 - 3 0 ^ { 9 1 }}$ & Ringold: Water Table & Senianntraliy & $8 / 1 / 95$ \\
\hline $\mathrm{W} 18-31^{9 T}$ & Ringold: Water Table & Sentannualiy & $8 / 1 / 95$ \\
\hline $119-91^{96}$ & Ringold: Water Fable & Semijammtaliy & $7 / 31 / 95$ \\
\hline $129-32^{97}$ & Ringold: Water Table & Semjannuat ty & $7 / 31 / 95$ \\
\hline $119-12^{83}$ & Ringold: Nater Table & Senfiantuatiy & NS \\
\hline
\end{tabular}

Notes: Shrding denotes upgradient wells. Superscript following well number denotes the year of completion of the well.

Wells are screened etther in sand and gravel of the Hanford formation or in matrix-supported si]ty sandy gravel of the Ringold Formation.

Wel1 in middle of WHA B-BX-BY and not clearly upgradient or downgradient. Therefore, data from sampling/analyses in this we]1 are not used in statistical evaluations.

part of 200-BP-1 operable unit network. Not sampled regularly as RCRA we]1s.

HS * not sampied. 
Table 15-2. RCRA Water Level Measurement Report Single-Shell Tanks, Third Quarter 1995.

(sheet 1 of 8 )

\begin{tabular}{|c|c|c|c|c|}
\hline Well & Bate & $\begin{array}{l}\text { Depth to } \\
\text { water (ft) }\end{array}$ & $\begin{array}{l}\text { Water } \\
\text { eTevation } \\
\text { (ft) }\end{array}$ & $\begin{array}{c}\text { Jevel } \\
\text { above msl } \\
\text { (i) }\end{array}$ \\
\hline \multicolumn{5}{|c|}{ 241-A/AX Tank Farm Mells } \\
\hline 299-E24-19 & $\begin{array}{l}7 / 25 / 95 \\
8 / 03 / 95 \\
8 / 16 / 95 \\
9 / 13 / 95\end{array}$ & $\begin{array}{l}292.25 \\
292.43^{\star} \\
292.30 \\
292.35\end{array}$ & $\begin{array}{l}401.40 \\
401.22 \\
401.35 \\
401.30\end{array}$ & $\begin{array}{l}122.35 \\
122.29 \\
122.33 \\
122.32\end{array}$ \\
\hline $299-€ 24-20$ & $\begin{array}{l}7 / 25 / 95 \\
8 / 02 / 95 \\
8 / 16 / 95 \\
9 / 13 / 95\end{array}$ & $\begin{array}{l}287.57 \\
287.92 \star \\
287.62 \\
287.68\end{array}$ & $\begin{array}{l}401.71 \\
401.36 \\
401.66 \\
401.60\end{array}$ & $\begin{array}{l}122.44 \\
122.33 \\
122.43 \\
122.41\end{array}$ \\
\hline $299-E 25-2$ & $\begin{array}{l}7 / 25 / 95 \\
8 / 16 / 95 \\
9 / 13 / 95\end{array}$ & $\begin{array}{l}273.82 \\
273.94 \\
273.94\end{array}$ & $\begin{array}{l}401.83 \\
401.71 \\
401.71\end{array}$ & $\begin{array}{l}122.48 \\
122.44 \\
122.44\end{array}$ \\
\hline 299-E25-40 & $\begin{array}{l}7 / 25 / 95 \\
8 / 01 / 95 \\
8 / 16 / 95 \\
9 / 13 / 95\end{array}$ & $\begin{array}{l}264.19 \\
264.16^{\star} \\
264.33 \\
264.30\end{array}$ & $\begin{array}{l}401.72 \\
401.75 \\
401.58 \\
401.61\end{array}$ & $\begin{array}{l}122.44 \\
122.45 \\
122.40 \\
122.41\end{array}$ \\
\hline $299-E 25-41$ & $\begin{array}{l}7 / 25 / 95 \\
8 / 02 / 95 \\
8 / 16 / 95 \\
9 / 13 / 95\end{array}$ & $\begin{array}{l}269.67 \\
269.88^{\star} \\
269.78 \\
269.79\end{array}$ & $\begin{array}{l}401.79 \\
401.58 \\
401.68 \\
401.67\end{array}$ & $\begin{array}{l}122.47 \\
122.40 \\
122.43 \\
122.43\end{array}$ \\
\hline 299-E25-46 & $\begin{array}{l}7 / 25 / 95 \\
8 / 01 / 95 \\
8 / 16 / 95 \\
9 / 13 / 95\end{array}$ & $\begin{array}{l}293.09 \\
293.03^{\circ} \\
293.17 \\
293.18\end{array}$ & $\begin{array}{l}401.72 \\
401.78 \\
401.64 \\
401.63\end{array}$ & $\begin{array}{l}122.44 \\
122.46 \\
122.42 \\
122.42\end{array}$ \\
\hline \multicolumn{5}{|c|}{ 24I-8/BX/BY Tank Farm Wells } \\
\hline 299-E33-1 & $\begin{array}{l}7 / 25 / 95 \\
8 / 16 / 95 \\
9 / 13 / 95\end{array}$ & $\begin{array}{l}230.65 \\
230.69 \\
230.63\end{array}$ & $\begin{array}{l}401.46 \\
401.42 \\
401.48\end{array}$ & $\begin{array}{l}122.37 \\
122.35 \\
122.37\end{array}$ \\
\hline 299-E,33-18 & $\begin{array}{l}7 / 25 / 95 \\
8 / 16 / 95 \\
9 / 13 / 95\end{array}$ & $\begin{array}{l}250.28 \\
250.35 \\
250.34\end{array}$ & $\begin{array}{l}401.58 \\
401.51 \\
401.52\end{array}$ & $\begin{array}{l}122.40 \\
122.38 \\
122.38\end{array}$ \\
\hline
\end{tabular}


Table 15-2. RCRA Hater Leve] Measurement Report Single-Shell Tanks, Third quarter 1995.

(sheet 2 of 8)

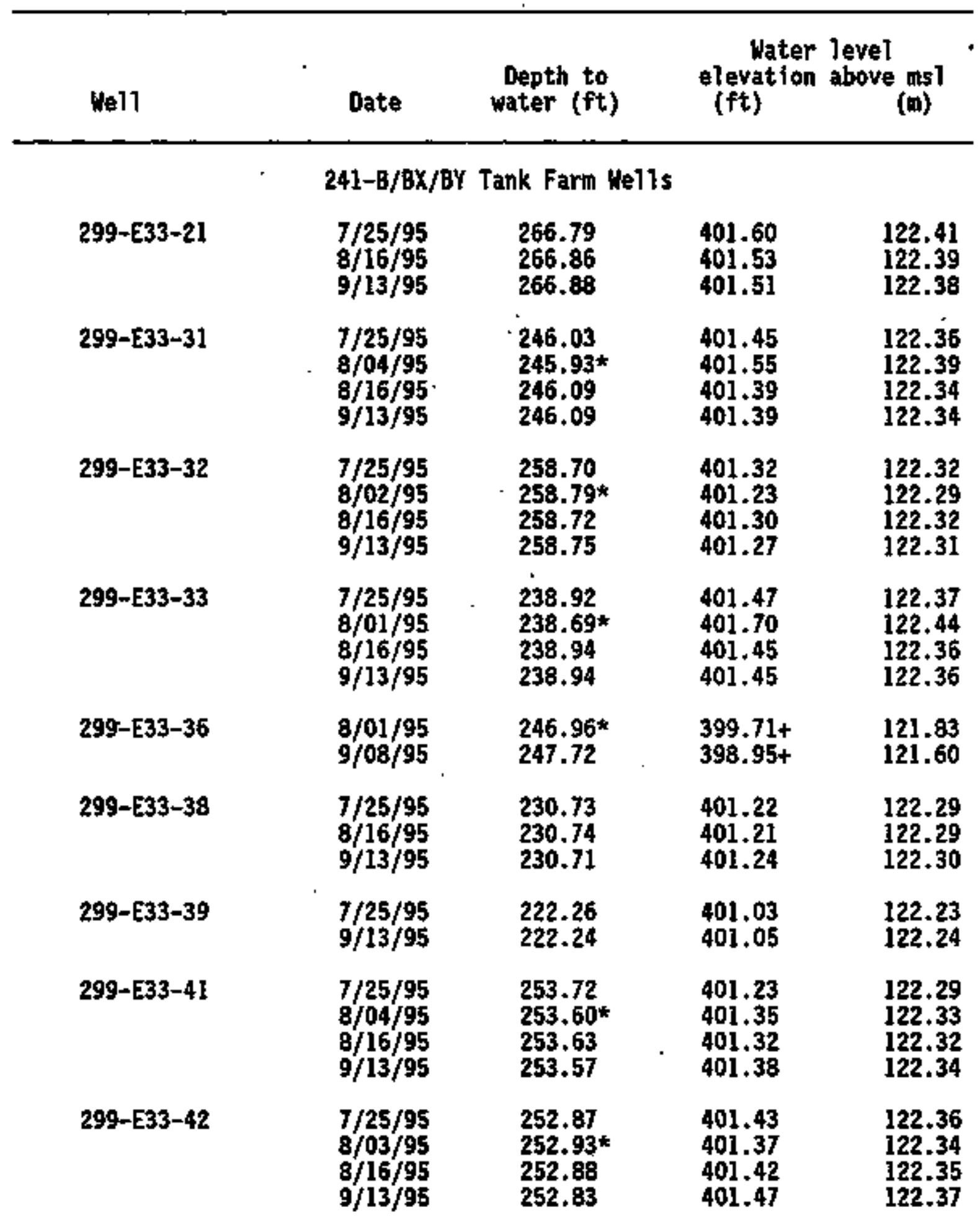


Table 15-2. RCRA Water Level Heasurement Report Single-Shell Tanks, Third Quarter 1995.

(sheet 3 of 8 )

\begin{tabular}{|c|c|c|c|c|}
\hline Wel? & Date & $\begin{array}{l}\text { Depth to } \\
\text { water (ft) }\end{array}$ & $\begin{array}{l}\text { Water } \\
\text { elevation } \\
\text { (ft) }\end{array}$ & $\begin{array}{l}\text { level } \\
\text { above msl } \\
\text { (m) }\end{array}$ \\
\hline \multicolumn{5}{|c|}{ 241-B/BX/BY Tank Farn Wel1s } \\
\hline 299-E33-43 & $\begin{array}{l}7 / 25 / 95 \\
8 / 03 / 95 \\
8 / 16 / 95 \\
9 / 13 / 95\end{array}$ & $\begin{array}{l}261.39 \\
261.47^{*} \\
261.36 \\
261.38\end{array}$ & $\begin{array}{l}401.29 \\
401.21 \\
401.32 \\
401.30\end{array}$ & $\begin{array}{l}122.31 \\
122.29 \\
122.32 \\
122.32\end{array}$ \\
\hline 299-E33-5 & $\begin{array}{l}7 / 25 / 95 \\
8 / 16 / 95 \\
9 / 13 / 95\end{array}$ & $\begin{array}{l}233.19 \\
233.23 \\
233.20\end{array}$ & $\begin{array}{l}401.53 \\
401.49 \\
401.52\end{array}$ & $\begin{array}{l}122.39 \\
122.37 \\
122.38\end{array}$ \\
\hline 299-E33-8 & $\begin{array}{l}7 / 25 / 95 \\
8 / 16 / 95 \\
9 / 13 / 95\end{array}$ & $\begin{array}{l}249.42 \\
249.46 \\
249.47\end{array}$ & $\begin{array}{l}401.57 \\
401.53 \\
401.52\end{array}$ & $\begin{array}{l}122.40 \\
122.39 \\
122.38\end{array}$ \\
\hline \multicolumn{5}{|c|}{ 241-C Tank Farm Wel1s } \\
\hline $299-E 27-12$ & $\begin{array}{l}7 / 25 / 95 \\
8 / 02 / 95 \\
8 / 16 / 95 \\
9 / 13 / 95\end{array}$ & $\begin{array}{l}259.58 \\
259.88 * \\
259.68 \\
259.66\end{array}$ & $\begin{array}{l}401.57 \\
401.27+ \\
401.47 \\
401.49\end{array}$ & $\begin{array}{l}122.40 \\
122.31 \\
122.37 \\
122.37\end{array}$ \\
\hline 299-E27-13 & $\begin{array}{l}7 / 25 / 95 \\
8 / 03 / 95 \\
8 / 16 / 95 \\
9 / 13 / 95\end{array}$ & $\begin{array}{l}267.58 \\
267.56 * \\
267.56 \\
267.63\end{array}$ & $\begin{array}{l}401.61 \\
401.63 \\
401.63 \\
401.56\end{array}$ & $\begin{array}{l}122.41 \\
122.42 \\
122.42 \\
122.40\end{array}$ \\
\hline $299-E 27-14$ & $\begin{array}{l}7 / 25 / 95 \\
8 / 02 / 95 \\
8 / 16 / 95 \\
9 / 13 / 95\end{array}$ & $\begin{array}{l}257.00 \\
257.09 * \\
257.08 \\
257.09\end{array}$ & $\begin{array}{l}401.54 \\
401.45 \\
401.46 \\
401.45\end{array}$ & $\begin{array}{l}122.39 \\
122.36 \\
122.37 \\
122.36\end{array}$ \\
\hline 299-E27-15 & $\begin{array}{l}7 / 25 / 95 \\
8 / 02 / 95 \\
8 / 16 / 95 \\
9 / 13 / 95\end{array}$ & $\begin{array}{l}251.69 \\
251.89 \star \\
252.09 \\
251.72\end{array}$ & $\begin{array}{l}401.18 \\
400.98 \\
400.78 \\
401.15+\end{array}$ & $\begin{array}{l}122.28 \\
122.22 \\
122.15 \\
122.27\end{array}$ \\
\hline 299-E27-7 & $\begin{array}{l}7 / 25 / 95 \\
8 / 02 / 95 \\
8 / 16 / 95 \\
9 / 13 / 95\end{array}$ & $\begin{array}{l}233.09 \\
233.72^{*} \\
233.23 \\
233.21\end{array}$ & $\begin{array}{l}401.78 \\
401.15 \\
401.64 \\
401.65\end{array}$ & $\begin{array}{l}122.46 \\
122.27 \\
122.42 \\
122.43\end{array}$ \\
\hline
\end{tabular}


Table 15-2. RCRA Vater Leve1 Heasurement Report Single-Shell Tanks, Third Quarter 1995.

(sheet 4 of 8 )

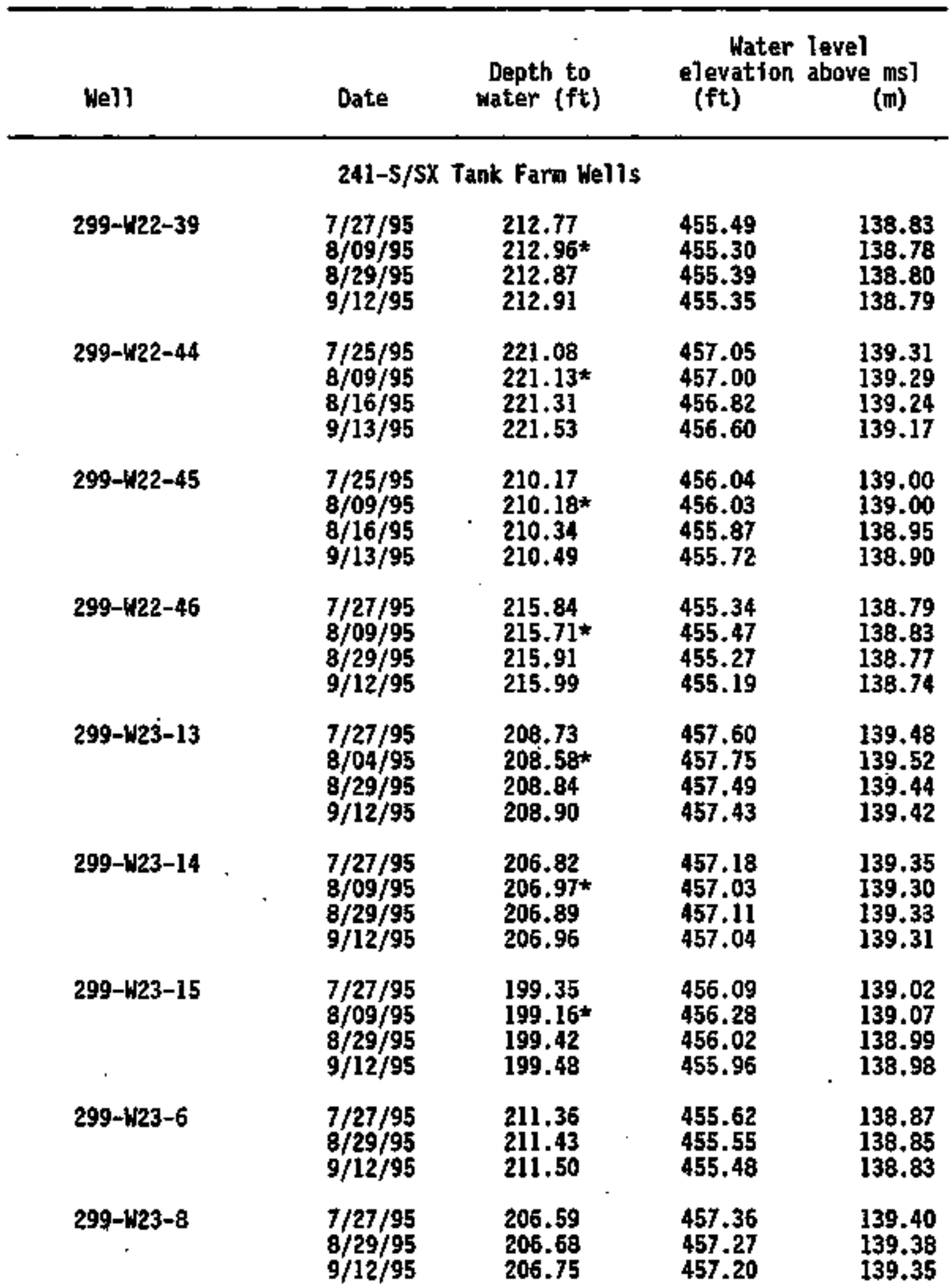


Table 15-2. RCRA Hater Level Heasurement Report Single-Shell Tanks, Third Quarter 1995. (sheet 5 of 8 )

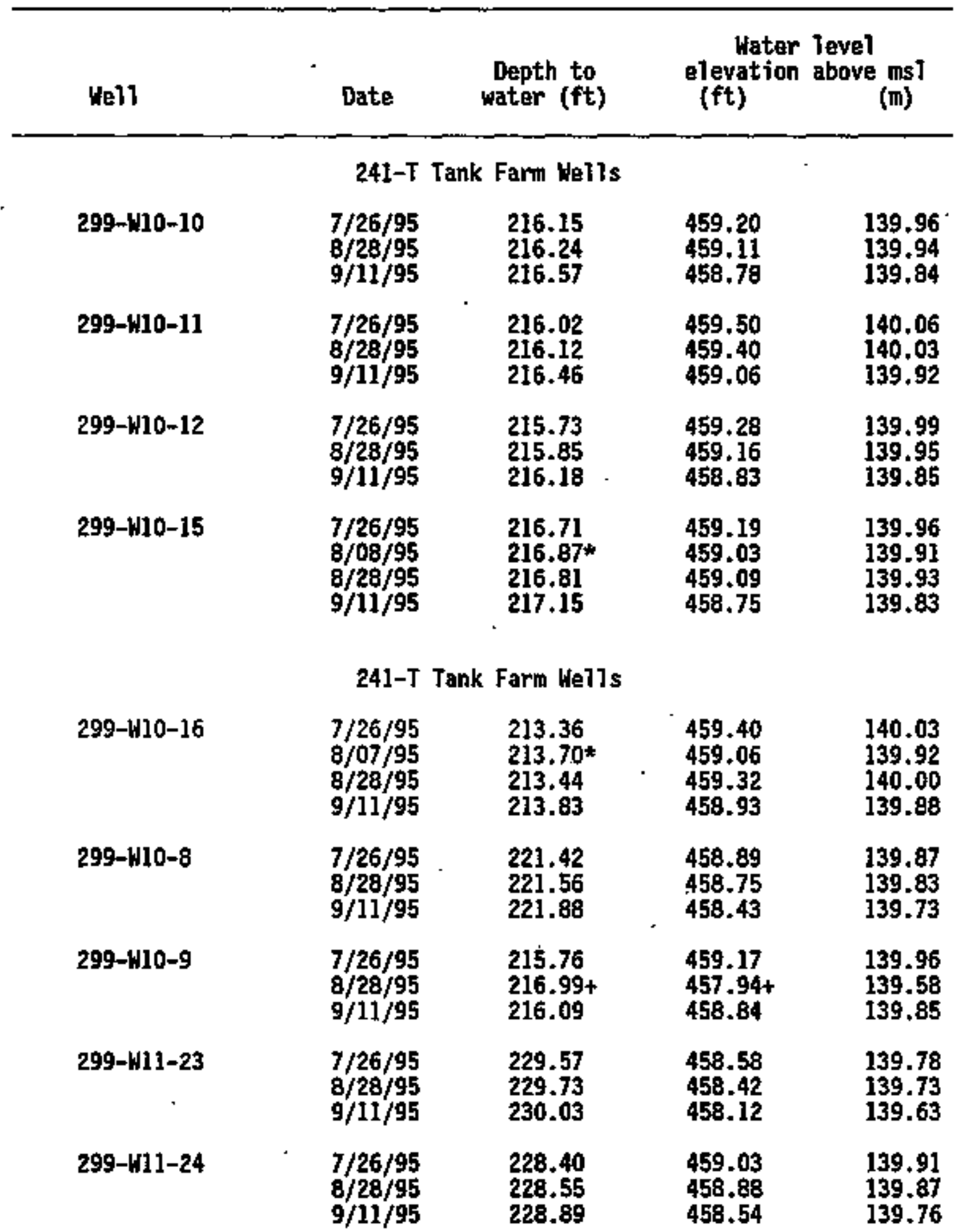


Tabie 15-2. RCPA Nater Level Measurenent Report Single-Shell Tanks, Third Quarter 1995.

(sheet 6 of 8 )

\begin{tabular}{|c|c|c|c|c|}
\hline Wel1 & Date & $\begin{array}{l}\text { Depth to } \\
\text { water (ft) }\end{array}$ & $\begin{array}{l}\text { Water } \\
\text { eleration } \\
\text { (ft) }\end{array}$ & $\begin{array}{l}\text { level } \\
\text { above ms1 } \\
\text { (m) }\end{array}$ \\
\hline & \multicolumn{2}{|c|}{ 241-T Tank Fard Wells } & . & \\
\hline $\begin{array}{c}\text { 299-W11-27 } \\
.\end{array}$ & $\begin{array}{l}7 / 26 / 95 \\
8 / 08 / 95 \\
8 / 28 / 95 \\
9 / 11 / 95\end{array}$ & $\begin{array}{l}226.47 \\
226.65 * \\
226.61 \\
226.91\end{array}$ & $\begin{array}{l}458.80 \\
458.62 \\
458.66 \\
458.36\end{array}$ & $\begin{array}{l}139.84 \\
139.79 \\
139.80 \\
139.71\end{array}$ \\
\hline \multirow[t]{2}{*}{ 299-W11-28 } & $\begin{array}{l}7 / 26 / 95 \\
8 / 10 / 95 \\
8 / 28 / 95 \\
9 / 11 / 95\end{array}$ & $\begin{array}{l}234.34 \\
234.30^{\star} \\
235.69+ \\
234.99\end{array}$ & $\begin{array}{l}459.14 \\
459.18 \\
457.79 \\
458.49\end{array}$ & $\begin{array}{l}139.95 \\
139.96 \\
139.53 \\
139.75\end{array}$ \\
\hline & \multicolumn{3}{|c|}{ 241-TX/TY Tank Farm Me11s } & \\
\hline 299-W10-17 & $\begin{array}{l}7 / 26 / 95 \\
8 / 07 / 95 \\
8 / 28 / 95 \\
9 / 11 / 95\end{array}$ & $\begin{array}{l}211.12 \\
211.50^{*} \\
211.20 \\
211.60\end{array}$ & $\begin{array}{l}459.72 \\
459.34 \\
459.64 \\
459.24\end{array}$ & $\begin{array}{l}140.12 \\
140.01 \\
140.10 \\
139.98\end{array}$ \\
\hline $299-410-18$ & $\begin{array}{l}7 / 26 / 95 \\
8 / 07 / 95 \\
8 / 28 / 95 \\
9 / 11 / 95\end{array}$ & $\begin{array}{l}210.50 \\
210.90^{*} \\
210.66 \\
211.15\end{array}$ & $\begin{array}{l}460.43 \\
460.03 \\
460.27 \\
459.78\end{array}$ & $\begin{array}{l}140.34 \\
140.22 \\
140.29 \\
140.14\end{array}$ \\
\hline $299-1114-12$ & $\begin{array}{l}7 / 26 / 95 \\
8 / 08 / 95 \\
8 / 28 / 95 \\
9 / 11 / 95\end{array}$ & $\begin{array}{l}209.44 \\
209.78^{\star} \\
209.77 \\
210.16\end{array}$ & $\begin{array}{l}461.08 \\
460.74 \\
460.75 \\
460.36\end{array}$ & $\begin{array}{l}140.54 \\
140.43 \\
140.44 \\
140.32\end{array}$ \\
\hline $299-415-12$ & $\begin{array}{l}7 / 26 / 95 \\
8 / 28 / 95 \\
9 / 11 / 95\end{array}$ & $\begin{array}{l}209.54 \\
209.67 \\
210.08\end{array}$ & $\begin{array}{l}460.53 \\
460.40 \\
459.99\end{array}$ & $\begin{array}{l}140.37 \\
140.33 \\
140.20\end{array}$ \\
\hline $299-415-13$ & $\begin{array}{l}7 / 26 / 95 \\
8 / 28 / 95 \\
9 / 11 / 95\end{array}$ & $\begin{array}{l}209.59 \\
209.81 \\
210.25\end{array}$ & $\begin{array}{l}460.53 \\
460.31 \\
459.87\end{array}$ & $\begin{array}{l}140.37 \\
140.30 \\
140.17\end{array}$ \\
\hline $299-115-22$ & $\begin{array}{l}7 / 26 / 95 \\
8 / 24 / 95 \\
8 / 28 / 95 \\
9 / 11 / 95\end{array}$ & $\begin{array}{l}208.94 \\
209.64 * \\
209.19 \\
209.65\end{array}$ & $\begin{array}{l}461.83 \\
461.13 \\
461.58 \\
461.12\end{array}$ & $\begin{array}{l}140.77 \\
140.55 \\
140.69 \\
140.55\end{array}$ \\
\hline
\end{tabular}


Table 15-2. RCRa Water Leve] Measurement Report single-She11 Tanks, Third Quarter 1995.

(sheet 7 of 8 )

\begin{tabular}{|c|c|c|c|c|}
\hline Well & Date & $\begin{array}{l}\text { Depth to } \\
\text { water (ft) }\end{array}$ & $\begin{array}{l}\text { Water } \\
\text { elevation } \\
\text { (ft) }\end{array}$ & $\begin{array}{c}\text { level } \\
\text { above msl } \\
\text { (m) }\end{array}$ \\
\hline \multicolumn{5}{|c|}{ 241-U Tank Fam Wells } \\
\hline $299-\$ 18-25$ & $\begin{array}{l}7 / 27 / 95 \\
7 / 31 / 95 \\
8 / 29 / 95 \\
9 / 12 / 95\end{array}$ & $\begin{array}{l}205.91 \\
205.86^{\star} \\
206.09 \\
206.23\end{array}$ & $\begin{array}{l}460.13 \\
460.18 \\
459.95 \\
459.81\end{array}$ & $\begin{array}{l}140.25 \\
140.26 \\
140.19 \\
140.15\end{array}$ \\
\hline 299-W18-30 & $\begin{array}{l}7 / 27 / 95 \\
8 / 01 / 95 \\
8 / 29 / 95 \\
9 / 12 / 95\end{array}$ & $\begin{array}{l}212.34 \\
212.26 \\
212.61 \\
212.81\end{array}$ & $\begin{array}{l}460.50 \\
460.58 \\
460.23 \\
460.03\end{array}$ & $\begin{array}{l}140.36 \\
140.38 \\
140.28 \\
140.22\end{array}$ \\
\hline 299-w18-31 & $\begin{array}{l}7 / 27 / 95 \\
8 / 01 / 95 \\
8 / 29 / 95 \\
9 / 12 / 95\end{array}$ & $\begin{array}{l}203.90 \\
202.84^{*}+ \\
204.11 \\
204.28 \\
.\end{array}$ & $\begin{array}{l}460.26 \\
461.32 \\
460.05 \\
459.88\end{array}$ & $\begin{array}{l}140.29 \\
140.61 \\
140.22 \\
140.17\end{array}$ \\
\hline 299-418-33 & $\begin{array}{l}7 / 27 / 95 \\
8 / 15 / 95 \\
8 / 29 / 95 \\
9 / 12 / 95\end{array}$ & $\begin{array}{l}209.33 \\
209.44 * \\
209.52 \\
209.65\end{array}$ & $\begin{array}{l}459.58 \\
459.47 \\
459.39 \\
459.26\end{array}$ & $\begin{array}{l}140.08 \\
140.05 \\
140.02 \\
139.98\end{array}$ \\
\hline 299-W19-1 & $7 / 27 / 95$ & 216.50 & 457.27 & 139.38 \\
\hline 299-W19-12 & $\begin{array}{l}7 / 27 / 95 \\
8 / 29 / 95 \\
9 / 12 / 95 \\
9 / 19 / 95\end{array}$ & $\begin{array}{l}212.82 \\
213.12 \\
213.65 \\
214.68^{*+}\end{array}$ & $\begin{array}{l}460.43 \\
460.13 \\
459.60 \\
458.57\end{array}$ & $\begin{array}{l}140.34 \\
140.25 \\
140.09 \\
139.77\end{array}$ \\
\hline 299-W19-21 & $\begin{array}{l}7 / 27 / 95 \\
8 / 15 / 95 \\
8 / 29 / 95 \\
9 / 12 / 95\end{array}$ & $\begin{array}{l}219.66 \\
219.88 * \\
219.95 \\
220.38\end{array}$ & $\begin{array}{l}459.13 \\
458.91 \\
458.84 \\
458.41\end{array}$ & $\begin{array}{l}139.94 \\
139.88 \\
139.85 \\
139.72\end{array}$ \\
\hline $299-W 19-27$ & $\begin{array}{l}7 / 27 / 95 \\
8 / 15 / 95 \\
8 / 29 / 95 \\
9 / 12 / 95\end{array}$ & $\begin{array}{l}224.40 \\
222.40 *_{+} \\
224.69 \\
224.90\end{array}$ & $\begin{array}{l}459.51 \\
461.51 \\
459.22 \\
459.01\end{array}$ & $\begin{array}{l}140.06 \\
140.67 \\
139.97 \\
139.91\end{array}$ \\
\hline 299-W19-31 & $\begin{array}{l}7 / 27 / 95 \\
7 / 31 / 95 \\
8 / 29 / 95 \\
9 / 12 / 95\end{array}$ & $\begin{array}{l}213.66 \\
213.68^{\star} \\
213.96 \\
214.17\end{array}$ & $\begin{array}{l}460.53 \\
460.51 \\
460.23 \\
460.02\end{array}$ & $\begin{array}{l}140.37 \\
140.36 \\
140.28 \\
140.21\end{array}$ \\
\hline
\end{tabular}


Table 15-2. RCRA Water Level Measurement Report Single-Shell Tanks, Third Quarter 1995. (sheet 8 of 8 )

\begin{tabular}{|c|c|c|c|c|}
\hline Well & Date & $\begin{array}{l}\text { Depth to } \\
\text { water (ft) }\end{array}$ & $\begin{array}{l}\text { Mater } \\
\text { elevation } \\
\text { (ft) }\end{array}$ & $\begin{array}{l}\text { level } \\
\text { above m51 } 1 \\
\text { (n) }\end{array}$ \\
\hline \multicolumn{5}{|c|}{ 241-U Tank Faral Wells } \\
\hline $299-W 19-32$ & $\begin{array}{l}7 / 27 / 95 \\
8 / 29 / 95 \\
9 / 12 / 95\end{array}$ & $\begin{array}{l}214.67 \\
214.93 \\
215.10\end{array}$ & $\begin{array}{l}460.23 \\
459.97 \\
459.80\end{array}$ & $\begin{array}{l}140.28 \\
140.20 \\
140.15\end{array}$ \\
\hline $299-419-6$ & $\begin{array}{l}7 / 27 / 95 \\
8 / 29 / 95 \\
9 / 12 / 95\end{array}$ & $\begin{array}{l}229.85 \\
230.03 \\
230.12\end{array}$ & $\begin{array}{l}456.75 \\
456.57 \\
456.48\end{array}$ & $\begin{array}{l}139.22 \\
139.16 \\
139.14\end{array}$ \\
\hline
\end{tabular}

NOTES: 1. Kater level elevations are calculated by subtracting the measured depth-to-water from the surveyed elevation for the well.

2. Depth-to-water values are transcribed from field records.

3. Measurements marked with an $1 * 1$ were taken at the time of sampling.

4. Measurements marked with a ' +1 are outside of the expected range, and are suspected of error. 
Table 15-3. Constituent List and Suinary of Results for the Single-She11 Tank Waste Management Area A-AX Data for Reporting Period July 1 through September 30, 1995.

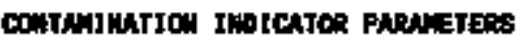

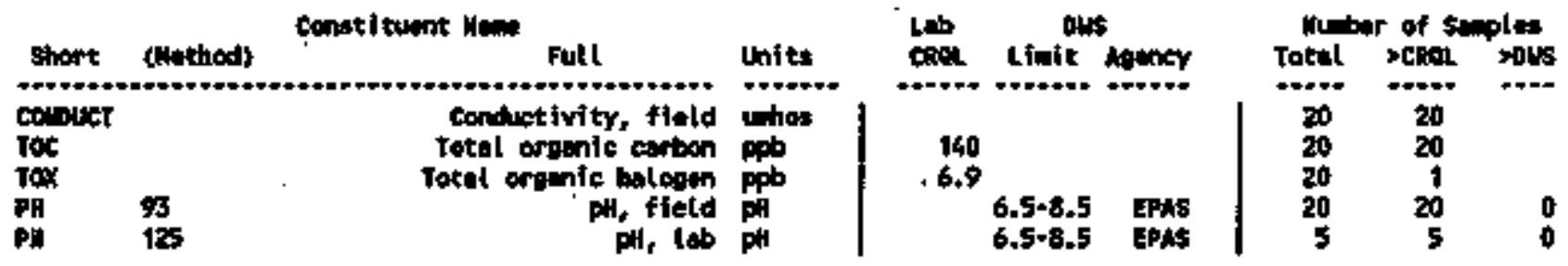

DRIWKHE UTER PMANETERS

\begin{tabular}{|c|c|c|c|c|c|c|c|c|c|c|}
\hline & & constiflunt wast & & & in & pr & & in & $r$ of -8 & ples \\
\hline stort & (Wethod) & & Full & Intst & Coal & Lifrit & Aoncy & Total & $\operatorname{xen}$ at & sons \\
\hline
\end{tabular}

SITE SPECIFIC MI oT:Ge COMSTITHENS

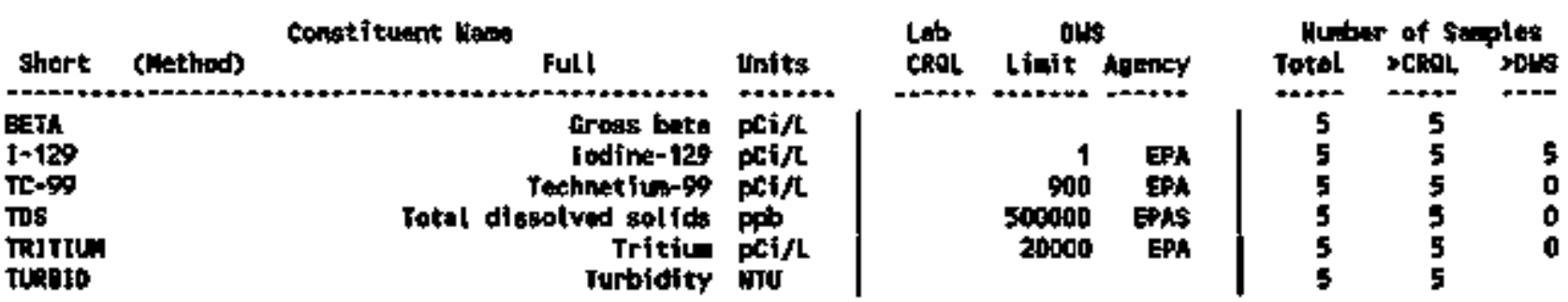

For explention of this teble, see stetion 1.4 of report. 
Table 15-4. Constituents with at Least One Detected Value for the Single-5he11 Tank Waste Management Area A-AX Data for Reporting Period July I through September 30, 1995.

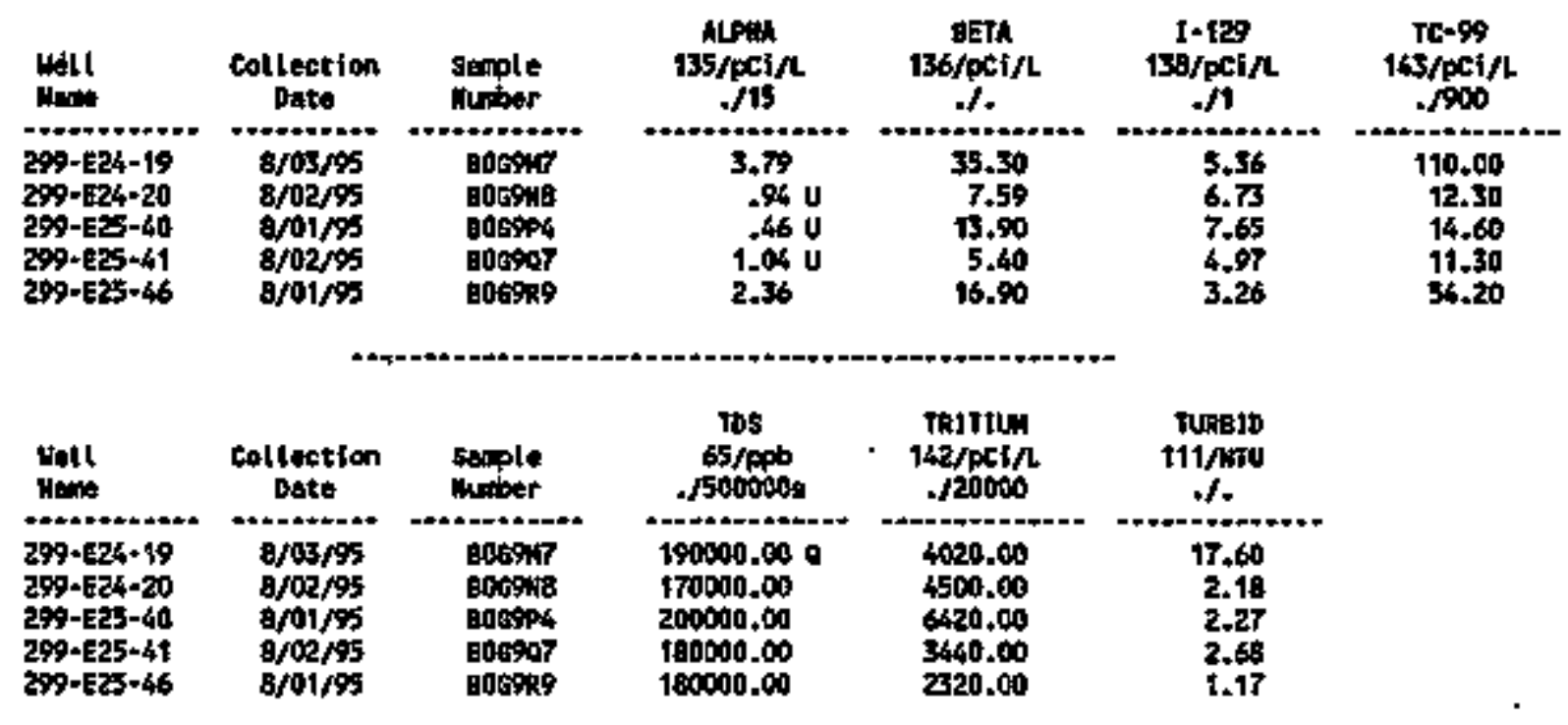

For exptantion of this table, aee section 1.4 of report. 
Table 15-5. Contanination Indicator Parameters for the Singlo-Shell Tank Waste Managenent Area A-AX Data for Reporting Period July 1 through September 30, 1995.

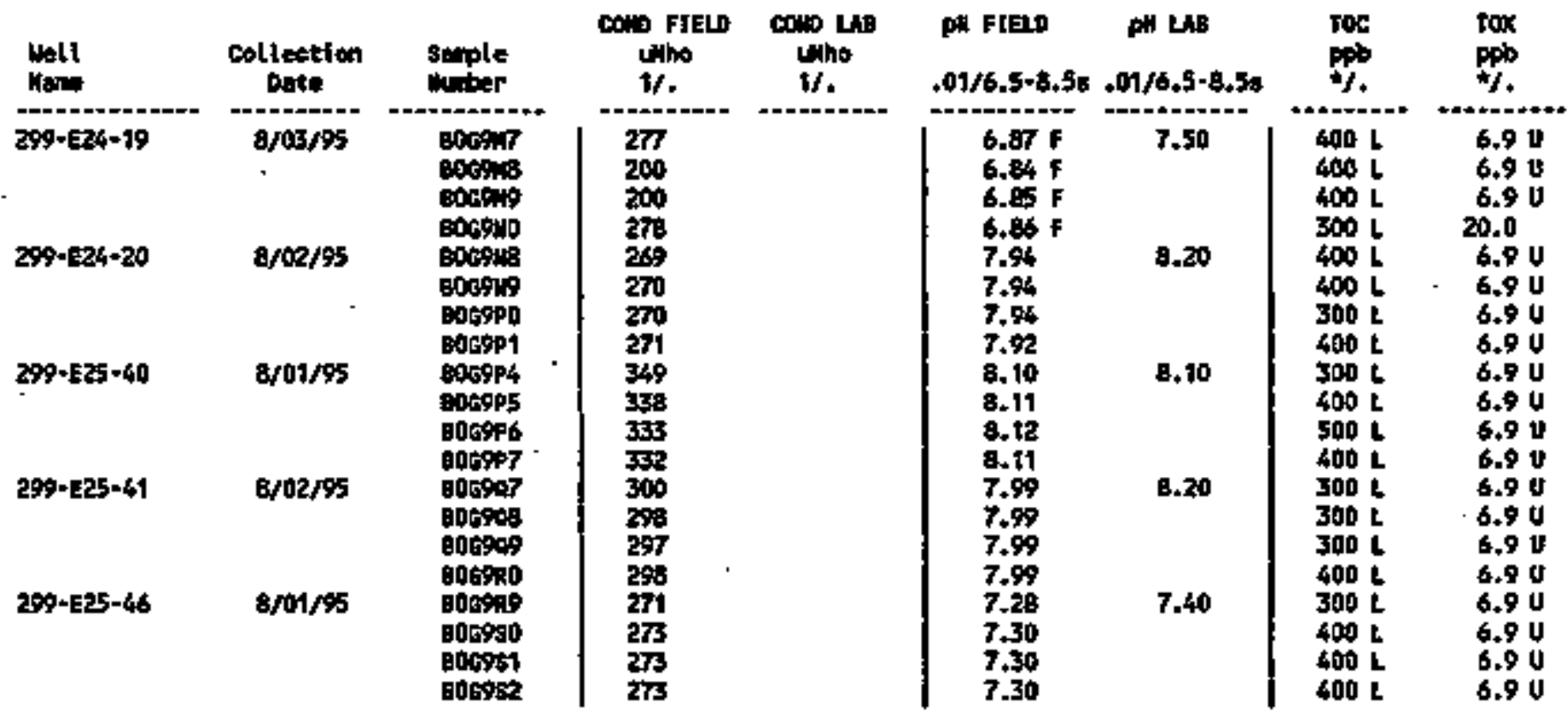

ontection Iinits for TOC and tox very depending on the performing loboratory. for explenation of this teble, 300 seetion 1.4 of roport. 
Table 15-6. Constituent List and Sumary of Results for the Sing Te-Shell Tank Waste Management Area B-BY-BX Data for Reporting Period July i through September 30, 1995.

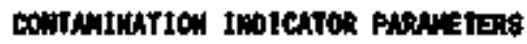

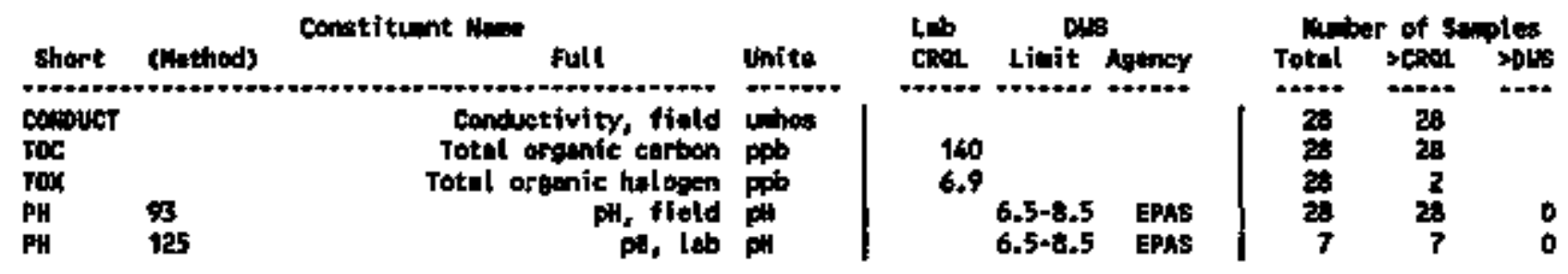

DRTMKING MTER PARNHETERS

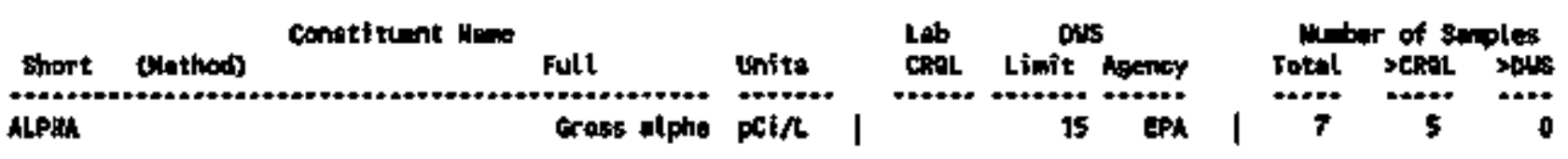

SITE SPEGIFIC AD OTHER COMSTITHETS

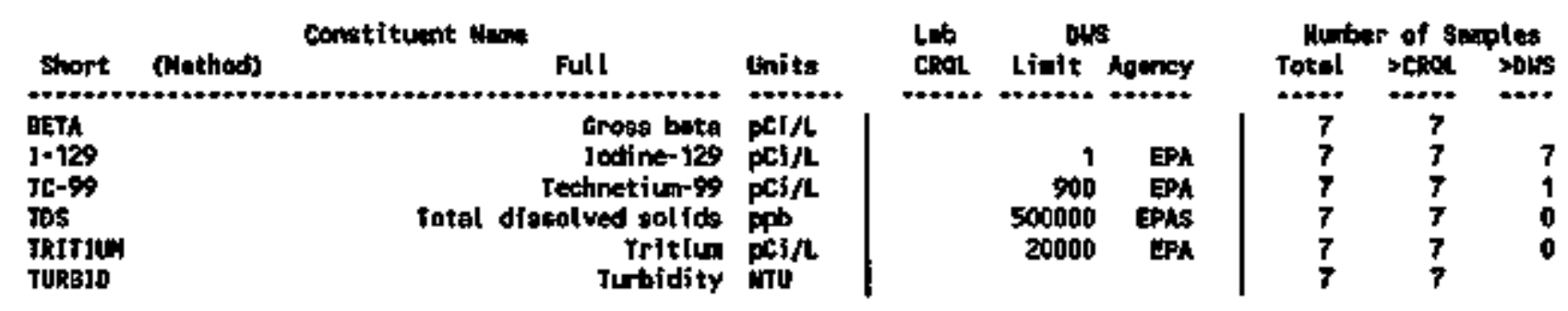

For explenation of this toble, oee section 1.4 of report. 
Table 15-7. Constituents with at Least One Detected Value for the Stngle-Shell Tank Waste Management Area B-BY-BX Data for Reporting Period July 1 through September 30, 1995.

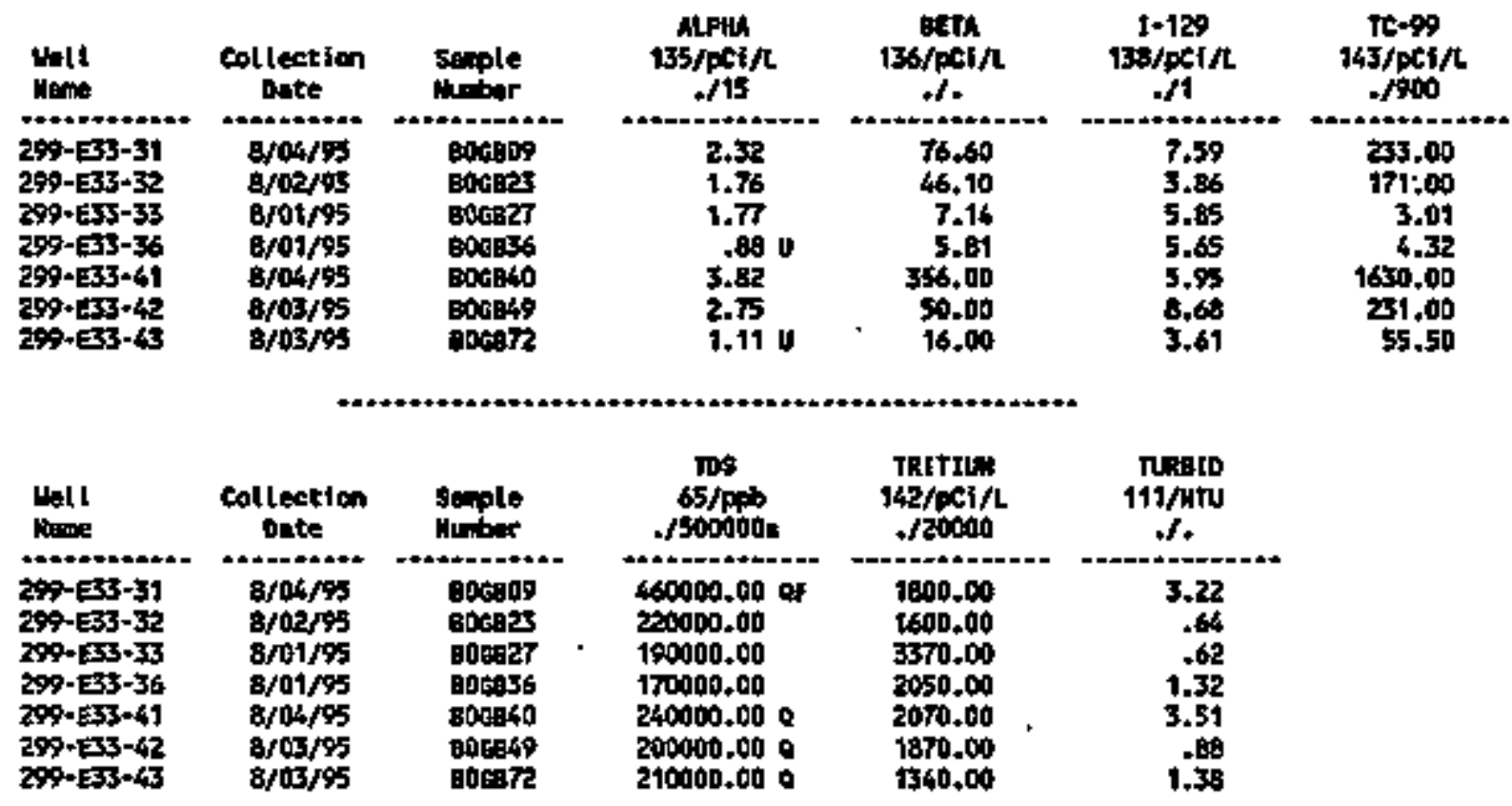

For explanation of thio teble, des section 1.4 of report. 
Table 15-8. Contamination Indfcator Parameters for the

Single-Shell Tank Waste Management Area B-BY-BX

Data for Reporting Period July 1

through September 30, 1995.

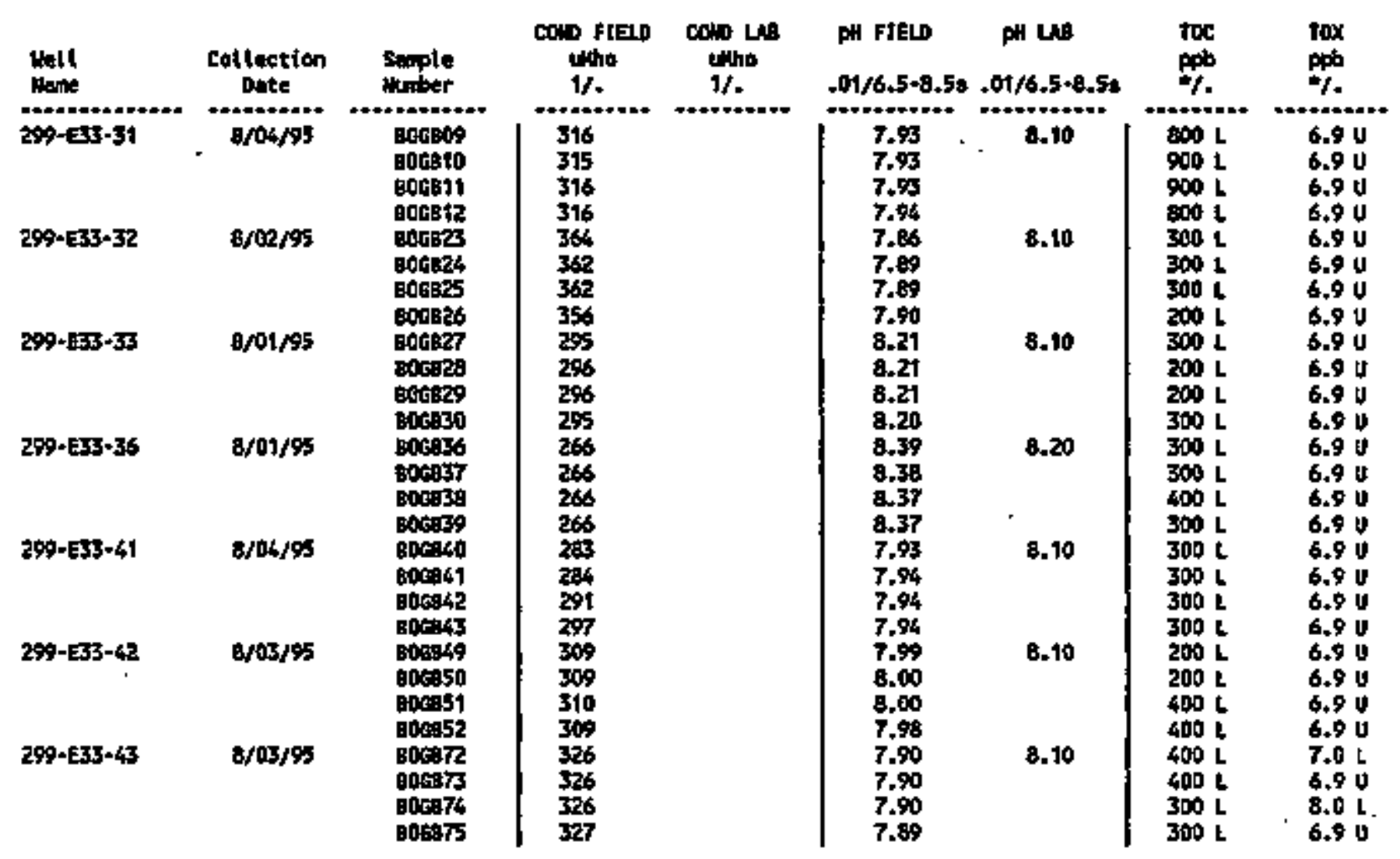

\footnotetext{
Detection liaits for TOC and TOX vary deperding on the perforning laboretory. For explanation of this tabte, see section 1.4 of report.
} 
Table 15-9. Constituent List and Sumary of Results for the Słngle-She11 Tank Maste Managewent Area C Data for Reporting Period July 1 through September 30, 1995.

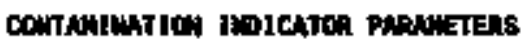

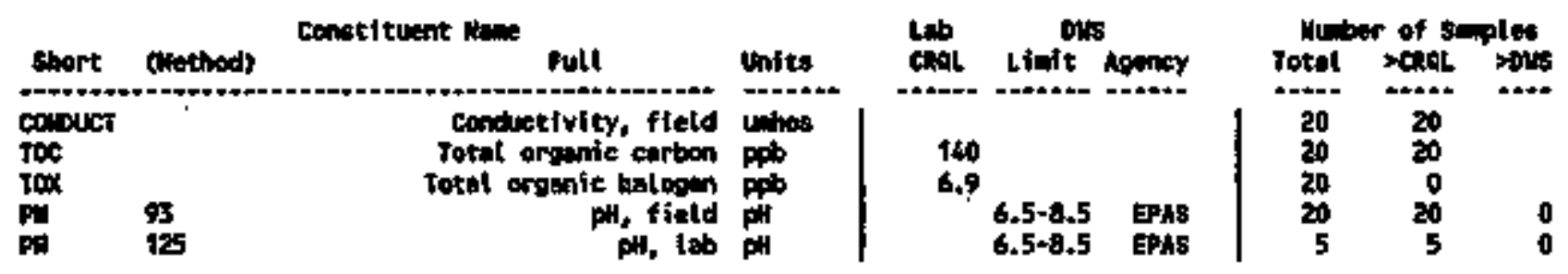

ERJMKIHB WTER PNRUETERS

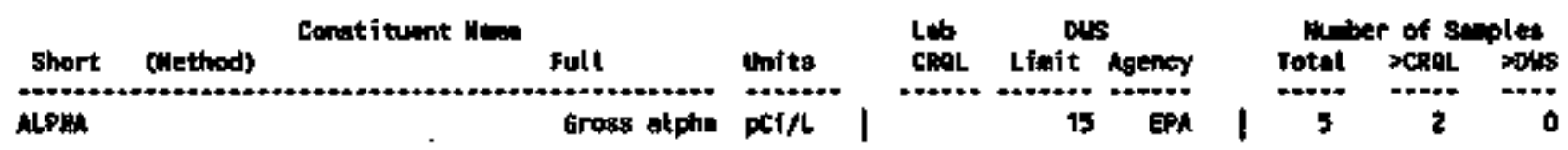

SITE SPECIFIC ND OTHER CONSTITLETS

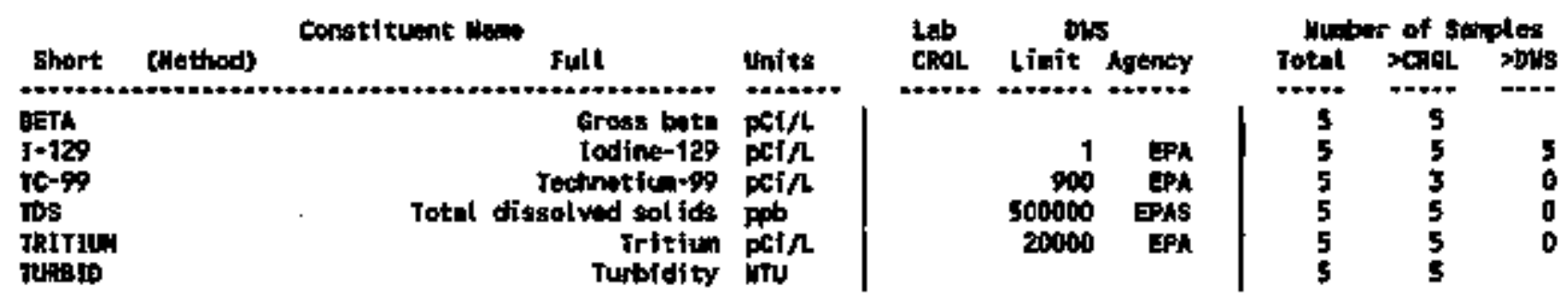

For explantion of this tabli, ses section 1,4 of report. 
Table 15-10. Constituents with at Least One Detected Va]ue for the Single-She]l Tank Waste Management Area C Data for Reporting Period Juty I through September 30, 1995.

\begin{tabular}{|c|c|c|c|c|c|c|}
\hline Well & $\begin{array}{c}\text { Codlection } \\
\text { Onte }\end{array}$ & 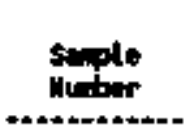 & $\begin{array}{c}\text { LPth } \\
135 / p \mathrm{pl} / \mathrm{h} \\
. / 15\end{array}$ & $\begin{array}{c}\text { BeTh } \\
136 / p 61 / L \\
A\end{array}$ & $\begin{array}{c}1-129 \\
138 / \mathrm{pCI} / \mathrm{h} \\
/ 1\end{array}$ & $\begin{array}{c}\text { TC-\$ } \\
143 / 961 / 2 \\
-1400\end{array}$ \\
\hline $\begin{array}{l}299-E 27-12 \\
299-E 27-13 \\
299-E 27-14 \\
299-E 27-15 \\
299-E 27-7\end{array}$ & $\begin{array}{l}8 / 02 / 95 \\
8 / 03 / 95 \\
8 / 02 / 95 \\
8 / 02 / 95 \\
8 / 02 / 95\end{array}$ & 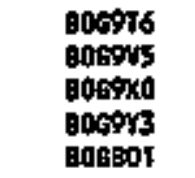 & $\begin{array}{r}.90 v \\
.94 v \\
1.16 u \\
1.46 \\
1.50\end{array}$ & $\begin{array}{r}7.18 \\
49.54 \\
29.50 \\
5.87 \\
8.39\end{array}$ & $\begin{array}{l}3.92 \\
4.37 \\
4.07 \\
7.09 \\
3.80\end{array}$ & $\begin{array}{r}4.18 \\
182.00 \\
7.40 \\
1.364 \\
.60 \mathrm{U}\end{array}$ \\
\hline
\end{tabular}

\begin{tabular}{|c|c|c|c|c|c|}
\hline Woll & $\begin{array}{c}\text { Colleation } \\
\text { Date }\end{array}$ & $\begin{array}{l}\text { serple } \\
\text { Antiber }\end{array}$ & $\begin{array}{c}\text { nos } \\
65 / 00 \mathrm{~b} \\
. \$ 00000 \mathrm{~s}\end{array}$ & $\begin{array}{l}\text { TRITIL } \\
\text { 162/peill } \\
\text { /20000 }\end{array}$ & $\begin{array}{c}\text { ruRato } \\
\text { 11t/wit } \\
\text { f }\end{array}$ \\
\hline $\begin{array}{l}299-E 27-12 \\
299-E 27-13 \\
249-E 27-14 \\
294-E 27-15 \\
279-E 27-7\end{array}$ & $\begin{array}{l}8 / 02 / 95 \\
8 / 03 / 93 \\
8 / 02 / 95 \\
8 / 02 / 95 \\
8 / 02 / 95\end{array}$ & 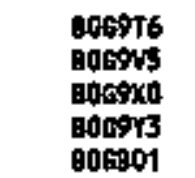 & $\begin{array}{l}170000.00 \\
170000.040 \\
230000.00 \\
220000.00 \\
170000.00\end{array}$ & $\begin{array}{r}860.00 \\
1830.00 \\
762.00 \\
624.00 \\
655.00\end{array}$ & $\begin{array}{l}.69 \\
3.16 \\
1.36 \\
2.16 \\
2.13\end{array}$ \\
\hline
\end{tabular}

For exphantion of this teble, see section 1.4 of report. 
Table 15-11: Contapination Indicator Parameters for the Single-Shell Tank Waste Management Area C

Data for Reporting Perlod July 1

through Septewber 30, 1995.

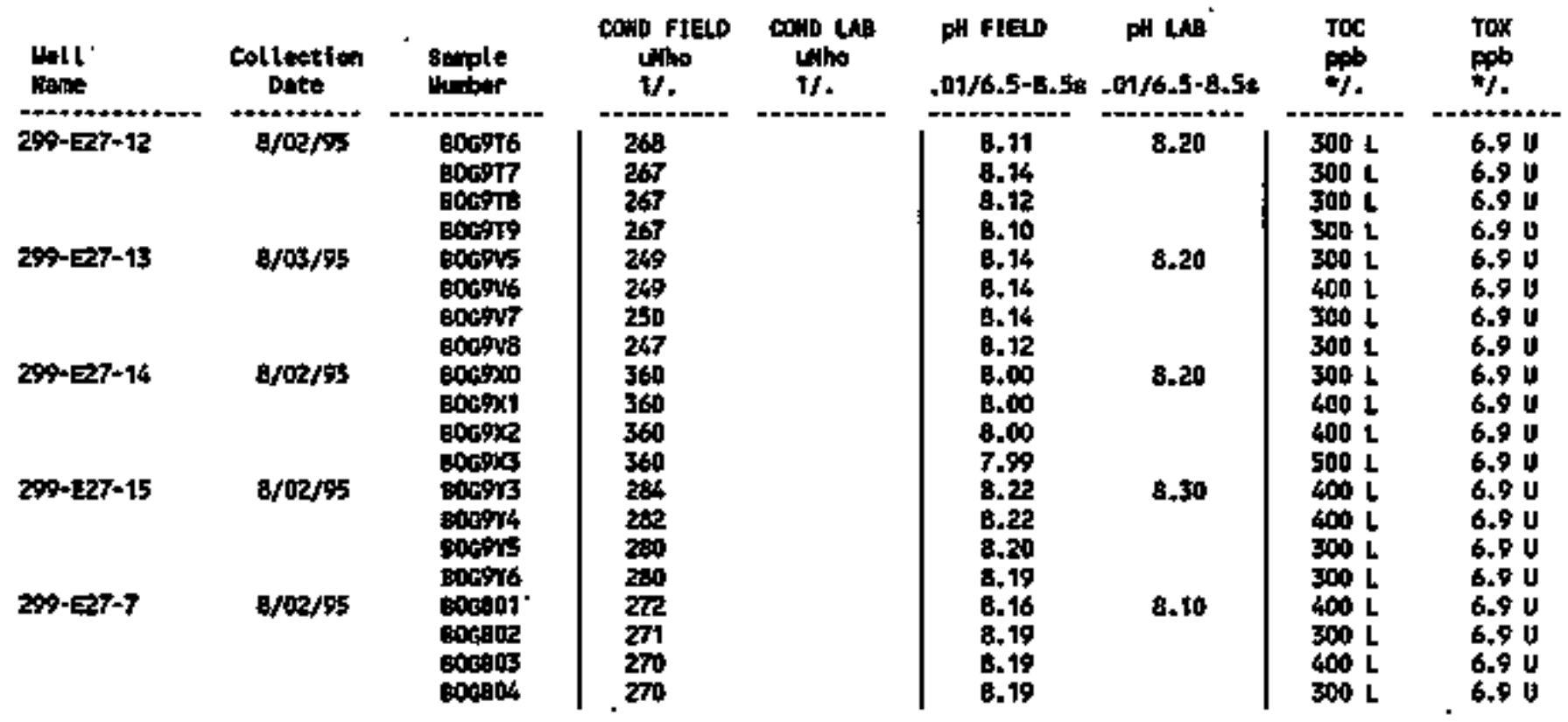

\footnotetext{
- Detection linits far ToC and TQX wary dapinding on the parforming laboratery. For explention of this teble, see sertion 1.4 of report.
} 
Table 15-12. Constituent List and Summary of Results for the Single-Shell Tank Waste Management Area S-SX Data for Reporting Period July 1 through September 30, 1995.

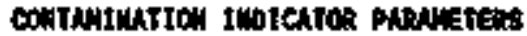

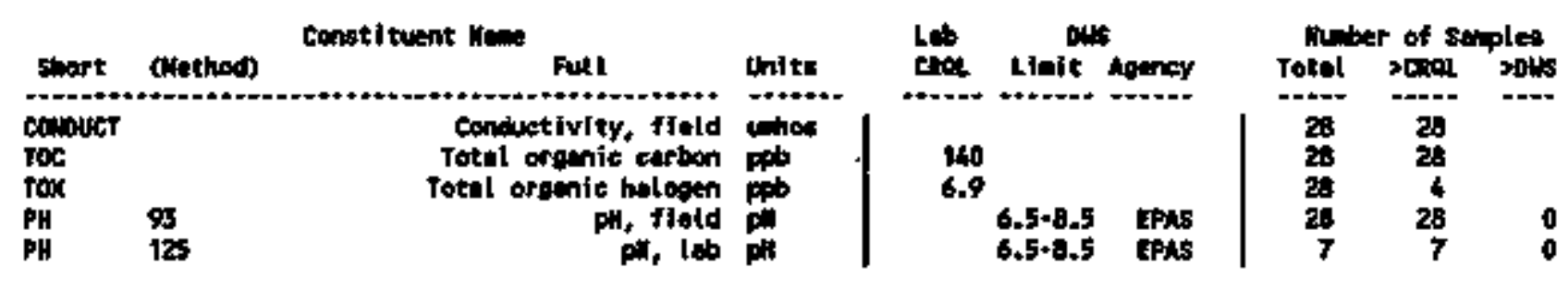

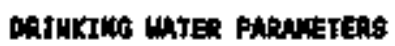

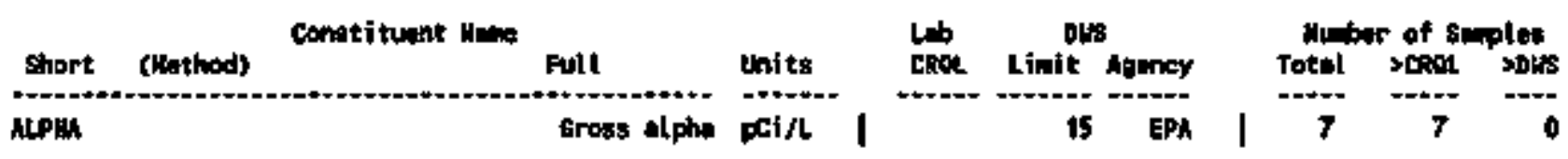

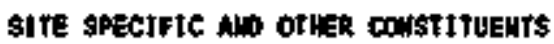

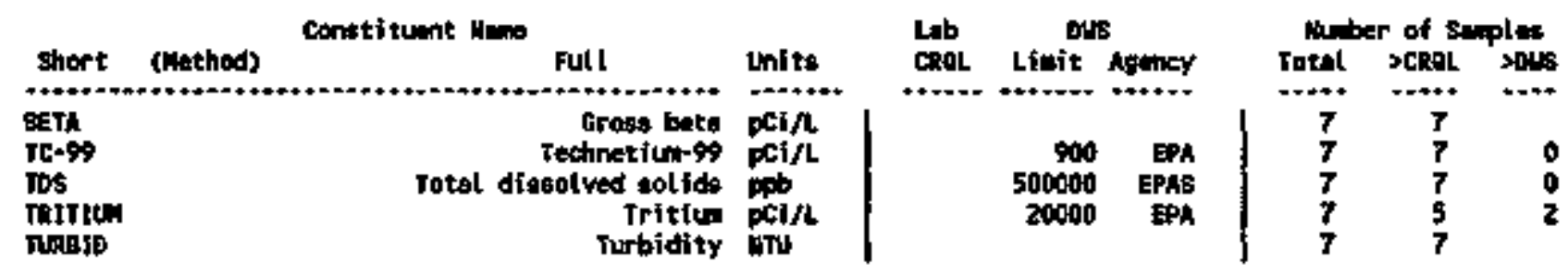

For explanation of this table, see section 1.4 of report. 
Table 15-13. Constituents with at Least One Detected Value for the Single-Shell Tank Waste Managentent Area S-SX Data for Reporting Perlod July 1 through September 30, 1995.

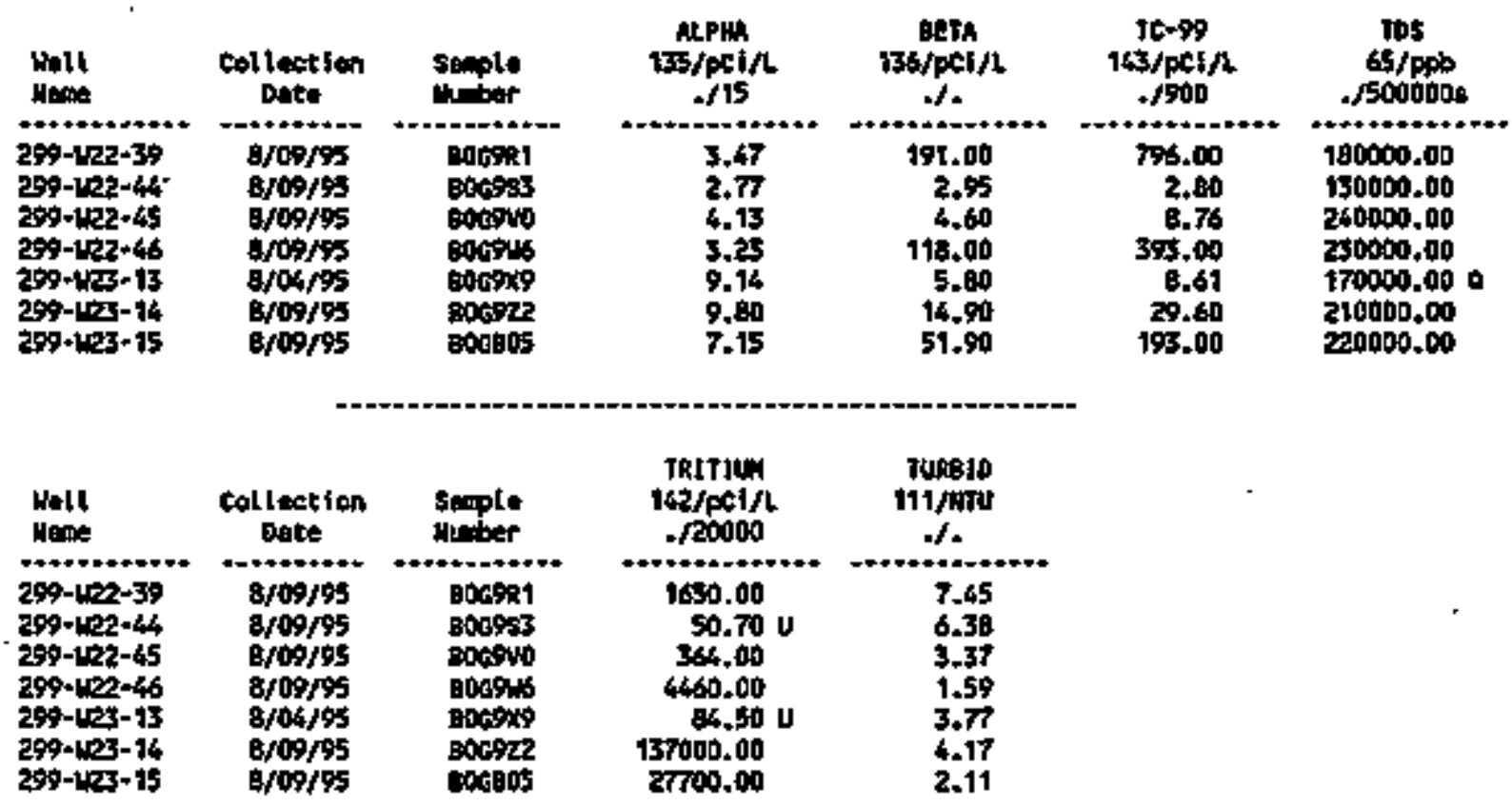

For explentition of this table, see section 1.4 of report. 
Table 15-14. Contamination Indicator Parameters for the Single-She1l Tank Waste Management Area S-SX Data for Reporting Period July 1 through September 30, 1995.

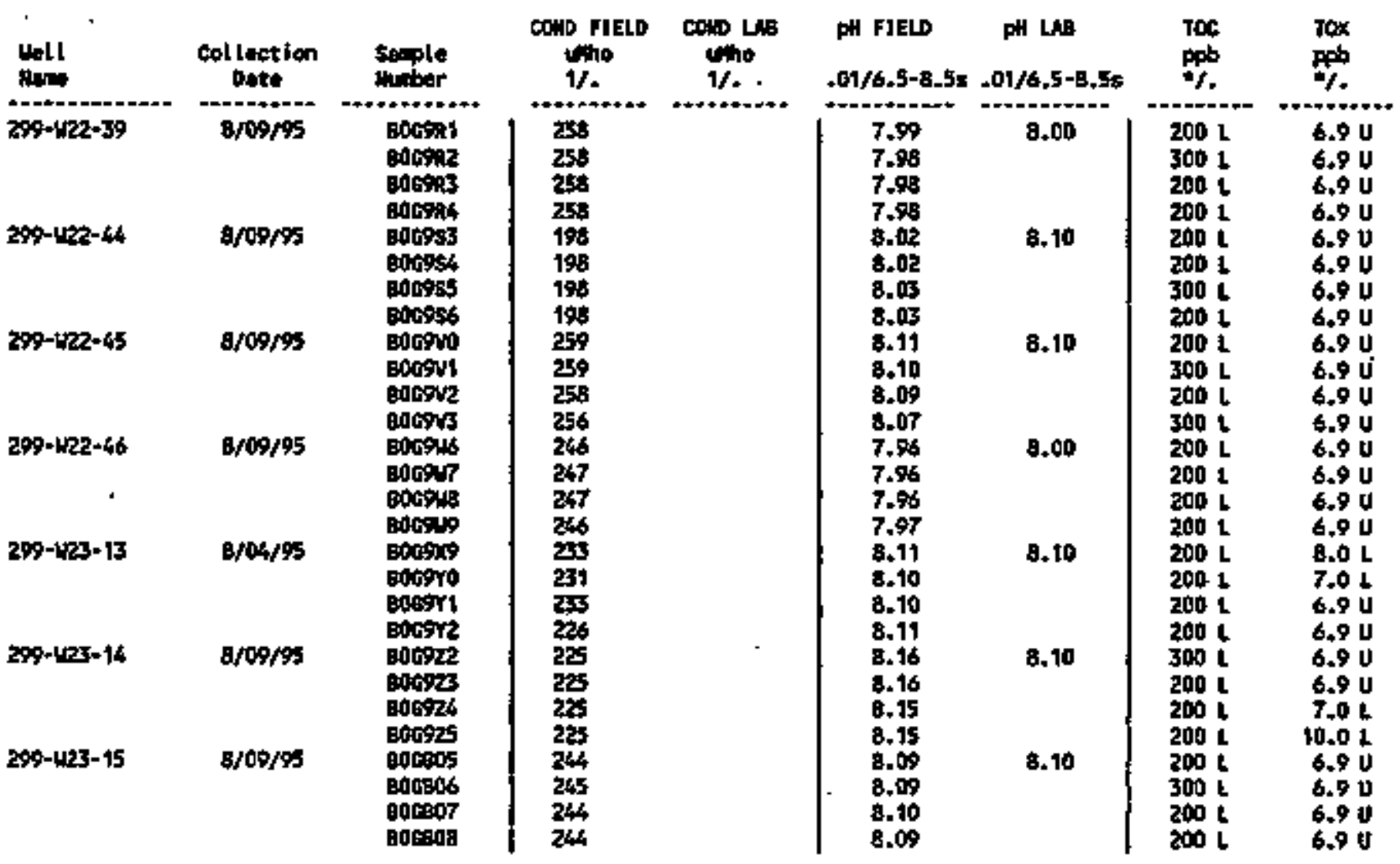

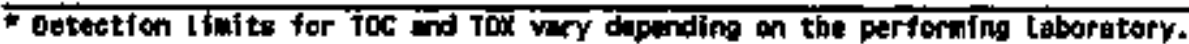
For explanation of this toble, an section 1.4 of repere. 
Table 15-15. Constituent List and Summary of Results for the Single-SheII Tank Naste Management Area T Data for Reporting Period July 1 through Septenber 30, 1995.

(sheet 1 of 2)

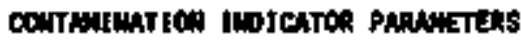

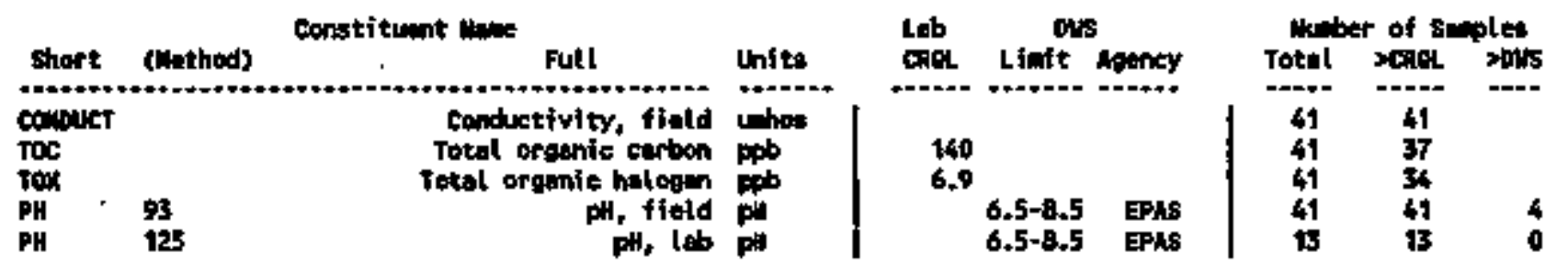

DRINKIHE LTER PARMITERS

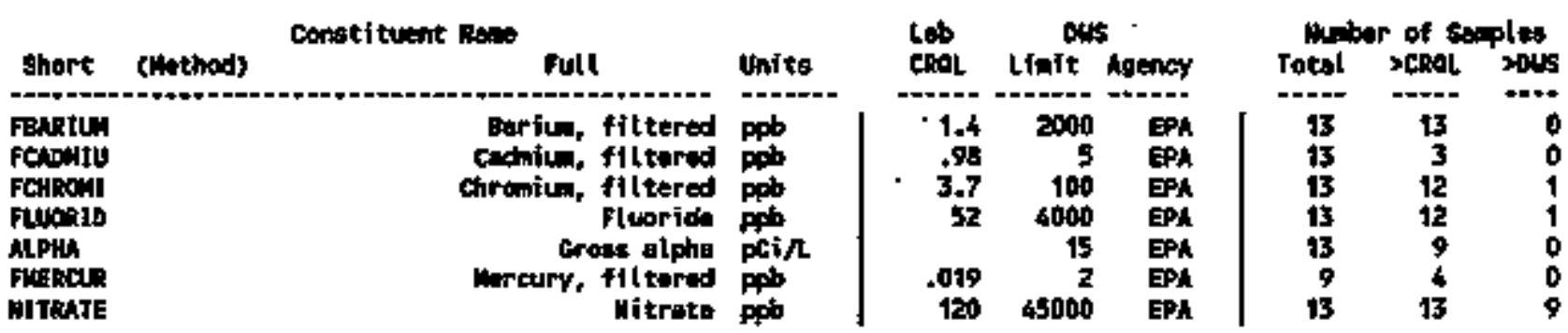

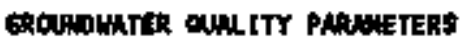

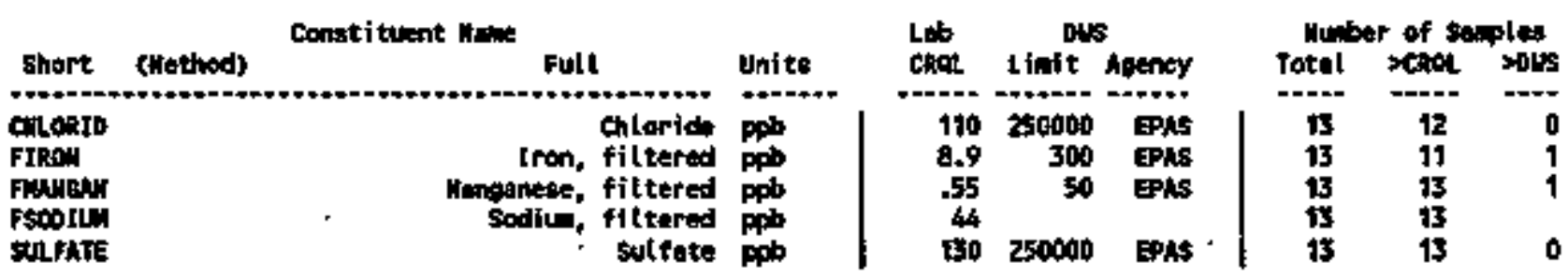

S[TE SPECLF]C AWD OTHER CCHSTITUANS

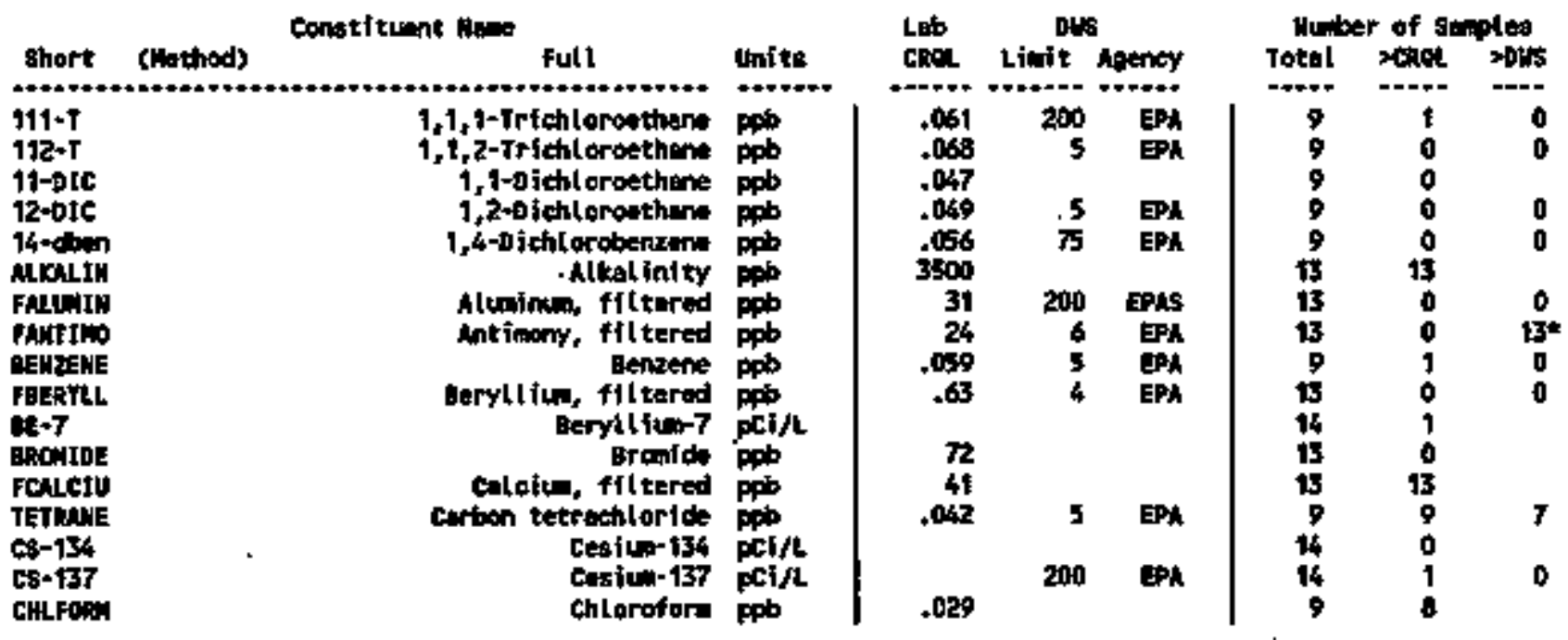


Table 15-15. Constituent List and Sunnary of Results for the Single-She1] Tank Maste Management Area T Data for

Reporting Period July I through September 30, 1995.

(sheet 2 of 2)

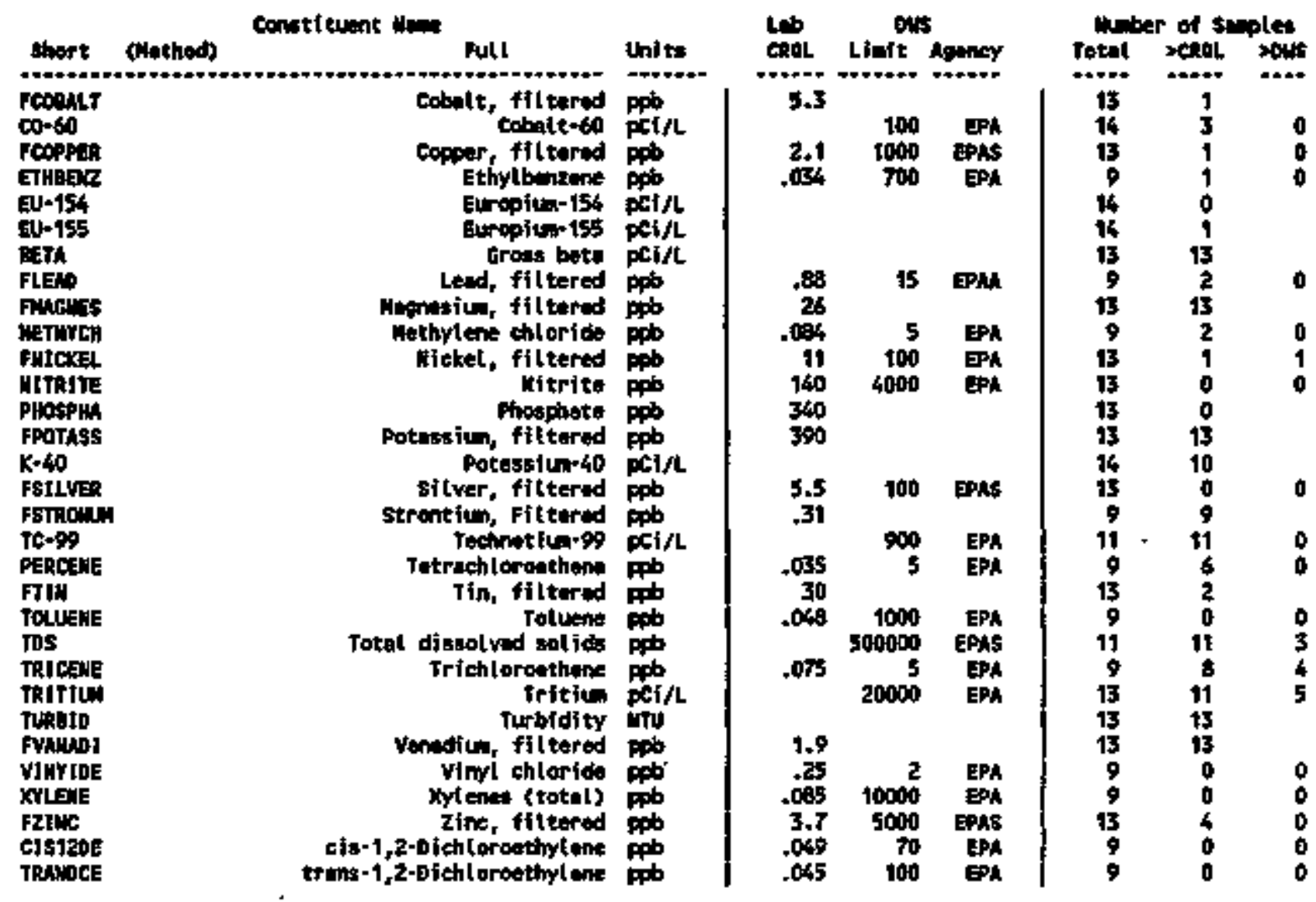

For explenation of this table, seit section 1.4 of report. 
Table 15-16. Constituents with at Least One Detected Value for the Single-Shell Tank Waste Hanagement Area T Data for Reporting Perjod July 1 through September 30, 1995.

(sheet 1 of 7 )

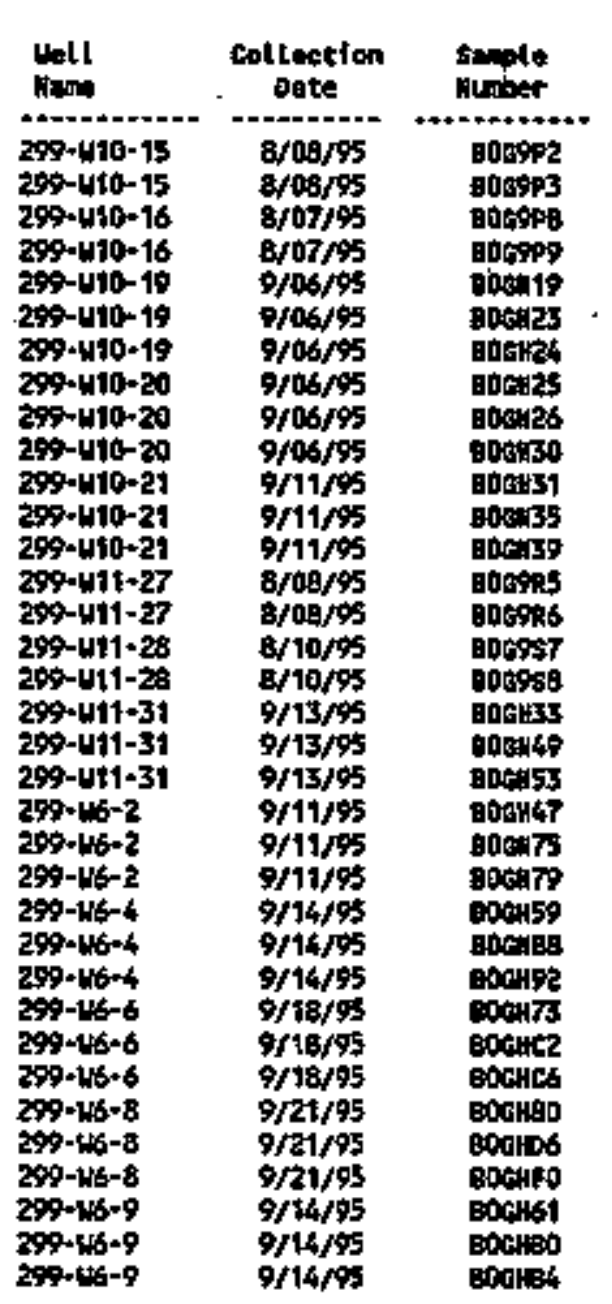

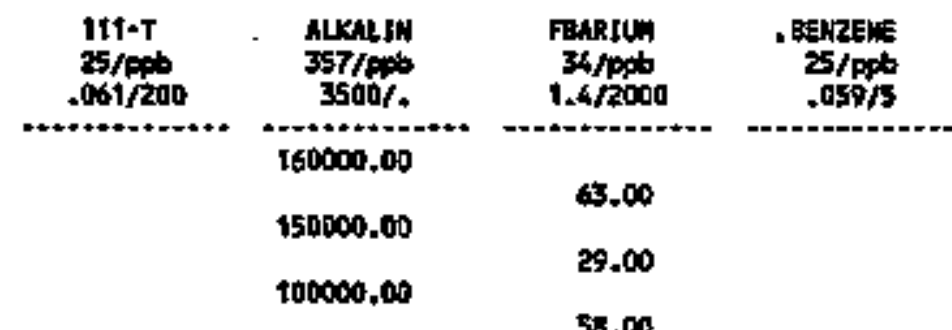

$.06 \mathrm{u}$
$.06 \mathrm{u}$

.060 $.06 \mathrm{~V}$

.06

$\$ 5.00$

90100.00 $.06 \mathrm{~V}$

150000.00

4900

160000.00

48,00

36.60

.46

130000,00

.11 L

51_00

$.06 \mathrm{y} 120000.00$

$.06 v$

$\begin{array}{lll}.056 & 36,00 & .06 U\end{array}$

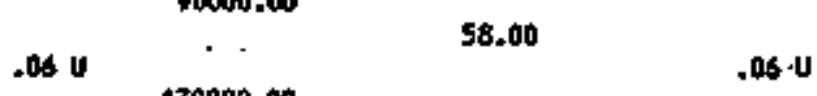

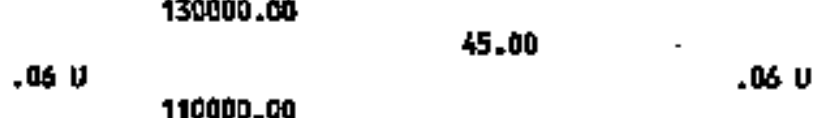

42.00

.060

120000.00

ot 0

\$3.01

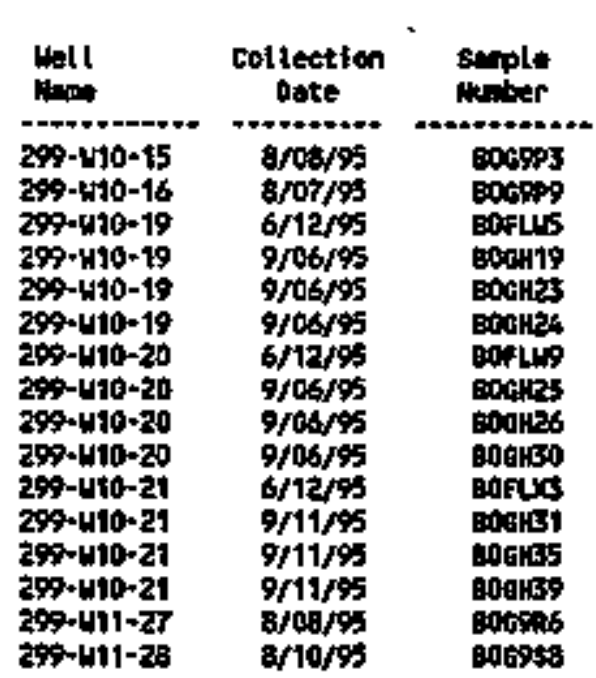

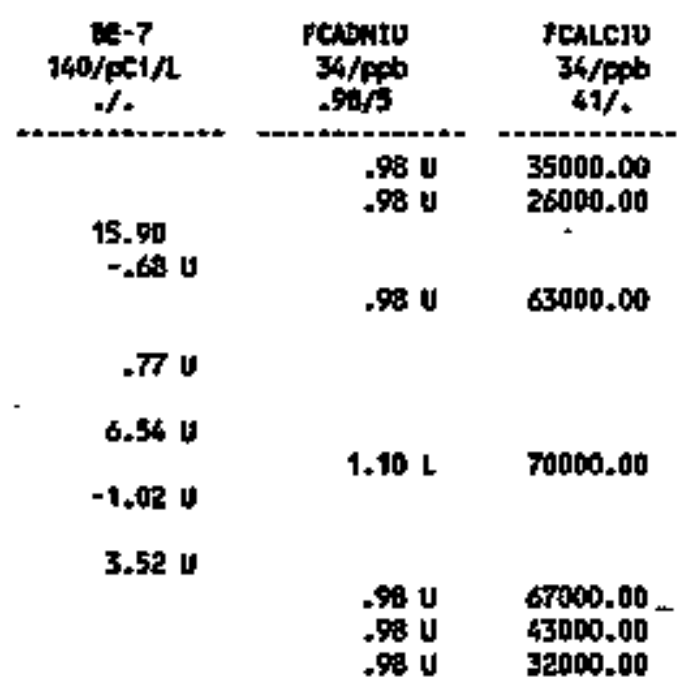


TabTe 15-16. Constituents with at Least One Detected Value for the Singie-Shell Tank Maste Management Area T Data for Reporting Period July 1 through September 30, 1995.

(sheet 2 of 7 )

\begin{tabular}{|c|c|c|c|c|c|c|}
\hline Iot & $\begin{array}{c}\text { Colfection } \\
\text { oute }\end{array}$ & somple & $\begin{array}{c}\text { AE-7 } \\
140 / \mathrm{PCI} / \mathrm{L} \\
+/\end{array}$ & $\begin{array}{l}\text { FCNaHIL } \\
34 / 496 \\
.99 / 5\end{array}$ & $\begin{array}{c}\text { FonLCIU } \\
36 / p \text { b } \\
61 \%\end{array}$ & $\begin{array}{l}\text { TEtPANE } \\
\text { 2/ppb } \\
.062 / 5\end{array}$ \\
\hline 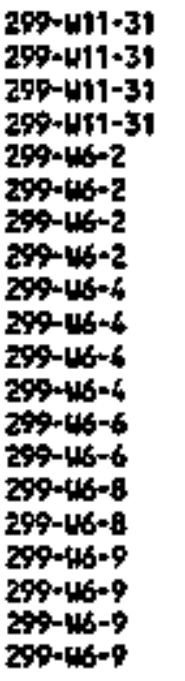 & $\begin{array}{l}6 / 12 / 95 \\
9 / 13 / 95 \\
9 / 13 / 95 \\
9 / 13 / 95 \\
6 / 14 / 95 \\
9 / 11 / 95 \\
9 / 11 / 95 \\
9 / 11 / 95 \\
6 / 12 / 95 \\
9 / 14 / 45 \\
9 / 14 / 95 \\
9 / 14 / 95 \\
9 / 18 / 55 \\
9 / 18 / 95 \\
9 / 21 / 95 \\
9 / 21 / 95 \\
6 / 13 / 95 \\
9 / 14 / 95 \\
9 / 14 / 95 \\
9 / 14 / 95\end{array}$ & 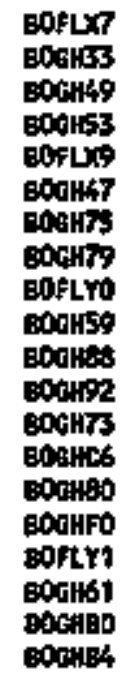 & $\begin{array}{l}6.62 \mathrm{U} \\
3.42 \mathrm{~V} \\
-1.24 \mathrm{U} \\
-4.79 \mathrm{U} \\
-6.59 \mathrm{U} \\
3.17 \mathrm{U}\end{array}$ & $\begin{array}{l}.96 \mathrm{U} \\
.98 \mathrm{U} \\
.98 \mathrm{U} \\
.96 \mathrm{U} \\
.00 \mathrm{~L}\end{array}$ & $\begin{array}{l}61000.00 \\
59000.00 \\
42000,00 \\
300000.00 \\
30000.00 \\
49000.00\end{array}$ & $\begin{array}{r}110.000 \\
290.000 \\
.0610 \\
.040 \\
240.000\end{array}$ \\
\hline $\operatorname{moll}_{\operatorname{lam}}$ & $\begin{array}{c}\text { Collectlon } \\
\text { Dote }\end{array}$ & $\begin{array}{l}\text { Stpple } \\
\text { Hutpor }\end{array}$ & $\begin{array}{c}c s-137 \\
140 / / 0 \mathrm{pc} 5 / \mathrm{L} \\
. / 200\end{array}$ & $\begin{array}{c}\text { CHLOK1D } \\
124 / \mathrm{pob} \\
110 / 2500000\end{array}$ & $\begin{array}{l}\text { 멭ak } \\
25 / p \text { b } \\
.02 \% / .\end{array}$ & $\begin{array}{l}\text { FCHROWH } \\
34 / p \text { b } \\
3.7 / 100\end{array}$ \\
\hline 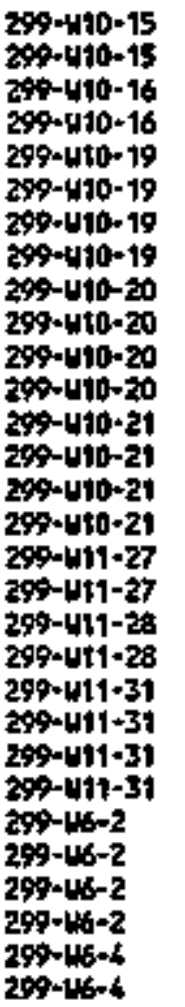 & 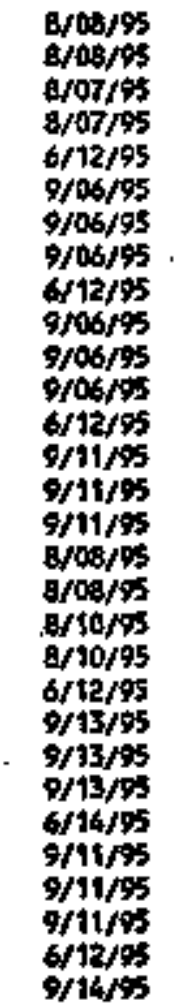 & 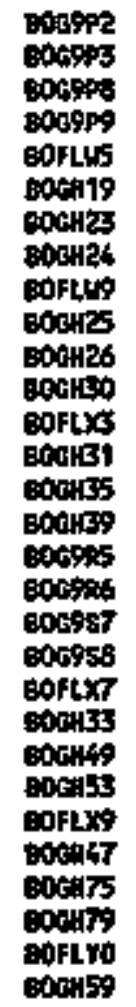 & $\begin{array}{r}.21 \mathrm{v} \\
.74 \mathrm{v} \\
-.08 \mathrm{v} \\
.86 \mathrm{v} \\
-.11 \mathrm{v} \\
.05 \mathrm{v}\end{array}$ & $\begin{array}{l}14000.000 \\
2400.00 \\
28000.000\end{array}$ & $\begin{array}{l}12.00 \\
38.00 \mathrm{D}\end{array}$ & $\begin{array}{l}13.00 \\
31.00 \\
11.00 \mathrm{~L} \\
56.00\end{array}$ \\
\hline
\end{tabular}


Table 15-16. Constituents with at Least One Detected Value for the Single-Shell Tank Maste Management Area T Data for Reporting Period July 1 through Septenber 30, 1995.

(sheet 3 of 7 )

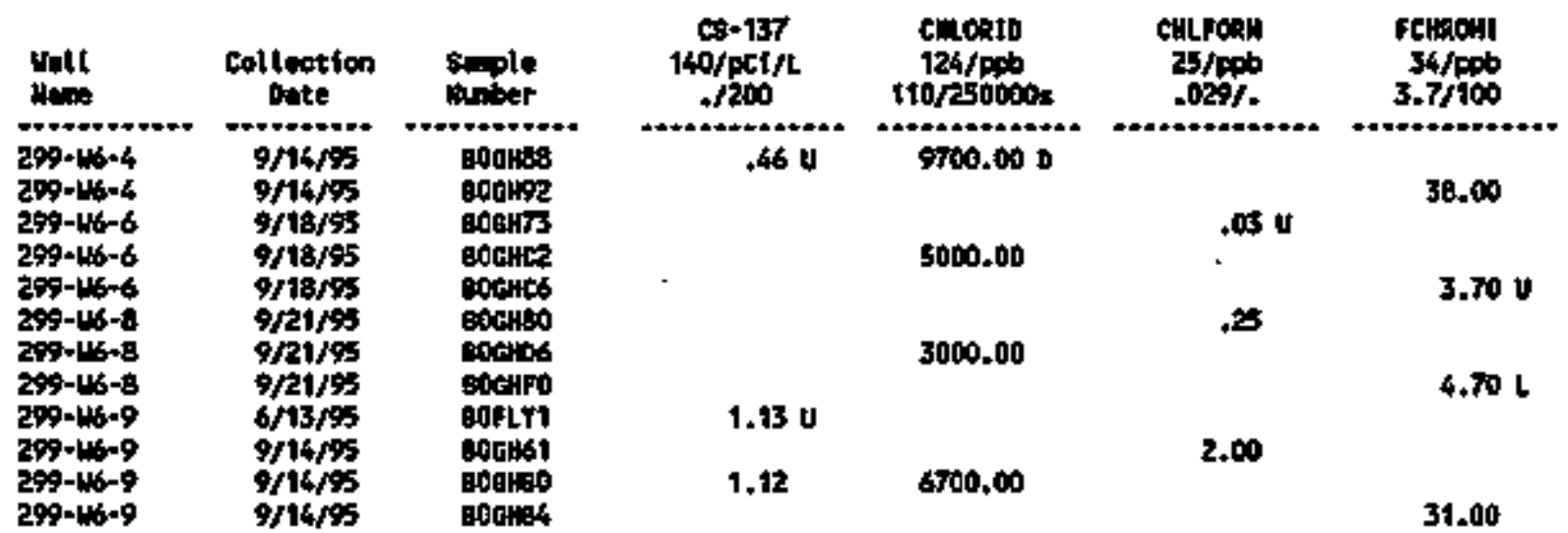

\begin{tabular}{|c|c|c|c|c|c|c|}
\hline Nelt & $\begin{array}{c}\text { Collection } \\
\text { Date }\end{array}$ & $\begin{array}{l}\text { sonple } \\
\text { thuber }\end{array}$ & $\begin{array}{l}\text { PCoAtit } \\
34 / \mathrm{mb} \\
\mathbf{3 . 3 \%}\end{array}$ & $\begin{array}{c}c 0-60 \\
140 / 0 \mathrm{DC} / \mathrm{C} \\
. / 100\end{array}$ & $\begin{array}{c}\text { FCoppleR } \\
34 / \mathrm{ppb} \\
2.1 / 1000 \mathrm{~s}\end{array}$ & $\begin{array}{c}\text { Efleenz } \\
2 \$ / p p b \\
.054 / 700\end{array}$ \\
\hline 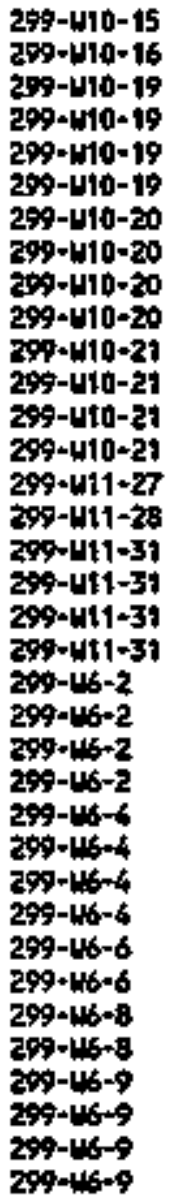 & 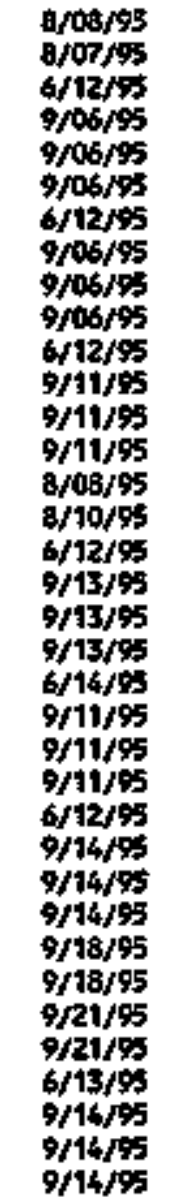 & 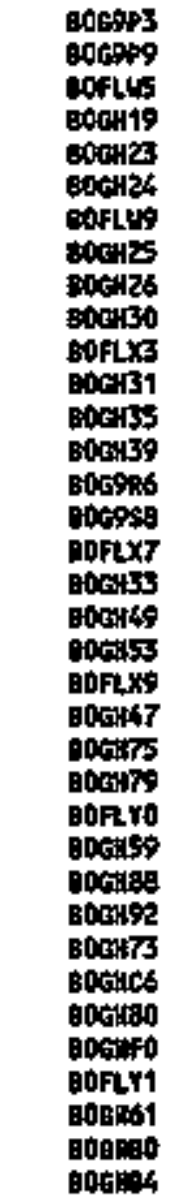 & $\begin{array}{l}5.30 \mathrm{U} \\
5.30 \mathrm{U} \\
5.30 \mathrm{U} \\
5.30 \mathrm{U} \\
5.30 \mathrm{U} \\
5.30 \mathrm{U}\end{array}$ & $\begin{array}{r}1.16 \mathrm{U} \\
-.79 \mathrm{~V} \\
-.21 \mathrm{U} \\
.96 \mathrm{U} \\
-.47 \mathrm{U} \\
.74 \mathrm{U} \\
.52 \mathrm{U} \\
1.54 \\
-.32 \mathrm{U} \\
1.24 \mathrm{U} \\
1.40 \\
-1.75 \mathrm{U}\end{array}$ & $\begin{array}{l}2.10 \mathrm{U} \\
2.10 \mathrm{U} \\
2.10 \mathrm{U} \\
2.10 \mathrm{~V}\end{array}$ & $\begin{array}{l}.03 \mathrm{U} \\
.03 \mathrm{U} \\
.03 \mathrm{U} \\
.03 \mathrm{U}\end{array}$ \\
\hline
\end{tabular}


Table 15-16. Constituents with at Least One Detected Value for the single-Shel1 Tank Waste Management Area T Data for Reporting Period Juty I through September 30, 1995. (sheet 4 of 7)

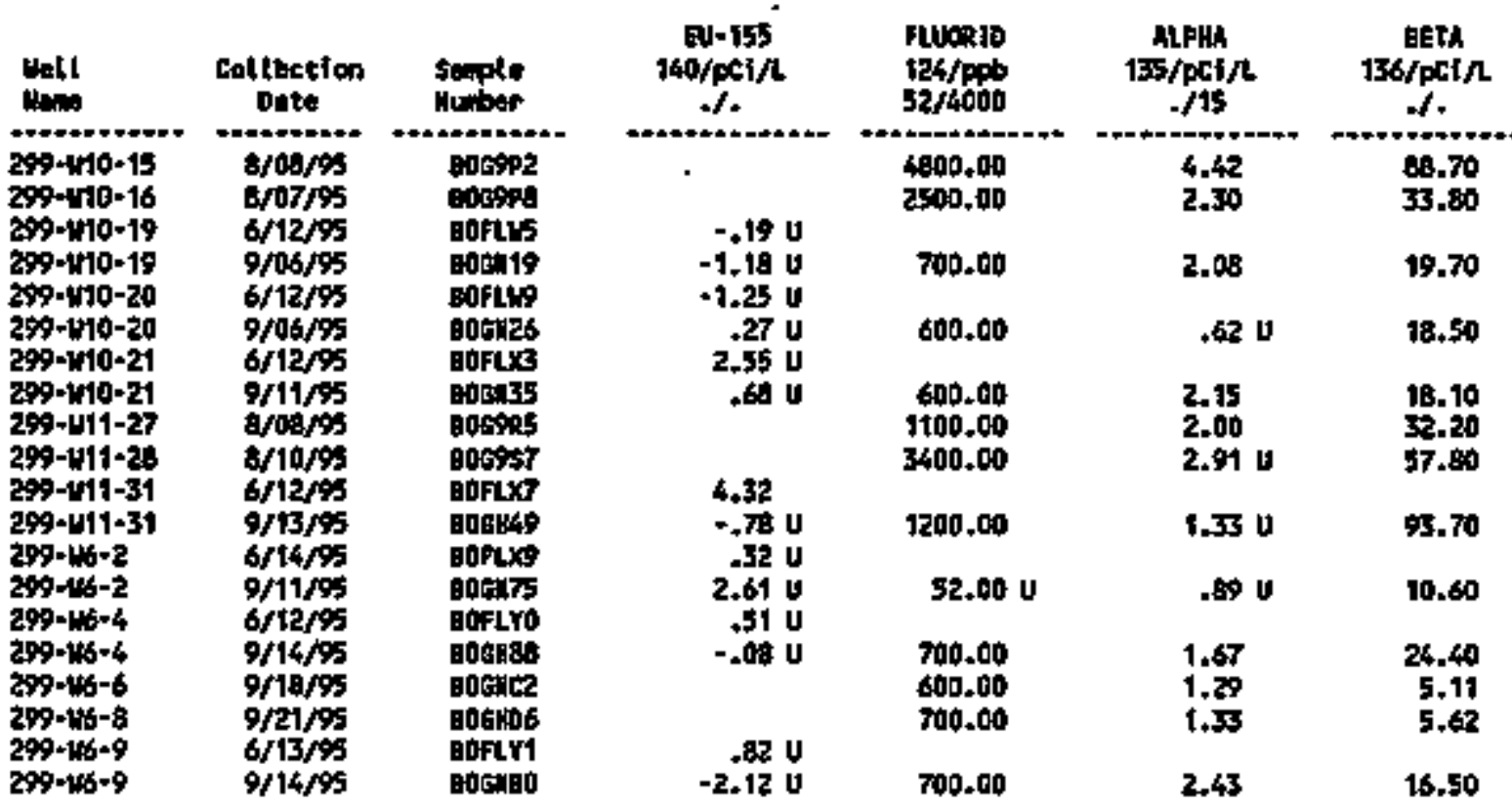

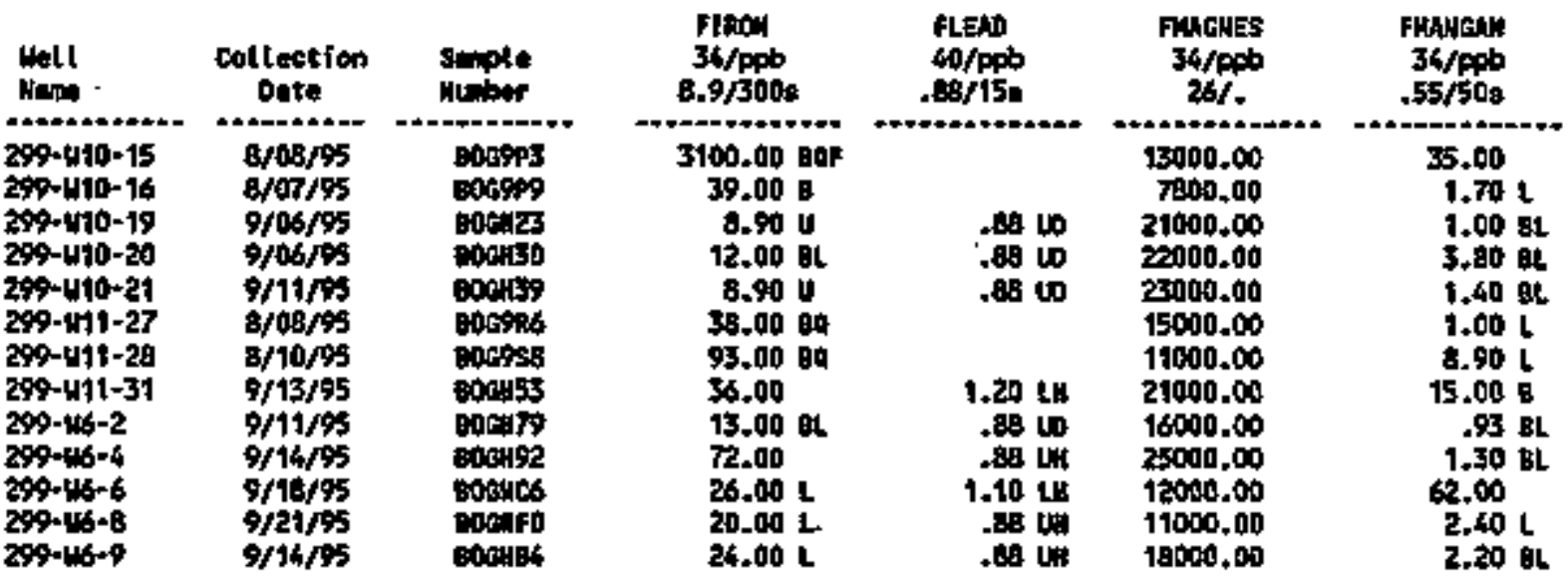


Table 15-16. Constituents with at Least One Detected Value for the Single-Shell Tank Naste Wanagenent Area $T$ Data for Reporting Period July 1 through September 30, 1995. (sheet 5 of 7)

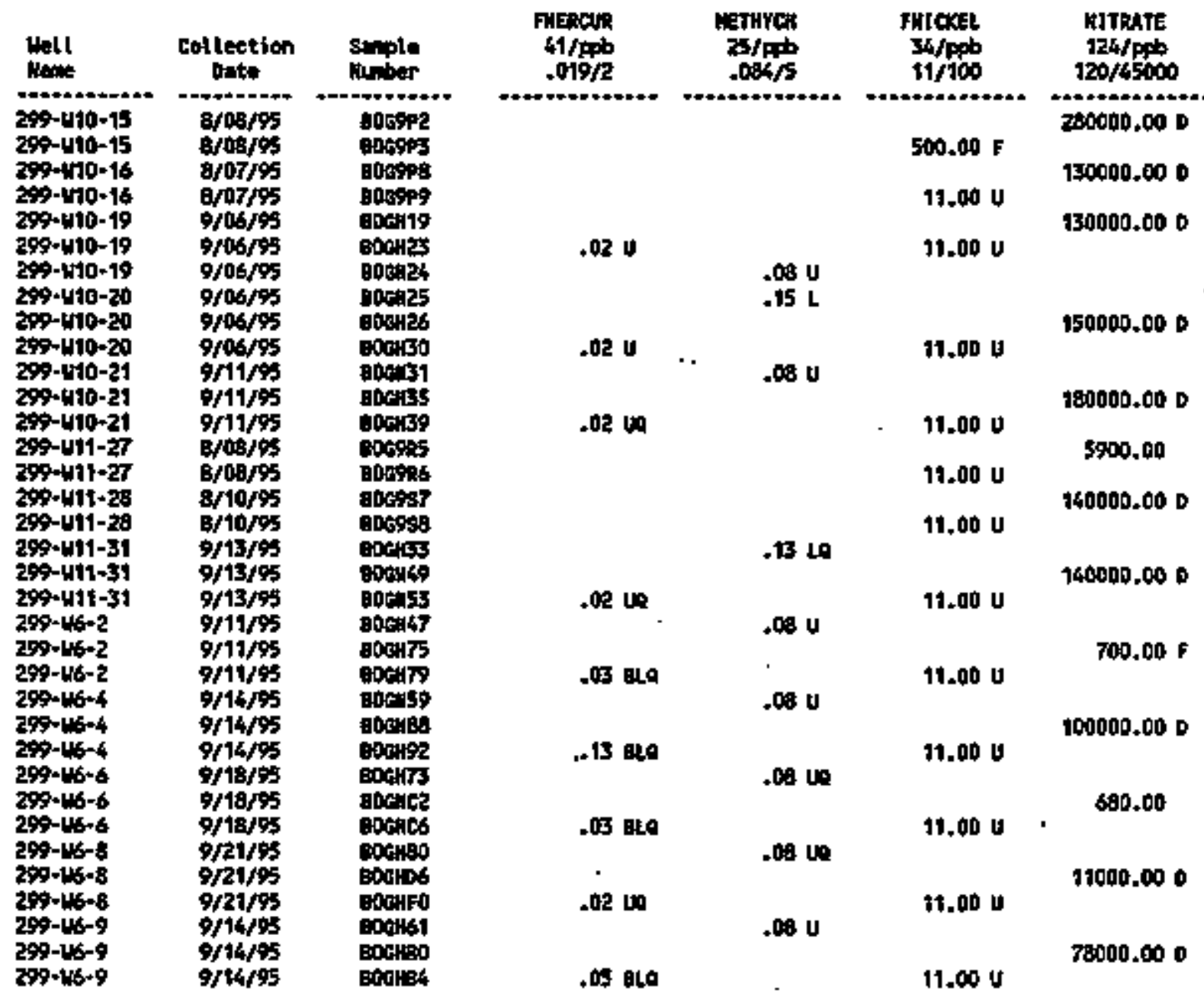

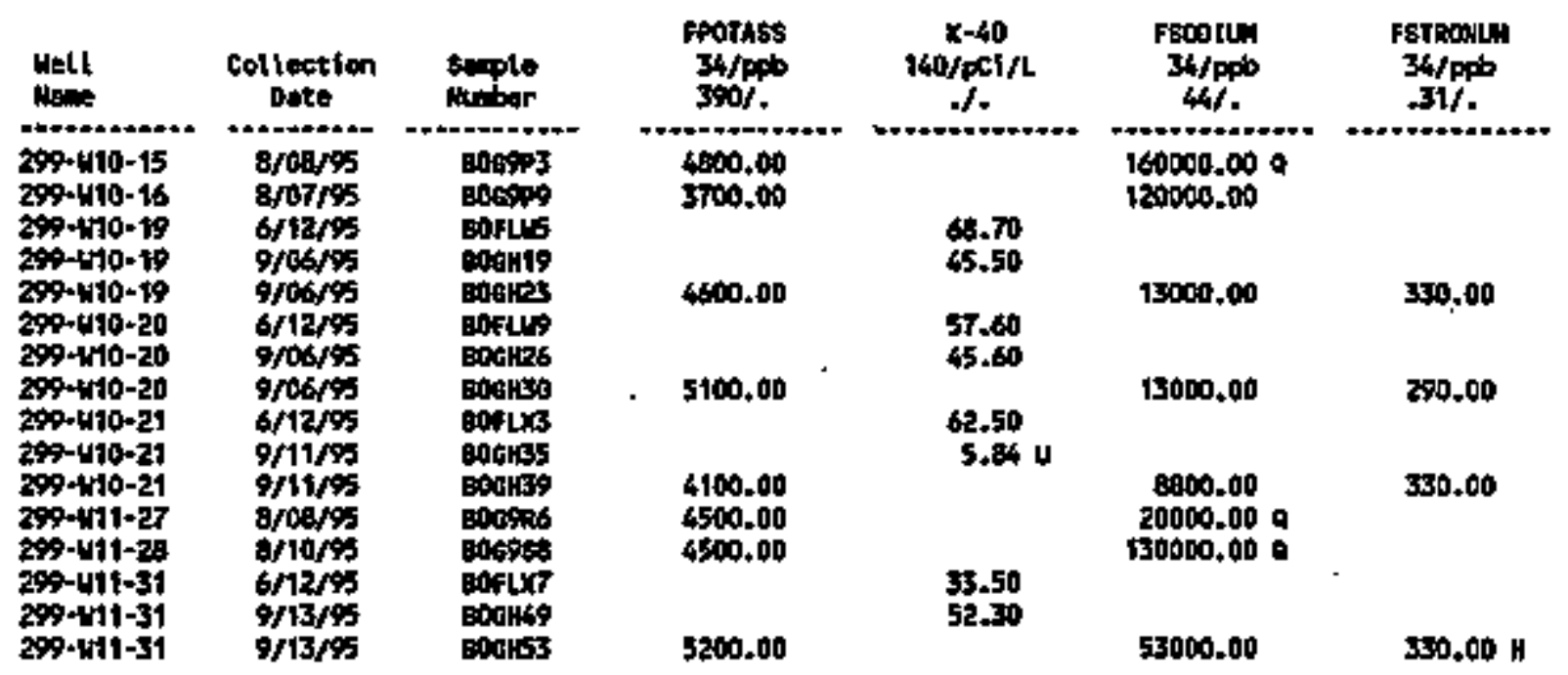


Table 15-16. Constituents with at least One Detected Value for the Single-She11 Tank Waste Managenent Area T Data for Reporting Period July 1 through September 30, 1995. (sheet 6 of 7 )

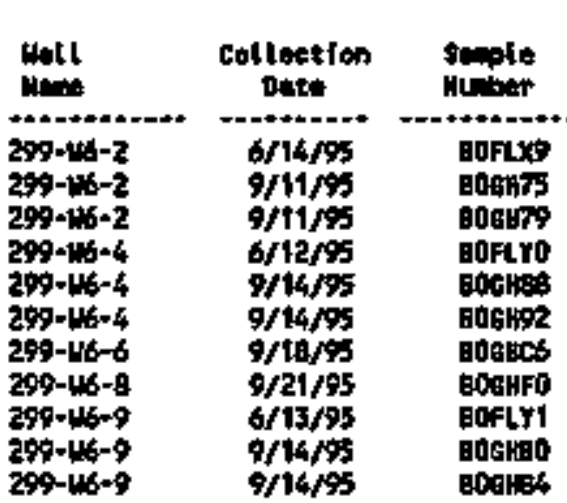

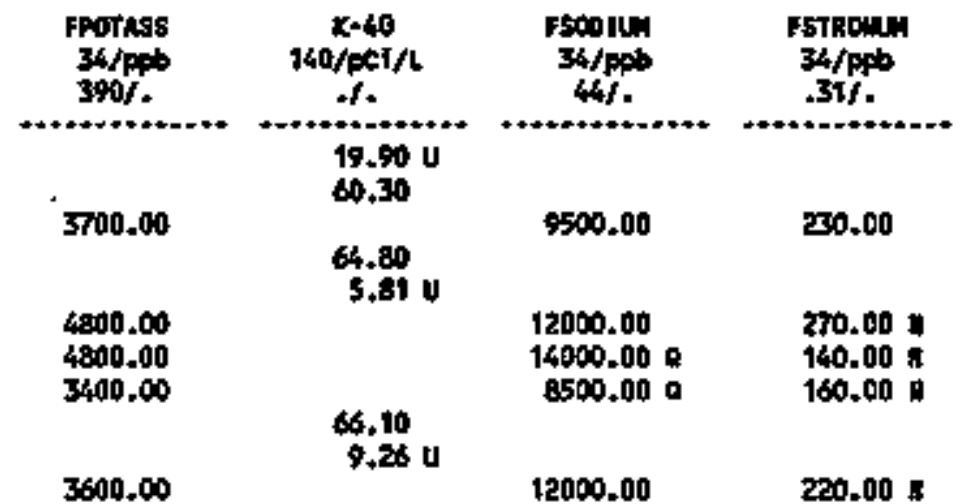

Nell

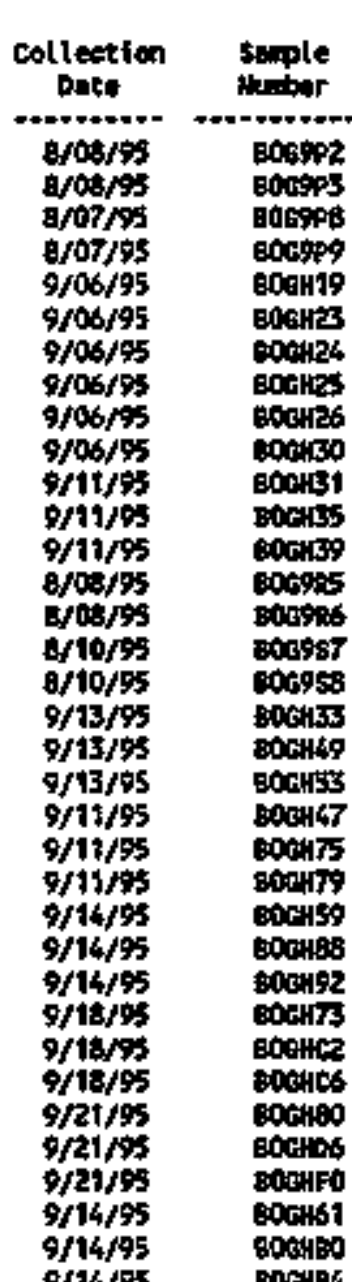

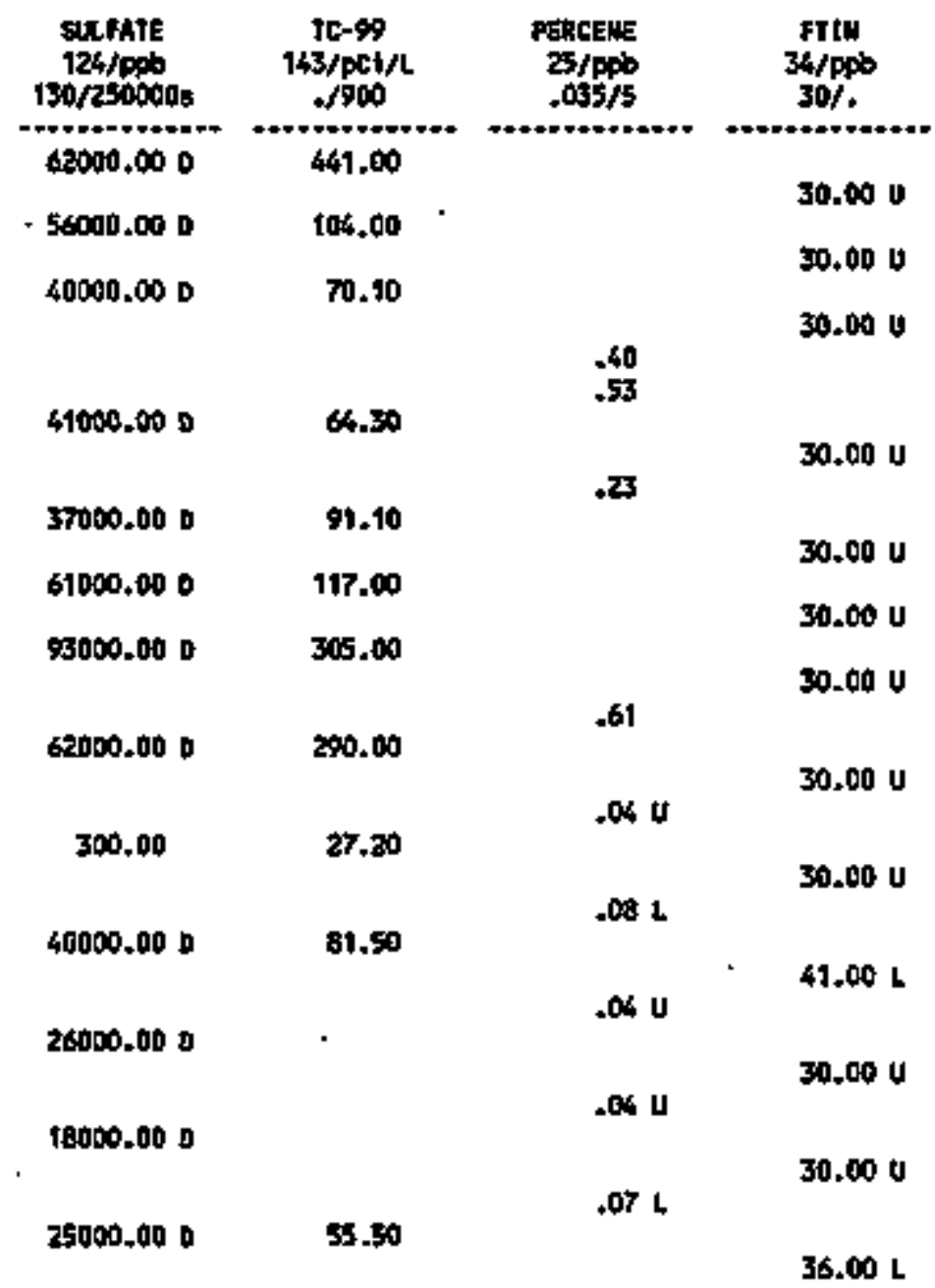


Table 15-16. Constituents with at Least One Oetected Va]ue for the Single-Shell Tank Waste Management Area T Data for Reporting Period July 1 through September 30, 1995. (sheet 7 of 7 )

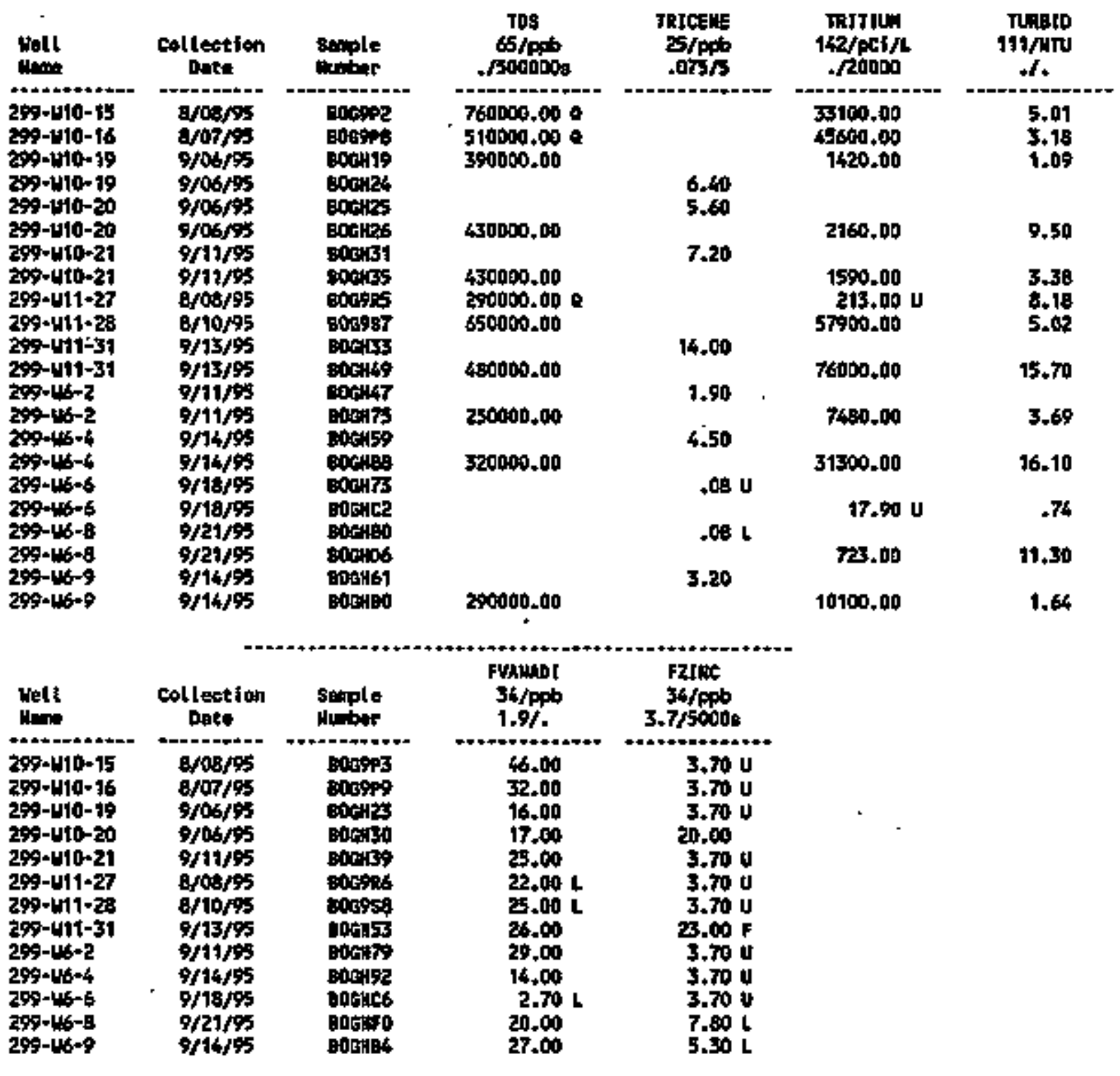

For explenation of this treble, Eas saction 1,4 of report. 
Table 15-17. Contamination Indicater Parameters for the Single-Shel1 Tank Waste Management Area T

Data for Reporting Perlod July 1

through September 30, 1995.

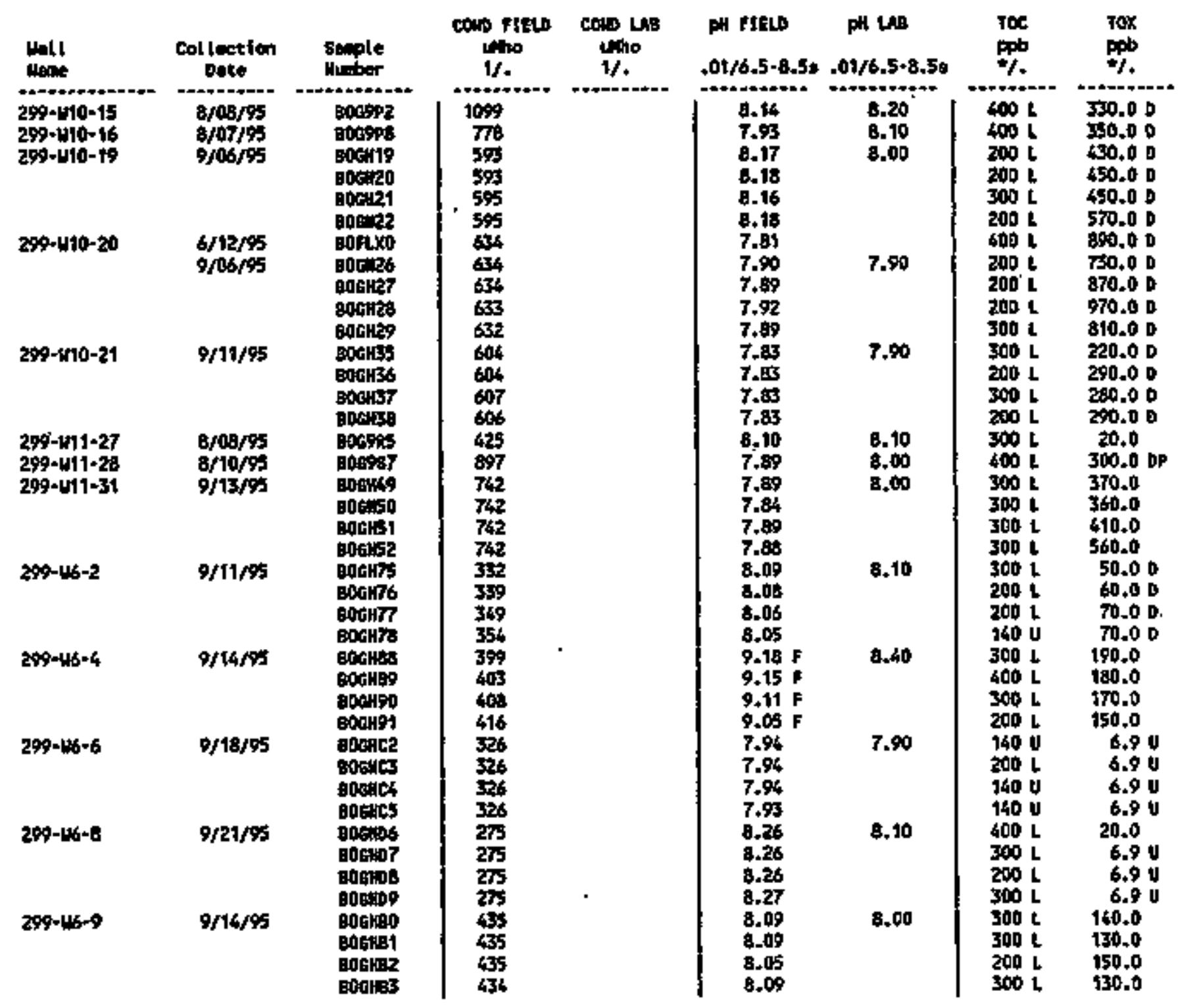

- Detetetion Linite for Toc and Tox vory dipending on the perforning loboratory. For explanation of thie table, sew sansion 1.4 of repert. 
Table 15-18. Constituent List and Subrary of Results for the Single-She11 Tank Waste Management Area TX-TY Data for

Reporting Period July 1 through September 30, 1995.

(sheet 1 of 2)

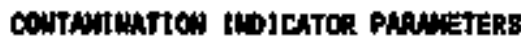

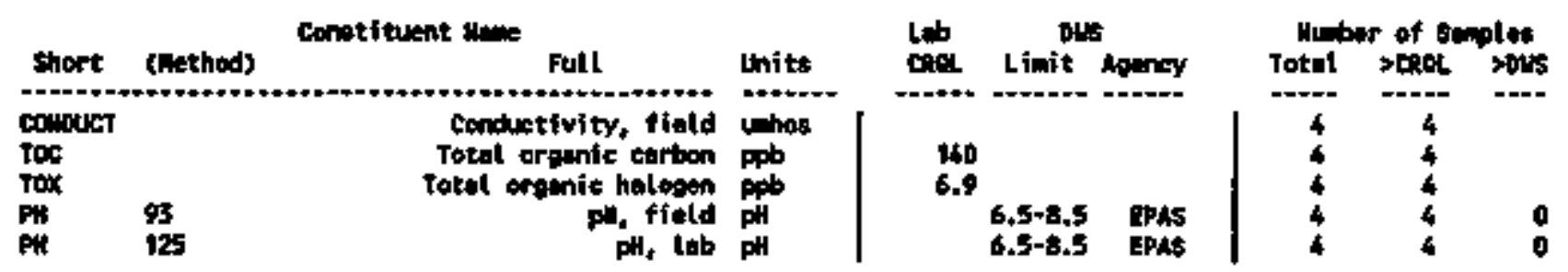

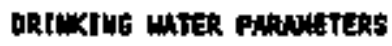

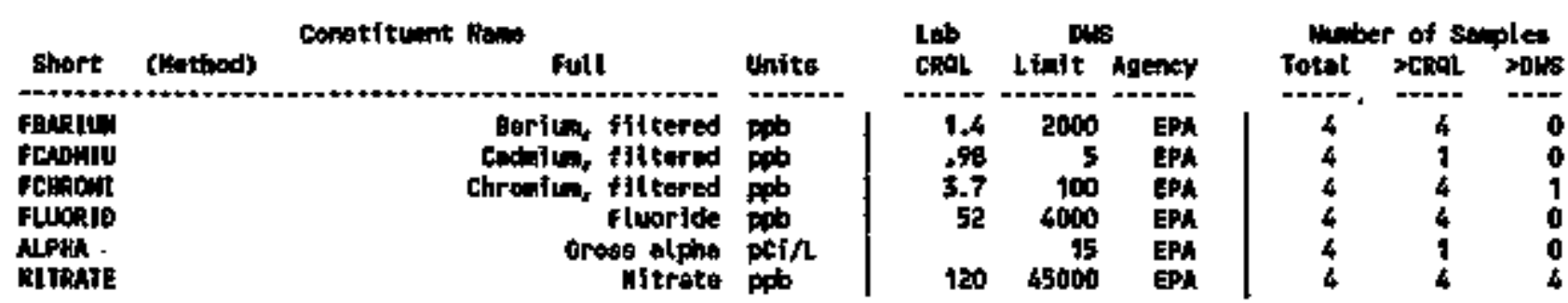

Godionter oux ITY PAotiters

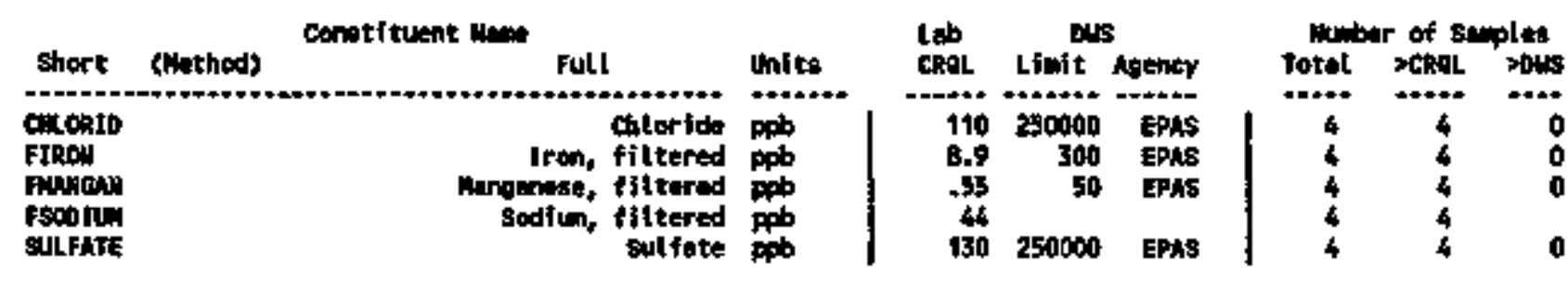

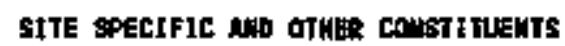

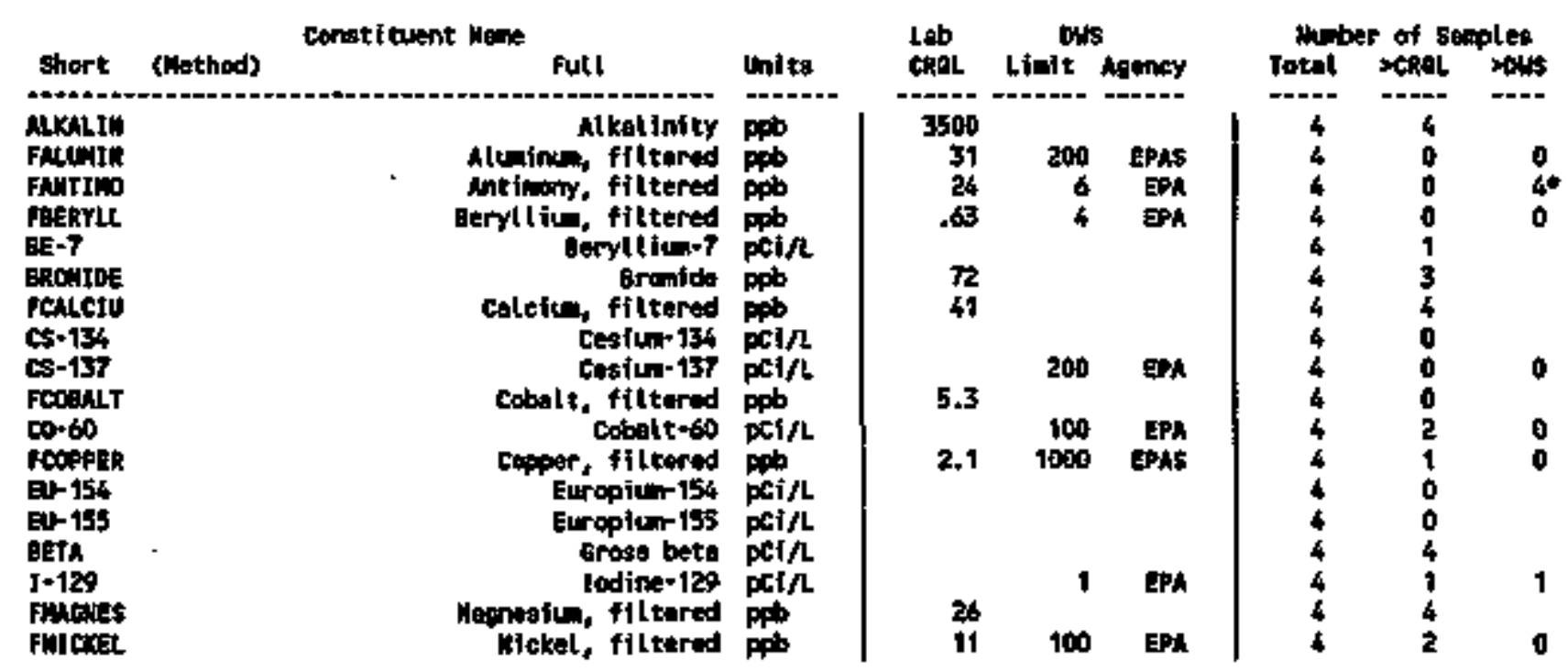


Table 15-18. Const ftuent List and Summary of Results for the Single-Shell Tank Waste Managertent Area TX-TY Data for Reporting Perlod July 1 through September 30, 1995.

(sheet 2 of 2)

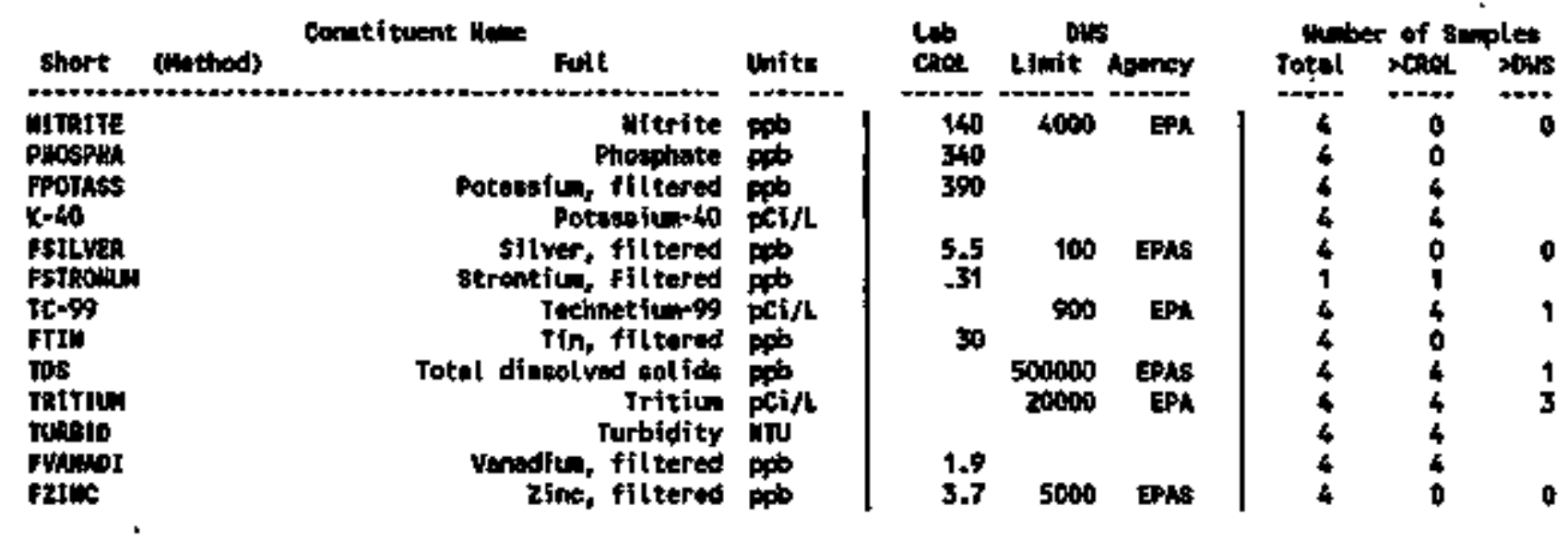

Far explanation of this toble, see section 1,4 of report. 
Tabte 15-19. Constituents with at Least One Detected Value for the Single-Sheil Tank Waste Nanagenent Area TX-TY Data for

Reporting Period July 1 through September 30, 1995.

(sheet 1 of 2)

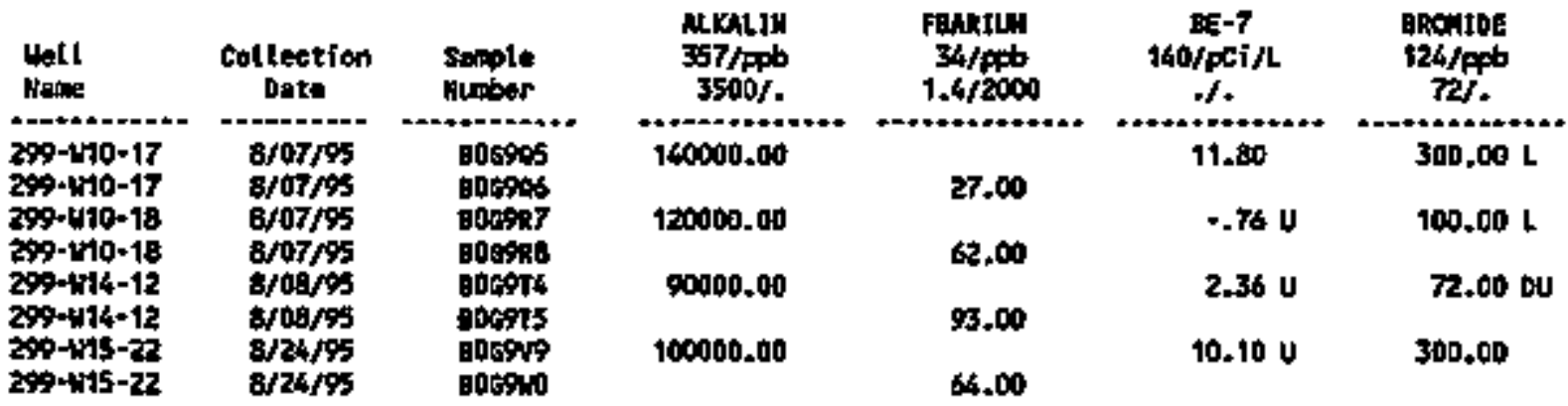

\begin{tabular}{|c|c|c|c|c|c|c|}
\hline Well & $\begin{array}{l}\text { collection } \\
\text { Date }\end{array}$ & $\begin{array}{l}\text { S-ivite } \\
\text { Hutioer }\end{array}$ & $\begin{array}{l}\text { Fentwits } \\
34 / \text { ppt } \\
\text { tya/s }\end{array}$ & $\begin{array}{c}\text { FChLett } \\
34 / \text { pob } \\
41 / \%\end{array}$ & $\begin{array}{c}\text { CHLORLD } \\
\text { 126/ppo } \\
\text { 110/250000s }\end{array}$ & $\begin{array}{r}\text { FCKROy! } \\
34 / \mathrm{ppb} \\
3,7 / \text { tob }\end{array}$ \\
\hline $\begin{array}{l}299-410-17 \\
29-1+10-17 \\
299-10-18 \\
299-410-18 \\
299-14-12 \\
299-414-12 \\
299-415-22 \\
299+415-22\end{array}$ & $\begin{array}{l}2 / 107 / 95 \\
2 / 07 / 95 \\
8 / 07 / 95 \\
8 / 07 / 95 \\
2 / 08 / 95 \\
8 / 00 / 95 \\
8 / 24 / 95 \\
8 / 26 / 95\end{array}$ & 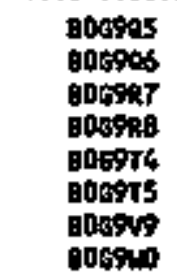 & $\begin{array}{l}.80 \mathrm{U} \\
.86 \mathrm{U} \\
.98 \mathrm{U} \\
1.30 \mathrm{~L}\end{array}$ & $\begin{array}{l}23000,00 \\
31000.00 \\
120000.00 \\
64000.00\end{array}$ & $\begin{array}{l}37000.000 \\
36000.000 \\
64000.000 \\
35000.000\end{array}$ & $\begin{array}{c}43.008 \\
5.70 \mathrm{~A} \\
250.00 \\
6.90 \mathrm{~L}\end{array}$ \\
\hline
\end{tabular}

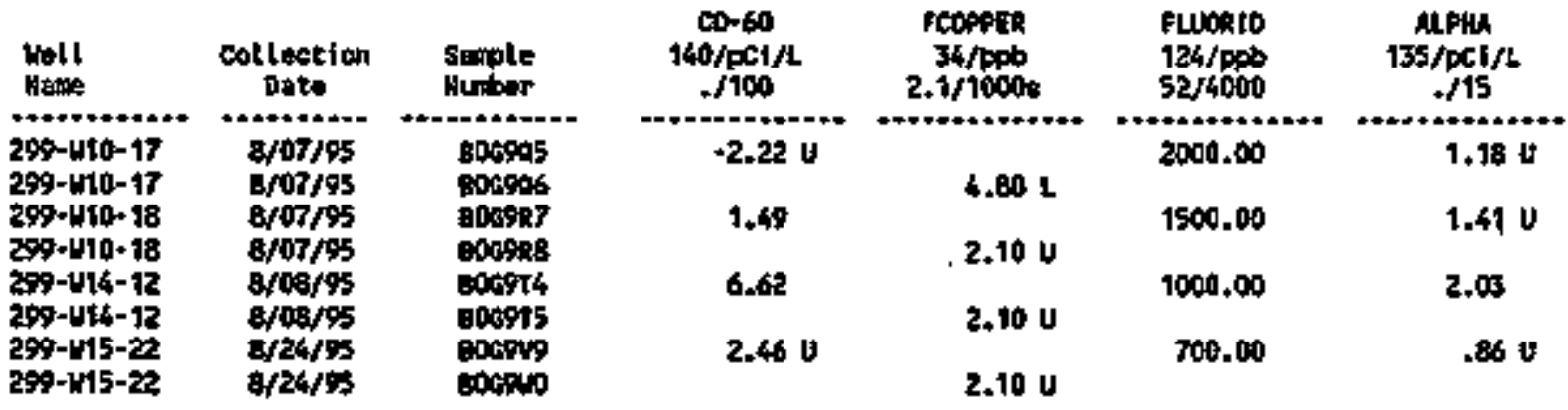

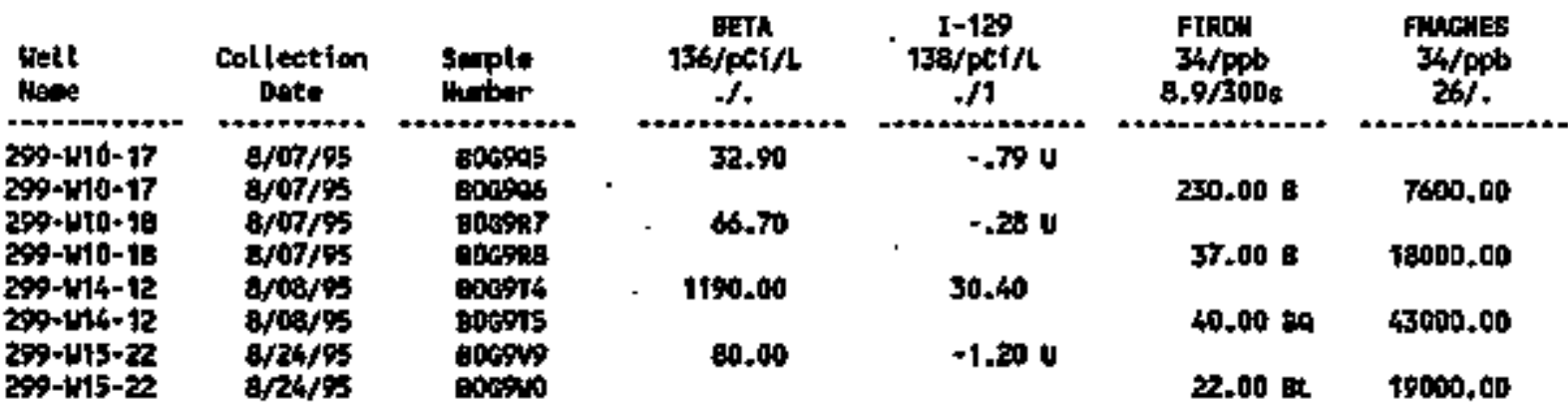


TabTe 15-19. Constiturnts with at Least One Detected Value for the single-Shell Tank Waste Management Area TX-TY Data for Reporting Period July 1 through September 30, 1995. (sheet 2 of 2)

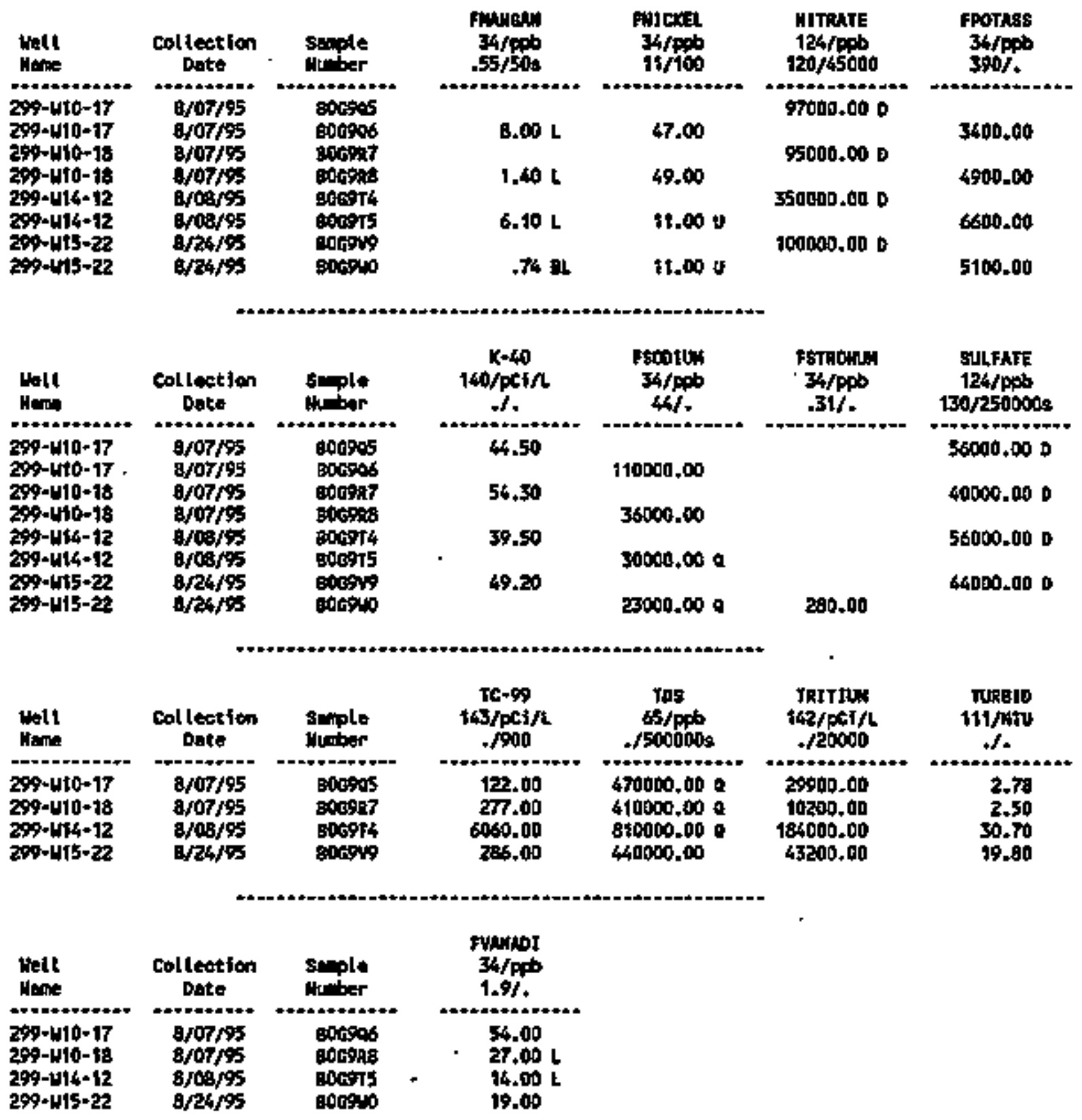

For explention of this table, aes Fection 9.4 of report. 
Table 15-20. Contamination Indicator Parameters for the Single-Shell Tank Waste Management Area TX-TY Data for Reporting Period July 1 through September 30, 1995.

\begin{tabular}{|c|c|c|c|c|c|c|c|c|}
\hline $\begin{array}{l}\text { Yell } \\
\text { Mans: }\end{array}$ & $\begin{array}{l}\text { Collection } \\
\text { bote }\end{array}$ & $\begin{array}{l}\text { 8smplo } \\
\text { Hiaper }\end{array}$ & $\begin{array}{c}\text { conto FIElo } \\
\text { whe } \\
\text { i/. }\end{array}$ & $\begin{array}{c}\text { cond Lut } \\
\text { whe } \\
\text { I/. }\end{array}$ & $\begin{array}{l}\text { DN FIELb } \\
\text {.01/6.5-8.50 }\end{array}$ & $\begin{array}{l}\text { DN LA } \\
\text {.01/6.5-8.50 }\end{array}$ & $\begin{array}{l}\text { Toc } \\
\text { ppt } \\
* / .\end{array}$ & $\begin{array}{l}\text { Tox } \\
\mathrm{ppb} \\
\mathrm{t} .\end{array}$ \\
\hline $\begin{array}{l}299-110-17 \\
299-w 10-18 \\
299-w 14-12 \\
299-415-22\end{array}$ & $\begin{array}{l}8 / 07 / 45 \\
8 / 07 / 95 \\
8 / 08 / 95 \\
8 / 24 / 95\end{array}$ & 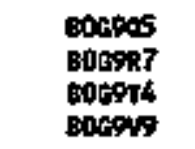 & $\begin{array}{r}726 \\
620 \\
1213 \\
622\end{array}$ & & $\begin{array}{l}8.06 \\
7.49 \\
7.68 \\
8.02\end{array}$ & $\begin{array}{l}8.10 \\
8.10 \\
7.80 \\
7.90\end{array}$ & $\begin{array}{l}300 \mathrm{~L} \\
300 \mathrm{~L} \\
400 \mathrm{~L} \\
300 \mathrm{~L}\end{array}$ & $\begin{array}{l}330.00 \\
100.000 \\
170.0 \\
550.00\end{array}$ \\
\hline
\end{tabular}

Detection Ifilits for TOC and TOX very deperding on the performing laboratory. For explenation of this tcile, seip section is of, report. 
Table 15-21. Constituent List and Summary of Results for the

Single-She7] Tank Waste Management Area U Data for

Reporting Period July 1 through September 30, 1995.

(sheet 1 of 2 )

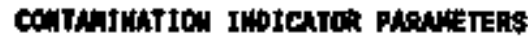

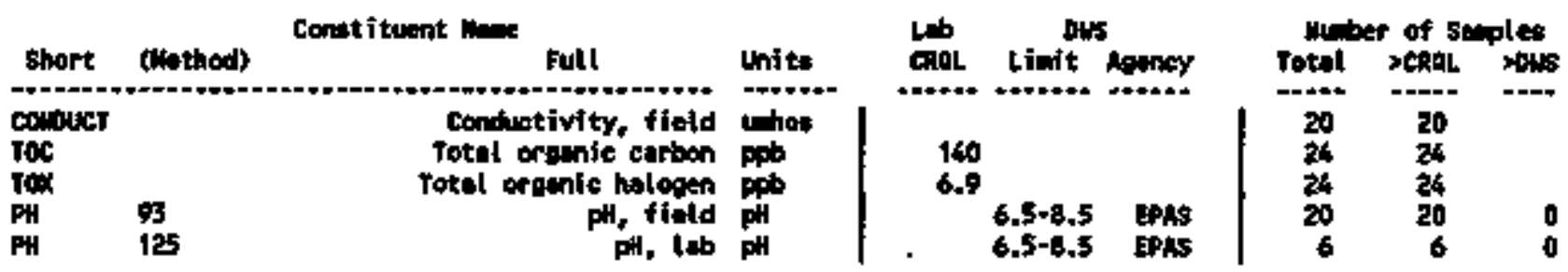

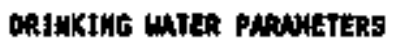

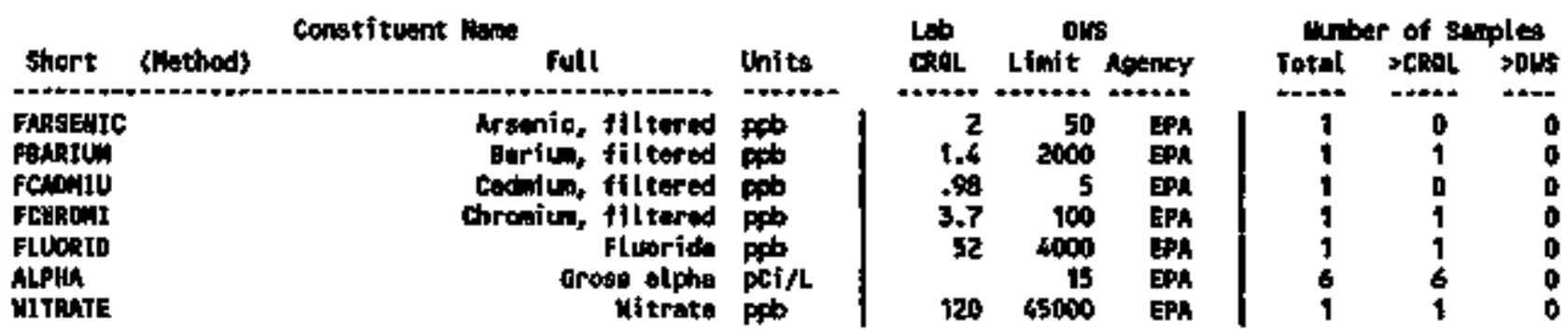

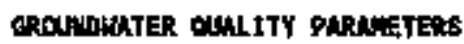

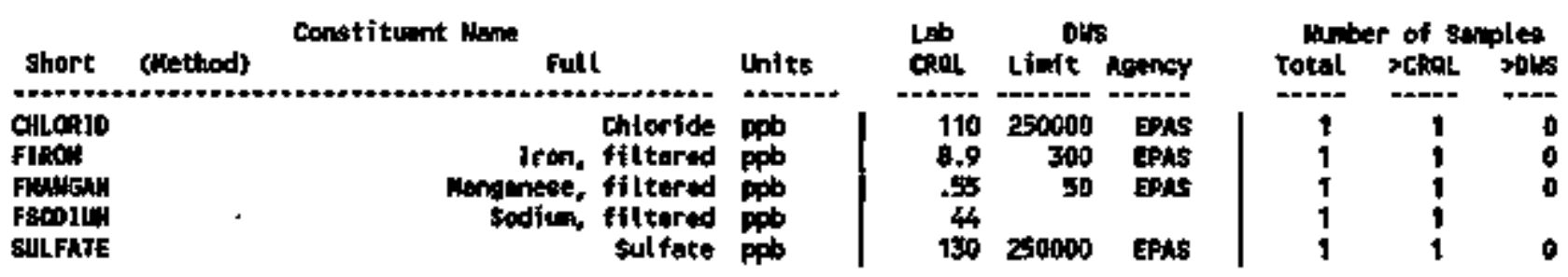

SITE SFECIFIC NAD DTHER CONSIITUENTS

\begin{tabular}{|c|c|c|c|c|c|c|c|c|c|}
\hline & & Constituent home & & Lab & Wh & & Wun & of 5 & plast \\
\hline short & (Wethod\} & Futl & Units. & GRAL & Linit & Apency & Total & $>0+0$ & T0145 \\
\hline FALIBAR & & Alunimu, fjltared & $\mathbf{F}^{\mathbf{b}}$ & 31 & 2010 & EPas & 1 & 0 & 0 \\
\hline Fantimo & & int juon, filtered & $F b$ & 24 & 6 & $E P A$ & 1 & 0 & 1 \\
\hline FaEktrLL & & Boryllium, filtored & ppo & .63 & 4 & EPh & 1 & ก & 0 \\
\hline BRoitloe & & Escontick & pab & 72 & & & 1 & 0 & \\
\hline Fentets & & Colefun, filtered & $\mathbf{p o b}$ & 41 & & & 1 & $j$ & \\
\hline FCOANT & & cobstt, filtered & pps & 5.3 & & & 1 & 0 & \\
\hline FCOPPER & & Copper, filtered & ppo & 2.1 & 1000 & EPAS & 1 & 0 & 0 \\
\hline geth & & troes bete & $\mathrm{pcih}$ & & & & 6 & 5 & \\
\hline PHICGAEL & 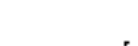 & $\begin{array}{l}\text { Ragmesilu, fittored } \\
\text { Niokel, fittered }\end{array}$ & $\operatorname{lp}_{\infty}^{\infty}$ & 36 & & & 7 & 1 & \\
\hline MITRJTE & & 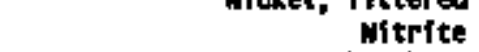 & pos & 140 & 4000 & EPA & 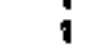 & 0 & 0 \\
\hline PHaspH & & Pho:phate & phos & 340 & & & 1 & D & \\
\hline FPoThss & & Poteselun, fittered & Prb & 390 & & & $?$ & 1 & \\
\hline Fotivet & & silver, filtered & path & 5.5 & 100 & $\begin{array}{l}\text { EPAS } \\
\text { EPR }\end{array}$ & 6 & $\frac{1}{5}$ & 0 \\
\hline FTIM & & tin, fittared & PF & 30 & & & 1 & 0 & \\
\hline tos & & $\begin{array}{l}\text { Tatal difeolved sotids } \\
\text { TrIttis }\end{array}$ & ppt & & $\begin{array}{r}500000 \\
20000\end{array}$ & $\begin{array}{l}\text { EPAS } \\
\text { ER }\end{array}$ & F & 6 & $\begin{array}{l}0 \\
0\end{array}$ \\
\hline Tula & & Turb? & & & & & 5 & 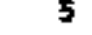 & \\
\hline
\end{tabular}


Tabie 15-21. Constituent List and Summary of Results for the Single-Shell Tank Waste Management Area U Data for Reporting Period July 1 through September 30, 1995. (sheet 2 of 2)

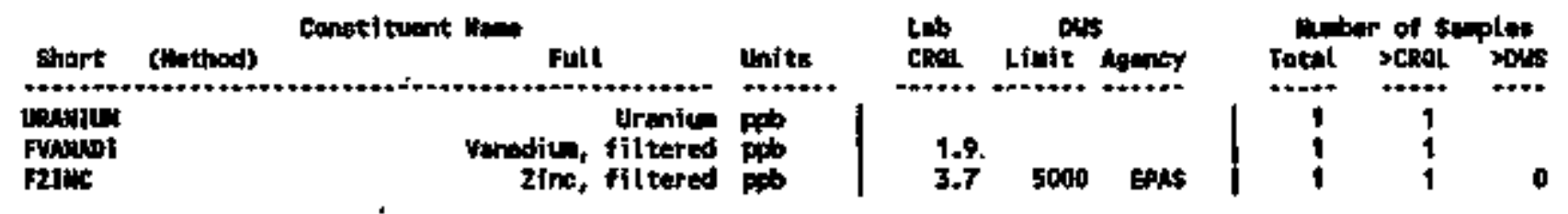

- For explantion of this tebte, sect Section 1.4 of teport. 
TabTe 15-22. Constituents with at Least one Detected Va]ue for the Single-Shell Tank Waste Management Area U Data for Reporting Period July 1 through September 30, 1995. (sheet 1 of 2)

\begin{tabular}{|c|c|c|c|c|c|c|}
\hline thetl & $\begin{array}{c}\text { Colliction } \\
\text { Dete }\end{array}$ & Sopite & 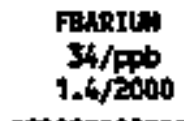 & $\begin{array}{c}\text { Fallery } \\
34 / \text { bis } \\
41 / .\end{array}$ & $\begin{array}{c}\text { CHLoRlo } \\
124 / \mathrm{ppb} \\
\text { t16/70009s }\end{array}$ & $\begin{array}{r}\text { ferRoin } \\
34 / \mathrm{pob} \\
3.7 / 100\end{array}$ \\
\hline 299-U19-32 & $7 / 51 / 9$ & B06sJB & 62,00 & 40000,00 & & \\
\hline
\end{tabular}

\begin{tabular}{|c|c|c|c|c|c|c|}
\hline thell & $\begin{array}{c}\text { Collection } \\
\text { Date }\end{array}$ & Somple & $\begin{array}{l}\text { Futgid } \\
124 / \text { ppb } \\
52 / 4000\end{array}$ & $\begin{array}{c}\text { AtPHh } \\
135 / \mathrm{pCI} / \mathrm{L} \\
. / 15\end{array}$ & $\begin{array}{c}\text { BEtA } \\
\text { \$3S/pCI/L } \\
+/ 2\end{array}$ & $\begin{array}{c}\text { freoh } \\
\text { 36/pob } \\
8.9 / 5006\end{array}$ \\
\hline $\begin{array}{l}299-118-25 \\
299-118-25 \\
299-118-30 \\
299-118-31 \\
299-419-31 \\
299-119-32 \\
290+119-3\end{array}$ & 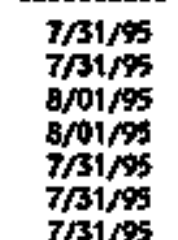 & 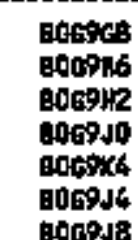 & 800.00 & $\begin{array}{r}1.13 \\
2.29 \\
-1.57 \\
5.96 \\
2.12 \\
6.53\end{array}$ & $\begin{array}{c}3.24 \\
4.28 \\
1.51 \\
5.80 \\
13.00 \\
20.50\end{array}$ & \\
\hline
\end{tabular}
$299+1419-32$ $7 / 31 \mathrm{As}$ boco요

\begin{tabular}{|c|c|c|c|c|c|}
\hline $\begin{array}{c}\text { Collection } \\
\text { Date }\end{array}$ & $\begin{array}{l}\text { Serple } \\
\text { funber }\end{array}$ & $\begin{array}{c}\text { Fuckes } \\
36 / p \text { pb } \\
26 / .\end{array}$ & $\begin{array}{l}\text { FWhowll } \\
\text { 34/ppb } \\
55 / 50 \mathrm{sa}\end{array}$ & $\begin{array}{l}\text { FNICKEL } \\
34 / p \mathrm{pb} \\
11 / 100\end{array}$ & $\begin{array}{c}\text { HITRATE } \\
124 / \mathrm{ppb} \\
120 / 45000\end{array}$ \\
\hline $\begin{array}{l}7 / 31 / 95 \\
7 / 31 / 45\end{array}$ & $\begin{array}{l}\text { nots } \\
\text { nosous }\end{array}$ & 12000.60 & 47.00 & 83.00 & 1700.00 \\
\hline
\end{tabular}

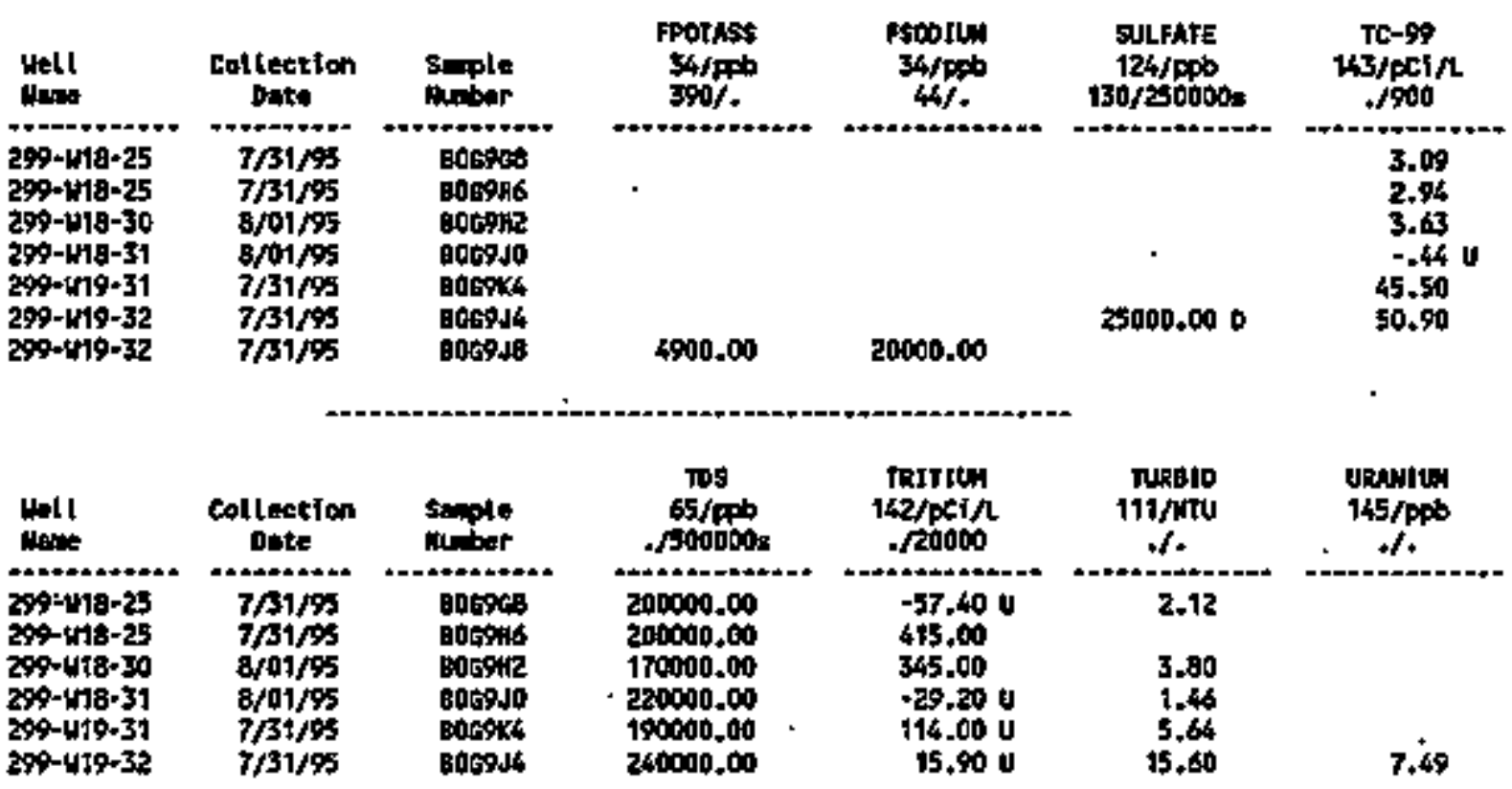




\section{DOE/RL-95-69-3}

Table 15-22. Constituents with at Least One Detected Value for the Single-5he17 Tank Waste Management Area U Data for Reporting Perlod July I through September 30, 1995. (sheet 2 of 2)

\begin{tabular}{|c|c|c|c|c|}
\hline Velt & $\begin{array}{c}\text { Colfection } \\
\text { Dete }\end{array}$ & sapple & $\begin{array}{l}\text { Fwathot } \\
34 / p \% 6 \\
1.9 \%\end{array}$ & $\begin{array}{c}\text { f21uc } \\
34 / \mathrm{pptb} \\
3.7 / 5000 \mathrm{~s}\end{array}$ \\
\hline $299-119-32$ & $7 / 3 t / 95$ & 6009 J8 & $11.00 \mathrm{~L}$ & $100.00 \mathrm{~B}$ \\
\hline
\end{tabular}

For explenation of this trble, seit section 7,4 of report. 
Table 15-23. Contamination Indicator Parameters for the Single-Shell Tank Waste Managenent Area U

Data for Reporting Period July 1

through Septenber 30, 1995.

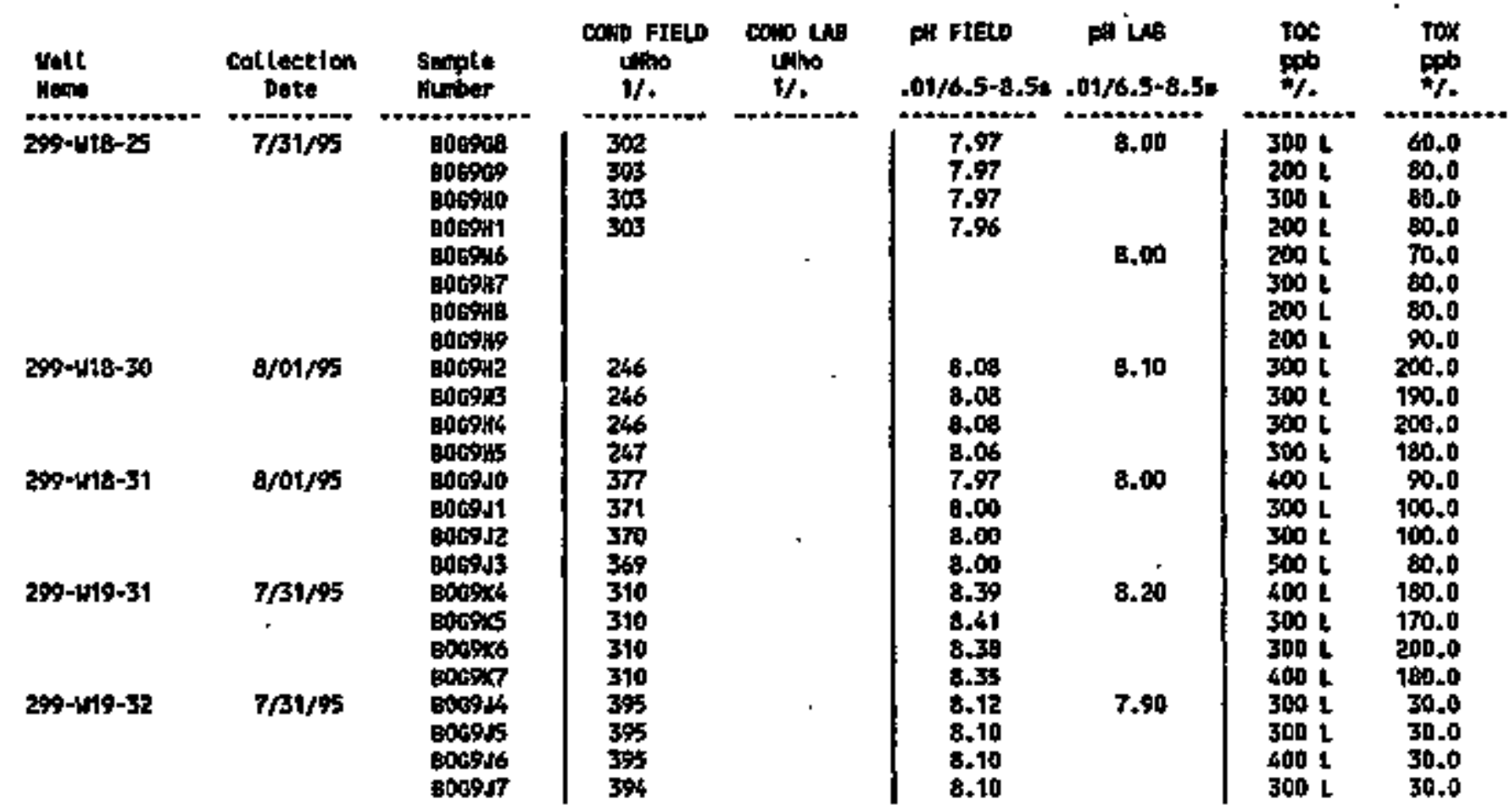

"Detection IInits for ioc and Tox vary depending on the pertorning laboretory. For explention of this table, ate section 1.4 of report. 
DOE/RL-95-69-3

This page intentionally left blank. 
DOE /RL-95-69-3

contarts

16.0300 AREA PROCESS TRENCHES $\ldots \ldots \ldots \ldots \ldots \ldots \ldots$

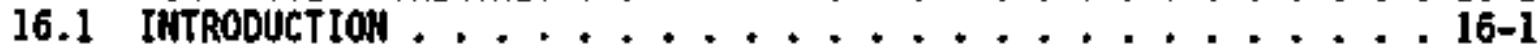

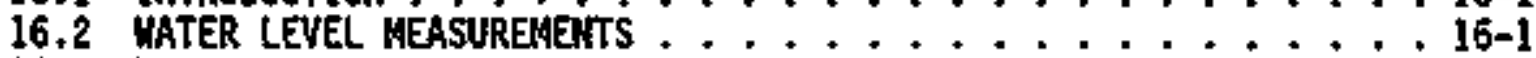

16.3 WATER CHEHISTRY DATA .................... 16-1 
DOE/RL-95-69-3

\section{LIST OF FIGURES}

16-1 Monitoring Hell Location Map for the 300 Area Process Trenches . 16-2

\section{LIST OF TABLES}

16-1 Manitoring Well Purpose and Sampling Schedule

for the 300 Area Process Trenches Network ............ 16-3

16-2 RCRA Water Leve] Measurement Report 300 Area Process Trenches, Third quarter $1995 \ldots . . \ldots$. . . . . . . . . . . . . . . 
DOE $/ R L-95-69-3$

\title{
16.0 300 AREA PROCESS TREMCHES
}

\author{
3. W. Lindberg \\ Nestinghouse Hanford Company
}

\subsection{INTRODUCTION}

The groundwater near the 300 Area Process Trenches (APTs) (316-5) has been monitored by a RCRA we11 network since June 1985. The groundwater monitoring is being conducted under interin-status regulations (40 CFR 265) and is an assessment-7eve] progran (Scha]la 1988). A revised groundwater monitoring plan has been prepared (Lindberg 1995). The revised plan will take effect 7 ater this year when the faciltty goes into a compliance monitoring program under final status.

The 300 APTs are located in the northern portion of the 300 Area and were used for the disposal of wastewater from various 300 Area operations. Administrative isolation of the trenches has been completed and all discharges to the trenches have been terminated. Complete physical isolation of the trenches occurred in January 1995 . Just before the trenches were taken out of use, the wastewater primarily consisted of cooling water, with 5 mall quantities of nonhazardous maintenance and process waste.

Monitoring wells were constructed in response to a Consent Agreenent and Compliance onder (Ecology and EPA 1986). Locations of groundwater wells currently used to monitor the facility are shown in Figure 16-1. Table 16-1 jists the wells of the monitoring network and provides information about their relative location, the portion of the aquifer sampled, sampling frequency, and last sampling date. Hine of the 11 wells in the monitoring network are RCRAstandard wefls; the other two are older, noncompliant wells.

\subsection{MATER LEVEL HEASUREMERTS}

Water levels are measured quarterly and at the time of sampling in selected 300 Area wells. Water level measurements obtained during the third quarter of 1995 are presented in Table 16-2.

\subsection{WATER ChEKISTRY DATA}

Water chemistry samples are collected seriannualiy fron al1 but one of the 300 APT monttoring wells. The 7 ast sampling took place during the second quarter, so no data were obtained from these weils for the third quarter. (See Table 16-1 for the sampling schedule.) Samples are collected four times per year from well 399-1-17A to provide near-trench nonitoring of contaninants. However, this woll was sampled on October 5, 1995, which was beyond the cut-off of the third quarter reporting period, so no data are reported for this well. 
Figure 16-1. Monitoring Nell Location Map for the 300 Area Process Trenches.

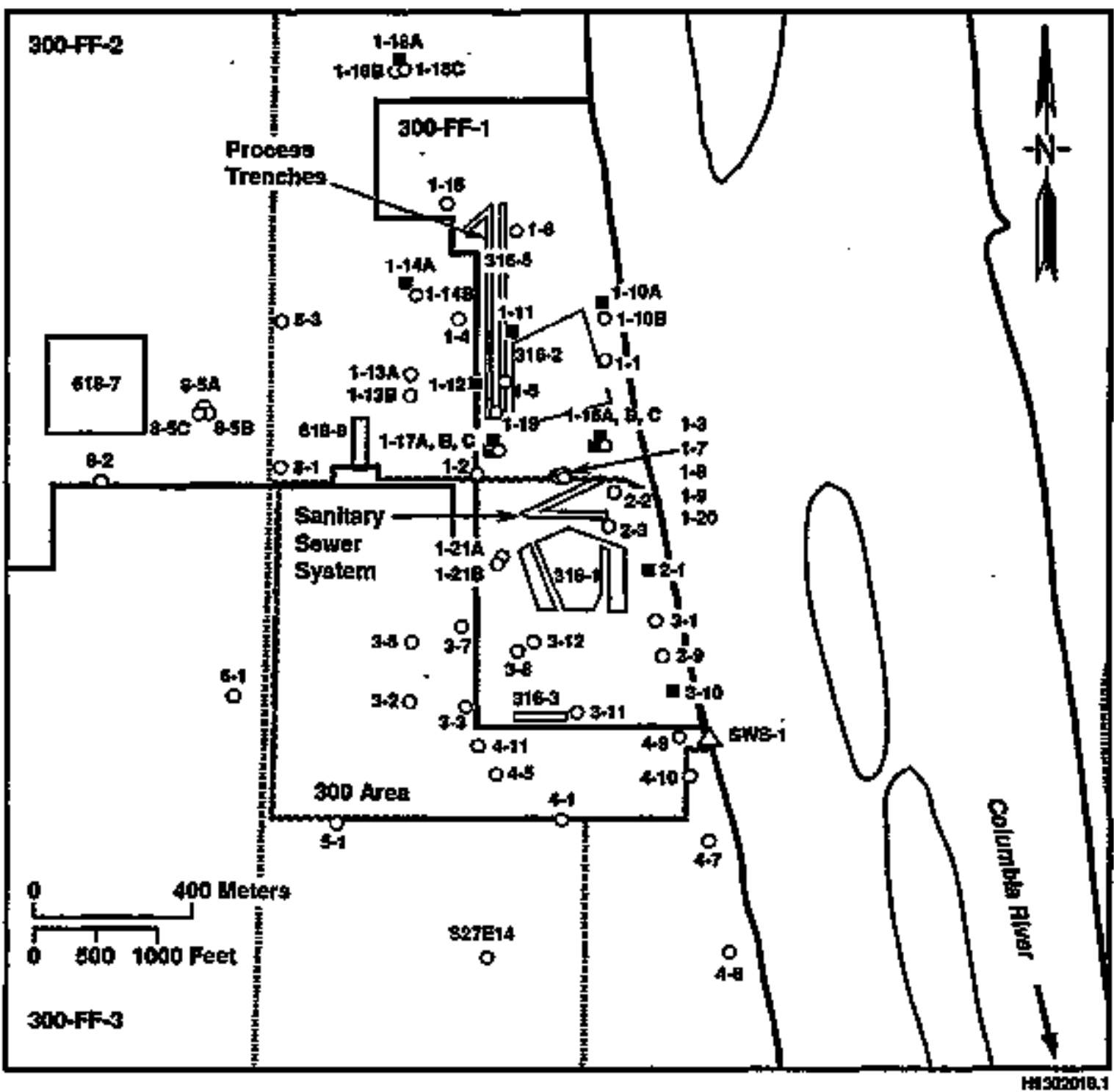

\% 1-12 Well Location and Number (Wells Prefixed by 39s-,
Excepl Those Eeglming with $s$ are Praflxed wh 699-)

- 4-7 Mionllowing Notwark Well

$\Delta$ sws-1 Surface-Water Monitoring Stalion

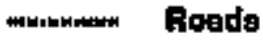


Table 16-1. Monitoring Well Purpose and Sampling ScheduTe for the 300 Area Process Trenches Hetwork.

\begin{tabular}{|c|c|c|c|c|}
\hline $\begin{array}{l}\text { Wel7 no. } \\
(399-)\end{array}$ & $\begin{array}{l}\text { Relative } \\
\text { position }\end{array}$ & Hydrogeologic unit & $\begin{array}{l}\text { Sample } \\
\text { frequency }\end{array}$ & $\begin{array}{l}\text { Last sampling } \\
\text { date(s) }\end{array}$ \\
\hline $1-10 A$ & Downgradient & $\begin{array}{l}\text { Hanford/Ringold: } \\
\text { Water Table }\end{array}$ & Semiannually & $06 / 01 / 95$ \\
\hline $1-11$ & Adjacent & $\begin{array}{l}\text { Hanford/Ringold: } \\
\text { Hater Table }\end{array}$ & Semiannually & $06 / 05 / 95$ \\
\hline $1-12$ & Downgradient & $\begin{array}{l}\text { Hanford/Ringold: } \\
\text { Water Table }\end{array}$ & Semiannual1y & $06 / 01 / 95$ \\
\hline 1-14A & Adjacent & $\begin{array}{l}\text { Hanford: Water } \\
\text { Table }\end{array}$ & Semiannually & $06 / 01 / 95$ \\
\hline $1-16 A$ & Downgradient & $\begin{array}{l}\text { Ringold: Water } \\
\text { Table }\end{array}$ & Semiannual1y & $05 / 01 / 95$ \\
\hline $1-16 B$ & Downgradient & $\begin{array}{l}\text { Ringold: Botton of } \\
\text { Unconfined Aquifer }\end{array}$ & Semianntally & $06 / 02 / 95$ \\
\hline $1-17 A$ & Downgradient & $\begin{array}{l}\text { Ringold: Water } \\
\text { Table }\end{array}$ & $\begin{array}{l}\text { Four Times } \\
\text { Per Year }\end{array}$ & $\begin{array}{l}04 / 05 / 95 \\
06 / 01 / 95 \\
10 / 05 / 95\end{array}$ \\
\hline 1-17B & Downgradient & $\begin{array}{l}\text { Ringold: Bottom of } \\
\text { Unconfined Aquifer }\end{array}$ & Semfannua\}]y & $06 / 02 / 95$ \\
\hline $1-18 A$ & Upgradient & $\begin{array}{l}\text { Ringold: Water } \\
\text { Table }\end{array}$ & Semiannualiy & $06 / 01 / 95$ \\
\hline $2-1^{4}$ & Downgradient & $\begin{array}{l}\text { Hanford/Ringold: } \\
\text { Water Table }\end{array}$ & Semjannual7y & $06 / 01 / 95$ \\
\hline $3-10^{n}$ & Downgradjent & $\begin{array}{l}\text { Hanford: Water } \\
\text { Table }\end{array}$ & Semiannually & $06 / 05 / 95$ \\
\hline
\end{tabular}

Hote: Hydrogeologic units include the sandy gravels of the Hanford formation and si]ty sands of the Ringold Formation. Water levels are measured in all wells monthly.

Wells 399-2-1 and 399-3-10 are not RCRA standard wells. The results are used as a suppiemental source of data only. 
Table 16-2, RCRA Water Level Measurement Report 300 Area Process Trenches, Third Quarter 1995. (sheet 1 of 3 )

\begin{tabular}{|c|c|c|c|c|}
\hline Well & Date & $\begin{array}{l}\text { Depth to } \\
\text { water (ft) }\end{array}$ & $\begin{array}{l}\text { Water } \\
\text { elevation } \\
\text { (ft) }\end{array}$ & $\begin{array}{l}\text { leve1 } \\
\text { above ms1 } \\
\text { (m) }\end{array}$ \\
\hline \multicolumn{5}{|c|}{ Wells Monitoring the Top of the Unconfined Aquifer } \\
\hline $399-1-1$ & $9 / 05 / 95$ & 35.95 & 340.71 & 103.85 \\
\hline $399-1-10 \mathrm{~A}$ & $9 / 05 / 95$ & 32.86 & 340.79 & 103.87 \\
\hline $399-1-11$ & $9 / 05 / 95$ & 36.34 & 341.38 & 104.05 \\
\hline $399-1-12$ & $9 / 05 / 95$ & 43.44 & 340.97 & 103.93 \\
\hline $399-1-14 A$ & $9 / 05 / 95$ & 41.40 & 341.83 & 104.19 \\
\hline $399-1-15$ & $9 / 05 / 95$ & 37.69 & 341.84 & 104.19 \\
\hline $399+1-16 \mathrm{~A}$ & $9 / 05 / 95$ & 40.91 & 340.56 & 103.80 \\
\hline $399-1-17 \mathrm{~A}$ & $9 / 05 / 95$ & 36.62 & 340.82 & 103.88 \\
\hline $399-1-18 A$ & $9 / 05 / 95$ & 48.30 & 342.53 & 104.40 \\
\hline $399-1-19$ & $9 / 05 / 95$ & 33.71 & 340.93 & 103.92 \\
\hline $399-1-3$ & $9 / 05 / 95$ & 44.04 & 340.67 & 103.84 \\
\hline $399-1-4$ & $9 / 05 / 95$ & 38.97 & 341.61 & 104.12 \\
\hline $399-1-5$ & $9 / 05 / 95$ & 38.84 & 340.93 & 103.92 \\
\hline $399-1-7$ & $9 / 05 / 95$ & 44.95 & 340.65 & 103.83 \\
\hline $399-1-8$ & $9 / 05 / 95$ & 44.18 & 340.70 & 103.85 \\
\hline $399-2-1$ & $9 / 06 / 95$ & 34.83 & 340.40 & 103.75 \\
\hline $399-2-2$ & $9 / 05 / 95$ & 37.01 & 340.51 & 103.79 \\
\hline $399-2-3$ & $9 / 05 / 95$ & 34.92 & 340.50 & 103.78 \\
\hline $399-3-1$ & $9 / 05 / 95$ & 44.13 & 340.26 & 103.71 \\
\hline $399-3-10$ & $9 / 05 / 95$ & 45.13 & 340.22 & 103.70 \\
\hline
\end{tabular}


Table 16-2. RCRA Water Level Measurement Report 300 Area Process Trenches, Third Quarter 1995. (sheet 2 of 3 )

Well Date $\quad \begin{gathered}\text { Depth to } \\ \text { water (ft) }\end{gathered} \quad \begin{gathered}\text { Nater level } \\ \text { eTevation above msl } \\ \text { (ft) }\end{gathered}$

Wells Monitoring the Top of the Unconfined Aquifer

$\begin{array}{lllll}399-3-12 & 9 / 05 / 95 & 47.25 & 340.79 & 103.87 \\ 399-3-6 & 9 / 05 / 95 & 51.96 & 340.84 & 103.89 \\ 399-3-9 & 9 / 05 / 95 & 47.81 & 340.27 & 103.71 \\ 399-4-1 & 9 / 05 / 95 & 55.32 & 340.28 & 103.72 \\ 399-4-10 & 9 / 05 / 95 & 38.50 & 340.04 & 103.64 \\ 399-4-11 & 9 / 05 / 95 & 63.93 & 340.52 & 103.79 \\ 399-4-7 & 9 / 05 / 95 & 38.55 & 340.01 & 103.64 \\ 399-5-1 & 9 / 05 / 95 & 54.37 & 341.16 & 103.99 \\ 399-6-1 & 9 / 05 / 95 & 47.13 & 341.66 & 104.14 \\ 399-8-1 & 9 / 05 / 95 & 54.63 & 341.50 & 104.09 \\ 399-8-2 & 9 / 05 / 95 & 55.33 & 342.69 & 104.45 \\ 399-8-3 & 9 / 05 / 95 & 52.82 & 341.93 & 104.22 \\ 699-\$ 27-614 & 9 / 05 / 95 & 61.81 & 337.87 & 102.98\end{array}$

Wells Monitoring the Bottom of the Unconfined Aquifer

$\begin{array}{lllll}399-1-16 \mathrm{~B} & 9 / 05 / 95 & 40.39 & 340.75 & 103.26 \\ 399-1-17 \mathrm{~B} & 9 / 05 / 95 & 36.72 & 341.0 \mathrm{~B} & 103.96 \\ 399-1-18 \mathrm{~B} & 9 / 05 / 95 & 47.19 & 342.70 & 104.45\end{array}$


Table 16-2. RCRA Nater Leve1 Measurenent Report 300 Area Process Trenches, Thtrd quarter 1995. (sheet 3 of 3 ).

\begin{tabular}{ccccc}
\hline Hell & Date & $\begin{array}{c}\text { Water level. } \\
\text { Depth to } \\
\text { water (ft) }\end{array}$ & $\begin{array}{c}\text { Wevation above msl } \\
\text { (ft) }\end{array}$ \\
\hline & Nells Monttoring the Conftned Aquifer & \\
$399-1-17 C$ & $9 / 05 / 95$ & 4.76 & 373.30 & 113.78 \\
$399-1-18 \mathrm{C}$ & $9 / 05 / 95$ & 44.65 & 343.36 & 104.66 \\
$399-1-9$ & $9 / 05 / 95$ & 27.58 & 357.18 & 108.87 \\
\hline
\end{tabular}

NOTES: 1. Hater level elevations are calculated by subtracting the noasured depth-to-water fron the surveyed elevation for the well.

2. Depth-to-water values are transcribed from field records.

3. Measurements marked with an ' $"$ ' were taken at the time of sampling.

4. Heasurements marked with a ' $t$ ' are outside of the expected range, and are suspected of error. 
DOE/RL-95-69-3

CoHtarts

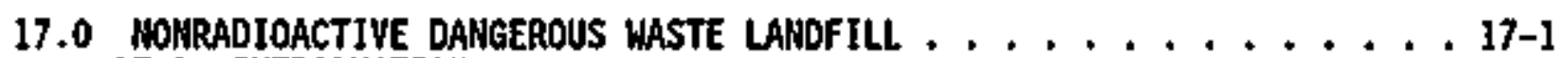

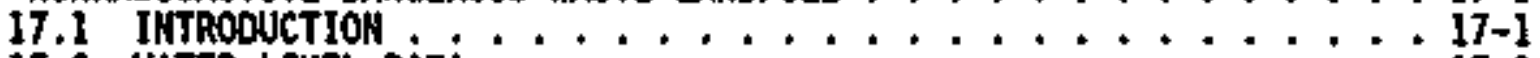

17.2 WATER LEVEL DATA . . . . . . . . ........ 17-1

17.3 WATER CHEMISTRY DATA . . . . . . . . . . . 17-1 


\section{LIST OF FIGURES}

17-1 Well Location Map for the Nonradioact fve Dangerous Waste Landfill and \$ol id Waste Landfil1 ....... 17-2

\section{LIST OF TABLES}

17-1 Monitoring Well Purpose and Sampling Schedule for the Nanradioactive Dangerous Waste LandfiIt Network . . . . . . 17-3

17-2 RCRA Water Leve] Measurement Report WRDNL, Third Quarter 1995 $\ldots$ 17-4

17-3 Constituent List and Summary of Results for the NRON Landfill Data for Reporting Period July 1 through September $30,1995 \ldots \ldots \ldots \ldots$

17-4 Constituents with at Least one Detected value for the NROM Landfill Data for Reporting Period July 1 through September $30,1995 \ldots \ldots \ldots$

17-5 Contamination Indicator Parameters for the NRDW Landfi11 Data for Reporting Period July 1 through September $30,1995 \ldots \ldots \ldots$ 17-13 


\author{
F. H. Hodges
}

Westinghouse Hanford Company

\title{
17.1 IHTRODUCTIOH
}

The Nonradioactive Dangerous Waste Landfill (NRDWL) is part of the Hanford Central Landfill. The NRDNL is Jocated approxfmately $5.6 \mathrm{~km}$ (3.5 ai) southeast of the 200 East Area. The MRONL, covered under the Consent Agreement and Compliance Onder (Ecology and EPA 1986), is currently under an interin-status, detection-7evel groundwater monitoring progran (HiC 1993c). The groundwater monitoring network consists of three upgradient we11s and six downgradient wells (Table 17-1). Two upgradient and five downgradient wells are screened at the top of the water tabie. One upgradient and one downgradient well are screened at the top of a low-permeability unit, approximately $21.3 \mathrm{~m}(70 \mathrm{ft}$ ) below the top of the water table. Figure 17-1 shows well locations. It should be noted that well 699-26-34A on Figure 17-1 was previously naned 699-26-34.

\subsection{MATER LEVEL. DATA}

Water level measurements were carried out on a monthly schedule for nine NROWl. nonitoring we11s, the nine monttoring wells of the adjacent Sol id Waste Landfil1, and in several nearby non-RCRA we11s. Results of the water level measurements are reported in Table 17-2.

\subsection{MATER CHEMISTRY DATA}

The monitoring network for the NRDWL is on a semiannuar sampling schedule. The network was sampled during August 1995 and results from that sampling are included in this report. The next scheduled sampling for the MRDWL network is in February 1996 . Thts report contains water level and groundwater chemistry data for the third quarter of CY 1995. The results are reported in Tables 17-3 and 17-4.

Va]ues for specific conductance, field $\mathrm{pH}, \mathrm{TOC}$, and TOX reported for this quarter do not exceed the critical means for this site. 
Figure 17-1. We1l Location Map for the Nonradioactive Dangerous Waste Landfill and Sol id Waste Landfiti.

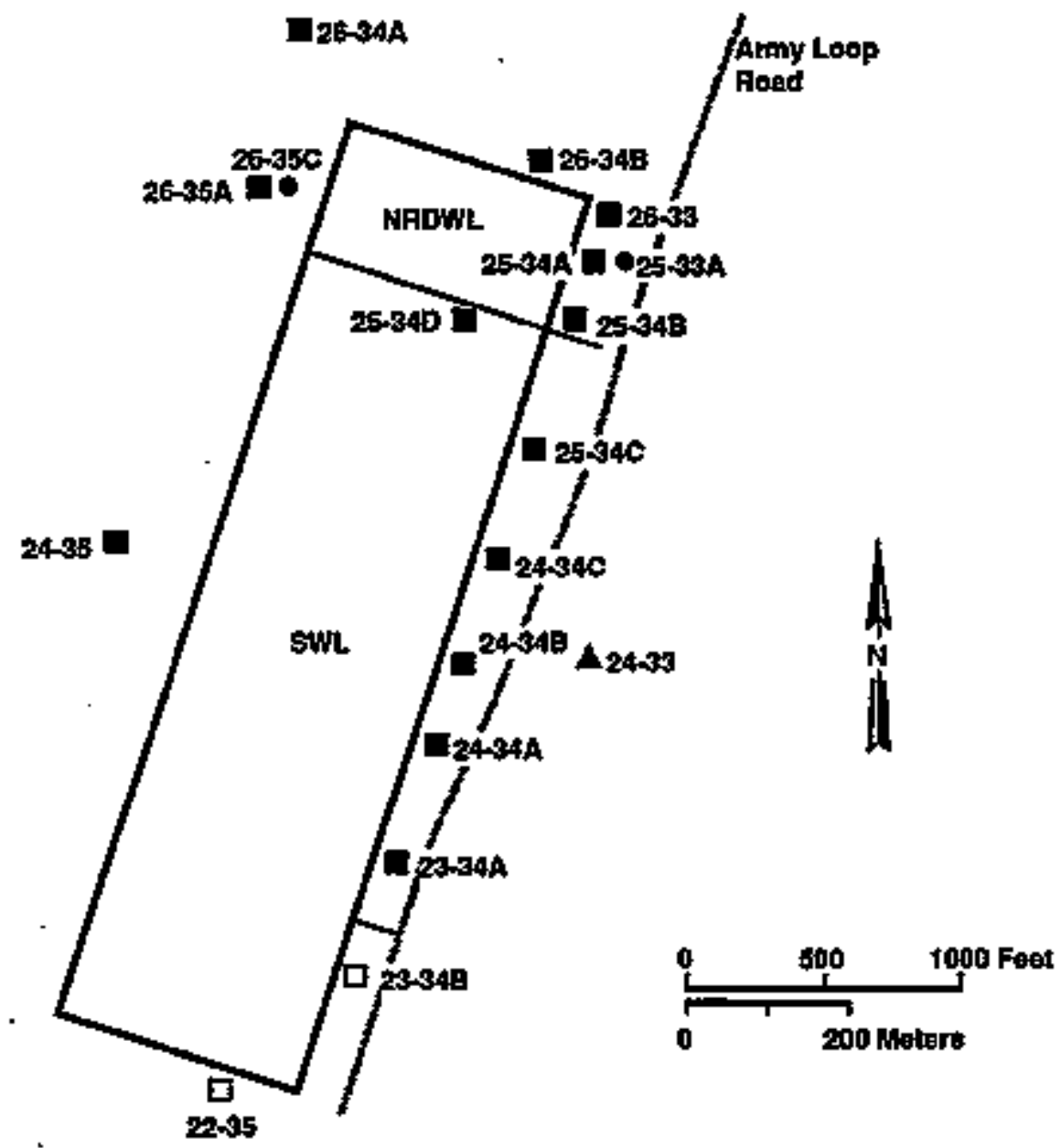

Well completed at the lop of the tuneonitimed aquiter

- Welt completed In the uppor fingold Formalion

- Well not contitrueted to RCRA speciicallong

ㅁ RCRA wells cortploted in bato 1993.

MfDWh Monradloactrve Dangerous Waste Landfil

SWL Solid Waste Landfill

All wells preflxed by 609 . 
Table 17-1. Monitoring Well Purpose and Sampling Schedule for the Nonradioactive Dangerous Waste Landfill Network.

\begin{tabular}{|c|c|c|c|c|}
\hline $\begin{array}{l}\text { Well no. } \\
(699-)\end{array}$ & $\begin{array}{l}\text { Relative } \\
\text { position }\end{array}$ & Hydrogeologic unit & $\begin{array}{c}\text { Sanple } \\
\text { frequency }\end{array}$ & $\begin{array}{l}\text { Sample date, } \\
\text { 3rd Qtr } 1995\end{array}$ \\
\hline $26-34 \mathrm{~A}$ & Upgradient & $\begin{array}{l}\text { Hanford: Water } \\
\text { Tab]le }\end{array}$ & Semi annua $11 y$ & $8 / 10 / 95$ \\
\hline $26-35 A$ & Upgradient. & $\begin{array}{l}\text { Hanford: Water } \\
\text { Table }\end{array}$ & Semiannual1 $1 y^{1}$ & $8 / 11 / 95$ \\
\hline $26-35 C$ & Upgradient & Top of Ringold & Semi annual $1 y$ & $8 / 11 / 95$ \\
\hline 25-33A & Downgradient & Top of Ringold & Semi annualiy & $8 / 14 / 95$ \\
\hline $25-34 A$ & Downgradient & $\begin{array}{l}\text { Hanford: Water } \\
\text { Table }\end{array}$ & Semiannually & $8 / 11 / 95$ \\
\hline $25-34 \mathrm{~B}$ & Downgradient & $\begin{array}{l}\text { Hanford: Water } \\
\text { Table }\end{array}$ & Semtannually & $8 / 11 / 95$ \\
\hline $25-340$ & Downgradient & $\begin{array}{l}\text { Hanford: Water } \\
\text { Table }\end{array}$ & Semiannualiy & $8 / 15 / 95$ \\
\hline $26-33$ & Downgradient & $\begin{array}{l}\text { Hanford: Water } \\
\text { Table }\end{array}$ & Seniannually & $8 / 10 / 95$ \\
\hline $26-34 B$ & Downgradient & $\begin{array}{c}\text { Hanford: Water } \\
\text { Table }\end{array}$ & Semiannual $1 y$ & $8 / 10 / 95$ \\
\hline
\end{tabular}

Note: Hydrogeologic units include the sandy gravels of the Hanford formation and silty sands of the Ringold Formation. Water levels are measured monthiy in all wells.

1. Also sampled as part of the network for the Soljd Waste Landfill. 
Tab]e 17-2. RCRA Water Level Measurement Report NRDWL, Third Quarter 1995. (sheet 1 of 3 )

\begin{tabular}{|c|c|c|c|c|}
\hline Well & Date & $\begin{array}{l}\text { Depth to } \\
\text { water (ft) }\end{array}$ & $\begin{array}{l}\text { Water } \\
\text { elevation } \\
\text { (ft) }\end{array}$ & $\begin{array}{c}\text { level } \\
\text { above nts] } \\
\text { (ti) }\end{array}$ \\
\hline \multicolumn{5}{|c|}{ NRDinl Wells konftoring the Top of the } \\
\hline $699-25-34 A$ & $\begin{array}{l}7 / 21 / 95 \\
8 / 11 / 95 \\
8 / 30 / 95 \\
9 / 22 / 95\end{array}$ & $\begin{array}{l}129.74 \\
129.22 * \\
130.11 \\
130.20\end{array}$ & $\begin{array}{l}400.57 \\
401.09 \\
400.20 \\
400.11\end{array}$ & $\begin{array}{l}122.09 \\
122.25 \\
121.98 \\
121.95\end{array}$ \\
\hline $699-25-348$ & $\begin{array}{l}7 / 21 / 95 \\
8 / 11 / 95 \\
8 / 30 / 95 \\
9 / 22 / 95\end{array}$ & $\begin{array}{l}128.59 \\
129.33^{*} \\
128.50 \\
128.54\end{array}$ & $\begin{array}{l}400.81 \\
400.07 \\
400.90 \\
400.86\end{array}$ & $\begin{array}{l}122.17 \\
121.94 \\
122.19 \\
122.18\end{array}$ \\
\hline $699-25-340$ & $\begin{array}{l}7 / 21 / 95 \\
8 / 15 / 95 \\
8 / 30 / 95 \\
9 / 22 / 95\end{array}$ & $\begin{array}{l}136.99 \\
137.05 * \\
136.91 \\
136.99\end{array}$ & $\begin{array}{l}400.92 \\
400.86 \\
401.00 \\
400.92\end{array}$ & $\begin{array}{l}122.20 \\
122.18 \\
122.22 \\
122.20\end{array}$ \\
\hline $699-26-33$ & $\begin{array}{l}7 / 21 / 95 \\
8 / 10 / 95 \\
8 / 30 / 95 \\
9 / 22 / 95\end{array}$ & $\begin{array}{l}134.94 \\
134.63^{*} \\
134.92 \\
135.04\end{array}$ & $\begin{array}{l}400.72 \\
401.03 \\
400.74 \\
400.62\end{array}$ & $\begin{array}{l}122.14 \\
122.23 \\
122.15 \\
122.11\end{array}$ \\
\hline $699-26-34 A$ & $\begin{array}{l}7 / 21 / 95 \\
8 / 10 / 95 \\
8 / 30 / 95 \\
9 / 22 / 95\end{array}$ & $\begin{array}{l}127.59 \\
121.26 * \\
127.90 \\
127.67\end{array}$ & $\begin{array}{l}400.81 \\
407.14 \\
400.50 \\
400.73\end{array}$ & $\begin{array}{l}122.17 \\
124.10 \\
122.07 \\
122.14\end{array}$ \\
\hline $699-26-348$ & $\begin{array}{l}7 / 21 / 95 \\
8 / 10 / 95 \\
8 / 30 / 95 \\
9 / 22 / 95\end{array}$ & $\begin{array}{l}129.34 \\
129.40^{*} \\
129.25 \\
129.42\end{array}$ & $\begin{array}{l}400.93 \\
400.87 \\
401.02 \\
400.85\end{array}$ & $\begin{array}{l}122.20 \\
122.19 \\
122.23 \\
122.18\end{array}$ \\
\hline $699-26-35 A$ & $\begin{array}{l}7 / 21 / 95 \\
8 / 11 / 95 \\
8 / 30 / 95 \\
9 / 22 / 95\end{array}$ & $\begin{array}{l}131.80 \\
131.30^{*} \\
131.81 \\
131.98\end{array}$ & $\begin{array}{l}400.86 \\
401.36 \\
400.85 \\
400.68\end{array}$ & $\begin{array}{l}122.18 \\
122.33 \\
122.18 \\
122.13\end{array}$ \\
\hline
\end{tabular}

MRONL. Wells Menitoring the Upper Ringold

699-25-33A

$\begin{array}{llll}7 / 21 / 95 & 128.13 & 400.84 & 122.18 \\ 8 / 14 / 95 & 128.28 * & 400.69 & 122.13 \\ 8 / 30 / 95 & 128.05 & 400.92 & 122.20 \\ 9 / 22 / 95 & 128.09 & 400.88 & 122.19\end{array}$


Table 17-2. RCRA Water Level Measurement Report NRDWL, Third Quarter 1995. (sheet 2 of 3 )

\begin{tabular}{|c|c|c|c|c|}
\hline We11 & Date & $\begin{array}{l}\text { Depth to } \\
\text { water (ft) }\end{array}$ & $\begin{array}{l}\text { Water } \\
\text { elevatjon } \\
\text { (ft) }\end{array}$ & $\begin{array}{c}\text { level } \\
\text { above msl } \\
\text { (m) }\end{array}$ \\
\hline \multicolumn{5}{|c|}{ NRDilL Wells Monitoring the Upper Ringold } \\
\hline $699-26-35 C$ & $\begin{array}{l}7 / 21 / 95 \\
8 / 11 / 95 \\
8 / 30 / 95 \\
9 / 22 / 95\end{array}$ & $\begin{array}{l}131.77 \\
131.10^{*} \\
131.69 \\
131.83\end{array}$ & $\begin{array}{l}400.91 \\
401.58 \\
400.99 \\
400.85\end{array}$ & $\begin{array}{l}122.20 \\
122.40 \\
122.22 \\
122.18\end{array}$ \\
\hline \multicolumn{5}{|c|}{ Other We]ls Associated with NROHL } \\
\hline $699-20-20$ & $\begin{array}{l}7 / 21 / 95 \\
8 / 30 / 95 \\
9 / 22 / 95\end{array}$ & $\begin{array}{l}106.35 \\
106.27 \\
106.31\end{array}$ & $\begin{array}{l}399.23 \\
399.31 \\
399.27\end{array}$ & $\begin{array}{l}121.69 \\
121.71 \\
121.70\end{array}$ \\
\hline $699-20-39$ & $\begin{array}{l}7 / 21 / 95 \\
8 / 30 / 95 \\
9 / 22 / 95\end{array}$ & $\begin{array}{l}138.81 \\
138.77 \\
138.69\end{array}$ & $\begin{array}{l}401.17 \\
401.21 \\
401.29\end{array}$ & $\begin{array}{l}122.28 \\
122.29 \\
122.31\end{array}$ \\
\hline $699-22-35$ & $\begin{array}{l}7 / 21 / 95 \\
8 / 14 / 95 \\
8 / 30 / 95 \\
9 / 22 / 95\end{array}$ & $\begin{array}{l}133.15 \\
133.20 * \\
133.06 \\
133.15\end{array}$ & $\begin{array}{l}400.82 \\
400.77 \\
400.91 \\
400.82\end{array}$ & $\begin{array}{l}122.17 \\
122.15 \\
122.20 \\
122.17\end{array}$ \\
\hline $699-23-34 A$ & $\begin{array}{l}7 / 21 / 95 \\
8 / 14 / 95 \\
8 / 30 / 95 \\
9 / 22 / 95\end{array}$ & $\begin{array}{l}131.99 \\
132.04^{\star} \\
131.90 \\
131.95\end{array}$ & $\begin{array}{l}400.87 \\
400.82 \\
400.96 \\
400.91\end{array}$ & $\begin{array}{l}122.19 \\
122.17 \\
122.21 \\
122.20\end{array}$ \\
\hline $699-23-348$ & $\begin{array}{l}7 / 21 / 95 \\
8 / 14 / 95 \\
8 / 30 / 95\end{array}$ & $\begin{array}{l}132.65 \\
132.53 * \\
132.57\end{array}$ & $\begin{array}{l}400.84 \\
400.97 \\
400.93\end{array}$ & $\begin{array}{l}122.18 \\
122.22 \\
122.20\end{array}$ \\
\hline $699-24-33$ & $\begin{array}{l}7 / 21 / 95 \\
8 / 14 / 95 \\
8 / 30 / 95 \\
9 / 22 / 95\end{array}$ & $\begin{array}{l}123.39 \\
123.33^{*} \\
123.30 \\
123.37\end{array}$ & $\begin{array}{l}400.89 \\
400.94 \\
400.97 \\
400.90\end{array}$ & $\begin{array}{l}122.19 \\
122.21 \\
122.22 \\
122.19\end{array}$ \\
\hline $699-24-34 A$ & $\begin{array}{l}7 / 21 / 95 \\
8 / 14 / 95 \\
8 / 30 / 95 \\
9 / 22 / 95\end{array}$ & $\begin{array}{l}133.02 \\
132.89 * \\
132.93 \\
132.99\end{array}$ & $\begin{array}{l}400.86 \\
400.99 \\
400.95 \\
400.89\end{array}$ & $\begin{array}{l}122.18 \\
122.22 \\
122.21 \\
122.19\end{array}$ \\
\hline
\end{tabular}


Table 17-2. RCRA Water Level Measurement Report MRDil, Third Quarter 1995. (sheet 3 of 3)

\begin{tabular}{|c|c|c|c|c|}
\hline Hel1 & Date & $\begin{array}{l}\text { Depth to } \\
\text { water (ft) }\end{array}$ & $\begin{array}{l}\text { Water } \\
\text { efevation } \\
\text { (ft) }\end{array}$ & $\begin{array}{c}\text { level } \\
\text { above ms) } \\
\text { (m) }\end{array}$ \\
\hline \multicolumn{5}{|c|}{ Other Nells Associated with NRDLL } \\
\hline $699-24-348$ & $\begin{array}{l}7 / 21 / 95 \\
8 / 15 / 95 \\
8 / 30 / 95 \\
9 / 22 / 95\end{array}$ & $\begin{array}{l}132.61 \\
132.66^{*} \\
132.52 \\
132.57\end{array}$ & $\begin{array}{l}400.89 \\
400.84 \\
400.98 \\
400.93\end{array}$ & $\begin{array}{l}122.19 \\
122.18 \\
122.22 \\
122.20\end{array}$ \\
\hline $699-24-34 C$ & $\begin{array}{l}7 / 21 / 95 \\
8 / 14 / 95 \\
8 / 30 / 95 \\
9 / 22 / 95\end{array}$ & $\begin{array}{l}131.71 \\
131.59 * \\
131.63 \\
131.69\end{array}$ & $\begin{array}{r}400.88 \\
401.00 \\
400.96 \\
400.90\end{array}$ & $\begin{array}{l}122.19 \\
122.22 \\
122.21 \\
122.19\end{array}$ \\
\hline $699-24-35$ & $\begin{array}{l}7 / 21 / 95 \\
8 / 11 / 95 \\
8 / 30 / 95 \\
9 / 22 / 95\end{array}$ & $\begin{array}{l}137.92 \\
137.99 * \\
137.82 \\
137.99\end{array}$ & $\begin{array}{l}400.89 \\
400.82 \\
400.99 \\
400.82\end{array}$ & $\begin{array}{l}122.19 \\
122.17 \\
122.22 \\
122.17\end{array}$ \\
\hline $699-25-34 C$ & $\begin{array}{l}7 / 21 / 95 \\
8 / 14 / 95 \\
8 / 30 / 95 \\
9 / 22 / 95\end{array}$ & $\begin{array}{l}134.58 \\
134.65 * \\
134.50 \\
134.58\end{array}$ & $\begin{array}{l}400.88 \\
400.81 \\
400.96 \\
400.88\end{array}$ & $\begin{array}{l}122.19 \\
122.17 \\
122.21 \\
122.19\end{array}$ \\
\hline $699-28-40$ & $\begin{array}{l}7 / 21 / 95 \\
8 / 30 / 95 \\
9 / 22 / 95\end{array}$ & $\begin{array}{l}158.19 \\
158.18 \\
158.20\end{array}$ & $\begin{array}{l}401.25 \\
401.26 \\
401.24\end{array}$ & $\begin{array}{l}122.30 \\
122.30 \\
122.30\end{array}$ \\
\hline $699-31-31$ & $\begin{array}{l}7 / 21 / 95 \\
8 / 30 / 95 \\
9 / 22 / 95\end{array}$ & $\begin{array}{r}128.52 \\
128.43 \\
128.51\end{array}$ & $\begin{array}{l}400.80 \\
400.89 \\
400.81\end{array}$ & $\begin{array}{l}122.16 \\
122.19 \\
122.17\end{array}$ \\
\hline $699-34-39 A$ & $\begin{array}{l}7 / 21 / 95 \\
8 / 30 / 95 \\
9 / 22 / 95\end{array}$ & $\begin{array}{l}135.69 \\
135.71 \\
135.75\end{array}$ & $\begin{array}{l}401.38 \\
401.36 \\
401.32\end{array}$ & $\begin{array}{l}122.34 \\
122.33 \\
122.32\end{array}$ \\
\hline
\end{tabular}

NOTES: 1. Hater leve] elevations are calculated by subtracting the measured depth-to-water from the surveyed. elevation for the well.

2. Depth-to-water values are transcribed from field records.

3. Measurements marked wt th an $" *$ ' were taken at the time of sampling.

4. Measurements marked with a ' + ' are outside of the expected range, and are suspected of error. 
Tab]e 17-3. Constituent List and Surmary of Results for the NRDW Landfill Data for Reporting Period July 1 through September 30, 1995.

(sheet 1 of 2)

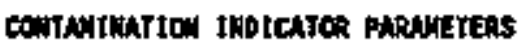

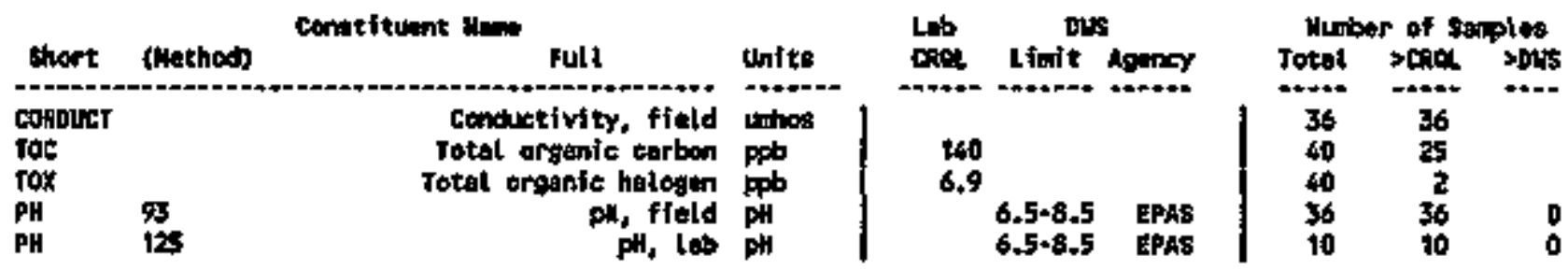

ORTHKIME WATER PARUITERS

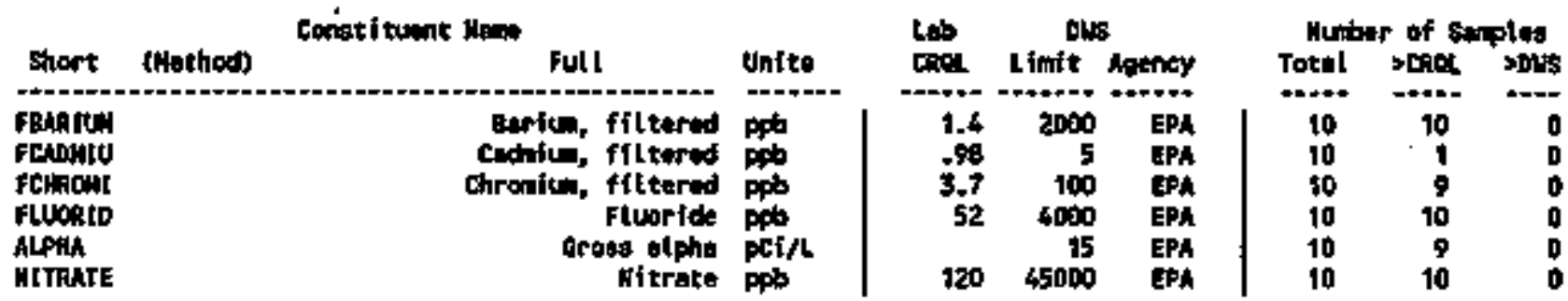

Hounoluter aut: IT PARANETERS

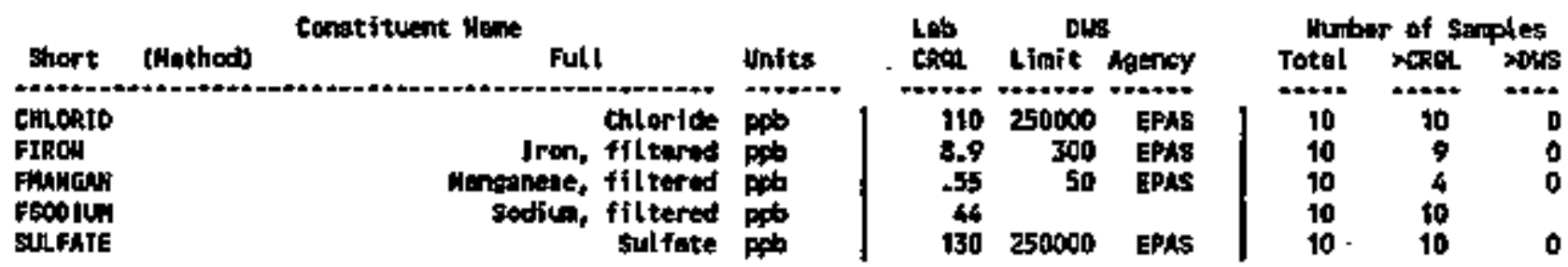

SITE SPECIFIC AVD OTHER CONBTITUEATS

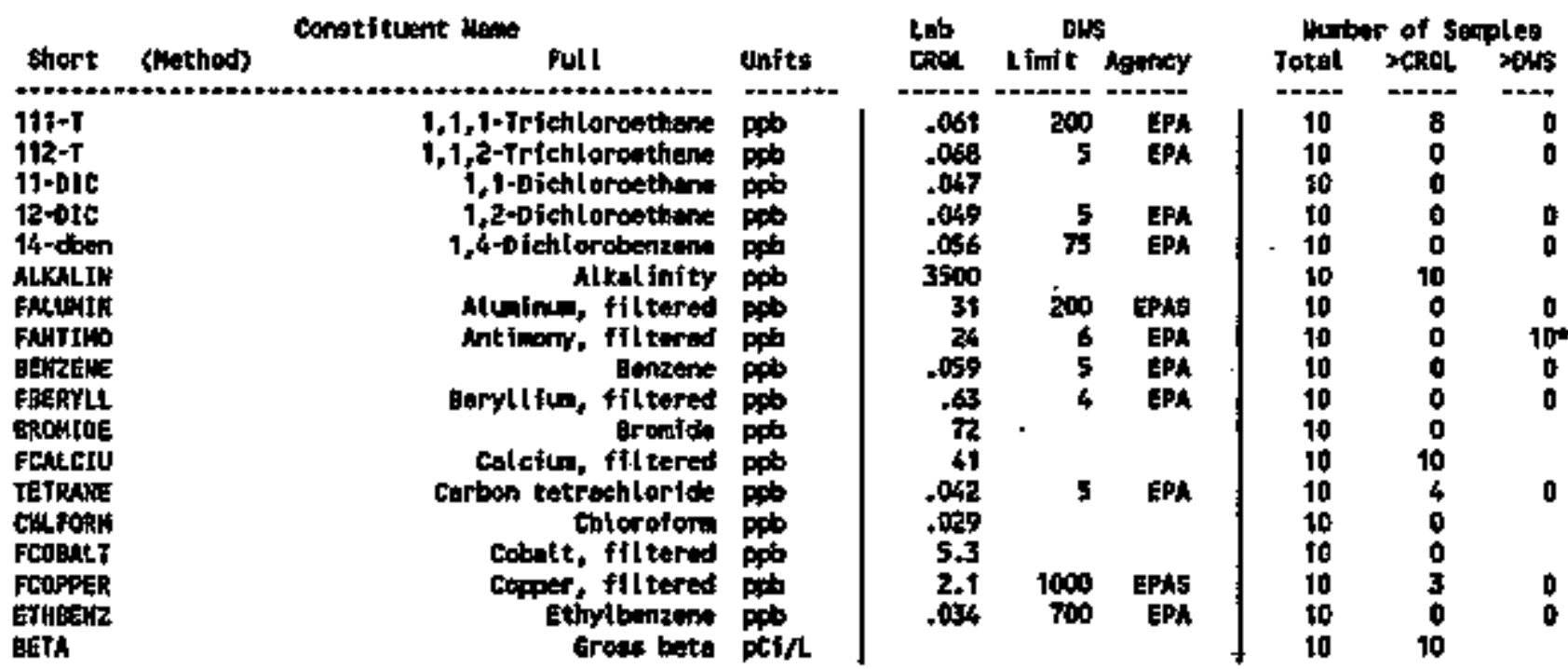


Table 17-3. Constituent List and Sunnary of Results for the NRDW Landfill Data for Reporting Period July 1 through September 30, 1995.

(sheet 2 of 2)

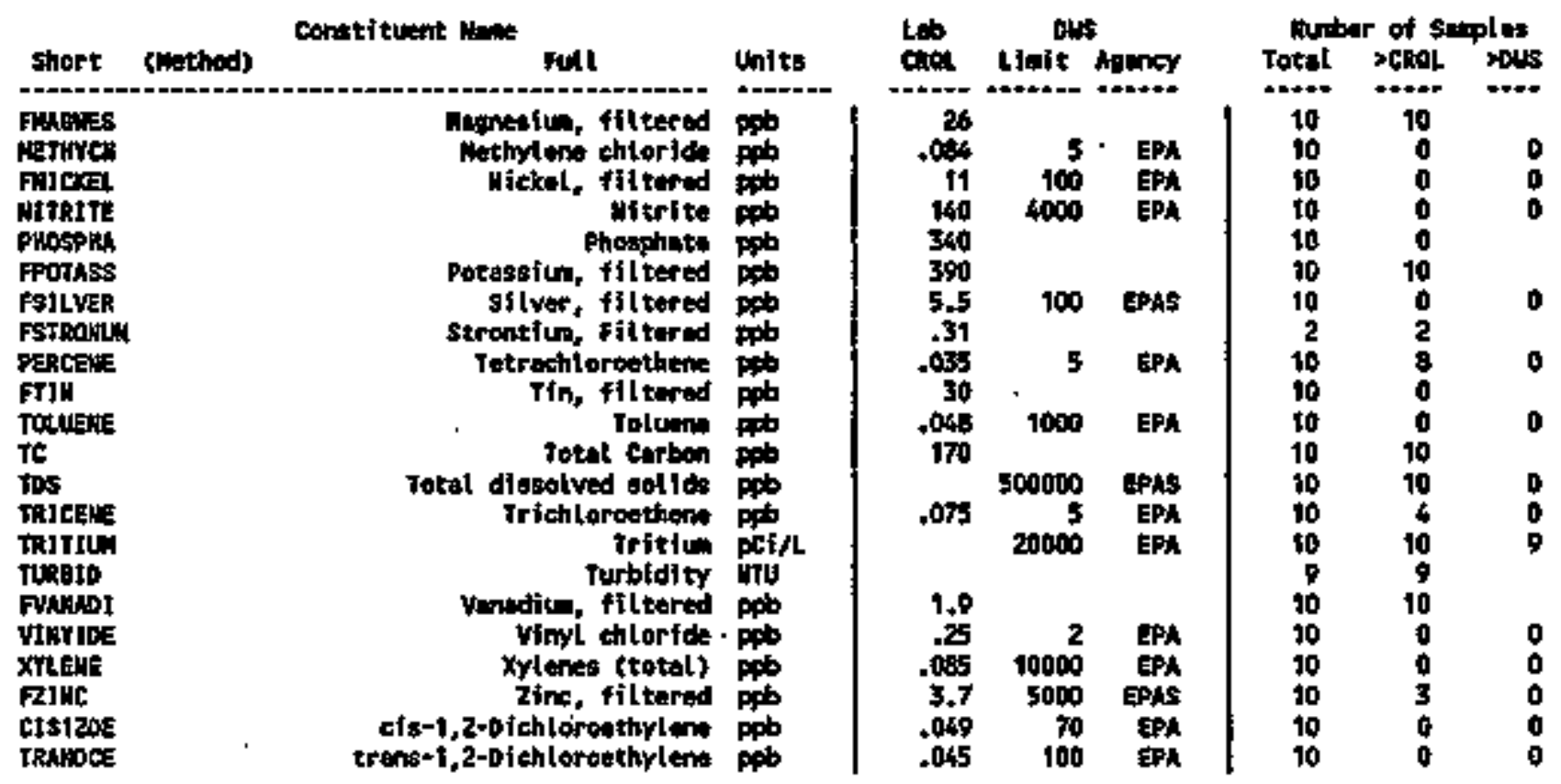


Table 17-4. Constituents with at Least One Detected Value for the NRDW Landfill Data for Reporting Peried

July 1 through September 30, 1995.

\begin{tabular}{|c|c|c|}
\hline $\begin{array}{l}\text { Hell } \\
\text { rome }\end{array}$ & $\begin{array}{l}\text { Gollection } \\
\text { Date }\end{array}$ & $\begin{array}{l}\text { Supple } \\
\text { inter }\end{array}$ \\
\hline 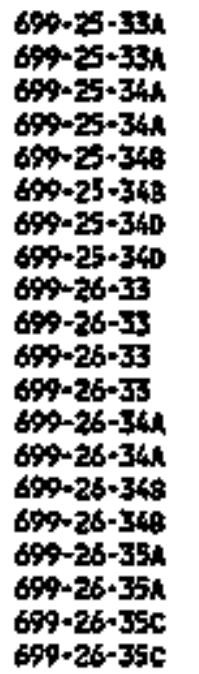 & $\begin{array}{l}8 / 14 / 95 \\
6 / 14 / 95 \\
8 / 11 / 95 \\
8 / 11 / 95 \\
8 / 11 / 95 \\
8 / 11 / 95 \\
2 / 15 / 95 \\
8 / 15 / 95 \\
8 / 10 / 95 \\
8 / 10 / 95 \\
8 / 10 / 95 \\
8 / 10 / 95 \\
8 / 10 / 95 \\
8 / 10 / 95 \\
8 / 10 / 95 \\
8 / 10 / 95 \\
8 / 11 / 95 \\
8 / 11 / 95 \\
8 / 11 / 95 \\
8 / 11 / 95\end{array}$ & 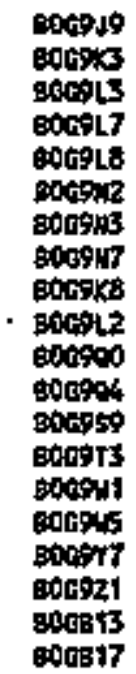 \\
\hline
\end{tabular}

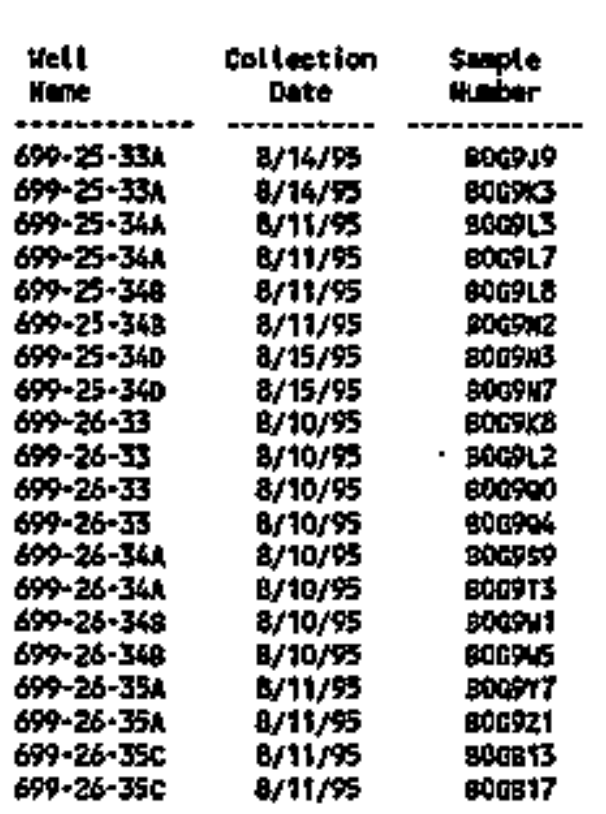
(sheet 1 of 4 )

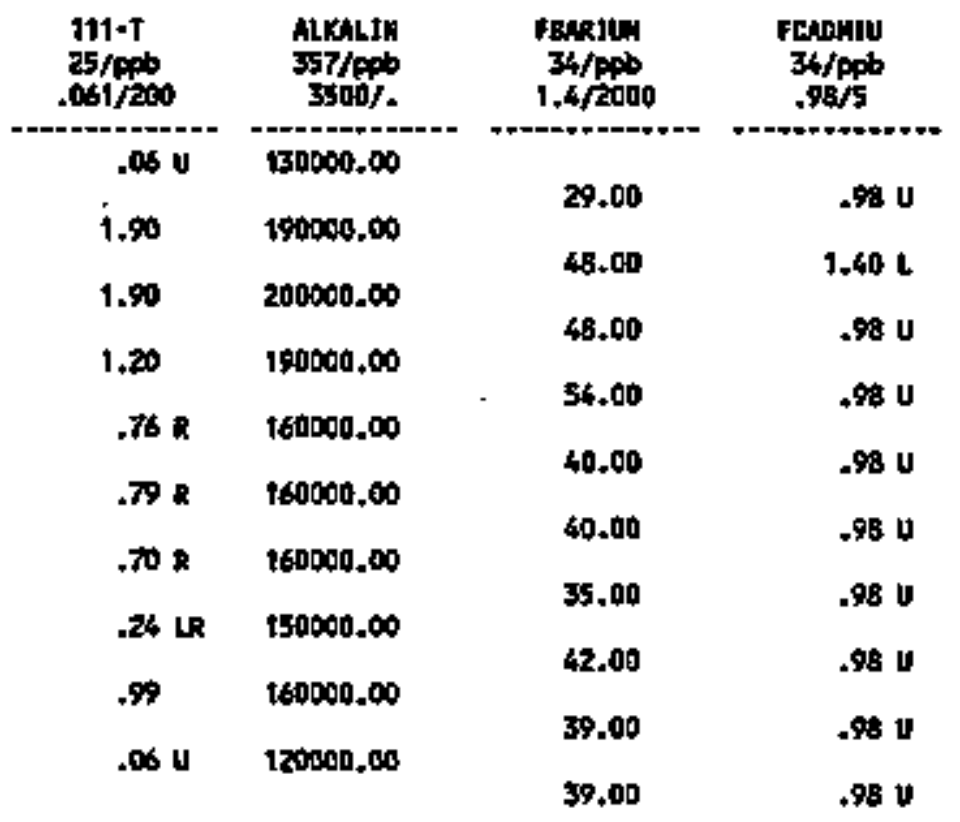

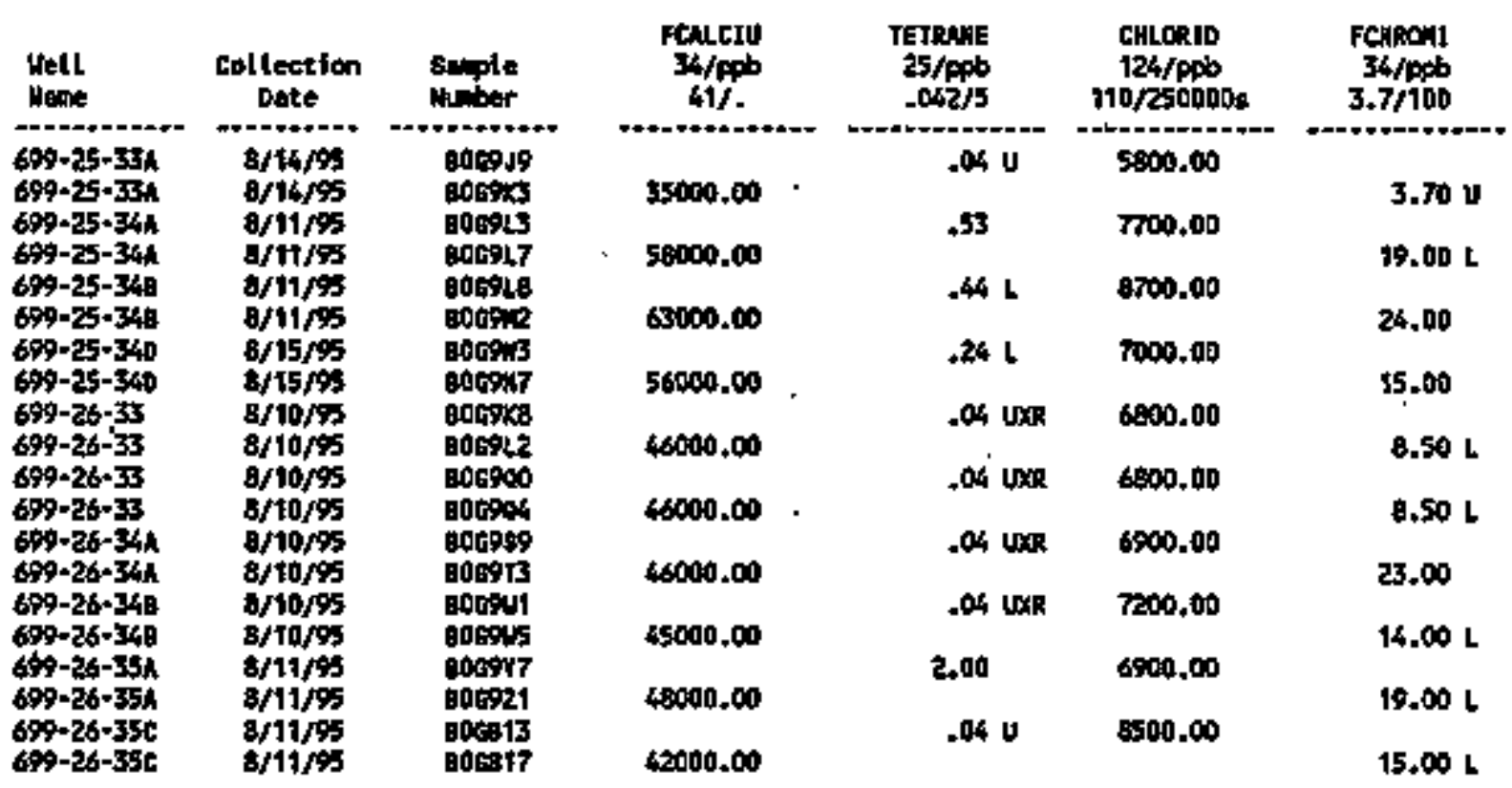


Table 17-4. Constituents with at Least One Detected Value for the NRDW Landfill Data for Reporting Period July 1 through September 30, 1995.

(sheet 2 of 4 )

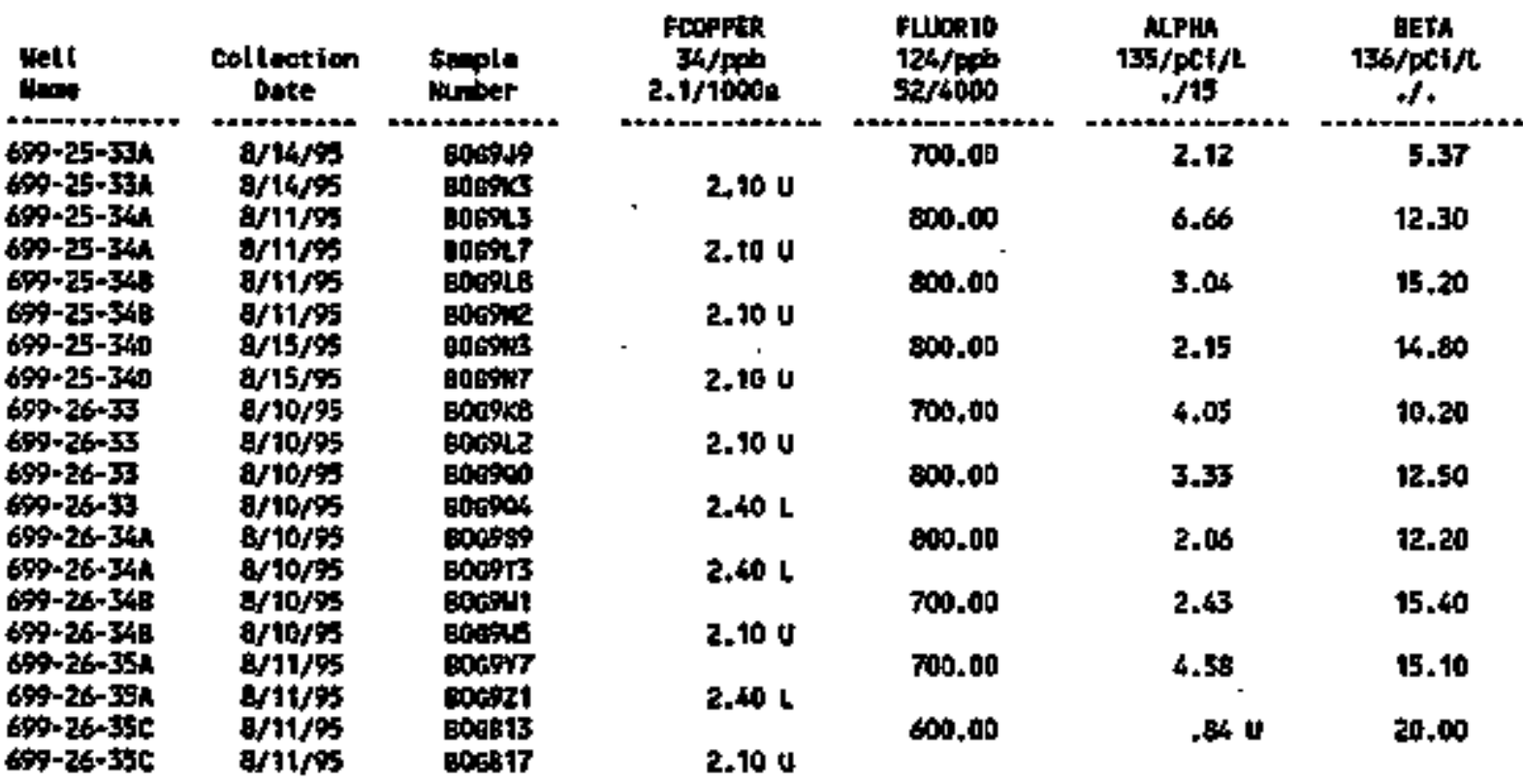

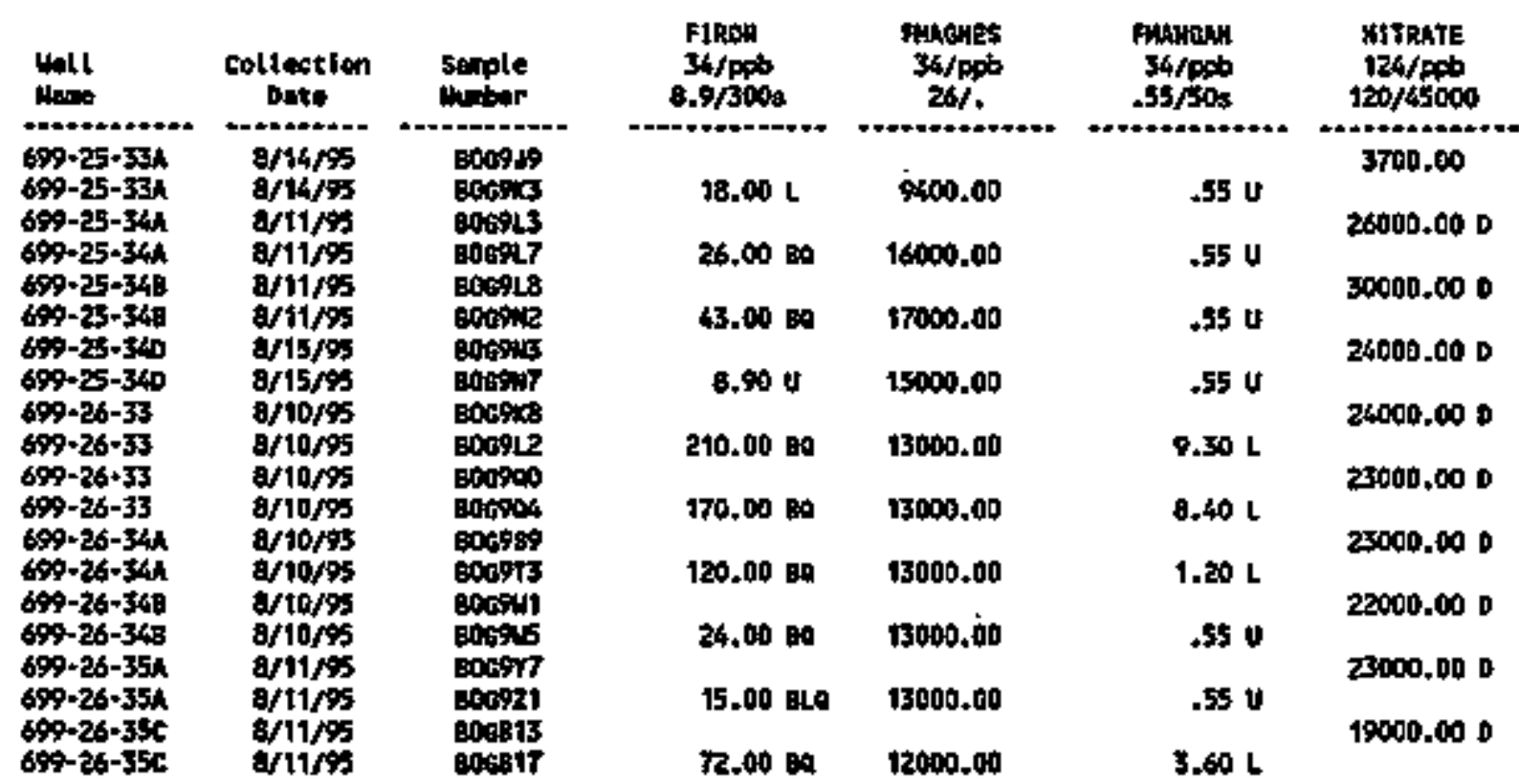


Table 17-4. Constituents with at Least One Detected Value for the NROW Landfil7 Data for Reporting Period July 1 through September 30, 1995.

\begin{tabular}{|c|c|c|c|c|c|c|}
\hline Moll & $\begin{array}{c}\text { Collection } \\
\text { Date }\end{array}$ & suple & $\begin{array}{l}\text { peptass } \\
34 / p o b \\
300 \% .\end{array}$ & $\begin{array}{c}150010 \% \\
34 / 1006 \\
44 / .\end{array}$ & $\begin{array}{c}\text { Fstroois } \\
34 / p p b \\
31 \%\end{array}$ & $\begin{array}{c}\text { SulfATE } \\
\text { 126/pepb } \\
130 / 25000 \mathrm{~s}\end{array}$ \\
\hline 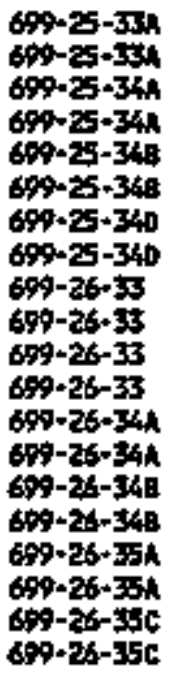 & 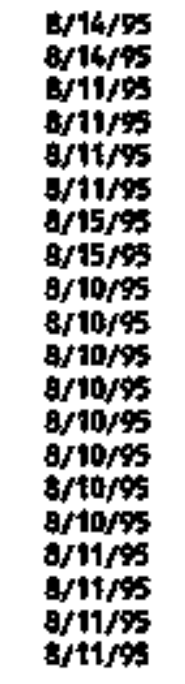 & 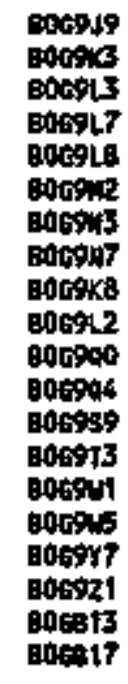 & $\begin{array}{r}5800.00 \\
7100.00 \\
7500.00 \\
7400.00 \\
6400.00 \\
6600.00 \\
6400.00 \\
6100.00 \\
6800.00 \\
5700.00\end{array}$ & $\begin{array}{l}19000.000 \\
32000.000 \\
26000.000 \\
25000.000 \\
23500.000 \\
24000.000 \\
230000.000 \\
22000.000 \\
23000.000 \\
180000.000\end{array}$ & 200.00 & $\begin{array}{l}21000.000 \\
54000.000 \\
52000.000 \\
38000.000 \\
34000.000 \\
37000.000 \\
37000.000 \\
40000.0100 \\
370000.000 \\
46000.000\end{array}$ \\
\hline $\begin{array}{l}\text { Hell } \\
\text { Hewoe }\end{array}$ & $\begin{array}{l}\text { Cotlection } \\
\text { Date }\end{array}$ & $\begin{array}{l}\text { Simpte } \\
\text { nuntor }\end{array}$ & $\begin{array}{l}\text { PERCENE } \\
25 / 996 \\
.055 / 5\end{array}$ & $\begin{array}{c}16 \\
113 / \mathrm{ppb} \\
170 / .\end{array}$ & $\begin{array}{c}\text { TOS } \\
65 / \mathrm{pab} \\
.50000 \mathrm{~s}\end{array}$ & $\begin{array}{l}\text { TR1CEME } \\
25 / p / 56 \\
.075 / 5\end{array}$ \\
\hline $\begin{array}{l}699-25-334 \\
699-25-344 \\
699-25 \cdot 346 \\
699-25-340 \\
699-26-33 \\
699-26-33 \\
699-26-34 k \\
699-26-34 \mathrm{~B} \\
699-26-35 \mathrm{~A} \\
699-26-35 \mathrm{c}\end{array}$ & $\begin{array}{l}8 / 14 / 95 \\
8 / 11 / 95 \\
8 / 11 / 95 \\
8 / 15 / 95 \\
8 / 10 / 95 \\
8 / 10 / 95 \\
8 / 10 / 95 \\
8 / 10 / 95 \\
8 / 11 / 95 \\
8 / 11 / 95\end{array}$ & 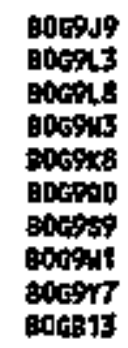 & $\begin{array}{l}.04 \mathrm{U} \\
.69 \\
1.00 \\
.58 \\
.18 \mathrm{LR} \\
.20 \mathrm{LR} \\
.22 \mathrm{LR} \\
.04 \mathrm{LR} \\
.30 \\
.04 \mathrm{U}\end{array}$ & $\begin{array}{l}35000.00 \\
51000.00 \mathrm{D} \\
52000.00 \mathrm{D} \\
47000.000 \\
40000.00 \mathrm{D} \\
40000.00 \mathrm{D} \\
41000.00 \mathrm{D} \\
38000.000 \\
41000.00 \mathrm{D} \\
28000.00\end{array}$ & $\begin{array}{l}2000000.00 \\
390000.00 \\
390000.00 \\
350000.00 \\
340000.00 \\
330000.00 \\
330000.00 \\
310000.00 \\
310000.00 \\
270000.00\end{array}$ & $\begin{array}{l}.08 \mathrm{U} \\
.34 \\
.40 \\
.31 \\
.08 \mathrm{UR} \\
.08 \mathrm{UR} \\
.08 \mathrm{UR} \\
.08 \mathrm{UR} \\
.08 \mathrm{UR} \\
.13 \mathrm{~L} \\
.09 \mathrm{U}\end{array}$ \\
\hline
\end{tabular}


Table 17-4. Constituents with at Least One Detected Value for the IRRD Landfill Data for Reporting Period July 1 through September 30, 1995 .

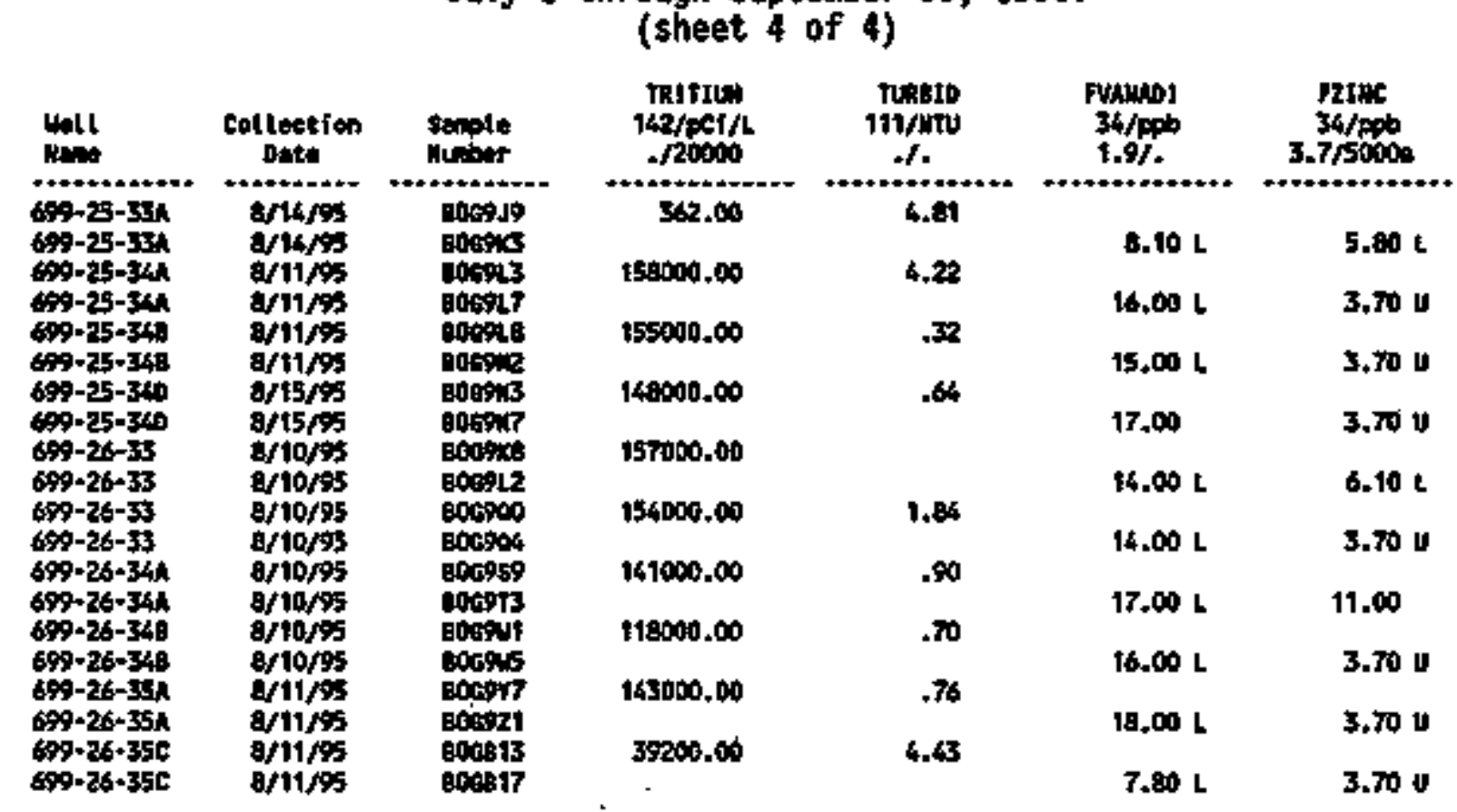

For explangtion of this teble, ses siction 1.4 of report. 
Table 17-5. Contamination Indicator Parameters for the HRow Landfi]l Data for Reporting Period July 1 through September 30, 1995.

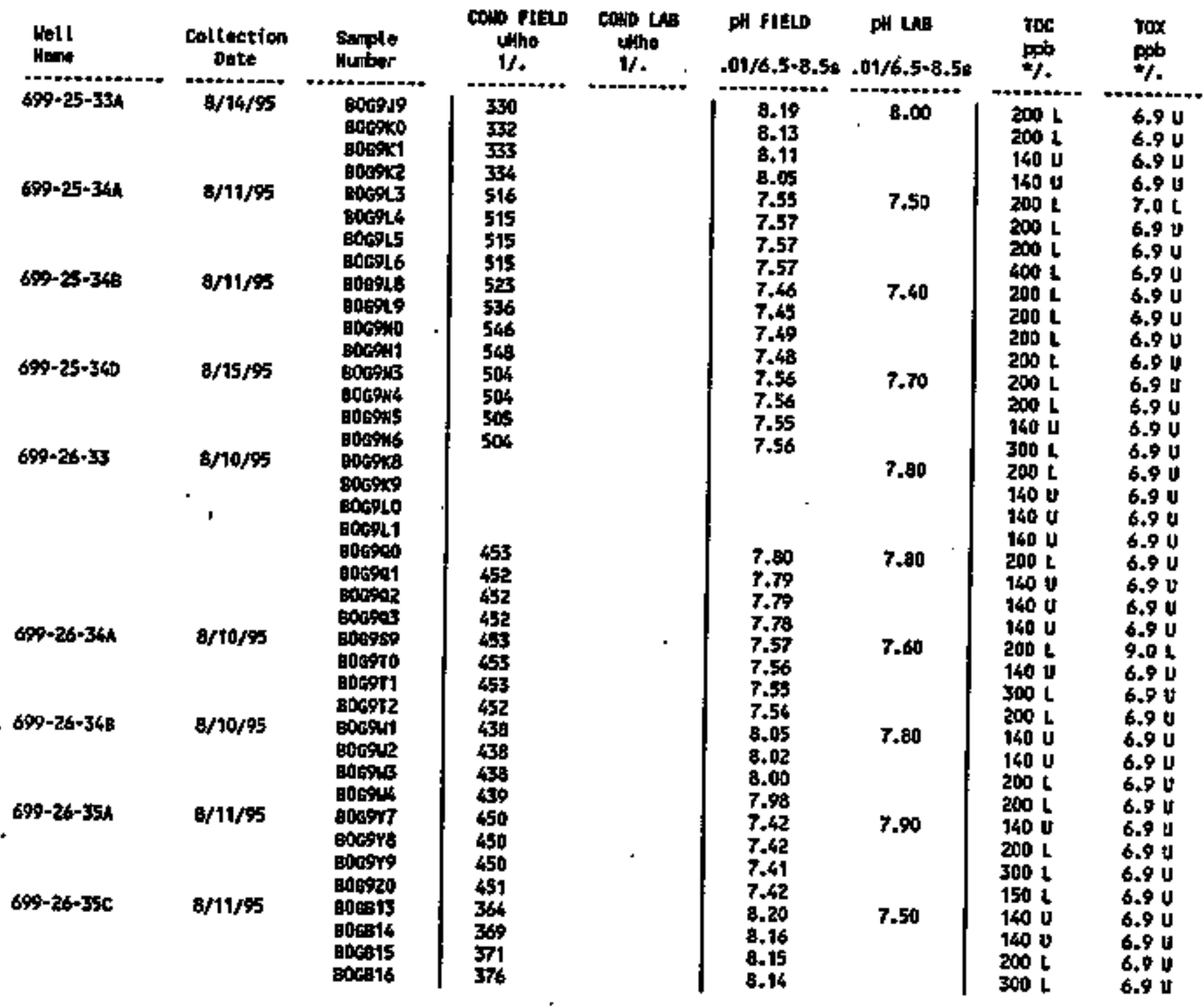

Datection linits for Toc and Tox vary depending on the perforing laboratory. for expluriation of this thbte, atis section 1.6 of report. 


\section{DOE/RL-95-69-3}

This page intentionalty left b]ank. 


\subsection{REFEREICES}

40 CFR 141, "National Primary Drinking Water Regulations," Code of Federal Regulations, as amended.

40 CFR 143, "National Secondary Drinking Water Regulations," Code of Federal Reguiations, as arended.

40 CFR 265, "Interim Status Standards for Owners and Operators of Hazardous Waste Treatment, Storage, and Disposal Facilities." Code of Federal Regulations, as amended.

Anderson, J. D., 1990, A History of the 200 Area Tank Farns: Westinghouse Henford Company, WHC-KR-0132, Westinghouse Hanford Company, Richland, Washington.

APHA, 1989, Standard Methods for Evaluation of Nater and Wasterater, 17th ed., American Public Health Association, Washington, D.C.

ASTM, 1991, Annual Book of ASTH Standands, Vol. 11.01, Water and Enviromenta1 .Technology, Philadelphia, Pennsylvania.

Caggiano, J. A., and C. J. Chou, 1993, Interim-Status Groundwater Quality Assessment P7 an for the Single Shell Tank Waste Management Areas $T$ and TX-TY: Richland, Washington, West inghouse Hanford Company, WHC-SD-EN-AP-132, Rev. O, West inghouse Hanford Company, Rich] and, Washington.

Caggiano, J. A., and S. M. Goodwin, 1991, Interim-Status Groundwater Monitoring Plan for the Single-Shel7 Tanks, WHC-SD-EN-AP-012, Rev, 1, Westinghouse Hanford Company, Richland, Washington.

Chamness, M. A., S. P. Luttrel1, and S. Dudziak, 1989, 40 CFR 265 Interis Status Ground-Water Nonitoring Plan for the 2101-N Pond, PNL-6851, Pacific Nortimest Laboratory, Richland, Washington.

Chou, C. J., G. L. Kasza, and R. B. Mercer, 1990, Interis-Status Groundwater Quality Assessment Plan for the 216-A-29 Ditch, MHC-SD-EN-AP-031, Rev. 0, Westinghouse Hanford Company, Richland, Washington.

DOE-RL, 1991, 183-H Solar Evaporation Basins Closure/Post-Closure Plan, DOE/RL-88-04, Rev. 3 (page changes only), U.S. Departwent of Energy, Richland Operations Office, Richland, Washington.

DOE-RL, 1994, Annual Report for RCRA Groundwater Nonitoring Projects at Hanford Site facilities for 1993, DOE/RL-93-8B, U.S. Department of Energy, Richiand Operations Office, Richland, Washington.

DOE-RL, 1995, Annual Report for RCRA Groundwater Nonftoring Projects at Hanford site facilities for 1994, DOE/RL-94-136, Rev. 0, U.S. Departnent of Energy, Richland Operations Office, Richland, Washington. 
DOE-RL, 1995, Annua Report for RCRA Groundwater Honitoring Projects at Hanford sfte facflities for 1994, D0E/RL-94-136, U.S. Departwent of Energy, Richland Operations, Office, Richland, Washington.

Ecology, 1994, Dangerous Waste Portion of the Resource Conservation and Recovery Act Pendt for the Treatment, Storage, and Disposal of Dangerous Waste, Permit Ho. WA7890008967, effective September 28, Washington State Department of Ecology, Olyapia, Washington.

Ecology and EPA, 1986, Consent Agreenent and Coinpliance Order, Ecology Mo. DE B6-133, Washington State Department of Ecology and the U.S. Environmental Protection Agency, Olympia, Washington.

Ecology, EPA, and DOE, 1994, Hanford Federal Facility Agreement and Consent order, Washington State Department of Ecology, U.S. Environmental Protection Agency, and U.S. Department of Energy, 0lywpia, Nashington.

EPA, 1979, Nethods for Chenical Analysis of Water and Waste, EPA-600/4-79-020, U.S. Environmental Protection Agency, Environmental Monitoring and Support Laboratory, Cincinnati, ohio.

EPA, 1986a, RCRA Groundwater Honitoring Technical Enforcenent Guidance Document, OSWER-9950.1, U.S. Environimental Protection Agency, Washington, D.C.

EPA, 1986b, Test Methods for Evaluating Solid Waste, SW-846, Third Edition, U.S. Environmental Protection Agency, Washington, D.C.

Harris, S. F., 1990, Groundirater Quality Assessment Plan for the 216-8-3 Pond Systen, WHC-SD-EN-AP-030, Rev. 0, Westinghouse Hanford Company, Richland, Washington.

Hartman, M. J., 1991, Groundwater Nonitoring Plan for the 100-D Ponds, WHC-SD-EN-AP-048, Rev. O, Mestinghouse Hanford Company, Richland, Washington.

Hartman, M. J., 1993, Groundwater Honitoring Plan for the 1301-N and 1325-N Liquid Waste Disposal facilities, MHC-5D-EH-AP-038, Rev. 1, Westinghouse Hanford Company, Richland, Washington.

Jensen, E. J., S. P. Airhart, M. A. Chamness, T. J. Gilmore, D. R. Hewcomer, and K. R. Oster, 1989, 40 CFR 265 Interin-Status Ground-Water Nonitoring Plan for the Single-Shell Tanks, MHC-SD-EH-AP-012, Hest inghouse Hanford Company, Richland, Washington

Kasza, G. L., and S. M. Goodwin, 1991, Groundwater Monitoring PIan for the 216-A-29 Ditch, WHC-SD-EN-AP-045, Rev. 0, Westinghouse Hanford Company, Richland, Washington.

Lindberg, J. W., 1995, Groundwater Monitoring Plan for the [RCRA] 300 Area Process Trenches, WHC-SD-EN-AP-185, Westinghouse Hanford Company, Richland, Washington. 
PAL, 1989, 40 CFR 265 Interin-Status Indicator-Evaluation Groundwater Nonitoring Plan for the 216-6-63 Trench, PNL-6862, Pacific Northwest Laboratery, Richland, Washington.

RCW 248, "Pubitic Water Supplies," Revised Code of Washington, as amended.

Resource Conservation and Recovery Act of 1976, 42 USC 6901, et seq.

Schalla, R., 1988, Revised Ground-Water Honitoring Compliance Plan for the 300 Area Process Trenches, PHL-6671, Pacific Northwest Laboratory, Richland, Washington.

Schmidt, J. H., A. R. Johnson, S. M. McKinney, C. J. Perkins, and C. R. Mebb, 1992, Westinghouse Hanford Company Environnental Survefil lance Annuat Report, WHC-EP-0573, Westinghouse Hanford Company, Richt and, Washington.

Sweeney, M. D., 1994, Interim-Status Groundwater Monitoring P7an for the 216-B-3 Pond Systen, WHAC-SD-EN-AP-013, Rev. 1, West inghouse Hanford Company, Richland, Washington.

Thornton, E. C., 1995, "2101-M Pond," in Annual Report for RCRA Groundwater Nonitoring Projects at Hanford Site facilities for 1994, DOE/RL-94-136, U.S. Department of Energy, Richland, Washington.

WAC 173-160, "Winimum Standards for Construction and Maintenance of Ne11s," Washington Adainistrative code, as amended.

WAC 173-200, "Water Quality Standards of the State of Washington," Washington Adainistrative Code, as amended.

WAC 173-303, "Dangerous Waste Regulations," Washington Administrative Code, as amended.

HHC-CH-7-8, Environaental Engineering and Geotechnology Function Procedures, Voluwe 4, Section 2.1, Rev. 1, Westinghouse Hanford Company, Richland, Hashington.

WHC, 1990a, Interin-Status Groundwater Monitoring Plan for the 216-5-10 Pond and Ditch, WHC-SD-EN-AP-018, Rev. 0, Hestinghouse Hanford Company,

Richl and, Washington.

HAC, 1990b, Interim-Status Groundwater Monitoring Plan for the 216-U-12 Crib, WHC-SD-EN-AP-019, Rev. 0, Westinghouse Hanford Campany, Richland, Washington.

WHC, 1991, Interin Status Groundwater Nonitoring PIan for the 200 East Area Liquid Effluent Retention Facility, WHC-SD-EN-AP-024, Rev. 1,

Westinghouse Hanford Company, Richland, Washington.

WC, 1992a, Generic Specifications--Groundwater Nonitoring Wells, WHC-S-014, Rev. 7, Westinghouse Hanford Company, Richland, Washington. 
WHC, 1992b, Quality Assurance Project PI in for RCRA Groundwater Monitoring Actfvities, WHC-SD-EN-QAPP-Dol, Rev. 2, Mestinghouse Hanford Company, Richl and, Washington.

WHC, 1993a, Interia-Status Groundwater Qualfty Assessaent Plan for the 216-U-12 Crib, WHC-SD-EH-AP-108, Rev, 0, West' Inghouse Hanford Company, Rich1 and, Washington.

WHC, 1993b, Interia Status Groundwater Monitoring Plan for the Nonradioactive Dangerous Waste Landfill, Hanford, Washington, WHC-SD-EH-AP-026, Rev. 0, Westinghouse Hanford Company, Richland, Washington.

WHC, 1993c, Radionuclide and Chemical Inventorfes for the Single-Shell Tanks: Report Prepared for Westinghouse Hanford Company by WASTREN, InC., WHC-SD-Wh-TI-565, Rev. 1, Westinghouse hanford Company, Richland, Washington. 


\section{DISTRIBUTION}

Nuber of coptes

\section{OFFSITE}

1

U.S. Enviraneiental_Protection Agency 7125 wift BTyd., Suite 5

Richland, Washington 99352

D. R. Sherwood

2

Uashington State Departnent of Ecology Kennewick Branch

1315 W. 4th

Kennewick, Washington 99336-6018

S. Leja

J. Wallace

1

Westinolhouse_Sayannah_River Company P. O. Box A

Aiken, South Carolina 29801

R. C. Tuckfield

ONSITE

10

U.S. Department of Energy. Bichland Operations of fice

R. D. Freeberg

M. J. Furman (4)

R. 6. Mcteod

R. P. Saget

T. K. Teynor

RL Pub]ic Reading Roon (2)

H4-83

$57-55$

H4-83

66-52

\$7-55

H2-53

6

Bechtel Hanford, Inc.

K. R. Fecht

H6-04

B. H. Ford

H4-85

A. J. Knepp

H4-85

J. Rugg

H4-79

1. C. Swanson

H6-04

ERC

H6-07

1

ICF-Kaiser Hanford

K. D. Johnson

64-07 
DOE/RL-95-69-3

DISTRIBUTIOA (cont)

Hunber of Conies

OUSITE

4

Pacific Northwest Laboratory

S. P. Luttre11

B. E. Opitz

D. L. Stewart

Technical Files

K6-96

K6-79

X6-96

$\mathrm{Kl}-11$

1

BUST_Geotech

J. R. Brodeur

HI- 49

33

Westinghouse Hanford Company

S. E. Campbel1

T4-05

D. J. Carrel1

$57-30$

C. J. Chou

H6-06

I. G. Gardner

C. J. Geier

S3-24

A. K. McDowell

R2-36

S. M. Price

RI-51

R. W. Reddinger

J. S. Schmid

H6-23

T4-05

R. R. Thompson

H6-06

B. A. Williams

S. A. Willians (10)

H6-32

H. T. York

H5-06

H6-06

H6-32 Centra] Files

A3-88

EPIC (7)

H6-08

OSTI (2)

Tank Farms Information

A3-36

Center

Rl-20 